WCH-85

Rev. 0

River Corridor Closure Contract

\title{
100 Area and 300 Area Component of the RCBRA Fall 2005 Data Compilation
}

\section{May 2006}

\section{Washington Closure Hanford}

Prepared for the U.S. Department of Energy, Richland Operations Office Office of Assistant Manager for River Corridor

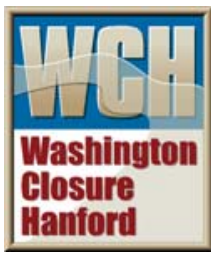


TRADEMARK DISCLAIMER

Reference herein to any specific commercial product, process, or service by trade name, trademark, manufacturer, or otherwise, does not necessarily constitute or imply its endorsement, recommendation, or favoring by the United States Government or any agency thereof or its contractors or subcontractors.

This report has been reproduced from the best available copy. Available in paper copy and microfiche.

Available for a processing fee to U.S. Department of Energy and its contractors from:

U.S. Department of Energy

Office of Scientific and Technical Information

P.O. Box 62

Oak Ridge, TN 37831-0062

(865) $576-8401$

fax: (865) $576-5728$

email: reports@adonis.osti.gov

online ordering: http://www.doe.gov/bridge

Available for sale to the public, in paper, from:

U.S. Department of Commerce

National Technical Information Service

5285 Port Royal Road

Springfield, VA 22161

(800) 553-6847

fax: (703) 605.6900

email: orders@ntis.fedworld.gov

online ordering: http://www.ntis.gov/ordering.htm

Printed in the United States of America

DISCLM-5.CHP (11/99) 


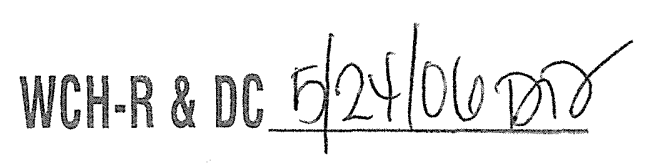

WCH- 85

Rev. 0

OU: N/A

TSD: N/A

ERA: N/A

\section{STANDARD APPROVAL PAGE}

Title: $\quad 100$ Area and 300 Area Component of the RCBRA Fall 2005 Data Compilation

Author Name: J. M. Queen

Approval: $\quad$ E. T. Feist, Director

End State and Final Closure Project

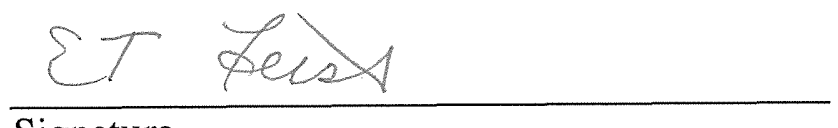

Signature

$5-24-06$

Date

The approval signatures on this page indicate that this document has been authorized for information release to the public through appropriate channels. No other forms or signatures are required to document this information release. 

Rev. 0

\section{River Corridor Closure Contract}

\section{Area and 300 Area Component of the RCBRA Fall 2005 Data Compilation}

May 2006

Author:

J. M. Queen 



\section{TABLE OF CONTENTS}

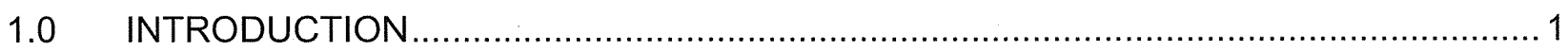

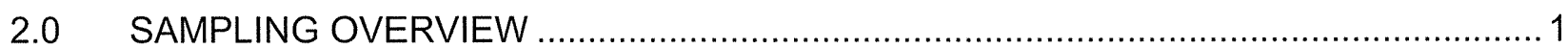

$2.1 \quad$ MULTI-INCREMENT SAMPLING ........................................................ 1

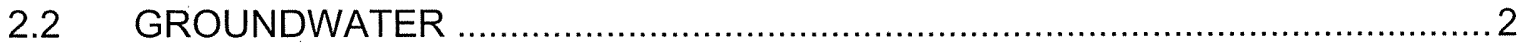

2.3 BIOTA AND RIVER SITE PREPARATION .............................................. 3

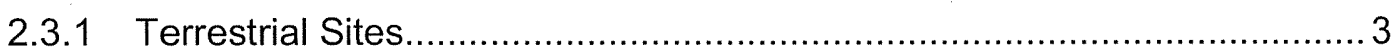

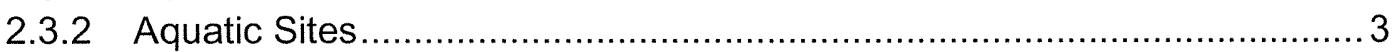

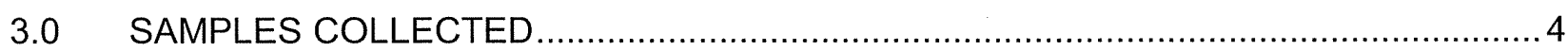

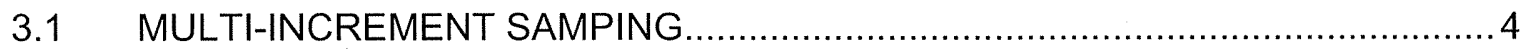

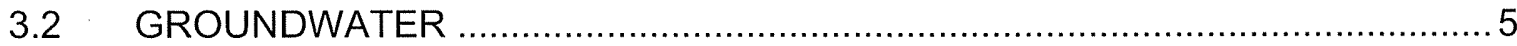

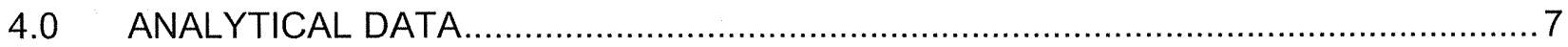

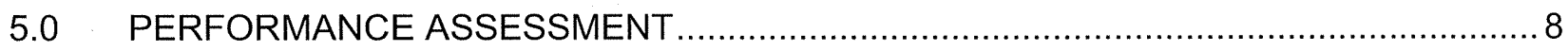

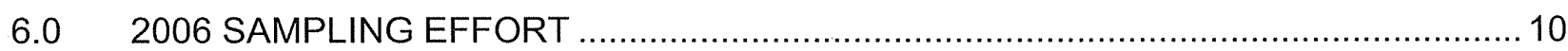

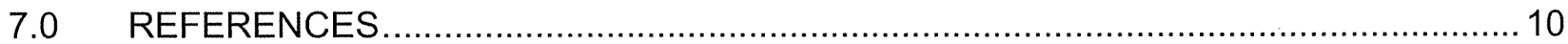

\section{APPENDICES}

A. $\quad 100 / 300$ AREA MULTI-INCREMENTAL SAMPLING STUDY PLOTS ......................... A-i

B. FALL 2005 MULTI-INCREMENT SAMPLING DESCRIPTION ..................................

C. $\quad 100 / 300$ AREA GROUNDWATER SAMPLING WELL LOCATIONS .......................... C-i

D. LOCATIONS OF MULTI-INCREMENT SAMPLING INVESTIGATION AREAS AND

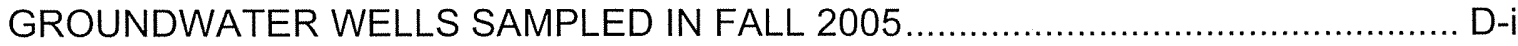

E. DATA SUMMARY OF MULTI-INCREMENT SAMPLING RESULTS ............................. E-i

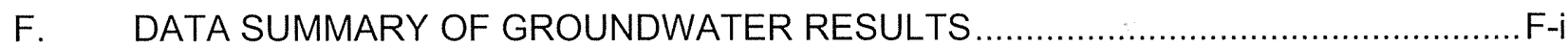

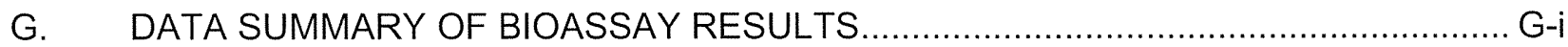




\section{TABLES}

1. Summary of MIS Collected During Fall 2005......................................................... 4

2. Summary of Groundwater Samples Collected During Fall 2005...............................5

3. Summary of Analytical Suite and Indicator Constituents for Upland Soil for the Fall 2005

Effort.

4. Summary of Analytical Suite and Indicator Constituents for Riparian Zone Soil and Groundwater for the Fall 2005 Effort.................................................................... 8

5. Sampling Schedule for Biota and River Components. ....................................... 10

\section{FIGURE}

1. Multi-increment Soil Sampling Performance Assessment Flow Chart. ......................... 9 


\section{ACRONYMS}

EAS Environmental Assessment Services

MIS multi-increment sampling

RCBRA River Corridor Baseline Risk Assessment 
WCH-85

Rev. 0 


\subsection{INTRODUCTION}

The purpose of this report is to provide a brief description of the sampling approaches, a description of the samples collected, and the results for the fall 2005 sampling event. This report presents the methods and results of the work to support the 100 Area and 300 Area Component of the River Corridor Baseline Risk Assessment (RCBRA). The scope of this project can be found in the 100 Area and 300 Area Component of the RCBRA Sampling and Analysis Plan (DOE-RL 2006). The results from the fall 2005 and spring 2006 sampling will be used to complete a baseline risk assessment on the environmental conditions remaining after completion of the interim remedial actions on the Comprehensive Environmental Response, Compensation, and Liability Act of 1980 waste sites in the 100 and 300 Areas of the Hanford Site. The risk assessment will help determine if the interim remedial actions are protective of the ecological and human receptors found at the site. The results of the assessment will be used to support risk management decision-making for final records of decision.

Upland, riparian, and near-shore environments along the southern and western shores of the Columbia River will be sampled for soil, groundwater, surface water, seep water, aquifer tube water, sediment, and biota. As of December 31, 2005, a portion of the soil and groundwater samples have been collected. Additionally, from October through December 2005, site preparations have been made to conduct biota and river sampling. This document provides a synopsis of the field work conducted from October 13 through December 29, 2005, and a compilation of the analytical results from the sampling.

\subsection{SAMPLING OVERVIEW}

Groundwater sampling and multi-increment sampling (MIS) of soil was conducted in the fall 2005. Groundwater sampling was conducted between October 13 and December 29, 2005. Soil sampling began on October 26 and was complete on November 29, 2005. The sampling effort is described further in the following subsections.

\subsection{MULTI-INCREMENT SAMPLING}

MIS is a sampling methodology used to more accurately estimate the true average concentrations of constituents in an investigation area through collection of a limited number of samples. The RCBRA used MIS to evaluate contaminant concentrations in the soil fraction less than or equal to $2 \mathrm{~mm}$ (0.8 in.) in size, consistent with the "Model Toxics Control Act - Cleanup" regulation (Washington Administrative Code 173-340). Each investigation area was evaluated by collecting five MIS. The coordinates of each site including their total size are presented in Appendix $A$. The results of the analyses will be evaluated through a performance assessment to determine the number of MIS that are required for RCBRA soil sampling. The performance assessment will be provided to U.S. Department of Energy, U.S. Environmental Protection Agency, and Washington State Department of Ecology as a letter report. 
Approximately 50 increments were collected for each sample collected within the identified upland and riparian zone investigation areas. Five samples were collected from each upland (approximately 1 ha [2.5 mi]) and riparian investigation area (200 m [656 ft] long of varying width). The shapes of the investigation areas varied depending on site-specific conditions and the shape of the associated waste site. Vegetation and organic matter of varying amounts were present at the site and were removed upon sample collection. Root and biological masses were separated during the sieving process after capturing the attached soil. To facilitate the sieving process, a stiff nylon bristle brush was used. Once the samples were collected, they were brought back to the warehouse (Building 3728) where they were stored in a refrigerator at $4^{\circ} \mathrm{C}$, and prepared for shipment.

Laboratory subsampling of each of the five multi-increment samples was performed to obtain the analytical soil sample. Samples were sent to the CH2M HILL Analytical Services Laboratory in Corvallis, Oregon, for preparation to be sent to Lionville Laboratory, Inc. in Exton, Pennsylvania (chemical constituents) or Eberline Services in Richmond, California (radiological constituents). Samples had an approximate 45-day turnaround time. Samples for toxicity bioassays were sent to $\mathrm{CH} 2 \mathrm{M}$ HILL Analytical Services Laboratory in Corvallis, Oregon (for the Frog Embryo Teratogenesis Assay-Xenopus and Ceriodaphnia dubia tests). A more detailed summary describing the MIS field collection and laboratory sampling is found in Appendix B.

\subsection{GROUNDWATER}

Groundwater monitoring is currently being managed by Pacific Northwest National Laboratory and performed by Duratek Federal Services of Hanford, Inc. Samples were collected per Duratek Technical Services Procedure Number SP3-1, "Groundwater Sampling." Washington Closure Hanford joined in this work and collected additional samples for the risk assessment at the same time the monitoring samples are being collected. The appropriate sampling method and type of pump depended on whether the borehole to be sampled was a well or a piezometer. Depth to water was measured in accordance with SP3-1, "Groundwater Sampling," to the nearest $1 \mathrm{~mm}$ (0.04 in.) for metric tapes, on the groundwater sample report, in the field logbook, and on the Groundwater Measurement form. Drawdown was determined and, after removing the water-level tape from the well, a sheen or oil product was checked for while cleaning the tape. After calculating purge time and allowing the readings to stabilize, $\mathrm{pH}$, conductivity, and temperature were recorded at least three times (start, middle, and end of designated purge time). The coordinates of each site are presented in Appendix C.

Sample containers were filled in the following order unless otherwise specified: total organic halogens, total organic carbon, semivolatile organics, other unfiltered samples, and filtered samples. The sample bottles were slowly filled by placing the inner side of the sample bottle near the sampling manifold drop leg to prevent trapping any air bubbles. Splashing or agitating the water while the bottle is being filled was avoided. The outside of the container was wiped clean of any dirt, grime, or liquid after the sample was placed in the sample container. If required, the samples were placed in a cooler with ice. Once the samples were collected, they were brought back to the warehouse (Building 3728) where they were stored in a refrigerator at $4{ }^{\circ} \mathrm{C}$, and prepared for shipment. 
Samples were sent to Lionville Laboratory, Inc., in Exton, Pennsylvania (chemical constituents), Eberline Services in Richmond, California (radiological constituents), and Severn Trent Laboratories, Inc., in Richland, Washington (Cr-VI).

\subsection{BIOTA AND RIVER SITE PREPARATION}

From October through December 2005, Environmental Assessment Services (EAS) performed site preparations to conduct biota and river sampling.

\subsubsection{Terrestrial Sites}

From October through November 2005, the 45 upland and riparian sites were staked with metal fence posts; the upland sites were staked at each corner and center, and the riparian sites were staked at each end and center point. Environmental dosimeters were placed at each stake and will remain for 6 months. The upland sites were placed with the intent to maximize both relative homogeneity of habitat and regularity of shape (as square or rectangular as possible) to ease the planning, collection, and reporting of MIS and subsequent biota sampling. For those riparian sites where MIS was to be performed in the fall, pin flags were placed at the upper and lower edges of the riparian zone to help delineate the borders. Soil texture at each site was described using ASTM D2488-00, "Standard Practice for Description and Identification of Soils (Visual - Manual Procedure)." Federal and state threatened, endangered, and rare riparian and near-shore aquatic plant species were surveyed during October 2005 while the river flows were low.

\subsubsection{Aquatic Sites}

Thirty-seven aquatic sites were selected based on the known uranium, chromium, and strontium-90 groundwater plumes, results of the 2005 conductivity survey, and past biota sampling locations and results (especially clams). The sampling sites were located throughout the 300 Area (for uranium plume), 100-D and 100-K Areas (for chromium plumes), and $100-\mathrm{N}$ Area (for strontium plume) to capture a range of conditions. At each of the sites (including the ten 100-NR-2 sampling locations), horizontal pore water sampling tubes were placed 10 to $15 \mathrm{~cm}$ (4 to $6 \mathrm{in}$.) below the riverbed and allowed to settle for at least a week before sampling began (pore water sample collection began in January 2006).

Aquatic site setup began from November through December 2005 with the placement of Asiatic clam tubes and artificial substrate (macroinvertebrate) baskets. The Asiatic clams were collected from three locations below Priest Rapids Dam. One hundred and fifty clams were inserted into six mesh tubes, 25 per tube, that were placed at each of 27 aquatic sites for a total of 4,050 clams in 162 tubes. Clam tubes were not placed at the $100-\mathrm{N}$ Area because the $100-N R-2$ risk evaluation previously sampled the river biota during 2005 . Each clam was examined and measured; only live clams from $15 \mathrm{~mm}(0.6 \mathrm{in}$.) to $22 \mathrm{~mm}$ (0.8 in.) were used in the tubes. The tubes will remain in place for at least 6 months. Six artificial substrate baskets were also placed at 27 aquatic sites and will remain in place for at least 6 months to allow colonization by macroinvertebrates. Gravel ranging from $45 \mathrm{~mm}$ (1.8 in.) to $60 \mathrm{~mm}$ (2.3 in.) was placed in each basket. As with the clam tubes, the macroinvertebrate baskets were not placed at the 100-N Area. 


\subsection{SAMPLES COLLECTED}

Below are summary tables for MIS and groundwater sampling (Tables 1 and 2). Appendix D provides maps depicting the MIS and groundwater sampling sites for the 100 and 300 Areas, respectively.

\subsection{MULTI-INCREMENT SAMPING}

The MIS were collected between October 26 and November 29, 2005, at the following upland and riparian zone investigation areas:

- Upland Investigation Areas - 600-131, Pit 23 (a borrow pit reference area), 100-F-2, the Central Plateau (reference area), 116-DR-1 \& 2, and JA Jones

- Riparian Investigation Areas- Site \#3 Upriver 100-D Area, Site \#10 Downriver 100-D Area, and Site \#13 Vernita Bridge (reference area).

The sample identification number, date collected, investigation area, and habitat type for each MIS collected is listed in Table 1.

Table 1. Summary of MIS Collected During Fall 2005.

\begin{tabular}{|l|l|l|l|}
\hline Sample ID Numbers & Date Collected & \multicolumn{1}{|c|}{ Investigation Area } & Habitat Type \\
\hline J10DM5 \& 6 & $10 / 26 / 2005$ & $600-131$ & Upland \\
\hline J10DM7 - 9 & $10 / 27 / 2005$ & $600-131$ & Upland \\
\hline J10DN0 - 4 & $11 / 2 / 2005$ & Pit 23 & Upland \\
\hline J10DP0 - 4 & $11 / 3 / 2005$ & $100-F-2$ & Upland \\
\hline J10DR5 - 8 & $11 / 7 / 2005$ & Central Plateau & Upland \\
\hline J10DR9 & $11 / 8 / 2005$ & Central Plateau & Upland \\
\hline J10DR0 - 4 & $11 / 8 / 2005$ & $116-D R-1 ~ \& 2$ & Upland \\
\hline J10DT0 - 4 & $11 / 9 / 2005$ & JA Jones & Upland \\
\hline J10DP5 & $11 / 9 / 2005$ & Site \#3 Upriver 100-D & Riparian \\
\hline J10DP6 \& 7 & $11 / 10 / 2005$ & Site \#3 Upriver 100-D & Riparian \\
\hline J10DP8 \& 9 & $11 / 14 / 2005$ & Site \#3 Upriver 100-D & Riparian \\
\hline J10LR8 & $11 / 10 / 2005$ & Site \#3 Upriver 100-D & Riparian \\
\hline J10LJ0 \& 1 & $11 / 14 / 2005$ & Site \#10 Downriver 100-D & Riparian \\
\hline J10LJ2 & $11 / 15 / 2005$ & Site \#10 Downriver 100-D & Riparian \\
\hline J10LJ3 \& 4 & $11 / 16 / 2005$ & Site \#10 Downriver 100-D & Riparian \\
\hline J10DN5 - 9 & $11 / 29 / 2005$ & Site \#13 Vernita Bridge & Riparian \\
\hline
\end{tabular}

MIS = multi-increment sampling. 


\subsection{GROUNDWATER}

Groundwater samples were collected between October 13 and December 29, 2005, from the $100-\mathrm{D}, 100-\mathrm{F}, 100-\mathrm{H}, 100-\mathrm{K}, 100-\mathrm{N}$ and, 300 Areas. The sample identification number, date collected, well name, and reactor area for each groundwater sample collected are listed in Table 2.

Table 2. Summary of Groundwater Samples Collected During Fall 2005. (2 Pages)

\begin{tabular}{|c|c|c|c|c|}
\hline $\begin{array}{c}\text { Radiological }^{\mathrm{a}} \text { and } \\
\text { Chemical }^{\mathrm{b}} \\
\text { Sample ID }\end{array}$ & $\begin{array}{c}\mathrm{Cr}^{-\mathrm{VI}^{\mathrm{C}}} \\
\text { Sample ID }\end{array}$ & Date Collected & Well Name & Reactor Area \\
\hline J107Y9 & J107Y8 & $10 / 13 / 2005$ & $199-\mathrm{H} 4-5$ & $100-\mathrm{H}$ \\
\hline J10LD1 & J10LD4 & $11 / 9 / 2005$ & 199-D2-6 & $100-D$ \\
\hline J10LD2 & J10LD5 & $11 / 9 / 2005$ & 199-D3-2 & $100-D$ \\
\hline J10LR2 & J10LR7 & $11 / 10 / 2005$ & 199-D4-20 & $100-D$ \\
\hline J10LD3 & J10LD6 & $11 / 10 / 2005$ & 199-D4-22 & $100-D$ \\
\hline J10FR4 & J10FR7 & $11 / 7 / 2005$ & 199-D4-23 & $100-D$ \\
\hline J10LJ6 & J10LJ9 & $11 / 10 / 2005$ & 199-D5-13 & $100-D$ \\
\hline J10LJ7 & J10LK0 & $11 / 10 / 2005$ & 199-D5-16 & $100-D$ \\
\hline J10LJ8 & J10LK1 & $11 / 10 / 2005$ & 199-D5-17 & $100-D$ \\
\hline J10FR5 & J10FR8 & $11 / 7 / 2005$ & 199-D5-36 & $100-D$ \\
\hline J10FR3 & J10FR6 & $11 / 7 / 2005$ & 199-D5-43 & 100-D \\
\hline J10889 & $J 10890$ & $10 / 31 / 2005$ & 199-F5-1 & $100-\mathrm{F}$ \\
\hline $\mathrm{J} 10891$ & $\mathrm{~J} 10892$ & $10 / 13 / 2005$ & 199-F5-6 & $100-\mathrm{F}$ \\
\hline J10LT0 & J10LT4 & $11 / 22 / 2005$ & $199-\mathrm{H} 3-2 \mathrm{C}$ & $100-\mathrm{H}$ \\
\hline J10M14 & J10M18 & $11 / 21 / 2005$ & $199-\mathrm{H} 4-10$ & $100-\mathrm{H}$ \\
\hline J10M16 & $\mathrm{J} 10 \mathrm{M} 20$ & $11 / 21 / 2005$ & $199-\mathrm{H} 4-13$ & $100-\mathrm{H}$ \\
\hline J10M54 & J10M50 & $11 / 28 / 2005$ & $199-\mathrm{H} 4-16$ & $100-\mathrm{H}$ \\
\hline J10M55 & J10M51 & $11 / 28 / 2005$ & $199-\mathrm{H} 4-4$ & $100-\mathrm{H}$ \\
\hline J10V31 & J10V26 & $12 / 27 / 2005$ & $199-\mathrm{H} 4-46$ & $100-\mathrm{H}$ \\
\hline J10M58 & J10M60 & $11 / 28 / 2005$ & $199-\mathrm{H} 5-1 \mathrm{~A}$ & $100-\mathrm{H}$ \\
\hline J10M17 & $\mathrm{J} 10 \mathrm{M} 21$ & $12 / 1 / 2005$ & $199-\mathrm{H} 6-1$ & $100-\mathrm{H}$ \\
\hline J10F89 & J10F87 & $11 / 1 / 2005$ & $199-K-18$ & $100 \mathrm{~K}$ \\
\hline J10F88 & J10F86 & $11 / 1 / 2005$ & $199-K-20$ & $100-K$ \\
\hline J10D10 \& 11 & J10D08 \& 9 & $10 / 24 / 2005$ & $199-K-21$ & $100-K$ \\
\hline J10FC6 & J10FC7 & $11 / 1 / 2005$ & $199-K-27$ & $100-K$ \\
\hline
\end{tabular}


Table 2. Summary of Groundwater Samples Collected During Fall 2005. (2 Pages)

\begin{tabular}{|c|c|c|c|c|}
\hline $\begin{array}{l}\text { Radiological }^{\mathrm{a}} \text { and } \\
\text { Chemical }^{\mathrm{b}} \\
\text { Sample ID }\end{array}$ & $\begin{array}{c}C r-V^{c} \\
\text { Sample ID }\end{array}$ & Date Collected & Well Name & Reactor Area \\
\hline J10M48 & J10M49 & $11 / 21 / 2005$ & $199-N-64$ & $100-N$ \\
\hline J10D15 & J10D14 & $11 / 1 / 2005$ & $199-N-96 A$ & $100-N$ \\
\hline J10D12 & J10D13 & $11 / 1 / 2005$ & 199-N-99A & $100-N$ \\
\hline J10V30 & J10V25 & $12 / 15 / 2005$ & $399-1-11$ & 300 \\
\hline J10V28 & J10V23 & $12 / 21 / 2005$ & $399-1-15$ & 300 \\
\hline J10LR9 & J10LT3 & $12 / 21 / 2005$ & $399-1-2$ & 300 \\
\hline J10V29 & J10V24 & $12 / 15 / 2005$ & $399-2-2$ & 300 \\
\hline J10VH7 & J10VH4 & $12 / 29 / 2005$ & $399-3-6$ & 300 \\
\hline J10VH8 & J10VH5 & $12 / 29 / 2005$ & $399-4-1$ & 300 \\
\hline
\end{tabular}

${ }^{a}$ Analyzed by Eberline Services.

${ }^{b}$ Analyzed by Lionville Laboratory.

${ }^{\circ}$ Analyzed by Severn Trent Laboratory.

A fraction of the data packages are validated for compliance with quality assurance project plan requirements (DOE-RL 2006). Data evaluation is performed to determine if the laboratory carried out all steps required by the sampling and analysis plan and the laboratory contract governing the conduct of analysis and reporting of the data. This evaluation also examines the available laboratory data to determine if an analyte is present or absent in a sample and the degree of overall uncertainty associated with that determination. Data validation is done at Level $\mathrm{C}$ in accordance with validation procedures (FH 2004a and $\mathrm{FH} \mathrm{2004b).} \mathrm{This} \mathrm{level} \mathrm{of} \mathrm{data}$ validation includes the following:

- Verification of required deliverables

- Requested versus reported analyses

- Evaluation of requested versus achieved analyte detection limits

- Evaluation and qualification of results based on analytical holding times and sample preservation

- Verification of transcription errors

- Evaluation and qualification of results based on method blank result criteria

- Qualification of sample results based on matrix spike (as appropriate to the method) 
- Laboratory control sample and laboratory duplicate or matrix spike/matrix spike duplicate (as appropriate to the method)

- Evaluation and qualification of sample results based chemical or tracer recovery criteria (as appropriate to the method).

Field blanks, field duplicates, and field splits (if information is provided) will be examined. No other validation or calculation checks are performed. For the fall sampling, data from one MIS site and two groundwater wells were validated.

\subsection{ANALYTICAL DATA}

Upland and riparian soil and groundwater samples were analyzed for metals, radionuclides, semivolatile organic compounds, pesticides, and polychlorinated biphenyls. Tables 3 and 4 show the analytical suites and indicator constituents for upland and riparian soil and groundwater samples, respectively. The bioassays for these soils are run on Sandberg's bluegrass (Poa sandbergii) under ASTM 1963-02, "Standard Guide for Conducting Terrestrial Plant Toxicity Tests," and on nematodes (Caenorhabditis elegans) under ASTM E2172-01. A compilation of the analytical results is provided in Appendices $E$ through $G$ for MIS, groundwater, and bioassay results, respectively. (Note: Only the bioassay results for nematodes are included. The laboratory missed several critical measurements of the Sandberg's bluegrass tests and is re-running those bioassays.)

Table 3. Summary of Analytical Suite and Indicator Constituents for Upland Soil for the Fall 2005 Effort. *

\begin{tabular}{|l|l|}
\hline \multicolumn{1}{|c|}{ Analysis Suite } & \multicolumn{1}{c|}{ Indicator Constituents } \\
\hline Metals & $\mathrm{Sb}, \mathrm{As}, \mathrm{Cd}, \mathrm{Cr}, \mathrm{Cu}, \mathrm{Pb}, \mathrm{Li}, \mathrm{Ag}, \mathrm{U}, \mathrm{Zn}, \mathrm{Cr}-\mathrm{VI}$, and $\mathrm{Hg}$ \\
\hline Radionuclides & $\begin{array}{l}\text { Gamma energy analysis; isotopic plutonium, thorium, and uranium; and, total } \\
\text { radioactive strontium }\end{array}$ \\
\hline $\begin{array}{l}\text { Semivolatile organic } \\
\text { compounds }\end{array}$ & $\begin{array}{l}\text { Acenaphthene, acenaphthylene, anthracene, benzo(a)pyrene, } \\
\text { benzo(g,h,i)perylene, benzo(k)fluoranthene, chrysene, dibenz(a,h)anthracene, } \\
\text { fluoranthene, fluorine, naphthalene, phenanthrene, pyrene, } \\
\text { indeno(1,2,3-cd)pyrene }\end{array}$ \\
\hline $\begin{array}{l}\text { Polychlorinated } \\
\text { biphenyls and } \\
\text { pesticides }\end{array}$ & Aroclor-1232, -1242,-1245, and -1260 \\
\hline
\end{tabular}

* All the constituents reported for an analytical group are reported in Appendix $E$. 
WCH-85

Rev. 0

Table 4. Summary of Analytical Suite and Indicator Constituents for Riparian Zone Soil and Groundwater for the Fall 2005 Effort.*

\begin{tabular}{|l|l|}
\hline \multicolumn{1}{|c|}{ Analysis Suite } & \multicolumn{1}{c|}{ Indicator Constituents } \\
\hline Metals & $\begin{array}{l}\mathrm{Al}, \mathrm{Sb}, \mathrm{As}, \mathrm{Ba}, \mathrm{Be}, \mathrm{Cd}, \mathrm{Cr}, \mathrm{Cu}, \mathrm{Fe}, \mathrm{Pb}, \mathrm{Mn}, \mathrm{Ni}, \mathrm{Ag}, \mathrm{Sn}, \mathrm{U}, \mathrm{V}, \mathrm{Zn}, \\
\mathrm{Cr}-\mathrm{VI}, \text { and } \mathrm{Hg} .\end{array}$ \\
\hline Radionuclides & $\begin{array}{l}\mathrm{C}-14, \mathrm{Cs}-137, \mathrm{Co}-60, \mathrm{Ra}-226 \mathrm{~h}, \mathrm{Ra}-228 \mathrm{~h}, \mathrm{Th}-232, \mathrm{U}-233 / 234, \mathrm{U}-235, \\
\mathrm{U}-238, \mathrm{Sr}-90, \text { and } \mathrm{H}-3\end{array}$ \\
\hline Semivolatile organic compounds & Total petroleum hydrocarbon constituents \\
\hline $\begin{array}{l}\text { Polychlorinated biphenyls and } \\
\text { pesticides }\end{array}$ & Methoxychlor \\
\hline
\end{tabular}

* All the constituents reported for an analytical group are reported in Appendix $E$.

\subsection{PERFORMANCE ASSESSMENT}

The goal of MIS was to characterize average contaminant concentrations within each investigation area. The MIS technique is an efficient way to characterize large upland and riparian locations for potential ecological risk to populations. The MIS technique has also been shown to be a robust indicator of the mean, because the influence of "nuggets" or otherwise atypical soils is better characterized. The fall sampling event, which assessed soil at $20 \%$ of the investigation areas, representing all nine environments and site type combinations, was conducted as a performance assessment. The purpose of the performance assessment is to provide information on the 'between-sample' and 'between-investigation area' variability in contaminant concentrations. The results of the performance assessment are currently being used to evaluate the adequacy of the number of samples and increments per investigation area. The criteria that are used in the performance assessment are provided in Figure 1.

Between-sample variability was evaluated at $20 \%$ of the investigation areas during the performance assessment. The performance assessment occurred during the fall sampling events so that the results of the performance assessment will influence the soil sampling design for other investigation areas. The flowchart for the MIS is depicted in Figure 1. Figure 1 presents the performance assessment criteria for the MIS approach. Resultant data from the MIS event is currently being evaluated at several decision points to determine the adequacy of the sample design. Based on the results of the performance assessment, modifications may be made in the sample design to improve the confidence in the mean contaminant values for the investigation areas. The current draft performance assessment, which includes the State of Washington Department of Ecology's comments that have yet to be incorporated, is attached in Appendix $\mathrm{H}$. 
Figure 1. Multi-increment Soil Sampling Performance Assessment Flow Chart.

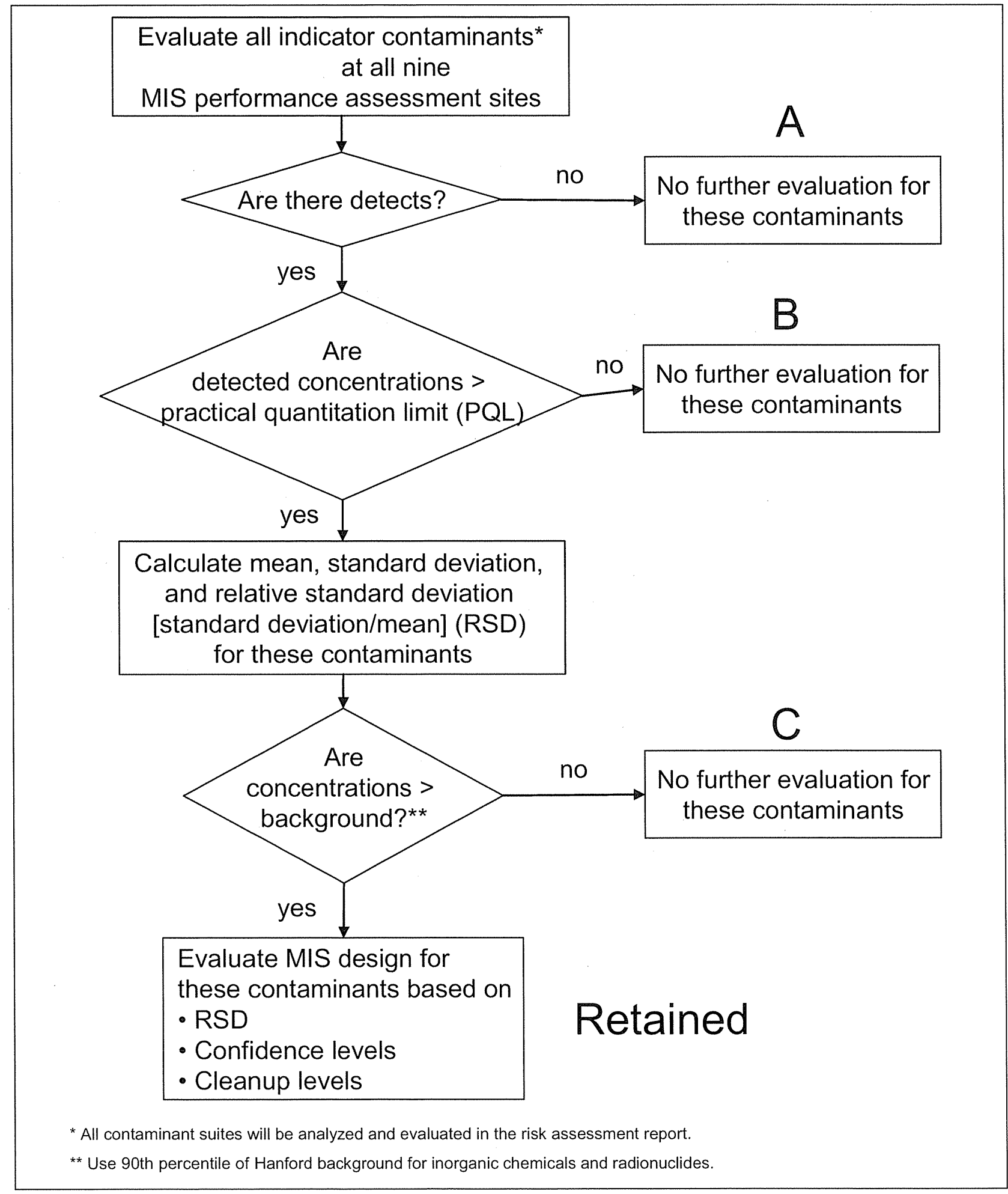




\subsection{SAMPLING EFFORT}

The remaining 36 MIS sites are scheduled to be sampled by summer 2006 upon approval of the MIS performance assessment results. Spring MIS effort intensity will be based on the results of the fall sampling effort. Up to five samples may be collected at each investigation area, depending on the variability of the fall sampling results. The remaining 32 groundwater monitoring wells are scheduled to be sampled by summer 2006. Groundwater will continue to be sampled by the same methods as described above.

EAS is conducting sampling for pore water, surface water, sediment, and biota. Over the next 6 months, EAS will collect fish and small mammal (mice) tissue, upland invertebrates, discrete soil samples, plant tissue, aquatic macroinvertebrates, bivalves (clams), and kingfisher crops. The tentative schedule for spring 2006 sampling is provided in Table 5. Samples will be sent to Lionville Laboratory, Inc., in Exton, Pennsylvania (chemical constituents), or Eberline Services in Richmond, California (radiological constituents).

Table 5. Sampling Schedule for Biota and River Components.

\begin{tabular}{|c|c|}
\hline Month (2006) & Sampling Activity \\
\hline January & Water and sediment \\
\hline February & Fish \\
\hline March/April & Upland biota \\
\hline July & Birds \\
\hline July/August & Macroinvertebrates/bivalves \\
\hline
\end{tabular}

\subsection{REFERENCES}

ASTM D2488-00, "Standard Practice for Description and Identification of Soils (Visual - Manual Procedure," American Society for Testing and Materials, West Conshohocken, Pennsylvania.

ASTM E1963-02, "Standard Guide for Conducting Terrestrial Plant Toxicity Tests," American Society for Testing and Materials, West Conshohocken, Pennsylvania.

ASTM E2172-01, "Standard Guide for Conducting Laboratory Soil Toxicity Tests with the Nematode Caenorhabditis elegans," American Society for Testing and Materials, West Conshohocken, Pennsylvania.

Comprehensive Environmental Response, Compensation, and Liability Act of 1980, 42 U.S.C. 9601 , et seq. 
DOE-RL, 2006, 100 Area and 300 Area Component of the RCBRA Sampling and Analysis Plan, DOE/RL-2005-42, Rev. 0, U. S. Department of Energy, Richland, Washington.

FH, 2004a, Data Validation Procedure for Chemical Analyses, HNF-20433, Rev. 0, Fluor Hanford, Inc., Richland, Washington.

FH, 2004b, Data Validation Procedure for Radiochemical Analyses, HNF-20434, Rev. 0, Fluor Hanford, Inc., Richland, Washington.

SP-3, "Groundwater Sampling," Duratek Federal Services of Hanford, Inc., Richland, Washington.

WAC 173-340, "Model Toxics Control Act -- Cleanup," Washington Administrative Code, as amended. 
WCH-85

Rev. 0 


\section{APPENDIX A}

\section{0/300 AREA MULTI-INCREMENT SAMPLING STUDY PLOTS}


WCH-85

Rev. 0

100 Area and 300 Area Component of the RCBRA Fall 2005 Data Compilation

May 2006

A-ii 
Table A-1. 100/300 Area Multi-Increment Sampling Study Plots. (2 Pages)

\begin{tabular}{|c|c|c|c|c|}
\hline Site ID & Fence Post & Northing* & Easting* & Area (ha) \\
\hline \multirow{5}{*}{$100-F-2$} & $A$ & 146945.480 & 579678.933 & \\
\hline & B & 147045.717 & 579683.083 & \\
\hline & $\mathrm{C}$ & 147051.515 & 579782.564 & 0.9965 \\
\hline & D & 146951.618 & 579779.074 & \\
\hline & Center & 147001.117 & 579728.068 & \\
\hline \multirow{5}{*}{ 116-DR-1 \& 2} & A & 152190.013 & 573945.875 & \\
\hline & B & 152339.401 & 574018.002 & \\
\hline & C & 152309.220 & 574069.379 & 0.9882 \\
\hline & $D$ & 152161.022 & 573998.988 & \\
\hline & Center & 152246.748 & 574007.817 & \\
\hline \multirow{5}{*}{$600-131$} & $A$ & 148885.055 & 577530.502 & \\
\hline & B & 149182.311 & 577387.730 & \\
\hline & $\mathrm{C}$ & 149197.469 & 577412.398 & 0.9873 \\
\hline & $\mathrm{D}$ & 148895.645 & 577559.306 & \\
\hline & Center & 149037.291 & 577475.261 & \\
\hline \multirow{5}{*}{ CP Ref2 } & A & 139745.965 & 563745.899 & \\
\hline & B & 139845.162 & 563741.992 & \\
\hline & $\mathrm{C}$ & 139845.253 & 563846.295 & 1.0109 \\
\hline & $D$ & 139746.033 & 563845.379 & \\
\hline & Center & 139793.869 & 563789.959 & \\
\hline \multirow{5}{*}{ JA Jones } & $A$ & 119959.352 & 593844.220 & \\
\hline & B & 119998.955 & 593815.944 & \\
\hline & C & 120065.687 & 593905.098 & 0.5493 \\
\hline & $D$ & 120025.691 & 593934.574 & \\
\hline & Center & 120013.908 & 593874.580 & \\
\hline \multirow{5}{*}{ Pit 23} & $A$ & 144806.051 & 572356.475 & \\
\hline & B & 144895.480 & 572314.278 & \\
\hline & C & 144943.846 & 572401.191 & 0.9830 \\
\hline & D & 144854.642 & 572443.697 & \\
\hline & Center & 144874.157 & 572381.012 & \\
\hline \multirow[t]{2}{*}{ Riparian \#3 } & A & 151713.927 & 572716.726 & \\
\hline & B & 151677.812 & 572639.650 & Transect \\
\hline
\end{tabular}


Table A-1. 100/300 Area Multi-Increment Sampling Study Plots. (2 Pages)

\begin{tabular}{|c|c|c|c|l|}
\hline Site ID & Fence Post & Northing $^{*}$ & Easting $^{*}$ & Area (ha) \\
\hline \multirow{3}{*}{ Riparian \#10 } & C & 151603.249 & 572594.851 & \\
\cline { 2 - 5 } & A & 153173.197 & 574031.855 & \\
\cline { 2 - 5 } & B & 153082.190 & 573987.873 & Transect \\
\hline \multirow{3}{*}{ Riparian \#13 } & C & 152990.041 & 573955.020 & \\
\cline { 2 - 5 } & A & 145404.948 & 558651.636 & \\
\cline { 2 - 5 } & C & 145345.115 & 558572.809 & Transect \\
\hline
\end{tabular}

*NAD 1983 Washington State Plan South 4602. 


\section{APPENDIX B}

\section{FALL 2005 MULTI-INCREMENT SAMPLING DESCRIPTION}


WCH-85

Rev. 0 


\section{B1.0 INTRODUCTION}

From October 26 to November 29, 2005, the 100 and 300 Areas component of the River Corridor Baseline Risk Assessment (RCBRA) multi-increment sampling (MIS) fall event was conducted. Nine investigation areas, including six upland and three riparian sites, were selected as part of the study and method performance evaluation as identified in the RCBRA sampling and analysis plan. As part of the performance evaluation, five parent soil masses were collected from each investigation site area. The field collection process was conducted in accordance to MIS standard operating procedures and Washington Closure Hanford sample handling, and chain-of-custody guidelines and procedures described in the RCBRA sampling analysis instructions (SAI).

This document provides details pertaining to each of the nine selected MIS investigation areas including site location and identification numbers, description and type, as well as any anomalies or difficulties encountered during field collection of the parent mass material. A brief description and case narrative are provided that describe existing conditions, site dimensions, number of grids selected, and grid area. The collection procedures employed are described for each site. Table B-1 provides data pertinent to the performance evaluation including random numbers generated for $\mathrm{X}$ - and $\mathrm{Y}$ - axes, mass of soil material collected, Hanford Environmental Information System (HEIS) identification numbers, and date collected for each site. For more detailed location information and photographs, refer to MIS Logbook number EL-1596. For quality assurance/quality control (QA/QC) and field decontamination of sampling equipment procedures implemented during the sample collection, refer to BHI-EE-01 Procedure Number 3.2.

As part of this study, all investigation areas included selected radiological, chemical, general chemistry, and physical property constituents for evaluation. The intent of this document is to present relevant data to support the performance evaluation.

\section{B2.0 UPLAND SITES}

The upland sites were selected to represent a cross section of all expected site types to be encountered during the RCBRA MIS. All investigation areas were delineated by a qualified environmental specialist by staking the four corner point locations. Each investigation area's four cardinal points were recorded using a Global Positioning System (GPS). All sites were as close to 1 hectare (ha) in size as possible and within representative habitat and vegetation study requirements. All sites selected were limited to rectangular shape design to reduce edging effect, maintain relatively homogeneous habitat, and facilitate grid pattern design. The grid pattern design for each site is in square meters and used to establish the number of rows and grid sections with each investigation area. Maintaining rectangular shape design resulted in the reduction of some sites to less than 1 ha. For detailed information on grid pattern design and the random number generation technique refer to the SAI for the RCBRA.

The number of soil collection points ranged between 49 and 52 increments and were collected using a random systematic approach ensuring uniform site coverage while minimizing potential 
hot spot area dimensions. Each soil increment was collected from the surface to a $15 \mathrm{~cm}$ (6 in.) depth and, when possible, consisted of equal fractions from all depth intervals. Each parent soil mass required a minimum air-dried mass of $1,390 \mathrm{~g}$ when $\mathrm{QA} / \mathrm{QC}$ analysis were not required for all analytical constituents and $1,810 \mathrm{~g}$ when $\mathrm{QA} / \mathrm{QC}$ sample analysis were required. To ensure adequate parent mass was collected for analysis, $2 \mathrm{~kg}$ was selected as the minimum representative soil parent mass to be collected. This is based on collecting a minimum of $40 \mathrm{~g}$ of $2 \mathrm{~mm}$ soil at each collection point from the targeted unsieved $100 \mathrm{~g}$ total increment. The increment target sizes were adjusted in the field as necessary based on site moisture estimates and the $2 \mathrm{~mm}$ soil fraction collected from trials at each site.

All collected material was sieved on site using a $2 \mathrm{~mm}$ sieve and only the $\leq 2 \mathrm{~mm}$ soil fraction was used for analysis. All samples were sieved into a dedicated plastic lined $19 \mathrm{~L}$ ( 5 gal) bucket using a nylon bristle brush to maintain the $2 \mathrm{~mm}$ cut fraction. During sieving, non-representative biological materials such as root mass, scat, leaves, insects etc., were removed to minimize non-soil intrusion into the parent soil mass. No attempt to capture or quantify the material fraction greater than $2 \mathrm{~mm}$ was conducted. All parent soil masses were collected, assigned unique HEIS identification numbers, labeled and placed into plastic bags, and custody sealed after collection. Table B-1 provides HEIS numbers, parent mass volumes, collection dates, $\mathrm{X}$ and $\mathrm{Y}$-axis random numbers, moisture content, field and lab weights for the following upland sites.

\section{B2.1 UPLAND INVESTIGATION AREA SITE 600-131}

On October 26,2005 , field collection procedures were implemented for the upland investigation area site $600-131$. The site had vegetation consisting of sparse grass and small sagebrush. The exposed surface area consisted of round river rock and sand. The site is $330 \mathrm{~m}$ long by $30 \mathrm{~m}$ wide ( $0.99 \mathrm{ha}$ ) and was divided into 52 grids; each grid section was $15 \mathrm{~m}$ wide by $13 \mathrm{~m}$ long. Each grid section was flagged at the corners using alternating colored flags. The $X$-axis coordinates were generated between 0 and 12 and the $Y$-axis coordinates between 0 and 14 .

Bulk soil collection of the parent mass started at T-post $A$ in the southwest corner of the site (N148885/E577530) and continued throughout all 52 grids using the previously generated random number locations. Five parent soil masses were collected over a consecutive 2-day period. Target mass for each parent soil mass was $2.0 \mathrm{~kg}$. An $8 \mathrm{~cm}$ (3-in.) hand auger was evaluated and yielded too much mass by one order of magnitude. Consequently, parent masses were collected using a calibrated shovel from a depth of 0 to $15 \mathrm{~cm}$ ( 0 to $6 \mathrm{in}$.).

Blackened soil was encountered during collection of J01DM6. The grid location was recorded and photographed before the soil increment was collected. Photos were taken documenting an example of typical root mass encountered during the collection of parent mass from this site. A concrete pad was discovered near the center of the site and excluded from the collection area. Both days were partially overcast with no rain. No other anomalies or difficulties were encountered during field collection of the parent mass materials. 


\section{B2.2 UPLAND INVESTIGATION AREA SITE PIT 23}

Pit 23 is approximately $84 \mathrm{~m}^{2}$; the size was reduced from $1 \mathrm{ha}^{2}$ to stay within the representative recovering borrow-pit vegetation area. The site had minimal vegetation with sparse grass and the exposed surface area was mostly sand and rock.

The investigation area was square in shape and divided into 49 grid sections in a 7 by 7 row configuration with each grid section $12 \mathrm{~m}^{2}$. Random numbers were generated between 0 and 11 for both the $X$ - and $Y$-axis values. Parent masses were collected using a calibrated shovel from a depth of 0 to $15 \mathrm{~cm}$ (0 to $6 \mathrm{in}$.). Five parent soil masses were collected on November 2, 2005. Bulk soil collection of the parent mass started at T-post C (N144943/E572401) and continued throughout all 49 grids at the previously generated random number locations. Minimum target parent mass to be collect was $2.0 \mathrm{~kg}$. The day was clear with no rain; no other anomalies or difficulties were encountered during field collection of the parent mass materials.

\section{B2.3 UPLAND INVESTIGATION AREA SITE 100-F-2}

The $100-F-2$ site area is 1 ha and is square. Due to site conditions and edging effect, the site was divided in 51 grid sections consisting of 2 rows of 8 grids and 5 rows of 7 grids; all grid sections sizes $12 \mathrm{~m}^{2}$. The site had heavy grass covering most of the area with few sparse areas of relatively low vegetation. In the areas with heavy grass, root mass extended below the surface down to $15 \mathrm{~cm}$ ( 6 in.) and retained higher moisture content than previously sampled sites. The root mass was separated from the collected soil with vigorous sieving. Random numbers were generated between 0 and 11 for both the $X$-and $Y$-axis values. Parent masses were collected using a calibrated shovel from a depth of 0 to $15 \mathrm{~cm}(0$ to $6 \mathrm{in}$.).

One parent soil mass was collected on November 3, 2005, and the remaining four parent masses were collected on November 7 and assigned unique HEIS numbers. Bulk soil collection of the parent mass started at T-post A (N146945/E579678) and continued throughout all 51 grid sections at the previously generated random number locations. Due to heavy rain on November 3, only parent mass J10DP0 was collected; collection resumed on November 7 for the remaining 4 parent masses. On November 7, a second 2-person soil collection team was added to the existing team. Each team consisted of one person to excavate down to $15 \mathrm{~cm}$ (6 in.) with a shovel and one person to collect a 15-cm (6-in.) core increment using a disposable

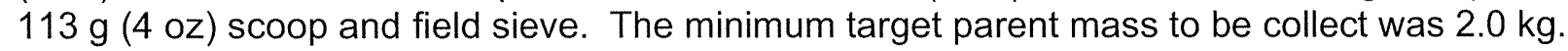
November 7 was clear with no rain.

\section{B2.4 UPLAND INVESTIGATION AREA SITE CENTRAL PLATEAU SITE CP-REF-2}

The Central Plateau Site CP-Ref-2 is $1 \mathrm{ha}^{2}$ and was divided into 49 grids in a 7 by 7 pattern; each grid was $14 \mathrm{~m}^{2}$ and soil collection occurred on November 7 and 8,2005 . The site (1 ha) was covered with dense large sagebrush and the exposed surface area was generally sandy with few rocks. The soil parent mass was collected using a $2.5-\mathrm{cm}$ (1-in.) outside diameter step core device. Two teams of two persons each completed the collection of five parent soil masses over a 2-day period. The target parent mass was increased to $2.5 \mathrm{~kg}$ to account for the larger bio-assay sample size requirements. Random numbers were generated between 0 and 13 for $X$ - and $Y$-axis coordinates. Both days were overcast in the morning and clear and partly sunny in the afternoon with no rain on either day. 


\section{B2.5 UPLAND INVESTIGATION AREA SITE 116-DR-1 \& 2}

Site 116-DR-1 \& 2 is $48 \mathrm{~m}$ by $156 \mathrm{~m}$ ( $1 \mathrm{ha}$ ) and was divided in 52 grid sections with 4 rows of 13 grids; each grid was $12 \mathrm{~m}^{2}$. The site had light vegetation consisting of grasses and sagebrush; the exposed surface area was sand and river rock. All five parent soil masses were collected on November 8, 2005. Collection was conducted with two teams using shovels and scoops. During collection it was noted that three strata layers existed within most increment soil cores of 0 to $15 \mathrm{~cm}$ ( 0 to 6 in.). Due to higher estimated moisture content, target soil masses were increased to $3.0 \mathrm{~kg}$. Random numbers were generated between 0 and 11 for the $X$ - and $Y$-axis coordinates. The day was slightly overcast in morning and clear with sun in the afternoon with no rain. No difficulties or anomalies were encountered during the collection process.

\section{B2.6 JA JONES UPLAND INVESTIGATION AREA SITE}

The JA Jones Site is $50 \mathrm{~m}$ by $110 \mathrm{~m}$ ( $0.55 \mathrm{ha})$ to keep the sampling and other site evaluations in the same relatively homogeneous habitat. The site was divided into 50 grids in a 5 by 10 pattern; each grid section was $10 \mathrm{~m}$ by $11 \mathrm{~m}$ and soil collection occurred on November 9, 2005. The site was a large excavation pit with very sparse vegetation consisting of grass and sagebrush with a few bitterbrush plants; the exposed surface area was generally rocky with sand. The sidewalls of the excavation area were included as part of the investigation area and 0 to $15-\mathrm{cm}$ ( 0 to 6 -in.) depth soil cores were collected from the sidewalls when dictated by random locations generated. The site was sampled using a $2.5-\mathrm{cm}$ (1-in.) outside diameter step core device for the collection of four parent soil masses, and a shovel and scoop was used to collect the single remaining parent soil mass. Two teams of two individuals completed the collection of five parent soil masses in 1 day. The target parent soil mass was $2.5 \mathrm{~kg}$. Random numbers were generated between 0 and 9 for the $X$-axis and from 0 to 10 for the $Y$-axis. The day was very foggy and overcast in the morning and clear and partly sunny in the afternoon with no rain that day. No difficulties or anomalies were encountered during the soil collection process.

\section{B3.0 RIPARIAN SITES}

The riparian sites were selected to represent a cross section of all expected site types to be encountered during the RCBRA MIS. The riparian and near-shore investigation area were delineated by a qualified environmental specialist by staking the boundaries with alternating colored pin flags at $4 \mathrm{~m}$ intervals. Each site's beginning (downriver), center, and end (upriver) points were recorded using a GPS. All sites were $200 \mathrm{~m}$ in length and of varying width based on the presence of riparian vegetation.

For each site, 50 incremental soil collection points were collected using a random systematic approach ensuring uniform site coverage while minimizing potential hot spot area dimensions. The $X$-axis random number was generated between 0 and 15 representing $0.25 \mathrm{~m}$ intervals, the 
$\mathrm{Y}$-axis was generated between 0 and $99 \%$ representing a percentage of the width of the riparian zone. After the first collection point, a systematic sampling approach was applied at each successive grid point: the addition of $30 \%$ to the previous $Y$-axis point towards the upland, employing a wrap-around approach after exceeding $100 \%$ of the width, using the same $X$-axis location for each successive grid. The $30 \%$ upland shift was employed to alleviate soil strata concerns present within riparian areas.

Due to the additional analytes required and associated QA/QC analysis for riparian sites, the target mass was increased to $2.5 \mathrm{~kg}$ as the minimum representative soil parent mass to be collected. All soil was sieved onsite using a $2-\mathrm{mm}$ sieve and only the soil fraction $\leq 2 \mathrm{~mm}$ was used for analysis. All samples were sieved into a dedicated plastic lined 19-L (5-gal) bucket using a nylon bristle brush to maintain the $2 \mathrm{~mm}$ cut fraction. Non-representative biological materials were removed prior to sieving to minimize non-soil intrusion into the parent soil mass. No attempt to quantify the soil fraction greater than $2 \mathrm{~mm}$ fraction was conducted.

All parent soil masses were collected, assigned unique HEIS identification numbers, labeled, placed into plastic bags, and were custody sealed after collection. Table B-1 provides HEIS numbers, parent mass volumes, collection dates and $\mathrm{X}$ - and $\mathrm{Y}$-axis random numbers for the following riparian sites.

\section{B3.1 ELEVATED \# 3 100-D RIPARIAN INVESTIGATION AREA SITE}

The Elevated \# 3 100-D riparian investigation area is $200 \mathrm{~m}$ long of varying width and was divided into fifty 4 -m-long grid sections. The selected investigation area has a unique curved shape consisting of an upriver water flow impact area of approximately $100 \mathrm{~m}$ with a jut of land delineating the upriver water impact side from the downriver slack water side. The investigation area has a steep slope and is covered with varying size rocks from pebbles to rip-rap and some larger boulders. The investigation area has varying degrees of vegetation from sparse to heavy. The downriver area had heavier vegetation near shore where as the upriver water impact area vegetation was predominately near the upper boundary and very sparse near shore. Sand deposits varied in the impact area with finer sand and high loam and organic content on the slack water side. Soil moisture content also varied, with the downriver slack water side having higher moisture content than the coarser sand on the upriver area. The lower and upper boundaries of the investigation area were delineated using alternating pink and green flagging at 4-m intervals on the corners of each grid section.

Soil collection for the five parent soil masses began on November 9, 2005, with two collection teams consisting of two personnel each and was completed November 14, 2005 . The parent soil masses were collected using shovels from a depth of 0 to $15 \mathrm{~cm}$ ( 0 to 6 in.); the cores from each increment were collected with 113-g (4-oz) scoops and then sieved. Prior to collecting each soil increment, the overburden rocks were removed to expose representative soil. During soil collection, the sieve plugged severely while collecting the downriver increments, whereas the upriver side sieved relatively freely. During subsequent collection procedures each team used dedicated buckets for sieving the upriver and downriver increments separately; this approach was used to alleviate difficulties encountered due to sieve blinding. Following parent mass collection, the total mass from each bucket was recorded and the amount of $\leq 2 \mathrm{~mm}$ particulate sized fractions was compared and found to be relatively equal. The two dedicated bucket contents were then combined to form a complete parent mass. 
Two distinct strata layers were observed during soil increment collection between the near-shore area and upper riparian boundary. Soil moisture content was estimated at approximately $20 \%$ and the parent soil target mass was $3.0 \mathrm{~kg}$. An equipment blank was collected from silica sand at this investigation area using a disposable scoop of sand and pouring it through a field-cleaned sieve into a plastic bag labeled HEIS \#J10LR8. During parent soil mass collection, the weather varied from very foggy in the morning to sunshine in the afternoon; no rain occurred during collection days.

\section{B3.2 LOW \# 10 RIPARIAN INVESTIGATION AREA SITE}

The riparian Low \# 10 investigation area is $200 \mathrm{~m}$ long with a varying width and was divided into fifty $4-m$-long grid sections. The selected investigation area is a flat, straight stretch along the river with moderate vegetation covering most of the site with the near shore having generally less dense vegetation and no vegetation near the low water line. The exposed areas have a uniform covering of river rock and sand. Soil collection for the five parent soil masses began on November 14, 2005, with one collection team consisting of three personnel. On November 15, 2005, a two person team collected one parent soil mass and three personnel completed the collection on November 16. The parent soil masses were collected using shovels from a depth of 0 to $15 \mathrm{~cm}$ (0 to $6 \mathrm{in}$.); the cores from each increment were collected with 227-g (8-oz) scoops and then sieved.

On the morning of November 15, 2005, the river level was above the lower boundary of the investigation area and the sample team had to postpone sampling until the water level receded. Prior to collecting each soil increment, the overburden rocks were removed to expose representative soil. During collection, the near-shore boundary soil increments were saturated and the 15-cm (6-in.) core holes filled with water after collection. Soil moisture content also varied with the near-shore area having higher moisture content than the upper boundary area. During soil collection, the sieve plugged severely while collecting the near-shore increments but the increments near the upper boundary sieved relatively freely. One team dug while two members collected the increment soil cores. One team member collected the near-shore $50 \%$ increments, while the other member collected upper $50 \%$ boundary increments; these were then combined at the end of collection and sieved to form the complete parent mass. The soil volume from each dedicated bucket was recorded separately and the volumes for each $\leq 2 \mathrm{~mm}$ fractions compared and found to be relatively equal.

During soil collection, the weather varied from very foggy in the morning to sunshine in the afternoon with light rain occurring only on the morning of November 16, 2005. Soil moisture content was estimated at approximately $20 \%$. As a result of an increase in moisture content, the parent soil target mass was increased to $3.0 \mathrm{~kg}$ on November 14, 2005, and increased to $3.5 \mathrm{~kg}$ on November 15, 2005. Two distinct strata layers were observed during soil collection: the near-shore area contained more sand and the upper riparian boundary consisted of an upper sand layer generally 0 to $10 \mathrm{~cm}(0$ to $4 \mathrm{in}$.) in depth and the subsequent 10 to $15-\mathrm{cm}$ (4 to 6 -in.) depth orange interval with clay properties. Root masses were generally denser near the upper riparian boundary. On November 15, 2005, one soil increment collection location had a white crystal-like substance of coarse granular material, photos were taken and the increment core was collected. The material had a rock-salt-like appearance under closer examination and did not appear to be crushed shells, but possibly was a crushed aggregate rock. 


\section{B3.3 VERNITA BRIDGE RIPARIAN INVESTIGATION AREA SITE}

The Vernita Bridge Riparian Reference Site 13 investigation area is $200 \mathrm{~m}$ long with a varying width and was divided into fifty 4 -m-long grid sections. The selected investigation area is a sloped, straight stretch along the river with heavy vegetation and large trees covering most of the area, with the near shore having generally less dense vegetation and smaller brush, with the least amount of vegetation near the low water line. The exposed areas had a non-uniform covering of drift debris up to $20 \mathrm{~cm}$ (8 in.) in depth near the high water line.

The collection of parent soil masses began on November 29, 2005, employing three personnel digging and collecting unsieved soil fractions and was completed the same day. Soil collection sieving was started November 29 and completed on November 30, 2005. Prior to collecting each soil increment the overburden rocks and drift debris were removed to expose representative soil. The parent soil masses were collected using shovels from a depth of 0 to $15 \mathrm{~cm}$ ( 0 to $6 \mathrm{in}$.). The cores from each increment were collected with full 227-g (8-oz) scoops as required target mass. All five parent masses were collected unsieved and the weight totals were recorded. During collection, three strata layers were observed: the near shore was saturated, the mid-level increments under trees were dry, and the upper-level increments had heavy root masses and the moisture content was frozen. Dead fish were observed within the investigation area, but no soil increments were collected near decaying fish carcasses.

No sieving was conducted during increment collection as a result of fluctuating river levels; on November 29. 2005 , the lower boundary was below water levels because of high levels of water released form the dam. Soil moisture content was estimated at approximately $20 \%$ and the parent soil target mass was estimated at $11 \mathrm{~kg}$ unsieved to collect a minimum volume of $3.0 \mathrm{~kg}$ after sieving. During soil collection, the weather varied from very foggy in the morning to sunshine in the afternoon; no rain occurred during collection days.

\section{B4.0 MULTI-INCREMENTAL SUB-SAMPLING}

The sub-sampling of parent soil masses was conducted in the Applied Science Laboratories (ASL) of $\mathrm{CH} 2 \mathrm{M} \mathrm{HILL}$ in Corvallis, Oregon. Soil parent masses were received under chain-of-custody and inspected with no anomalies recorded. After receiving parent soil masses, the masses were weighed "as received" and weights were recorded for moisture determination. The parent masses for each investigation area site were spread on individual pre-taped drying pans and initial weights and HEIS identifications were recorded on bench sheets. Each parent mass drying pan was maintained under limited access chain-of-custody in a monitored, temperature controlled environment area during the air-drying process. Each drying pan was tilled and weighed on a regular basis until a constant weight was obtained and recorded. Drying time varied from 1 to 4 days, depending on the moisture content and soil mass size.

After the soil masses reached a constant weight, trained personnel conducted incremental sub-sampling of parent soil masses using calibrated disposable scoops. Each scoop was calibrated to the specific sub-sample increment size required to produce the desired sample 
weights. Each sub-sample consisted of a minimum of 50 increments. Target sub-sample sizes were either 30 or $500 \mathrm{~g}$ depending on analytical method sample mass requirements.

A separate sample was created for each analytical method required, assigned a unique identification number based on the original HEIS number, and labeled with the analytical method and sub-sampled date. For each investigation area, all QA/QC samples for Matrix Spike (MS) Matrix Spike Duplicate (MSD) were created from the largest parent soil mass samples to ensure adequate material remained for each analytical method required. The final sample weight for each analytical method was recorded on sampling bench sheets. All completed sub-samples for each investigation area site were batched as a complete set and were recorded on chain-of-custodies with specific analysis instructions and packaged for shipment to the appropriate laboratory for analysis.

After sub-sampling for chemical and radiological parameters for each site investigation area was complete, the bioassay, general chemistry, and physical property composite samples were produced. One bioassay and one general chemistry composite sample representing each investigation area site were created. The bio-assay and general chemistry samples consisted of soil fractions from all five parent soil masses from an individual site investigation area. Each bioassay sample weighed $4 \mathrm{~kg}$ and the general chemistry and particle size samples weighed $1 \mathrm{~kg}$. Samples were maintained under chain-of-custody and retained for analysis and bio-assay by ASL.

A brief description of each investigation area parent mass sub-sampling and any anomalies or difficulties encountered is included. Individual sections for each investigation area identifying sample numbers, QA/QC samples, weights and dates are provided in Table B-2.

\section{B4.1 UPLAND AND RIPARIAN SITES}

All soil parent masses were air dried prior to sub-sampling. All sub-samples were generated by collecting 50 aliquots from each soil parent mass with a systematic uniform distribution system similar to that used in the field. For analytical methods and associated QA/QC procedures and MS/MSD requirements, refer to Instructions for MIS and Laboratory Sub-sampling document. The upland sites included analysis of polynuclear aromatic hydrocarbons (PAHs) (Method 8270) that were not required in the riparian investigation areas. The riparian sites included analysis for ion chromatography (IC) anions (Method 300.0) and $\mathrm{NO}^{2} / \mathrm{NO}^{3}$ analytes that were not required in upland investigation areas.

Each upland investigation area generated total of 56 sub-samples per site. Eberline Services received a total of 12 samples for radiological analysis; Lionville Laboratory Incorporated received 42 for chemical analysis; ASL maintained two samples. The riparian sites sub-sample number varied from 69 or 63 samples due to the addition of the equipment blank. Equipment blank number J10LR8 was processed with the sample set collected at site \#3 100-D and was handled with the same procedures. Eberline Services received a total of 12 samples for radiological analysis; Lionville Laboratory Incorporated received 49 or 55 (with Equipment Blank) for chemical analysis; ASL maintained two samples. No anomalies or difficulties were encountered during the sub-sampling procedures. Tables B-1 and B-2 show sample HEIS numbers, weights, dates, and specific analyses requested. Table B-3 shows the HEIS numbers generated for the nine investigation areas bio-assay, general chemistry, and physical properties composite samples. 
Table B-1. Parent Soil Mass Summary Information for the Fall 2005 Multi-Incremental Field Collection Effort. (2 Pages)

\begin{tabular}{|c|c|c|c|c|c|c|c|c|c|}
\hline 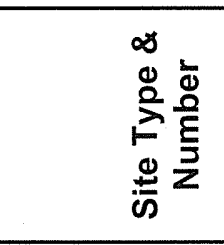 & 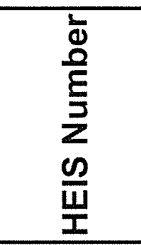 & $\frac{\bar{d}}{\frac{\delta}{0}}$ & 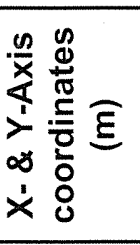 & 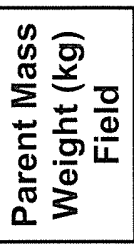 & 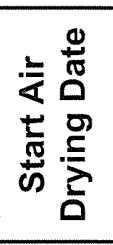 & 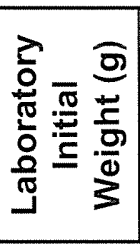 & 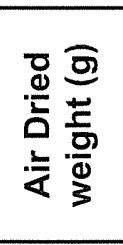 & 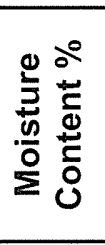 & 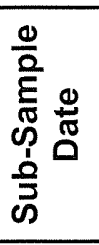 \\
\hline \multicolumn{10}{|l|}{ Upland Sites } \\
\hline \multirow{5}{*}{$600-131$} & J10DM5 & $10 / 26$ & 8,4 & 2.401 & $10 / 28$ & 2393 & 2356 & 1.55 & $10 / 31$. \\
\hline & J10DM6 & $10 / 26$ & 3,2 & 2.766 & $10 / 28$ & 2759 & 2720 & 1.41 & $10 / 31$ \\
\hline & J10DM7 & $10 / 27$ & 13,7 & 2.3 & $10 / 28$ & 2304 & 2261 & 1.88 & $10 / 31$ \\
\hline & J10DM8 & $10 / 27$ & 9,3 & 2.14 & $10 / 28$ & 2159 & 2120 & 1.83 & $10 / 31$ \\
\hline & J10DM9 & $10 / 27$ & 10,5 & 3.4 & $10 / 28$ & 3461 & 3393 & 1.97 & $10 / 31$ \\
\hline \multirow{5}{*}{ Pit 23} & J10DNO & $11 / 2$ & 11,2 & 4.08 & $11 / 5$ & 4078.2 & 3892.5 & 4.55 & $11 / 8$ \\
\hline & J10DN1 & $11 / 2$ & 7,6 & 2.94 & $11 / 5$ & 2945.9 & 2807.9 & 4.68 & $11 / 8$ \\
\hline & J10DN2 & $11 / 2$ & 9,4 & 3.27 & $11 / 5$ & 3266.4 & 3177.6 & 4.56 & $11 / 8$ \\
\hline & J10DN3 & $11 / 2$ & 3,8 & 2.725 & $11 / 5$ & 2667.8 & 2544 & 4.64 & $11 / 8$ \\
\hline & J10DN4 & $11 / 2$ & 1,2 & 2.534 & $11 / 5$ & 2535.9 & 2423.9 & 4.42 & $11 / 8$ \\
\hline \multirow{5}{*}{$100-\mathrm{F}-2$} & J10DP0 & $11 / 3$ & 3,7 & 2.94 & $11 / 10$ & 2998.7 & 2818.3 & 6.02 & $11 / 14$ \\
\hline & J10DP1 & $11 / 7$ & 5,11 & 3.04 & $11 / 10$ & 3079.2 & 2820.7 & 8.4 & $11 / 14$ \\
\hline & J10DP2 & $11 / 7$ & 9,2 & 1.81 & $11 / 10$ & 1782.2 & 1644.7 & 7.72 & $11 / 14$ \\
\hline & J10DP3 & $11 / 7$ & 1,8 & 3.37 & $11 / 10$ & 3359.5 & 3084.9 & 8.17 & $11 / 14$ \\
\hline & J10DP4 & $11 / 7$ & 10,5 & 2.91 & $11 / 10$ & 2913.6 & 2667.4 & 8.45 & $11 / 14$ \\
\hline \multirow{5}{*}{ CP-Ref-2 } & J10DR5 & $11 / 7$ & 5,10 & 2.61 & $11 / 11$ & 2619.3 & 2504.4 & 4.39 & $11 / 15$ \\
\hline & J10DR6 & $11 / 7$ & 12,3 & 3.14 & $11 / 11$ & 3153.5 & 3022.2 & 4.16 & $11 / 15$ \\
\hline & J10DR7 & $11 / 7$ & 7,9 & 3.06 & $11 / 11$ & 3105.3 & 2972 & 4.29 & $11 / 15$ \\
\hline & J10DR8 & $11 / 7$ & 13,5 & 2.48 & $11 / 11$ & 2484 & 2368.3 & 4.66 & $11 / 15$ \\
\hline & J10DR9 & $11 / 8$ & 4,5 & 3.08 & $11 / 11$ & 3095.7 & 2968.5 & 4.11 & $11 / 15$ \\
\hline \multirow{5}{*}{ 116-DR-1 \& 2} & J10DR0 & $11 / 8$ & 3,9 & 4.11 & $11 / 10$ & 4183.5 & 3869.1 & 7.52 & $11 / 15$ \\
\hline & J10DR1 & $11 / 8$ & 5,8 & 3.97 & $11 / 10$ & 4093.5 & 3813.8 & 6.83 & $11 / 15$ \\
\hline & J10DR2 & $11 / 8$ & 7,10 & 3.31 & $11 / 10$ & 3309.3 & 3096.2 & 6.44 & $11 / 15$ \\
\hline & J10DR3 & $11 / 8$ & 2,11 & 3.67 & $11 / 10$ & 3698.4 & 3456.6 & 6.54 & $11 / 15$ \\
\hline & J10DR4 & $11 / 8$ & 4,1 & 2.94 & $11 / 10$ & 2983.7 & 2779.3 & 6.85 & $11 / 15$ \\
\hline \multirow{5}{*}{ JA Jones } & J10DT0 & $11 / 9$ & 3,1 & 2.6 & $11 / 11$ & 2621.1 & 2516.1 & 4.01 & $11 / 16$ \\
\hline & J10DT1 & $11 / 9$ & 4,10 & 2.52 & $11 / 11$ & 2540.2 & 2440.2 & 3.94 & $11 / 16$ \\
\hline & J10DT2 & $11 / 9$ & 6,5 & 3.39 & $11 / 11$ & 3440.1 & 3293.1 & 4.27 & $11 / 16$ \\
\hline & J10DT3 & $11 / 9$ & 9,11 & 2.21 & $11 / 11$ & 2226.1 & 2130.9 & 4.28 & $11 / 16$ \\
\hline & J10DT4 & $11 / 9$ & 9,2 & 2.7 & $11 / 11$ & 2727 & 2613.3 & 4.17 & $11 / 16$ \\
\hline
\end{tabular}


Table B-1. Parent Soil Mass Summary Information for the Fall 2005 Multi-Incremental Field Collection Effort. (2 Pages)

\begin{tabular}{|c|c|c|c|c|c|c|c|c|c|}
\hline 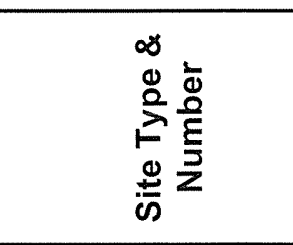 & 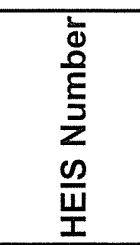 & 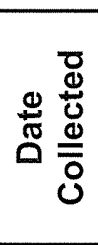 & 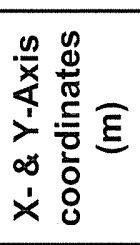 & 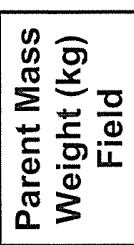 & 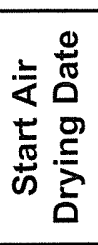 & 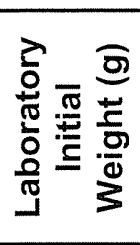 & 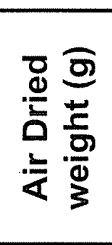 & 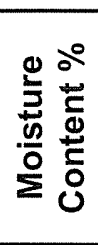 & 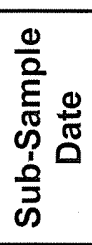 \\
\hline \multicolumn{10}{|l|}{ Riparian } \\
\hline \multirow{6}{*}{ Elevated \#3 100-D } & J10DP5 & $11 / 9$ & $1,10 \%$ & 3.54 & $11 / 12$ & 3613.2 & 3130.6 & 13.36 & $11 / 21$ \\
\hline & J10DP6 & $11 / 10$ & $7,73 \%$ & 4.84 & $11 / 18$ & 4657.1 & 4295.2 & 7.77 & $11 / 21$ \\
\hline & J10DP7 & $11 / 10$ & $3,31 \%$ & 4.35 & $11 / 18$ & 4364.4 & 3728.9 & 14.56 & $11 / 21$ \\
\hline & J10DP8 & $11 / 14$ & $5,20 \%$ & 4 & $11 / 18$ & 4016 & 3443.7 & 14.25 & $11 / 21$ \\
\hline & J10DP9 & $11 / 14$ & $14,57 \%$ & 3.06 & $11 / 18$ & 3105.1 & 2625.1 & 15.46 & $11 / 21$ \\
\hline & J10LR8 & $11 / 9$ & \multicolumn{2}{|c|}{ Equipment Blank } & $11 / 18$ & 1250 & 1249.7 & 0.02 & $11 / 21$ \\
\hline \multirow{5}{*}{ Low \#10 } & J10LJ0 & $11 / 14$ & $4,25 \%$ & 3.34 & $11 / 19$ & 3345.1 & 2798.6 & 16.34 & $11 / 28$ \\
\hline & J10LJ1 & $11 / 14$ & $8,95 \%$ & 3.5 & $11 / 19$ & 3508.6 & 2931.5 & 16.45 & $11 / 28$ \\
\hline & J10LJ2 & $11 / 15$ & $15,38 \%$ & 4.23 & $11 / 19$ & 4229.5 & 3527.9 & 16.59 & $11 / 28$ \\
\hline & J10LJ3 & $11 / 16$ & $13,17 \%$ & 3.1 & $11 / 19$ & 3573.3 & 2872 & 19.67 & $11 / 28$ \\
\hline & J10LJ4 & $11 / 16$ & $5,12 \%$ & 4.64 & $11 / 19$ & 4664.1 & 3895.6 & 16.48 & $11 / 28$ \\
\hline \multirow{5}{*}{$\begin{array}{l}\text { Vernita Bridge } \\
\text { Reference Site \#13 }\end{array}$} & J10DN5 & $11 / 30$ & $7,57 \%$ & 2.995 & $12 / 2$ & 2989 & 2430 & 18.7 & $12 / 6$ \\
\hline & J10DN6 & $11 / 29$ & $3,85 \%$ & 3.265 & $12 / 2$ & 3297.3 & 2661.7 & 19.28 & $12 / 6$ \\
\hline & J10DN7 & $11 / 29$ & $6,75 \%$ & 4.432 & $12 / 1$ & 4424.2 & 3497 & 20.96 & $12 / 6$ \\
\hline & J10DN8 & $11 / 29$ & $8,20 \%$ & 3.93 & $12 / 1$ & 3942.4 & 3178 & 19.39 & $12 / 6$ \\
\hline & J10DN9 & $11 / 29$ & $15,95 \%$ & 4.131 & $12 / 2$ & 4108.7 & 3229.4 & 21.4 & $12 / 6$ \\
\hline
\end{tabular}


Table B-2. - Analytes and number of samples collected from each parent mass including QA/QC control samples matrix spikes and duplicates are provided for the Fall 2005 Multi-incremental Sub-sampling effort. GEAs, RADs, Metals, Cr-VI, SVOAs, PAHs (Upland), IC Anions (Riparian), Pesticides, PCBs, and NO2/ NO3(Riparian) were analyzed for.

\begin{tabular}{|c|c|c|c|c|c|c|c|c|c|c|}
\hline 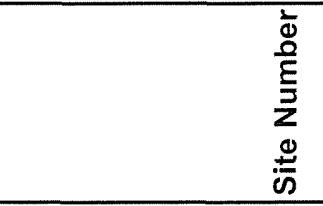 & 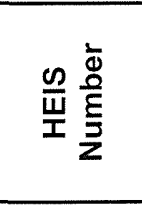 & 嵌 & $\frac{\rho}{\alpha}$ & $\frac{\frac{n}{\pi}}{\frac{\pi}{2}}$ & $\frac{1}{0}$ & 市 & 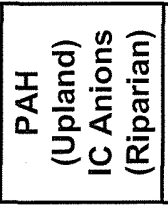 & $\frac{8}{0}$ & 罢 & $\begin{array}{l}\frac{m}{O} \\
\frac{2}{\Sigma} \\
\frac{O}{z}\end{array}$ \\
\hline \multicolumn{11}{|l|}{ Upland Sites } \\
\hline \multirow[t]{5}{*}{$600-131$} & J10DM5 & 1 & 1 & 3 & 3 & 1 & 1 & 1 & 1 & N/A \\
\hline & J10DM6 & 1 & 3 & 1 & 1 & 1 & 3 & 1 & 1 & N/A \\
\hline & J10DM7 & 1 & 1 & 1 & 1 & 1 & 1 & 1 & 1 & N/A \\
\hline & J10DM8 & 1 & 1 & 1 & 1 & 1 & 1 & 1 & 3 & N/A \\
\hline & J10DM9 & 1 & 1 & 1 & 1 & 3 & 1 & 3 & 1 & $N / A$ \\
\hline \multirow[t]{5}{*}{ Pit 23} & J10DNO & 1 & 3 & 3 & 1 & 1 & 1 & 1 & 1 & $N / A$ \\
\hline & J10DN1 & 1 & 1 & 1 & 3 & 1 & 1 & 1 & 3 & N/A \\
\hline & J10DN2 & 1 & 1 & 1 & 1 & 3 & 3 & 1 & 1 & $N / A$ \\
\hline & J10DN3 & 1 & 1 & 1 & 1 & 1 & 1 & 3 & 1 & $N / A$ \\
\hline & J10DN4 & 1 & 1 & 1 & 1 & 1 & 1 & 1 & 1 & N/A \\
\hline \multirow[t]{5}{*}{$100-F-2$} & J10DP0 & 1 & 3 & 3 & 1 & 1 & 1 & 1 & 1 & $\overline{N / A}$ \\
\hline & J10DP1 & 1 & 1 & 1 & 3 & 3 & 1 & 1 & 1 & $N / A$ \\
\hline & J10DP2 & 1 & 1 & 1 & 1 & 1 & 1 & 1 & 1 & $N / A$ \\
\hline & J10DP3 & 1 & 1 & 1 & 1 & 1 & 3 & 3 & 1 & N/A \\
\hline & J10DP4 & 1 & 1 & 1 & 1 & 1 & 1 & 1 & 3 & $\mathrm{~N} / \mathrm{A}$ \\
\hline
\end{tabular}




\begin{tabular}{|c|c|c|c|c|c|c|c|c|c|c|}
\hline 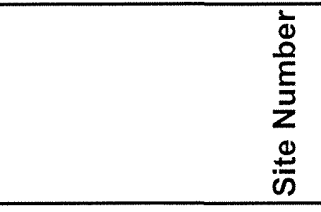 & 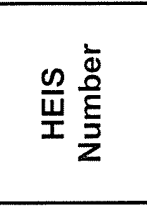 & 嵌 & 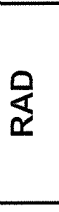 & $\frac{\frac{0}{\pi}}{\frac{0}{20}}$ & 京 & 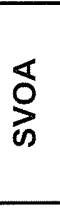 & 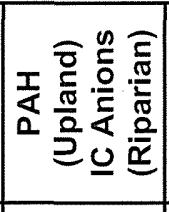 & $\begin{array}{l}\frac{n}{0} \\
\frac{0}{0} \\
\stackrel{0}{0} \\
0 \\
0 \\
0\end{array}$ & 罟 & 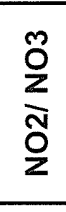 \\
\hline \multirow[t]{5}{*}{ CP-Ref-2 } & J10DR5 & 1 & 1 & 1 & 3 & 3 & 1 & 1 & 1 & N/A \\
\hline & J10DR6 & 1 & 1 & 1 & 1 & 1 & 3 & 1 & 1 & $\mathrm{~N} / \mathrm{A}$ \\
\hline & J10DR7 & 1 & 1 & 1 & 1 & 1 & 1 & 3 & 1 & $\mathrm{~N} / \mathrm{A}$ \\
\hline & J10DR8 & 1 & 1 & 1 & 1 & 1 & 1 & 1 & 3 & N/A \\
\hline & J10DR9 & 1 & 3 & 3 & 1 & 1 & 1 & 1 & 1 & $N / A$ \\
\hline \multirow[t]{5}{*}{ 116-DR-1 \& 2} & J10DR0 & 1 & 3 & 3 & 1 & 1 & 1 & 1 & 1 & $N / A$ \\
\hline & J10DR1 & 1 & 1 & 1 & 3 & 3 & 1 & 1 & 1 & N/A \\
\hline & J10DR2 & 1 & 1 & 1 & 1 & 1 & 3 & 3 & 1 & N/A \\
\hline & J10DR3 & 1 & 1 & 1 & 1 & 1 & 1 & 1 & 3 & N/A \\
\hline & J10DR4 & 1 & 1 & 1 & 1 & 1 & 1 & 1 & 1 & N/A \\
\hline \multirow[t]{5}{*}{ JA Jones } & J10DT0 & 1 & 3 & 1 & 1 & 1 & 1 & 1 & 1 & $N / A$ \\
\hline & J10DT1 & 1 & 1 & 3 & 1 & 1 & 1 & 1 & 1 & $N / A$ \\
\hline & J10DT2 & 1 & 1 & 1 & 3 & 3 & 1 & 1 & 1 & N/A \\
\hline & J10DT3 & 1 & 1 & 1 & 1 & 1 & 3 & 1 & 1 & N/A \\
\hline & J10DT4 & 1 & 1 & 1 & 1 & 1 & 1 & 3 & 3 & $N / A$ \\
\hline \multicolumn{11}{|l|}{ Riparian Sites } \\
\hline \multirow[t]{5}{*}{ Elevated \#3 100-D } & J10DP5 & 1 & 3 & 1 & 1 & 1 & 3 & 1 & 1 & 1 \\
\hline & J10DP6 & 1 & 1 & 3 & 3 & 1 & 1 & 1 & 1 & 1 \\
\hline & J10DP7 & 1 & 1 & 1 & 1 & 3 & 1 & 1 & 1 & 3 \\
\hline & J10DP8 & 1 & 1 & 1 & 1 & 1 & 1 & 3 & 1 & 1 \\
\hline & J10DP9 & 1 & 1 & 1 & 1 & 1 & 1 & 1 & 3 & 1 \\
\hline
\end{tabular}




\begin{tabular}{|c|c|c|c|c|c|c|c|c|c|c|}
\hline 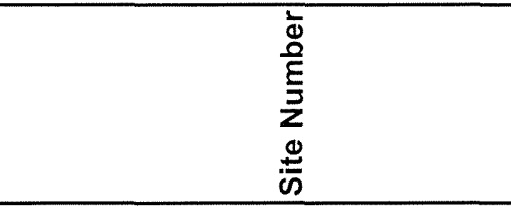 & 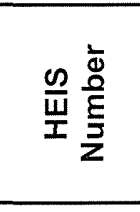 & 岃 & 定 & $\frac{\frac{\omega}{\pi}}{\frac{\pi}{\Sigma}}$ & 竝 & $\overbrace{\text { os }}^{\delta}$ & 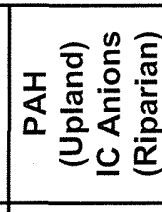 & 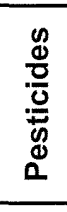 & $\begin{array}{l}\text { Oी } \\
0\end{array}$ & $\begin{array}{l}\text { o } \\
\text { ล̃ } \\
\text { ô }\end{array}$ \\
\hline & J10LR8 & N/A & N/A & 1 & 1 & 1 & 1 & 1 & 1 & 0 \\
\hline \multirow[t]{5}{*}{ Low \#10 } & J1OLJO & 1 & 3 & 1 & 1 & 1 & 1 & 1 & 1 & 1 \\
\hline & J10LJ1 & 1 & 1 & 3 & 3 & 1 & 1 & 1 & 1 & 1 \\
\hline & J10LJ2 & 1 & 1 & 1 & 1 & 3 & 3 & 1 & 1 & 1 \\
\hline & J10LJ3 & 1 & 1 & 1 & 1 & 1 & 1 & 3 & 1 & 1 \\
\hline & J10LJ4 & 1 & 1 & 1 & 1 & 1 & 1 & 1 & 3 & 3 \\
\hline \multirow[t]{5}{*}{ Vernita Bridge Reference Site \#13 } & J10DN5 & 1 & 1 & 1 & 1 & 1 & 1 & 1 & 1 & 1 \\
\hline & J10DN6 & 1 & 1 & 1 & 3 & 3 & 1 & 1 & 1 & 1 \\
\hline & J10DN7 & 1 & 3 & 3 & 1 & 1 & 1 & 1 & 1 & 1 \\
\hline & J10DN8 & 1 & 1 & 1 & 1 & 1 & 3 & 1 & 1 & 3 \\
\hline & J1ODN9 & 1 & 1 & 1 & 1 & 1 & 1 & 3 & 3 & 1 \\
\hline
\end{tabular}


Table B-3. HEIS Numbers: Composite Site Samples for Bio-Assay and Selected General Chemistry \& Physical Properties Analysis.

\begin{tabular}{|l|l|}
\hline \multicolumn{1}{|c|}{ Site } & HEIS NUMBER \\
\hline 600-131 & J10DW4 \\
\hline Pit 23 & J10DV4 \\
\hline 100-F-2 & J10DT8 \\
\hline CP-Ref-2 & J10DN8 \\
\hline 116-DR-1 \& 2 & J10DN7 \\
\hline JA Jones & J10DN9 \\
\hline Elevated \# 3 100-D & J10DN6 \\
\hline Low \# 10 & J10LJ5 \\
\hline Ref. Site \# 13 & J10DT9 \\
\hline
\end{tabular}




\section{APPENDIX C}

\section{0/300 AREA GROUNDWATER SAMPLING WELL LOCATIONS}


WCH-85

Rev. 0 
Table C-1. 100/300 Area Groundwater Sampling Risk Assessment Well Locations. (2 Pages)

\begin{tabular}{|l|c|c|}
\hline Well Name & Northing & Easting \\
\hline $199-\mathrm{D} 2-6$ & 151119.86 & 573000.21 \\
\hline $199-\mathrm{D} 3-2$ & 151165.653 & 572453.955 \\
\hline $199-\mathrm{D} 4-20$ & 151257.474 & 572794.042 \\
\hline $199-\mathrm{D} 4-22$ & 151539.293 & 572788.453 \\
\hline $199-\mathrm{D} 4-23$ & 151592.87 & 572672.455 \\
\hline $199-\mathrm{D} 5-13$ & 151955.183 & 573535.527 \\
\hline $199-\mathrm{D} 5-16$ & 151652.51 & 573917.45 \\
\hline $199-\mathrm{D} 5-17$ & 151322.83 & 573730.52 \\
\hline $199-\mathrm{D} 5-36$ & 151746.32 & 572909.823 \\
\hline $199-\mathrm{D} 5-43$ & 151269.43 & 573179.976 \\
\hline $199-\mathrm{F} 5-1$ & 147736.865 & 581250.123 \\
\hline $199-\mathrm{F} 5-6$ & 148041.992 & 580901.652 \\
\hline $199-\mathrm{H} 3-2 \mathrm{C}$ & 152750.302 & 577632.065 \\
\hline $199-\mathrm{H} 4-10$ & 153155.813 & 577827.209 \\
\hline $199-\mathrm{H} 4-13$ & 152595.273 & 578219.304 \\
\hline $199-\mathrm{H} 4-16$ & 152591.573 & 577981.91 \\
\hline $199-\mathrm{H} 4-4$ & 152853.956 & 578060.859 \\
\hline $199-\mathrm{H} 4-46$ & 152439.868 & 577883.861 \\
\hline $199-\mathrm{H} 4-5$ & 152939.783 & 577944.937 \\
\hline $199-\mathrm{H} 5-1 \mathrm{~A}$ & 152257.724 & 577650.08 \\
\hline $199-\mathrm{H} 6-1$ & 152247.63 & 578236.557 \\
\hline $199-\mathrm{K}-18$ & 147400.811 & 569353.687 \\
\hline $199-\mathrm{K}-20$ & 147687.239 & 569520.516 \\
\hline $199-\mathrm{K}-21$ & 147932.06 & 569769.902 \\
\hline $199-\mathrm{K}-27$ & 146763.797 & 569155.956 \\
\hline $199-\mathrm{N}-64$ & 149551.177 & 571561.412 \\
\hline $199-\mathrm{N}-96 \mathrm{~A}$ & 149800.762 & 591213.477 \\
\hline $199-\mathrm{N}-99 \mathrm{~A}$ & 150151.407 & \\
\hline $399-1-11$ & 116660.156 & 59398.332 \\
\hline $399-1-15$ & 116964.243 & \\
\hline $399-1-2$ & 116329.576 & \\
\hline
\end{tabular}


WCH-85

Rev. 0

Table C-1. 100/300 Area Groundwater Sampling Risk Assessment Well Locations. (2 Pages)

\begin{tabular}{|l|c|c|}
\hline Well Name & Northing $^{*}$ & Easting $^{*}$ \\
\hline $399-2-2$ & 116282.618 & 594385.728 \\
\hline $399-3-6$ & 115944.403 & 593927.103 \\
\hline $399-4-1$ & 115537.349 & 594274.1 \\
\hline
\end{tabular}

*NAD 1983 Washington State Plane South 4602 


\section{APPENDIX D}

LOCATIONS OF MULTI-INCREMENTAL SAMPLING INVESTIGATION AREAS AND GROUNDWATER WELLS SAMPLED IN FALL 2005 
WCH-85

Rev. 0 


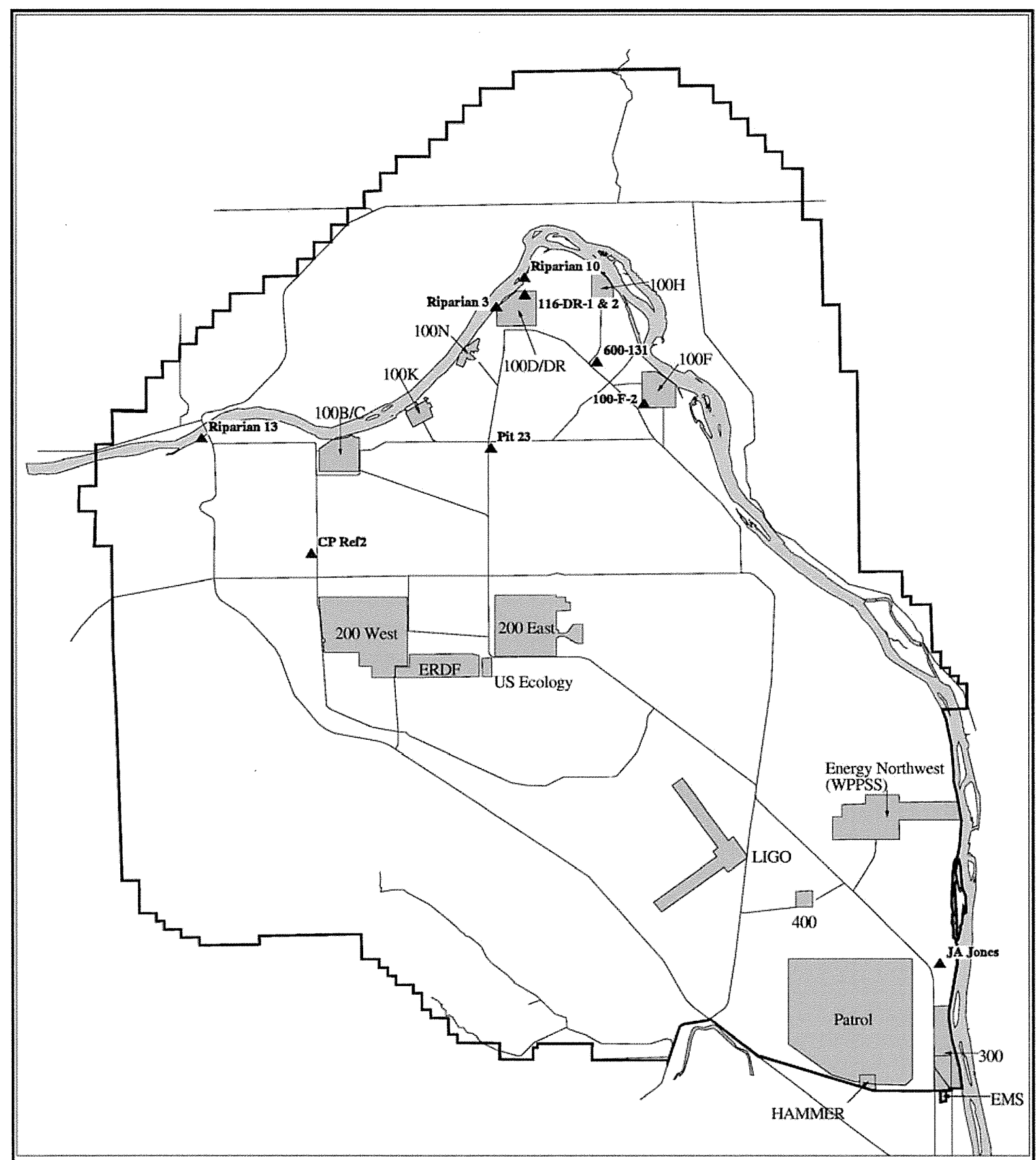

Fall Sampling Locations

- Study Plots

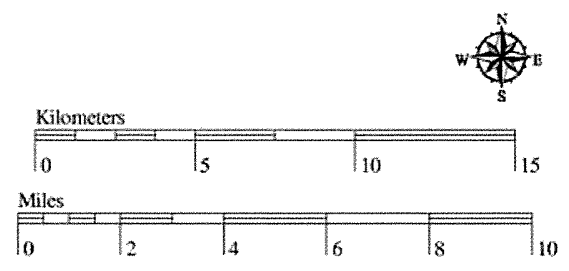

ERC:rkz:02/23/06:/gishome1/rkzufelt/fall_samp_locs_over.aml:Rev. 1 Database: 02/28/06 0:17 AM 


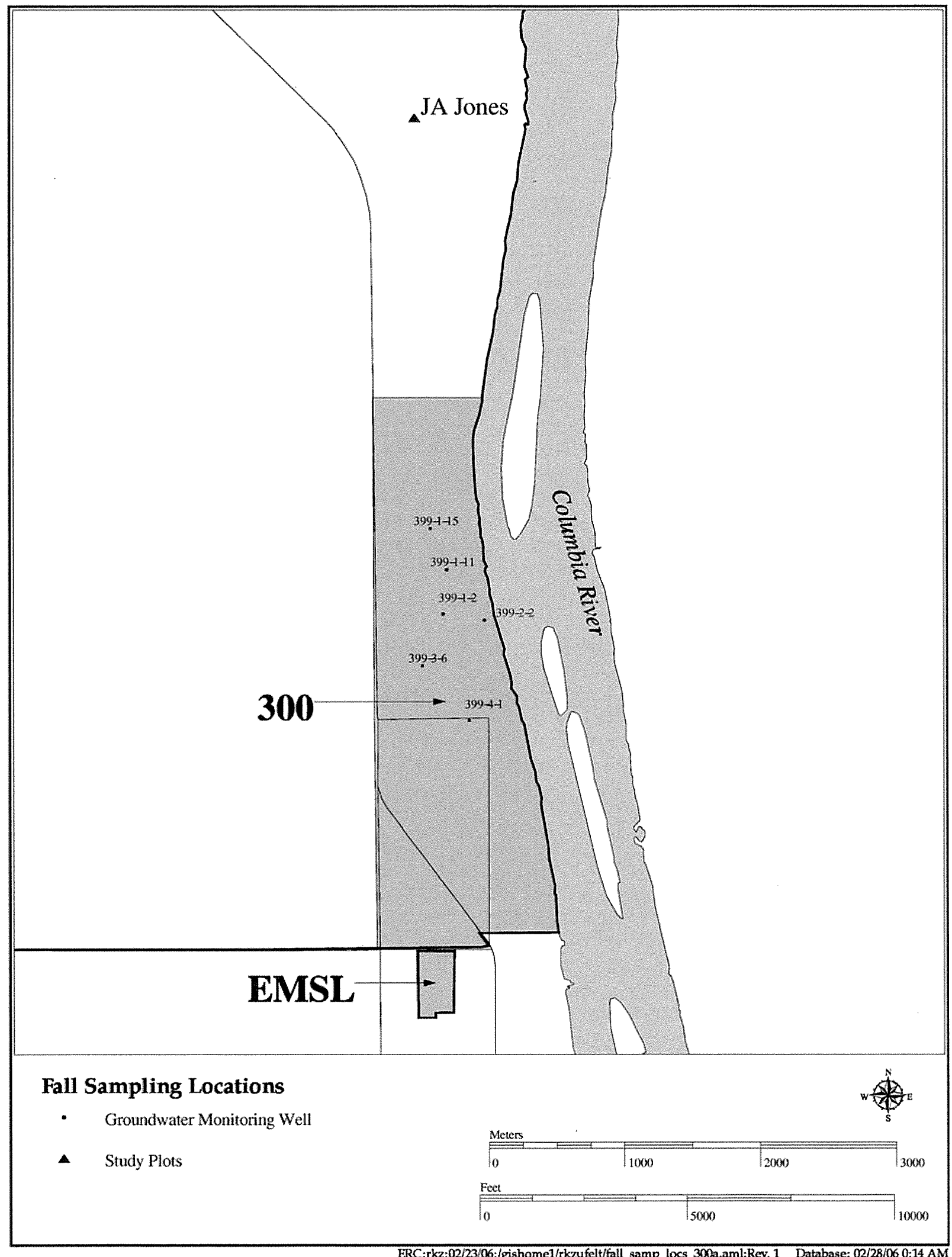

ERC:rkz:02/2306:/gishome1/rkzufelt/fall samp locs 300a.aml:Rev. 1 Database: 02/28/06 0:14 AM 


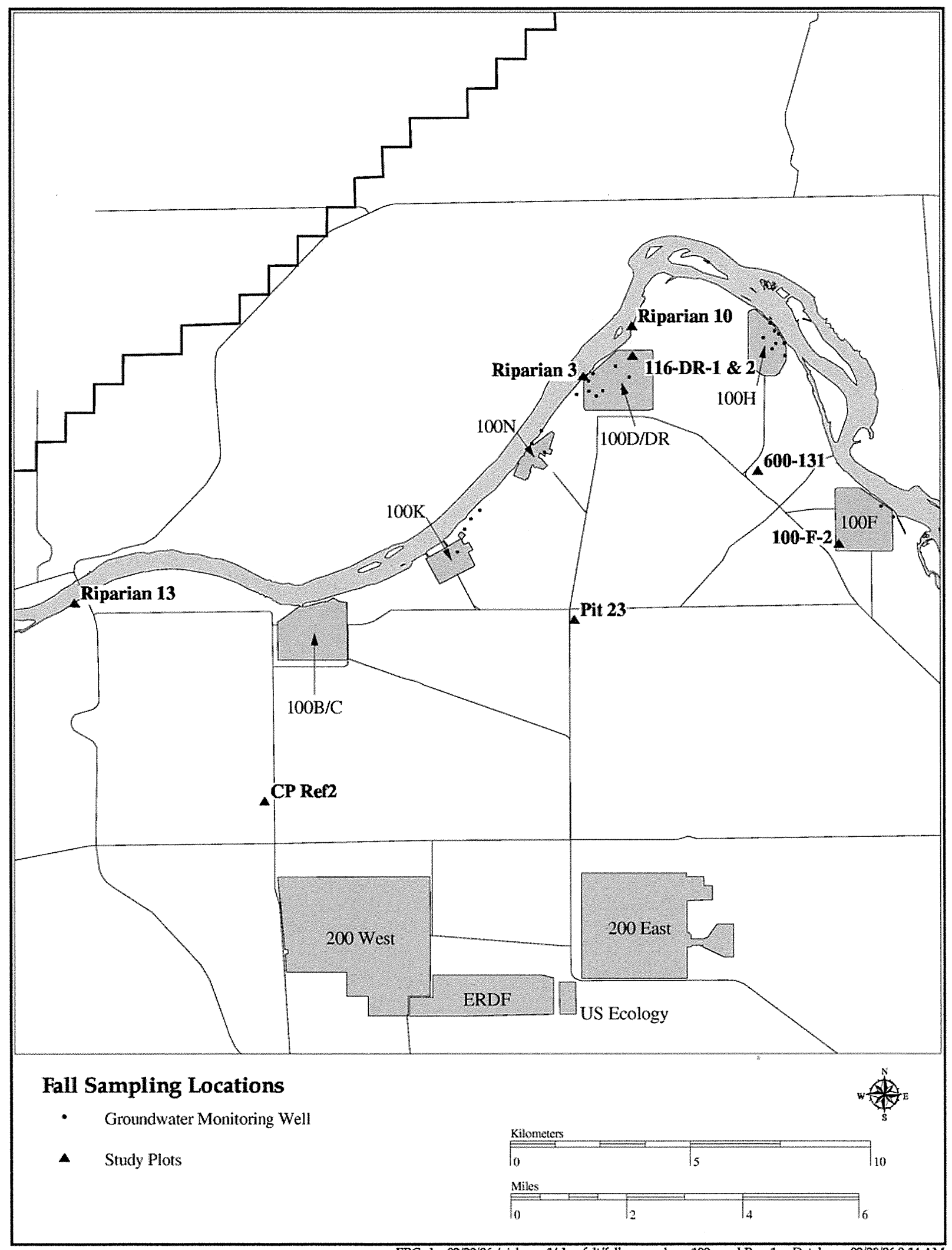

ERC:rkz:02/23/06:/gishome1/rkzufelt/fall_samp_locs_100a.aml:Rey. 1 Database: 02/28/06 0:14 AM 


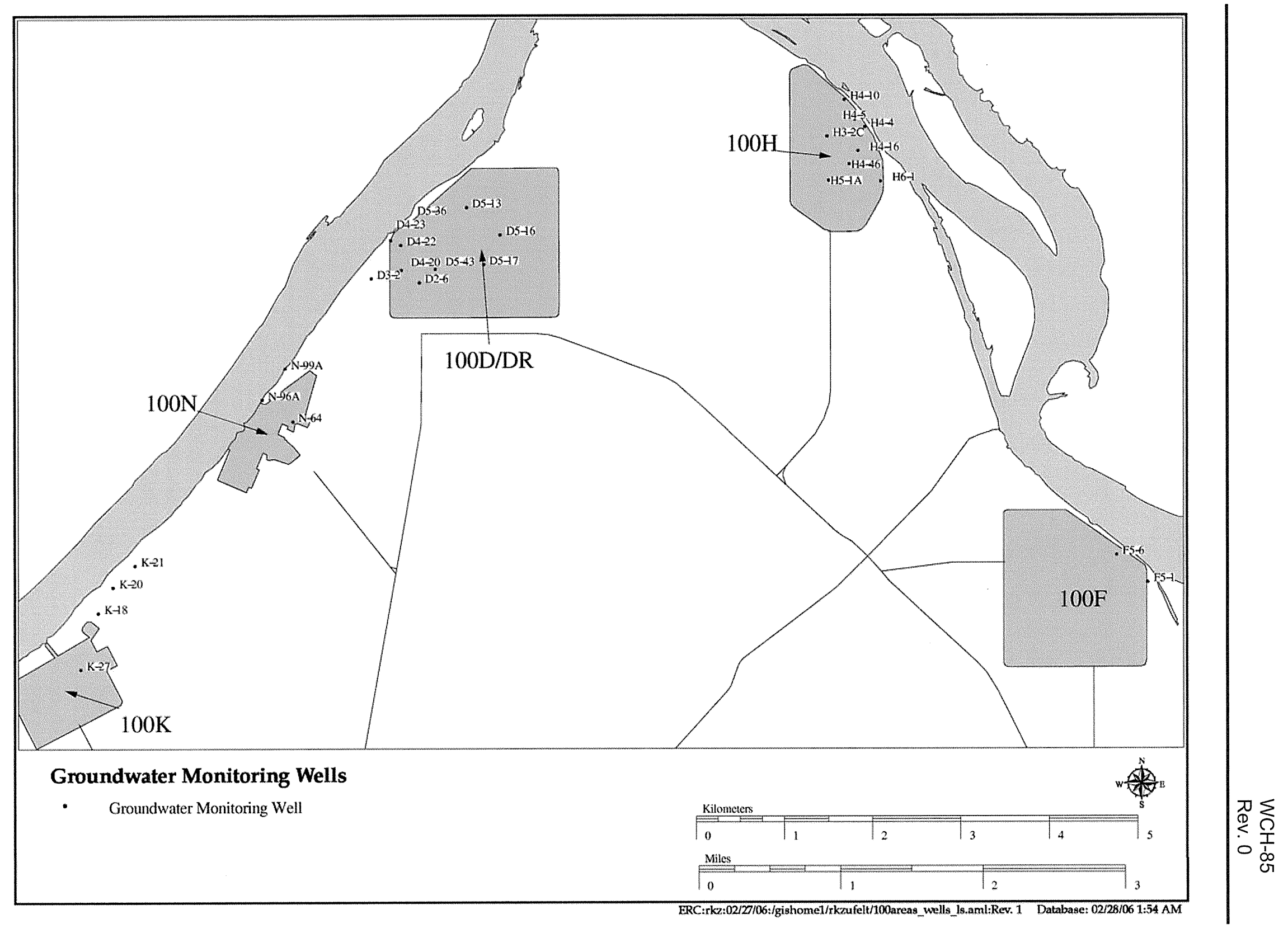




\section{APPENDIX E}

DATA SUMMARY OF MULTI-INCREMENTAL SAMPLING RESULTS 
WCH-85

Rev. 0 
MIS 05 SAMPLES

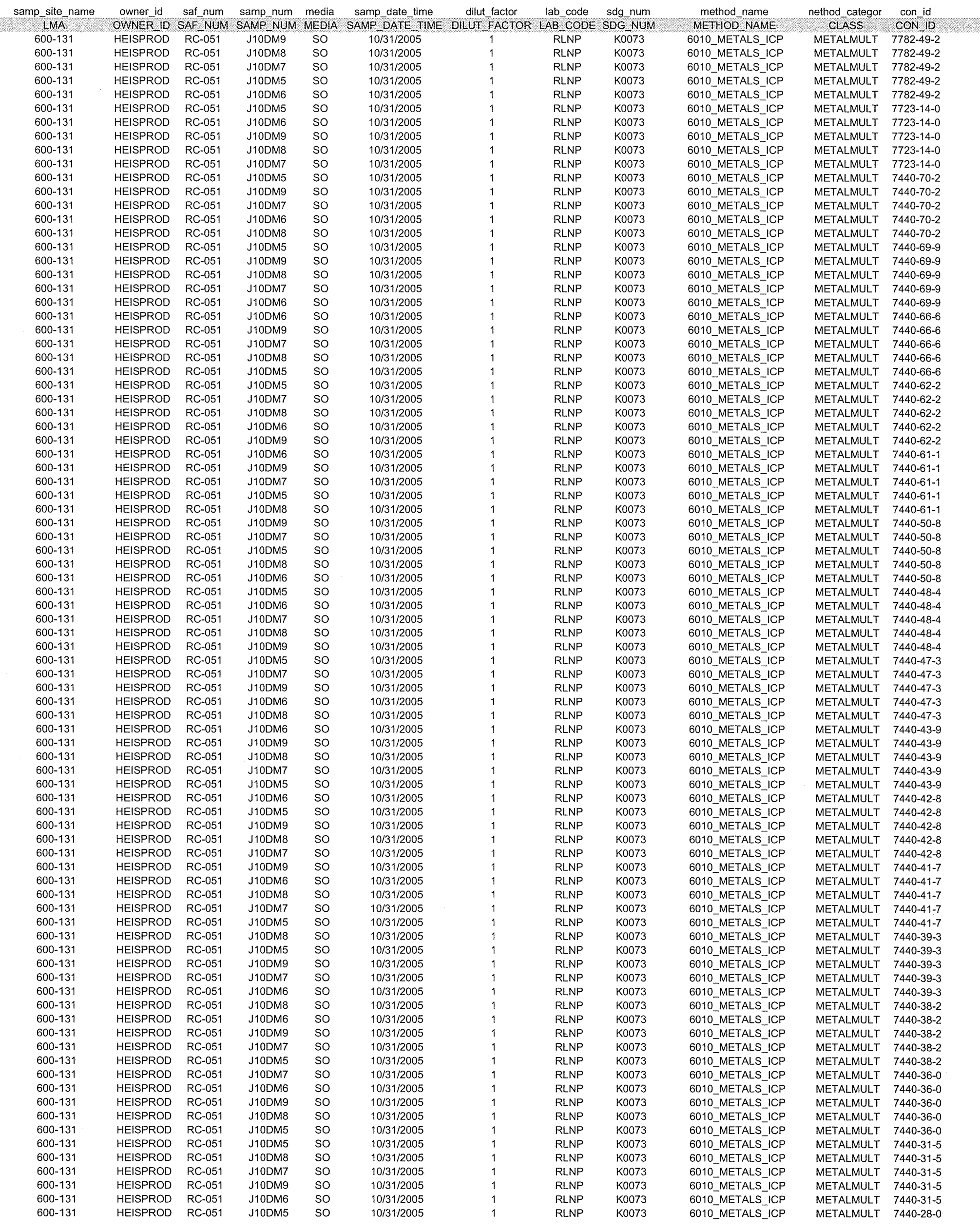

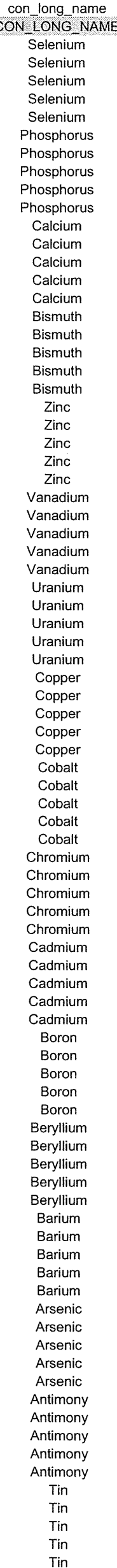

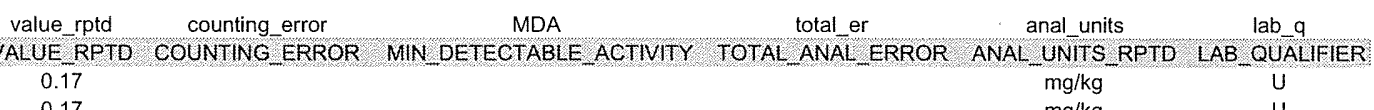

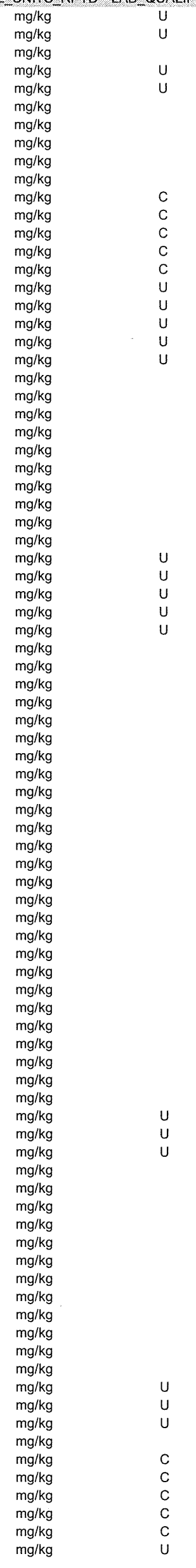


MIS_05 SAMPLES

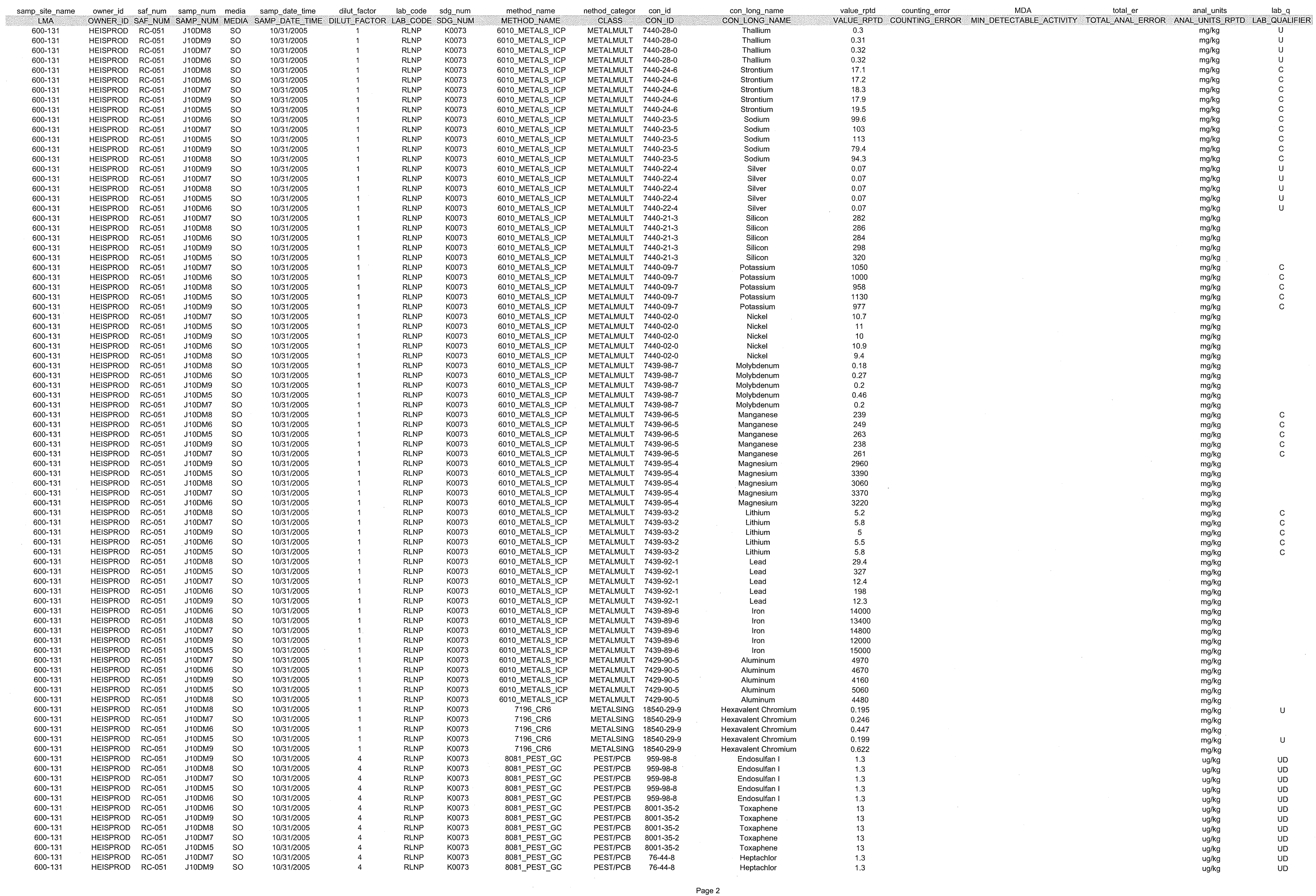


MIS_05 SAMPLES

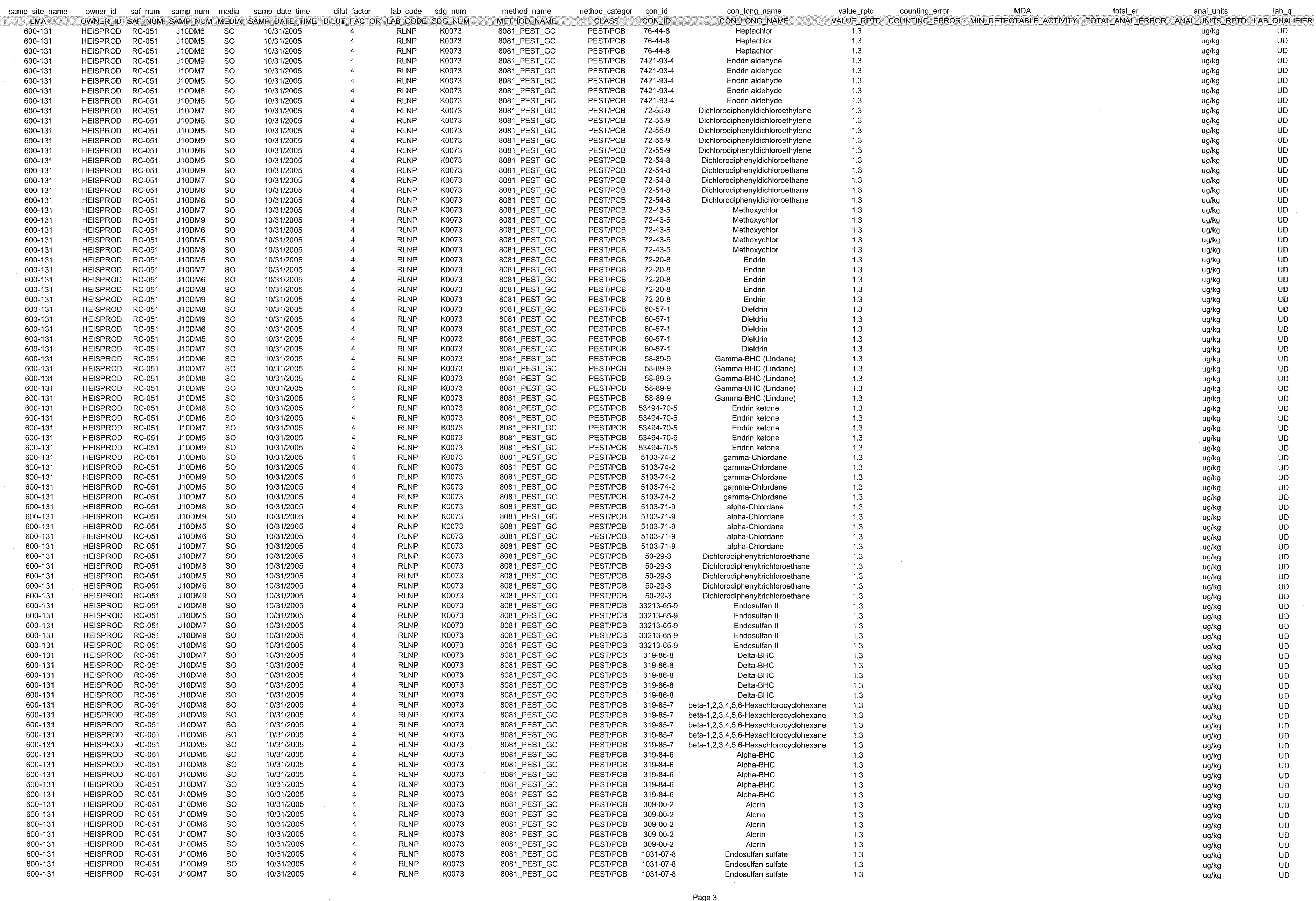


MIS_05 SAMPLES

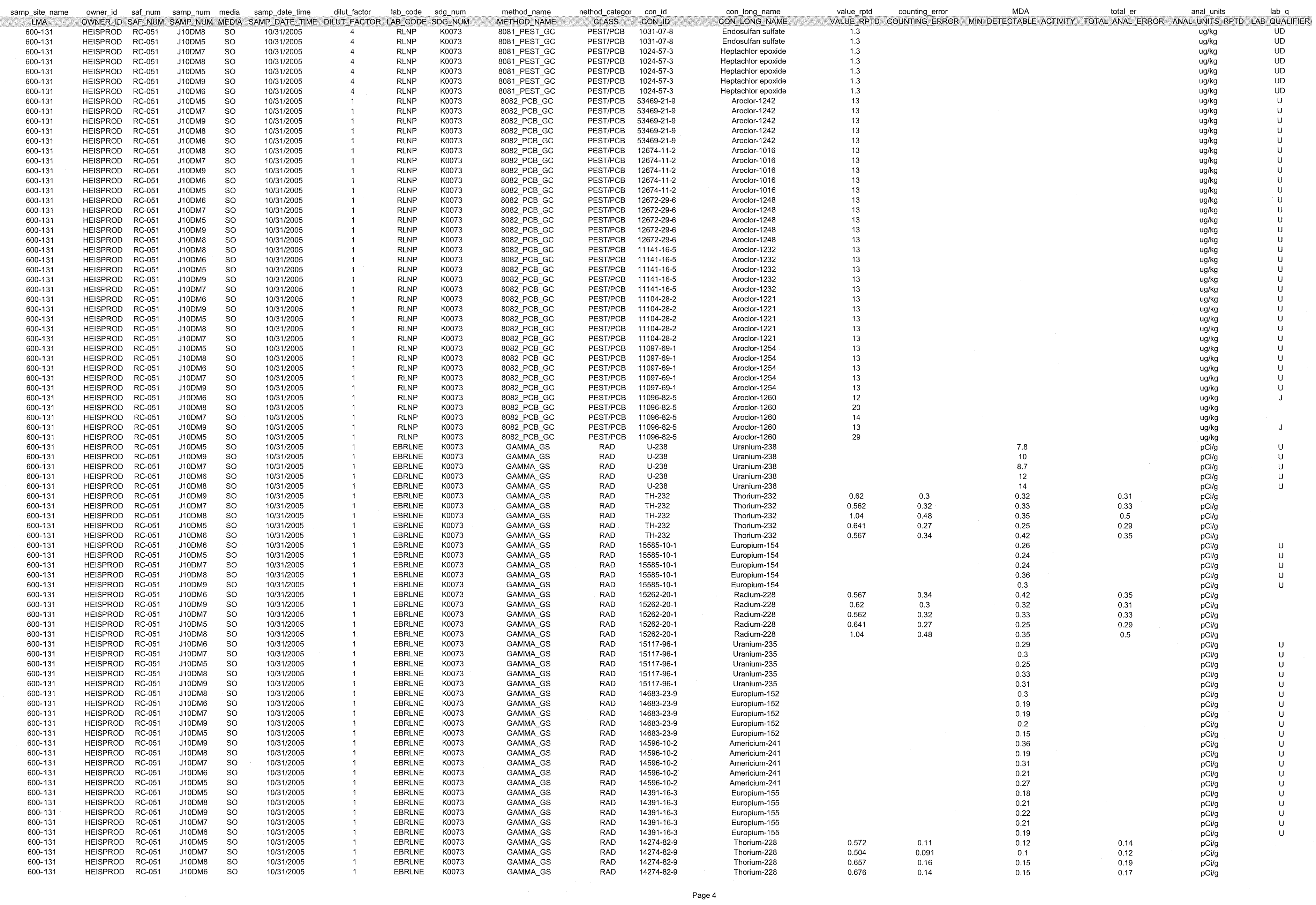




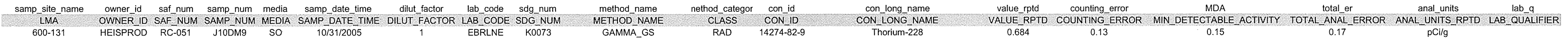

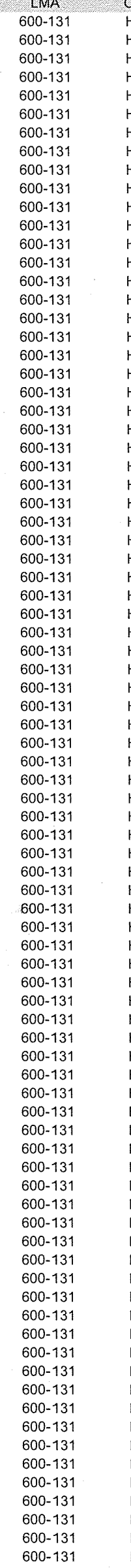

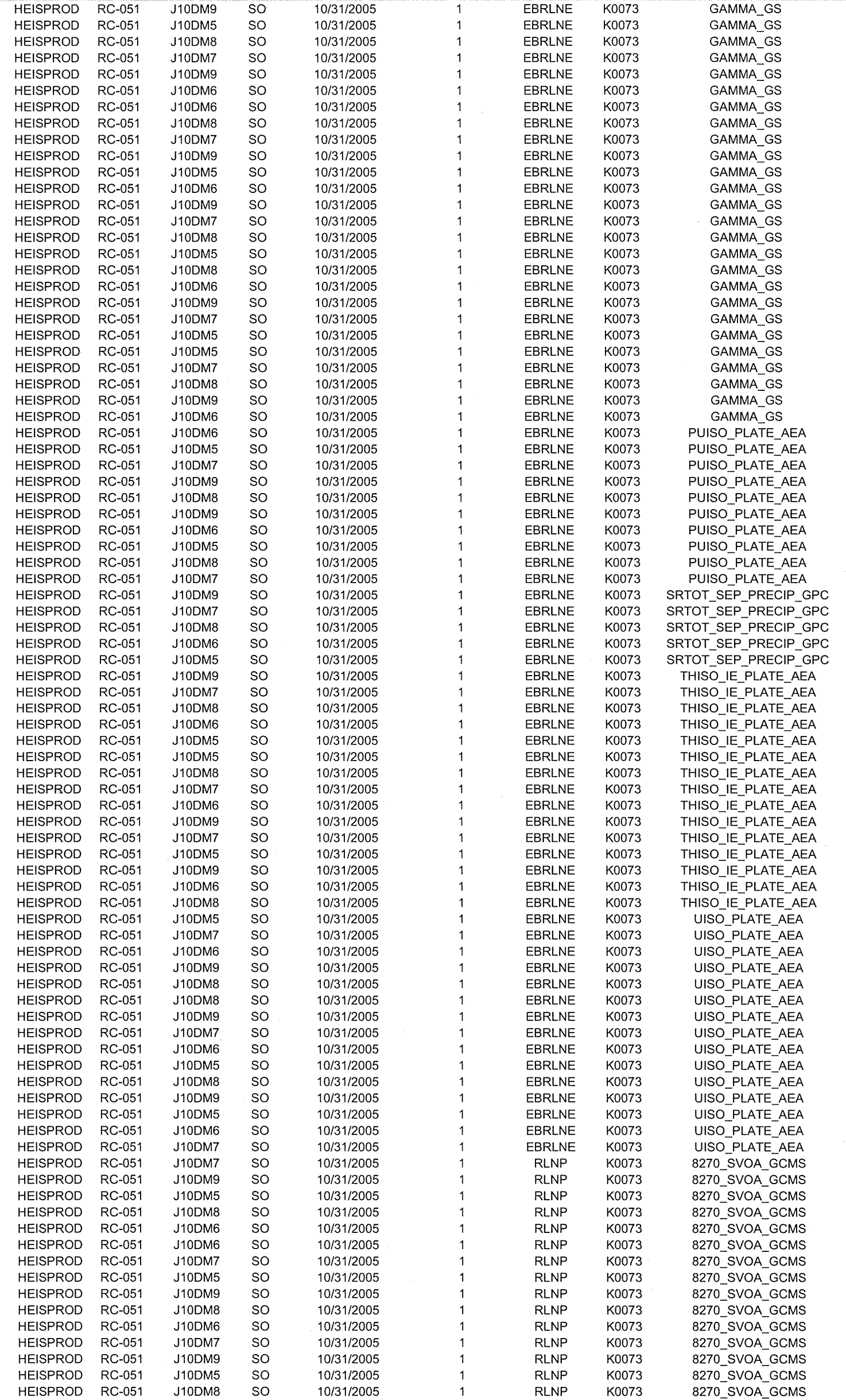

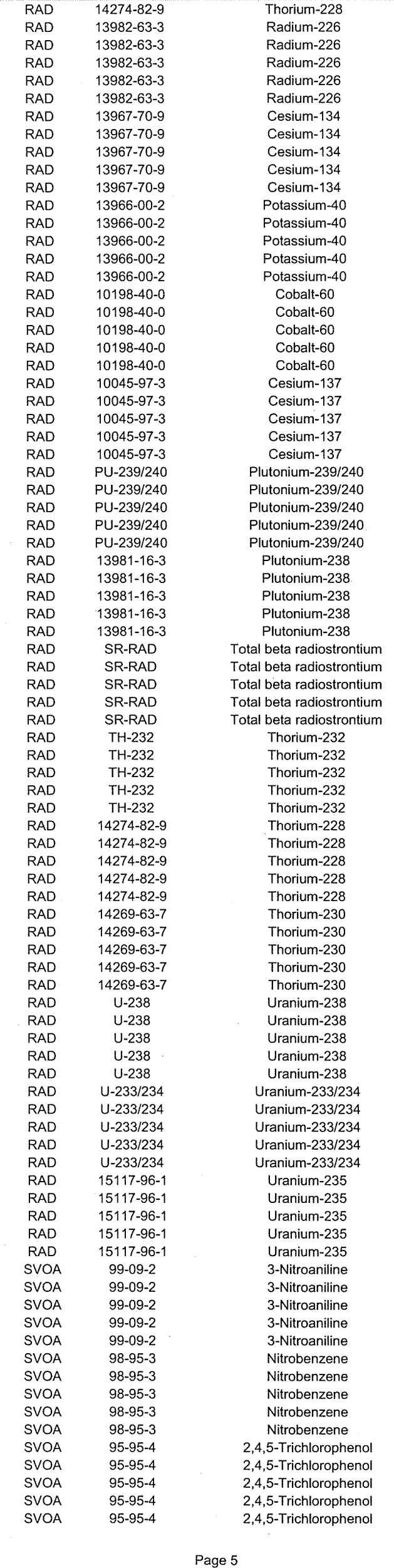

$\begin{array}{ll}0.684 & 0.13 \\ 0.488 & 0.13\end{array}$

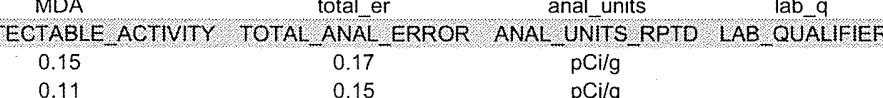


MIS_05 SAMPLES

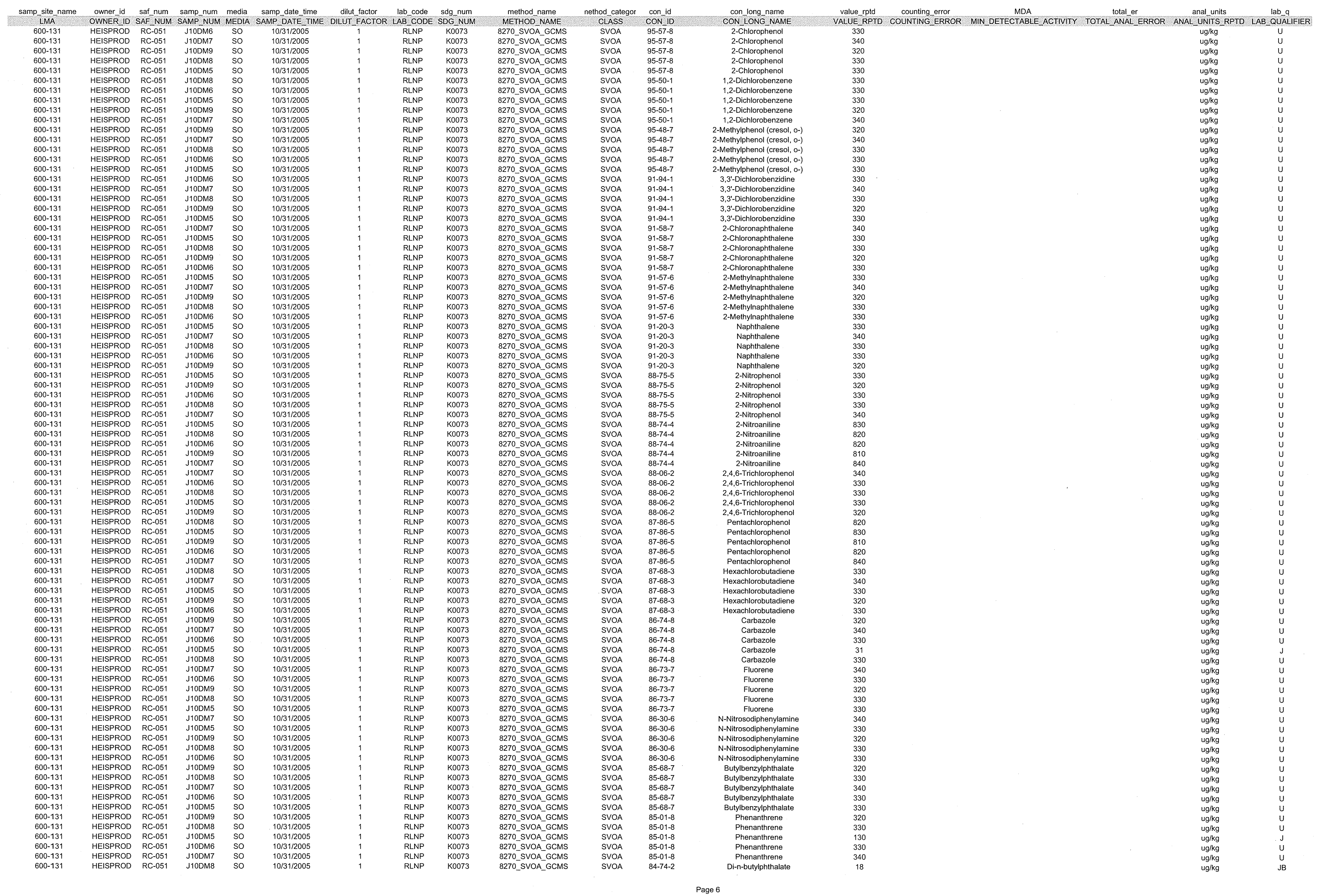


MIS_05 SAMPLES

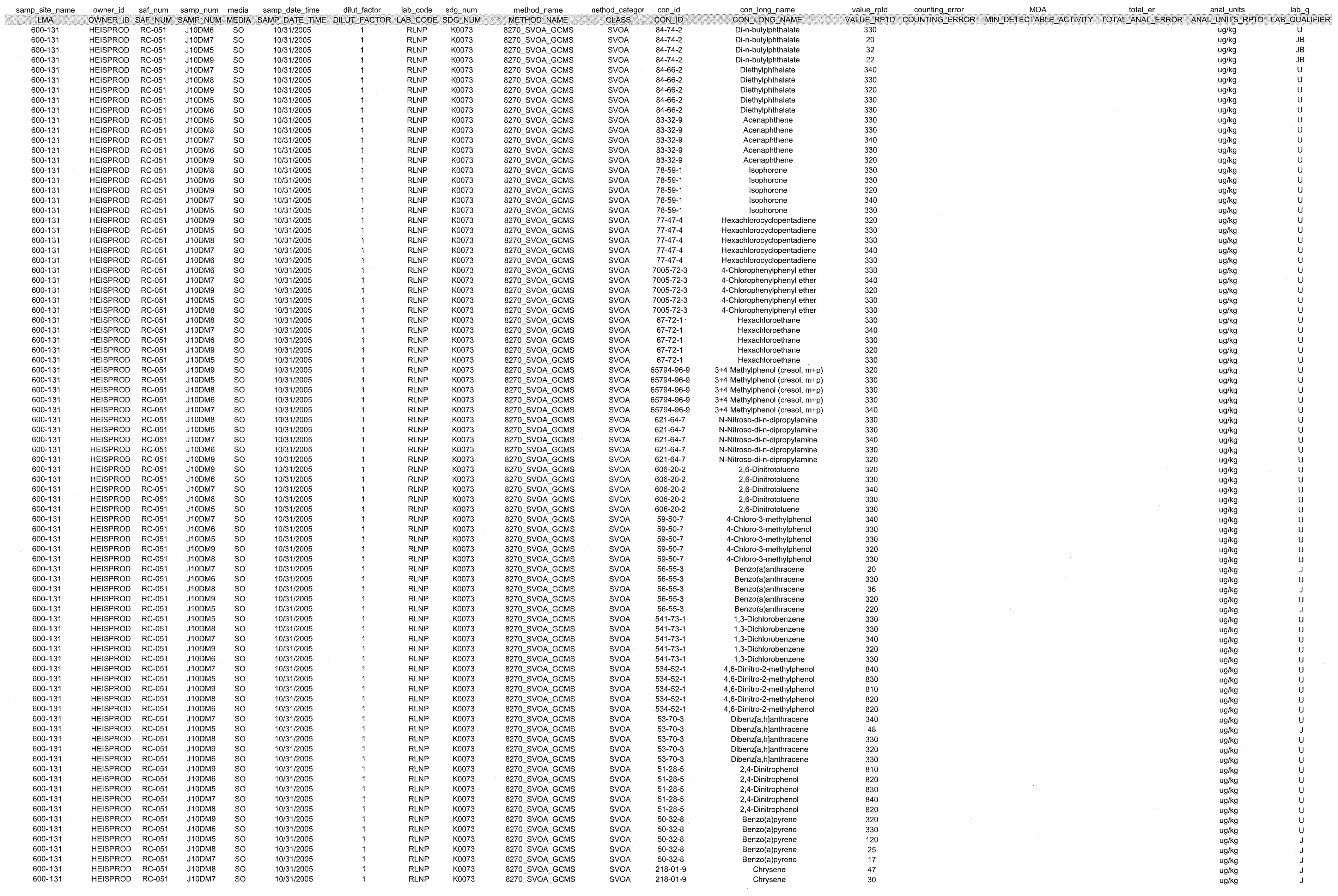




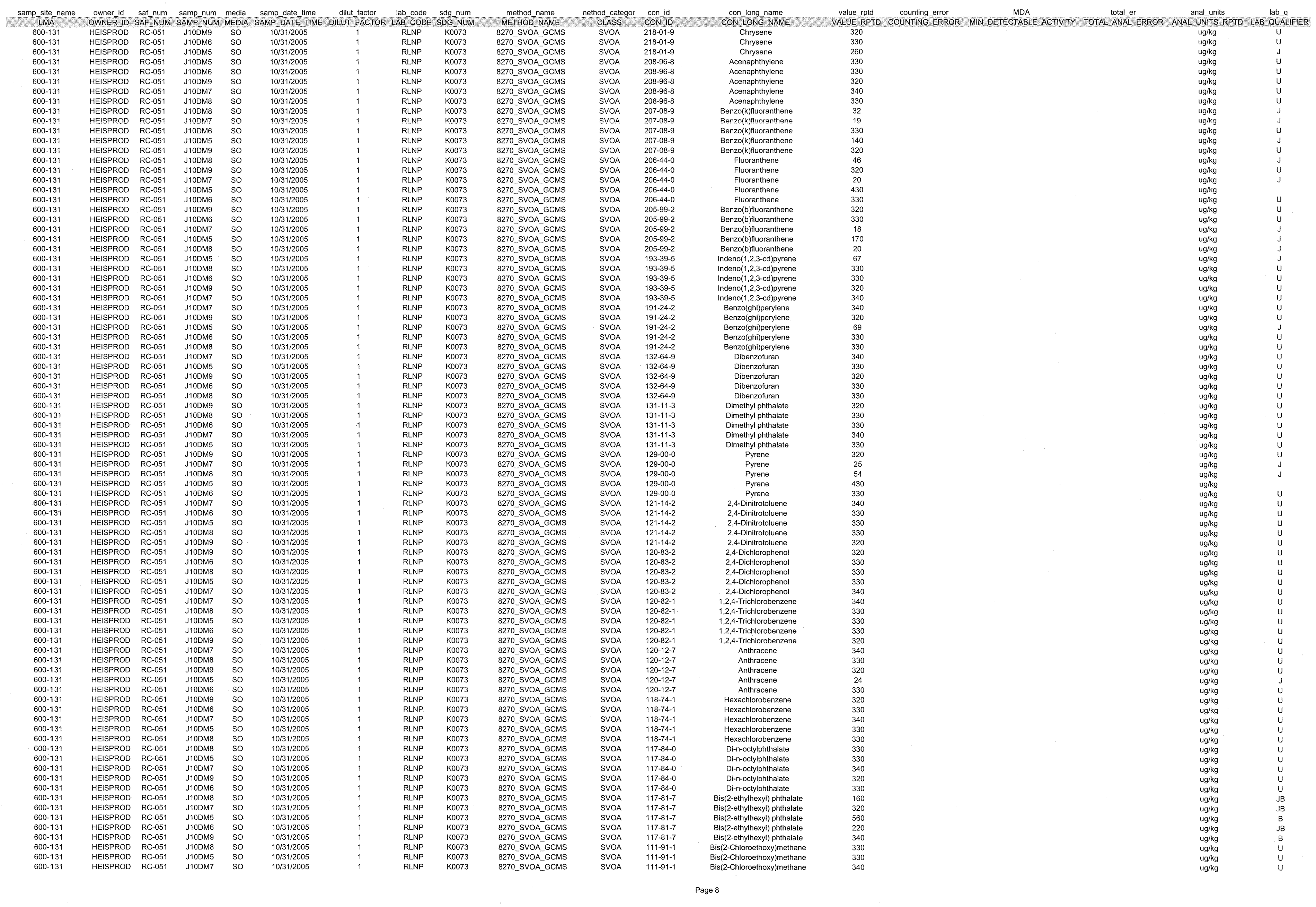


MIS_05 SAMPLES

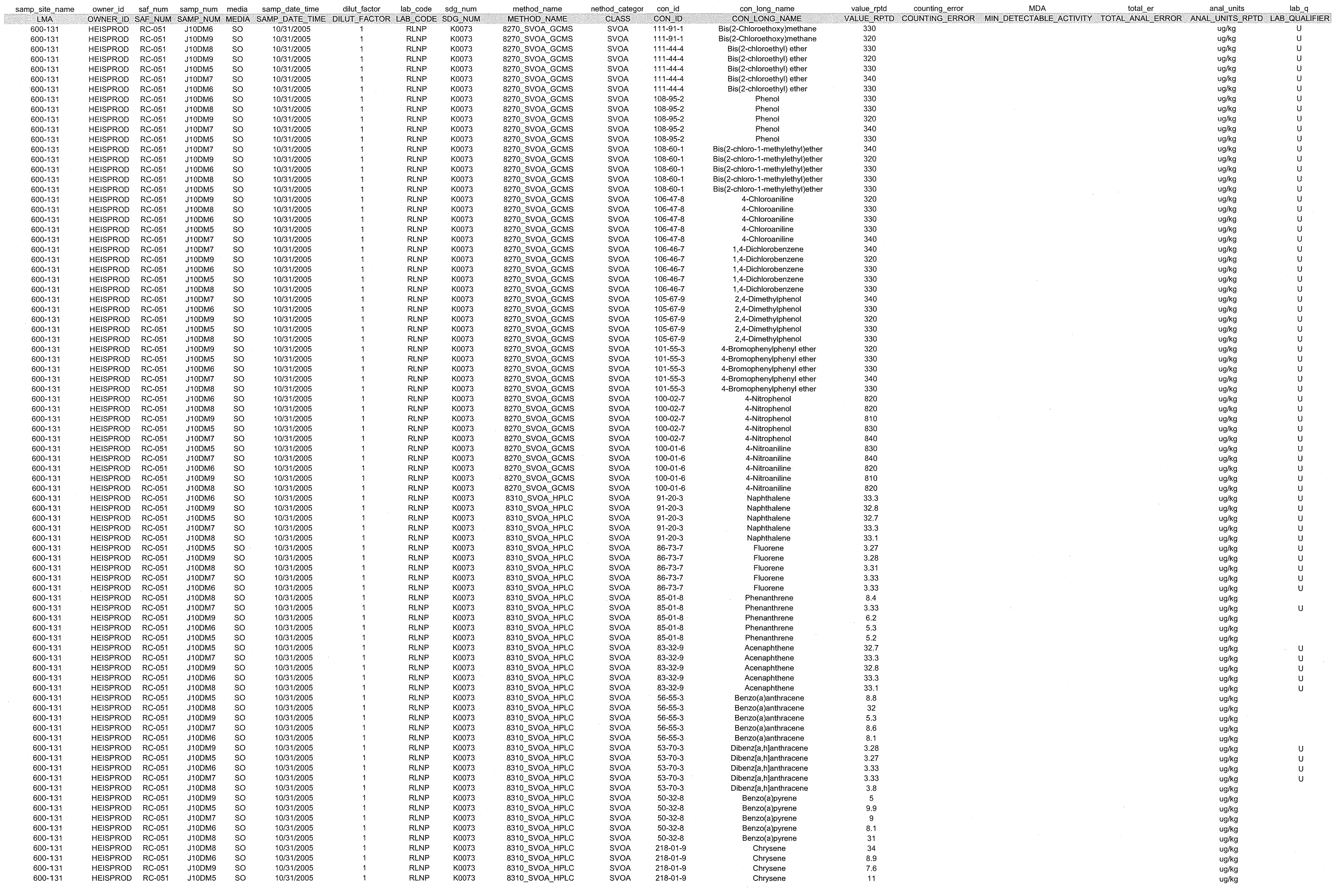

Page 9 
MIS_05 SAMPLES

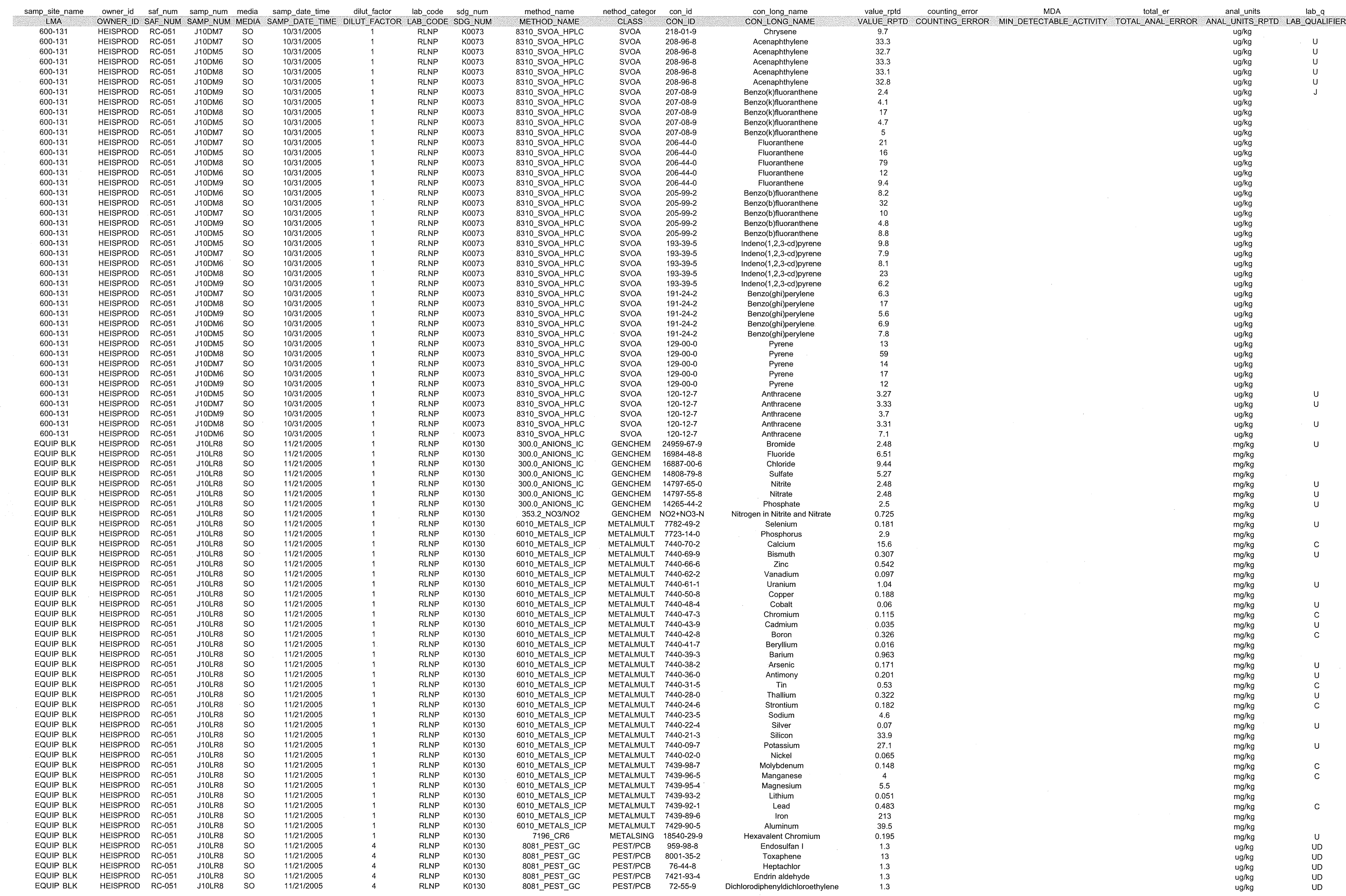


MIS 05 SAMPLES

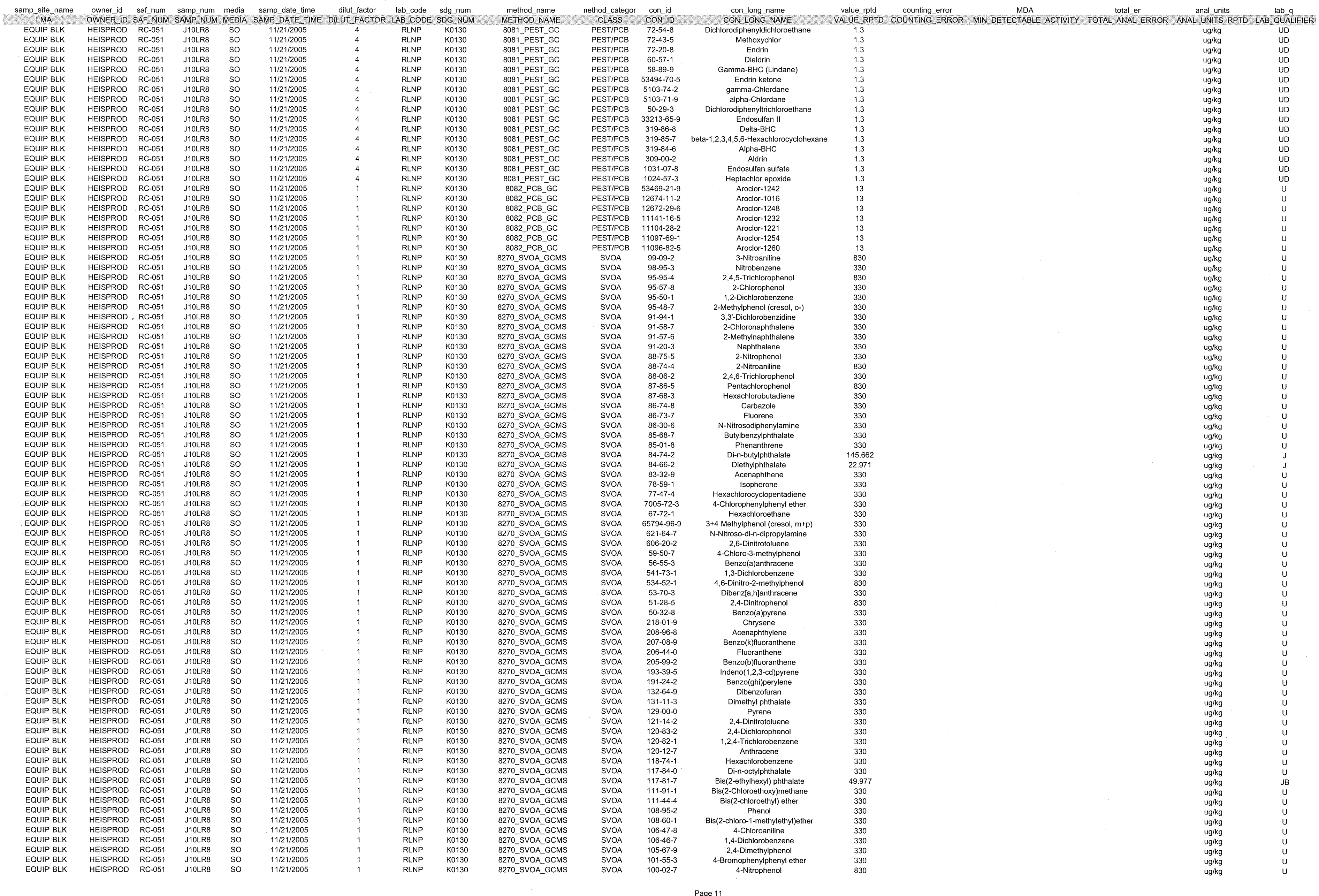


MIS_05 SAMPLES

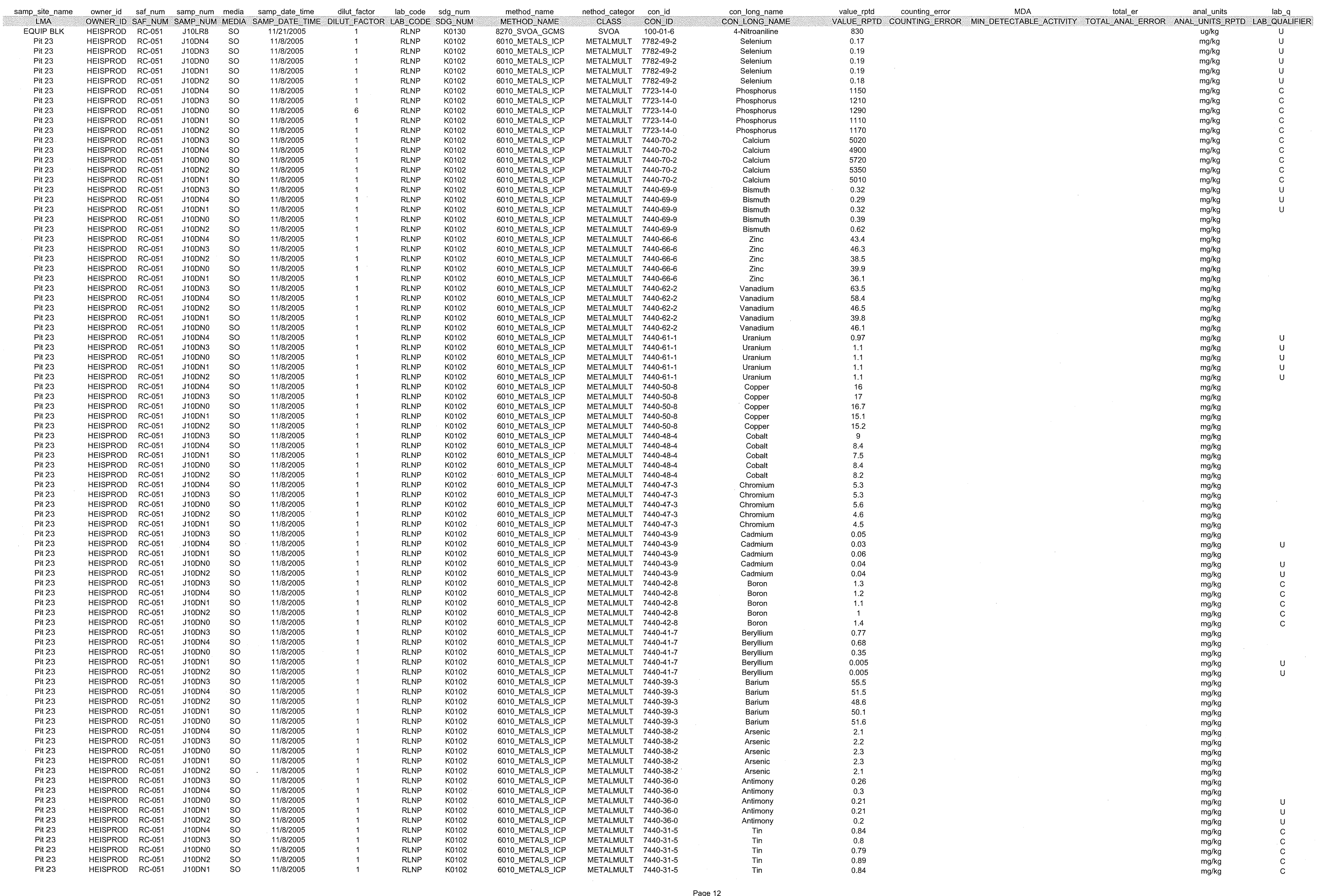


MIS_05 SAMPLES

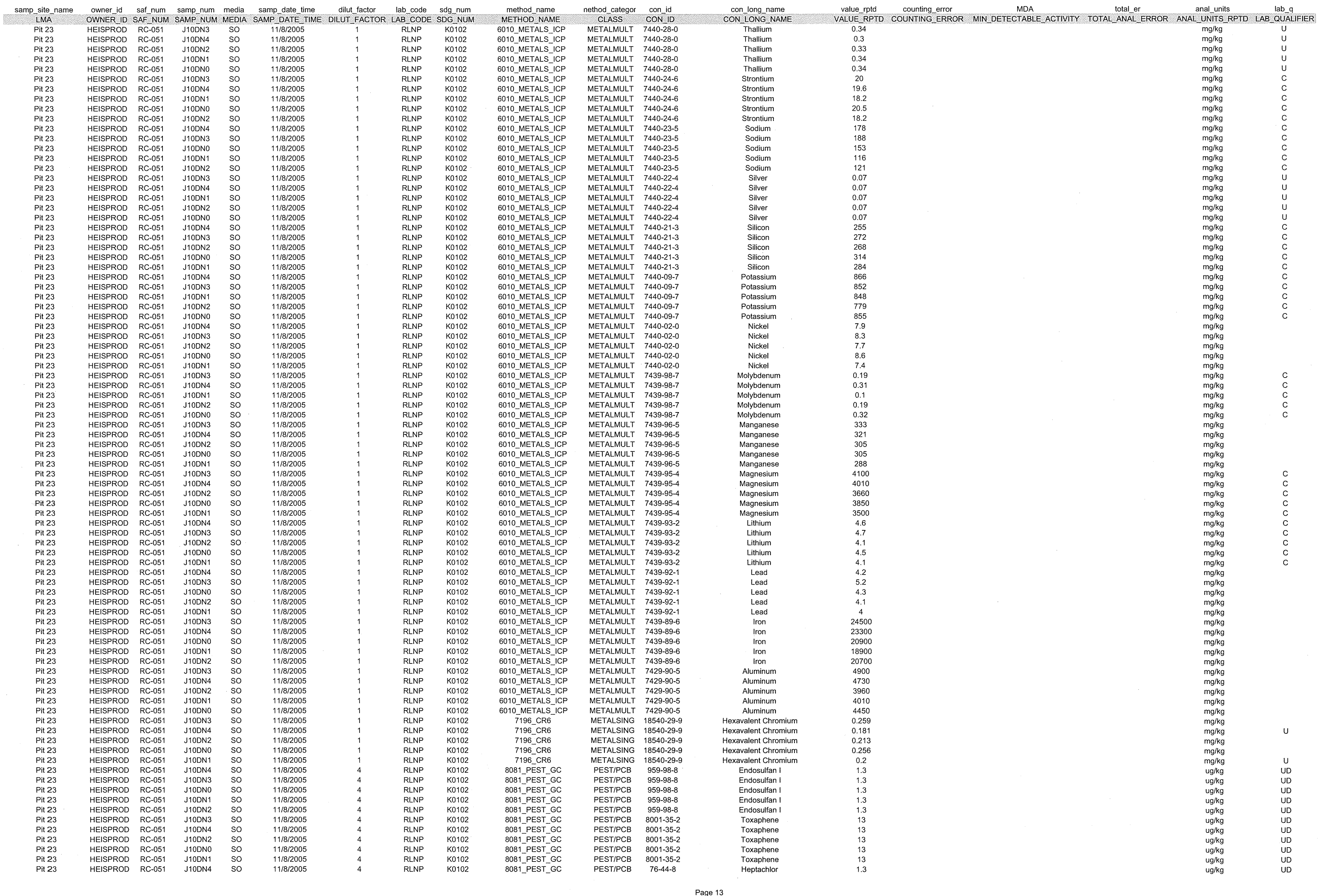


MIS_05 SAMPLES

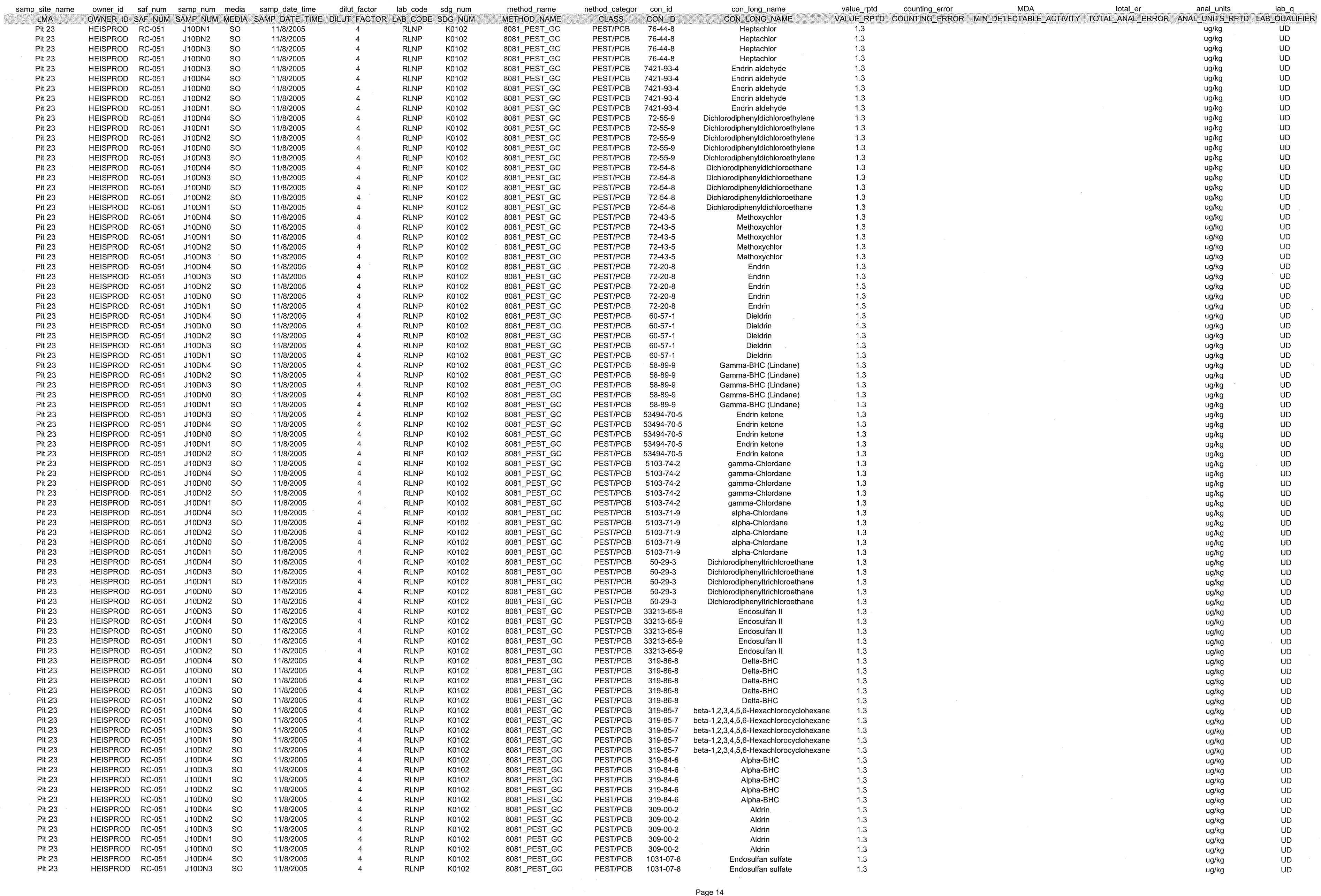


MIS_05 SAMPLES

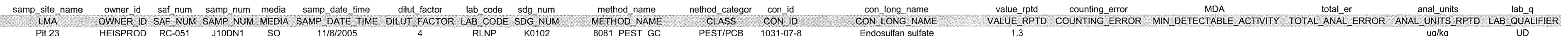

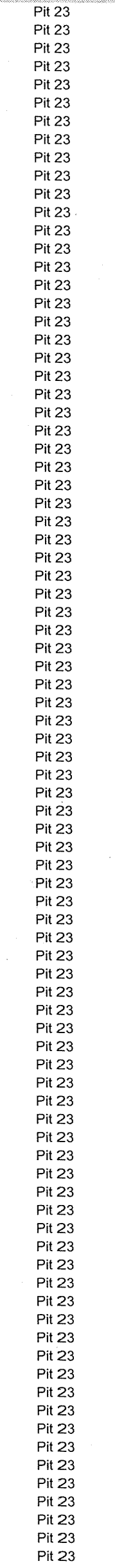

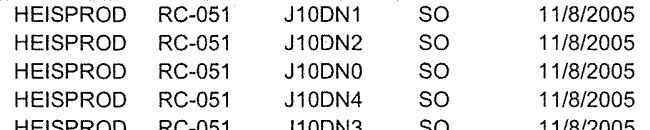

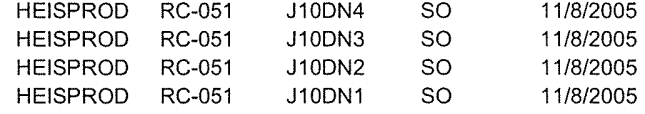

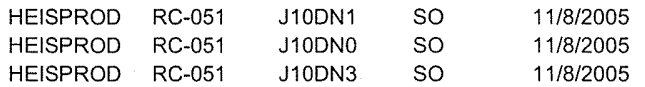

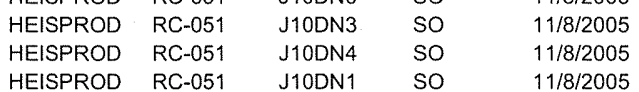

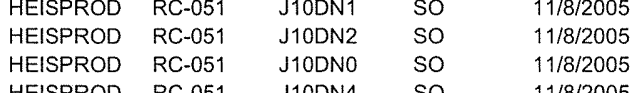

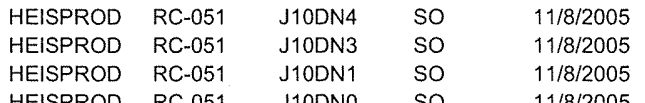

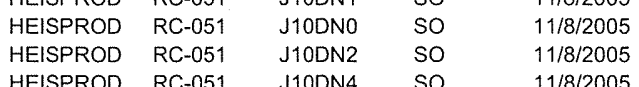

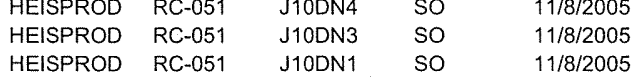

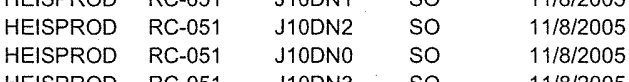

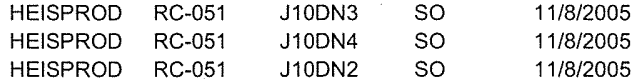

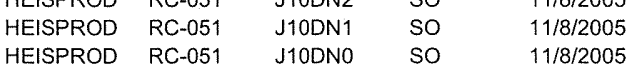

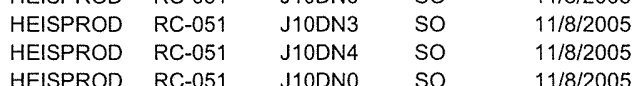

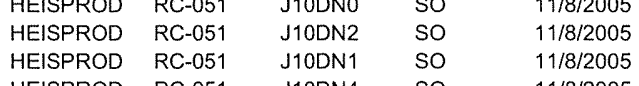

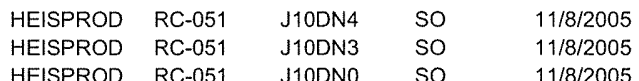

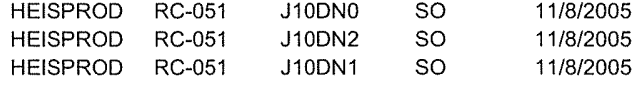

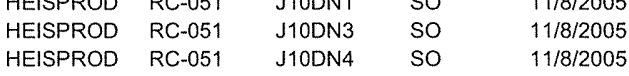

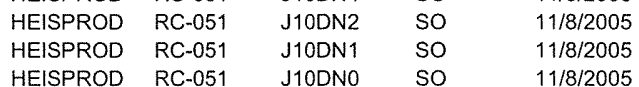

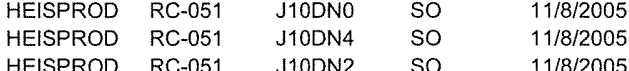

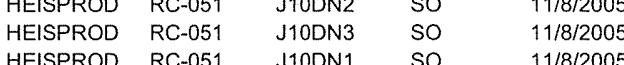

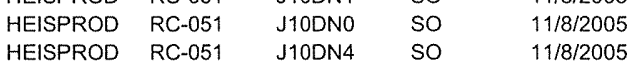

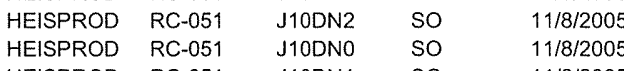

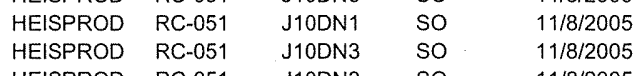
$\begin{array}{lllll}\text { HEISPROD } & \text { RC-051 } & \text { J10DN3 } & \text { SO } & 11 / 1822005 \\ \text { HEISPROD } & \text { RC-051 } & \text { J100N4 } & \text { SO } & 11 / 822005\end{array}$

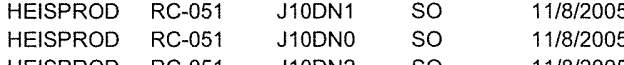

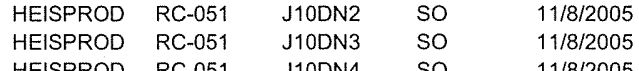

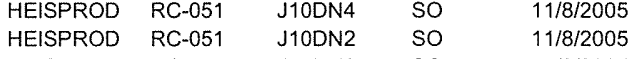

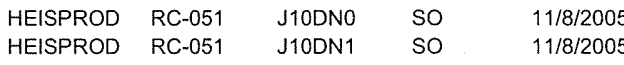

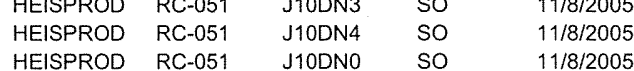

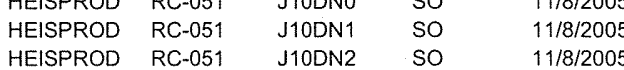

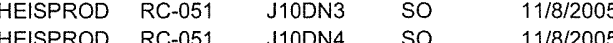

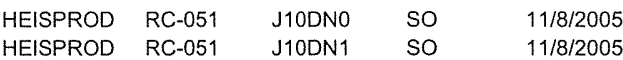

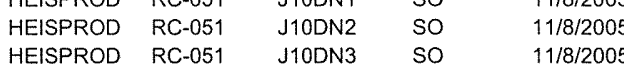

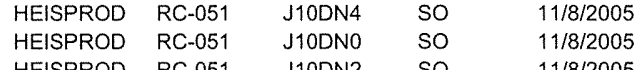

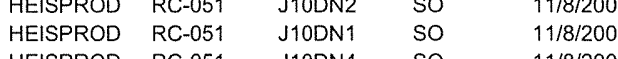
$\begin{array}{lllll}\text { HEISPROD } & \text { RC-051 } & \text { J10DN } 3 & \text { SO } & 11 / 8212005 \\ \text { SO } & 111822005\end{array}$

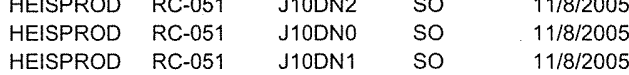

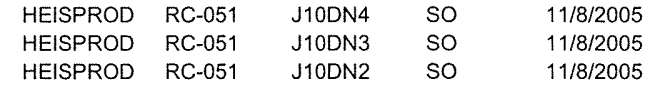

\begin{tabular}{|c|c|c|}
\hline NP & & \\
\hline & & 8081_PEST_GC \\
\hline $\begin{array}{l}\text { RLNPP } \\
\text { RLNP }\end{array}$ & $\begin{array}{l}\begin{array}{r}\text { K01022 } \\
\text { K0102 }\end{array}\end{array}$ & $\begin{array}{l}\text { 8081_PSTTGC } \\
\text { - } 0881 \text { PEST_GC }\end{array}$ \\
\hline $\begin{array}{l}\text { RLNP } \\
\text { RLNP }\end{array}$ & $\begin{array}{l}\text { Ko102 } \\
\text { K0102 }\end{array}$ & $\begin{array}{l}\text { 8081-PEST-GC } \\
\text { 8081- }\end{array}$ \\
\hline RLNP & K0102 & 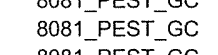 \\
\hline & $\begin{array}{l}\text { K01022 } \\
\text { K0102 }\end{array}$ & $\begin{array}{l}\text { 80811PPST GC } \\
\text { 808_PCB_GC }\end{array}$ \\
\hline RLNP & Ko102 & $\begin{array}{l}\text { 8082_PCB_GC } \\
\text { 8082 PCB GC }\end{array}$ \\
\hline & $\begin{array}{l}\text { K01022 } \\
\text { K0102 }\end{array}$ & $\begin{array}{l}\text { 8082-PCB_CC } \\
\text { 8082_PCB_CC }\end{array}$ \\
\hline $\begin{array}{l}\text { RLNP } \\
\text { RNPP }\end{array}$ & $\begin{array}{l}\text { K01022 } \\
\text { K0102 }\end{array}$ & $\begin{array}{l}\text { 8002_PC__GC } \\
08082 \text { PCB_GC }\end{array}$ \\
\hline & $\begin{array}{l}K_{0102} \\
K 0102\end{array}$ & 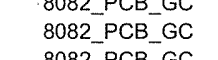 \\
\hline 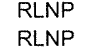 & 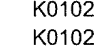 & $\begin{array}{l}\text { 8082_PCB_GC } \\
\text { 8082_PCB_GC }\end{array}$ \\
\hline & K0102 & 8082_PCB_GC \\
\hline 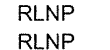 & $\begin{array}{l}\mathrm{K} 0102 \\
\text { K10202 }\end{array}$ & 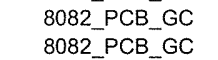 \\
\hline RLNP & $\begin{aligned} K 0102 \\
K 10102\end{aligned}$ & $\begin{array}{l}8082-P C B-G C \\
8082-P C\end{array}$ \\
\hline 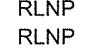 & 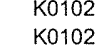 & $\begin{array}{l}\text { 8082_PCB_GC } \\
\text { 8082_PCB_GC }\end{array}$ \\
\hline & & $\begin{array}{l}\text { 8082_PC_-GC } \\
8082 \text { PCE GC }\end{array}$ \\
\hline RLNP & $\begin{array}{l}K 0102 \\
K 0102\end{array}$ & $\begin{array}{l}\text { 8082PCPC_CC } \\
\text { 8082_PCB_CC }\end{array}$ \\
\hline & 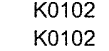 & $\begin{array}{l}\text { 8082_PCB_GC } \\
\text { 8082_PCB_GC }\end{array}$ \\
\hline & $\begin{aligned} K 0102 \\
K\end{aligned}$ & 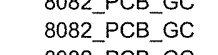 \\
\hline $\begin{array}{l}\text { RLNP } \\
\text { RLNP }\end{array}$ & $\begin{array}{l}\text { K0102 } \\
\text { K10202 }\end{array}$ & 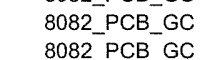 \\
\hline & 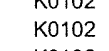 & 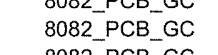 \\
\hline $\begin{array}{l}\text { RLNP } \\
\text { RLNP }\end{array}$ & $\begin{array}{l}\text { K0102 } \\
\text { K10202 }\end{array}$ & $\begin{array}{l}\text { 8082-PC_-GC } \\
8082 \text { PCB GC }\end{array}$ \\
\hline RLNF & $\begin{array}{l}K 0102 \\
K 10102\end{array}$ & 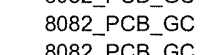 \\
\hline & 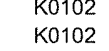 & $\begin{array}{l}\text { 8082_PCB_GC } \\
\text { 8082_PCB_GC }\end{array}$ \\
\hline & K0102 & 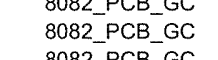 \\
\hline & 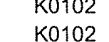 & $\begin{array}{l}\text { 8082-PCB-GC } \\
\text { 8082 PCB GC }\end{array}$ \\
\hline RLNP & K0102 & $\begin{array}{l}\text { 8002_PCB_GC } \\
\text { 8082_PCE_GC }\end{array}$ \\
\hline RLNP & K0102 & 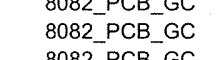 \\
\hline & 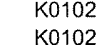 & $\begin{array}{l}\text { 8082-PCB_GC } \\
\text { GAMMA GS }\end{array}$ \\
\hline & K0102 & GAMMA_GS \\
\hline & Ko102 & $\begin{array}{l}\text { GAMMA_GS } \\
\text { GAMMA GS }\end{array}$ \\
\hline & $\begin{array}{l}\text { Ko102 } \\
\text { K0102 }\end{array}$ & $\begin{array}{l}\text { GAMMA_GS } \\
\text { GAMMAGS }\end{array}$ \\
\hline & & GAMMA_GS \\
\hline & K0102 & GAMMAGS \\
\hline & 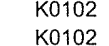 & $\begin{array}{l}\text { GAMMA-GS } \\
\text { GAMMA GS }\end{array}$ \\
\hline & K0102 & $\begin{array}{l}\text { GAMMA_GS } \\
\text { GAMMAGS }\end{array}$ \\
\hline & K0102 & GAMMA_GS \\
\hline & K0102 & $\begin{array}{l}\text { GAMMA-GS } \\
\text { GAMMA }\end{array}$ \\
\hline & $\begin{array}{l}K 102 \\
K 0102\end{array}$ & $\begin{array}{l}\text { GAMMA_GS } \\
\text { GAMMA GS }\end{array}$ \\
\hline & K0102 & $\begin{array}{l}\text { GAMMA_GS } \\
\text { GAMMAGS }\end{array}$ \\
\hline & K0102 & GAMMA_GS \\
\hline & K0102 & GAMMA_GS \\
\hline & $\begin{array}{l}\text { K0102 } \\
K 0102\end{array}$ & $\begin{array}{l}\text { GAMMA_GS } \\
\text { GAMMA GS }\end{array}$ \\
\hline & $\begin{aligned} \text { K010 } \\
\end{aligned}$ & $\begin{array}{l}\text { GAMMA_GS } \\
\text { GAMMAGS }\end{array}$ \\
\hline & K0102 & GAMMA_GS \\
\hline & $\begin{array}{r}k 102 \\
\text { Ko1 }\end{array}$ & GAMMA-GS \\
\hline & Kof102 & $\begin{array}{l}\text { GAMMA_GS } \\
\text { GAMMA GS }\end{array}$ \\
\hline & KO102 & GAMMA_GS \\
\hline & K0102 & GAMMAGS \\
\hline & K0102 & $\begin{array}{l}\text { GAMMA_GS } \\
\text { GAMMA GS }\end{array}$ \\
\hline & $\begin{array}{l}\text { Ko102 } \\
\text { K0102 }\end{array}$ & $\begin{array}{l}\text { GAMMA_GS } \\
\text { GAMMAGS }\end{array}$ \\
\hline & & GAMMA_GS \\
\hline & K0102 & GAMMA-GS \\
\hline & Kot & $\begin{array}{l}\text { GAMMA_GS } \\
\text { GAMMA GS }\end{array}$ \\
\hline & K0102 & GAMMA_GS \\
\hline & $\begin{array}{r}K_{0102} \\
\text { K0102 }\end{array}$ & $\begin{array}{l}\text { GAMMA_GS } \\
\text { GAMMA GS }\end{array}$ \\
\hline $\begin{array}{l}\text { ERRLNE } \\
\text { ERRLNE }\end{array}$ & K0102 & $\begin{array}{l}\text { GAMMA-GS } \\
\text { GAMMAGS }\end{array}$ \\
\hline & & GAMMA-GS \\
\hline & & GAMMA_GS \\
\hline & & A A \\
\hline & & \\
\hline & & \\
\hline
\end{tabular}

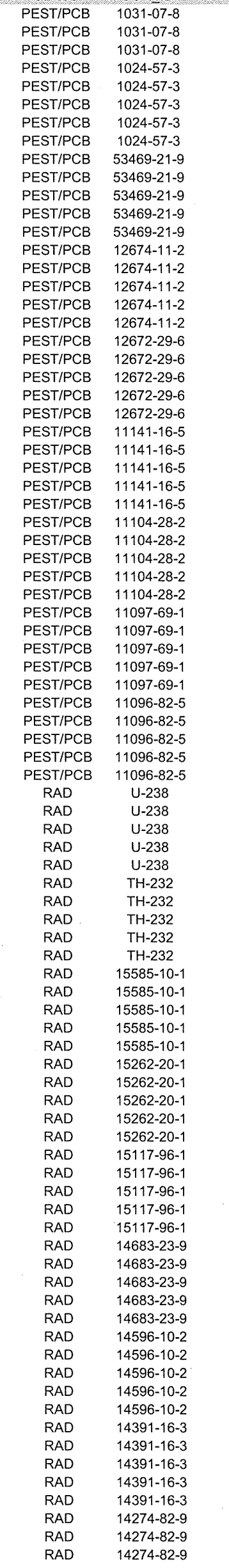

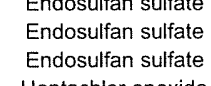

Heptachlor epoxide
Hepptachlor epoxide

Heplachlor epoxide
Heptachlor epoxide
Aroclor-1242 Aroclor-1242
Aroclor-1242 Aroclor-1242
Aroclor-10:10
Arocolor-1010

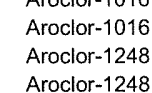

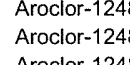
Aroclor-1248 Aroclor-1232
Aroclor-1232 Arodol-120232
Arocolor-1221 Aroclor-1221
Aroclor-1221
Alot-122

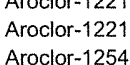
Aroclor-1254
AAroclor- 2254
A

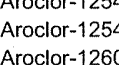
Aroclor-1260
Aroclor-1260 Arocolor-1260
Arocorl-1260
Aroclor-1260
U. Aroclor-1260
Uranium-238 Uranium-238
Uranium-238
Uranium-238
Urium-238 Uranium-238
Uranium-238
Thorium-232 Thorium-232
Thorium-232
Thorium-232

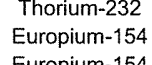

Europium-154
Radium-228
Radium-228
Radiumm-228
Radium-228

Radium-228
Uranium-235
Uranium-235
Uranium-235 
MIS_05 SAMPLES

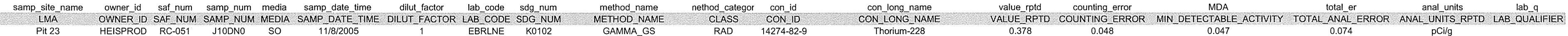

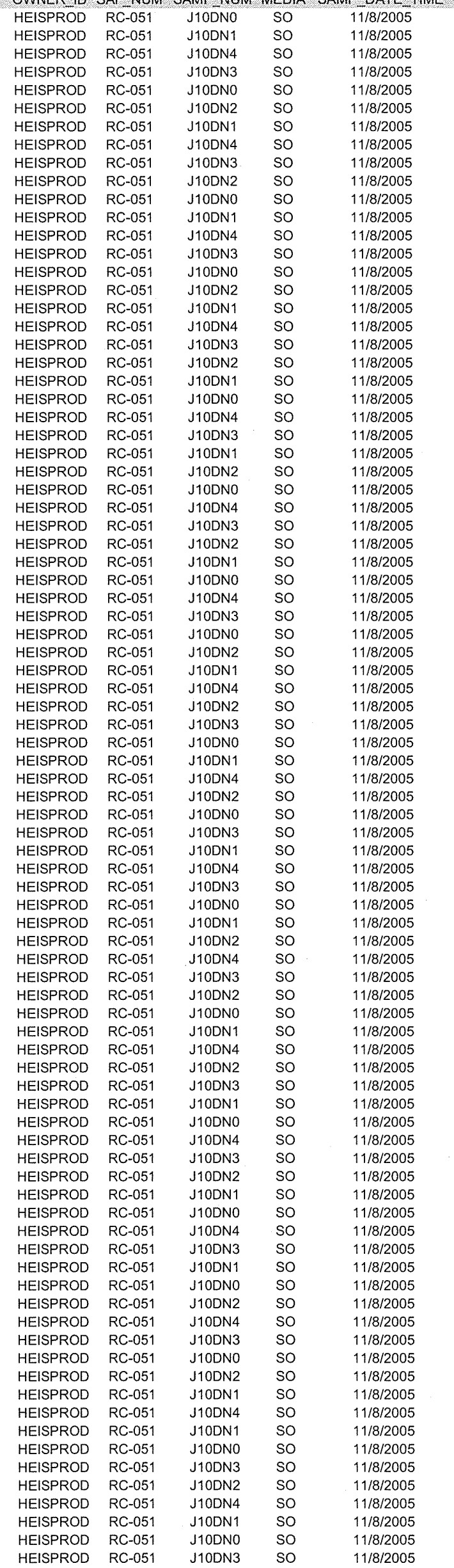

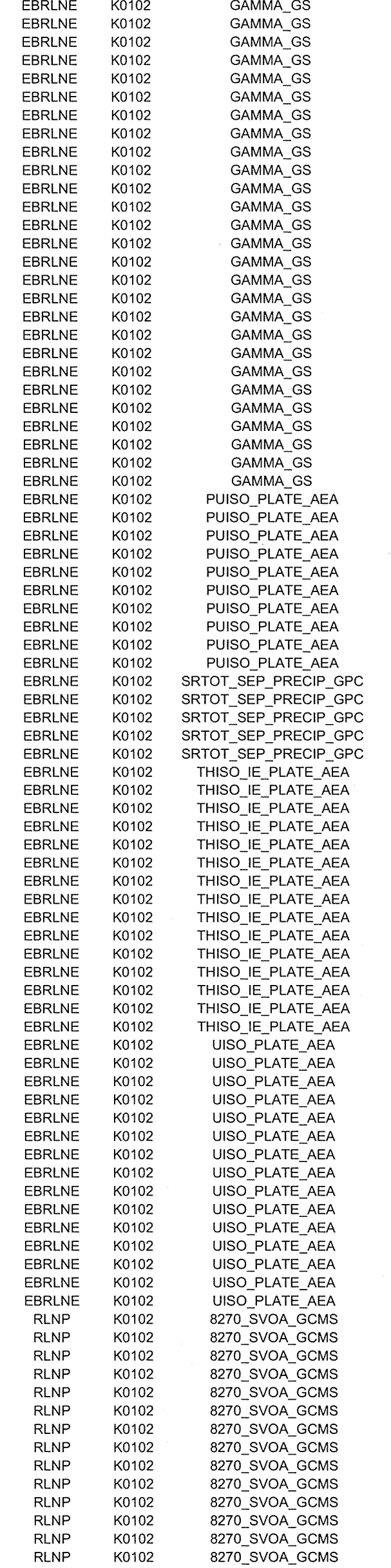

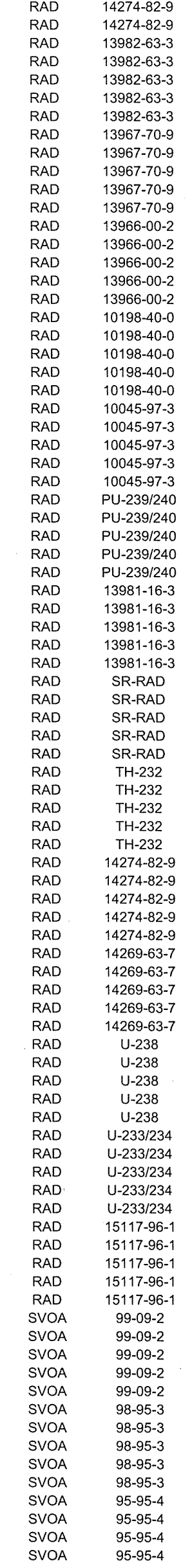

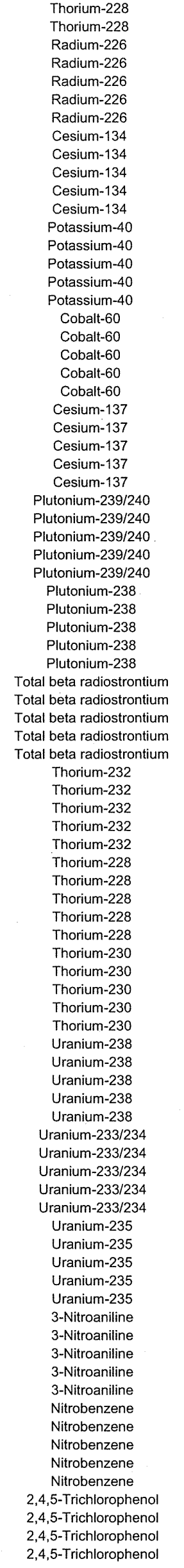

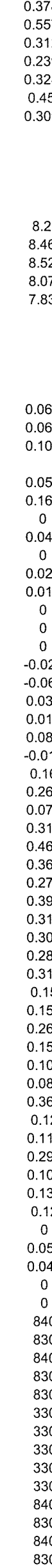

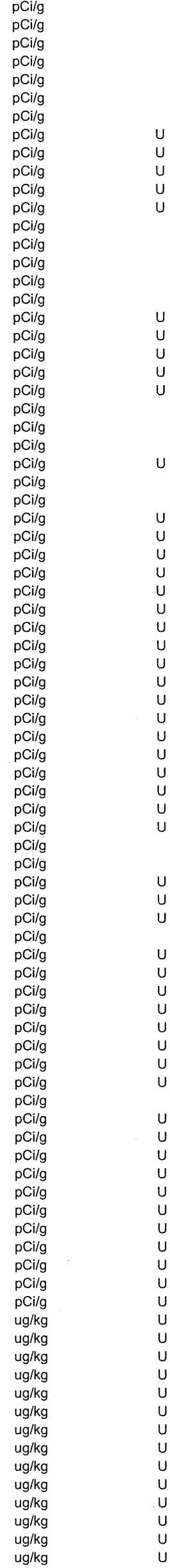




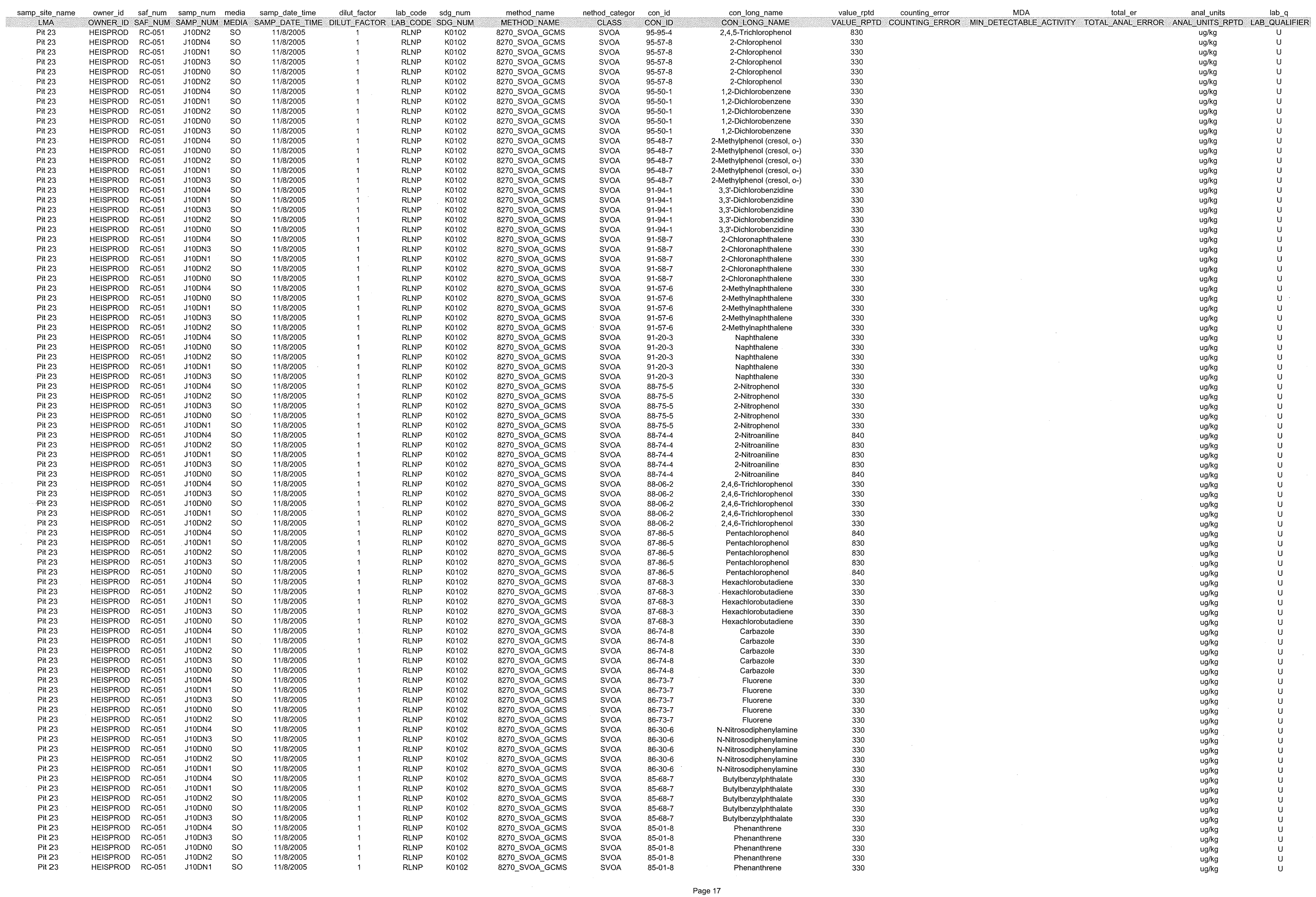


MIS_05 SAMPLES

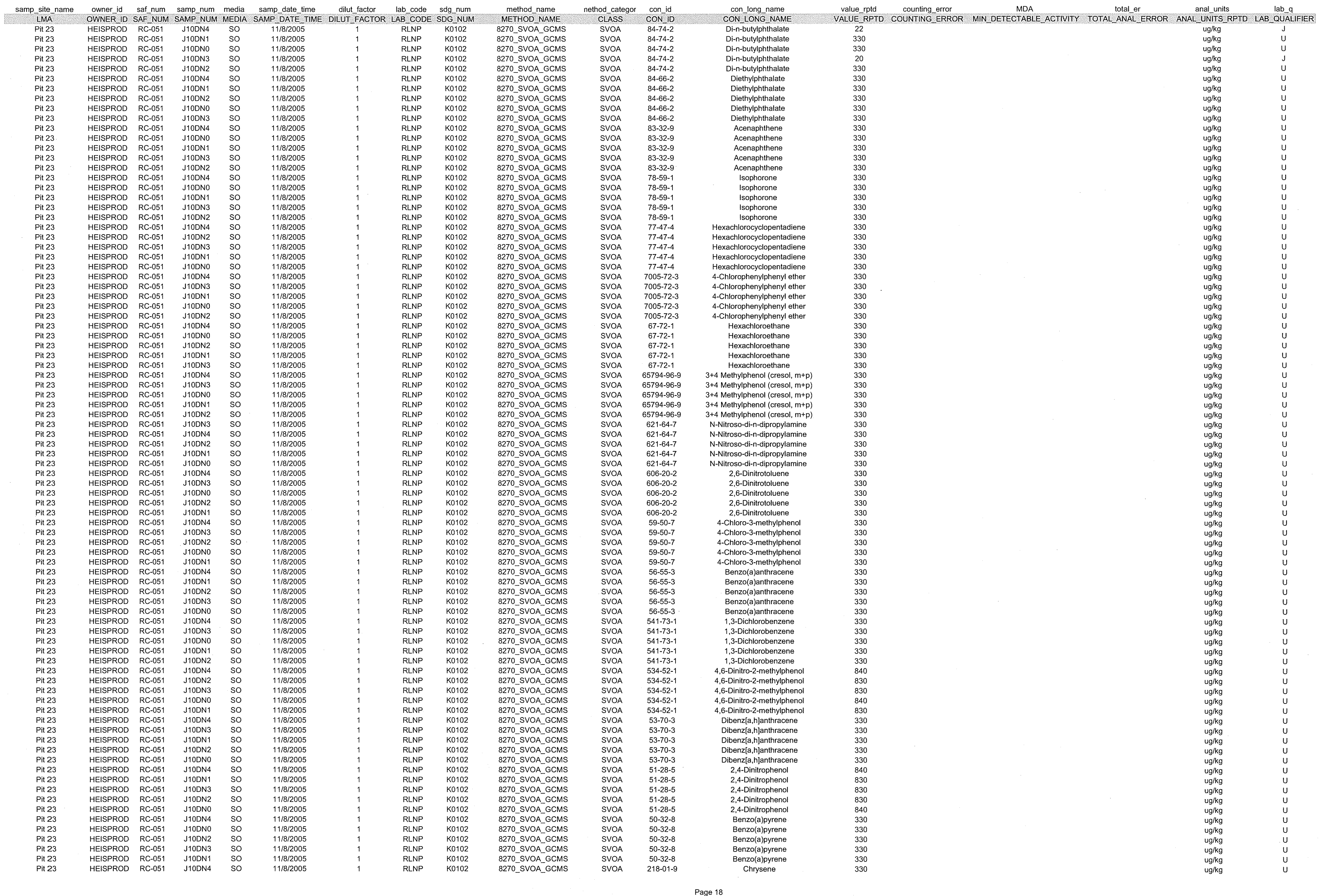


MIS_05 SAMPLES

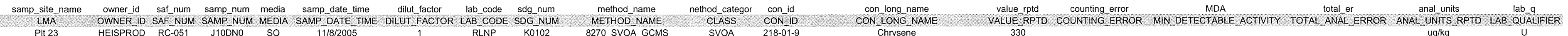

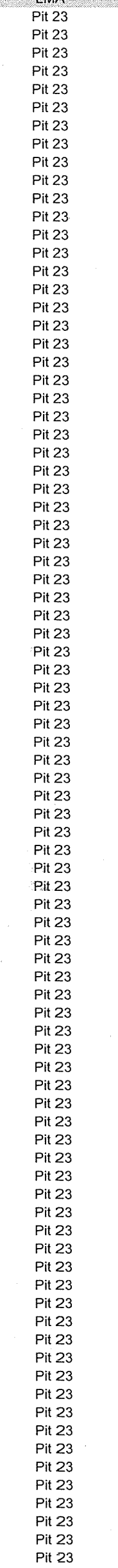

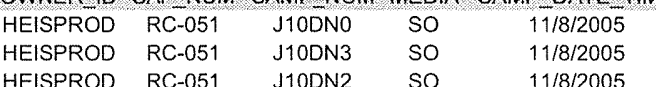

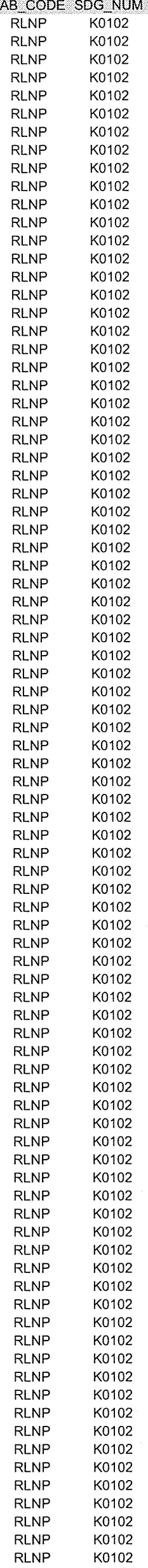

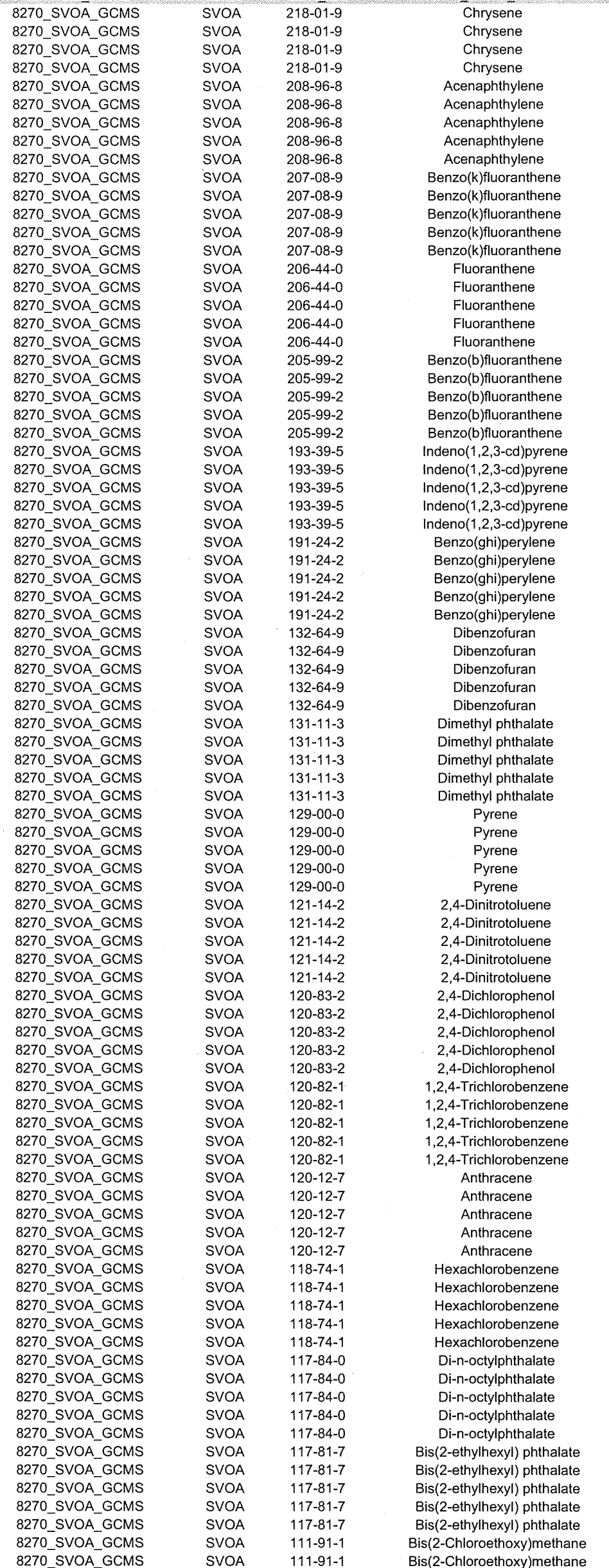


MIS_05 SAMPLES

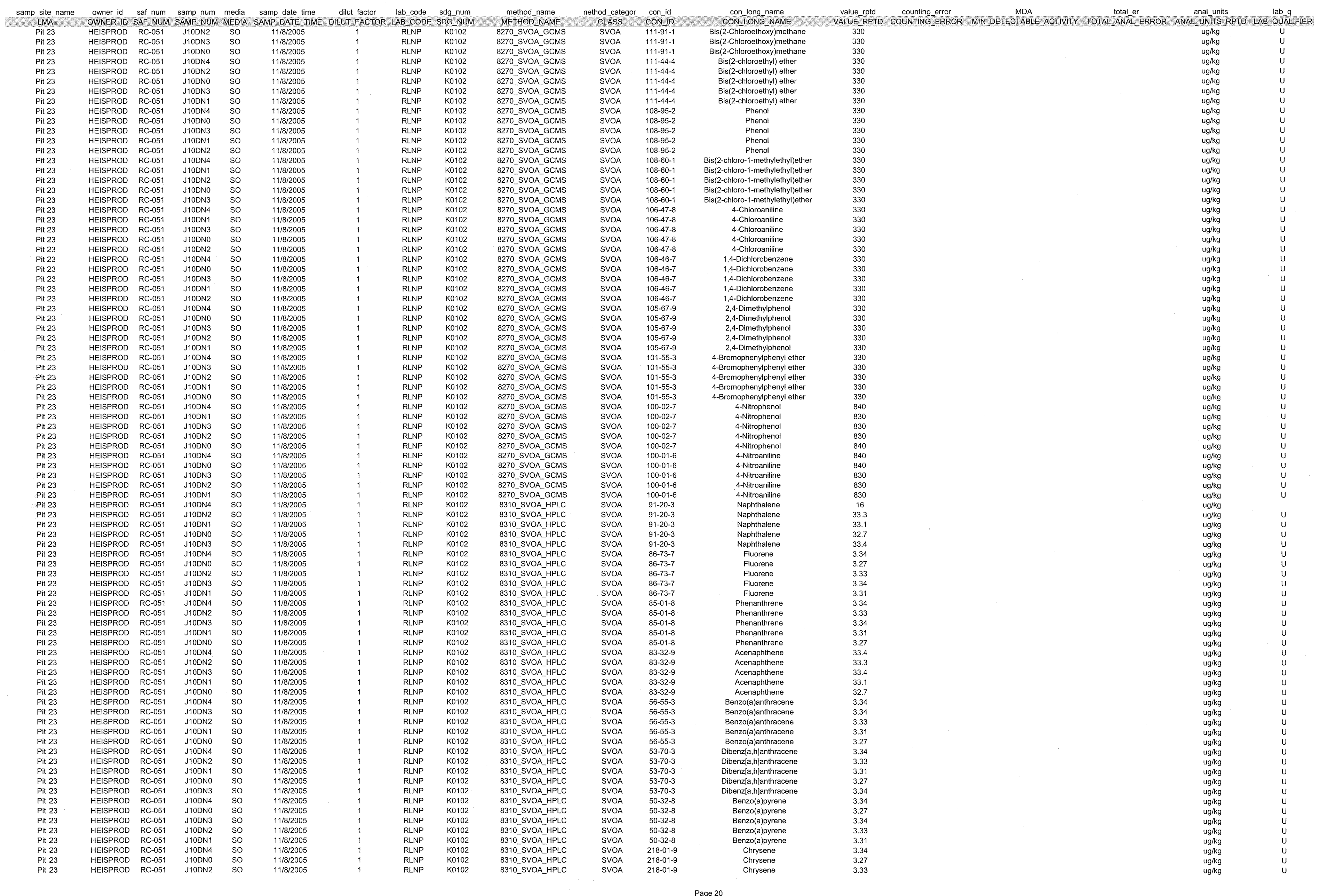




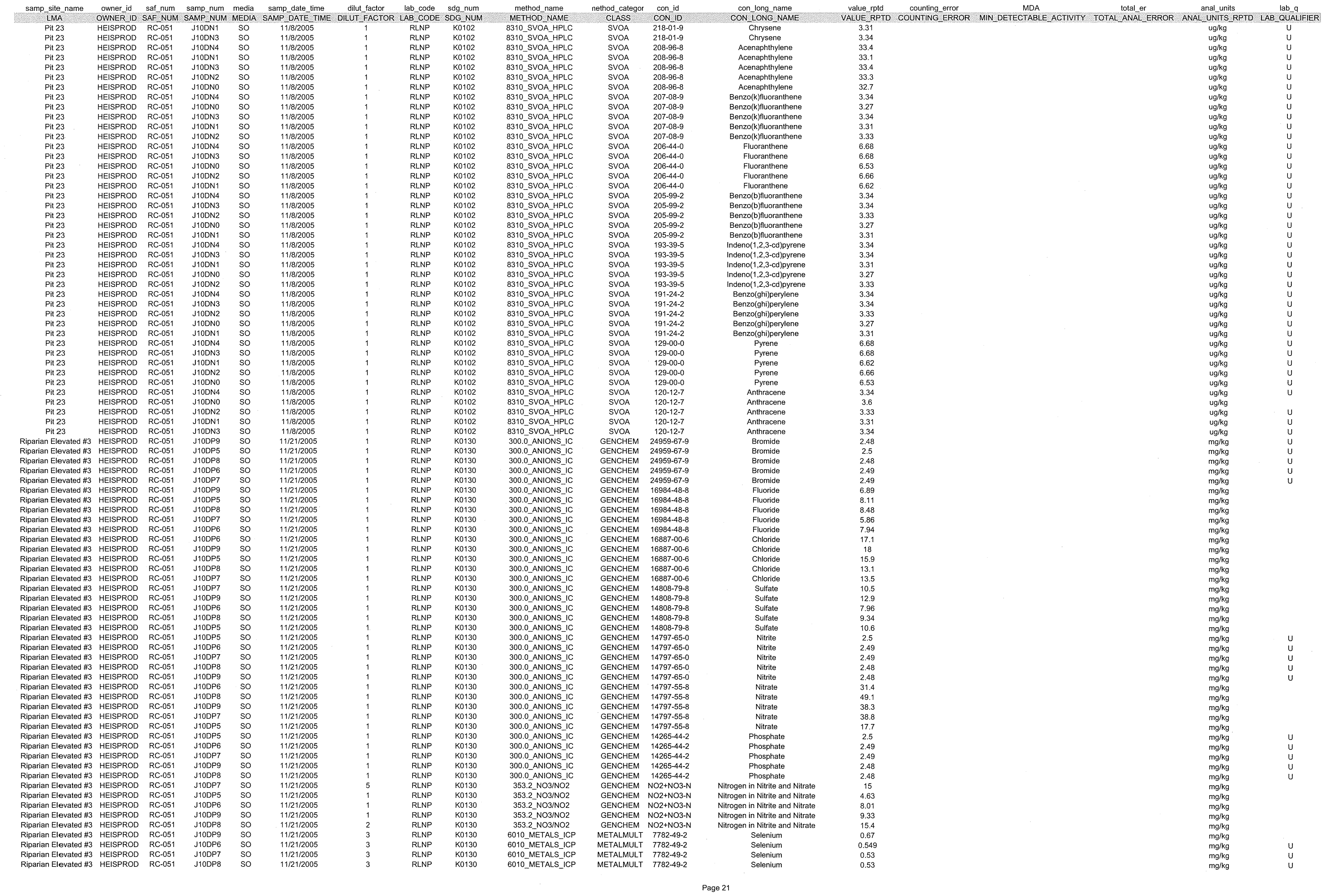


MIS_05 SAMPLES

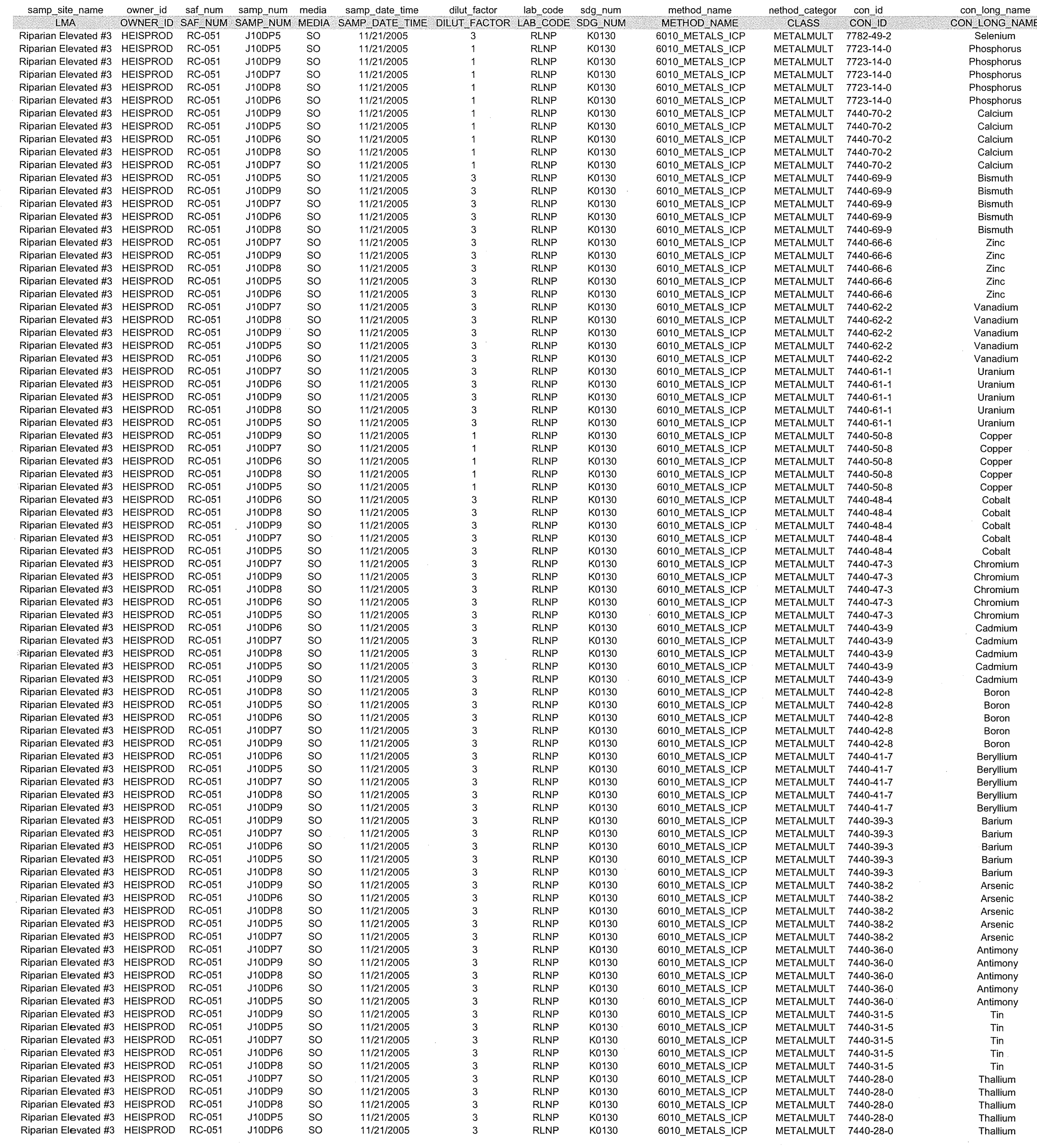

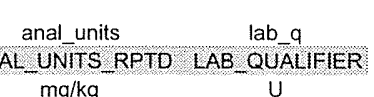

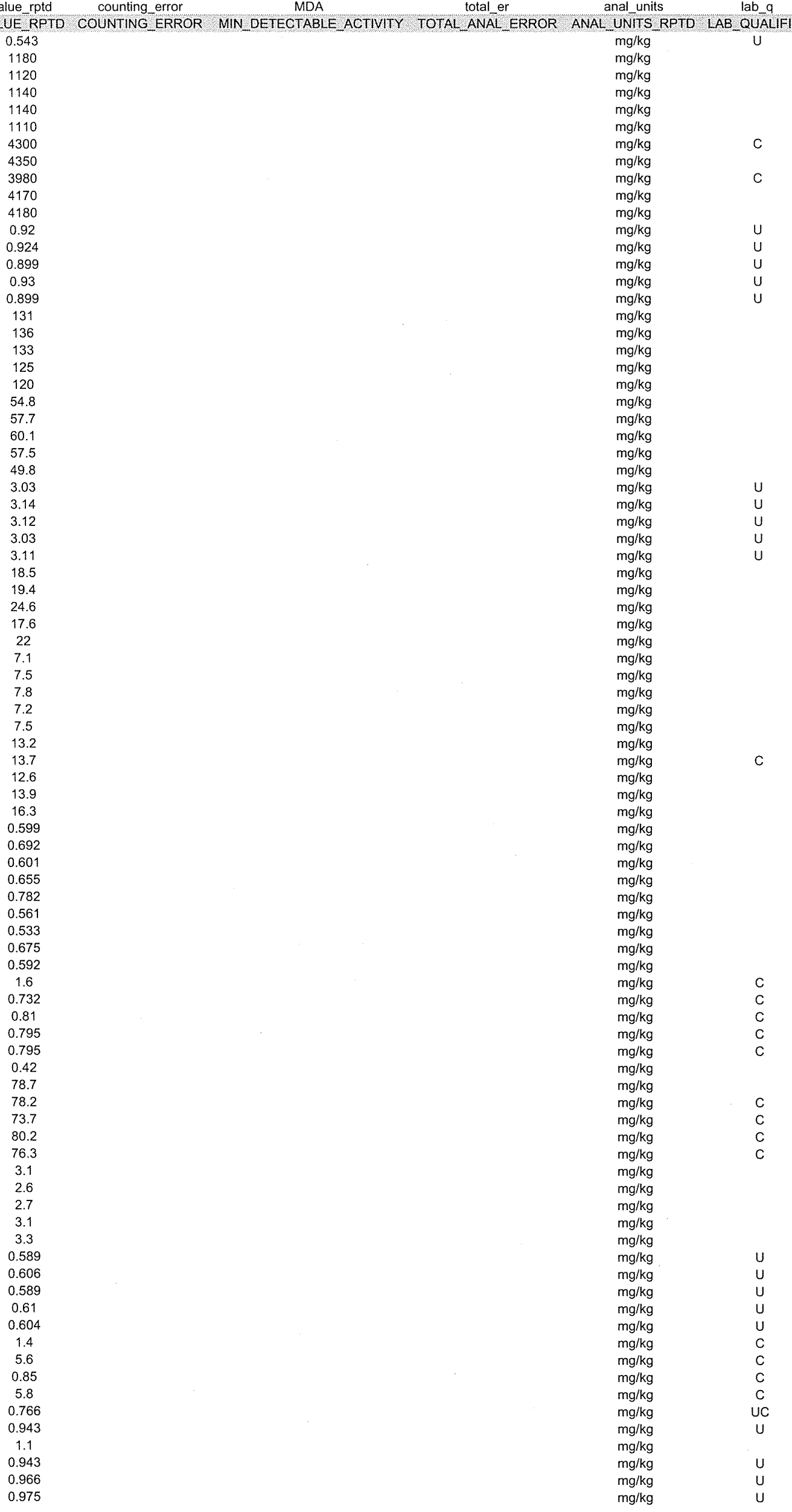




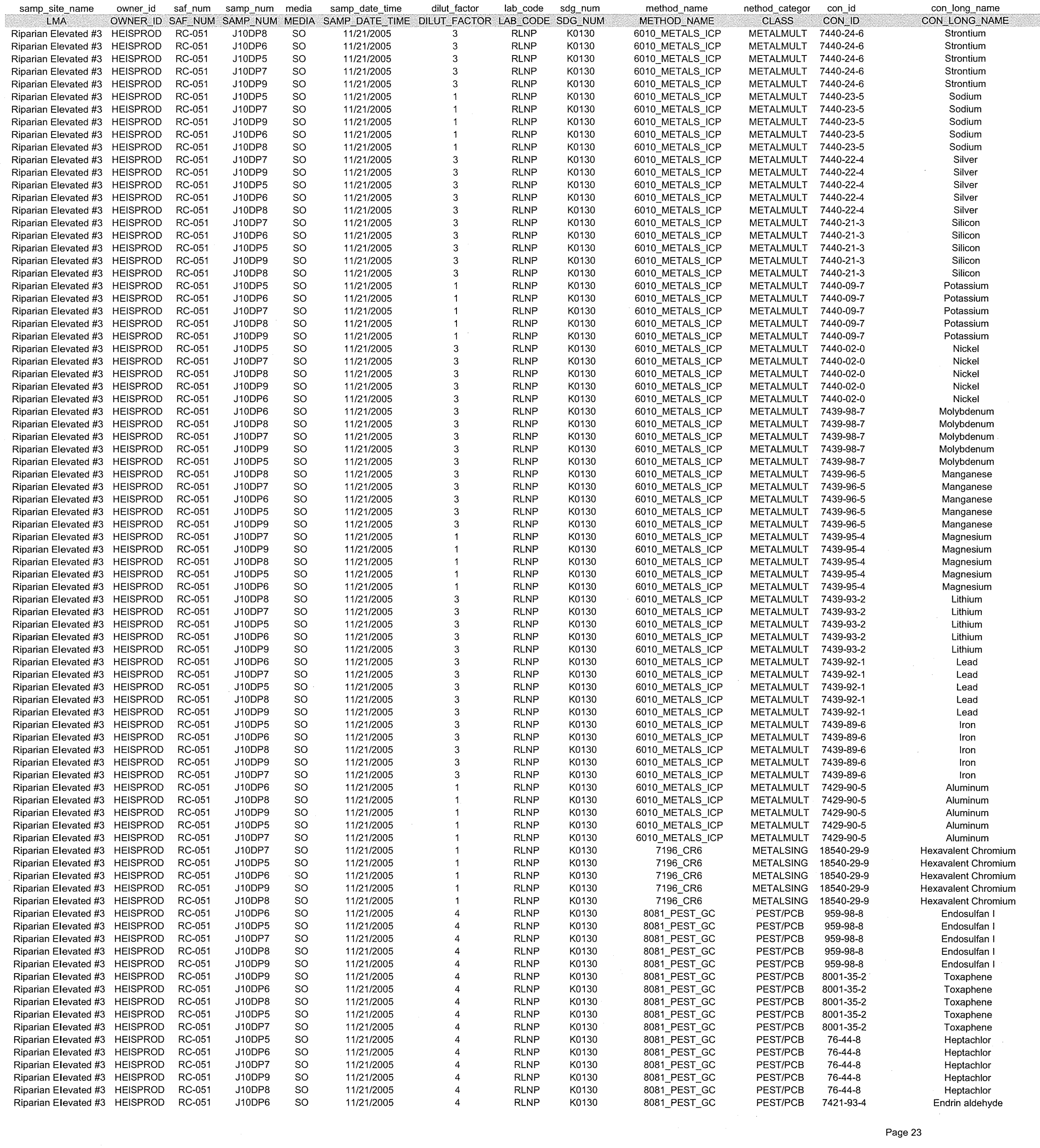

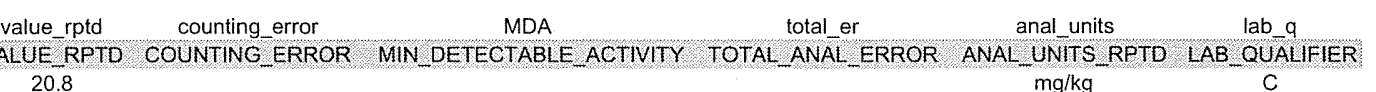

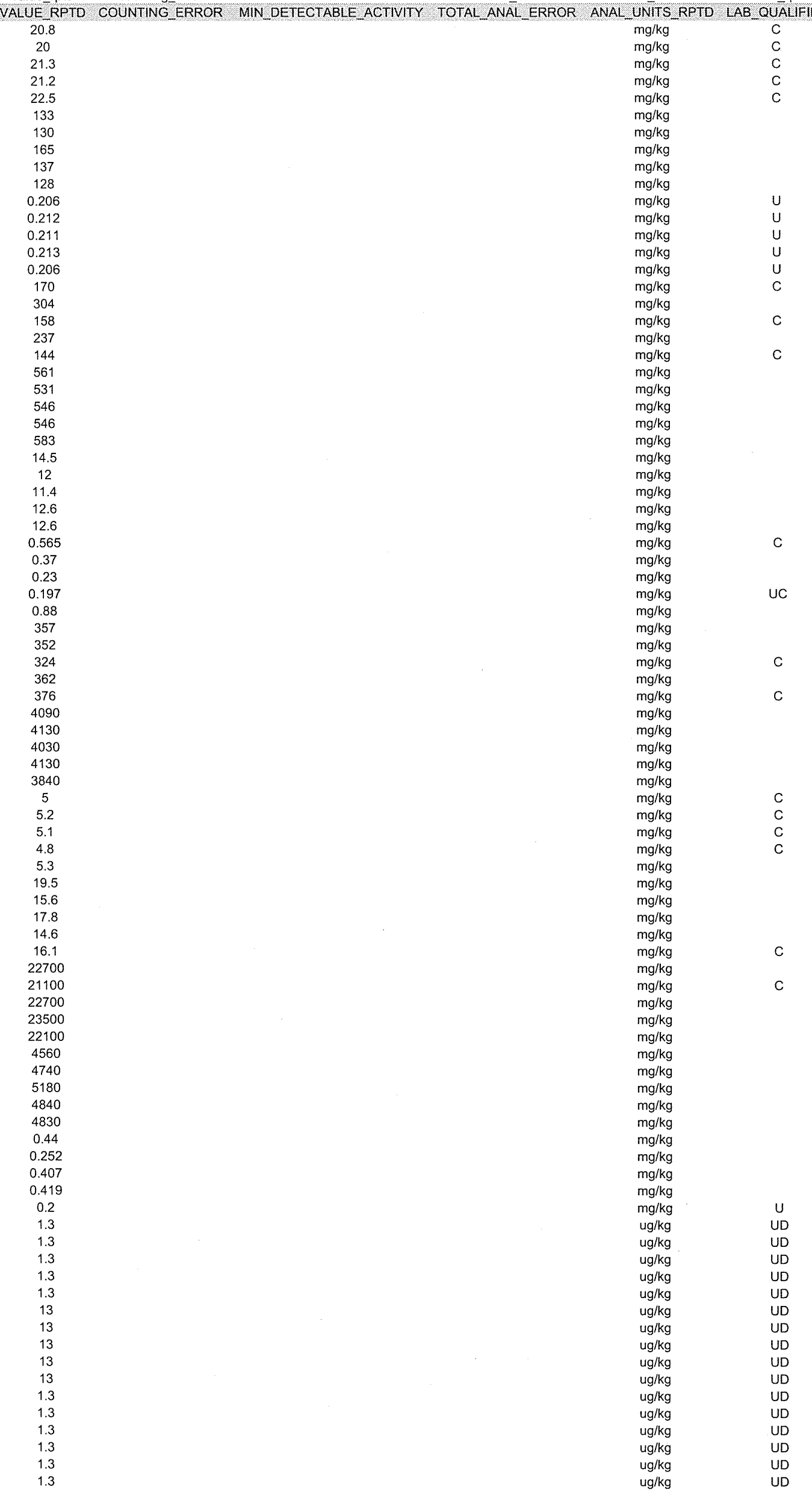


MIS_05 SAMPLES

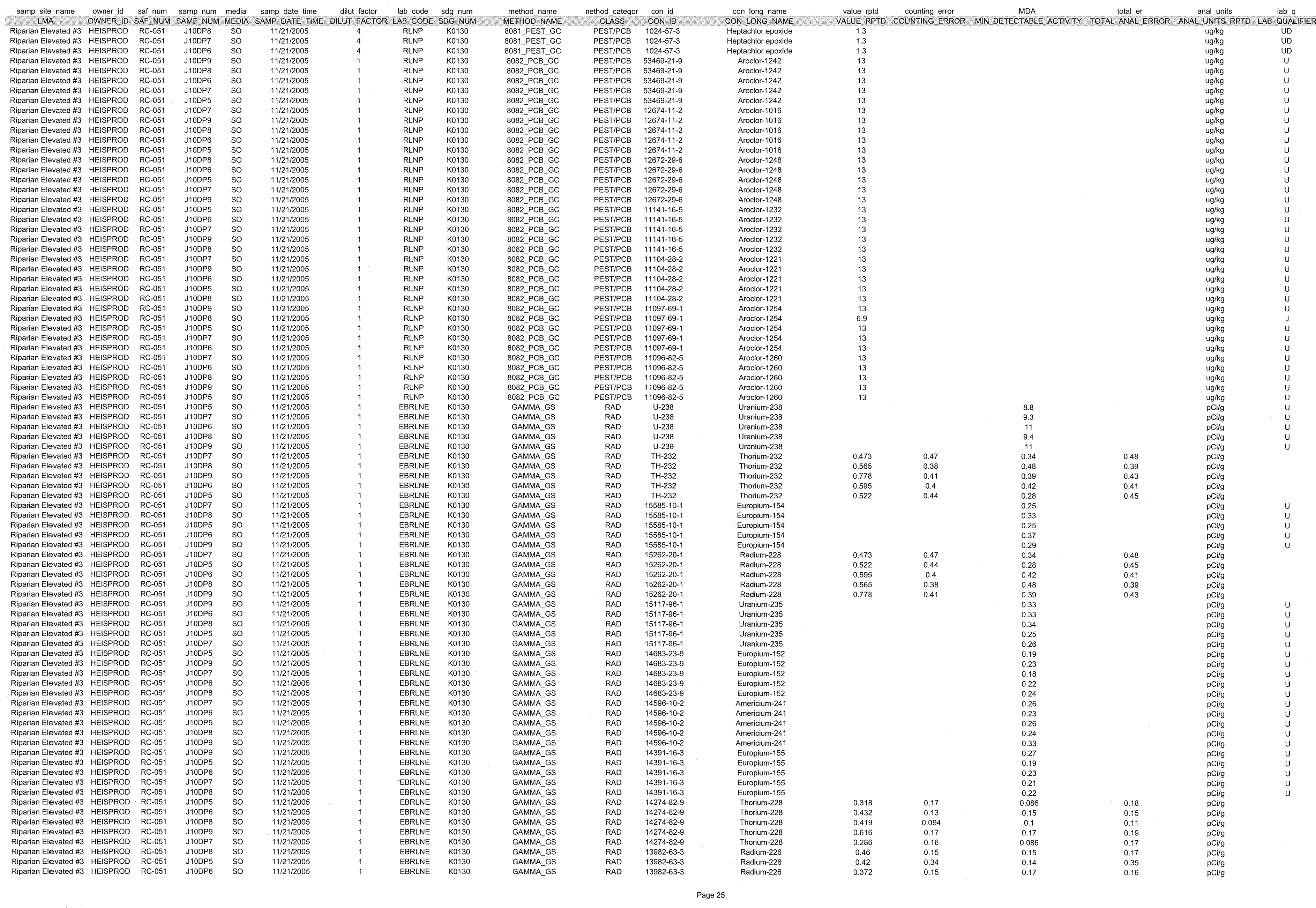




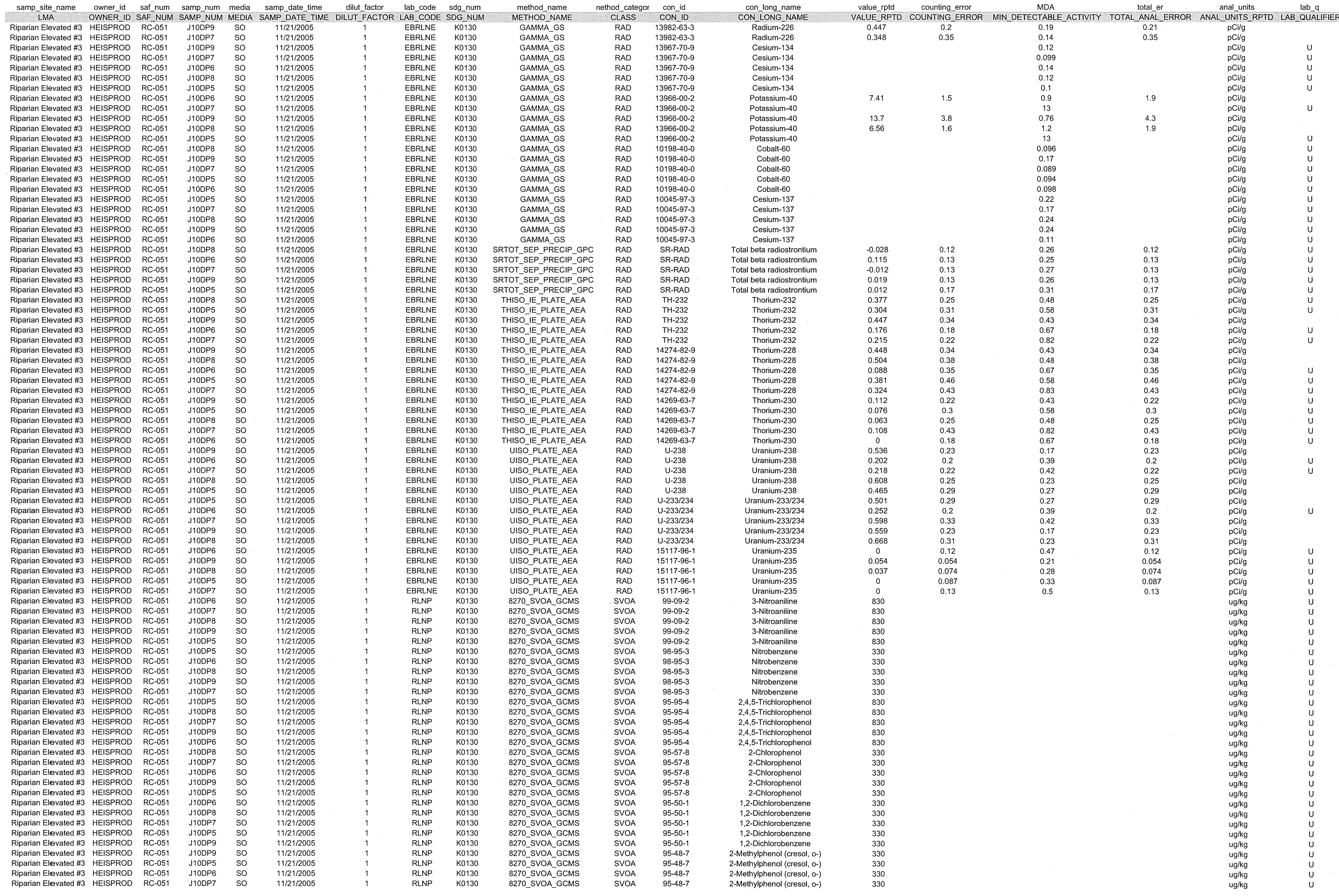




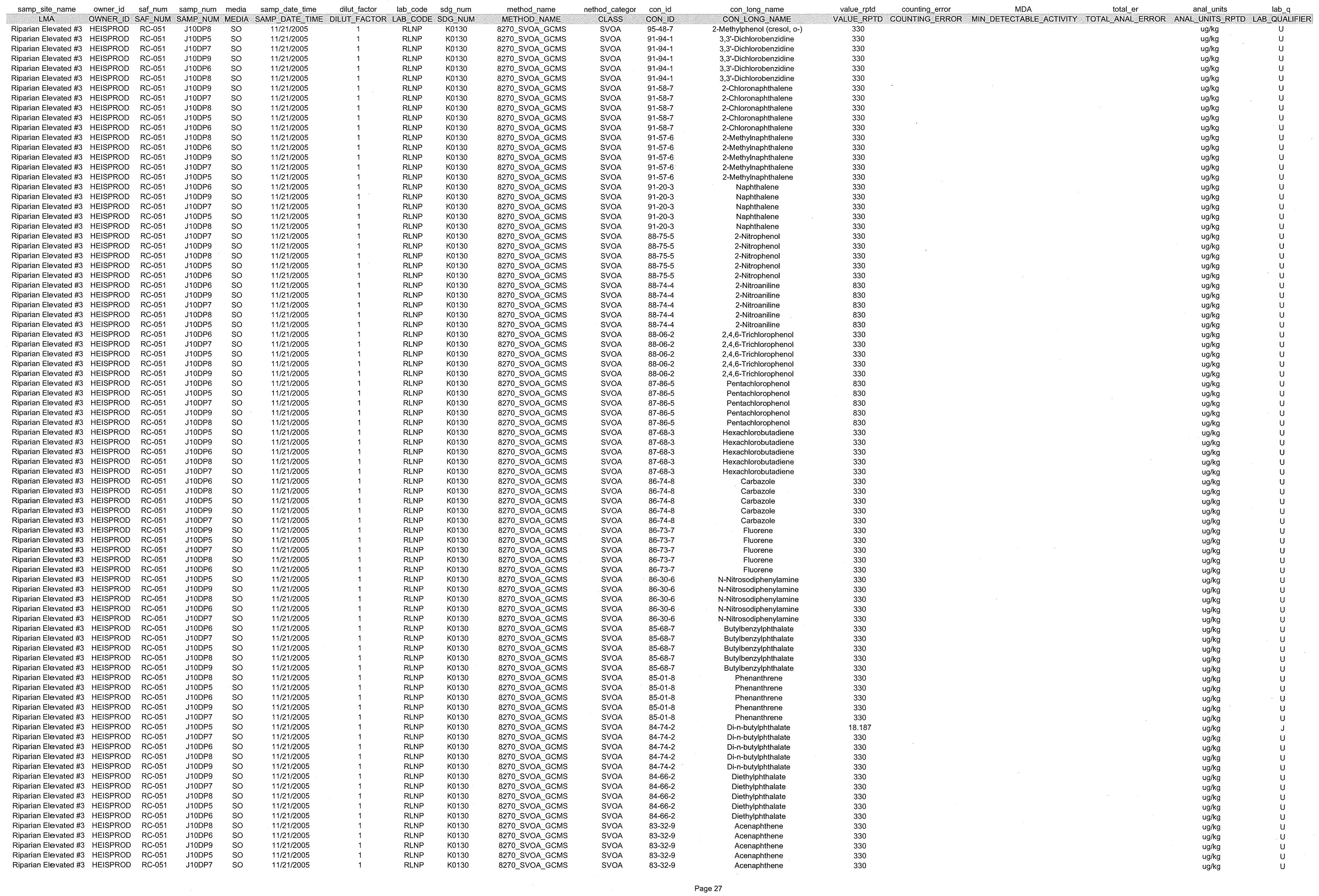


MIS_05 SAMPLES

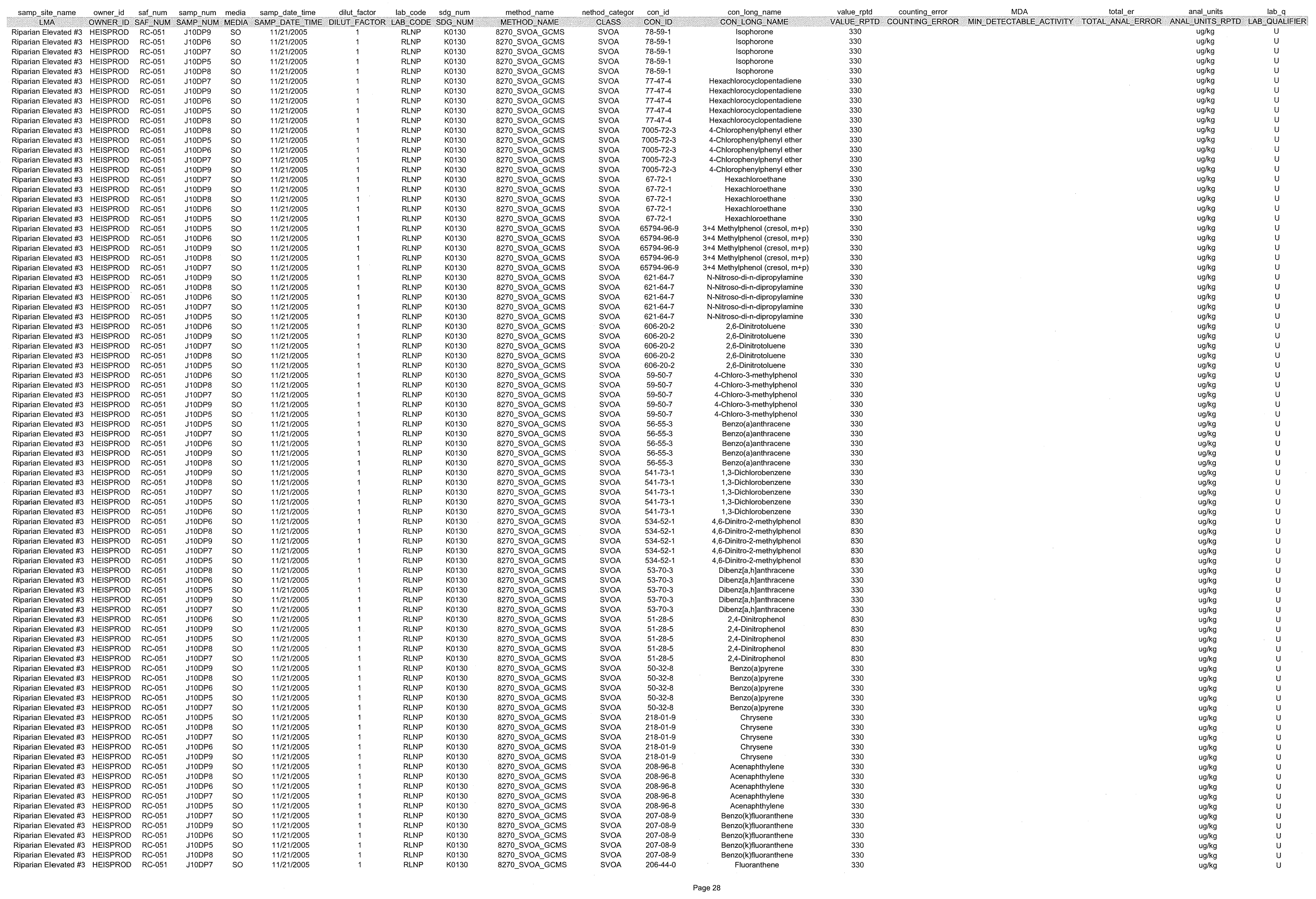


MIS_05 SAMPLES

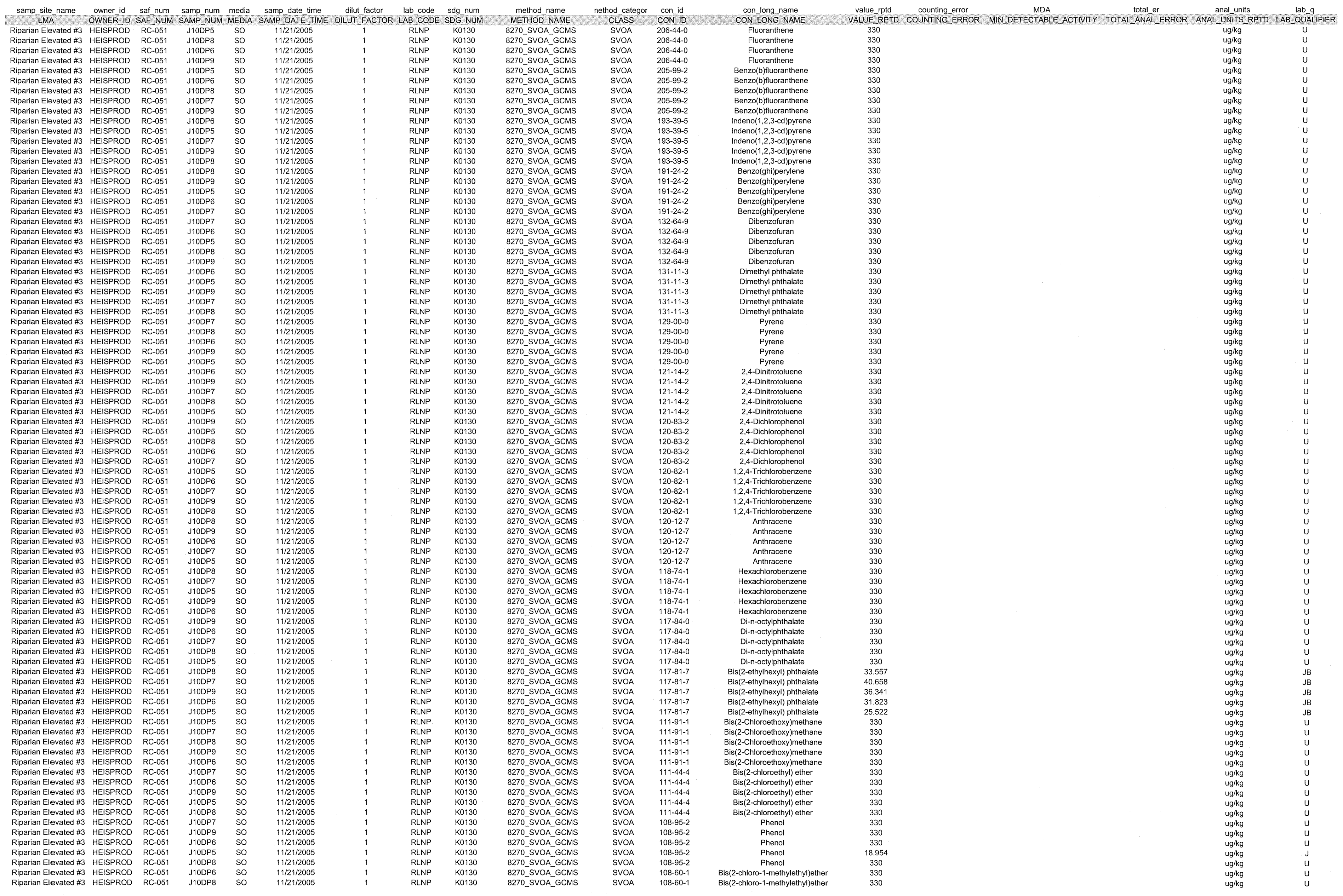




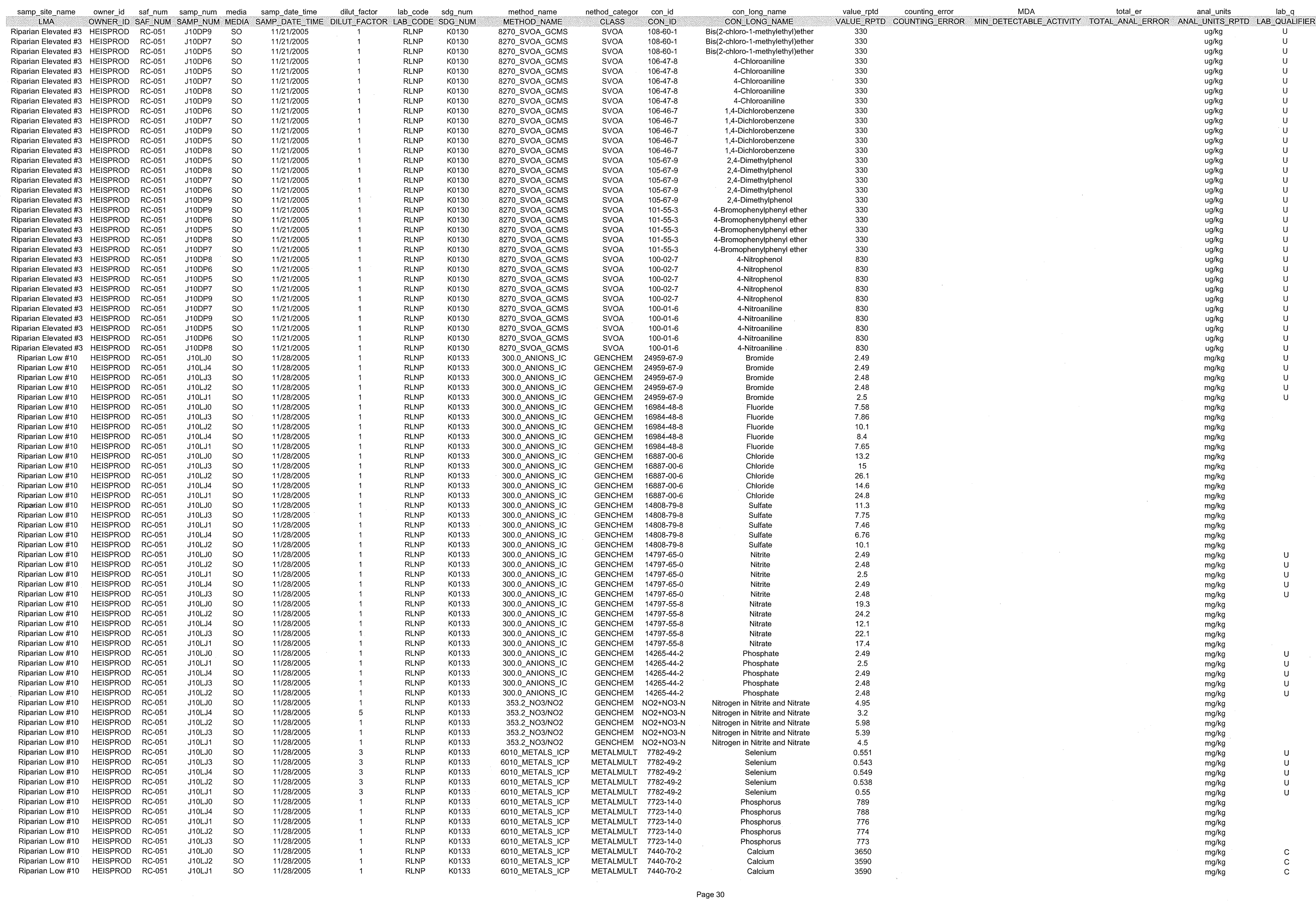




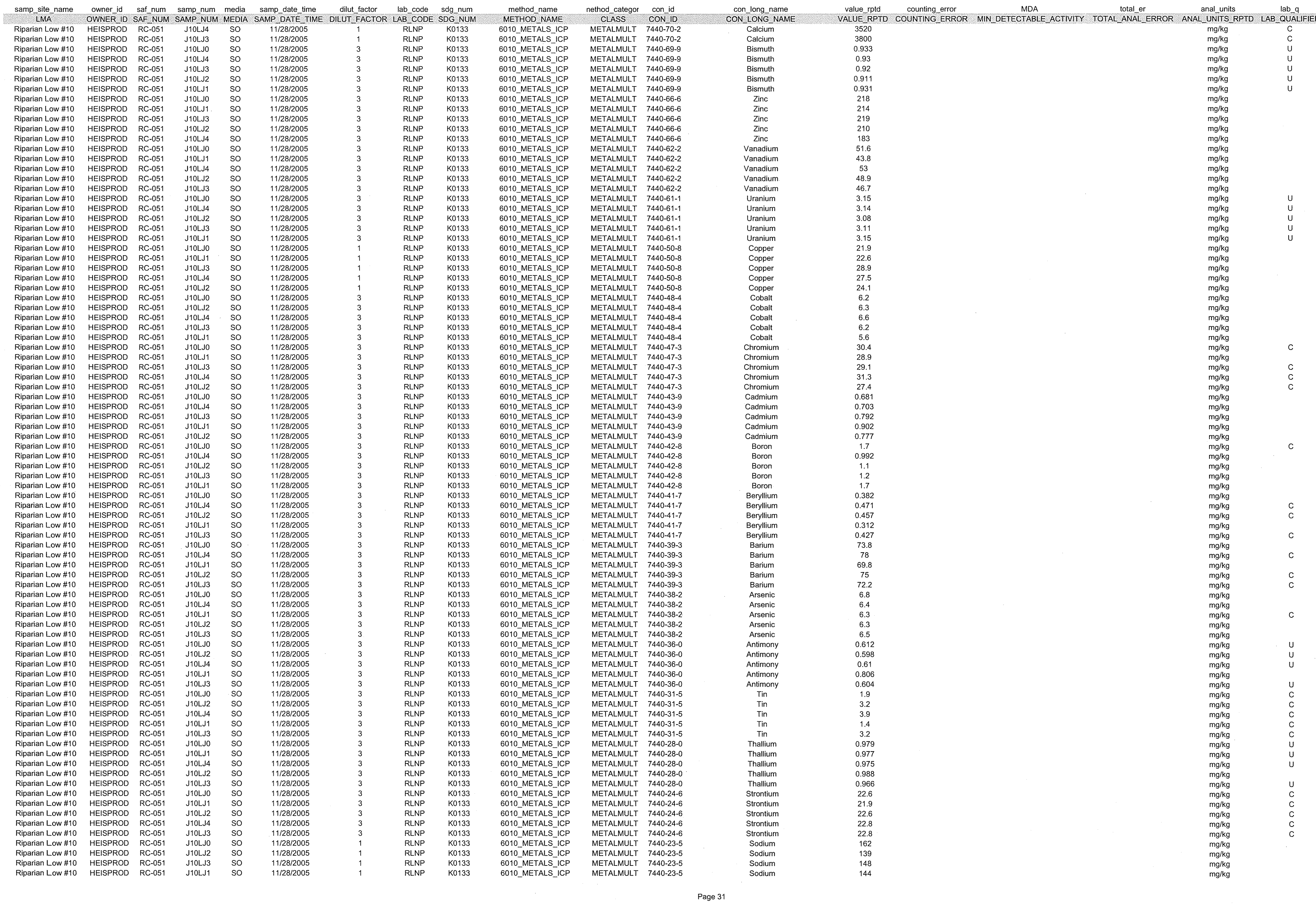




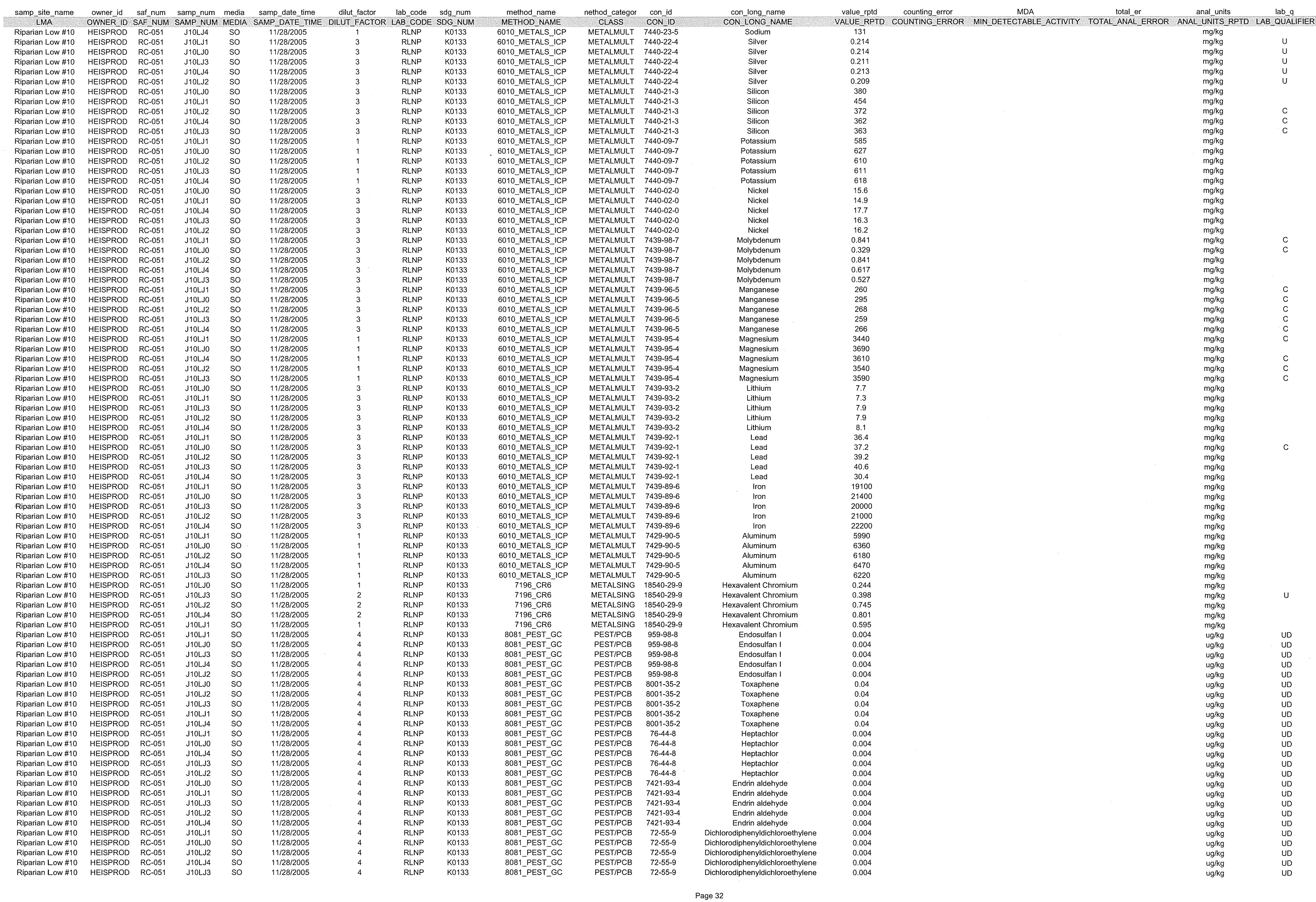


MIS_05 SAMPLES

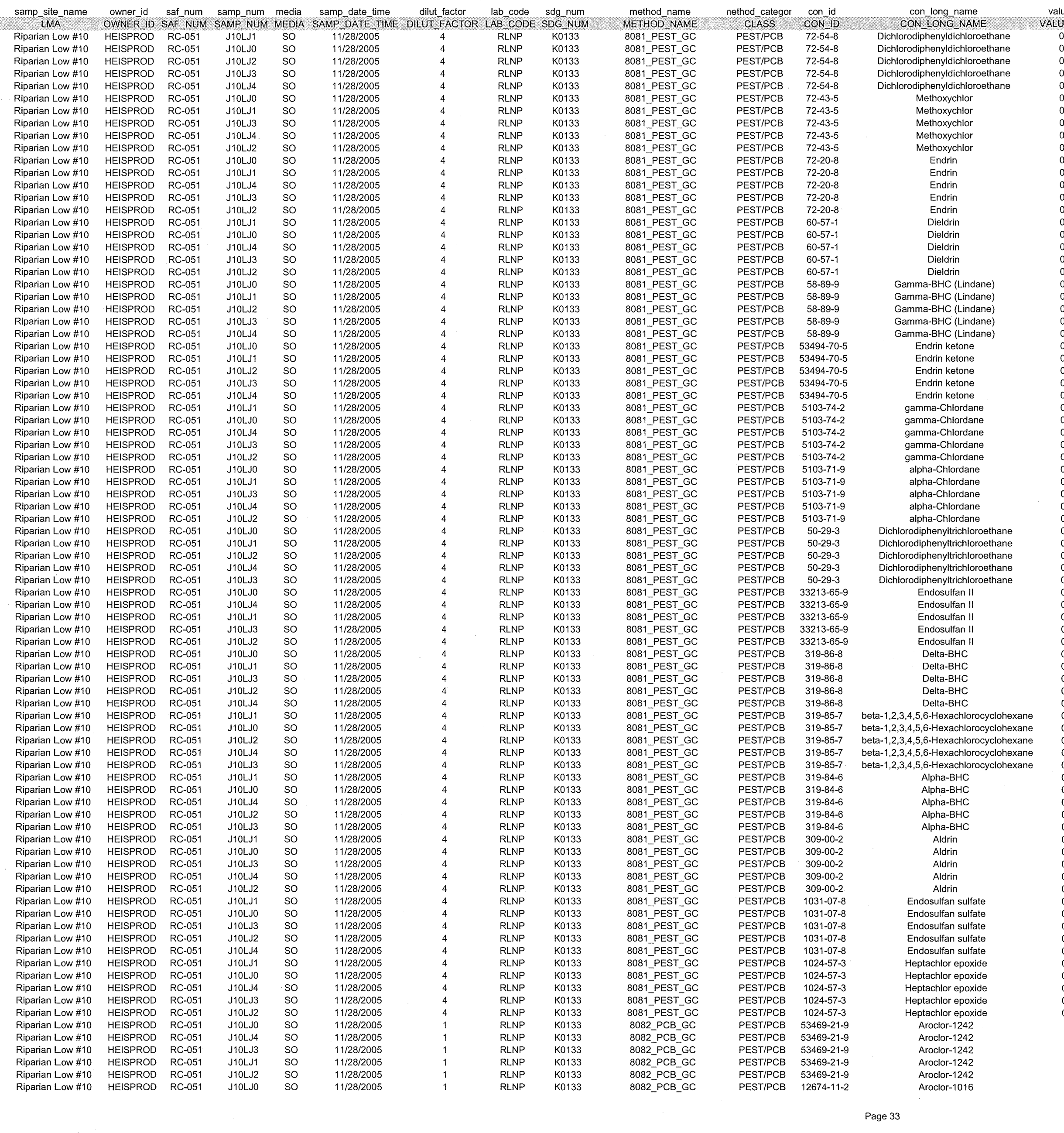

MDA
Countingerror

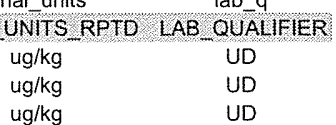

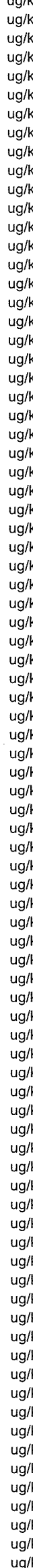


MIS_05 SAMPLES

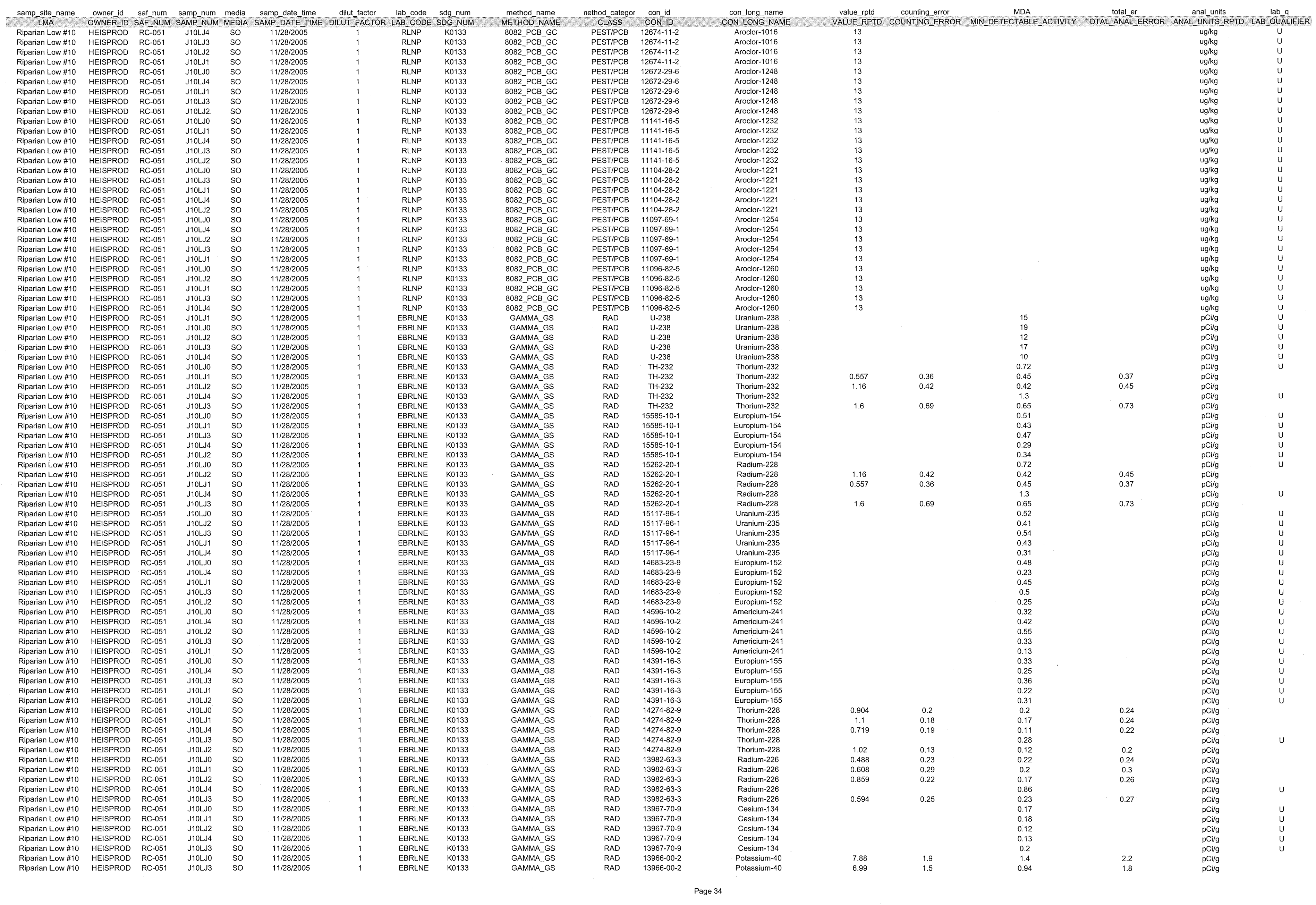




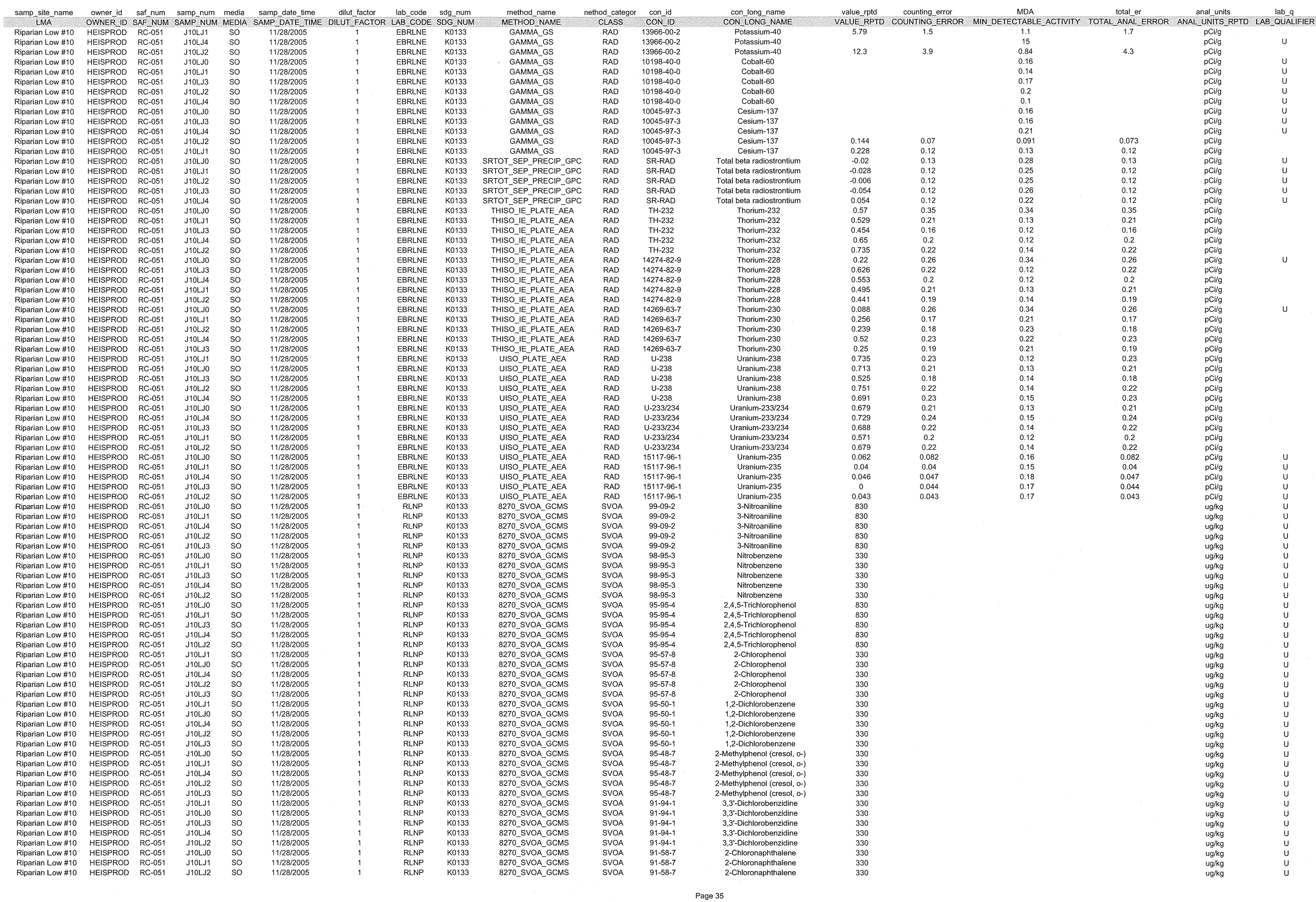




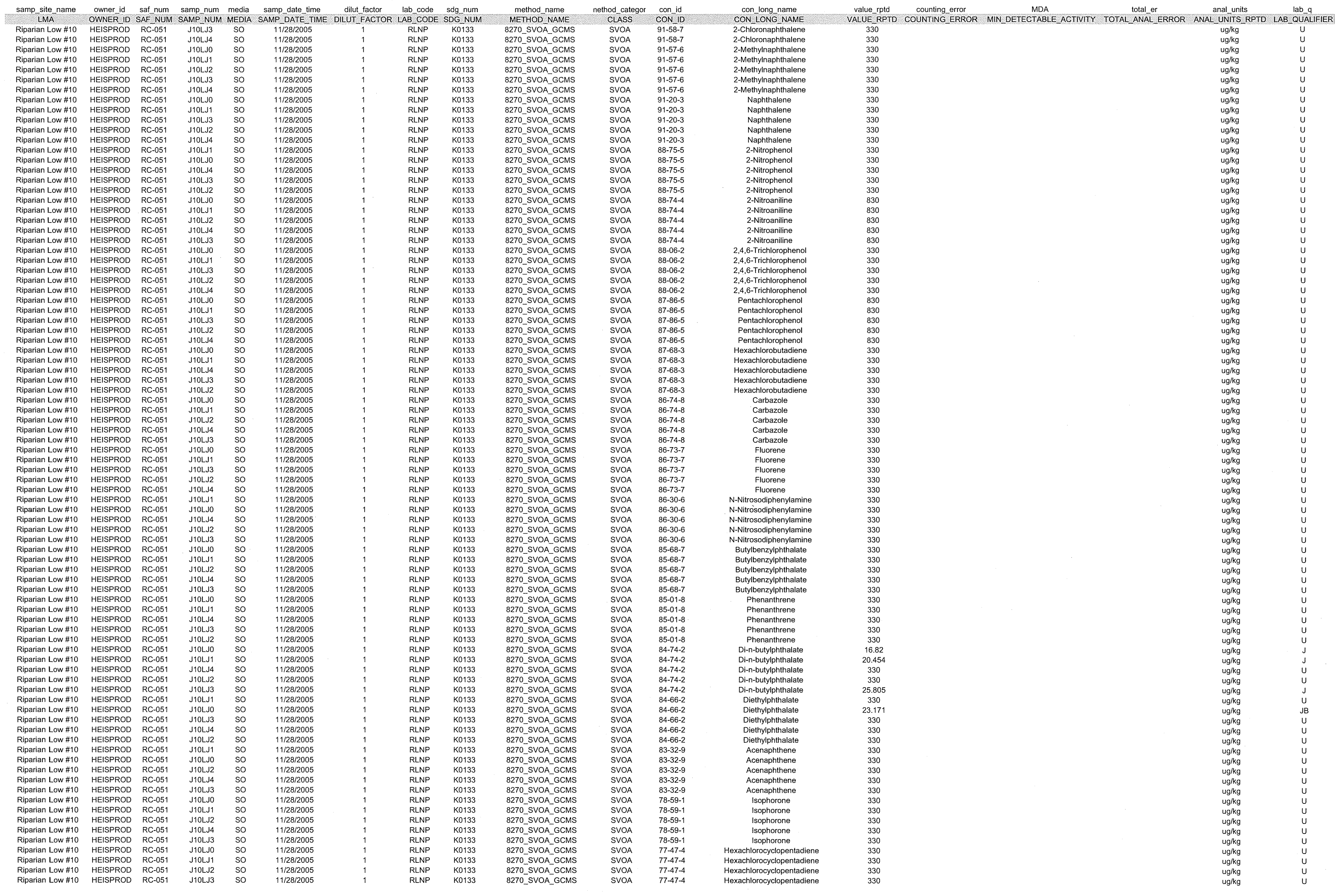




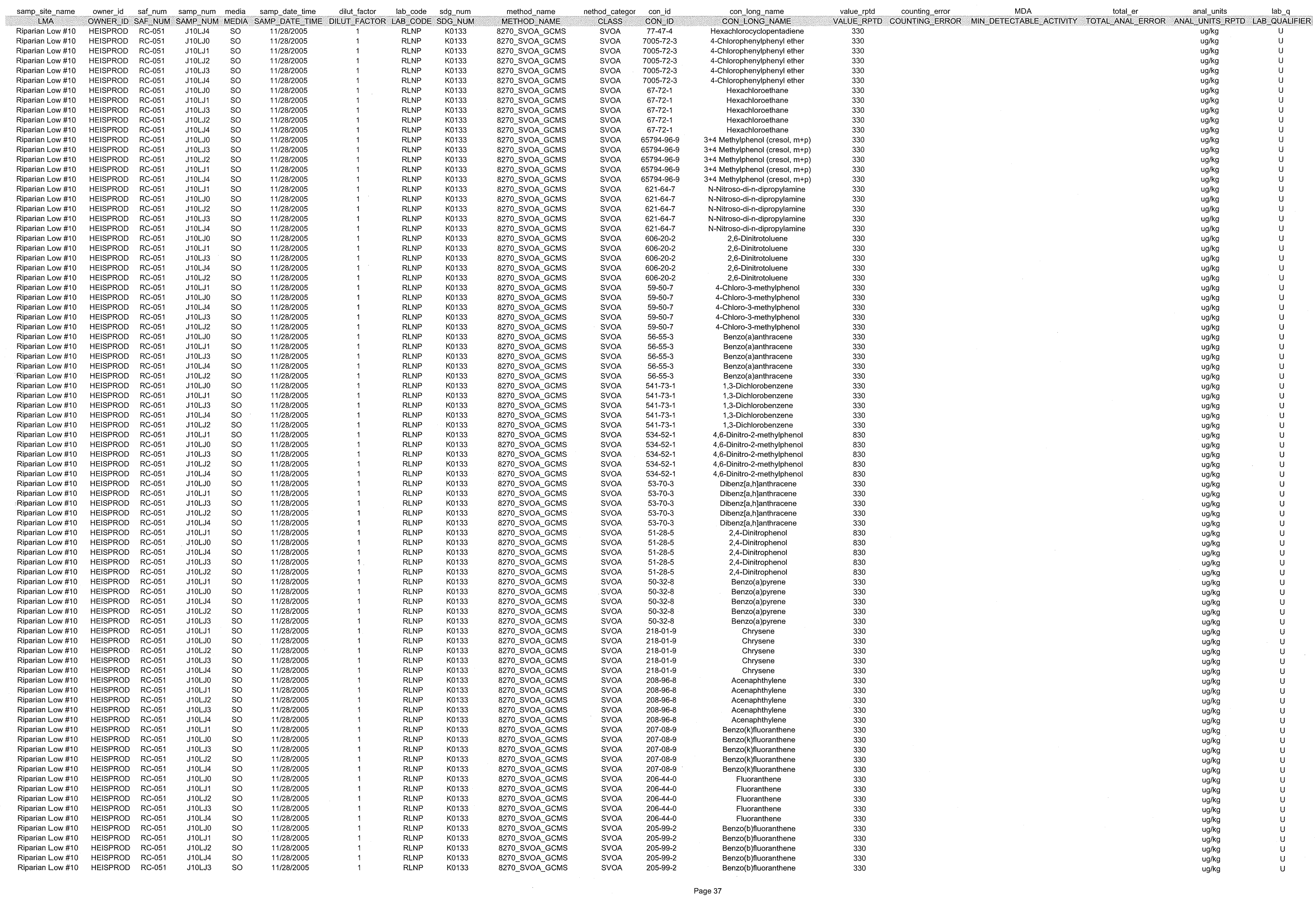




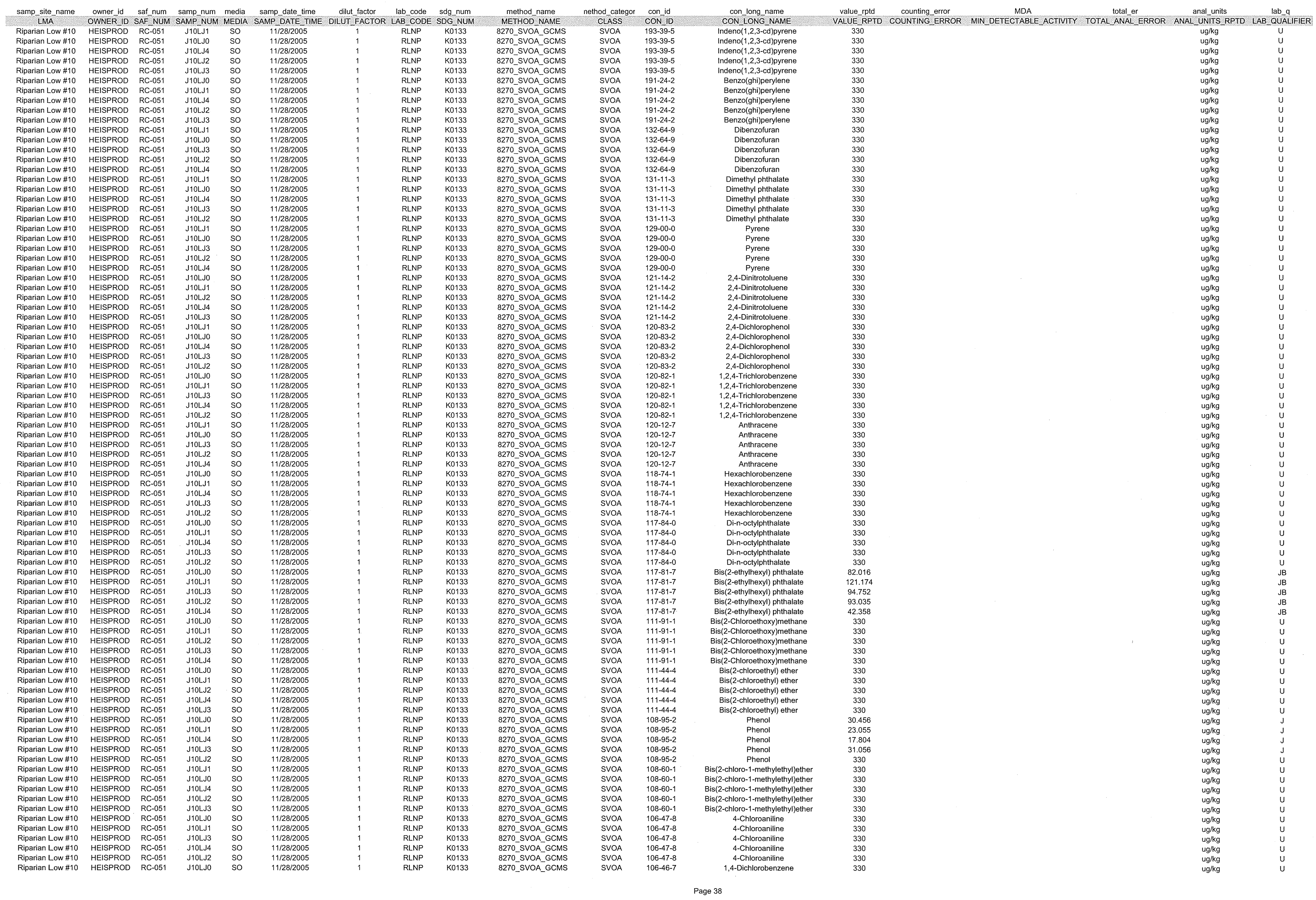


MIS_05 SAMPLES

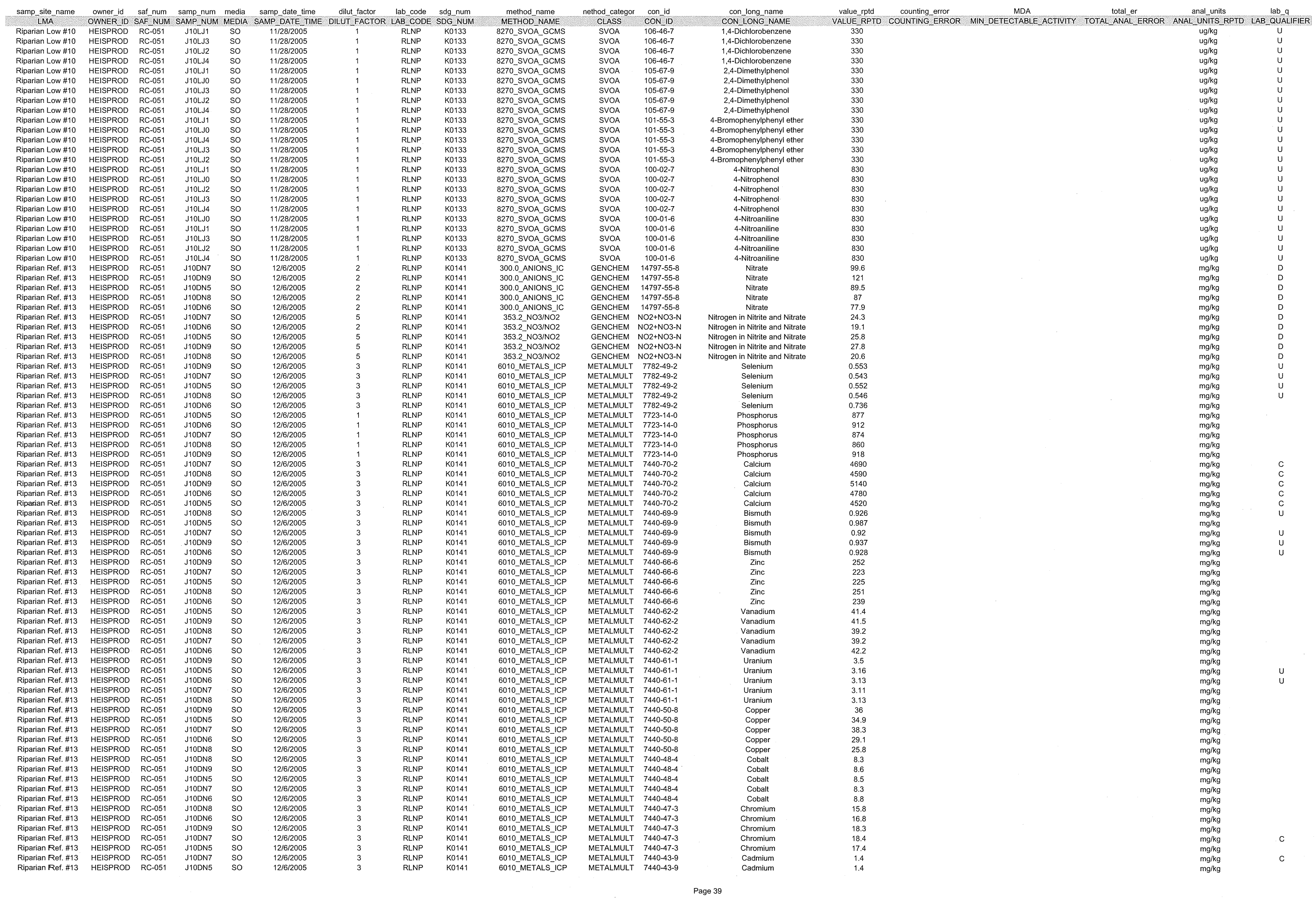


MIS_05 SAMPLES

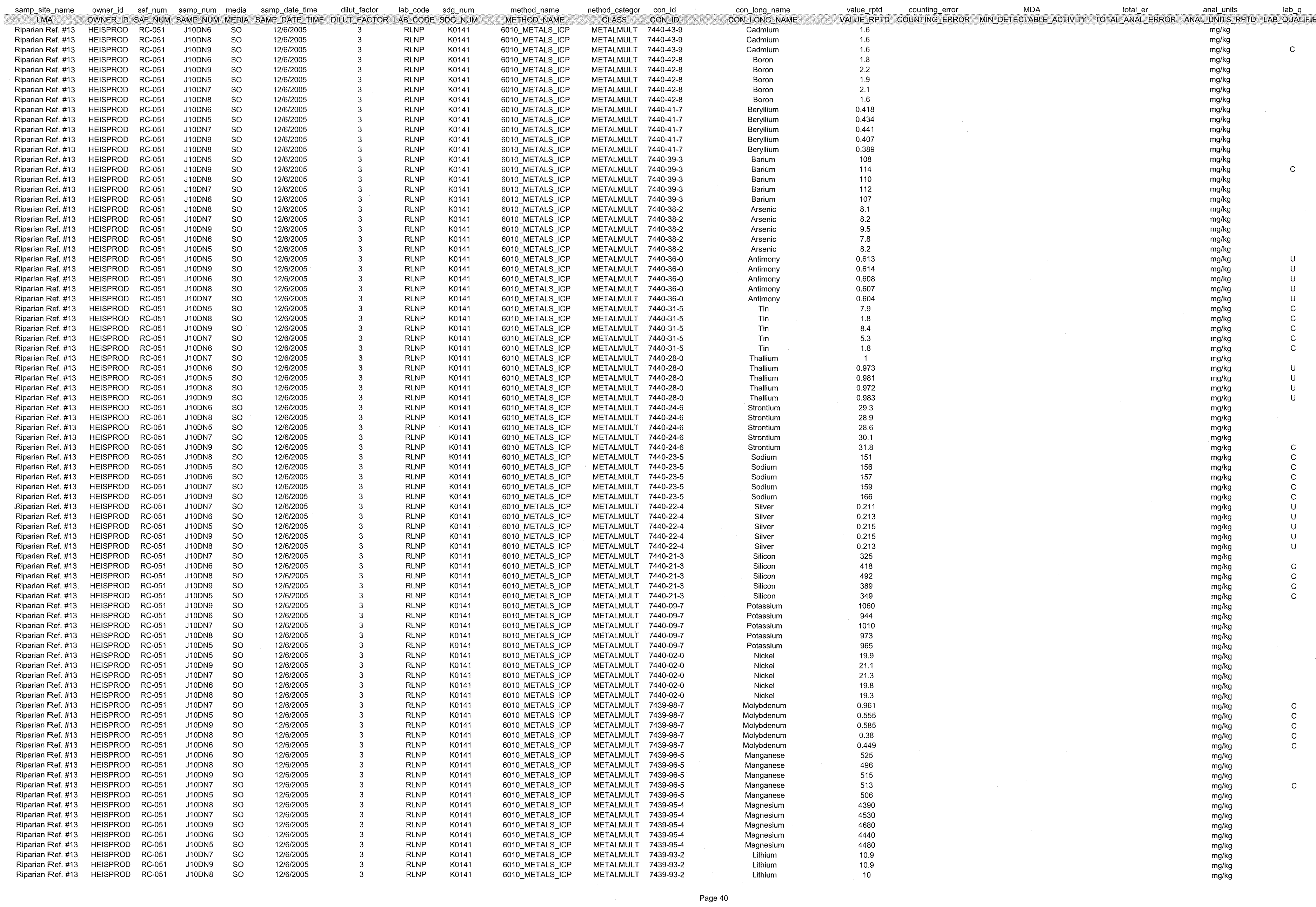


MIS_05 SAMPLES

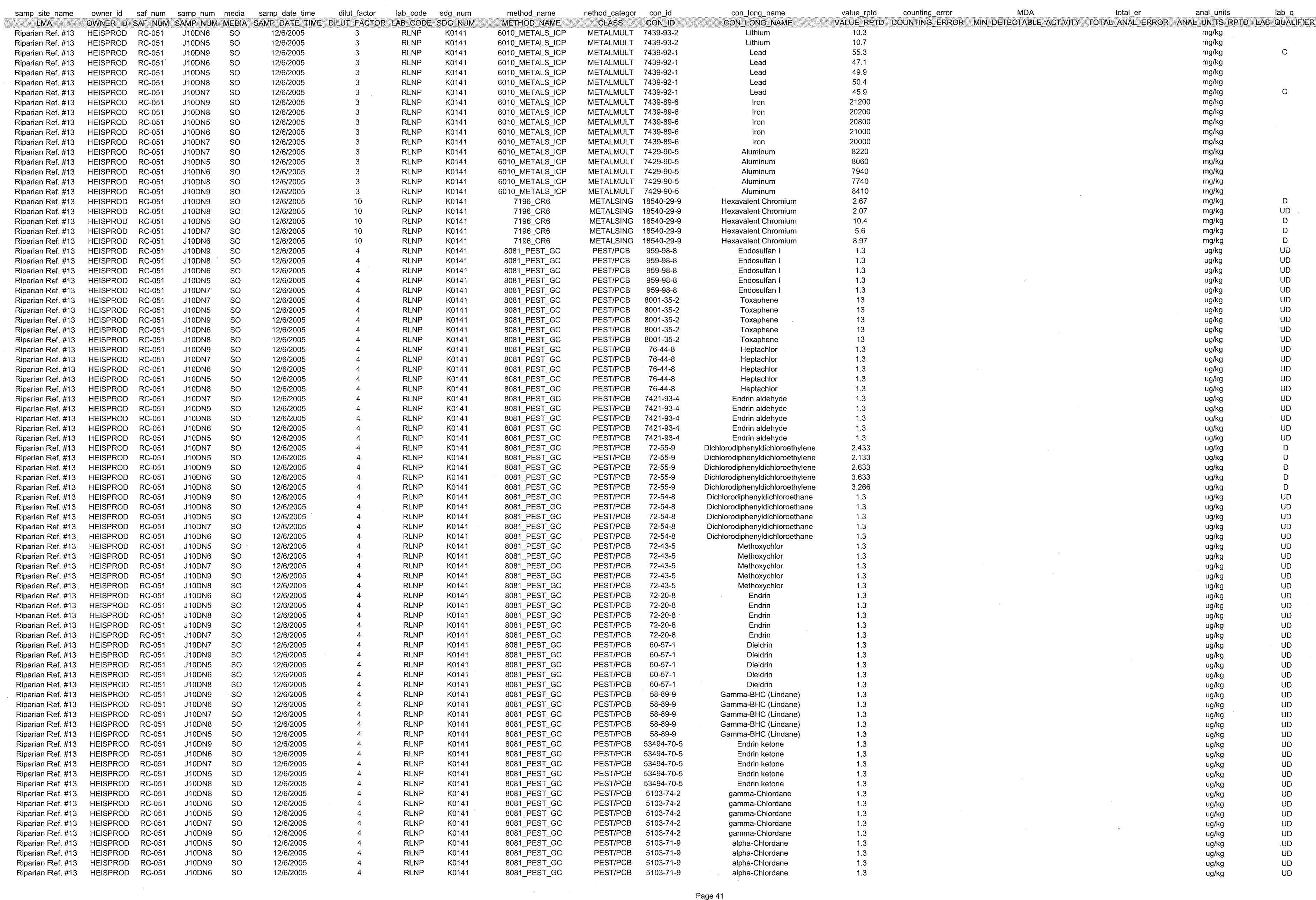


MIS_05 SAMPLES

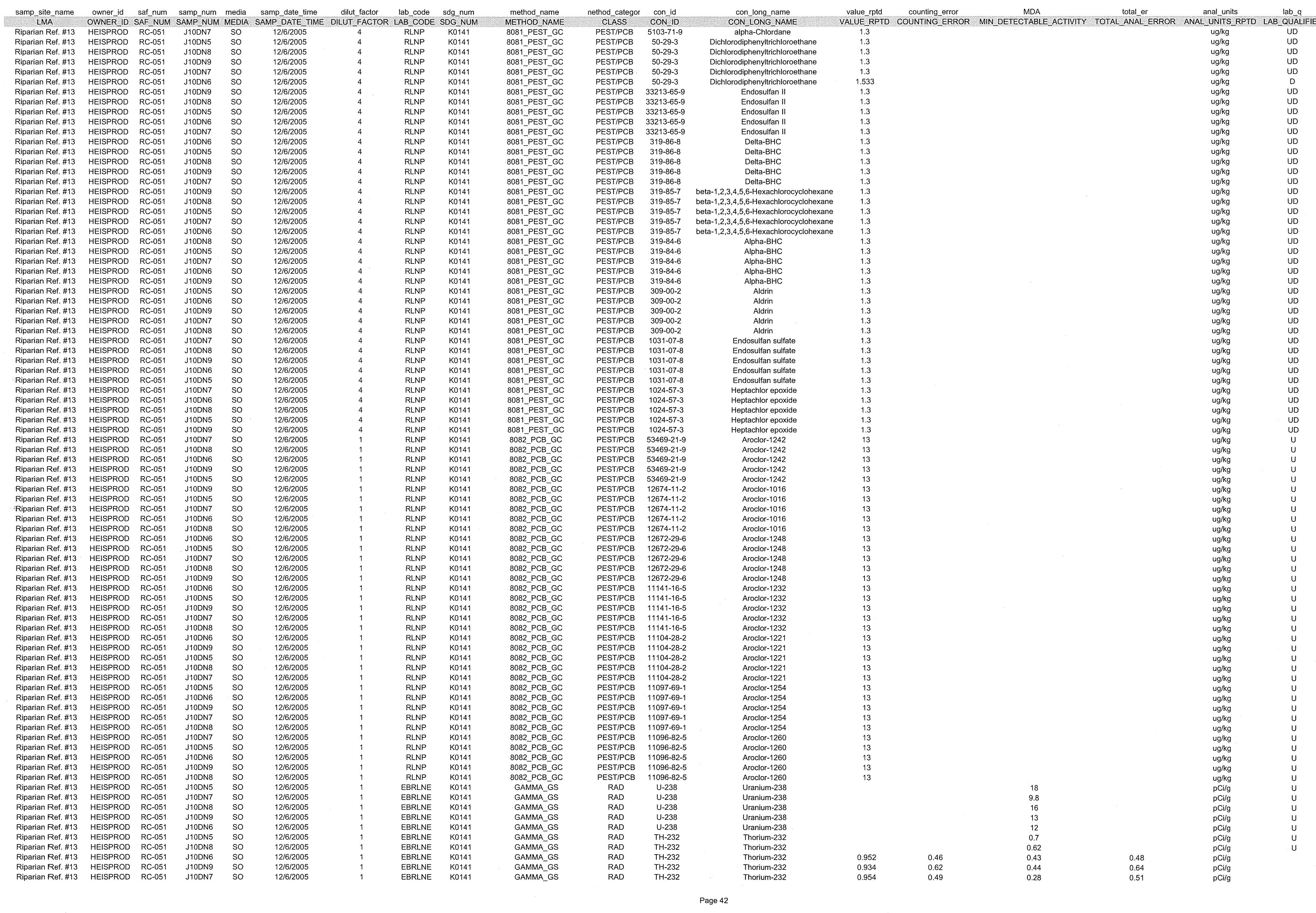


MIS_05 SAMPLES

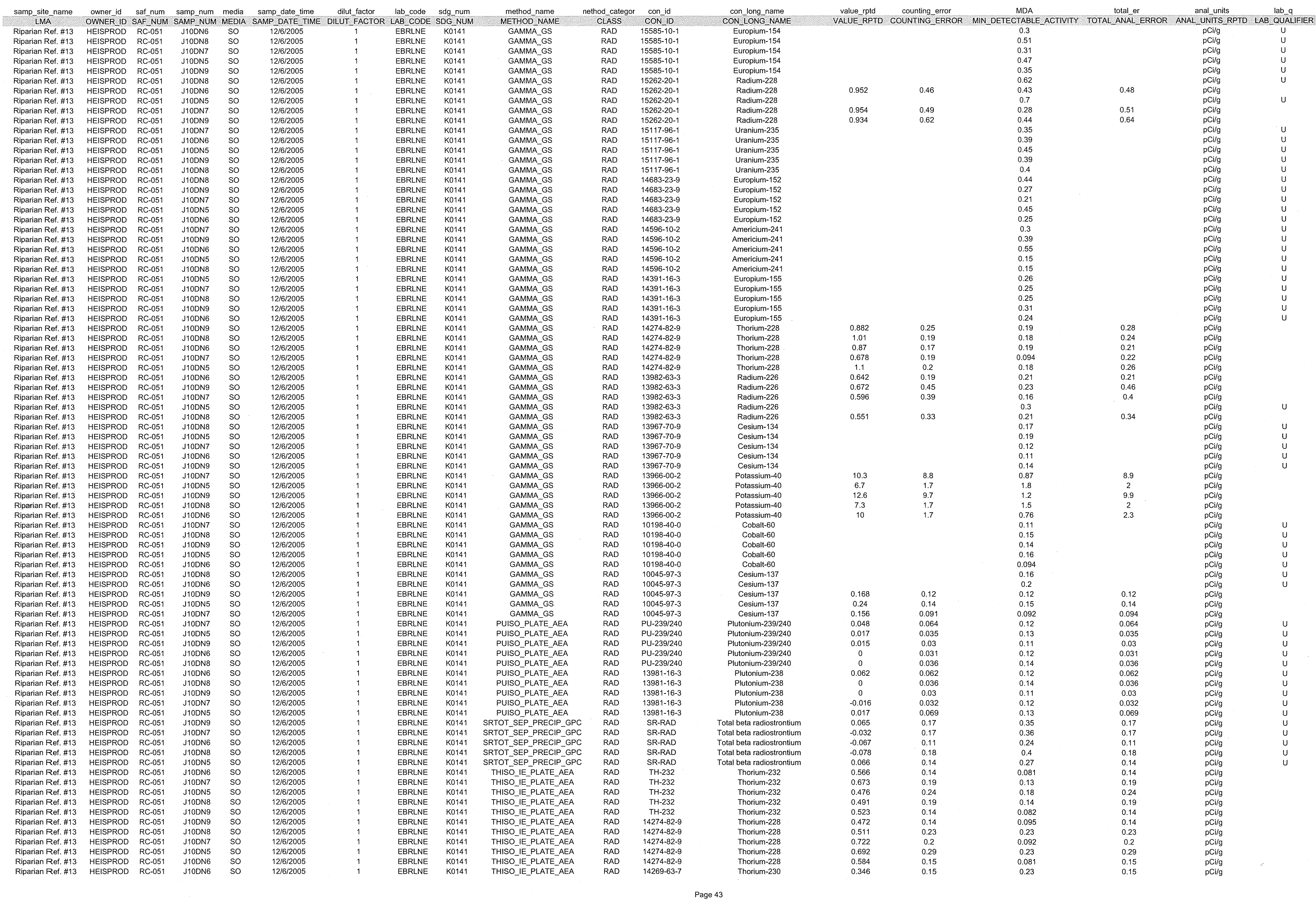




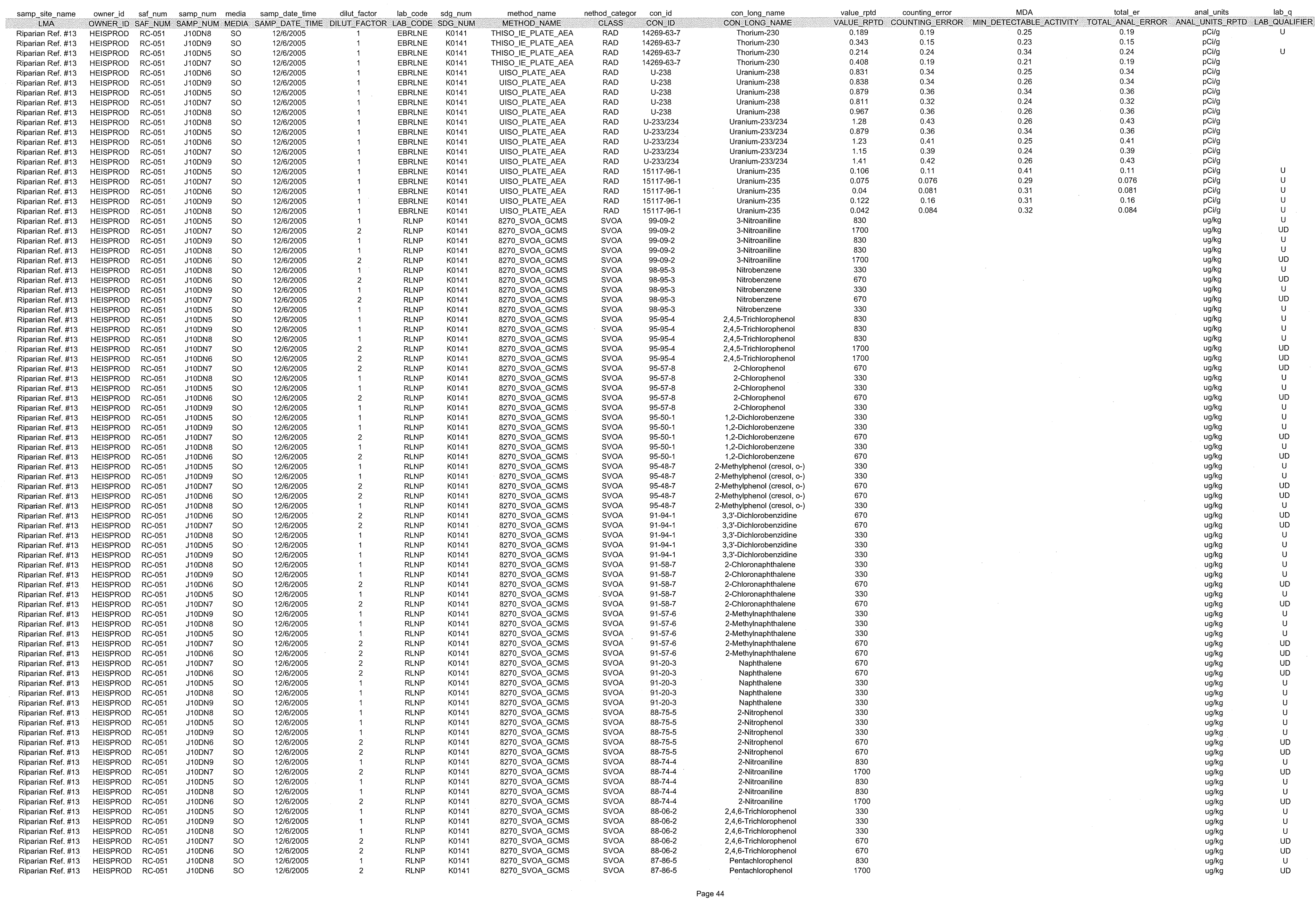


MIS_05 SAMPLES

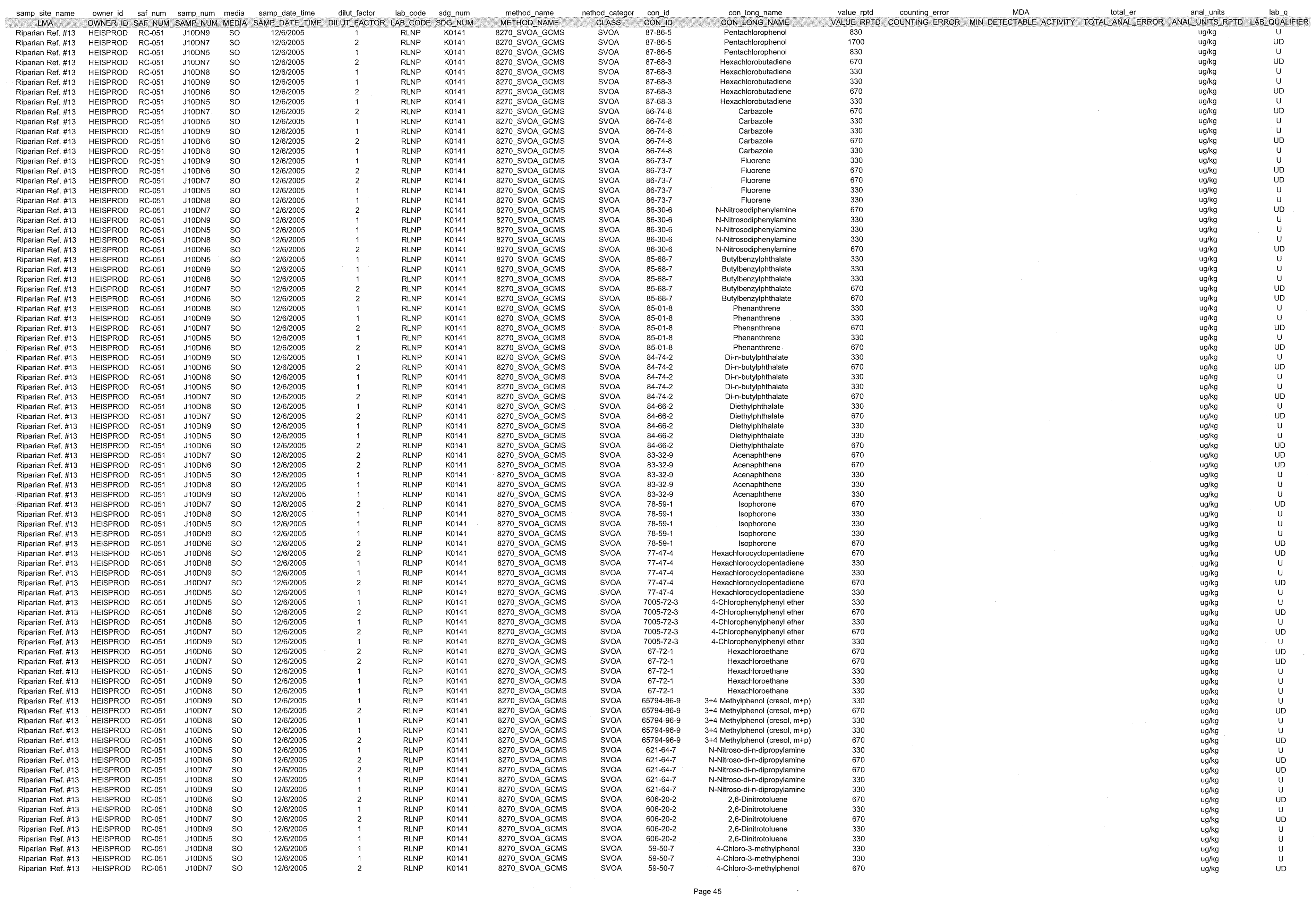


MIS_05 SAMPLES

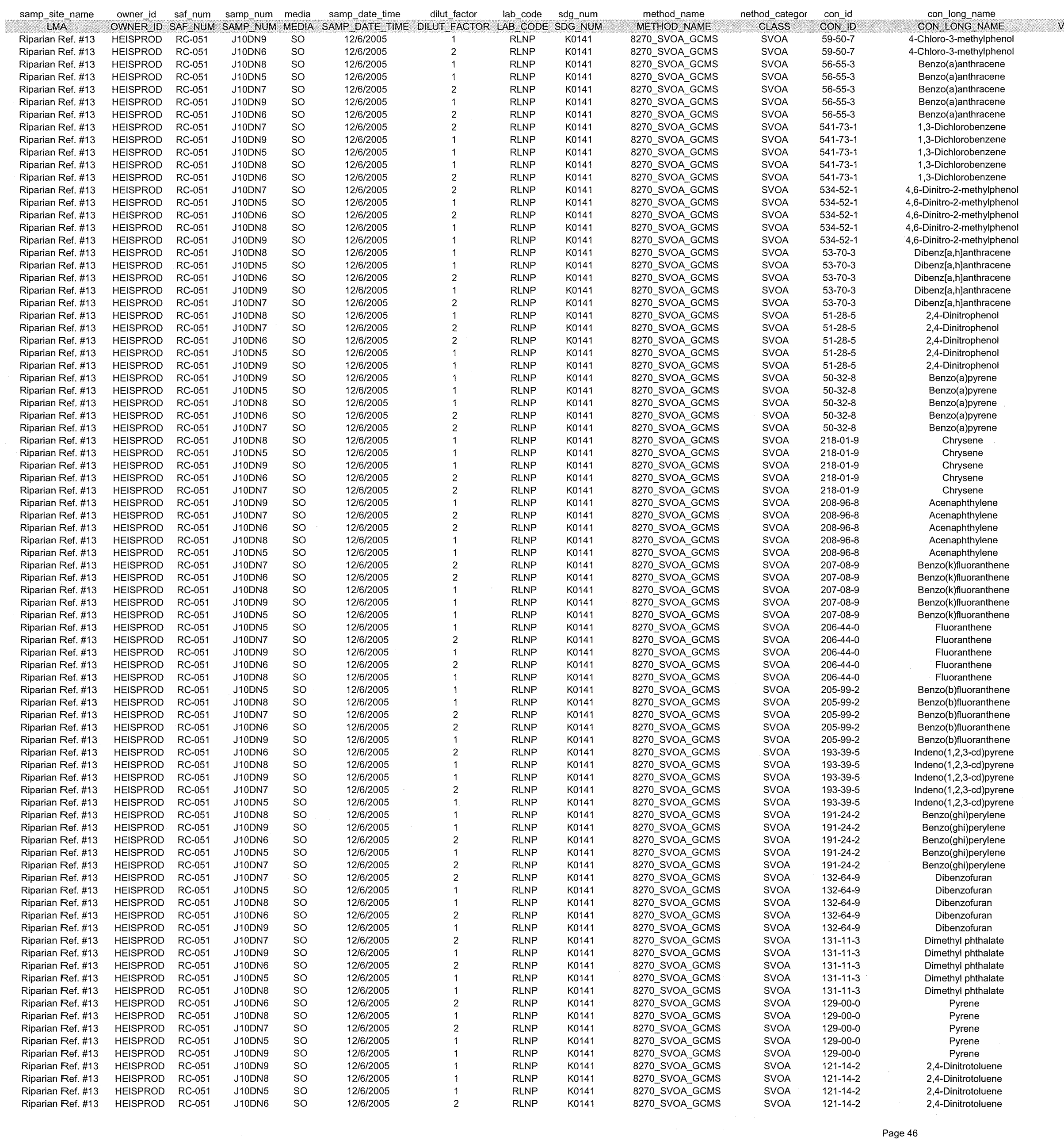

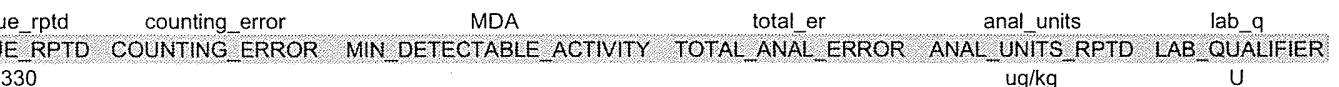
\begin{tabular}{l}
630 \\
670 \\
630 \\
330 \\
370 \\
670 \\
330 \\
670 \\
670 \\
330 \\
330 \\
330 \\
670 \\
6700 \\
780 \\
1700 \\
830 \\
830 \\
330 \\
330 \\
670 \\
330 \\
670 \\
830 \\
1700 \\
1700 \\
830 \\
830 \\
330 \\
330 \\
330 \\
670 \\
670 \\
330 \\
330 \\
330 \\
670 \\
670 \\
330 \\
670 \\
670 \\
330 \\
330 \\
670 \\
670 \\
330 \\
330 \\
330 \\
330 \\
670 \\
330 \\
670 \\
330 \\
330 \\
330 \\
670 \\
670 \\
330 \\
670 \\
330 \\
330 \\
670 \\
330 \\
330 \\
330 \\
670 \\
330 \\
670 \\
670 \\
330 \\
330 \\
670 \\
330 \\
670 \\
330 \\
670 \\
330 \\
330 \\
670 \\
330 \\
670 \\
330 \\
330 \\
330 \\
330 \\
330 \\
670 \\
67 \\
\hline
\end{tabular} 
MIS_05 SAMPLES

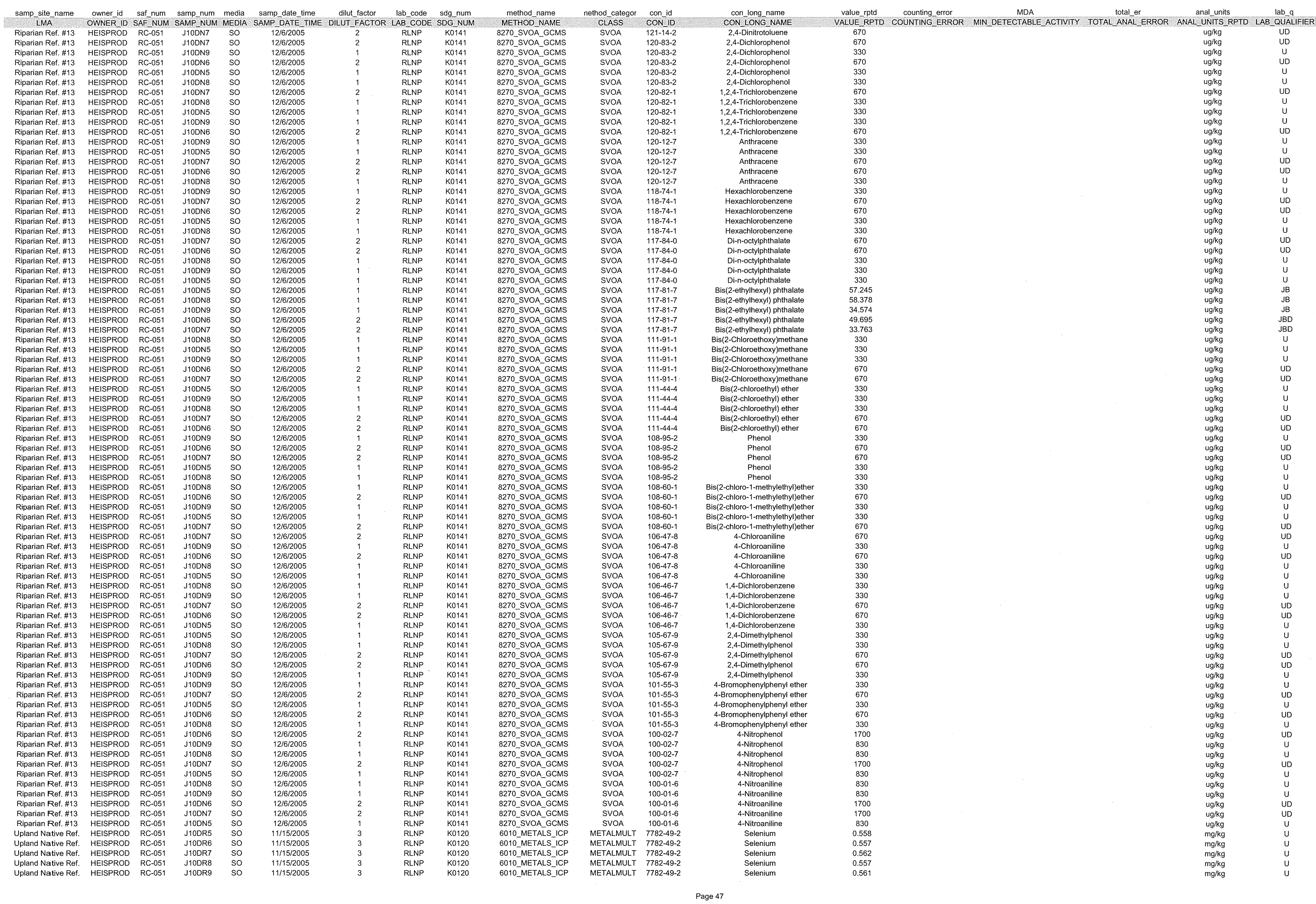


MIS_05 SAMPLES

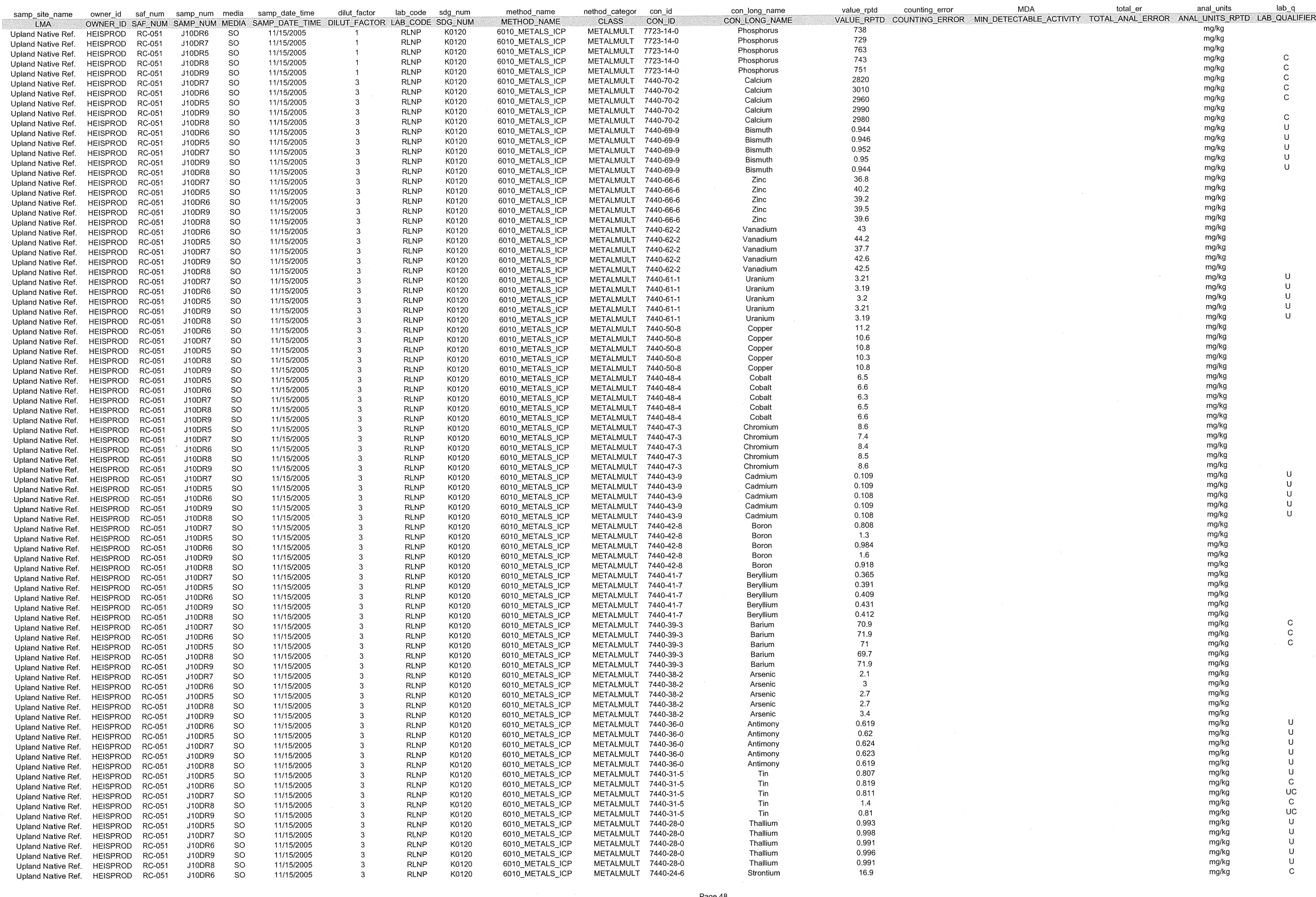


MIS_05 SAMPLES

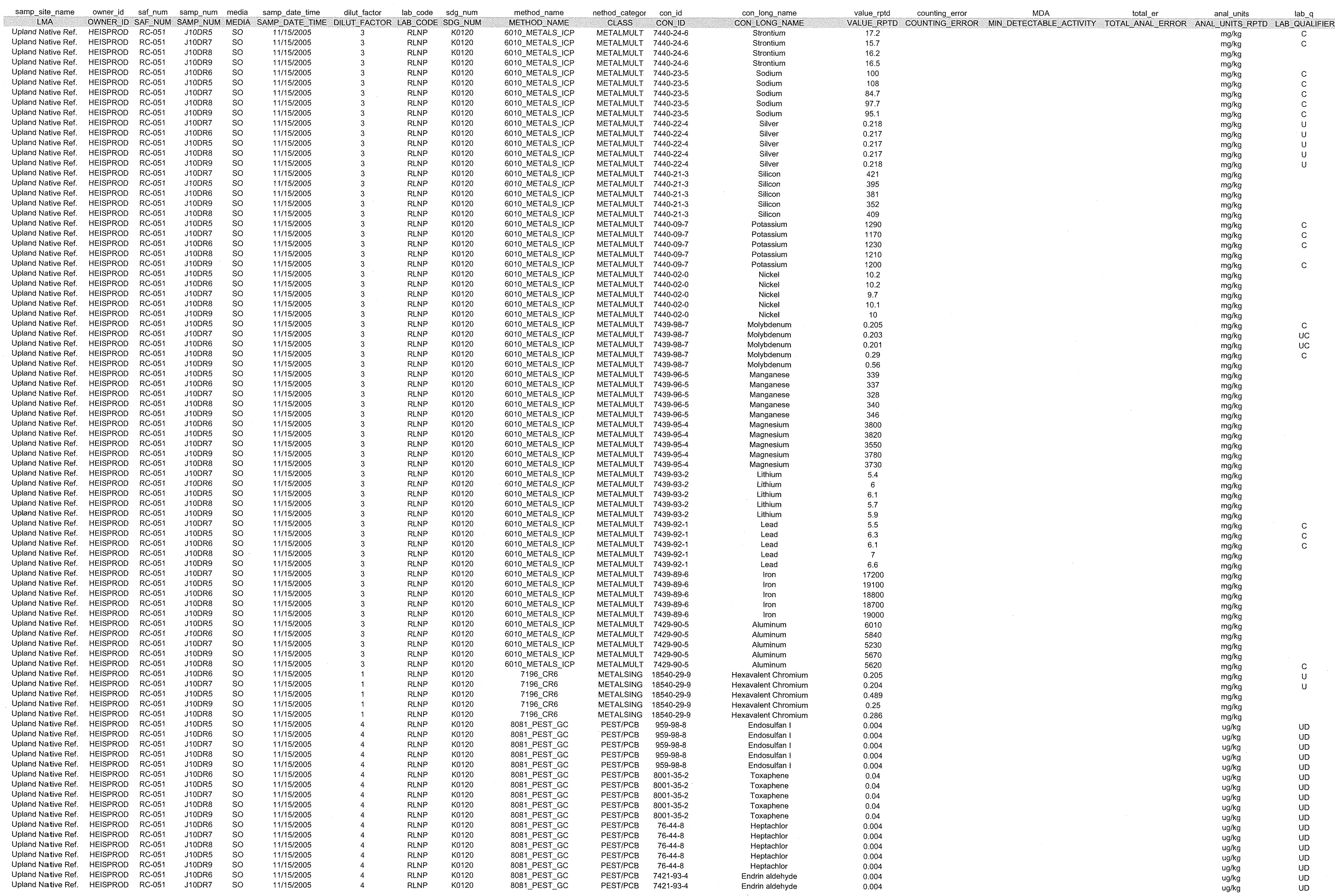


MIS_05 SAMPLES

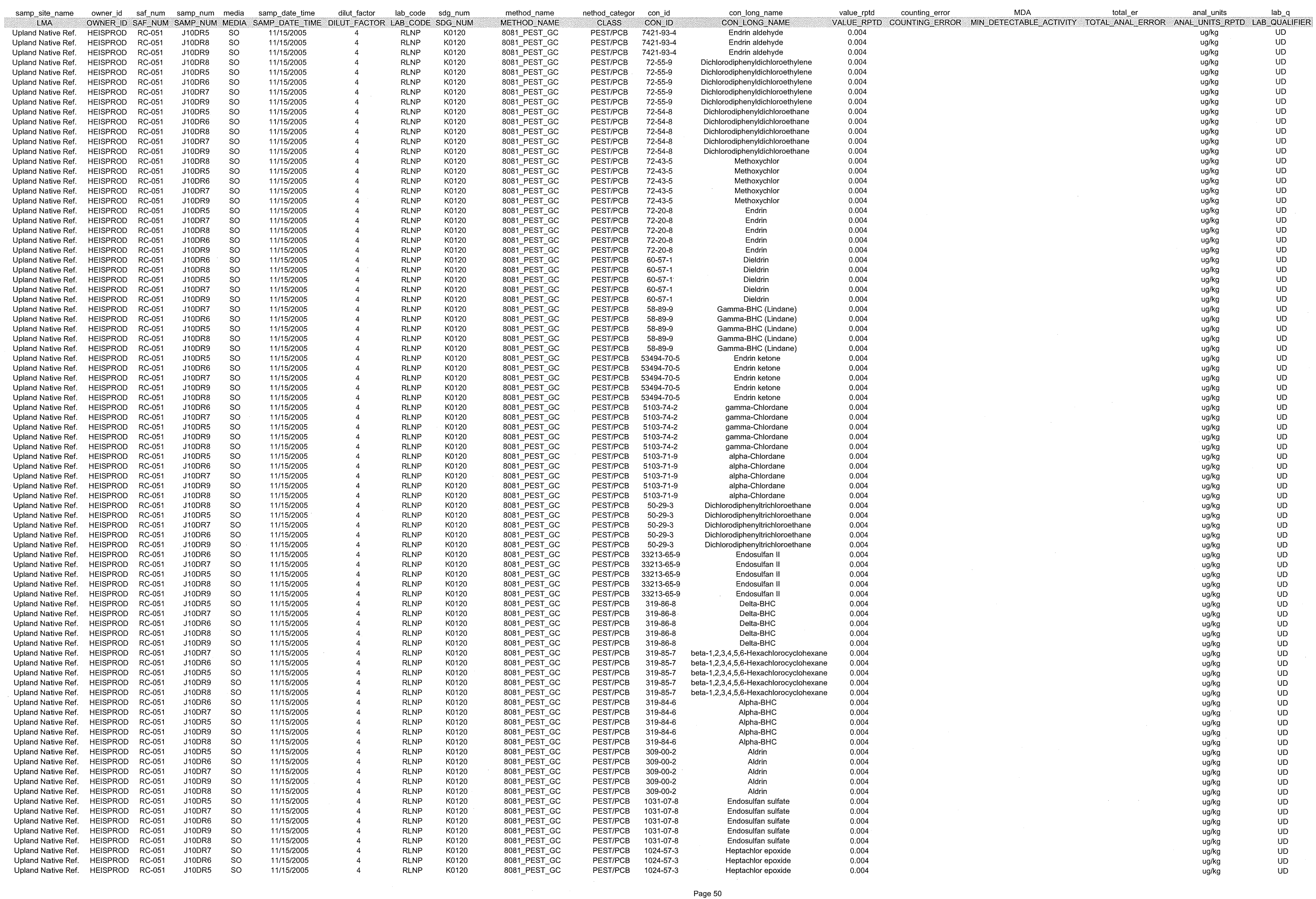


MIS_05 SAMPLES

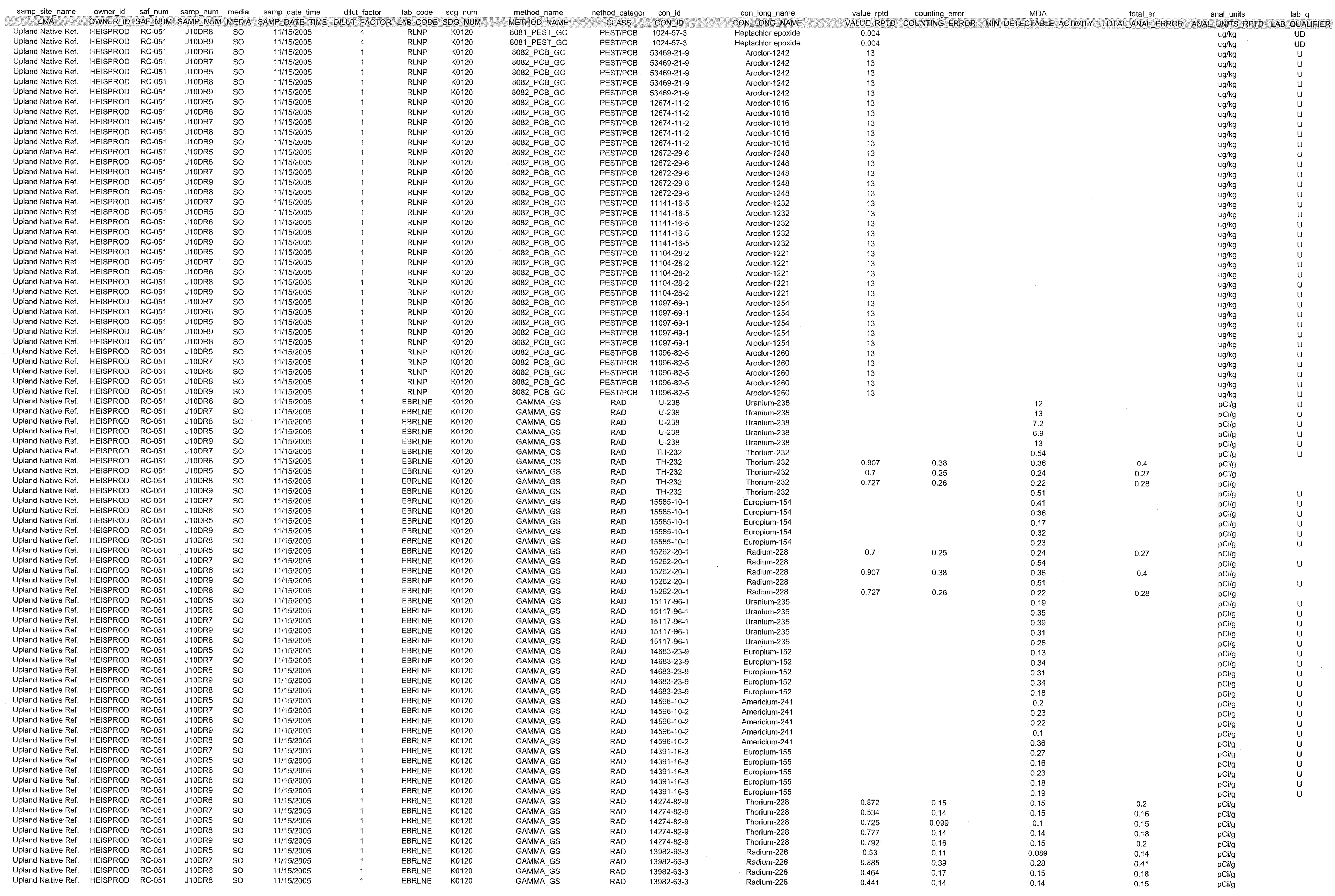




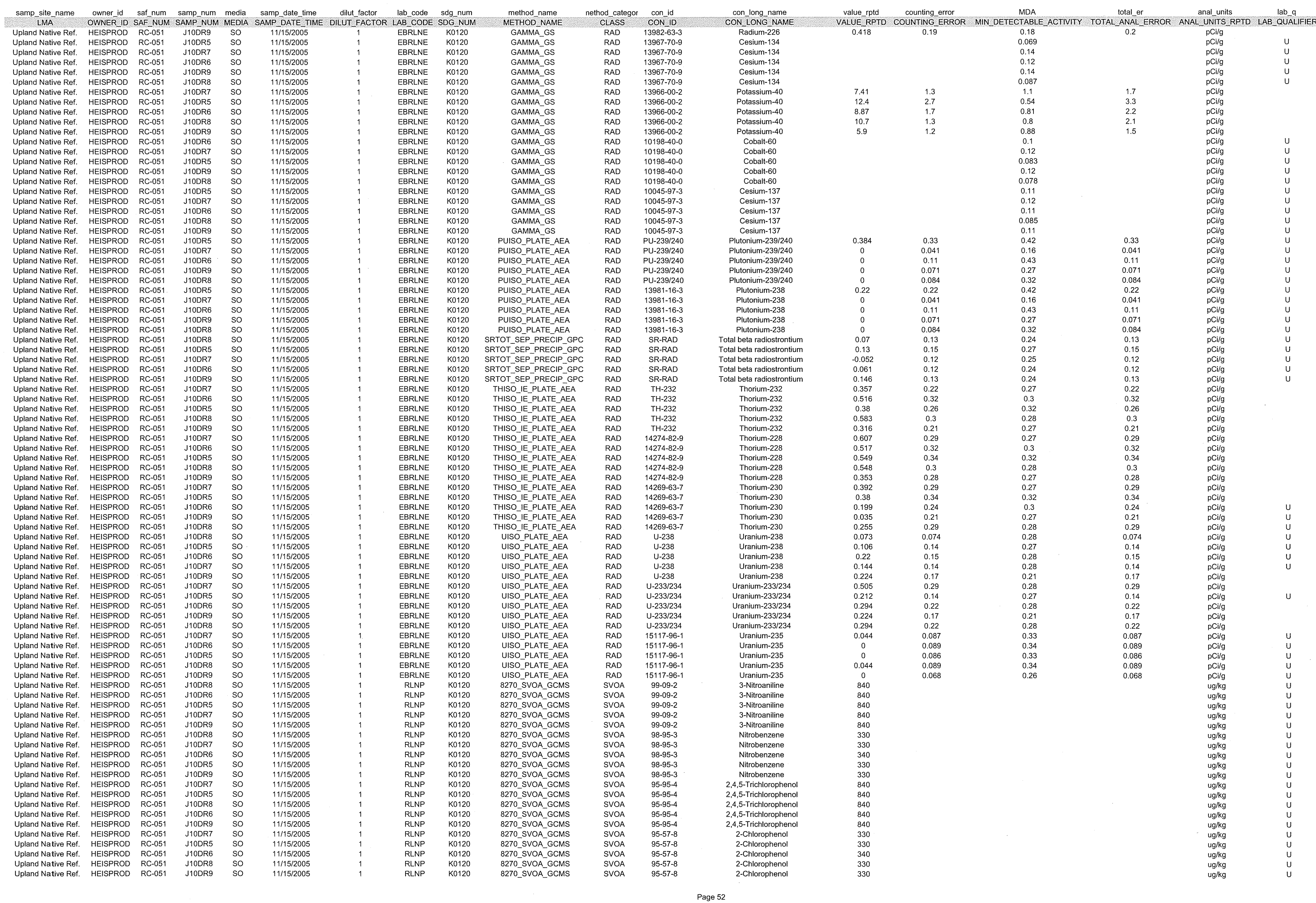




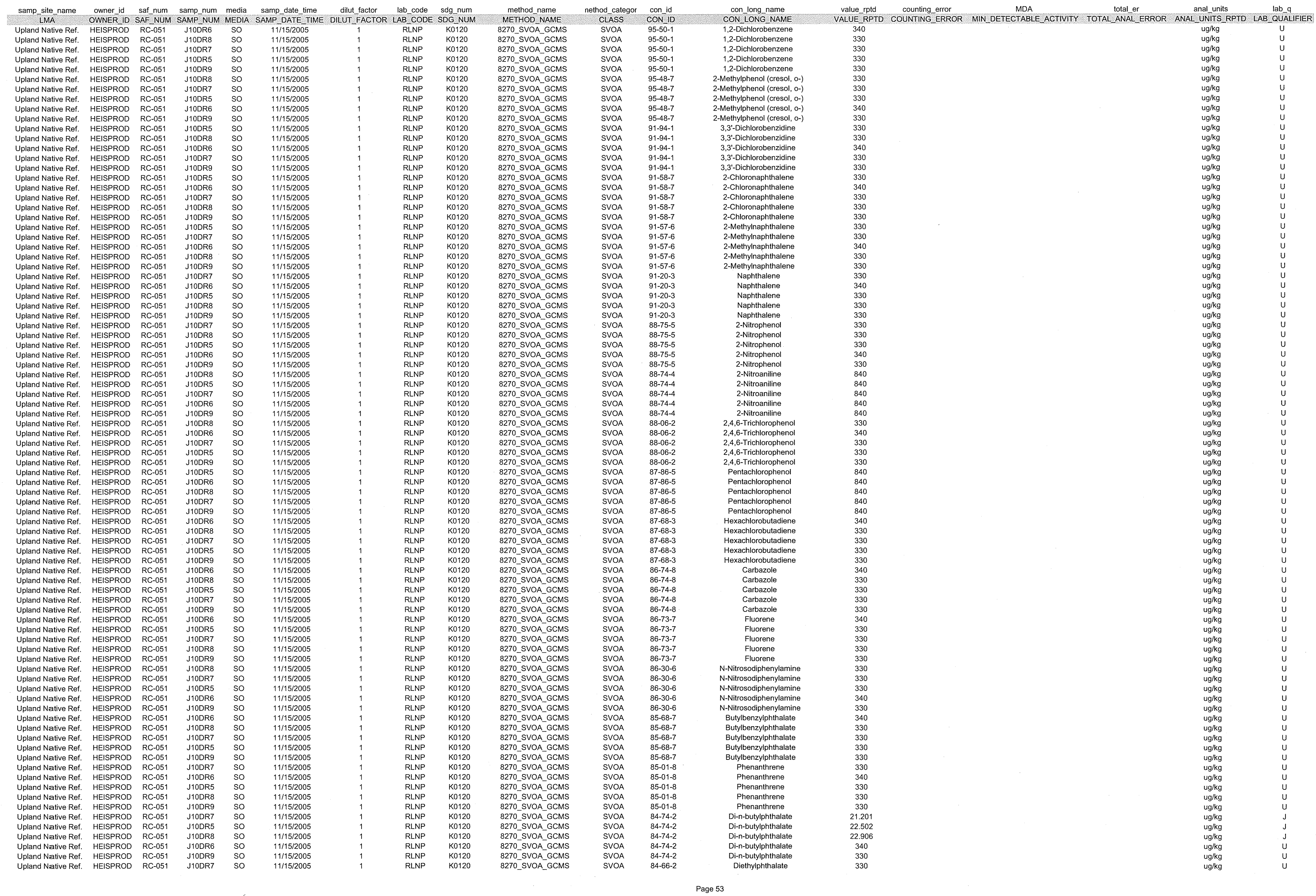




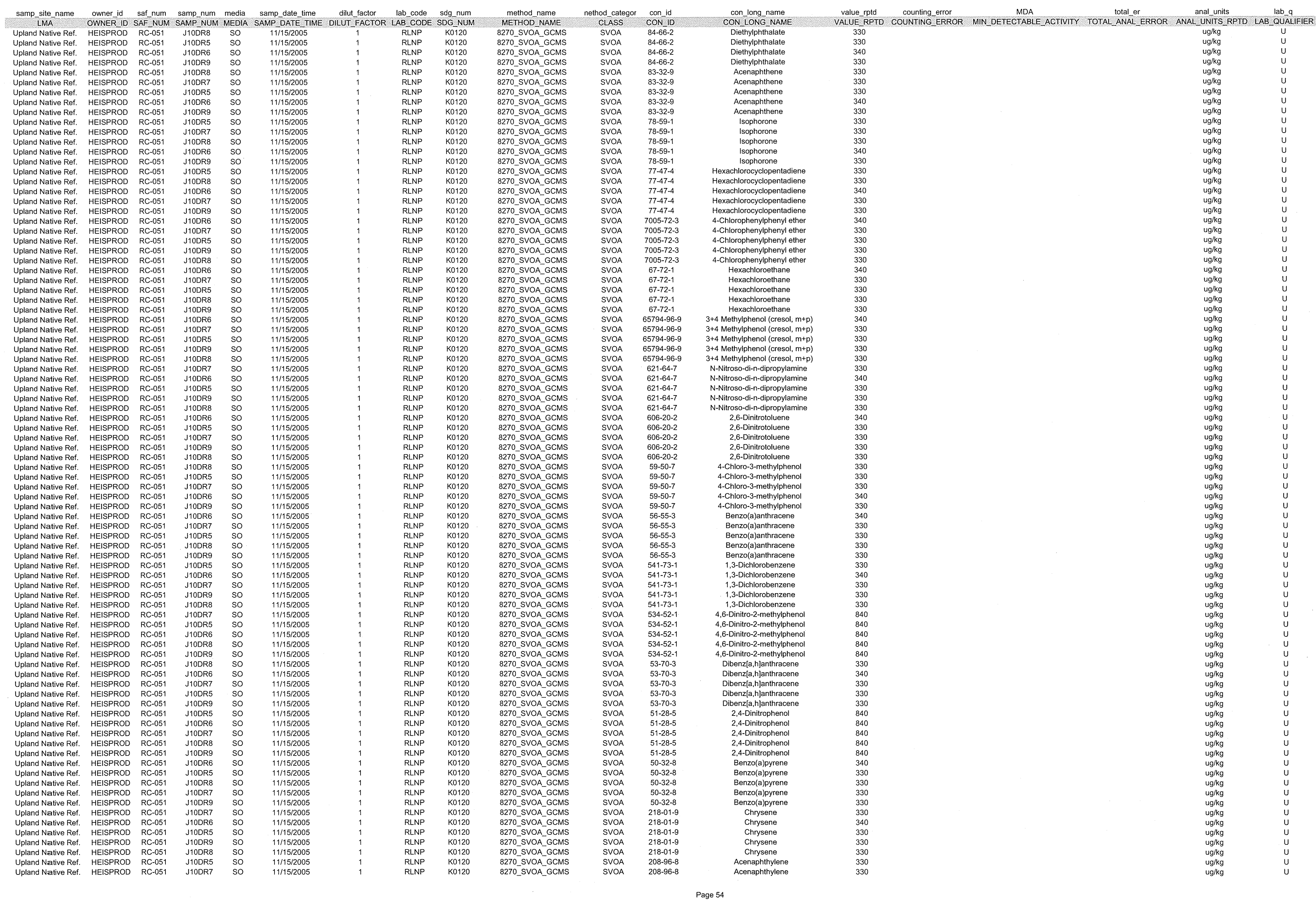


MIS_05 SAMPLES

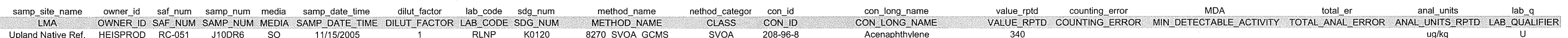
$171 / 1522005$
1111522005

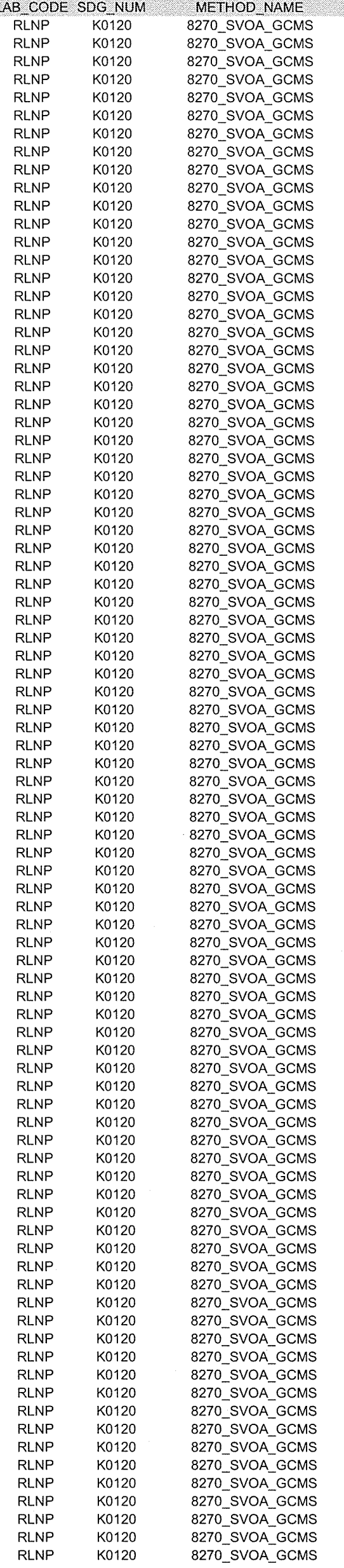

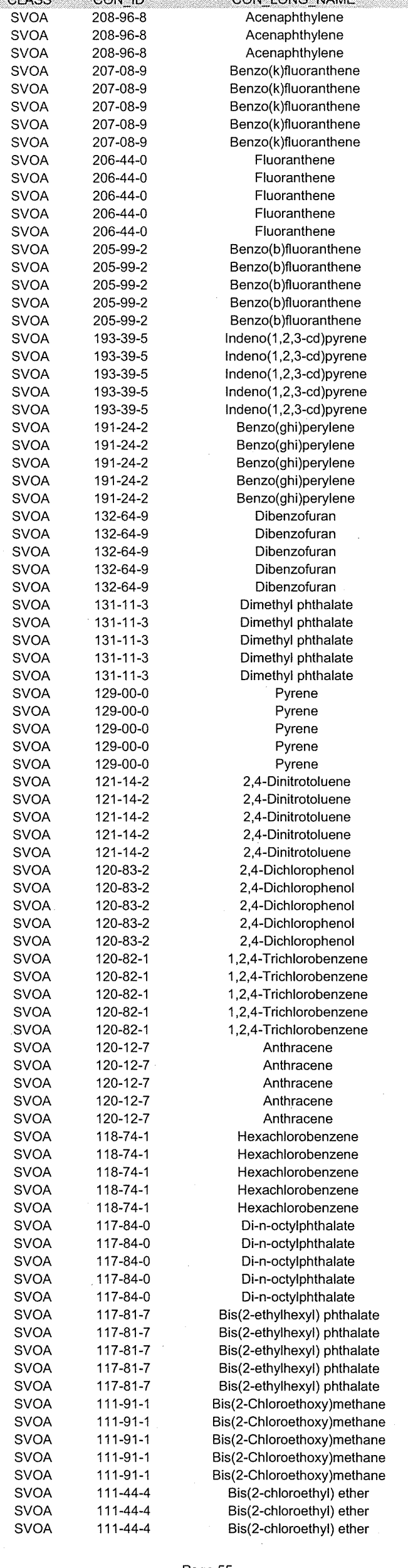


MIS_05 SAMPLES

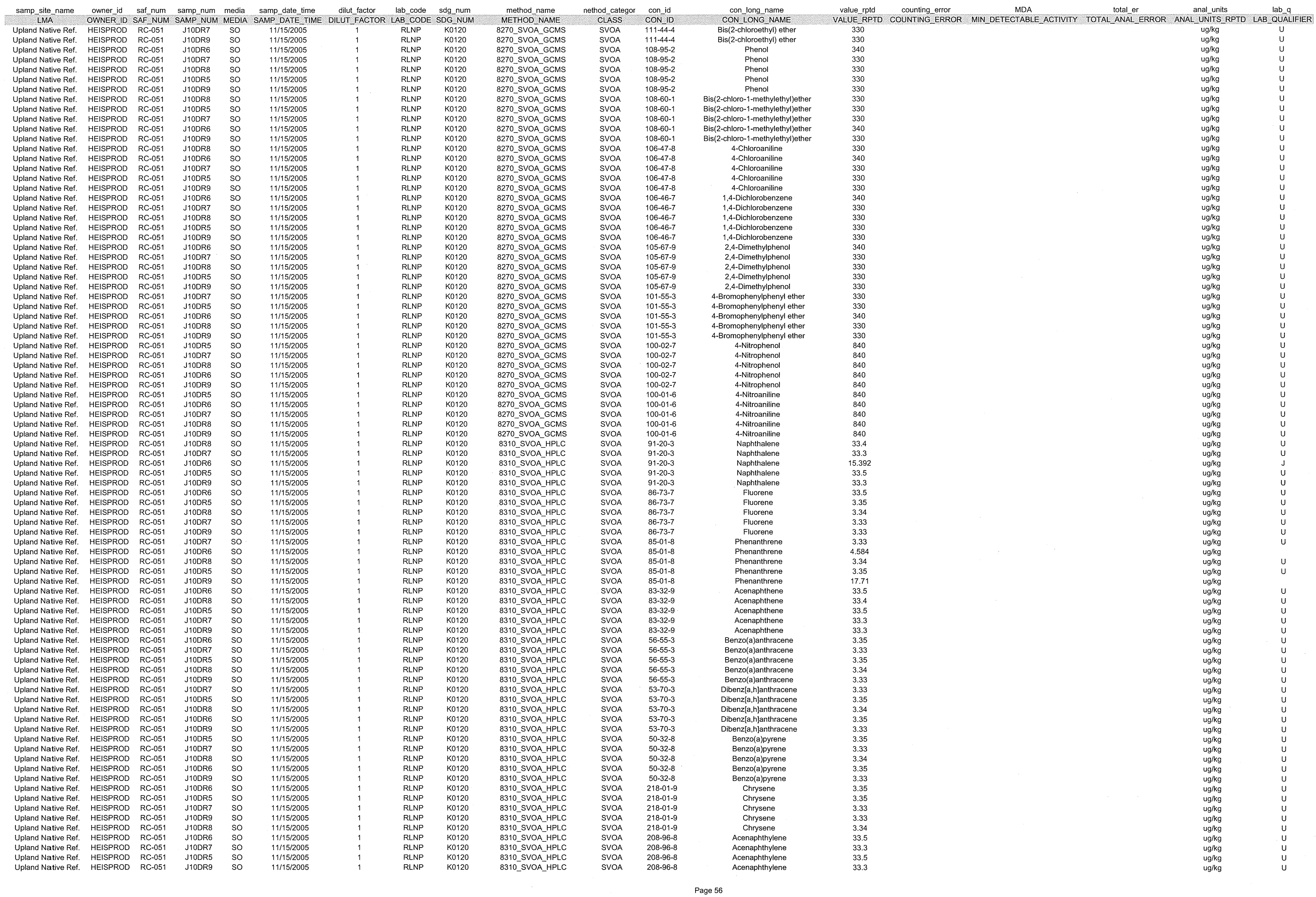


MIS_05 SAMPLES

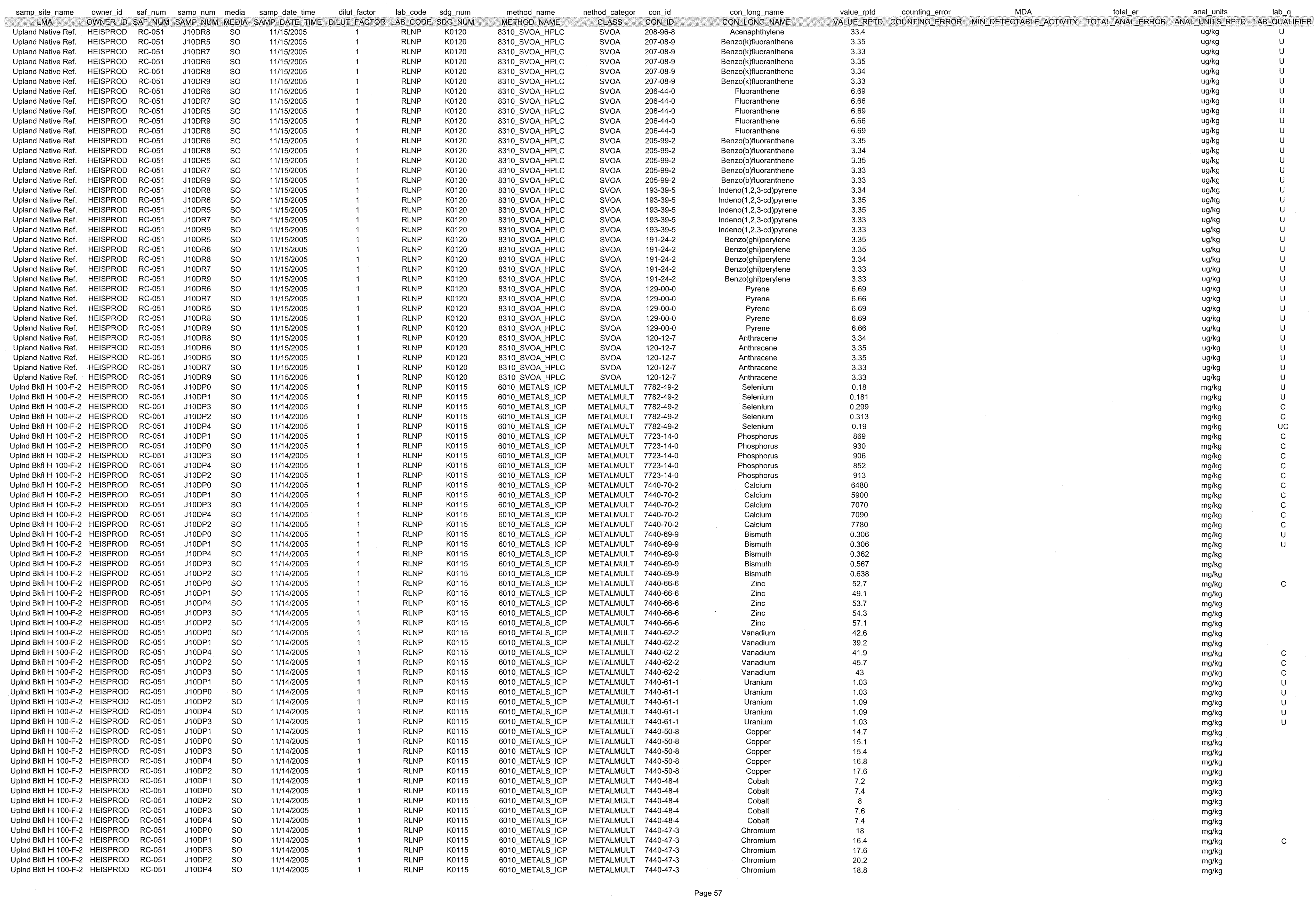


MIS_05 SAMPLES

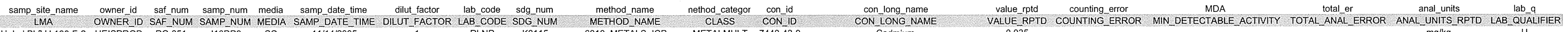

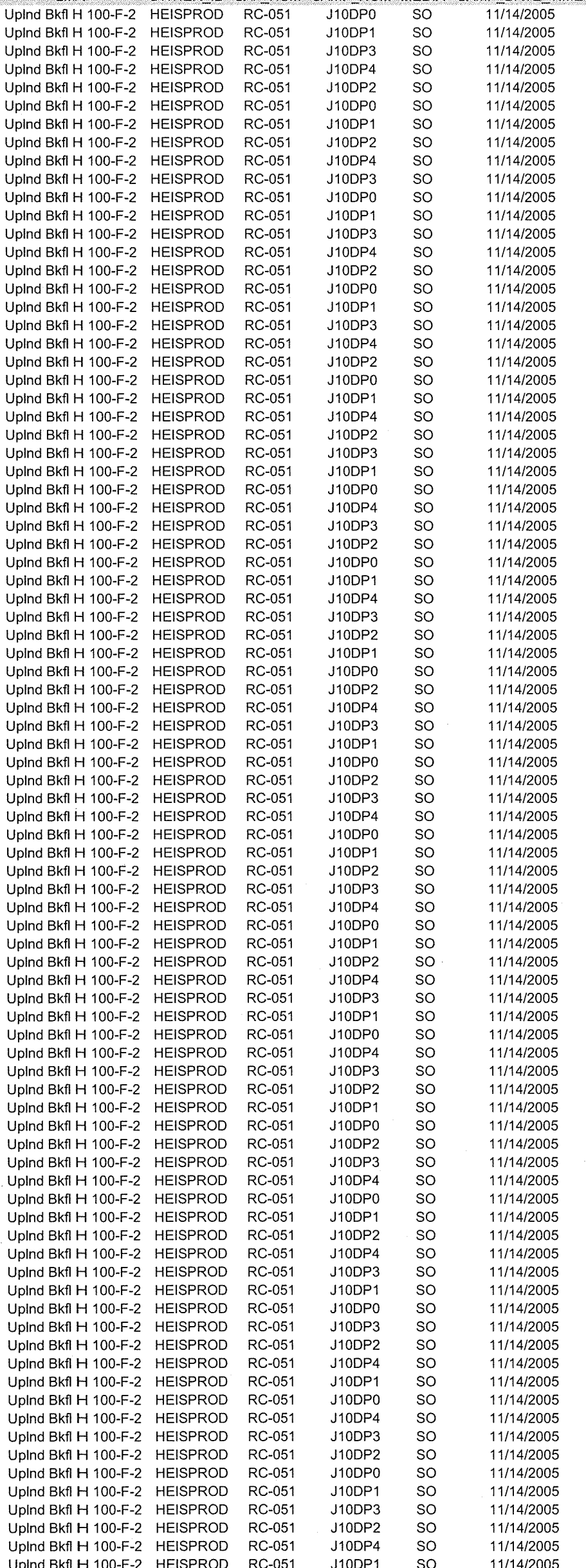

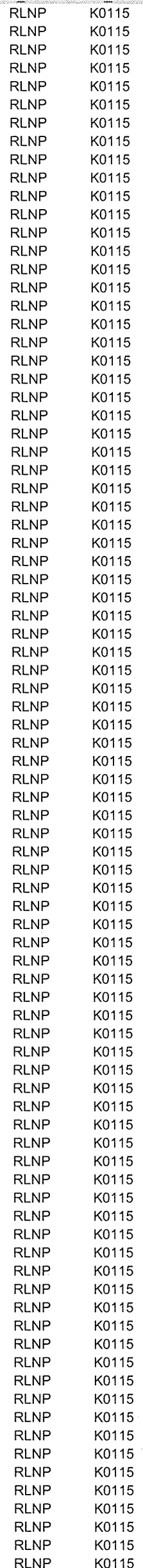

$\begin{array}{lll}\text { 6010_METALIICP } & \text { METALMULT } & \text { 7440-43-9 } \\ \text { 6010_METALSICP } & \text { METALMULT } & 7440-43-9\end{array}$

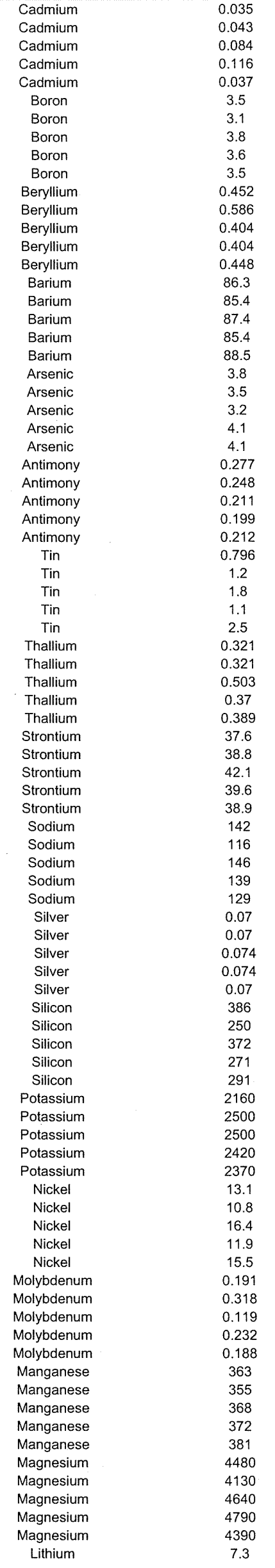

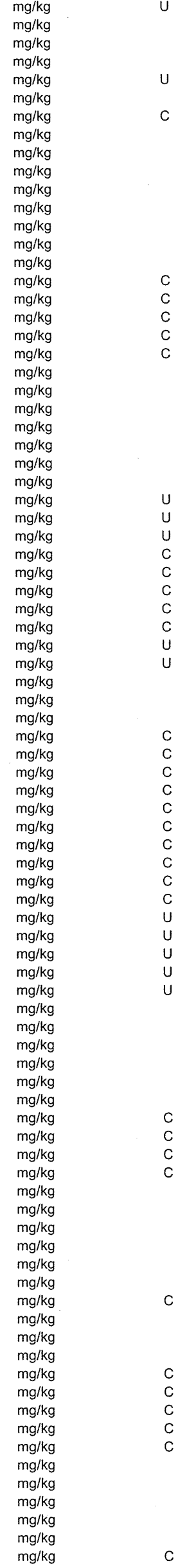

Page 58 
MIS_05 SAMPLES

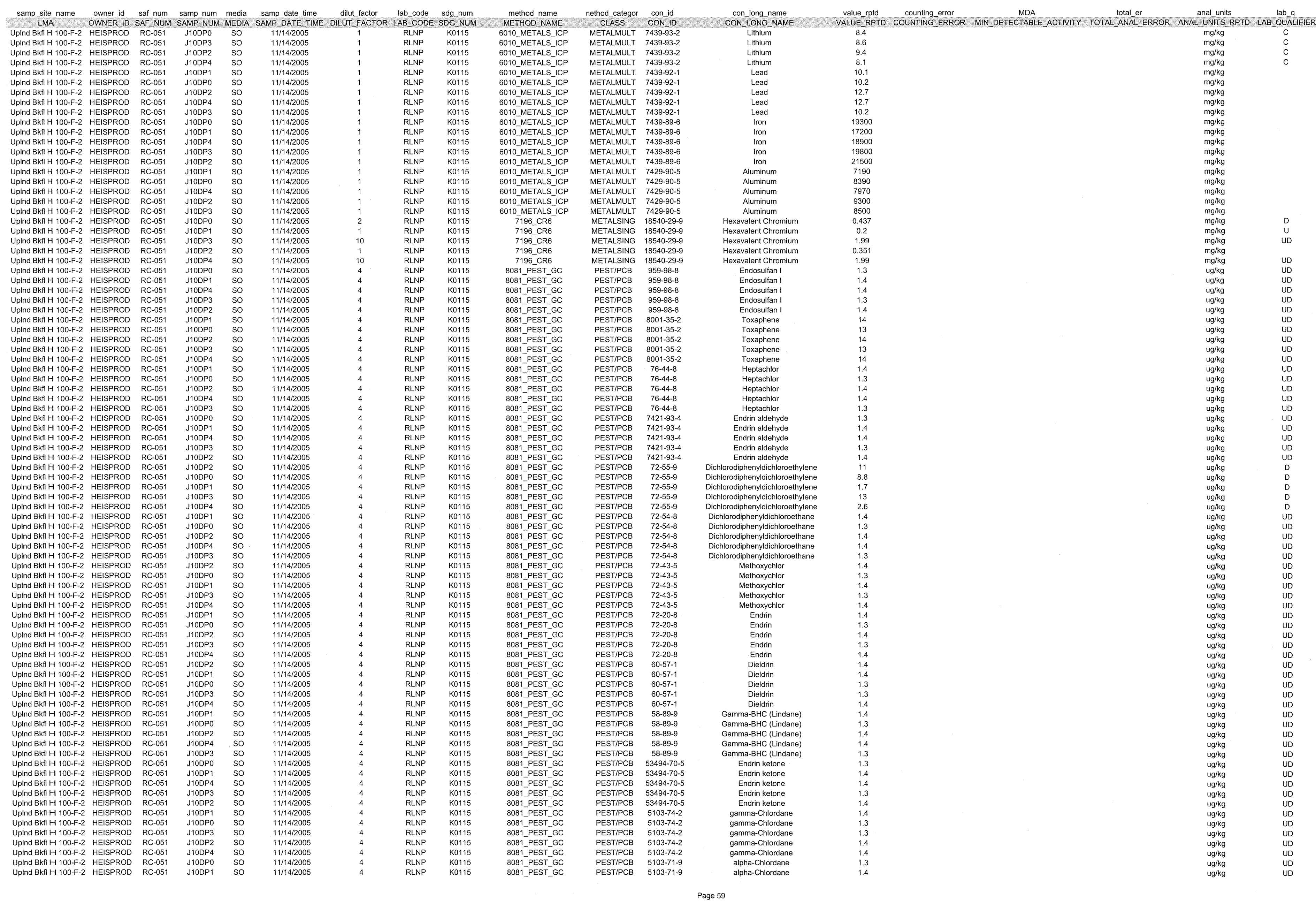


MIS_05 SAMPLES

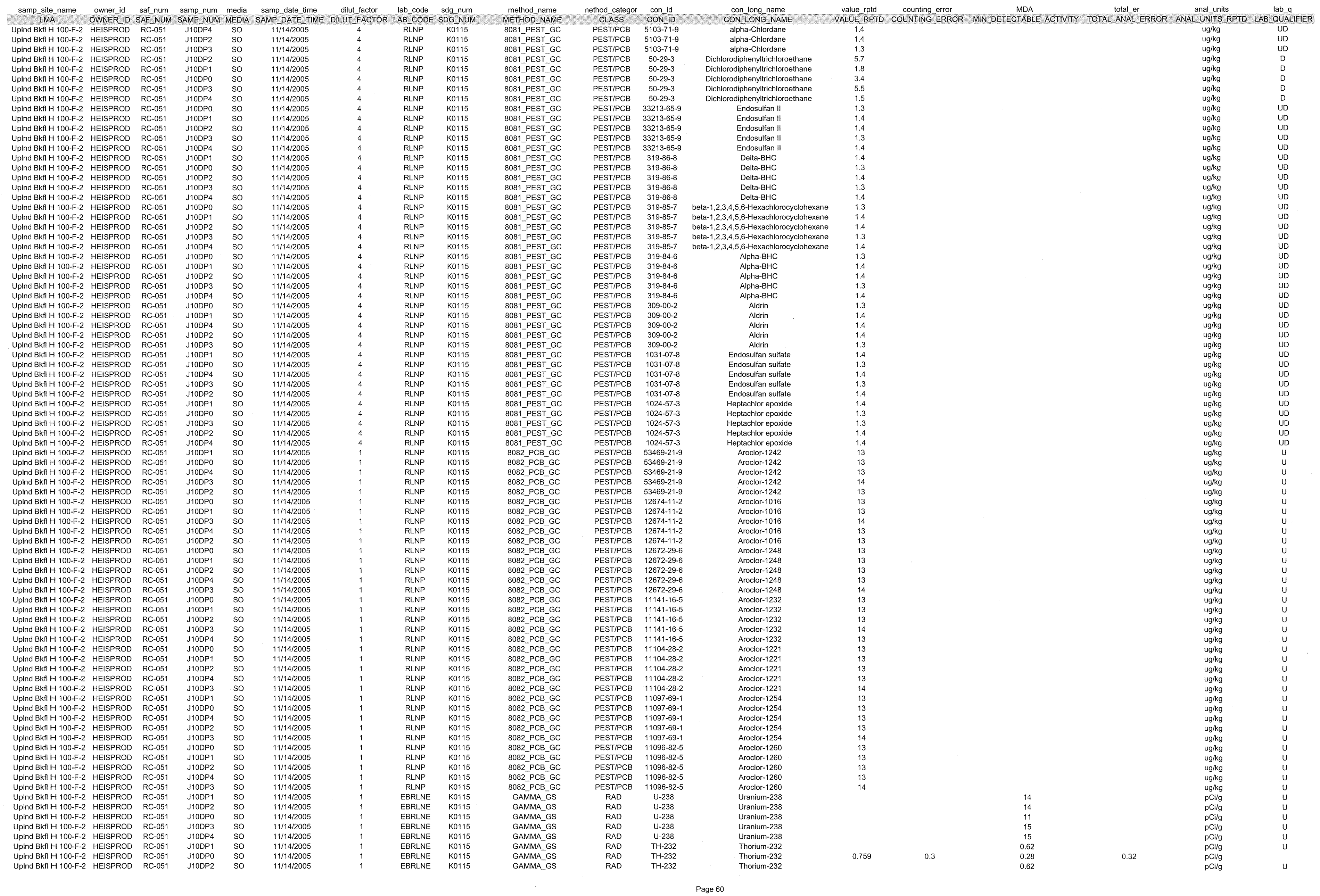


MIS_05 SAMPLES

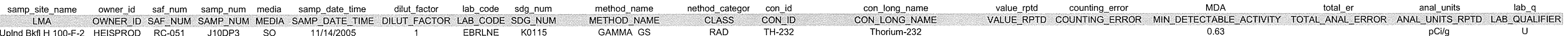

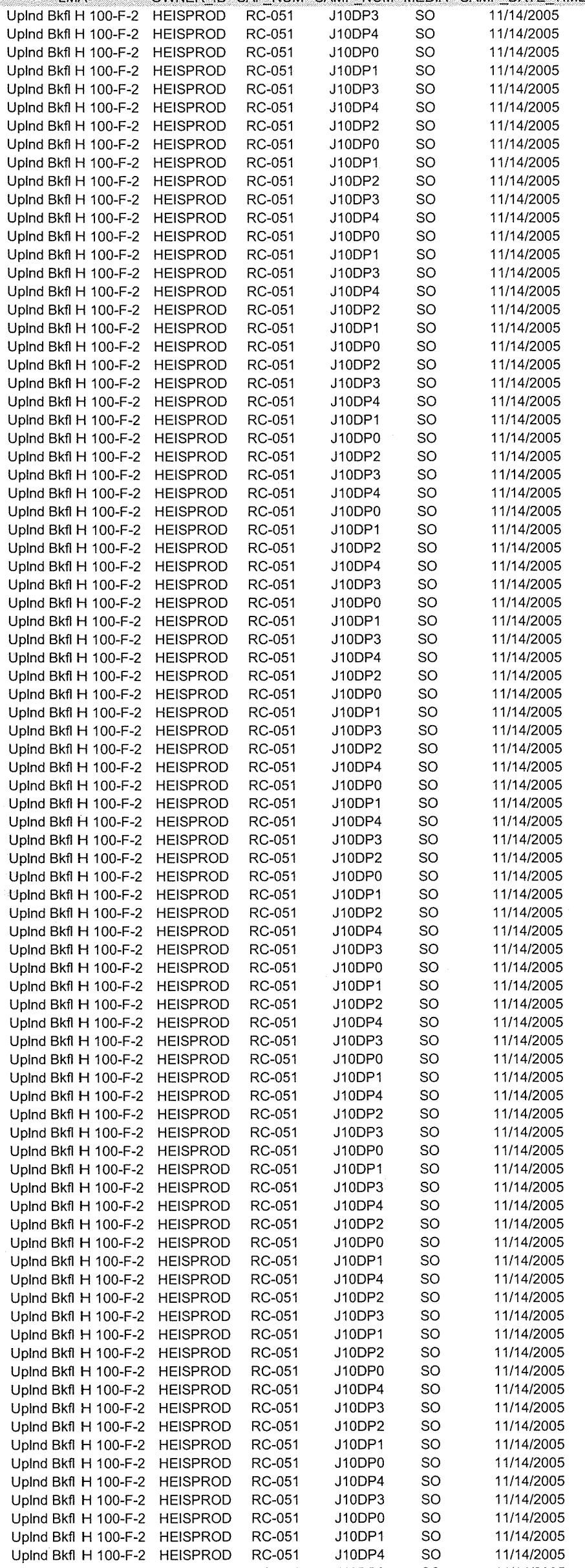

\begin{tabular}{|c|c|c|}
\hline ENRE & 年 & \\
\hline $\begin{array}{l}\text { EERLE } \\
\text { ERLLNE }\end{array}$ & $\begin{array}{l}\text { Ko115 } \\
\text { Ko115 }\end{array}$ & $\begin{array}{l}\text { GAMMA_GS } \\
\text { GAMMA GS }\end{array}$ \\
\hline & Ko115 & $\begin{array}{l}\text { GAMMA-GS } \\
\text { GAMM_GS }\end{array}$ \\
\hline $\begin{array}{l}\text { ERRLNE } \\
\text { ERRLNE }\end{array}$ & $\begin{array}{l}\text { Ko115 } \\
\text { Ko115 }\end{array}$ & $\begin{array}{l}\text { GAMMA_GS } \\
\text { GAMMA GS }\end{array}$ \\
\hline $\begin{array}{l}\text { E ERLNE } \\
\text { ERR }\end{array}$ & Ko115 & $\begin{array}{l}\text { GAMMA-GS } \\
\text { GAMM_GS }\end{array}$ \\
\hline $\begin{array}{l}\text { ERRLE } \\
\text { ERRLNE }\end{array}$ & $\begin{array}{l}\text { k0115 } \\
0115\end{array}$ & $\begin{array}{l}\text { GAMMA-GS } \\
\text { GAMM_GS }\end{array}$ \\
\hline & Ko115 & GAMMA GS \\
\hline $\begin{array}{l}\text { ERRLE } \\
\text { ERRLNE }\end{array}$ & 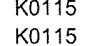 & $\begin{array}{l}\text { GAMMA-GS } \\
\text { GAMMA GS }\end{array}$ \\
\hline ERRLE & $\begin{array}{l}K 0115 \\
K 0115\end{array}$ & $\begin{array}{l}\text { GAMMAGS } \\
\text { GAMM_GS }\end{array}$ \\
\hline EBRLNE & Ko115 & $\begin{array}{l}\text { GAMMAGGS } \\
\text { GAMMA GS }\end{array}$ \\
\hline & $\begin{array}{l}\text { Ko115 } \\
\text { Ko115 }\end{array}$ & $\begin{array}{l}\text { GAMMA-GS } \\
\text { GAMM_GS }\end{array}$ \\
\hline & $\begin{array}{l}\text { Ko115 } \\
\text { Ko115 }\end{array}$ & $\begin{array}{l}\text { GAMMA-GS } \\
\text { GAMMA GS }\end{array}$ \\
\hline & $\begin{array}{l}\text { Ko115 } \\
\text { K0115 }\end{array}$ & $\begin{array}{l}\text { GAMMA_GS } \\
\text { GAMM_GS }\end{array}$ \\
\hline & Ko115 & 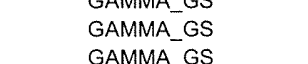 \\
\hline $\begin{array}{l}\text { RLNE } \\
\text { RLNE }\end{array}$ & $\begin{array}{l}K 0115 \\
\text { Ko115 }\end{array}$ & $\begin{array}{l}\text { GAMMA_GS } \\
\text { GAMM_GS }\end{array}$ \\
\hline & Ko115 & $\begin{array}{l}\text { GAMMA-GS } \\
\text { GAMMA GS }\end{array}$ \\
\hline RILE & 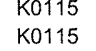 & $\begin{array}{l}\text { GAMMAGS } \\
\text { GAMM_GS }\end{array}$ \\
\hline & $\begin{array}{l}\text { K0115 } \\
\text { Ko115 }\end{array}$ & $\begin{array}{l}\text { GAMMA_GS } \\
\text { GAMMA GS }\end{array}$ \\
\hline RLNE & Ko115 & $\begin{array}{l}\text { GAMMAGS } \\
\text { GAMA-GS }\end{array}$ \\
\hline & 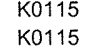 & $\begin{array}{l}\text { GAMMA_GS } \\
\text { GAMM_GS }\end{array}$ \\
\hline RLNE & Ko115 & $\begin{array}{l}\text { GAMMA_GS } \\
\text { GAMMA GS }\end{array}$ \\
\hline & $\begin{array}{l}K 0115 \\
K 0115\end{array}$ & $\begin{array}{l}\text { GAMMAGS } \\
\text { GAMMA_GS }\end{array}$ \\
\hline $\begin{array}{l}\text { EBLLNE } \\
\text { ERRLNE }\end{array}$ & $\begin{array}{l}k_{0115} \\
\text { k0115 }\end{array}$ & $\begin{array}{l}\text { GAMMA_GS } \\
\text { GAMMA GS }\end{array}$ \\
\hline EBRLNE & K0115 & $\begin{array}{l}\text { GAMMA_GS } \\
\text { GAMA }\end{array}$ \\
\hline $\begin{array}{l}\text { RLINE } \\
\text { RLNE }\end{array}$ & $\begin{array}{l}\text { Ko115 } \\
\text { Ko115 }\end{array}$ & $\begin{array}{l}\text { GAMMA_GS } \\
\text { GAMMA GS }\end{array}$ \\
\hline $\begin{array}{l}\text { RLNE } \\
\text { RINE }\end{array}$ & Ko115 & GAMMA_GS \\
\hline $\begin{array}{l}\text { E ERLLNE } \\
\text { EBRE }\end{array}$ & $\begin{array}{l}\text { Ko115 } \\
0115\end{array}$ & $\begin{array}{l}\text { GAMMAGS } \\
\text { GAMM_GS }\end{array}$ \\
\hline & $\begin{array}{l}\text { Ko115 } \\
\text { Ko115 }\end{array}$ & $\begin{array}{l}\text { GAMMA_GS } \\
\text { GAMMA GS }\end{array}$ \\
\hline 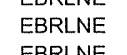 & Ko115 & GAMMA_GS \\
\hline & $\begin{array}{l}\text { Ko115 } \\
\text { Ko115 }\end{array}$ & $\begin{array}{l}\text { GAMMAGS } \\
\text { GAMM_GS }\end{array}$ \\
\hline $\begin{array}{l}\text { EBRLNE } \\
\text { EBRLNE }\end{array}$ & Ko115 & $\begin{array}{l}\text { GAMMAGS } \\
\text { GAMA-GS }\end{array}$ \\
\hline $\begin{array}{l}\text { S } \\
\text { EBRLNE }\end{array}$ & $\begin{array}{l}K 0115 \\
\text { Ko115 }\end{array}$ & $\begin{array}{l}\text { GAMMAGS } \\
\text { GAMM_GS }\end{array}$ \\
\hline $\begin{array}{l}\text { ERBLNE } \\
\text { EBRLNE }\end{array}$ & Ko115 & $\begin{array}{l}\text { GAMMA_GS } \\
\text { GAMMA GS }\end{array}$ \\
\hline & Ko115 & $\begin{array}{l}\text { GANMA_GS } \\
\text { GAMA_GS }\end{array}$ \\
\hline $\begin{array}{l}\text { RLLEN } \\
\text { RLNE }\end{array}$ & $\begin{array}{l}\text { Ko115 } \\
\text { K0115 }\end{array}$ & $\begin{array}{l}\text { GAMMA_GS } \\
\text { GAMMA GS }\end{array}$ \\
\hline & Ko115 & GAMMA-GS \\
\hline 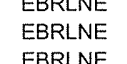 & $\begin{array}{l}\text { Ko115 } \\
\text { K0115 }\end{array}$ & $\begin{array}{l}\text { GAMMAGGS } \\
\text { GAMM_GS }\end{array}$ \\
\hline & $\begin{array}{l}\text { Ko115 } \\
\text { Ko115 }\end{array}$ & $\begin{array}{l}\text { GAMMA_GS } \\
\text { GAMMA GS }\end{array}$ \\
\hline $\begin{array}{l}\text { LNE } \\
\text { LNNE }\end{array}$ & $\begin{array}{l}20115 \\
\text { Ko115 }\end{array}$ & $\begin{array}{l}\text { GAMMA_GS } \\
\text { GAMM_GS }\end{array}$ \\
\hline & 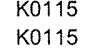 & $\begin{array}{l}\text { GAMMA_GS } \\
\text { GAMMAGS }\end{array}$ \\
\hline $\begin{array}{l}\text { ERLNE } \\
\text { ERRLE } \\
\text { ERLE }\end{array}$ & Ko115 & $\begin{array}{l}\text { PUISO PLATEAEAEA } \\
\text { PUISO PAE AEA }\end{array}$ \\
\hline 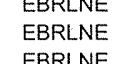 & K0115 & $\begin{array}{l}\text { PUISO_PLATE_AEA } \\
\text { PUEA ACA }\end{array}$ \\
\hline & 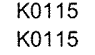 & $\begin{array}{l}\text { PUIIOPLPAAEAEAEA } \\
\text { PUISOPLATEAEA }\end{array}$ \\
\hline & Ko115 & PUISO_PLATE_AEA \\
\hline $\begin{array}{l}\text { ERRLNE } \\
\text { ERRLNE }\end{array}$ & 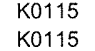 & $\begin{array}{l}\text { PUISO_PLATE_AEA } \\
\text { PUISO PLAEEAEA }\end{array}$ \\
\hline & K0115 & $\begin{array}{l}\text { PUISO_PLATE-AEA } \\
\text { PEAEA }\end{array}$ \\
\hline $\begin{array}{l}\text { ERRLLE } \\
\text { ERRLNE }\end{array}$ & 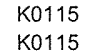 & $\begin{array}{c}\text { PUISO_PLATE_AEA } \\
\text { SRTOT SEP PRECIP GPC }\end{array}$ \\
\hline ERRLNE & $\begin{array}{l}k 0115 \\
k 0115\end{array}$ & SRTOT_SPPPRECIP_GPC \\
\hline $\begin{array}{l}\text { ERRLE } \\
\text { EBRLNE }\end{array}$ & 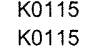 & $\begin{array}{l}\text { SRTO__SE_PPECIP_GPC } \\
\text { SRTOS_SPP_PRECIP_GPC }\end{array}$ \\
\hline & $\begin{array}{l}\text { Ko115 } \\
\text { K0115 }\end{array}$ & SRTOT_SEP_PRECIP_GPC \\
\hline & Ko115 & $\begin{array}{l}\text { IHISO_E__LAL_AEA } \\
\text { THISO_EPAATE_AEA }\end{array}$ \\
\hline & $\begin{array}{l}\text { K0115 } \\
\text { Ko115 }\end{array}$ & THISO_E-PLATE-AEA \\
\hline E & Ko115 & $\begin{array}{l}\text { THISO_IE_PLAAE- } \\
\text { THISO IE PLTE }\end{array}$ \\
\hline & & $\begin{array}{l}\text { THISO_E-PLATE_AEA } \\
\text { THEATEA }\end{array}$ \\
\hline & & SO_IE_PLATE_AEA \\
\hline & Ko115 & $\begin{array}{l}\text { PLAAE_AEA } \\
\text { PLATE_AEA }\end{array}$ \\
\hline
\end{tabular}

\begin{tabular}{|c|}
\hline 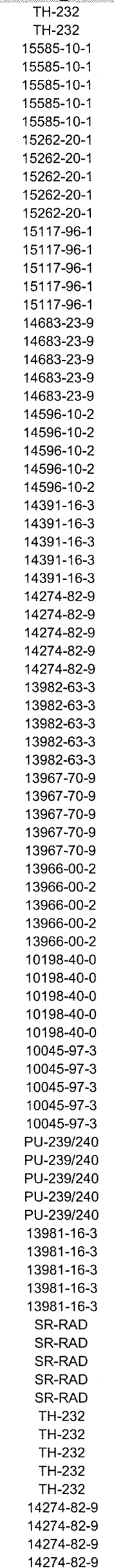 \\
\hline
\end{tabular}

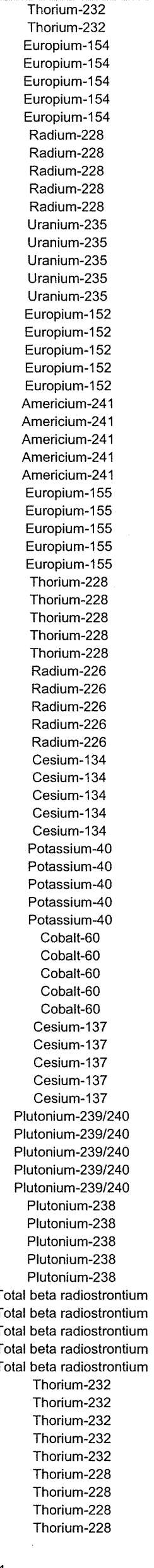


MIS_05 SAMPLES

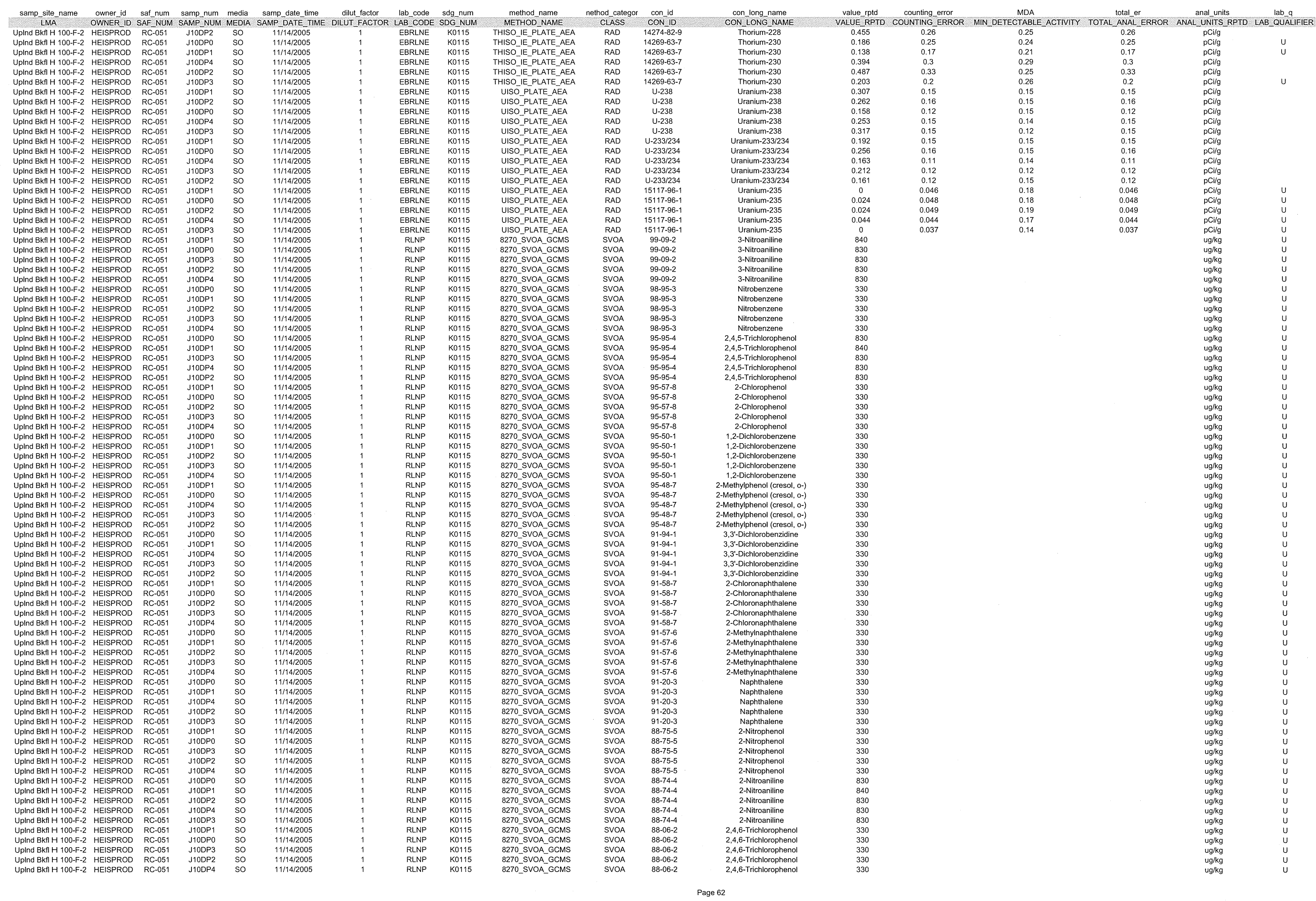


MIS_05 SAMPLES

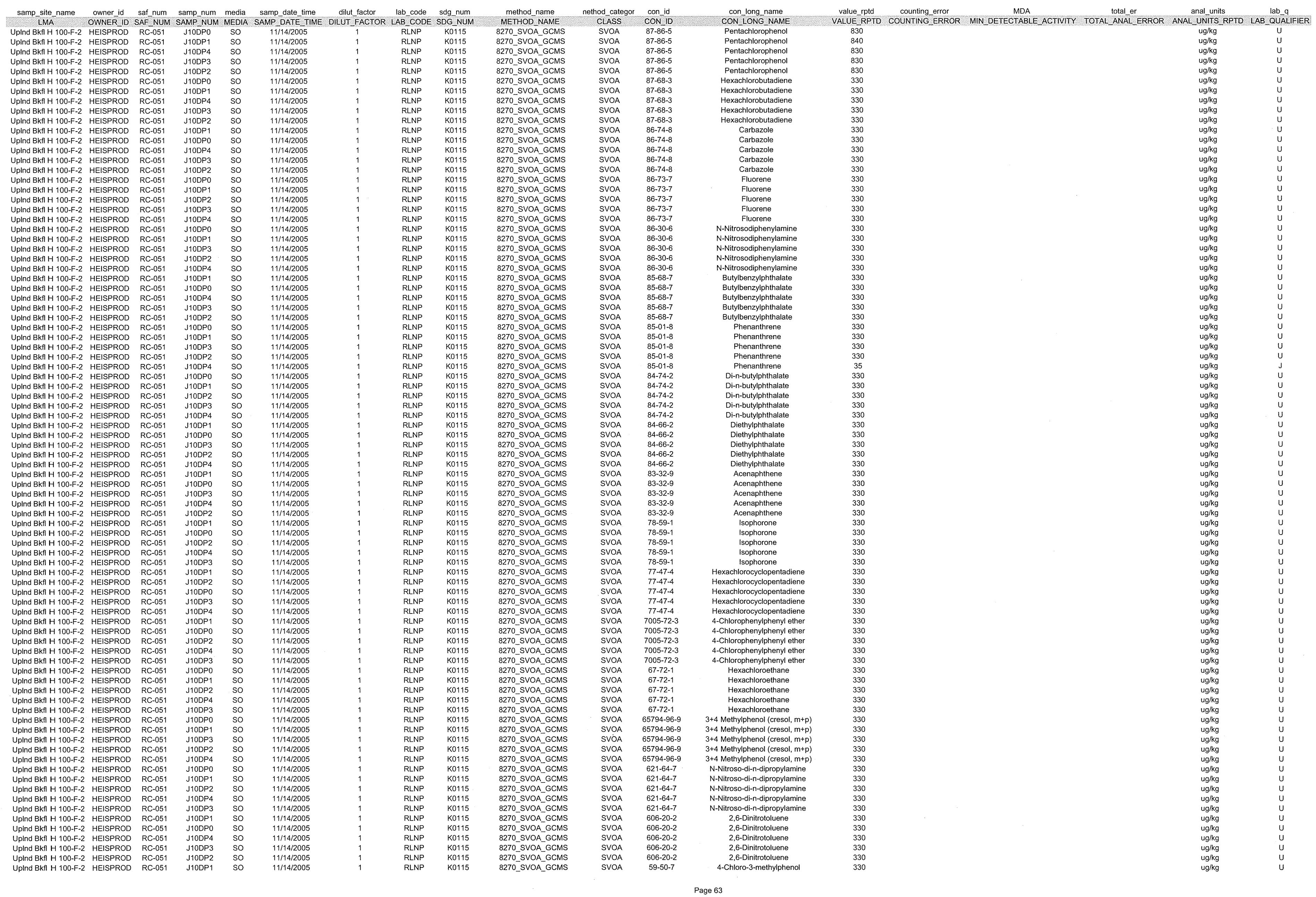




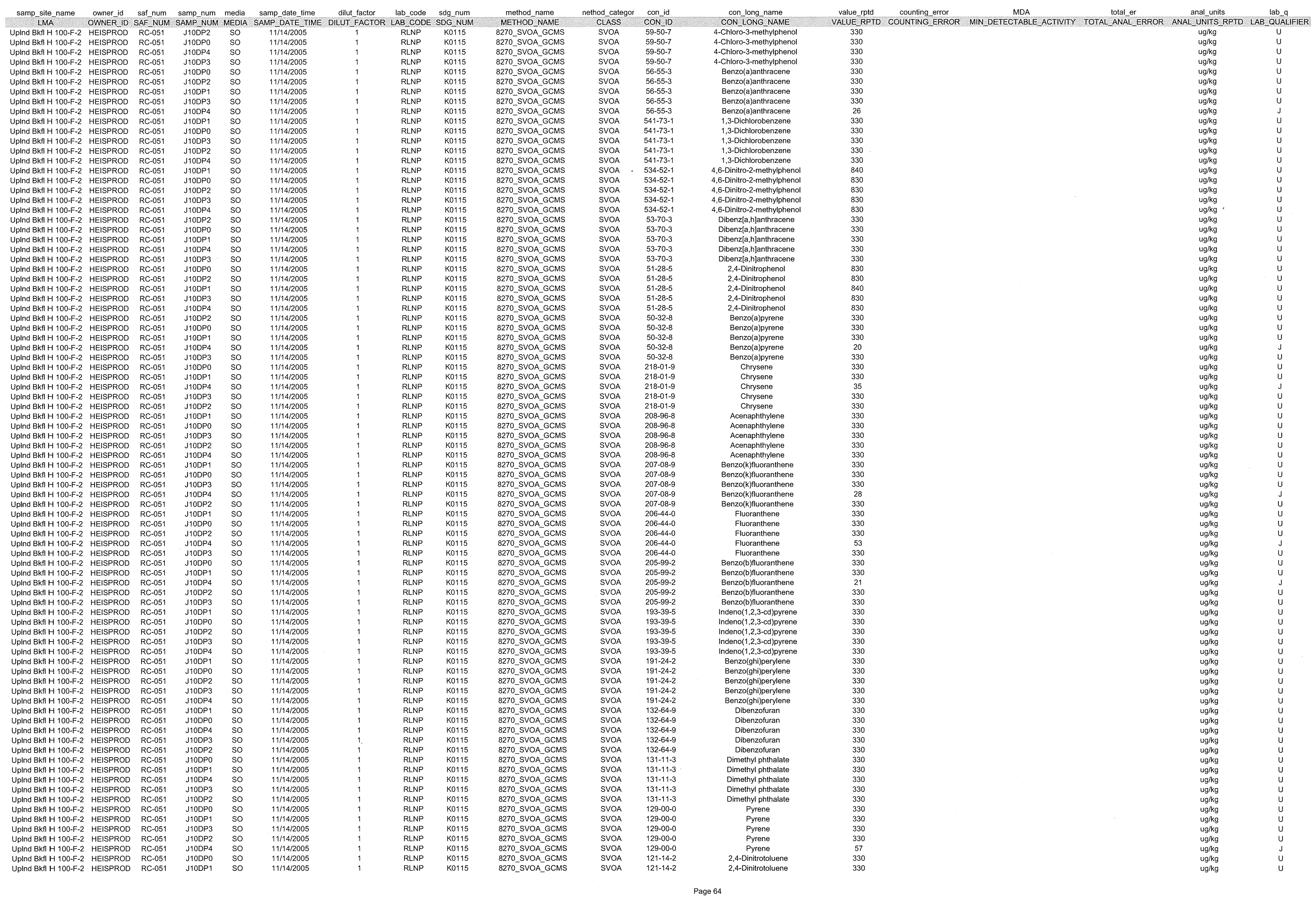




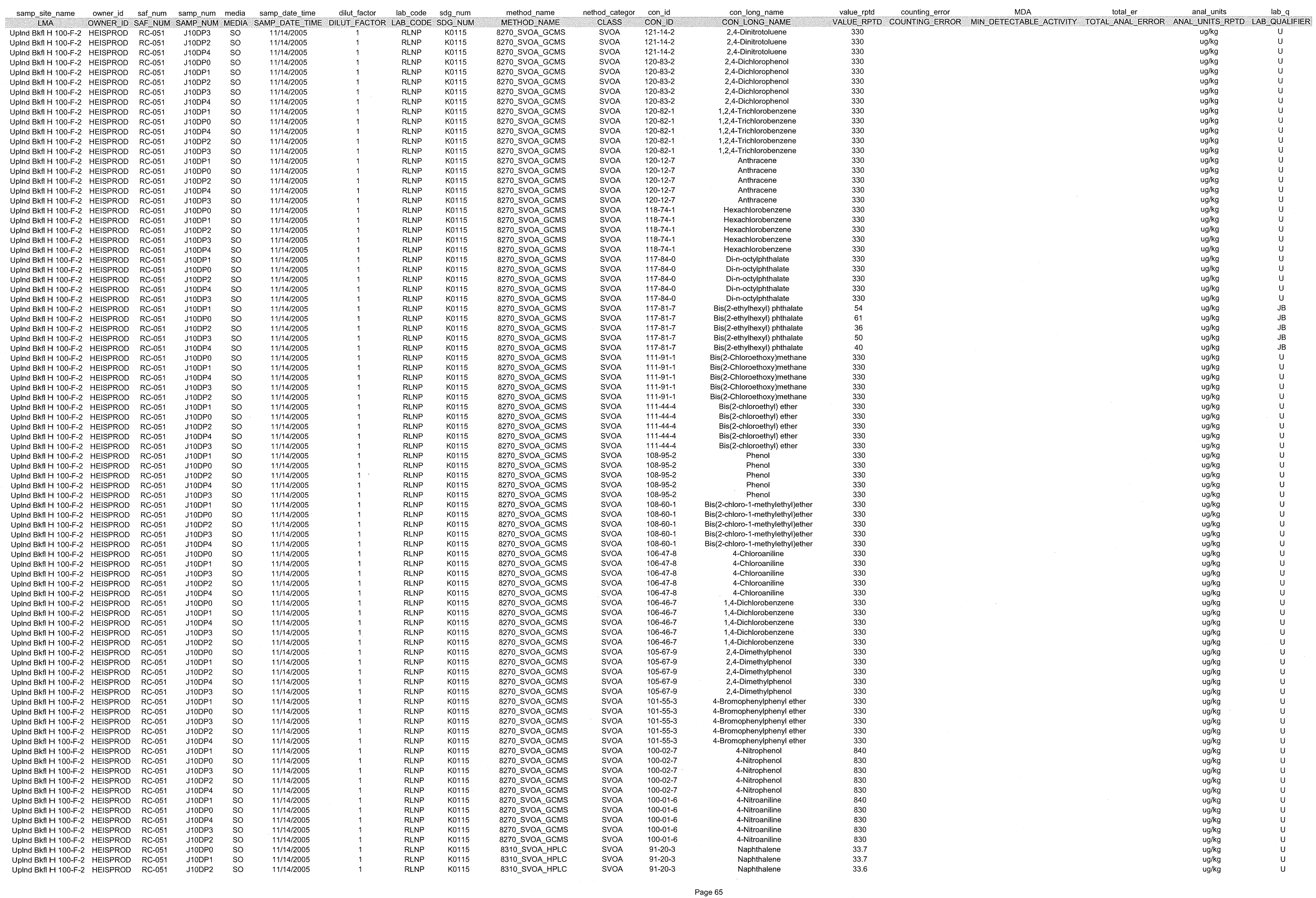


MIS_05 SAMPLES

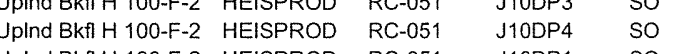

Upind BKRIH 100-F-2. HEISPRO
Upind BKI H 1 100-F-2 HEISPROO

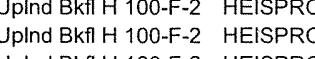

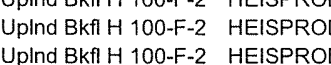

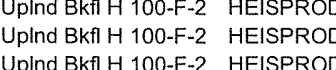

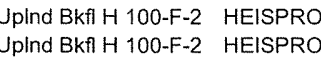

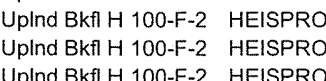

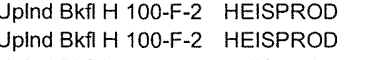

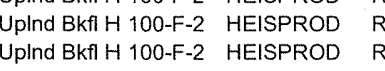

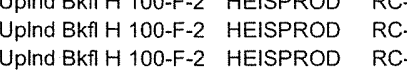

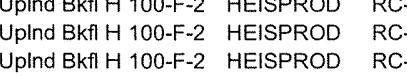

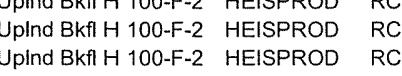

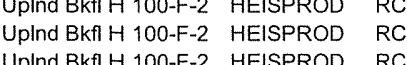

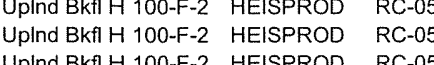

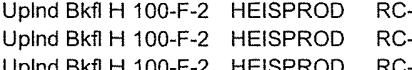

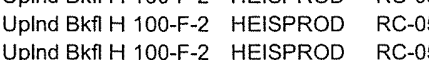

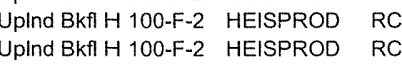

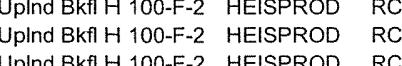

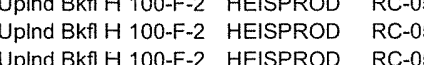

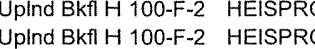

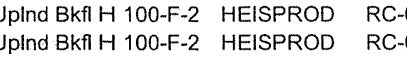

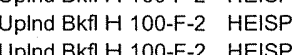

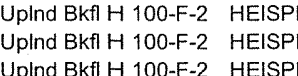
Uplind BkfH $H$ 100-F-2 HEISP

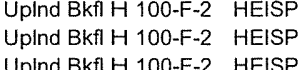

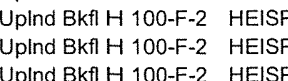

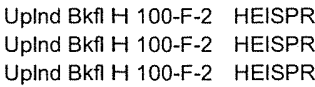

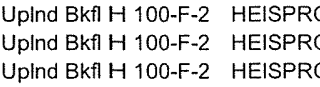

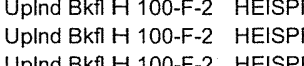

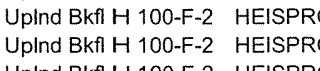

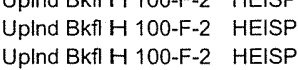

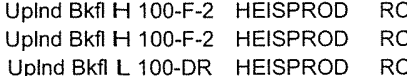

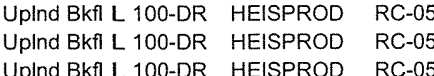

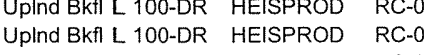

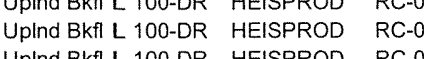

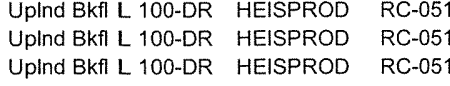

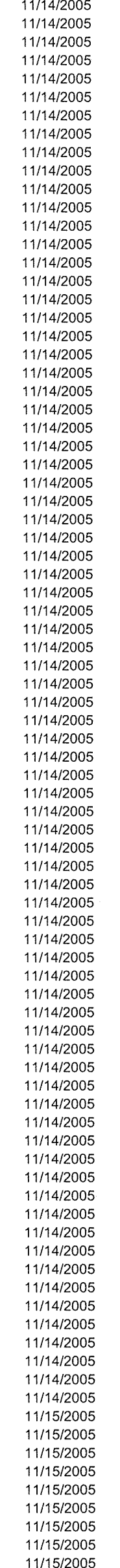

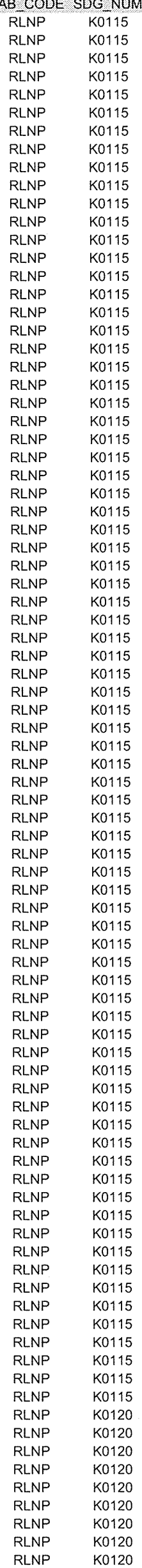

8310_SVOA-HPLC

$\begin{array}{ll}\text { SVOA } & 91-20.0 \\ \text { SVOA } & 91-20.3 \\ \text { SVOA } & 907-7\end{array}$

Con_long_name
CONLONG_NAME
Naphthalene
Naphthalene

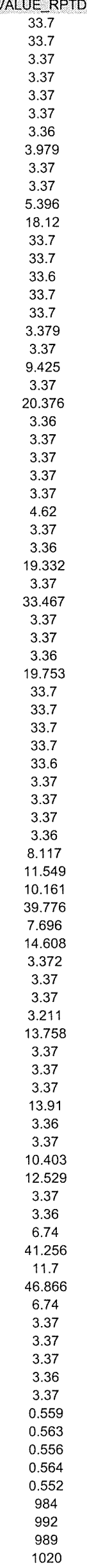

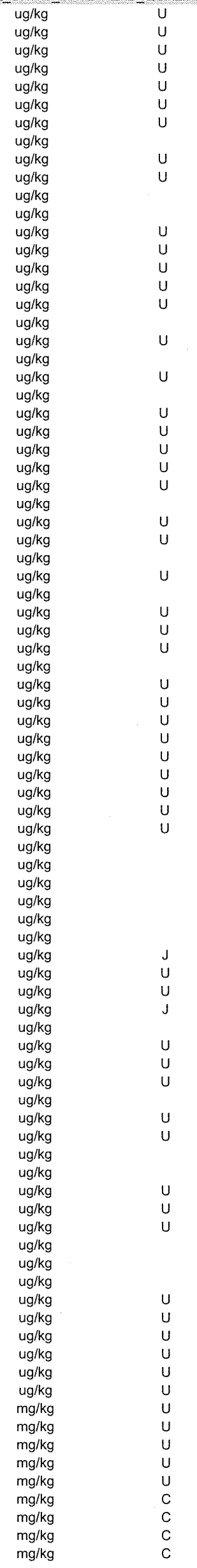

Page 66 
MIS_05 SAMPLES

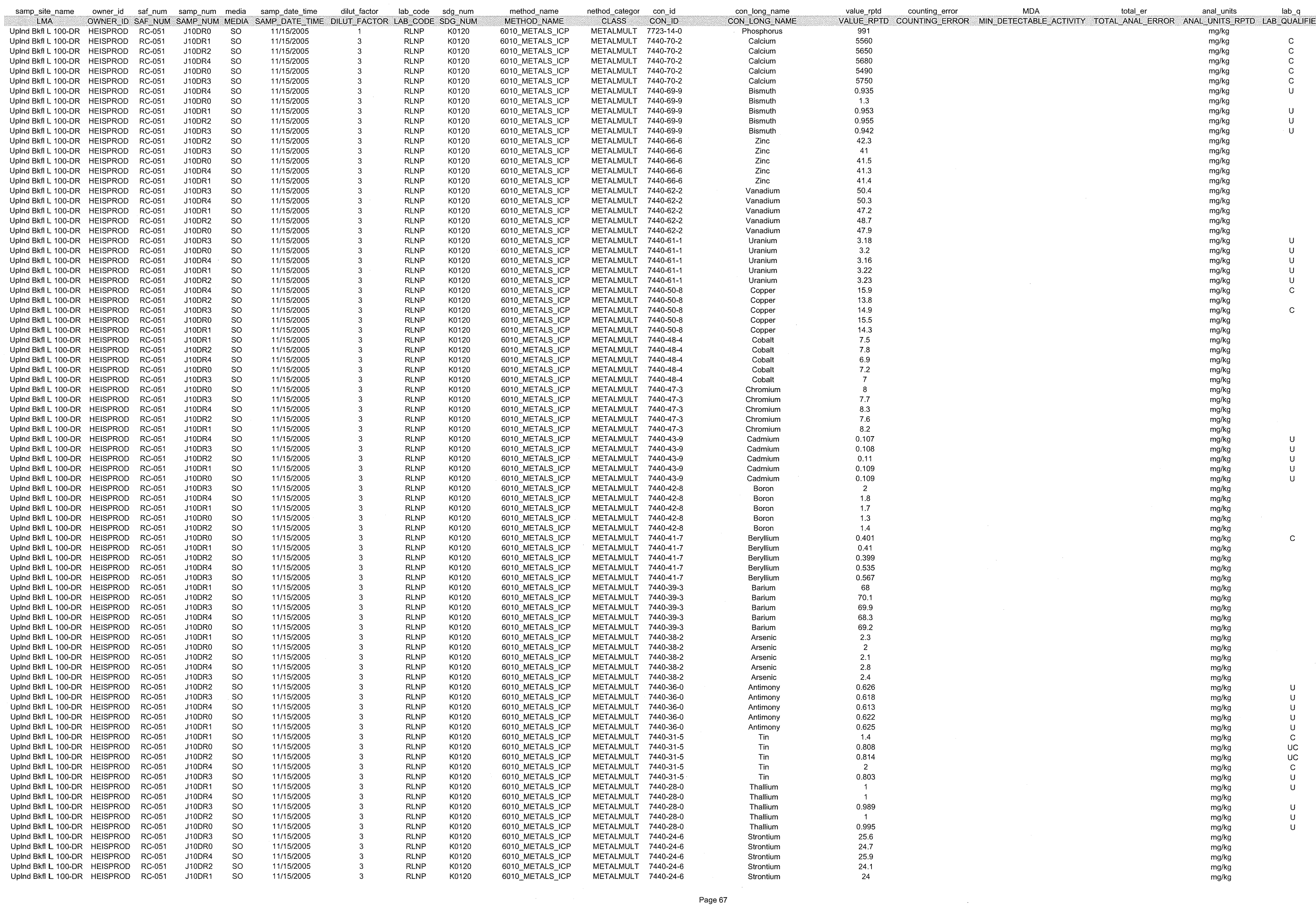


MIS_05 SAMPLES

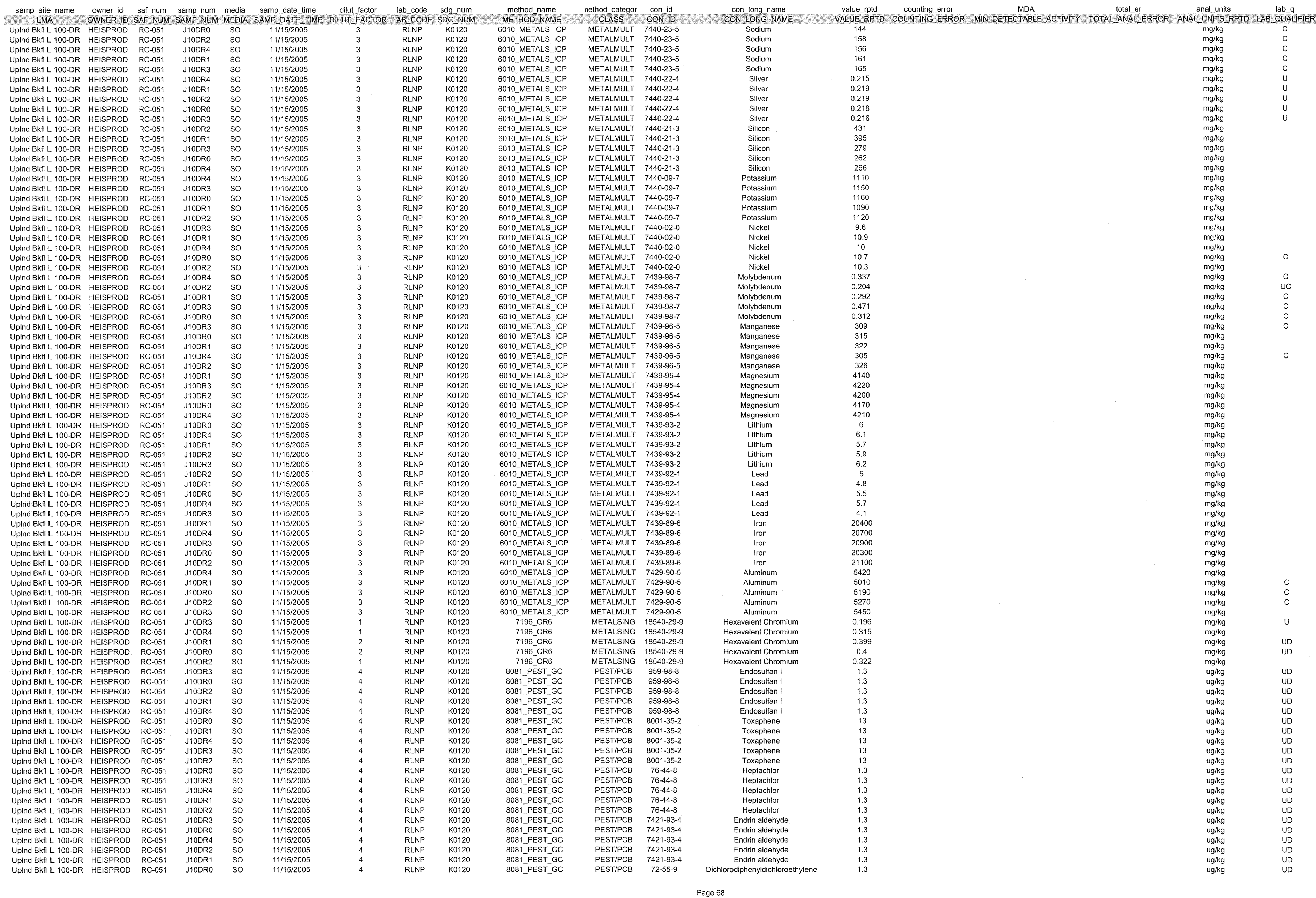


MIS_05 SAMPLES

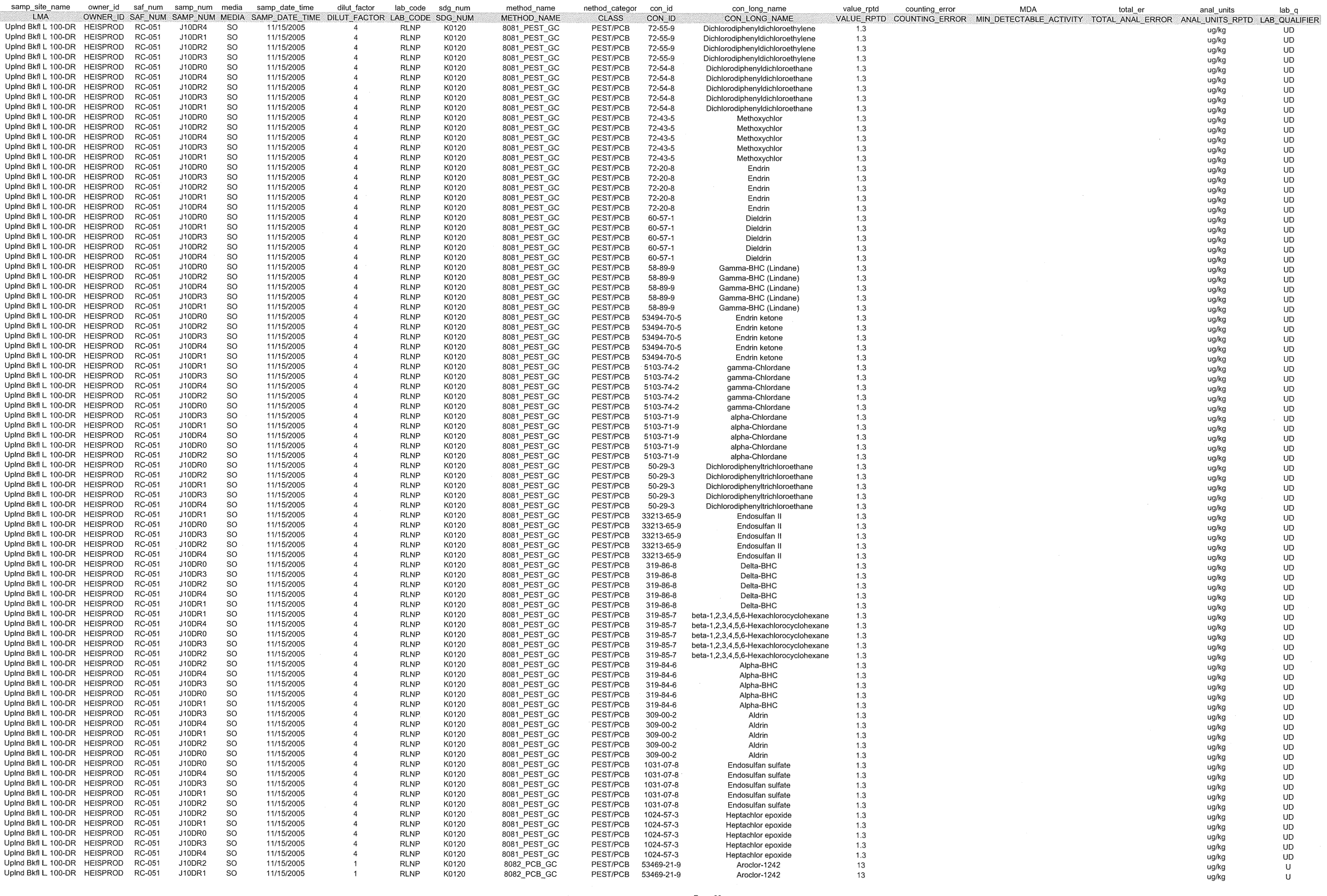


MIS_05 SAMPLES

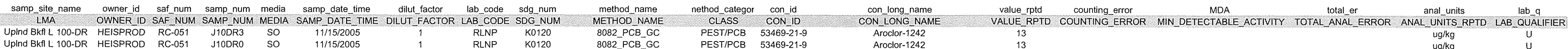

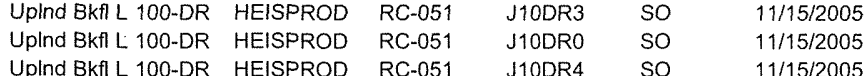

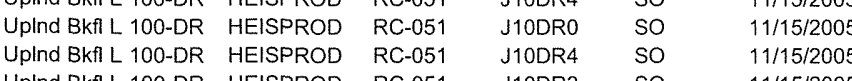

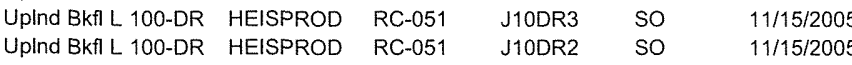

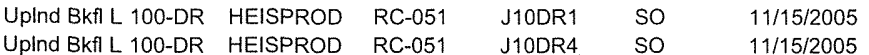

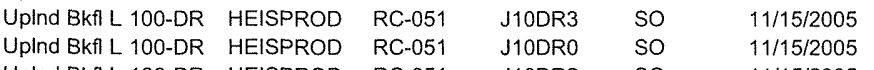

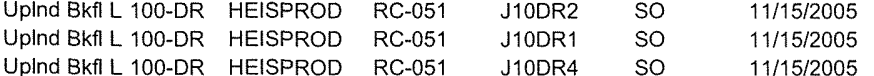

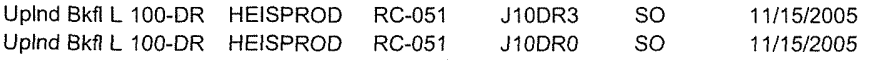

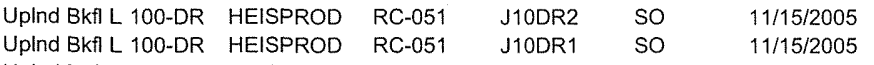

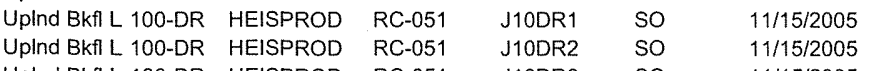

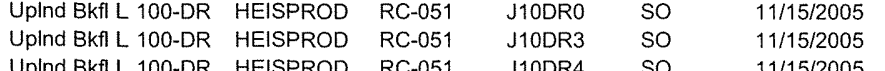

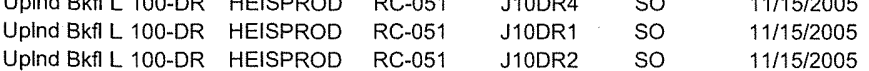

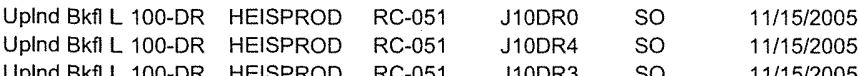

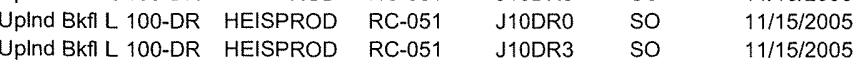

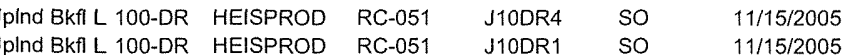

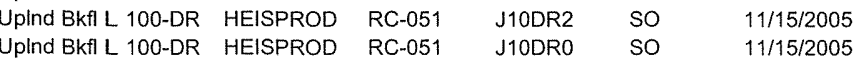
$\begin{array}{lllll} & \end{array}$ Wplnd BkT L 100-DR HEISPROD RCOSY R Uplin BKIL 100-DR HEISPROD RC $\begin{array}{llll} & 11 / 1525005 \\ & \end{array}$

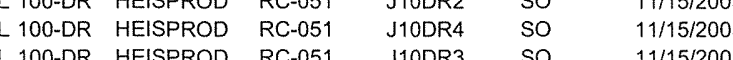
UPlnd BKA L L 100-DR HEISPROD RC-OS

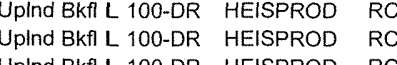
$\begin{array}{lllll} & 11 / 1 / 12005 \\ & 11 / 1 / 2005 \\ \end{array}$ $\begin{array}{ll} & \\ & \end{array}$

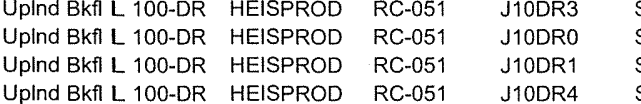

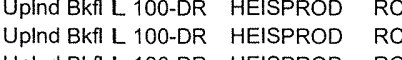

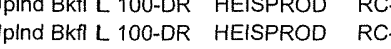

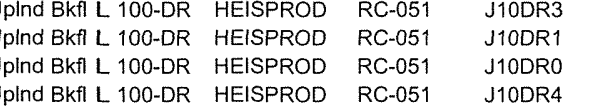

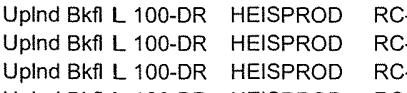

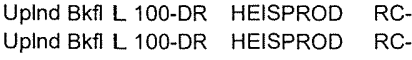

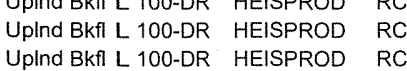

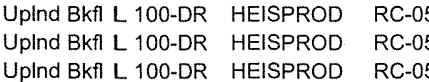

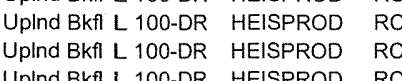

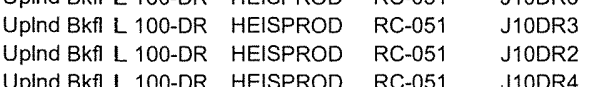

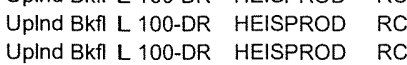
$\begin{array}{lll}\text { Uplind Bkf } L \text { L 100-DR } & \text { HEISPROD } & R C \\ \text { Uplind BkA L L 100-DR } & \text { HEISPROD } & R C \\ \text { UC } & \text { RC }\end{array}$

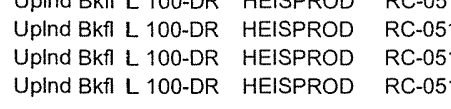

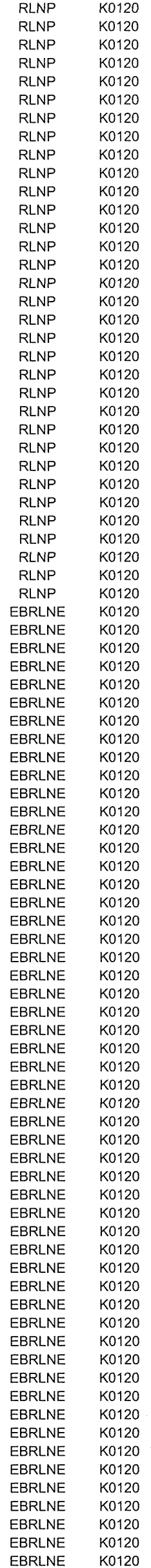

\begin{tabular}{|c|}
\hline 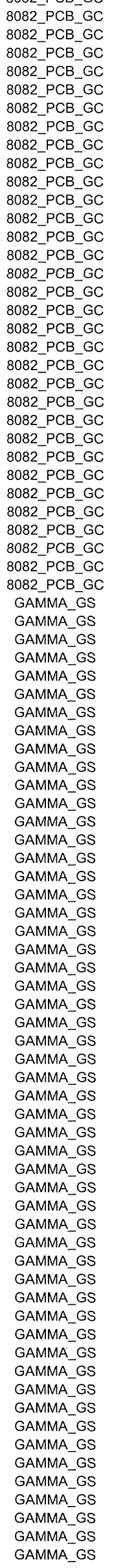 \\
\hline
\end{tabular}
$\begin{array}{ll}\text { PESTIPCB } & 53469-219 \\ \text { PESTPCCB } & 53469-21-9 \\ \text { PESTPCB } & 53469-1-9\end{array}$ 


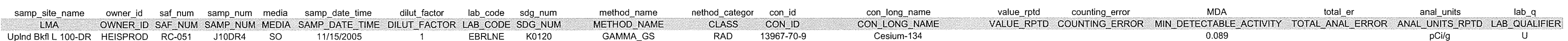

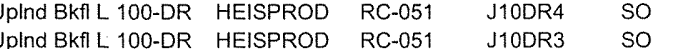

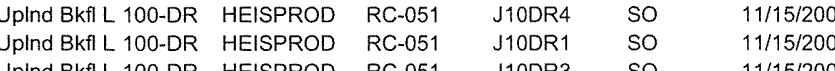

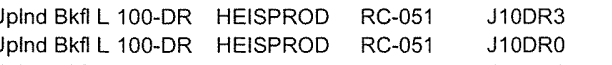

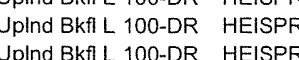

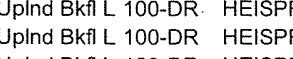
Upind $B k i L$ L 100-DR HEISP
Upind Bki $L$ 100-DR HEISP

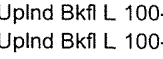
Upind BKRL $L$ 100-DR HEISP Uplnd BkAL 100-DR HEISPROD

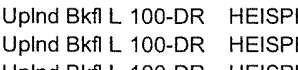
Uplnd BkfL L 100-DR HEISPRO

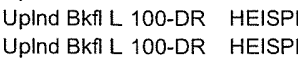

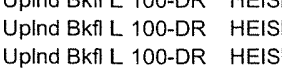

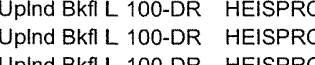
Upind BkTI L 100-DR HEISPROD
Upind BkR L 100-DR HEISPROD

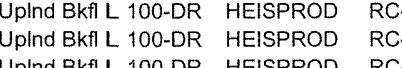

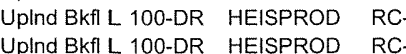

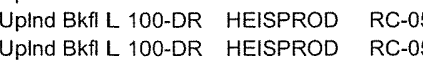

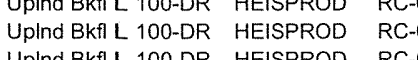
Upind BKR L 1 100-DR HEISPROD RC-OS

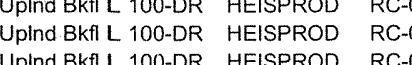

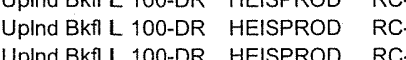

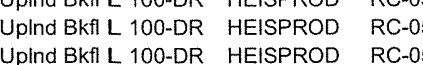

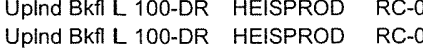

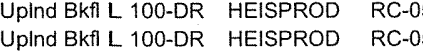

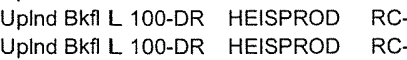

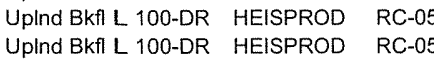

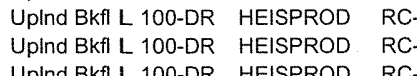

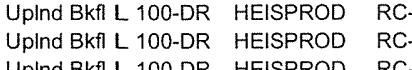

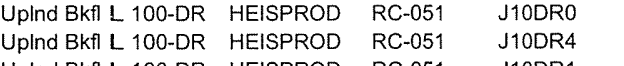

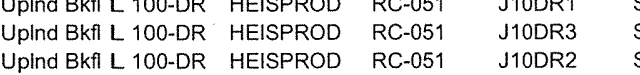
Uplind BKA L $100-$-DR HEISPROD RC-

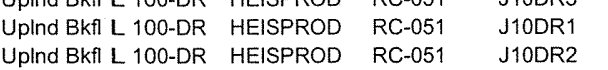

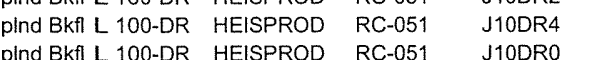

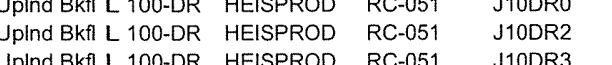

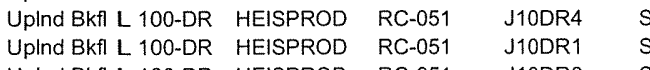

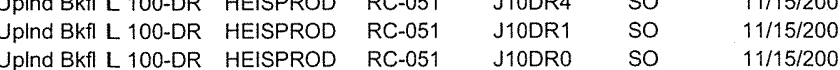

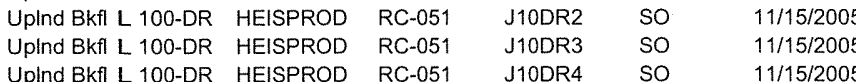

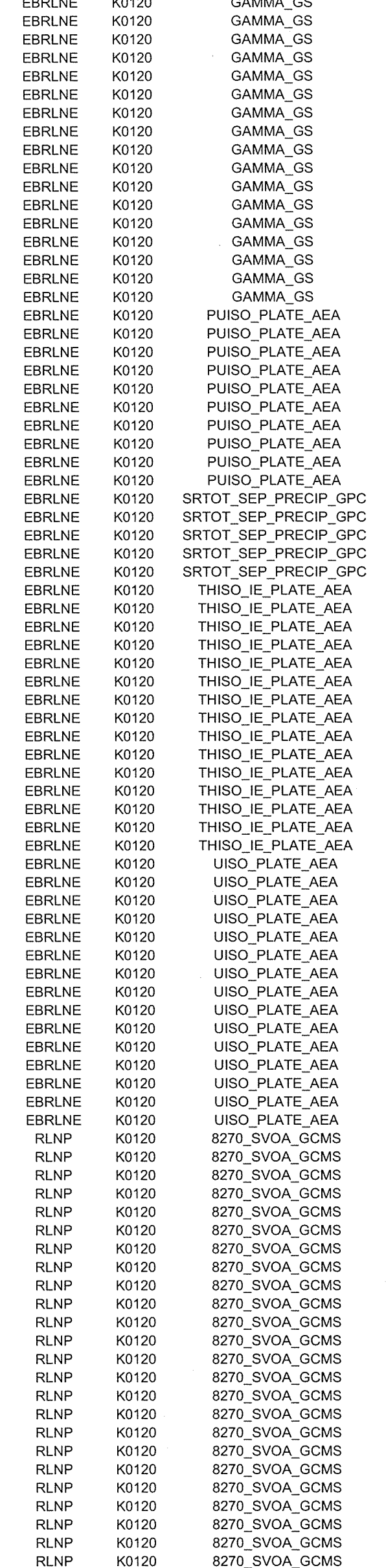




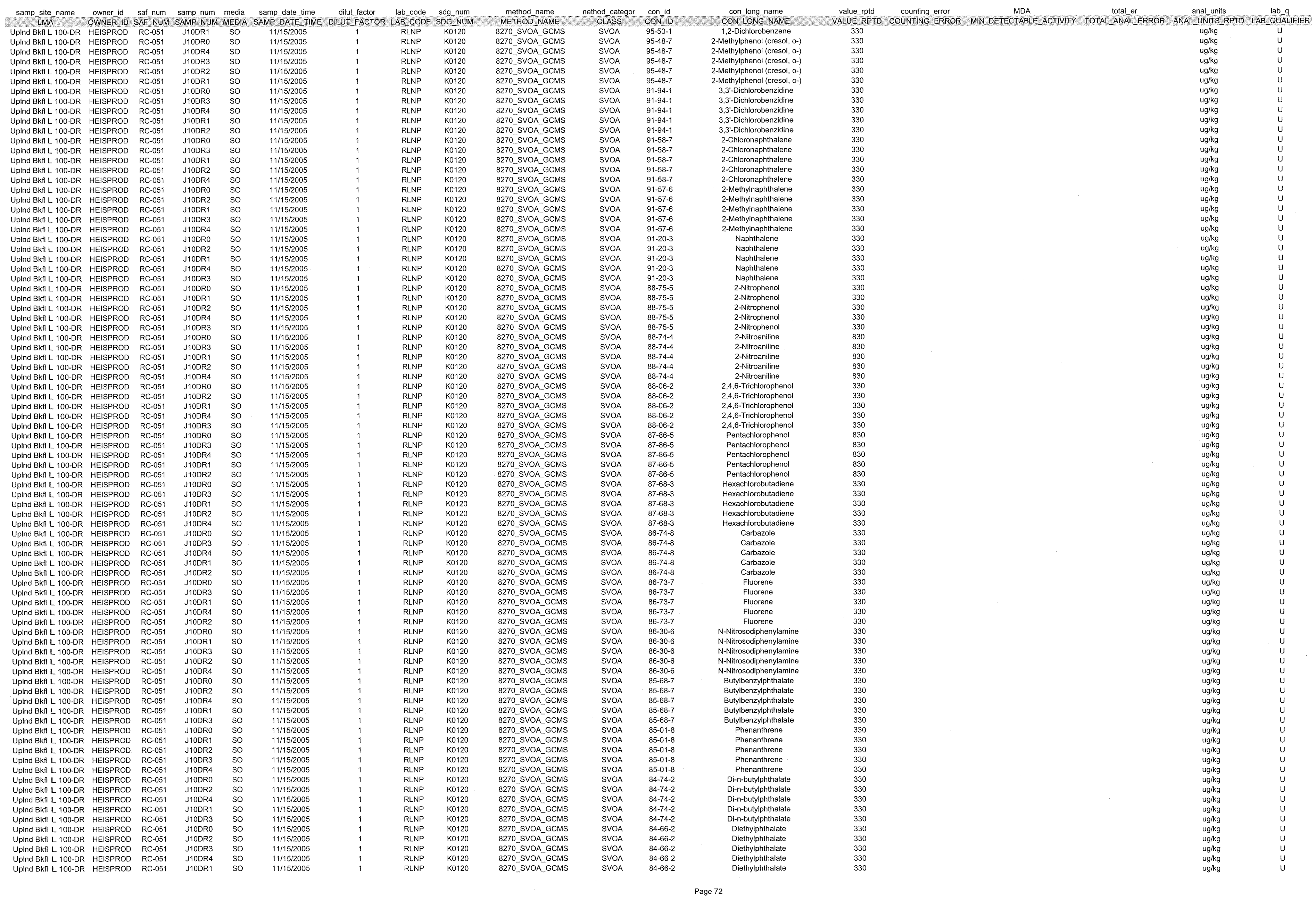


MIS_05 SAMPLES

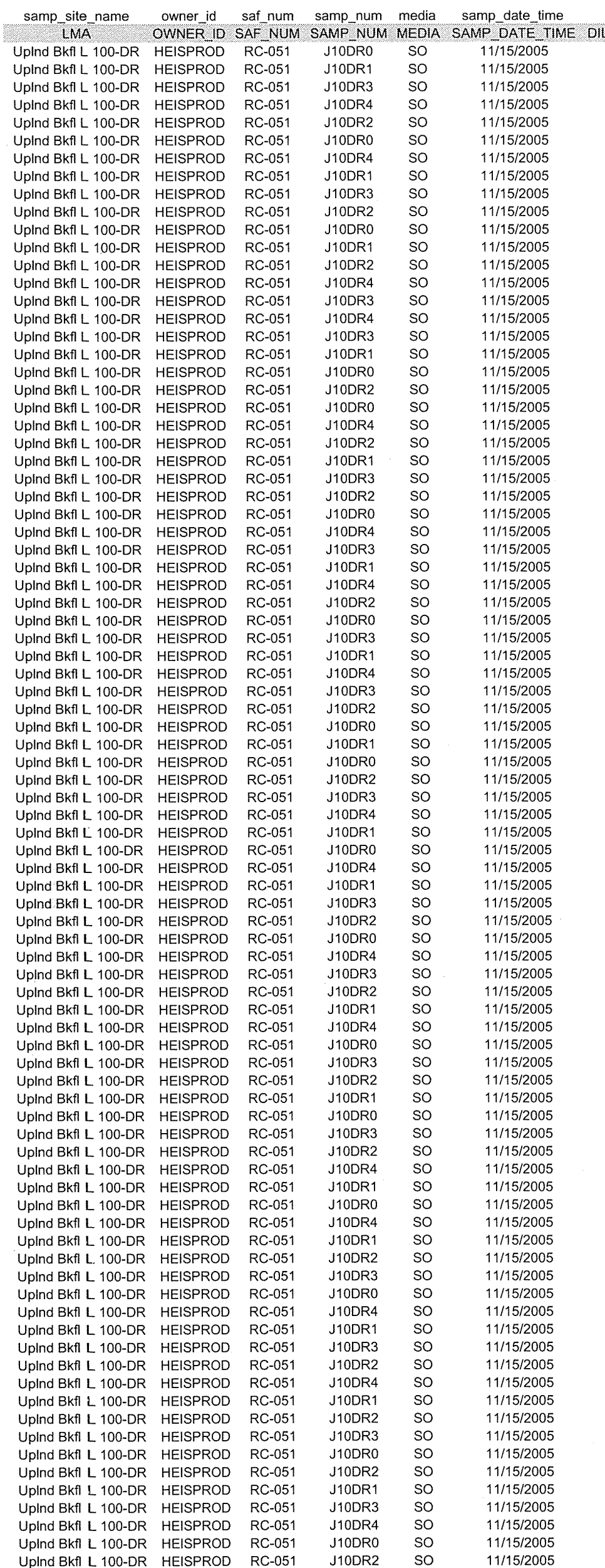
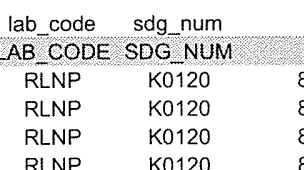

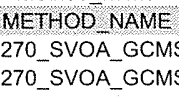
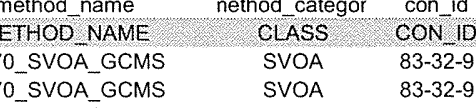

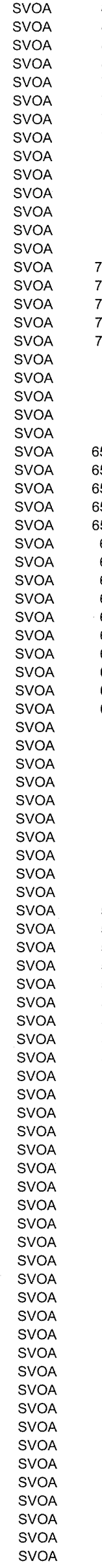

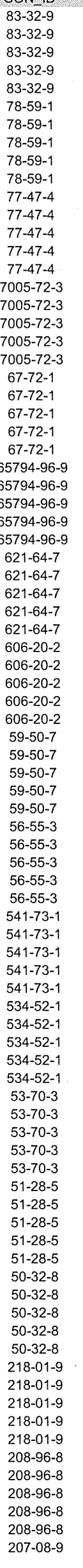

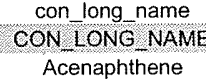

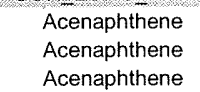

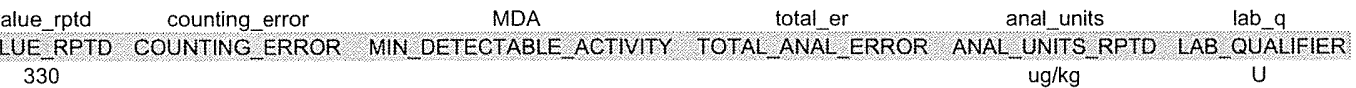




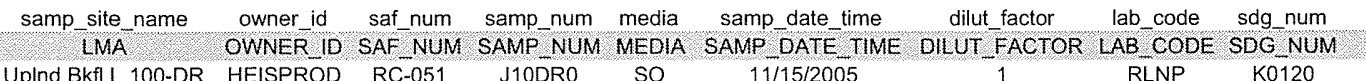

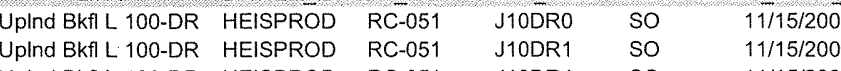

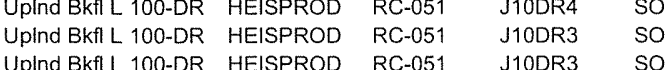

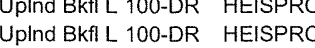

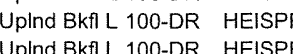

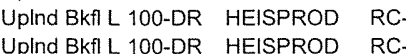

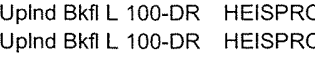

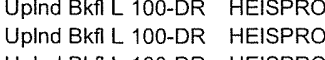

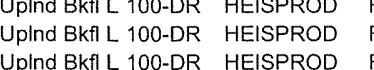

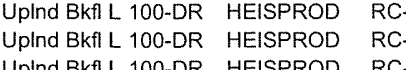

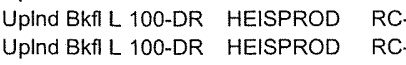

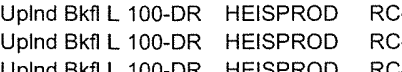

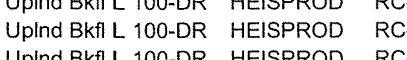

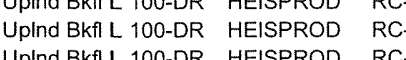

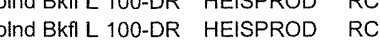

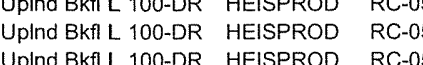

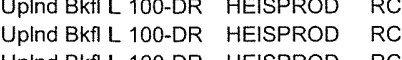

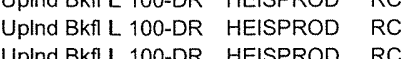

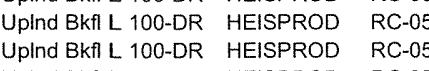

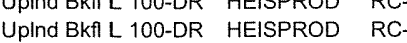

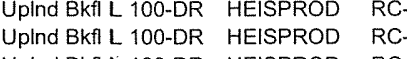

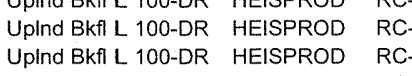

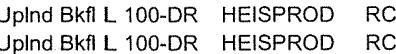

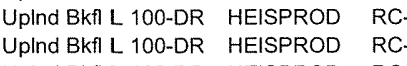

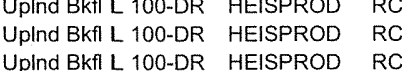

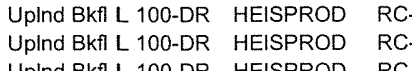

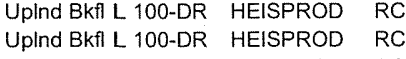

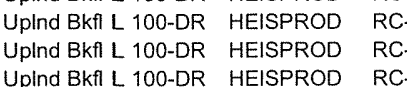

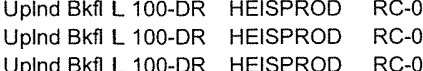

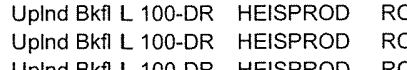

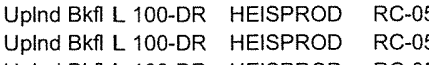

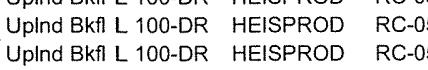

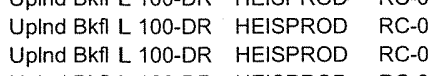

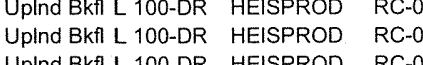

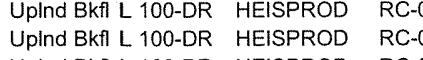

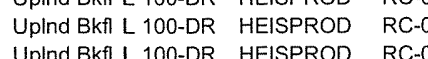

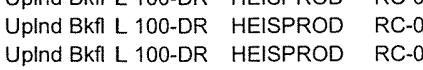

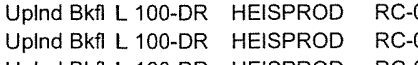

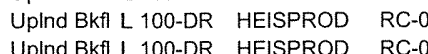

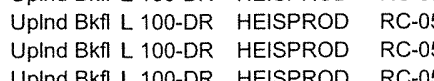

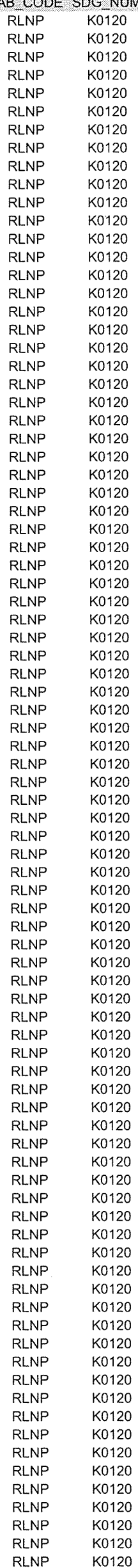

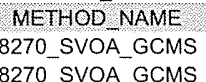

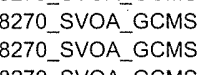
$8270-$ SVOA-GCMS
8270 SVOA GCMS 8270 -SVOA_GCMMS 8270 - SVOA-GCMS
8270 SVOA-GCMS

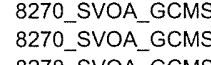
8270_SVVOA_GCMS 8270 SVOA-GCMS 827-SVVA-GCMS
8270-SVOA-GCMS

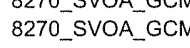

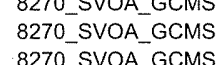
8270_SVOA_CCMS
8270_SVOA_GCCMS 827-SSVOA-GCMS
8270_SVOA-GCMS 8270 -SVOA_CCCMS

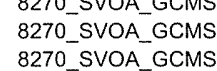
8270 - SVOA GCM 8270-SVOA-GCMS

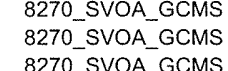

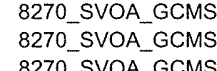
827-SVAO-GCMS
8270_SVOA-GCMS 8277-SVOA-GCMS
8270-SVOOA-GCMS

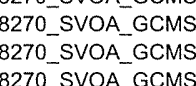

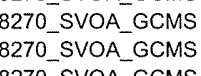

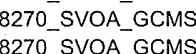
8270 SVOA-GCMS

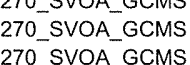

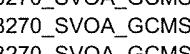
8270-SVoA-GCMMS

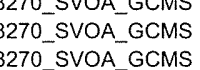

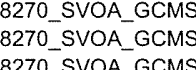

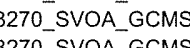
270 SVOA GCMS

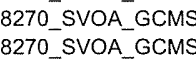

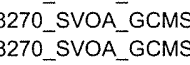
8270_SVOA_-GCMM
8270_-SVOA_GCCOS

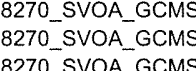

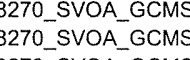

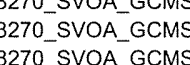

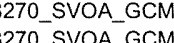

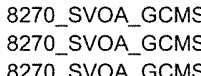

\begin{tabular}{|c|c|c|c|}
\hline 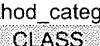 & CON ID & $\begin{array}{l}\text { Con Longhame } \\
\text { CON LNA }\end{array}$ & \\
\hline 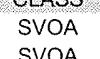 & 207-08-9 & Benzo(k)fluoranthene & \\
\hline & $\begin{array}{l}207-08-9 \\
200-989\end{array}$ & 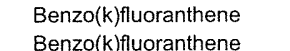 & $\begin{array}{l}330 \\
330\end{array}$ \\
\hline & $207-08-9$ & $\begin{array}{l}\text { Benzo(k)ifuorantinene } \\
\text { Benzo(k)fuoranthene }\end{array}$ & \\
\hline & 206-44-0 & Fluoranthene & $\begin{array}{l}330 \\
330\end{array}$ \\
\hline & $206-44-0$ & $\begin{array}{l}\text { Fluoranthene } \\
\text { Fluoranthene }\end{array}$ & 330 \\
\hline & $206-44-0$ & $\begin{array}{l}\text { Fluoranthene } \\
\text { Fluoranthene }\end{array}$ & 23.071 \\
\hline & $206-44-0$ & Fluoranthene & $\begin{array}{l}330 \\
330\end{array}$ \\
\hline & $205-99-2$ & Benzo(b)fluoranthene & 330 \\
\hline $\begin{array}{l}\text { VOA } \\
\text { VOA }\end{array}$ & 205-99-2 $209-2$ & $\begin{array}{l}\text { Benzo(b)fllforanthene } \\
\text { Benzzo(b)luoranthene }\end{array}$ & 330 \\
\hline & $205-99-2$ & $\begin{array}{l}\text { Betzzo(b)fluoranhinene } \\
\text { Benzo(b)fuoranthene }\end{array}$ & $\begin{array}{c}330 \\
330 \\
330\end{array}$ \\
\hline & 205-99-2 $2093-5$ & 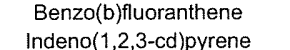 & $\begin{array}{l}330 \\
330\end{array}$ \\
\hline & 193-39-5 & 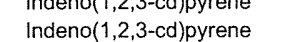 & $\begin{array}{c}330 \\
330 \\
330\end{array}$ \\
\hline & 1993-399.5 & Indeno( $(1,2,3$-cod) pyrene & 330 \\
\hline & 1933-39-5 & Indeno(1,2,3-2-cd)|yyrene & 330 \\
\hline VOA & 1951-24-2-2 & 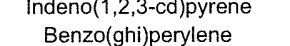 & $\begin{array}{l}330 \\
330\end{array}$ \\
\hline VOA & 191-24-2 & Benzo(ghi)perylene & $\begin{array}{l}330 \\
330\end{array}$ \\
\hline & $\begin{array}{l}191-2-2-2 \\
191-2-2\end{array}$ & & 330 \\
\hline & 191-24-2 & $\begin{array}{l}\text { Benzo(ghi)perylene } \\
\text { Benzooghilperliene }\end{array}$ & 330 \\
\hline & 132-64-9 & $\begin{array}{l}\text { Defbenzofuran } \\
\text { Dibutis }\end{array}$ & \\
\hline & $\begin{array}{l}132-64-9 \\
132,64.9\end{array}$ & Dibenzofuran & \\
\hline & $\begin{array}{l}132-64-9 \\
132-69-9\end{array}$ & Dibenzofuran & 330 \\
\hline & $\begin{array}{l}132-64-9 \\
132-649\end{array}$ & $\begin{array}{l}\text { Dibenzoforuan } \\
\text { Dibenzofuran }\end{array}$ & 330 \\
\hline & 131-11-3 & Dimethy phthalate & \\
\hline & 131-11-3 & $\begin{array}{l}\text { Dimenthy phathalate } \\
\text { Dithe }\end{array}$ & 330 \\
\hline & 131-11-3 & Dimethyl phthalate & 330 \\
\hline & $\begin{array}{l}131-1-3 \\
131-1-3\end{array}$ & $\begin{array}{l}\text { Dimethyly phthalate } \\
\text { Dimethyl phthalate }\end{array}$ & \\
\hline & & Plinene & $\begin{array}{l}330 \\
23.538\end{array}$ \\
\hline & $129-00-0$ & Pyrene & $\begin{array}{l}20.030 \\
330\end{array}$ \\
\hline & 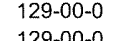 & Pyrene & 330 \\
\hline & 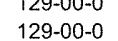 & & $\begin{array}{l}330 \\
330\end{array}$ \\
\hline & 121-14-2 & 2,4-Dinitrotoluene & $\begin{array}{l}330 \\
330\end{array}$ \\
\hline & 121-14-2 & 2,4-4Dinitrotoluene & 330 \\
\hline & & $\begin{array}{l}\text { 2,4-Dinititotoluene } \\
\text { 2,4-Dinitrotoluene }\end{array}$ & 年30 \\
\hline & 121-14-2 & 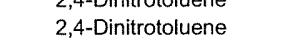 & $\begin{array}{l}330 \\
330 \\
330\end{array}$ \\
\hline & 120-83-2 & 2,4-Dichlorophenol & 330 \\
\hline & 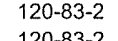 & 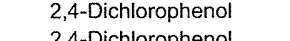 & 330 \\
\hline & & & $\begin{array}{l}330 \\
330\end{array}$ \\
\hline & 120-83-2 & 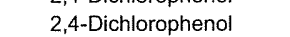 & $\begin{array}{l}330 \\
330\end{array}$ \\
\hline & 120-82-1 & 1,2,4-4richlorobenenzene & 330 \\
\hline & $\begin{array}{l}120-82-1 \\
120-82-1\end{array}$ & 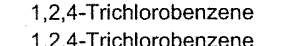 & 330 \\
\hline & & 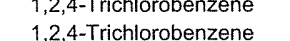 & $\begin{array}{l}330 \\
330\end{array}$ \\
\hline & $120-82-1$ & 1,2,4-4Tichlorobenzene & $\begin{array}{l}350 \\
330\end{array}$ \\
\hline & $120-12-7$ & Anthracene & 330 \\
\hline & $\begin{array}{l}120-12-7 \\
120-12-7\end{array}$ & $\begin{array}{l}\text { Anthracene } \\
\text { Anthracene }\end{array}$ & $\begin{array}{l}330 \\
330\end{array}$ \\
\hline & $120-12-7$ & $\begin{array}{l}\text { Antiracene } \\
\text { Anthracene }\end{array}$ & $\begin{array}{l}330 \\
330\end{array}$ \\
\hline & $120-12-7$ & Anthracene & 330 \\
\hline & $\begin{array}{l}118-74-1 \\
118-7-1\end{array}$ & Hexachlorobbenzene & 330 \\
\hline & $\begin{array}{l}118-74-1 \\
118-74-1\end{array}$ & $\begin{array}{l}\text { Hexachiorobonezzene } \\
\text { Hexachlobenzene }\end{array}$ & 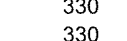 \\
\hline & $118-74-1$ & Hexachlorobenzene & $\begin{array}{l}330 \\
330\end{array}$ \\
\hline & 118-74-1 & Hexachlorobenzene & 330 \\
\hline & 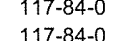 & 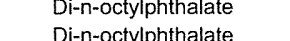 & $\begin{array}{l}330 \\
330\end{array}$ \\
\hline & $117-84-0$ & 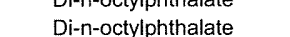 & $\begin{array}{l}330 \\
330\end{array}$ \\
\hline & $117-84-0$ & Di-n-octylphthalate & $\begin{array}{l}330 \\
330\end{array}$ \\
\hline & $\begin{array}{l}117-84-0 \\
117017\end{array}$ & Di-n-octyphithalate & 330 \\
\hline & $\begin{array}{l}117-81-7 \\
117-81-7\end{array}$ & 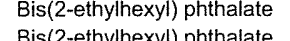 & $\begin{array}{l}36.491 \\
51777-175\end{array}$ \\
\hline & $117-81-7$ & 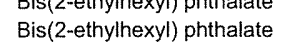 & \\
\hline & $117-81-7$ & (2-ethylhexyl) phthalate & 44. \\
\hline & 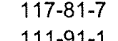 & Bis(2-ethylhexyl) phthalate & 86.621 \\
\hline & $\begin{array}{l}-111-91-1 \\
111-91\end{array}$ & 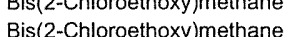 & $\begin{array}{l}330 \\
330\end{array}$ \\
\hline & $111-91-1$ & Bis (2-Chloroethoxyymethane & $\begin{array}{l}330 \\
330\end{array}$ \\
\hline & 111-91-1 & Bis(2-Chloroethoxy)methane & 330 \\
\hline & $\begin{array}{l}111-91-1 \\
111-4-4\end{array}$ & 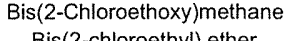 & $\begin{array}{l}330 \\
330\end{array}$ \\
\hline & $111-44-4$ & 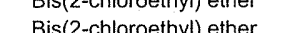 & $\begin{array}{l}330 \\
330\end{array}$ \\
\hline & 111-44-4 & Bis (2-chloroethy) ether & 330 \\
\hline & 111-44. & Bis(2-chloroethyl) ether & \\
\hline & $\begin{array}{l}108-44-4 \\
108-2\end{array}$ & 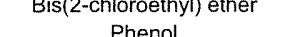 & 管 \\
\hline & 108-95-2 $>2>0$ & & ${ }^{330}$ \\
\hline
\end{tabular}

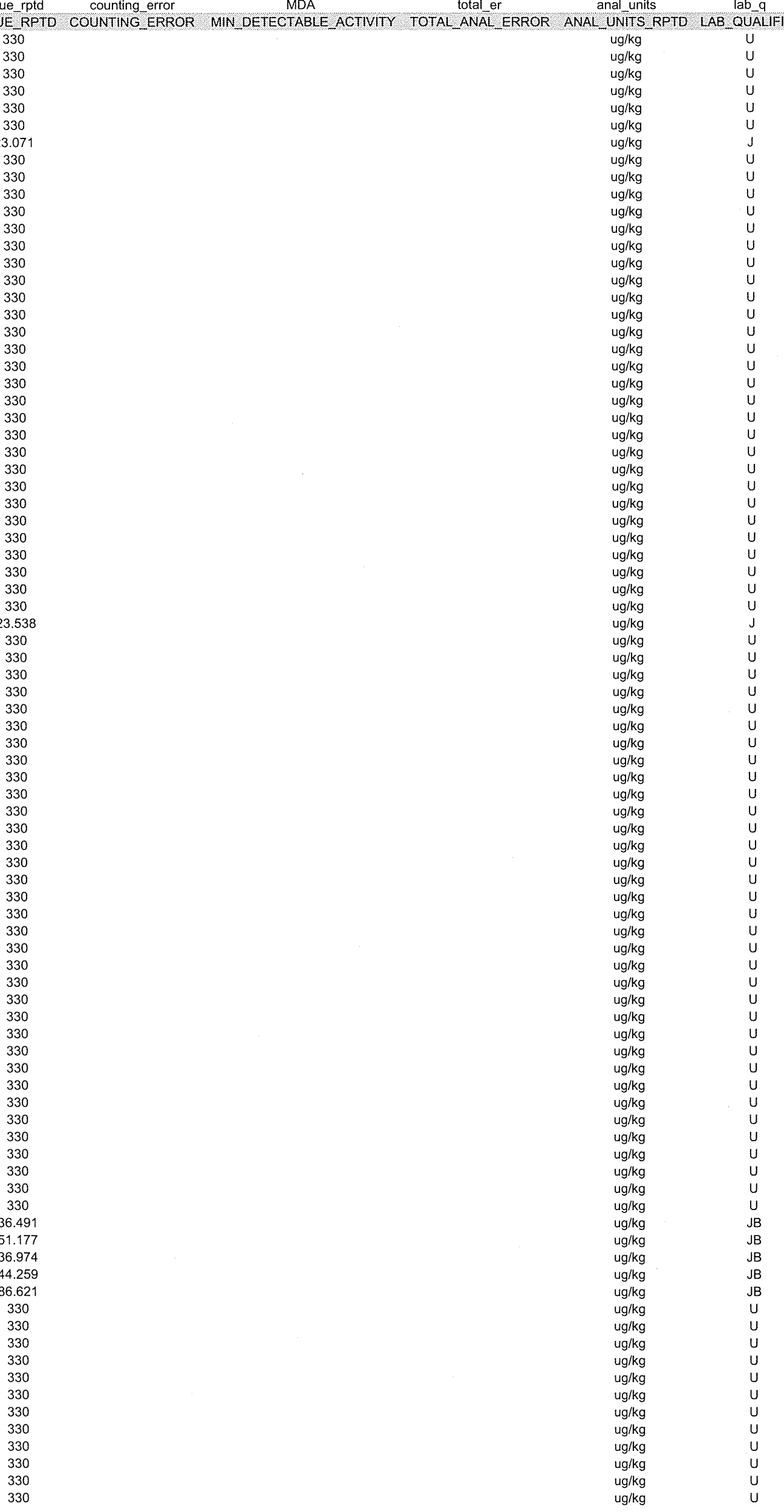


MIS_05 SAMPLES

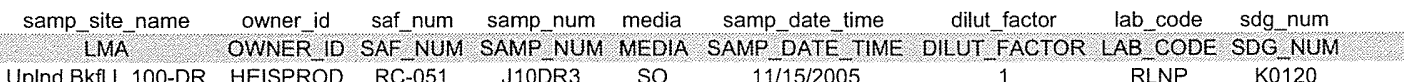

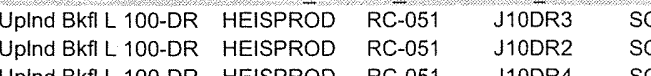
$\begin{array}{lll} & 111 / 152005 \\ 11 / 152005 \\ 50\end{array}$ Upind BKALL 100-DR HEISPROD

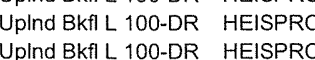

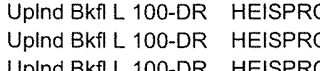
Uplnd B BRL L 100-DR HEISPROD
Upind BKAL L 100-DR HEISPROD

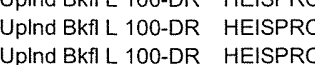

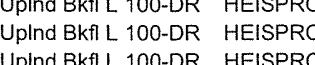
Uplnd BKA L L 100-DR HEISPROD
UDlnd BKAL 100-DR HEISPROD

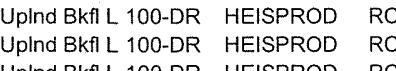

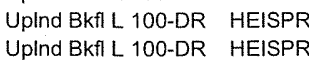

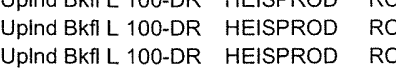

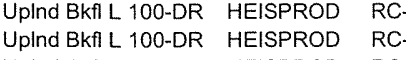

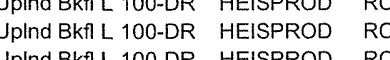

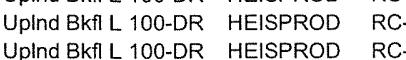

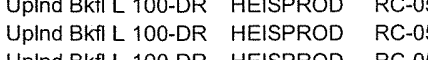

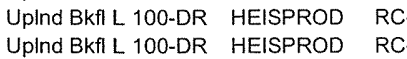
Uphnd BKR L L 100-DR HEISPROD RC-05

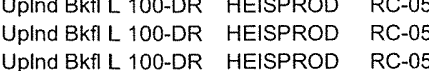

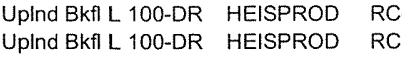

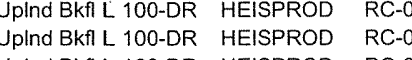
Uplind BKA L 100-DR HEIISPROD RC RC

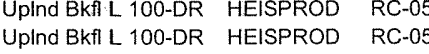

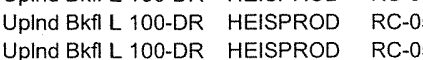

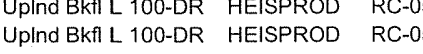

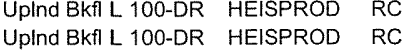

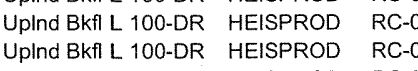

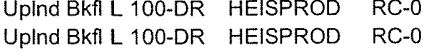

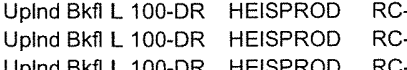

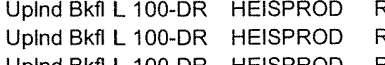

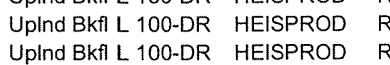

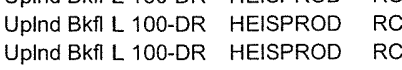

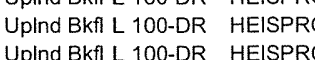

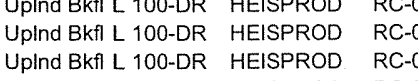

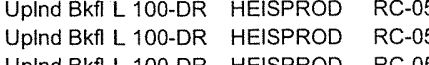

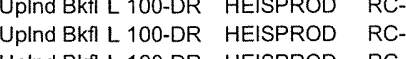

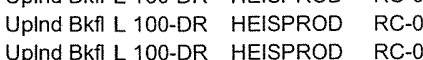

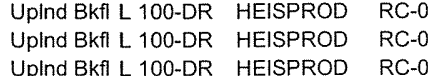

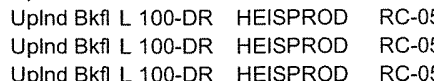

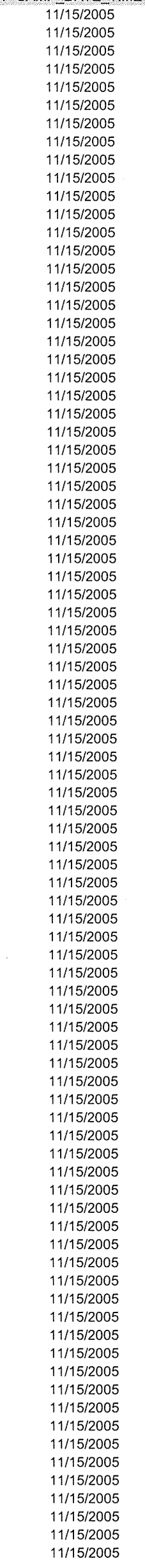

METHOD_NAME
8270_SOABGCMS
8270_SVOACGCMS 8270_SVOA_GCMS
3270_SVOA_GCMS 8270-SVA-GCMS
8270 SVVA-GCMS
8270 SCMS 8270 SVOA-GCMS
8720 SVOA GCMS 8270-SVOAAGCMS
8270_SVOA_GCMS 827-SVAO_CGMS
8270 SVOAGCMS

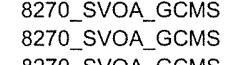
8270-SVOA_GCMS
8270_SVOA_GCMS 8270_SVOA_GCMS
8270_SVOA_GCMS 8270_SVOA_GCMS
8270 SVOA_CCMS
8270 SVIOACCSS 827-SVAOAGCCS
8270_SVOA_GCMS 8270_SVOA_GCMS
8270 SVOA_GCMS 8270_SVAA_GCMS
8270_SVOA_GCMS 8270_SVOA-GCMS
8270 SVOA_GCMS
8270 SVOOA GCMS 8270_SVOA_GCMS

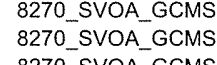
8270_SVOA_GCMS 8270-SVOA-GCMS
8277-SVOA-GMS
8270 SVOA_GCMS

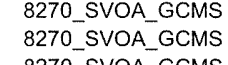

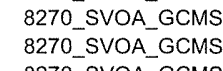
8270_-SVOA-GCMS
8270-SVOA-GCMS
8270 SVOA_GMS

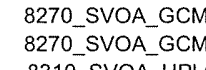
8310_SVOA_HPLC

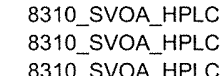
8310.SVOA-HPLC
8310_SVOA_HPLC 8310_SVVA_HPLC
8310_SSOA_HPLC 8310_SVVA_HPLC
8310_SVOA_HPLC 8310_SVOA_HPLC
8310 -SVOA_HPLC 8310_SVVA_HPLC
8310_SVOA_HPLC 8310-SVOA-HPCC
8310_SVOA_HPLC

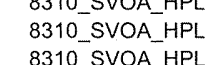
8310_SVOA_HPLC 8310_SVOA-HPCC
8310-SVOA HPLC
8310 SVOAA HLC

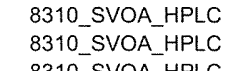
8310_SVOA_HPLC 8310_SVOA_HPLC 8310_SVAA_HPLC 8310-SVOA HPCLC
8310_SVOA HPLC
83100 SSOA HPPC 8310 SVOA HPCC
8310 SVOA HPLC

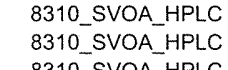

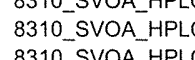
8310-SVAAAHPCC
8310_SVOA_HPLC 8310_SVOAAHPLC
8310-SVAA_HPLC 8310-SVOAAHLCC
8310 SVOA-HPLC
8310 SVOA 8310-SVOA_HPLC
8310_SVOA__PPLC 8310-SVOA-HPCC
8310.SVA_HLCC
8310 SVOA_HPLC

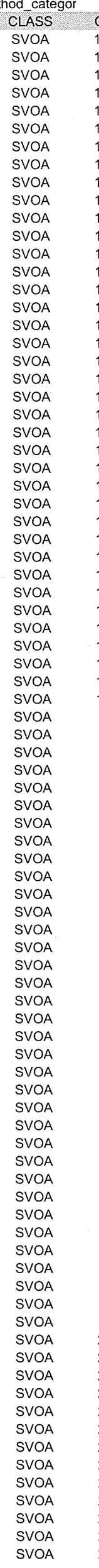

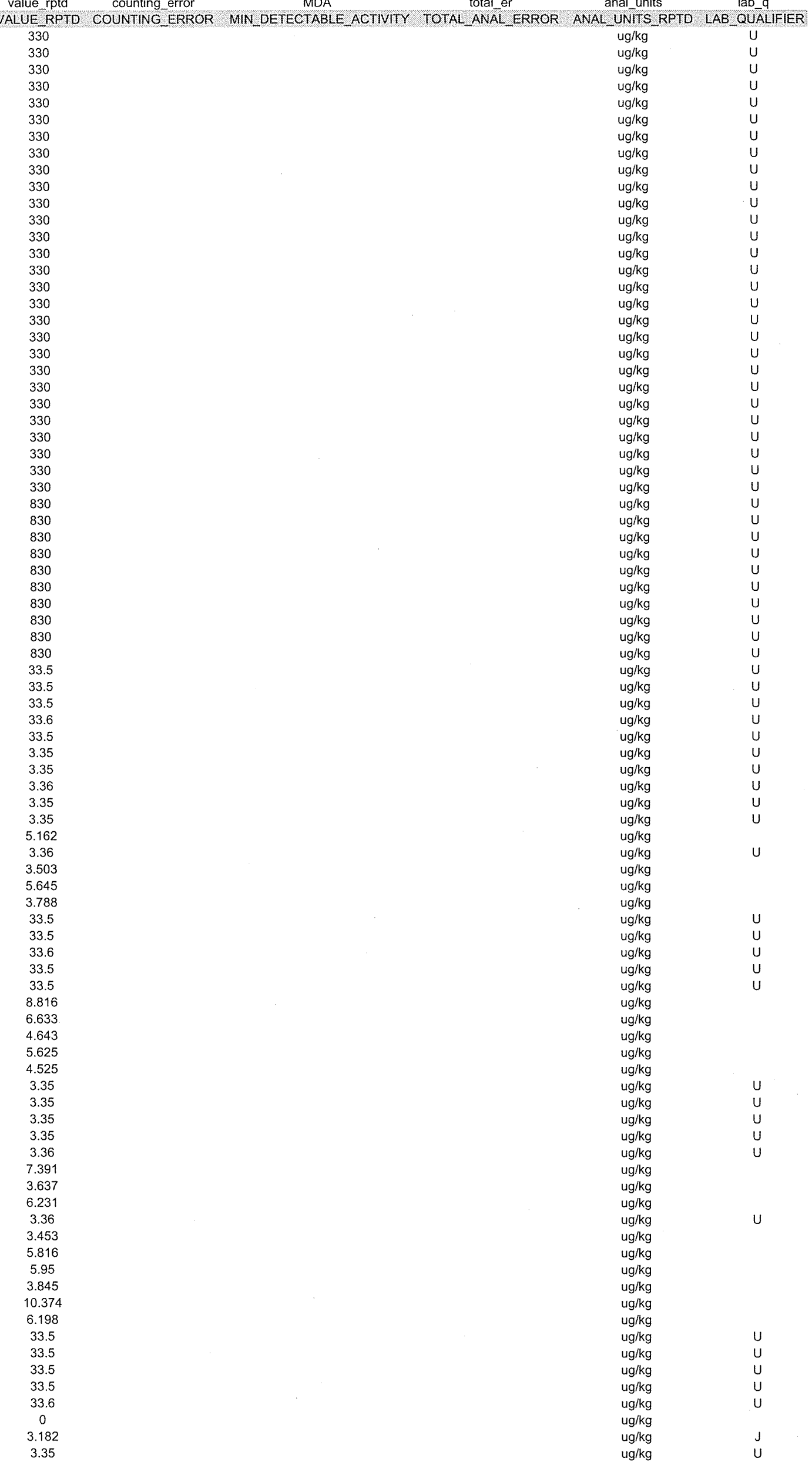


MIS_05 SAMPLES

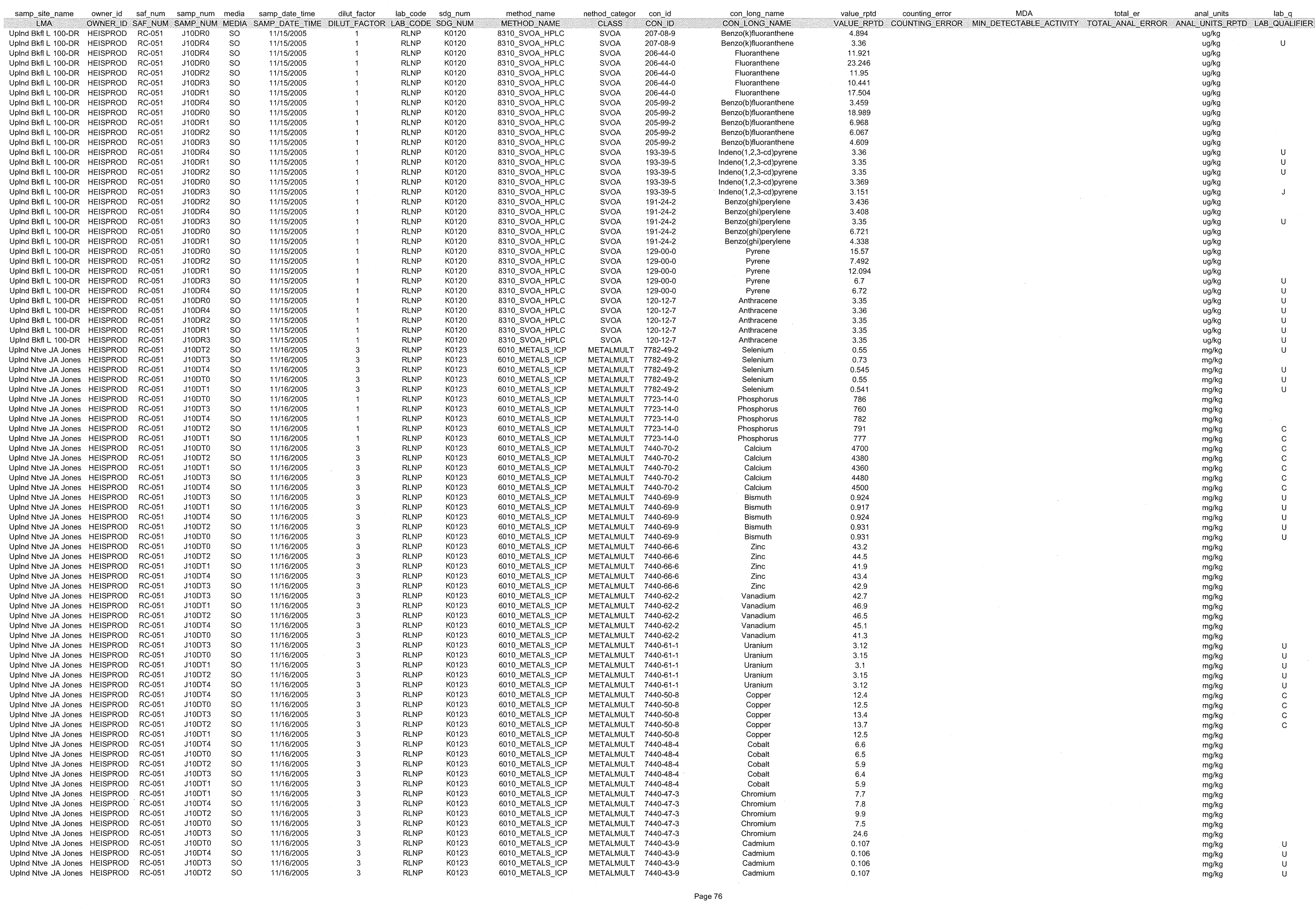


MIS_05 SAMPLES

samp_sile_name owner_id saf_num samp_num media samp_date_time dilut_factor lab__ode sdg num method_name nethod_categor con_id

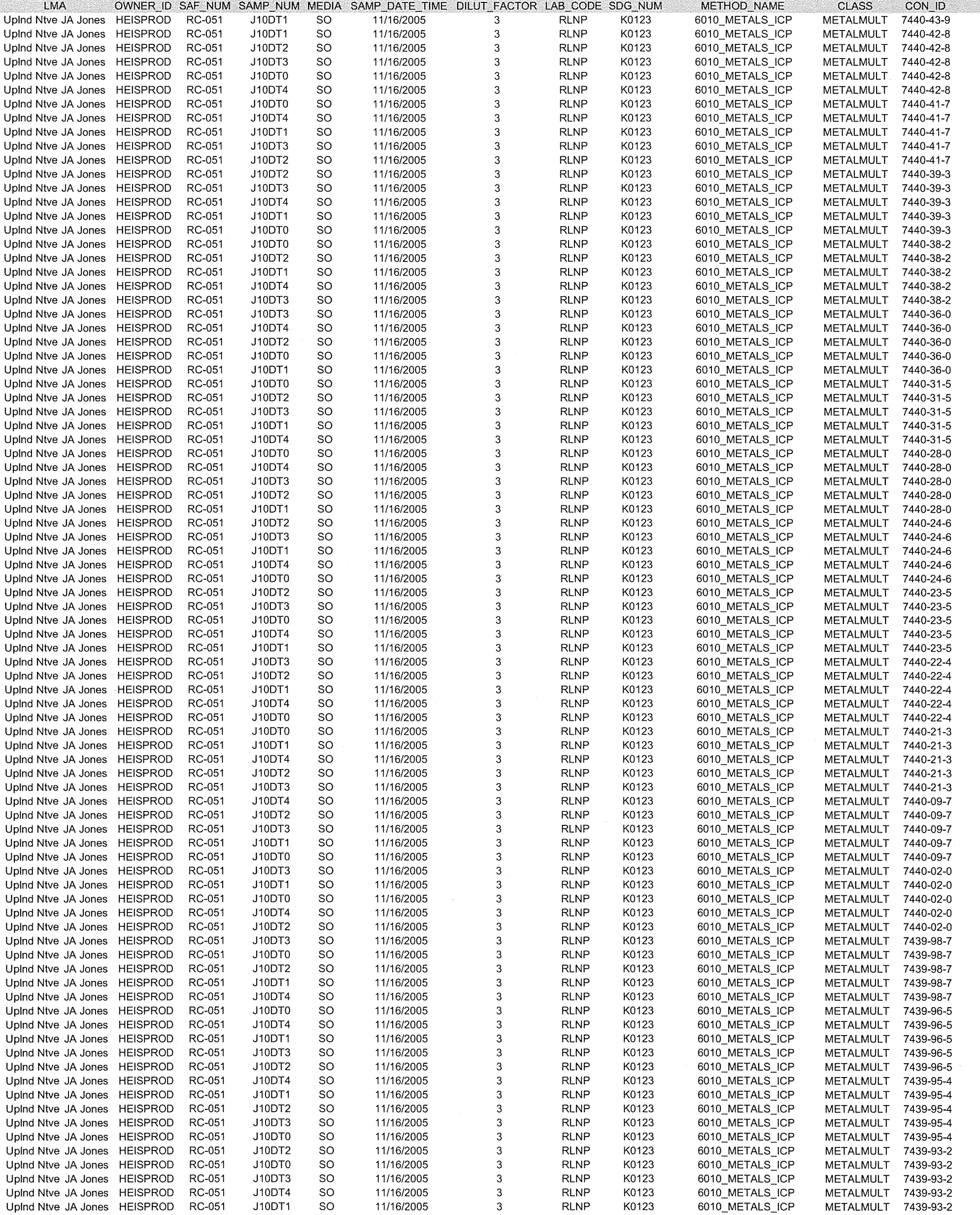

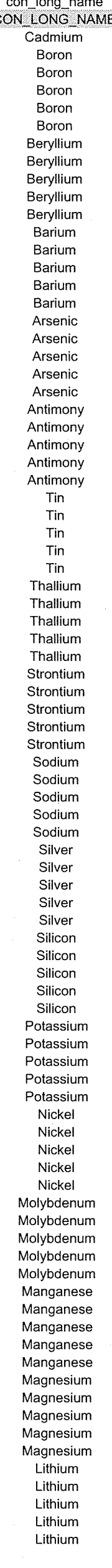

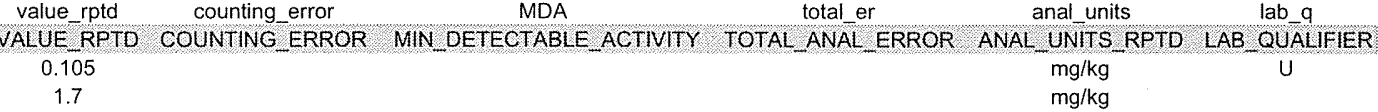

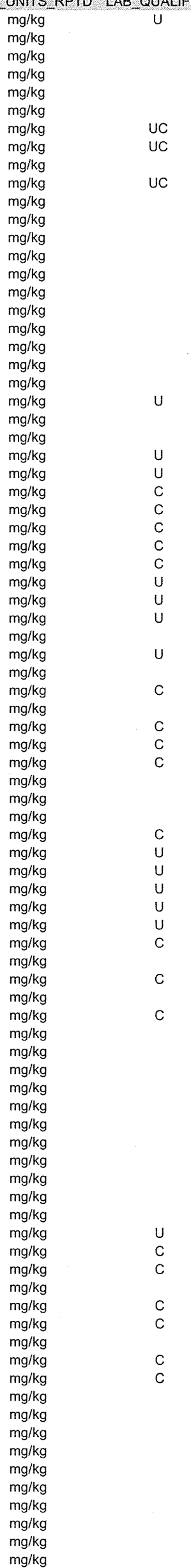




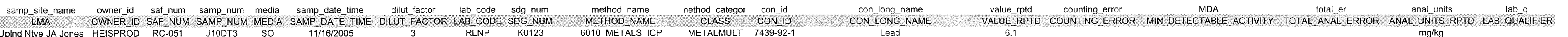

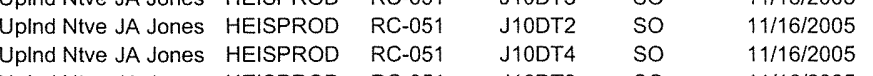

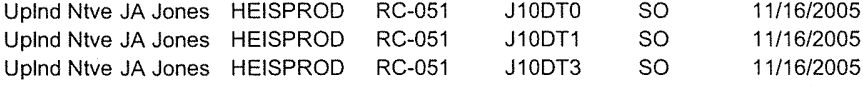

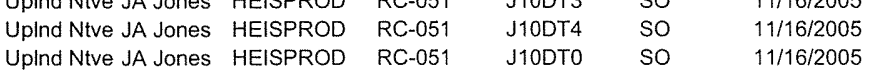

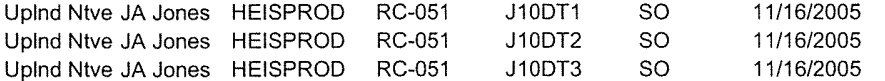

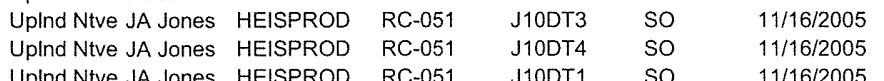

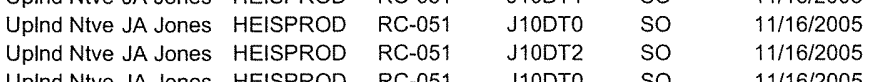

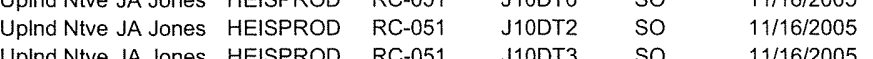

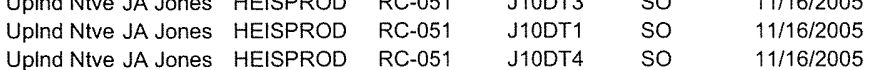

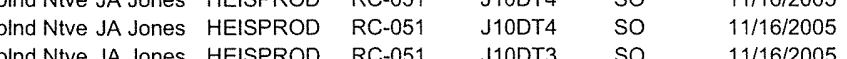

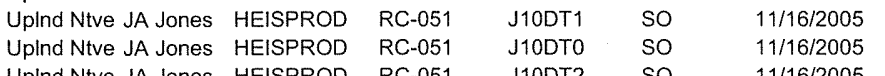

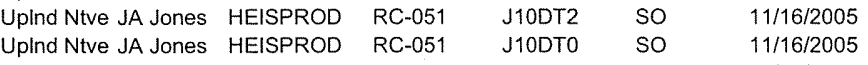

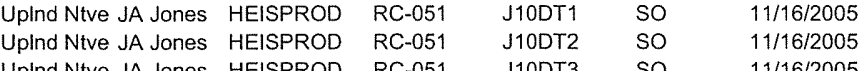

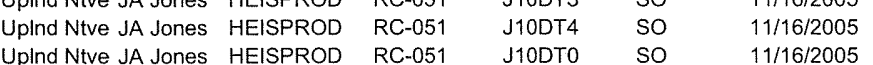

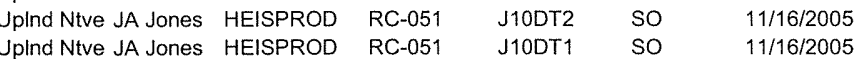

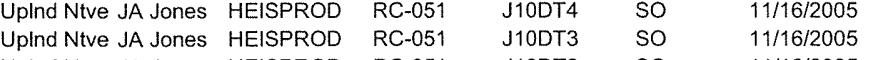

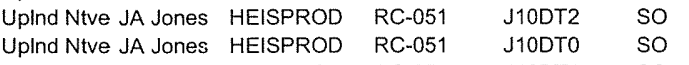

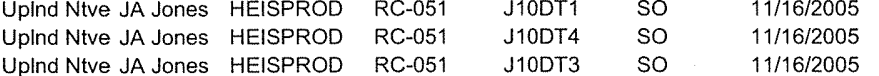

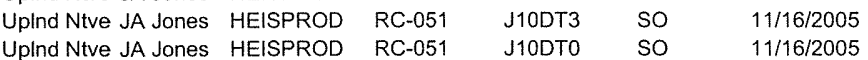

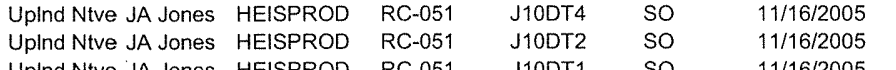
Uplind Nive JA Jones HEISPROD RC-OCI Ulind Nive JA Jones HEISPROD RC

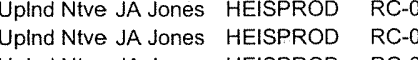
Uplind Nive JA Jones HEISPROD RC R Uplind Nive JA Jones HEISPROD RC RT

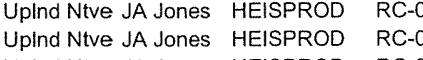

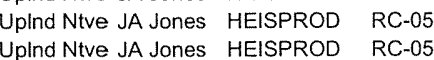
Upind Nive JA Jones HEISPROD RC-05

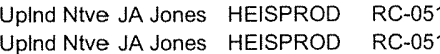

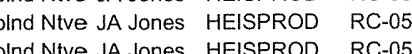
Uplnd Nive JA Jones HEISPROD RCOST Upind Nive JA Jones HEISPROD RC RTS Jind Nive JA Jones HEISPROD RC

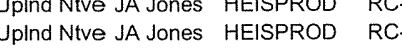

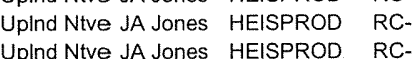

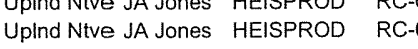

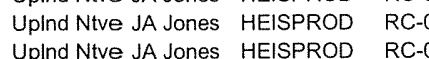

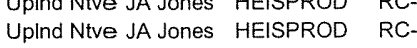

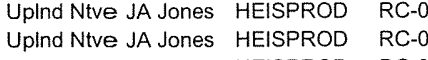

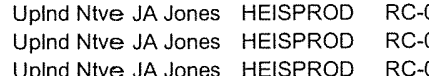

(10-Merats

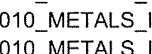

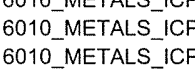

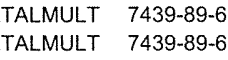

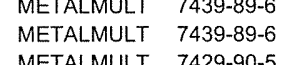

METAMULT
METALMULT T

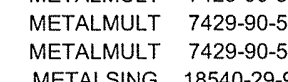

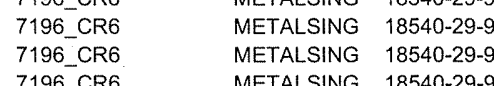

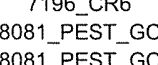

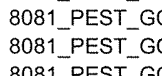

8081 -PEST-C

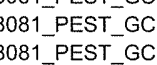

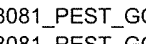

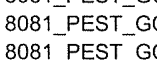

So83-PEST-CC

8081-PEST GC

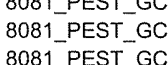

Bo81-_EST-GC

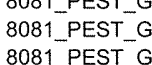

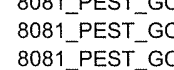

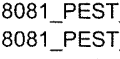

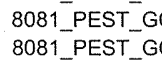

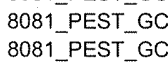

8081-PEST-GC

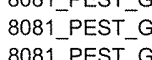

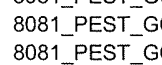

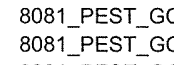

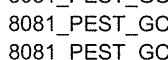

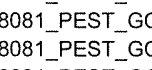

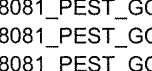

8081-PEST- $-C$

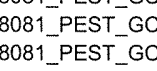

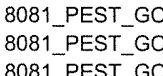

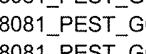

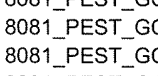

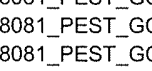

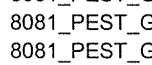

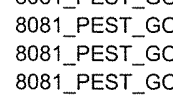

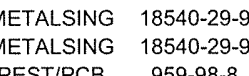

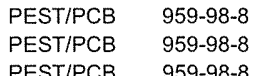

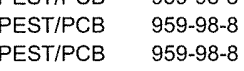

PESTPCB

\begin{tabular}{ll} 
PESTPCB & $8001-13-2$ \\
EESTPCB & $8001-155-2$ \\
\hline &
\end{tabular}

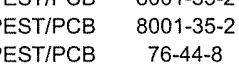

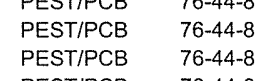

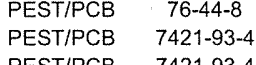

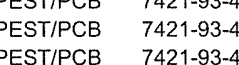

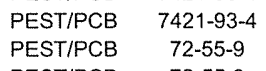

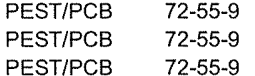

PESTPCO
PESTPCE

PESTTPCB
PESTIPCB
PESTIPCB
PESTPCB
PESTIPCB

PESTPCB
PESTPCB
PESPCE

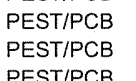

PESTPCB
PESTIPCB
PESTPCB
PESTIPCB

PESTPCB
PESTPCB
PESTPCB
PEST

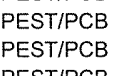

PESTPCB
PESTPCB
PESTPCBC

PESTPCB
PESTPCB
PESTTCB
PSETCS

PESTPCB
PESTPCB
PESTPPCE
PET

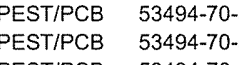

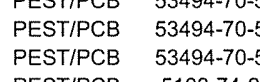

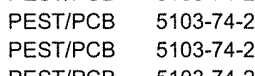

$\begin{array}{ll}\text { PESTPCB } & 5103-74-2 \\ \text { PESTPCB } & 5103-7.1-9\end{array}$

\begin{tabular}{ll} 
PESTPCB & $5103.71-9$ \\
\hline PESTPCB & $5103.71-9$ \\
\hline
\end{tabular}

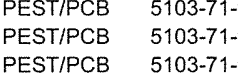

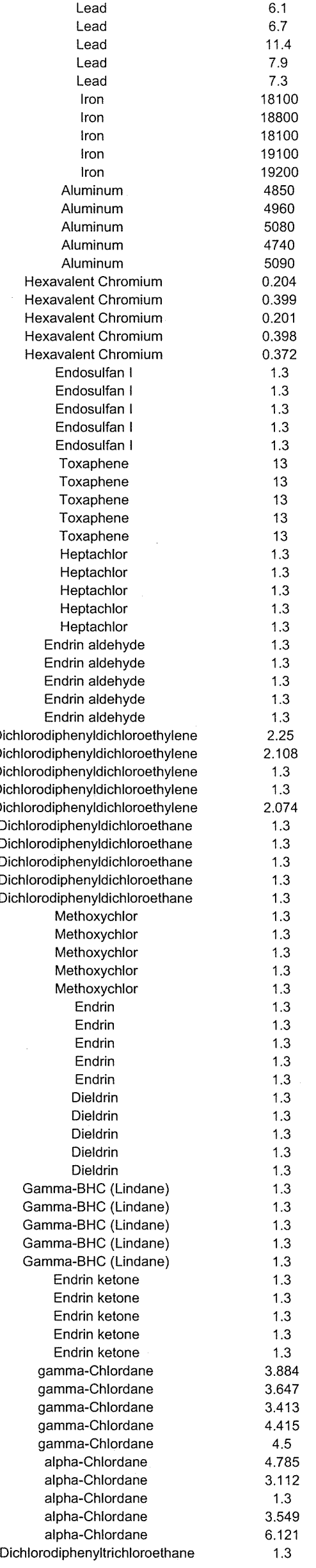


MIS_05 SAMPLES

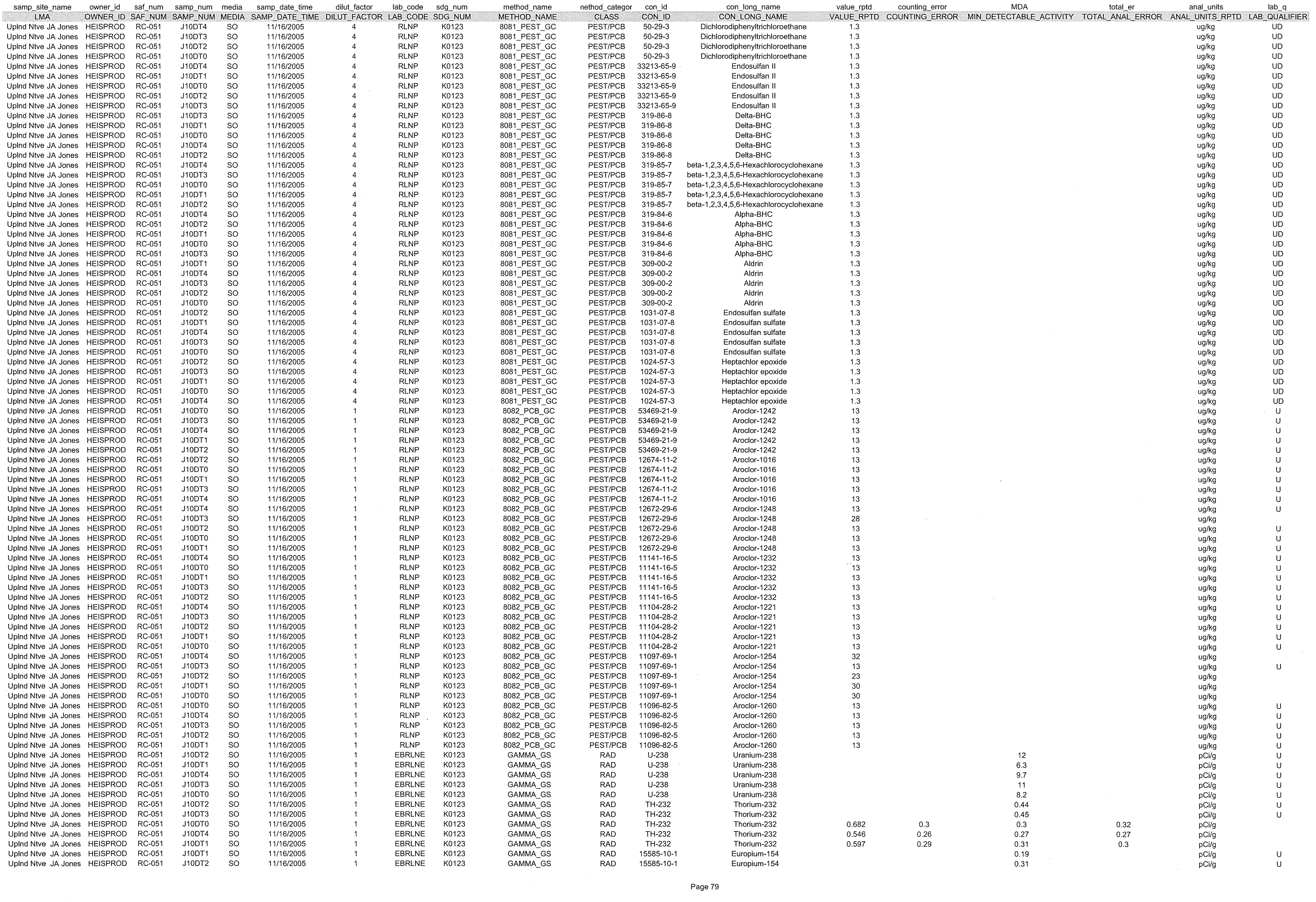




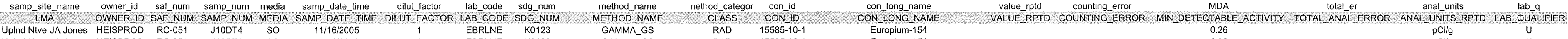

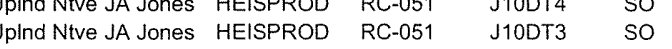

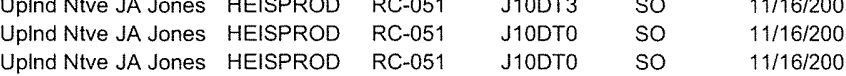

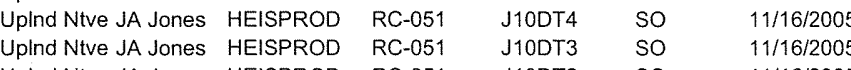

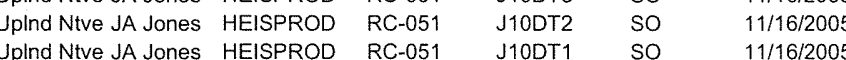

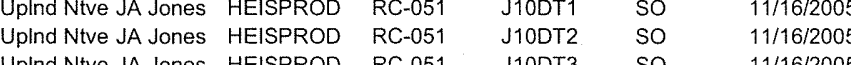

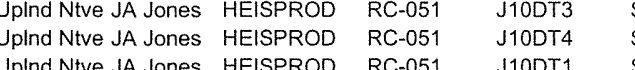

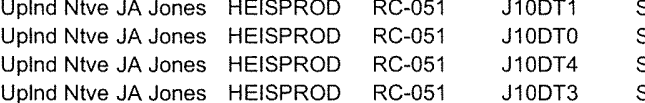

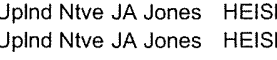

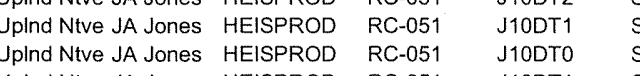
Uplnd Nive JA Jones HEISPROD RC-051 J10DT1

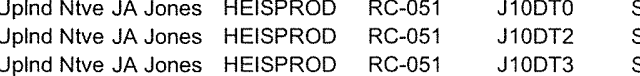
Whon Nve JA Jones Hes Heishor Uplind Nive JA Jones HEISPROD RC-051 J100T4

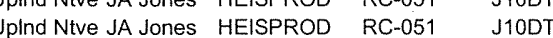
Uplnd Nive JA Jones HELISPROD
Uplnd Nive JA Jones HEISPROD

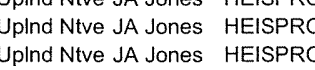

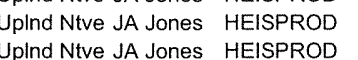

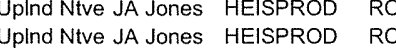

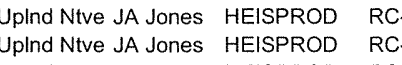
Uplind Nive JA Jones HEISPROD RC RCS

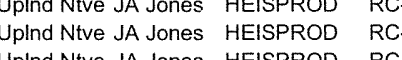
RC-051 J100T0 Uplind Nive JA Jones HEISPROD RC RT

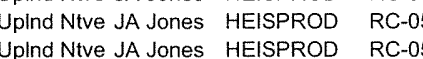

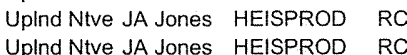
Jphnd Nive JA Jones HEISPROD
Uphnd Nive JA Jones HEISPROD

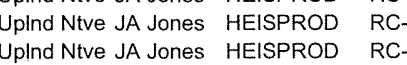

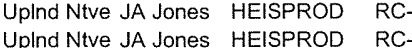

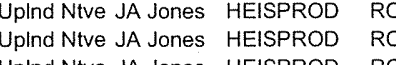
Uplnd Nve JA Junses HEESPROD RC-OS Uplind Nive JA Jones HEISPROD RC-05

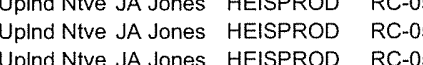

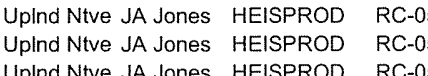
Uplnd NVE JA Jones HEISPROD
Upind Nve JA Jones HEISPROD
UC

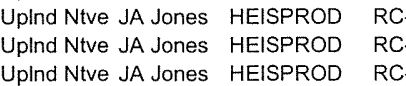

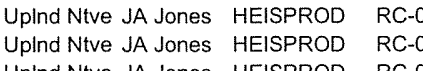

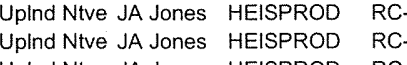

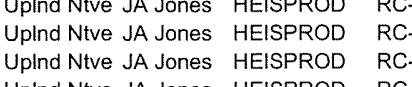

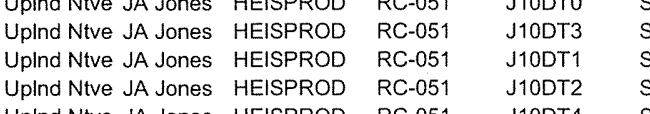

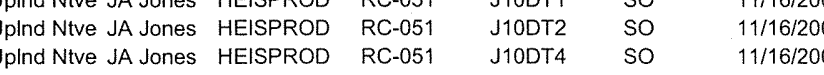

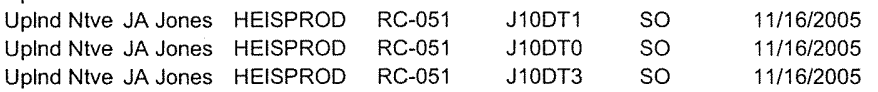

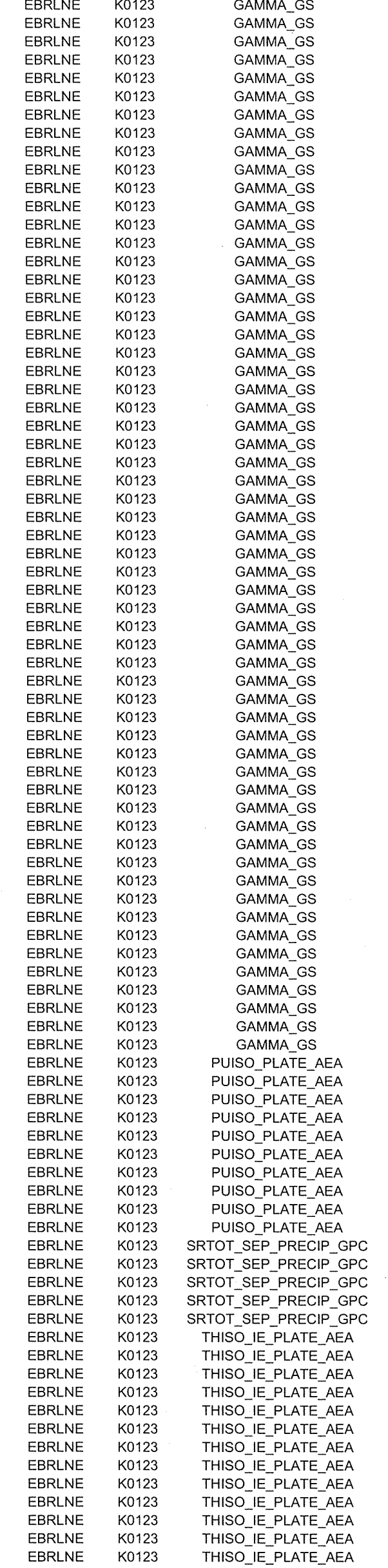

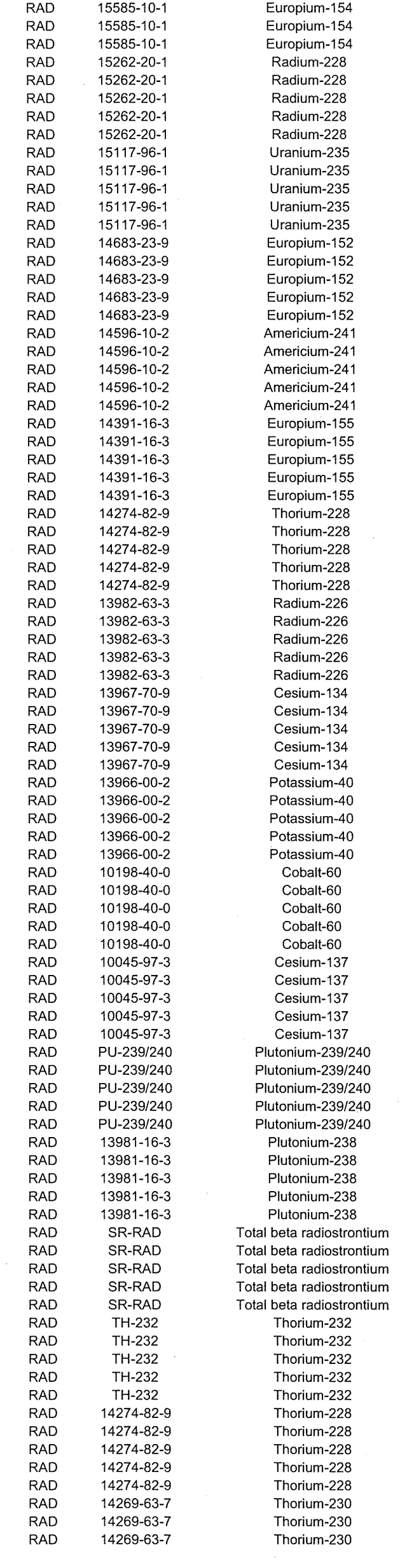

0.682
0.54

0.59

0.3
0.26 
MIS_05 SAMPLES

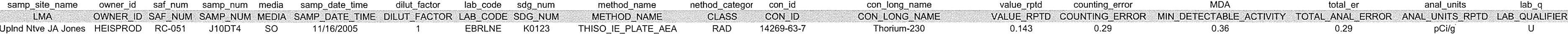

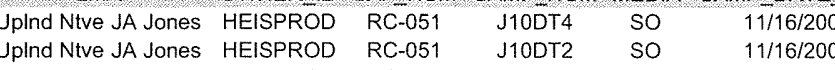

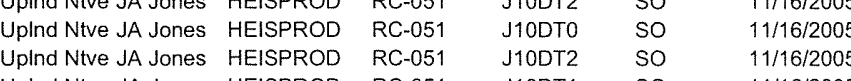

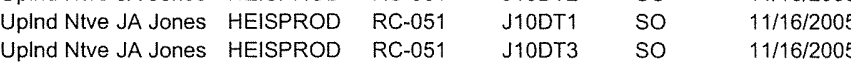

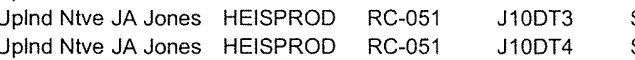
$\begin{array}{llll}\text { Uplnd Nive JA Jones HEISPROD } & R C-051 & \text { J100T1 } \\ \text { Ulind Nive JA Jones } \\ \text { HEISPROD }\end{array}$ Uind Nive JA Jones HEISPROD Uphnd Nive JA Jones HEISPRR
UDhnd Nive JA Jones HEISPRO Nive JA Jones HESPROD RCO51 J100T2 Uind Nive JA Jones HEISPROD RC RC \begin{tabular}{llll} 
& \\
\hline
\end{tabular} Ulind Nive JA Jones HEISPROD RC Uplnd Nive JA Jones HEISPROD
RCOS

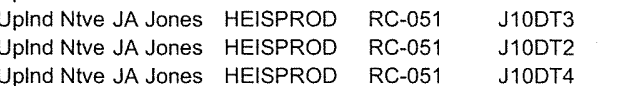
Ind Nive JA Jones HEISPROD RCOS

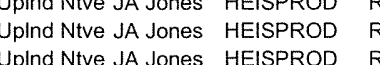
Uplnd Nive JA Jones HEISPROD RCDOS

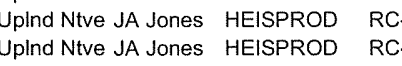
Uplnd Nive JA Jones HEISPROD RCST RTh Uphnd Nive JA Jones HEISPROD RCOSI Uplnd Nive JA Jones HEISPROD
UCC.
Uplnd Ntve JA Jones HEISPROD
RC.

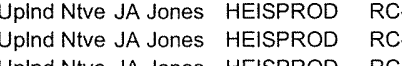
Uplnd Nive JA Jones HEISPROD RC RT

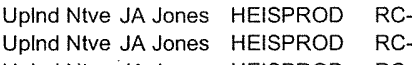
Uplnd Nive JA Jones HEISPROD RC-OSY Uplnd Nive J J Jones
Uplnd Nive JA Jones HEISPROD

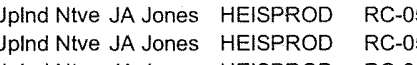

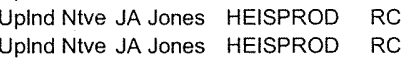
Uphn Nive JA Jones HESPROD
Uphnd Nive JA Jones HEISPROD RC-OS

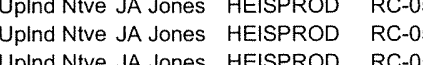

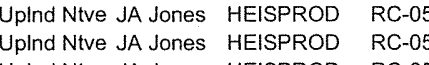

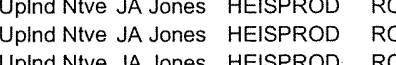

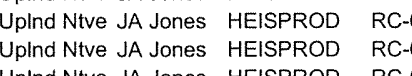
Upind Nive JA Jones HEISPROD RC

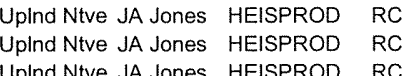

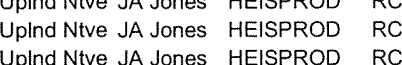

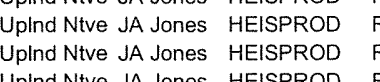

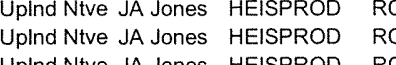

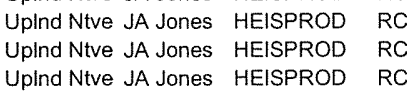

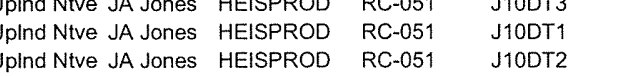

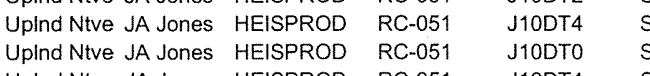

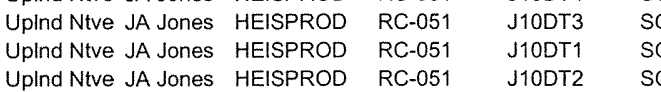

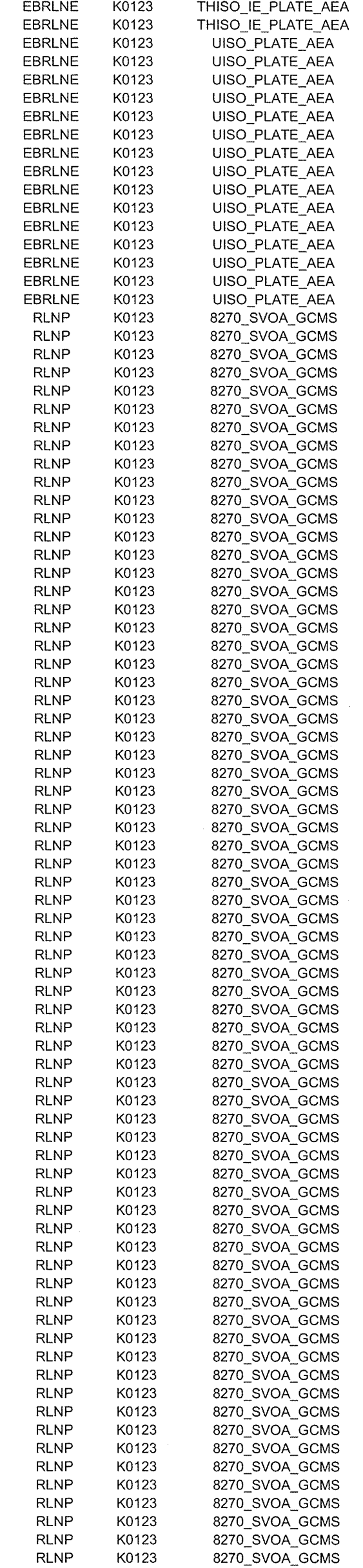

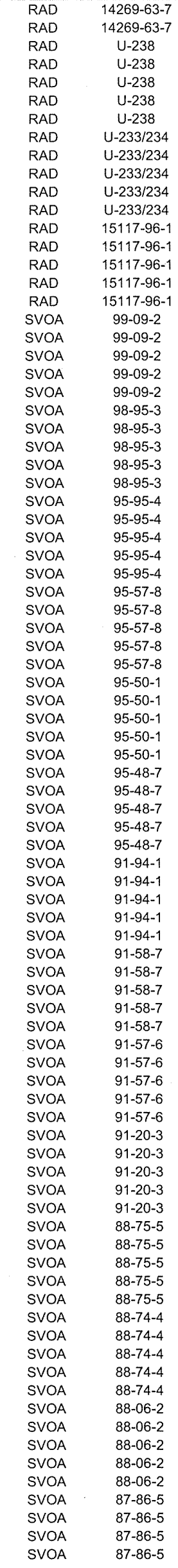

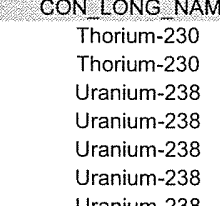


MIS_05 SAMPLES

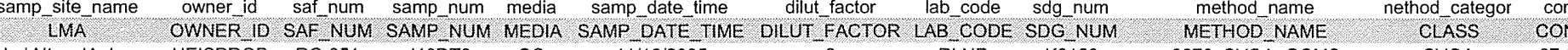

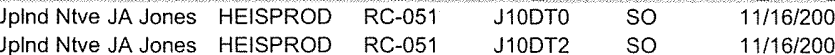

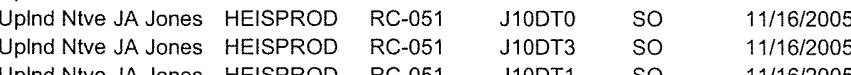

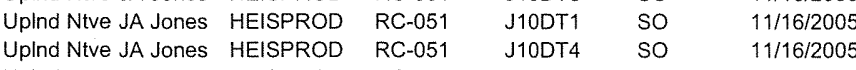
UPlnd Nive JA Jones HEISPROD RC-051 J100T1 SO 1 111/61/2005

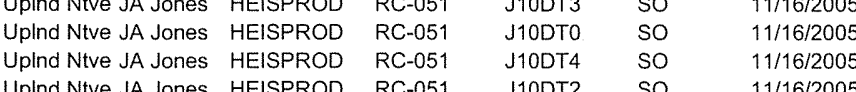

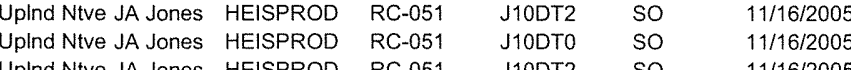

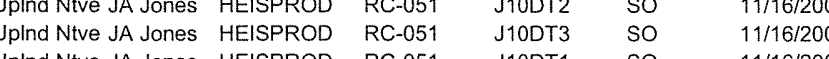

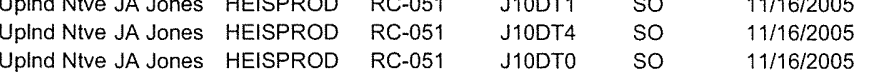

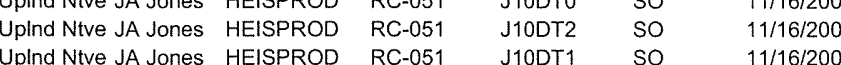

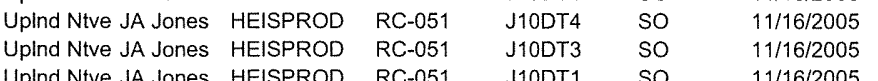

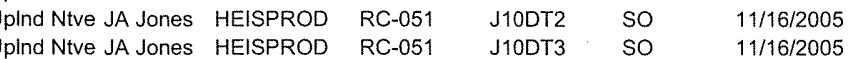
Uplnd Nive JA Jones HEISPROD RC-051 J100TO SO $11 / 166 / 2005$

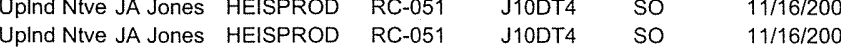

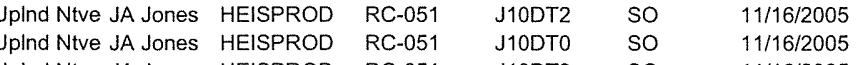

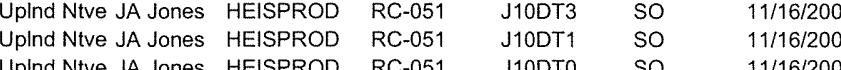

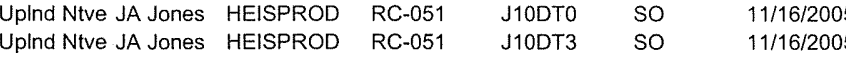

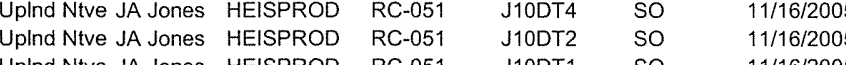

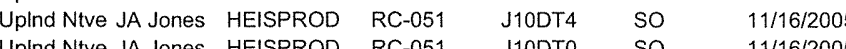

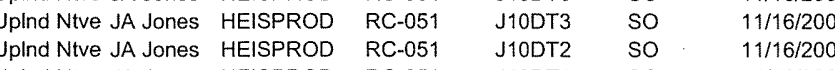

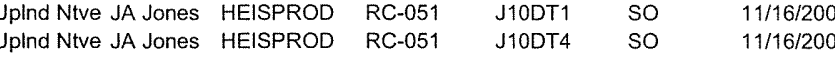

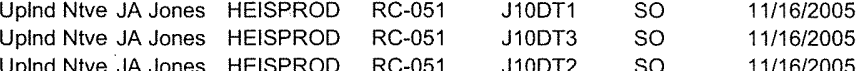

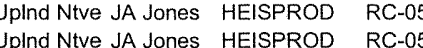
Uphnd Nve JA Jones HEISPROD RC-0S

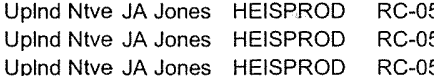

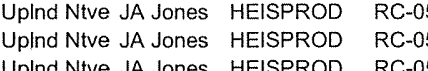

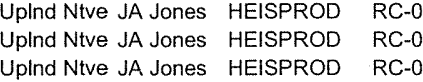
$\begin{array}{lll}\text { Uplnd Nive JA Jones } & \text { HEISPROD } & \text { RC-OS } \\ \text { Uplind Nive JA Jones } & \text { HEISPROD } & \text { RC-0.5 }\end{array}$

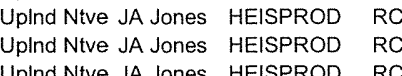

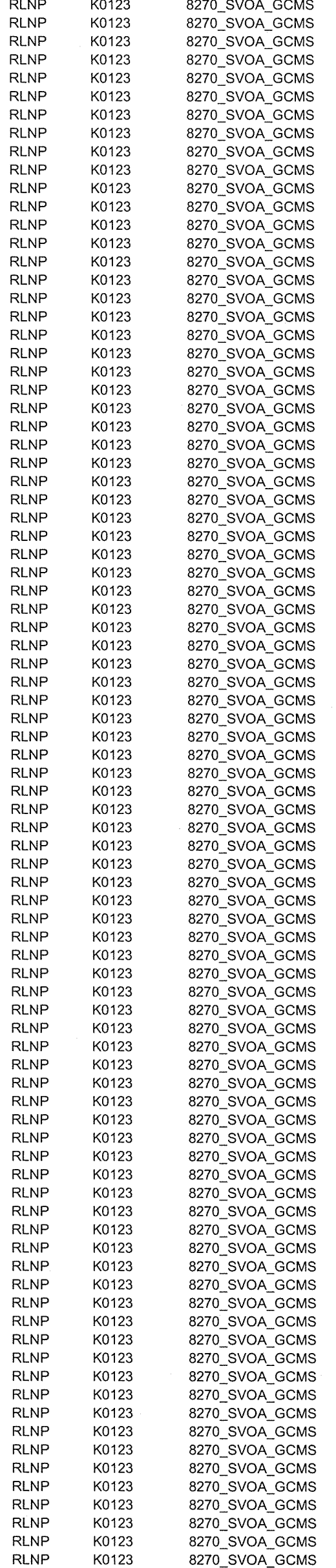

\begin{tabular}{|c|c|c|c|}
\hline & $\begin{array}{l}\operatorname{con} 10 \\
87-86-5\end{array}$ & $\begin{array}{l}\text { CON LONG NAME } \\
\text { Pentachlorophenol }\end{array}$ & 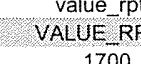 \\
\hline & $87-68-3$ & $\begin{array}{l}\text { Hexachlorobutadiene } \\
\text { Hont }\end{array}$ & 670 \\
\hline & - 87-68-3 & $\begin{array}{l}\text { Hexachlorobutadiene } \\
\text { Hexachlorobutadiene }\end{array}$ & $\begin{array}{l}670 \\
670\end{array}$ \\
\hline sv & $87-68-3$ & Hexachlorobutadiene & 660 \\
\hline & $\begin{array}{l}87-68-3 \\
88-74-8\end{array}$ & $\begin{array}{l}\begin{array}{l}\text { Hexachlorobutadiene } \\
\text { Carbazole }\end{array} \\
\text { a }\end{array}$ & $\begin{array}{l}670 \\
660\end{array}$ \\
\hline & $\begin{array}{l}80-74-8 \\
86-74-8\end{array}$ & $\begin{array}{l}\text { Carbaziole } \\
\text { Carbazle }\end{array}$ & $\begin{array}{l}660 \\
670\end{array}$ \\
\hline & $86-74-8$ & $\begin{array}{l}\text { Carbazole } \\
\text { Calus }\end{array}$ & 670 \\
\hline & $\begin{array}{l}86-74-8 \\
87-74-8\end{array}$ & Carbazole & 670 \\
\hline & $\begin{array}{l}86-74-8 \\
86-73-7\end{array}$ & $\begin{array}{l}\text { Carbazole } \\
\text { Fluorene }\end{array}$ & 670 \\
\hline & 86-73-7 & Fluorene & $\begin{array}{l}670 \\
670\end{array}$ \\
\hline & 86-73-7 & $\begin{array}{l}\text { Fluorene } \\
\text { Fluse }\end{array}$ & 要70 \\
\hline & $\begin{array}{l}86-73-7 \\
86-73-7\end{array}$ & $\begin{array}{l}\text { Fluorene } \\
\text { Fluorene }\end{array}$ & 660 \\
\hline & $\begin{array}{l}88-37-7 \\
86-30-6\end{array}$ & $\begin{array}{l}\text { Flluorene } \\
\text { N-Nitrosodiphenylamine }\end{array}$ & $\begin{array}{l}670 \\
670\end{array}$ \\
\hline & ${ }_{86-30-6}$ & N-Nitrosodiphenylanine & $\begin{array}{l}670 \\
670\end{array}$ \\
\hline & $86-30-6$ & N-Nitrosodiphenylamine & $\begin{array}{l}670 \\
660\end{array}$ \\
\hline & $86-30-6$ & $\begin{array}{l}\text { N-Nitrosodiphenylamine } \\
\text { N-Nitrosontinhentumino }\end{array}$ & 670 \\
\hline & $\begin{array}{l}86-3-3-6 \\
85-68-7\end{array}$ & 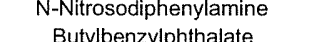 & $\begin{array}{l}670 \\
660 \\
660\end{array}$ \\
\hline & $\begin{array}{l}85-68-7 \\
85-6\end{array}$ & 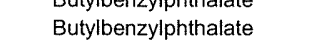 & $\begin{array}{l}660 \\
670\end{array}$ \\
\hline & 85-68-7 & Butylbenzy/phthalate & 670 \\
\hline & $\begin{array}{l}85-68-7 \\
85-6-7\end{array}$ & 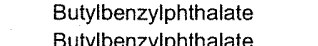 & 670 \\
\hline & $\begin{array}{l}85-6-6-1 \\
85-01-8\end{array}$ & $\begin{array}{l}\text { Butylbenzylphthalate } \\
\text { Phenanthrene }\end{array}$ & $\begin{array}{l}670 \\
670\end{array}$ \\
\hline & $85-01-8$ & $\begin{array}{l}\text { Prenanturene } \\
\text { Phenanthrene }\end{array}$ & 670 \\
\hline & $\begin{array}{l}85-01-8 \\
85-1-8\end{array}$ & $\begin{array}{l}\text { Phenanthrene } \\
\text { Phenanthrene }\end{array}$ & 670 \\
\hline & $85-01-8$ & $\begin{array}{l}\text { Phenanthrene } \\
\text { Phenter }\end{array}$ & $\begin{array}{l}670 \\
660\end{array}$ \\
\hline & 84-74-2 & Di-n-butylynthalate & 670 \\
\hline 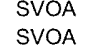 & $\begin{array}{l}84-7-2 \\
84-74-2\end{array}$ & 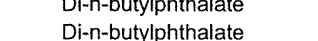 & $\begin{array}{l}44.294 \\
670\end{array}$ \\
\hline SVOA & 84-74-2 -1 & $\begin{array}{l}\text { Di-n-Duryphinalale } \\
\text { Di-n-buty|phthalate }\end{array}$ & 70 \\
\hline & 84-74-2 & $\begin{array}{l}\text { Di-n-butylyhthalate } \\
\text { nipthylpthalato }\end{array}$ & 60 \\
\hline & (84-66-2 $8-2$ & $\begin{array}{l}\text { Diethylphthalate } \\
\text { Diettyvhththalate }\end{array}$ & 70 \\
\hline & $\begin{array}{l}84-66-2 \\
84-2-2\end{array}$ & $\begin{array}{l}\text { Dienthyppintalate } \\
\text { Diethylpthalate }\end{array}$ & 70 \\
\hline & 84-66-2 & $\begin{array}{l}\text { Diethylphthalatate } \\
\text { Dintyly }\end{array}$ & 670 \\
\hline & $\begin{array}{l}84-6-2-2 \\
83-32-9\end{array}$ & $\begin{array}{l}\text { Diethylphthalate } \\
\text { Acenaphthene }\end{array}$ & 70 \\
\hline SVOA & $\begin{array}{l}83-32-9 \\
83-2309\end{array}$ & $\begin{array}{l}\text { Acenaphthene } \\
\text { Accononthon }\end{array}$ & 60 \\
\hline & $\begin{array}{l}83-3-3-9 \\
83-22-9\end{array}$ & $\begin{array}{l}\text { Accnaphthene } \\
\text { Acenaphthene }\end{array}$ & 70 \\
\hline & 83-32-9 19 & $\begin{array}{l}\text { Acenaphthene } \\
\text { Actine }\end{array}$ & 70 \\
\hline & $78-59-1$ & Isophorone & 70 \\
\hline & $\begin{array}{l}78.59-1 \\
77.501\end{array}$ & Isophorone & 70 \\
\hline & (78.59-1 & $\begin{array}{l}\text { IIsohhorone } \\
\text { sophorone }\end{array}$ & $\begin{array}{l}670 \\
660 \\
660\end{array}$ \\
\hline & 78-59-1 & $\begin{array}{l}\text { Isophorone } \\
\text { siste }\end{array}$ & 70 \\
\hline & 77-47-4 & Hexachlorocyclopentadiene & 70 \\
\hline & 77-47-4 $777-4$ & $\begin{array}{l}\text { Hexacchlorocyclopentadadiene } \\
\text { Hexachlbrococylonentadied }\end{array}$ & $\begin{array}{l}670 \\
670 \\
670-250\end{array}$ \\
\hline & $77-47-4$ & $\begin{array}{l}\text { nexactirorocycopentadere } \\
\text { Hexachlorocyclopentadiene }\end{array}$ & 70 \\
\hline & $77-47-4$ & Hexachlorocyclopentadien & \\
\hline & $\begin{array}{l}7055-72-3 \\
7005-72-3\end{array}$ & $\begin{array}{l}\text { 4-Chlorophenylyhenyl ether } \\
\text { 4-Chlorophennlyhenyl ther }\end{array}$ & $\begin{array}{l}670 \\
670\end{array}$ \\
\hline svo & $\begin{array}{l}7005-72-3 \\
7005-23.3\end{array}$ & $\begin{array}{l}\text { 4-Chlorophenylphenyl ether } \\
\text { - }\end{array}$ & 70 \\
\hline $\begin{array}{l}\text { svo } \\
\text { svo }\end{array}$ & $\begin{array}{l}70055-7 \cdot 3-3 \\
7005-72-3\end{array}$ & $\begin{array}{l}\text { 4-Chlorophennylyhennl ther } \\
\text { 4-Chlorophenyyphenyy ether }\end{array}$ & 700 \\
\hline $\begin{array}{l}\text { svc } \\
\text { svc }\end{array}$ & $\begin{array}{l}67-72-1 \\
67-72-1\end{array}$ & $\begin{array}{l}\text { Hexachloroethane } \\
\text { Hexachlorothane }\end{array}$ & $\begin{array}{l}670 \\
670\end{array}$ \\
\hline $\begin{array}{l}\text { SVOA } \\
\text { SVOA }\end{array}$ & $67-72-1$ & $\begin{array}{l}\text { Hexachoroenanane } \\
\text { Hexachloroethane }\end{array}$ & 670 \\
\hline & $6 r-r<-1$ & $\begin{array}{l}\text { Hexachloroethane } \\
\text { Hexachloroethane }\end{array}$ & 700 \\
\hline $\begin{array}{l}\text { SVOA } \\
\text { sVOA }\end{array}$ & $\begin{array}{l}65794-96-9 \\
6579-96-9\end{array}$ & $3+4$ Methylphenol (cresol, $m+p$ ) & $\begin{array}{l}600 \\
670 \\
670 \\
670\end{array}$ \\
\hline svo & $\begin{array}{l}659744-96-9 \\
65794-96-9\end{array}$ & $\begin{array}{l}3+4 \text { Methylphenol (cresol, } m+p) \text { ) } \\
3+4\end{array}$ & 70 \\
\hline & $65794-96-9$ & & 60 \\
\hline $\begin{array}{l}\text { SVOA } \\
\text { SVOA }\end{array}$ & $\begin{array}{l}\begin{array}{l}657744-96-9 \\
621-64-7\end{array} \\
0\end{array}$ & 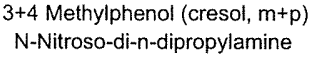 & $\begin{array}{l}670 \\
670\end{array}$ \\
\hline & $\begin{array}{l}621-64-7 \\
621-64-7\end{array}$ & $\begin{array}{l}\text { N-Nitroso-di-n-dipropylamine } \\
\text { NNitrocdi-dipons }\end{array}$ & 660 \\
\hline & $\begin{array}{l}621-1-64-7 \\
621-7\end{array}$ & $\begin{array}{l}\text { N-Nitroso-di-n-dipropplamine } \\
\text { N-Nitroso-din-dipropylamine }\end{array}$ & $\begin{array}{l}670 \\
670\end{array}$ \\
\hline $\begin{array}{l}\text { svo } \\
\text { svo }\end{array}$ & $\begin{array}{l}621-64-7 \\
600-20-2\end{array}$ & N-Nitroso-di-n-dipropylamine & 670 \\
\hline & $\begin{array}{l}-600-20-2 \\
606-20-2\end{array}$ & 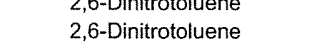 & $\begin{array}{l}670 \\
660\end{array}$ \\
\hline $\begin{array}{l}\text { SVOA } \\
\text { SVOA }\end{array}$ & $\begin{array}{l}606-20-2 \\
600-20-2\end{array}$ & $\begin{array}{l}\text { 2,6-Dinitrotoluene } \\
2,6 \text {-Dinitotlouene }\end{array}$ & 70 \\
\hline sv & $\begin{array}{l}-60-20-2 \\
606-20-2\end{array}$ & $\begin{array}{l}\text { 2, } 2 \text {-bunirirofouene } \\
\text { 2,6-Dinitrotoluene }\end{array}$ & 670 \\
\hline $\begin{array}{l}\text { svc } \\
\text { svo }\end{array}$ & $\begin{array}{l}59-50-7 \\
59-50-7\end{array}$ & $\begin{array}{l}\text { 4-Chloro-3-methylphenol } \\
\text { 4-Chloro-3-methylohenol }\end{array}$ & \\
\hline & 59-50-7 & 4-Chloro-3-3-methylphenol & 70 \\
\hline & 59-50-7 & 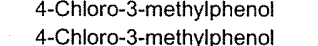 & \\
\hline
\end{tabular}

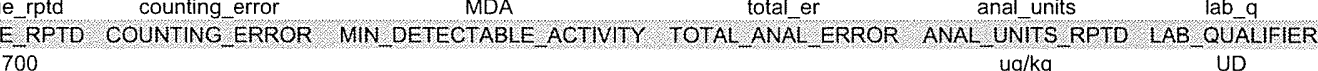


MIS_05 SAMPLES

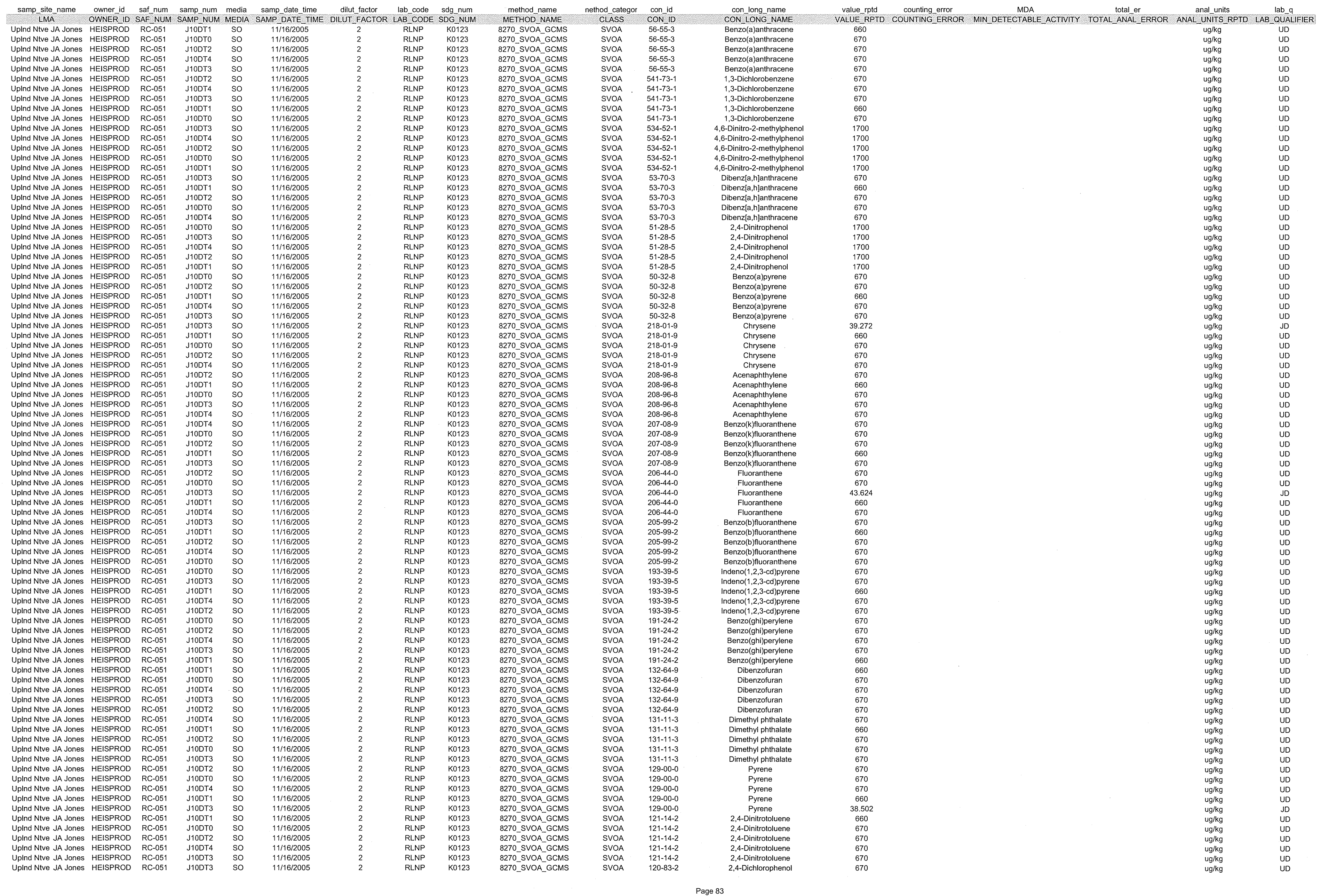


MIS_05 SAMPLES

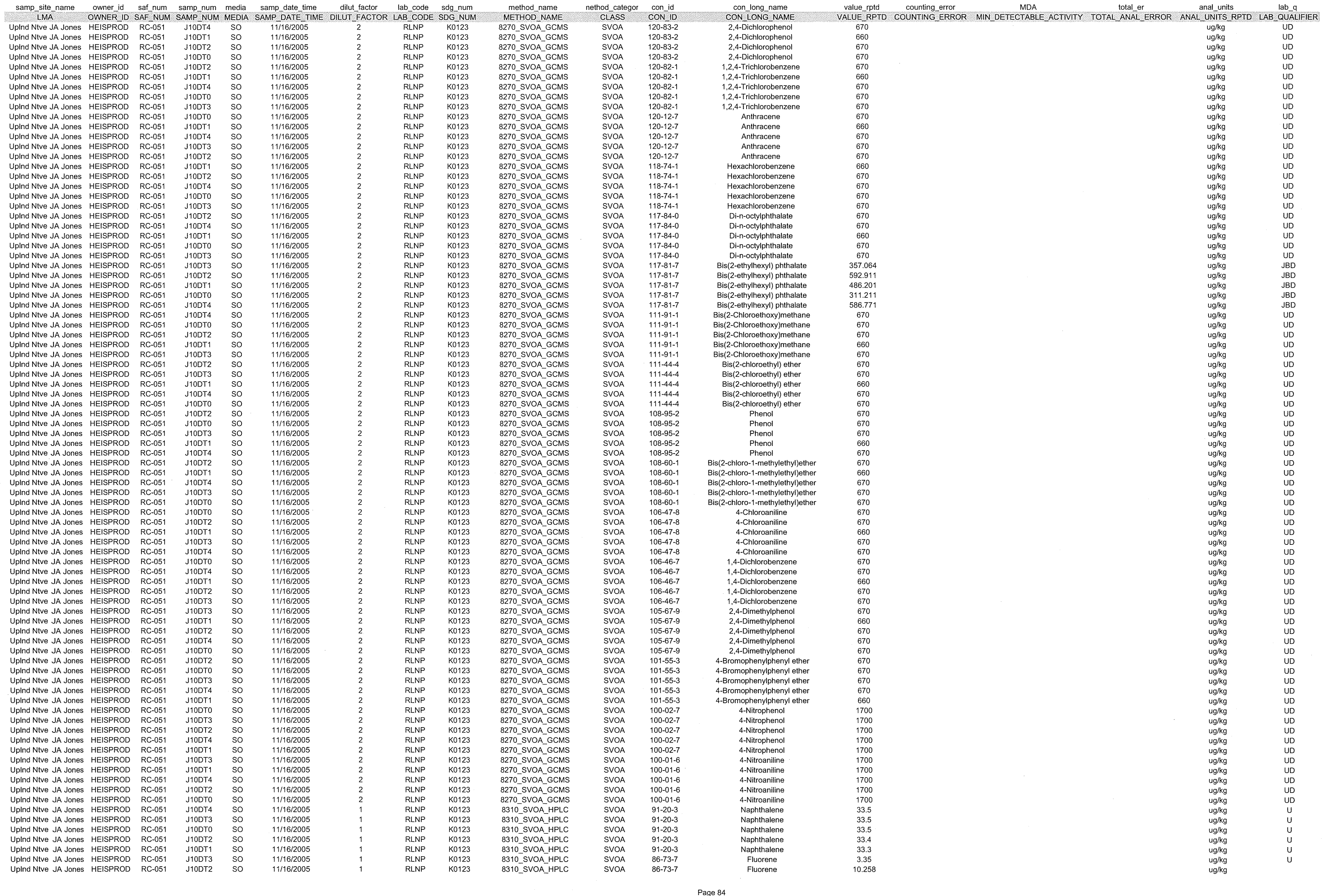




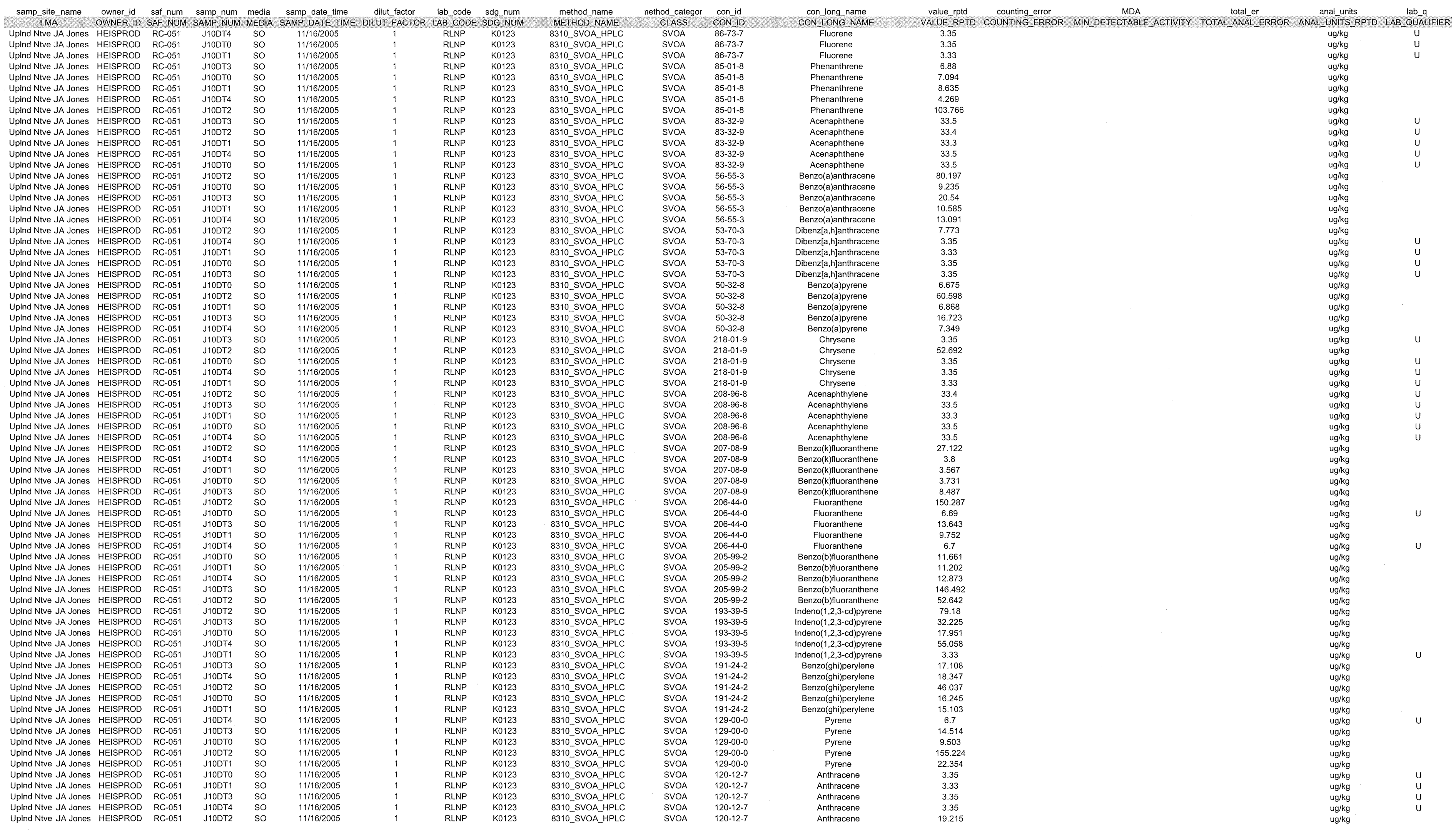


WCH-85

Rev. 0

\section{APPENDIX F}

DATA SUMMARY OF GROUND WATER RESULTS 
WCH-85

Rev. 0 


\begin{tabular}{|c|c|c|c|c|c|c|c|c|c|c|c|c|c|c|c|c|c|c|c|c|c|c|}
\hline & & & & & & MEDDA & & & & SDG_NUM & METHOD NAME & CLASS & CON ID $k$ & ION_LONG NAMV V & VALUE,RPTD & BUNTING ER & dETECTABLEAAT & & & WAB_ QUALIFEF & & \begin{tabular}{|l|l|} 
EW_COORD \\
\end{tabular} \\
\hline $\begin{array}{l}A 4636 \\
A 4636\end{array}$ & $199-H 4-5$ & & HESPROD & $\begin{array}{lll}R C-048 \\
\end{array}$ & J107\%8 & GW & $13-$ Oct-05 & 1 & STLRL & J00002 & 7196 CR6 & METALSING & $18540-29-9$ & Chromium & 0.0167 & & & & $\mathrm{mg} / \mathrm{L}$ & & 152939.783 & 577944.937 \\
\hline $\begin{array}{l}A 4636 \\
A 4636\end{array}$ & $199-44-5$ & & HEISPROD & RC-048 & J10748 & $\mathrm{GW}$ & $13-0 \mathrm{ct}-05$ & & FIELD & & $\begin{array}{c}D \\
D\end{array}$ & PHYSICAL & CONDUCT & Conductance & 583 & & & & uslcm & & 152939.783 & 577944.937 \\
\hline $\begin{array}{l}A 6436 \\
A 4636\end{array}$ & $199-44-5$ & & HEISPROD & $\begin{array}{ll}R C-048 \\
R C-048\end{array}$ & $\begin{array}{l}\text { J107Y8 } \\
\text { J10778 }\end{array}$ & GW & $\begin{array}{l}\frac{13-.0 t-05}{13}-0.05 \\
13.05\end{array}$ & & FIELD & & $\begin{array}{l}\mathrm{D} \\
\mathrm{T}\end{array}$ & $\begin{array}{l}\text { PHYSICAL } \\
\text { PHSICAI }\end{array}$ & PH & $\begin{array}{l}\text { Measurement } \\
\text { Tomperatiue }\end{array}$ & 7.69 & & & & $\mathrm{pH}$ & & 152939.783 & 577944.937 \\
\hline A4636 & $\begin{array}{l}199-14-5 \\
199-H 4-5\end{array}$ & & $\begin{array}{l}\text { HEISPROD } \\
\text { HEISPROD }\end{array}$ & $\begin{array}{l}\text { RC-048 } \\
\text { RC-048 }\end{array}$ & $\begin{array}{ll}\text { J10778 } \\
\text { J10778 }\end{array}$ & GW & $\begin{array}{l}\text { 13-0.0t-105 } \\
\text { 13-0ct-05 }\end{array}$ & & $\begin{array}{l}\text { FEED } \\
\text { FIELD }\end{array}$ & & $\begin{array}{l}\text { TEMP FLD } \\
\text { TY FID }\end{array}$ & $\begin{array}{l}\text { PHYSICAL } \\
\text { PYYSICAI }\end{array}$ & TUE E & $\begin{array}{l}\text { Temperature } \\
\text { Teurite }\end{array}$ & $\frac{18.4}{218}$ & & & & $\begin{array}{ll}\text { DeggC } \\
\text { DNT }\end{array}$ & & $\begin{array}{l}152939.783 \\
1529373\end{array}$ & 577944.937 \\
\hline A4636 & 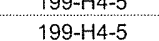 & & HESPROD & 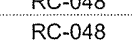 & J107rg & ${ }_{G W}$ & 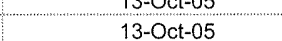 & & RLNP & & cMS & SVOA & (0) & $\begin{array}{l}\text { 4-vitroanilife } \\
\end{array}$ & $\frac{2.10}{25}$ & & & & wgol & & 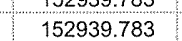 & 5 57799444.937 \\
\hline A4636 & $199-44-5$ & & HEISPROD & RC-048 & J107Y9 & GW & 13-0ct-05 & 1 & RLNP & K0046 & CMS & SVOA & $100-02-7$ & 4-Nitrophenol & 25 & & & & ugh & $u$ & 152939.783 & 5779444.937 \\
\hline A4636 & $199-44-5$ & & HEISPROD & RC-048 & J107Y9 & Gw & 13--oct-05 & 1 & EBRLNE & K0046 & $906.0 \mathrm{H}$ - 3 LSC & RAD & 10028-17-8 & Tritium & 2900 & 170 & 170 & 340 & pcil & & 152939.783 & 577944.937 \\
\hline $\begin{array}{ll}A 4636 \\
\text { A4663 }\end{array}$ & 199-H4-5 & & HEISPROD & RC-048 & 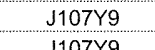 & GW & 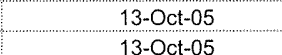 & 1 & EBRLNE & Ko046 & GAMMAGS & RAD & 10045-97-3 & Cesium-137 & & & 21 & & pcil & $u$ & 152939.783 & 5777944.937 \\
\hline $\begin{array}{l}\text { A46636 } \\
\text { A4636 }\end{array}$ & $\begin{array}{l}199-94-5 \\
199-44-5\end{array}$ & & $\begin{array}{l}\text { HEISPROD } \\
\text { HEISPROD }\end{array}$ & $\begin{array}{l}\text { RC-048 } \\
\text { RC-048 }\end{array}$ & $\begin{array}{l}\text { J10799 } \\
\text { J107999 }\end{array}$ & $G W$ & $\begin{array}{l}\text { 13-OCt-0.5 } \\
\text { 13-coct-05 }\end{array}$ & $\frac{1}{1}$ & $\begin{array}{l}\text { RLNP } \\
\text { EBRLNE }\end{array}$ & $\begin{array}{l}\text { K0044 } \\
\text { K0046 }\end{array}$ & $\begin{array}{l}\text { CMS } \\
\text { GAMMA GS }\end{array}$ & SVOA & $\frac{101-55-3}{1098-40-0}$ & $\begin{array}{l}\text { Bromophenylyh } \\
\text { Colabltro }\end{array}$ & 10 & & 20 & & ugh/ & $\frac{u}{u}$ & $\begin{array}{l}\frac{152939.783}{159293783} \\
150\end{array}$ & $\begin{array}{l}577944.937 \\
577937\end{array}$ \\
\hline $\begin{array}{l}A 43636 \\
A 4636\end{array}$ & $\begin{array}{l}199-94-5 \\
199-H 4-5\end{array}$ & & $\begin{array}{l}\text { HEISPROD } \\
\text { HEISPROD }\end{array}$ & $\begin{array}{l}\text { RC-048 } \\
\text { RC-048 }\end{array}$ & $\begin{array}{l}\text { J10779 } \\
\text { J10779 }\end{array}$ & GW & $\begin{array}{l}13-0.0-505 \\
\text { 13-0ct-05 }\end{array}$ & 1 & EBRLNE & $\begin{array}{l}\text { K00046 } \\
\text { K0046 }\end{array}$ & $\begin{array}{l}\text { GAMMA GS } \\
\text { C }\end{array}$ & $\begin{array}{l}\text { RAD } \\
\text { PEST/PCB }\end{array}$ & $1024-47-3$ & $\begin{array}{l}\text { Cobabltibo } \\
\text { epoxide }\end{array}$ & 0.05 & & & & $\begin{array}{l}\text { pCCin } \\
\text { ugli }\end{array}$ & u & $\begin{array}{l}1529339.983 \\
152939.783\end{array}$ & 5 5779944.939.937 \\
\hline $\begin{array}{l}A 46636 \\
A 4636\end{array}$ & 199-H4-34-5 & & HEISPROD & RC-048 & J107r9 & GW & 13-Oct-05 & 1 & RLNP & 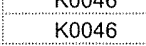 & $c$ & PESTIPCB & $1031-07-8$ & suxfate & 0.05 & & & & ugh & u & 152939.783 & 577944.937 \\
\hline A4636 & $199 \cdot-44-5$ & & HEISPROD & RC-048 & J10779 & Gw & 13-.0ct-05 & 1 & RLNP & K0046 & CMS & SVOA & $105-67-9$ & Dimethyphenol & 10 & & & & ug/h & u & 152939.783 & 577944.937 \\
\hline $\begin{array}{ll}A 4636 \\
A 4636\end{array}$ & $\begin{array}{l}199 \cdot-44-5 \\
199-94-5\end{array}$ & & $\begin{array}{l}\text { HEISPROD } \\
\text { HEIPROD }\end{array}$ & $\begin{array}{l}\text { RC-048 } \\
\text { RC-048 }\end{array}$ & $\begin{array}{l}\text { J107Y9 } \\
\text { J110799 }\end{array}$ & $\frac{\mathrm{GW}}{\mathrm{GW}}$ & $\begin{array}{l}\text { 13-oct-05 } \\
\text { 13-0ct-05 }\end{array}$ & 1 & RLNP & Ko046 & CMS & SVOA & $106-46-7$ & Dichlorobenzen & 10 & & & & ugh & $u$ & 152939.783 & 577944.937 \\
\hline $\begin{array}{l}\text { A46636 } \\
\text { A4636 }\end{array}$ & $199-44-5$ & & HEISPROD & 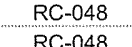 & $\begin{array}{ll}510799 \\
10779\end{array}$ & GW & 13-oct-05 & 1 & RLNP & Ko046 & CMS & SVOA & $106-47-8$ & 4-Chloroaniline & 10 & & & & ug/L & u & 152939.783 & 5779444.937 \\
\hline $\begin{array}{l}\text { A4636 } \\
\text { A4366 }\end{array}$ & $\begin{array}{l}199-14-5 \\
199-H 4-5\end{array}$ & & $\begin{array}{l}\text { HESPRROD } \\
\text { HESPROD }\end{array}$ & $\begin{array}{l}\text { RC-0.-048 } \\
\text { RC.048 }\end{array}$ & 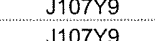 & $\frac{G N}{G W}$ & $\begin{array}{l}\text { 13-0.ct-to5 } \\
\text { 13--0ct-05 }\end{array}$ & 1 & 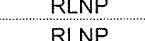 & $\begin{array}{l}K_{0.046} \\
K_{0.046}\end{array}$ & $\begin{array}{l}\text { Cins } \\
\text { cis }\end{array}$ & $\begin{array}{l}\text { SVOA } \\
\text { SVOA }\end{array}$ & $\begin{array}{l}1006-00-1 \\
10-85-2\end{array}$ & 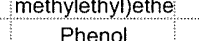 & $\frac{10}{10}$ & & & & 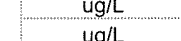 & u & 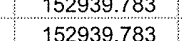 & $\begin{array}{l}577944.937 \\
577944.937\end{array}$ \\
\hline $\begin{array}{l}A 40636 \\
A 4636\end{array}$ & $199-\mathrm{H} 4 \mathrm{-5}-5$ & & HEISPROD & RC-048 & J107Y9 & $G W$ & 13-0ct-05 & 1 & RLNP & Koo46 & 8082_PCB_GC & PEST/PCB & $\frac{110006-82-5}{1109-5}$ & Aroctor-1260 & 0.4 & & & & ugh & $u$ & 152939.783 & 5779944.937 \\
\hline A4636 & $199-\mathrm{H} 4-5$ & & HEISPROD & RC-048 & J107Y9 & GW & 13-0ct-05 & 1 & RLNP & Ko046 & 8082_PCB_GC & PEST/PCB & $11097-69-1$ & Aroclor-1254 & 0.4 & & & & ugh & $u$ & 152939.783 & 577944.937 \\
\hline $\begin{array}{l}A 4636 \\
\text { A4663 }\end{array}$ & $\begin{array}{l}199 \cdot-44-5 \\
10004-5\end{array}$ & & HEISPROD & RC-048 & J107Y9 & GW & 13-OCt-05 & 1 & RLNP & Ko046 & CMS & SVOA & 111-44.4 & chloroethyl) & 10 & & & & ugh & u & 152939.783 & 577944.937 \\
\hline & $199-14-5$ & & HEISPROD & $\begin{aligned} R C-048 \\
\text { RC-048 }\end{aligned}$ & $\begin{array}{ll}107 \times 9 \\
110779\end{array}$ & GW & 13-0ct-05 & & RLNP & K0046 & $\begin{array}{c}\text { CMS } \\
8083 \text { CMC }\end{array}$ & $\begin{array}{l}\text { SVOA } \\
\text { PESTPCB }\end{array}$ & $\frac{111-91-1}{1104-28-2}$ & 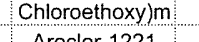 & 10 & & & & ugh & u & $\begin{array}{l}152939.783 \\
155030783\end{array}$ & 577944.937 \\
\hline $\begin{array}{ll}A 4636 \\
A 4636\end{array}$ & $\begin{array}{l}199-444-5 \\
109-44-5\end{array}$ & & $\begin{array}{l}\text { HEISPROD } \\
\text { HEISPROD }\end{array}$ & $\begin{array}{ll}R^{R C-048} \\
\text { RC.048 }\end{array}$ & $\begin{array}{l}5107 \times 9 \\
1107 \times 9\end{array}$ & GW & $\begin{array}{l}\text { 13-0ct-105 } \\
\text { 13-0ct-05 }\end{array}$ & $\frac{1}{1}$ & $\begin{array}{l}R I N P \\
R I N P\end{array}$ & $\begin{aligned} K 00046 \\
K 0046\end{aligned}$ & $\begin{array}{l}8082 \text { PCEGCG } \\
8082 \text { PCB GC }\end{array}$ & $\begin{array}{l}\text { PESTPCB } \\
\text { PEST/PCB }\end{array}$ & $\begin{array}{l}11104-28-2 \\
11141-16-5\end{array}$ & Aroctor-1221 & 0.4 & & & & $\begin{array}{l}\text { gugh } \\
\text { uglh }\end{array}$ & u & $\begin{array}{l}1529393.783 \\
15293.783\end{array}$ & $\begin{array}{l}577944.937 \\
5770937\end{array}$ \\
\hline $\begin{array}{ll}A 40630 \\
A 4636\end{array}$ & 199--H4-5-5 & & HEISPROD & $\begin{array}{l}\text { RL-048 } \\
\text { RC-048 }\end{array}$ & J107Y9 & GW & 13-.0ct-05 & & RLNP & Koo46 & CMS & SVOA & $117-81-7$ & ethylhexyl) & $\begin{array}{l}0.4 \\
10\end{array}$ & & & & ugh & u & 152939.783 & S577944.937 \\
\hline A4636 & $1999-44-5$ & & HEISPROD & RC-048 & J107Y9 & GW & 13-0ct-05 & 1 & RLNP & K0046 & CMS & SVOA & $177-84-0$ & octyphinhalate & 10 & & & & ugl & u & 152939.783 & 577944.937 \\
\hline A4636 & $199-44-5$ & & HEISPROD & RC-048 & J107Y9 & GW & 13-0ct-05 & 1 & RLNP & K0046 & CMS & SVOA & $118-74-1$ & ene & 10 & & & & ught. & $u$ & 1529399.783 & 577944.937 \\
\hline A4636 & $199-44-5$ & & HEISPROD & RC-048 & J107r9 & GW & 13-0ct-05 & 1 & RLNP & K0046 & CMS & SVOA & 120-122-7 & Anthracene & 10 & & & & ugll & u & 152939.783 & 577944.937. \\
\hline $\begin{array}{l}\text { A46636 } \\
\text { A4636 }\end{array}$ & $\begin{array}{l}199-14-5 \\
199-14-5\end{array}$ & & HEISPROD & RC-048 & $\begin{array}{ll}110799 \\
10779\end{array}$ & sw & $\begin{array}{l}\text { 13-coct-05 } \\
\text { 13-0c-05 }\end{array}$ & $\frac{1}{1}$ & RLNP & K0046 & CMS & SVOA & $120-82-1$ & Trichlorobenzzen. & 10 & & & & uglt & $u$ & 152939.783 & 577944.937 \\
\hline A46360 & (199--H4-5 & & HEISPROD & 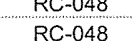 & 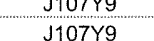 & $G W$ & 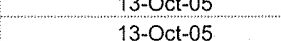 & & $\begin{array}{l}\text { RLNP } \\
\text { RINP }\end{array}$ & K0046 & CMS & $\begin{array}{l}\text { SOOA } \\
\text { SONA }\end{array}$ & $\begin{array}{l}120-83-2 \\
102-2\end{array}$ & Dichlorophenenol & 10 & & & & ugh & & $\begin{array}{l}152939.9783 \\
51520302\end{array}$ & 5779944.937 \\
\hline A4636 & $199--44-5$ & & HEISPROD & 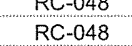 & J107r9 & GW & 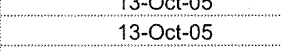 & & RLNP & K0046 & 8082 PCB GC & PESTPCB & $12677-2.6$ & A & 104 & & & & 年 & u & 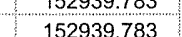 & 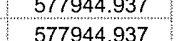 \\
\hline A4636 & 199-H4-5 & & HESPROD & RC-048 & J107r9 & GW & 13-Oct-05 & & RLNP & K0046 & 8082 PCB GC & PEST/PCB & $12674-11-2$ & Aroclor-1016 & 0.4 & & & & ugh & 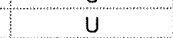 & 152939.783 & $\begin{array}{l}57 / 9494.937 \\
577944.937\end{array}$ \\
\hline A46366 & $199-44-5$ & & HEISPROD & RC-048 & J107Y9 & GW & 13--0ct-05 & & RLNP & K0046 & CMS & SVOA & $129-00-0$ & Pyrene & 10 & & & & ugh & & 152939.783 & 577944.937 \\
\hline $\begin{array}{l}\text { A46636 } \\
\text { A4636 }\end{array}$ & $199-44-5$ & & HEISPROD & RC-048 & J10779 & ow & 13-0ct-05 & & RLNP & K0046 & CMS & SVOA & $131-11-3$ & phthalate & 10 & & & & ught & $J$ & 152939.783 & 5777944.937 \\
\hline $\begin{array}{l}\text { A4636 } \\
A 4636\end{array}$ & $\begin{array}{l}199-144.5 \\
1094-5\end{array}$ & & $\begin{array}{l}\text { HESSRPOD } \\
\text { HESPROD }\end{array}$ & 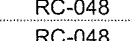 & $\begin{array}{lll}510779 \\
10779\end{array}$ & $\frac{G W}{G W}$ & 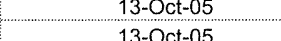 & & $\begin{array}{l}\text { RRNAN } \\
\text { ERPINE }\end{array}$ & $\begin{array}{l}K_{0.046} \\
K 0006\end{array}$ & $\begin{array}{l}\text { CMS } \\
\text { GAMA GS }\end{array}$ & $\begin{array}{l}\text { SVAA } \\
\text { BaD }\end{array}$ & $\begin{array}{l}132-24.9 \\
1396-0.2\end{array}$ & 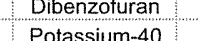 & 10 & & 200 & & $\begin{array}{l}\text { ugh } \\
\text { ocin }\end{array}$ & 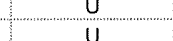 & $\begin{array}{l}152939.983 \\
15293983\end{array}$ & 577944.937 \\
\hline A4636 & 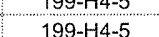 & & HESROD & RC-0.08 & J1079 & $G \mathrm{G}$ & 13--0ct-05 & & EBRLNE & K0046 & GAMMA GS & RAD & 年 & Berllum-7 & & & $\frac{960}{160}$ & & (C) & Jx & 15293939.683 & $\begin{array}{l}577944.931 \\
5794937\end{array}$ \\
\hline A4636 & $199-14-5$ & & HEISPROD & RC-048 & J107Y9 & GW & 13-0ct-05 & & EBRLNE & K0046 & GAMMA_GS & RAD & 13967-48-1 & Ruthenium-106 & & & 140 & & pCilh & u & 1522399.783 & 577944.937 \\
\hline A4636 & $199-H 4-5$ & & HEISPROD & RC-048 & J107r9 & GW & 13-0ct-15 & 1 & $\begin{array}{l}\text { EBRLNE } \\
\end{array}$ & Ko046 & GAMMA_GS & RAD & $13967-70-9$ & & & & 23 & & pcill & u & 1522939.783 & 577944.937 \\
\hline A4636 & $199 \cdot-44-5$ & & HEISPROD & RC-048 & J107Y9 & GW & 13-Oct-05 & 1 & $\begin{array}{l}\text { EBRLNE } \\
\text { EPRDNEF }\end{array}$ & K0046 & UC & RAD & $13982-63-3$ & Radium-226 & 0.818 & 1.1 & 1.9 & 1.1 & pcilh & u & 152939.783 & 5779944.937 \\
\hline $\begin{array}{l}\text { A4636 } \\
\text { A4636. }\end{array}$ & $\frac{199-14-5}{199-H 4-5}$ & & $\begin{array}{l}\text { HEISPROD } \\
\text { HEISPROD }\end{array}$ & $\begin{array}{l}\text { RC-048 } \\
\text { RC-048 }\end{array}$ & $\begin{array}{l}\text { J107Y9 } \\
\text { J107Y9 }\end{array}$ & $\frac{G W}{G W}$ & $\begin{array}{l}13-\text { oct-05 } \\
\text { 13-0ct-05 }\end{array}$ & & $\begin{array}{l}\text { EBRRLE } \\
\text { EBRLNE }\end{array}$ & $\begin{array}{l}\text { K00046 } \\
\text { K0046 }\end{array}$ & $\begin{array}{l}\text { GAMMA GS } \\
\text { GAMMA GS }\end{array}$ & $\begin{array}{l}\text { RAD } \\
\text { RAD }\end{array}$ & $\begin{array}{l}\frac{13982-633-3}{1434-35-6} \\
14\end{array}$ & Radium-226 & & & 33 & & pCin & & $\begin{array}{l}152939.783 \\
1502029783\end{array}$ & 577944.937 \\
\hline $\begin{array}{ll}A 466636 \\
A 4636\end{array}$ & $\frac{199-94-5}{199-14-5}$ & & $\begin{array}{l}\text { HEISPROD } \\
\text { HEISPROD }\end{array}$ & $\begin{array}{l}\text { RC-048 } \\
\text { RC-048 }\end{array}$ & $\begin{array}{l}\text { Jitory9 } \\
\text { jory }\end{array}$ & GW & 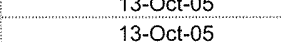 & & $\begin{array}{l}\text { EBRLNE } \\
\text { RLNP }\end{array}$ & K0046 & GAMMACGS & $\begin{array}{l}\text { RAD } \\
\text { GENCHEM }\end{array}$ & $\begin{array}{l}142344-355-6 \\
14265-44-2\end{array}$ & $\begin{array}{l}\text { Antimonyy-1255 } \\
\text { Phosphate }\end{array}$ & & & 40 & & pcine & 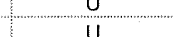 & 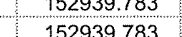 & $\begin{array}{l}577944.937 \\
5770937\end{array}$ \\
\hline A4636 & $199-H 4-5$ & & HEISPROD & $\begin{array}{l}\text { RC-048 } \\
\text { RC- } 048\end{array}$ & J107r9 & GW & 13--oct-05 & 1 & EBRLNE & Koout6 & TE AEA & $\begin{array}{l}\text { GENAD } \\
\text { RAD }\end{array}$ & $\begin{array}{l}142659-4-63-2-7 \\
1469\end{array}$ & $\begin{array}{l}\text { Thonosphater } \\
\text { The }\end{array}$ & 0.065 & 0.13 & 0.25 & 0.13 & pcil & $u$ & 152939.783 & 年 \\
\hline A4636 & $199--H 4-5$ & & HEISPROD & RC-048 & J107Y9 & GW & 13-0ct-05 & & EBRLNE & Ko046 & GAMMA_GS & RAD & $14274-82-9$ & Thorium-228 & & & 20 & & pcil & $u$ & 152939.783 & 577944.937 \\
\hline A4636 & $199-H 4-5$ & & HEISPROD & RC-048 & $\begin{array}{l}110779 \\
107700\end{array}$ & GW & 13-oct-05 & & EBRLNE & K0046 & TEEAEA & RAD & $14274-82-9$ & Thorium-228 & 0.066 & 0.066 & 0.25 & 0.066 & pcint & $u$ & 1529399.783 & 5777944.937 \\
\hline A4636 & $199-H 4-5$ & & HEISPROD & RC-048 & J107Y9 & GW & 13-Oct-05 & & EBRLNE & K0046 & GAMMA GS & RAD & $14391-16-3$ & Europium-155 & & & 45 & & pcin & $u$ & 152939.783 & 5779944.937 \\
\hline A4636 & $\begin{array}{l}199-144-5 \\
190+044-5\end{array}$ & & HEISPROD & $\begin{array}{l}\text { RC- }-048 \\
\text { Rc }\end{array}$ & $\begin{array}{l}\text { J10779 } \\
\text { J10799 }\end{array}$ & GW & 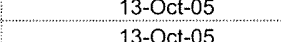 & & 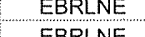 & K00046 & GAMMA GS & RAD & $145566-10-2$ & Americium-241 & & & 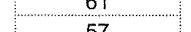 & & pelin & $u$ & $\begin{array}{l}1592939.733 \\
1520273\end{array}$ & 577994.937 \\
\hline 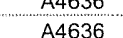 & $\begin{array}{l}199-74-5 \\
199-H 4-5\end{array}$ & & $\begin{array}{l}\text { HEISPROD } \\
\text { HEISPROD }\end{array}$ & $\begin{array}{l}\text { RC-048 } \\
\text { RC-048 }\end{array}$ & J10779 & GW & $\begin{array}{l}\text { 13-0ct-1-15 } \\
\text { 13-Oct-05 }\end{array}$ & 10 & $\begin{array}{l}\text { EBRLNE } \\
\text { RINP }\end{array}$ & Ko04646 & GAMMA_GS & GEACHEM & $\begin{array}{l}14683-23-9 \\
1479-55-8\end{array}$ & Europiumatis2 & 65.4 & & 57 & & pcin & u & 15293939.983 & 5779944.937 \\
\hline A4636 & $199 \cdot-H 4-5$ & & & RC-048 & J10779 & GW & 13-oct-05 & & RLNP & K0046 & $\frac{\pi}{16}$ & GENCHEM & 147797-65-0 & Nitrale & 0.25 & & & & $m g h$ & 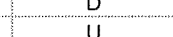 & 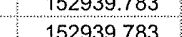 & 577944.937 \\
\hline A4636 & $199 \cdot-H 4-5$ & & HEISPROD & RC-048 & J107Y9 & $\mathrm{GW}$ & 13.-cct-05 & 10 & RLNP & Ko046 & 16 & GENCHEM & $14808-79-8$ & Sulfate & 66 & & & & moll & D & 1529399783 & 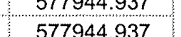 \\
\hline A4636 & $199-14-5$ & & HEISPROD & RC-048 & J107Y9 & GW & 13-oct-05 & 1 & EBRLNE & Koo46 & GAMMA GS & RAD & $15117-96-1$ & Uranium-235 & & & 63 & & pcil & u & $\frac{152939.783}{15293}$ & 577744.957 .937 \\
\hline A4636 & $199-44-5$ & & HEISPROD & RC-048 & J107Y9 & GW & 13-Oct-05 & & EBRLNE & K0046 & AEA & RAD & $15117-96-1$ & Uranium-235 & 0.185 & 0.15 & 0.28 & 0.15 & pCil & $u$ & 152939.783 & 577944.937 \\
\hline $\begin{array}{l}A 4636 \\
A 4636\end{array}$ & $199-44-5$ & & HEISPROD & RC-048 & J107Y9 & GW & 13-oct-05 & & EBRLNE & K0046 & GAMMA GS & RAD & $\begin{array}{l}15262-20-1 \\
15252-20-1\end{array}$ & Radium-228 & 0 & (2) & & 07 & pcint & u & 152939.783 & 577944.937 \\
\hline & $\begin{array}{l}199-744-5 \\
19094-5\end{array}$ & & $\begin{array}{l}\text { HEESPRDD } \\
\text { HESPRPOD }\end{array}$ & 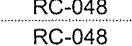 & $\begin{array}{l}510799 \\
10799\end{array}$ & $G W$ & 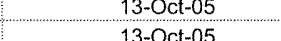 & $?$ & $\begin{array}{l}\text { EBBLLNE } \\
\text { ERBPINE }\end{array}$ & $\begin{array}{l}\text { K00046 } \\
\text { K0046 }\end{array}$ & DGAC & RAD & 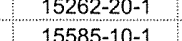 & 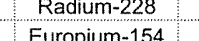 & -0.185 & 0.13 & 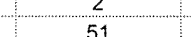 & 0.13 & pCili & un & 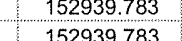 & 577944.937 \\
\hline A4636 & 199-74-5 & & HESTSROD & RC-048 & (1079 & GW & 13-0ct-05 & 10 & REN & Konots & 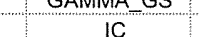 & GECAEM & 16858-00-16 & 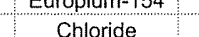 & 248 & & o1 & & puli & D & 1593939.9383 & 577944.937 \\
\hline A4636 & $199-44-5$ & & HEISPROD & RC- 048 & J107Y9 & GW & 13-oct-05 & 1 & RLNP & K0046 & $\frac{\pi}{16}$ & GENCHEM & $16984-48-8$ & Fluoride & 0.25 & & & & mglh & u & 152939.783 & 577944.937 \\
\hline A4636 & $199-14-5$ & & HEISPROD & RC-048 & J107Y9 & GW & 13-Oct-05 & & RLNP & Ko046 & CMS & SVOA & $191-24-2$ & ene & 10 & & & & ugh & $u$ & 152939.783 & 577944.937 \\
\hline A4636 & $199-44-5$ & & HEISPROD & RC-048 & J107Y9 & GW & 13-Oct-05 & & $\begin{array}{l}\text { RLNP } \\
\text { RINP }\end{array}$ & K0046 & CMS & SVOA & $\begin{array}{l}193-39-5 \\
20-590 .-2\end{array}$ & $\begin{array}{c}\text { cdlpyrene } \\
\text { thene }\end{array}$ & 10 & & & & ugh & $u$ & $\begin{array}{l}152939.783 \\
152020702\end{array}$ & 577944.937 \\
\hline A46636 & $\begin{array}{l}199-744-5 \\
1999-5\end{array}$ & & & $\begin{array}{l}\text { RC- }-048 \\
\text { RC-048 }\end{array}$ & $\begin{array}{l}.10799 \\
\text { J10799 }\end{array}$ & $G W$ & 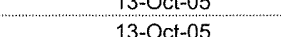 & & $\frac{R \text { RLNP }}{\text { RLNP }}$ & & $\frac{C M S}{\text { CMS }}$ & SVOA & $2025-9-9-2$ & $\begin{array}{l}\text { thene } \\
\text { Fluoranthen }\end{array}$ & 10 & & & & ogn & 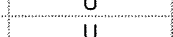 & $\begin{array}{l}152939.783 \\
515203978383\end{array}$ & 577944.937 \\
\hline A6436 & 1995-94-5.-5 & & HESTSROD & RC-068 & J1079 & $G w$ & 13-0.0-505 & & RLN & K0046 & CMS & $\begin{array}{l}\text { SSVAA } \\
\text { SSOA }\end{array}$ & $200-7408-9$ & 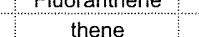 & 10 & & & & vol & 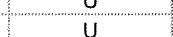 & 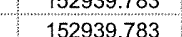 & $\begin{array}{r}57944.937 \\
57794.937\end{array}$ \\
\hline A4636 & $199-44-5$ & & HEISPROD & RC-048 & J107799 & GW & 13-0ct-05 & & RLNP & K0046 & CMS & SVOA & $208-96-8$ & Acenaphithyenen & 10 & & & & oglh. & $\bar{u}$ & 152939.783 & 577944.937 \\
\hline A4636 & $199-H 4-5$ & & HEISPROD & RC-048 & J107r9 & GW & 13--Oct-05 & & RLNP & K0046 & CMS & SVOA & $218-01-9$ & Chrysene & 10 & & & & ugh & $u$ & 1529399.783 & 577944.937 \\
\hline A4636 & $199-H 4-5$ & & HEISPROD & $\begin{aligned} \text { RC-048 } \\
\text { RC-018 }\end{aligned}$ & $\begin{array}{l}5107 Y 9 \\
107799\end{array}$ & GW & 13-Oct-05 & & RLNP & K0046 & 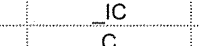 & GENCHEM & $24959-67-9$ & Bromide & 0.25 & & & & mglh & & $\begin{array}{l}152939.783 \\
16020270302\end{array}$ & 577944.937 \\
\hline & $195-745$ & & HESTSROD & 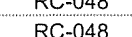 & $\begin{array}{l}510799 \\
\text { J10779 }\end{array}$ & $G w$ & 13-0.0-1050 & & RLN & Kove46 & $c_{c}$ & $\begin{array}{l}\text { PESTIPCB } \\
\text { PESTPC }\end{array}$ & $\begin{array}{l}309-00-2 \\
39-84.6\end{array}$ & $\begin{array}{l}\text { Aldrin } \\
\text { Alloba-BHC }\end{array}$ & - & & & & ogre & 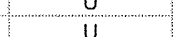 & 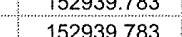 & 577944.9377 \\
\hline A4636 & $195-14-5$ & & HESPRPOD & RC-048 & (1079 & GW & 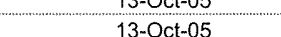 & & RLNP & K0046 & $c_{c}$ & PESPCB & $\begin{array}{l}313-9-6-5 \\
319-5\end{array}$ & 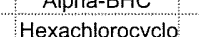 & - 0.05 & & & & . & 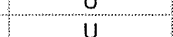 & 1593939.78 & $\begin{array}{l}579794.937 \\
5579493\end{array}$ \\
\hline A4636 & $199 \cdot-44-5$ & & HEISPROD & RC-048 & J107r9 & $\mathrm{GW}$ & 13-.0ct-05 & & RLNP & K0046 & $c_{c}$ & PEST/PCB & $319-86-8$ & Delta-BHC & 0.05 & & & & gigh & u & 152939.783 & 577944.937 \\
\hline A4636 & $199 \cdot-\mathrm{H} 4-5$ & & HEISPROD & RC-048 & J107799 & GW & 13-Oct-05 & & RLNP & K0046 & $c$ & PESTIPCB & 33213-55-9 & Endosulfan II & 0.05 & & & & uglh & $u$ & 152939.783 & 577944.937 \\
\hline A4636 & $199 \cdot-44-5$ & & HEISPROD & $\begin{array}{l}\text { RC- }-048 \\
\text { Re }\end{array}$ & J10779 & $\mathrm{GW}$ & 13-0ct-105 & & RINP & K00046 & chescis & PESTPCB & $50-29-3$ & litichloroethane & 0.05 & & & & ugh & $u$ & 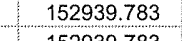 & 577944.937 \\
\hline 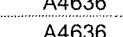 & $\begin{array}{l}199-74-5 \\
190.44-5\end{array}$ & & HEISPROD & RC-048 & 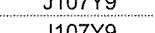 & $G N$ & 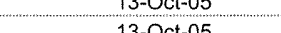 & & RLN & 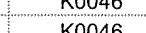 & CNS & & 50 & 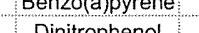 & 10 & & & & ugh & 0 & 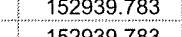 & 577944.937 \\
\hline A4636 & $199-74-5$ & & HISTSROD & RC- & (10707 & GW & 13-0ct-105 & & RLNP & K0046 & No & PEST & 5103-1-1-9 & Chlolordanto & 20 & & & & ught & u & 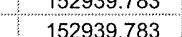 & 577944.937 \\
\hline A4636 & $199-44-5$ & & HEISPROD & RC-048 & J107r9 & GW & 13-0ct-05 & & RLNP & K0046 & $c$ & PEST/PCB & $5103774-2$ & Chlordane & 0.05 & & & & ugh & u & 1522939.783 & 577944.937 \\
\hline A4636 & $199-H 4-5$ & & HEISPROD & RC-0 & J107Y9 & GW & 13-.0ct-05 & & RLNP & Ko046 & CMS & DA & $53-70-3$ & racene & 10 & & & & igh & $u$ & 1522939.783 & 577944.937 \\
\hline $\begin{array}{l}A 4636 \\
A 4636\end{array}$ & $\begin{array}{l}199-H 4-5 \\
199-H 4-5\end{array}$ & & $\begin{array}{l}\text { HEISPROD } \\
\text { HISPRODD }\end{array}$ & $\begin{array}{l}\text { RC-048 } \\
\text { RC-048 }\end{array}$ & $\begin{array}{l}\text { J10799 } \\
\text { J10799 }\end{array}$ & $\begin{array}{c}G W \\
G W\end{array}$ & $\begin{array}{l}13 \text { 13-0ct-1-05 } \\
\text { 13-0c-05 }\end{array}$ & & $\begin{array}{l}\text { RLLP } \\
\text { RLNP }\end{array}$ & $\begin{array}{l}\text { K00046 } \\
\text { K0046 }\end{array}$ & $\begin{array}{c}\text { CMS } \\
8082 \text { PCB C }\end{array}$ & $\begin{array}{l}\text { SVAA } \\
\text { PESTPPCB }\end{array}$ & $\begin{array}{l}534-52-1 \\
53499-21-9\end{array}$ & 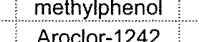 & $\begin{array}{l}25 \\
0.4 \\
0.4\end{array}$ & & & & $g / L$ & $\frac{u}{u}$ & $\begin{array}{l}152939.783 \\
5520237983\end{array}$ & 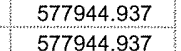 \\
\hline $\begin{array}{l}A 4636 \\
A 4636\end{array}$ & $\frac{199-H 4-5}{199-H 4-5}$ & & HEISPROD & $\begin{array}{l}\text { RC- } \\
\text { RC-048 }\end{array}$ & J107ry & GW & 13-OCct-05 & & RLNP & K0040 & & PEST/PCB & $\begin{array}{l}534994-1-9-9 \\
5340-5\end{array}$ & 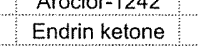 & 0.4 & & & & $\frac{u g h}{\text { ugh }}$ & $u$ & $\begin{array}{l}1529399.1835 \\
15293.783\end{array}$ & 5779444.937 \\
\hline
\end{tabular}



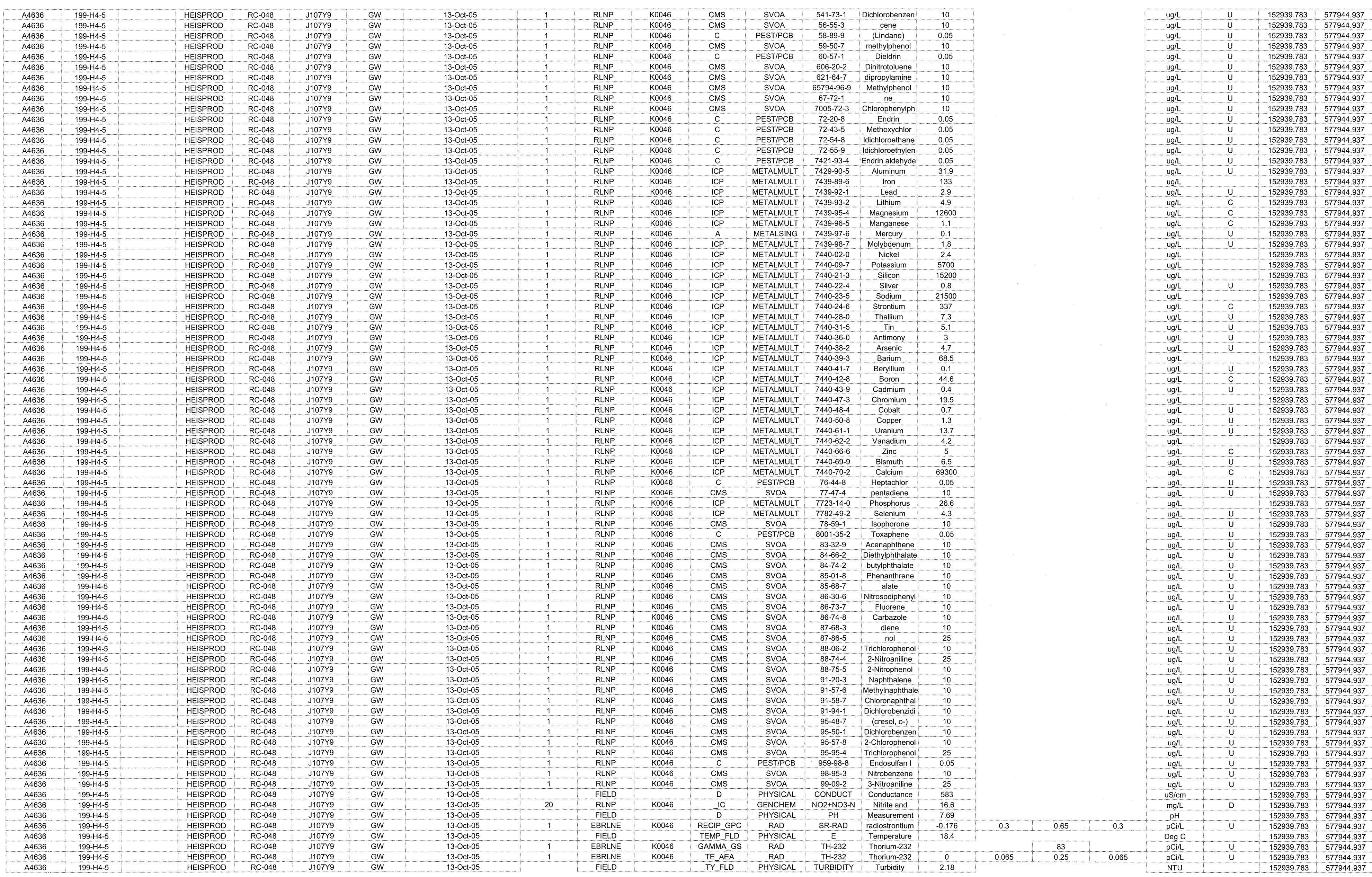


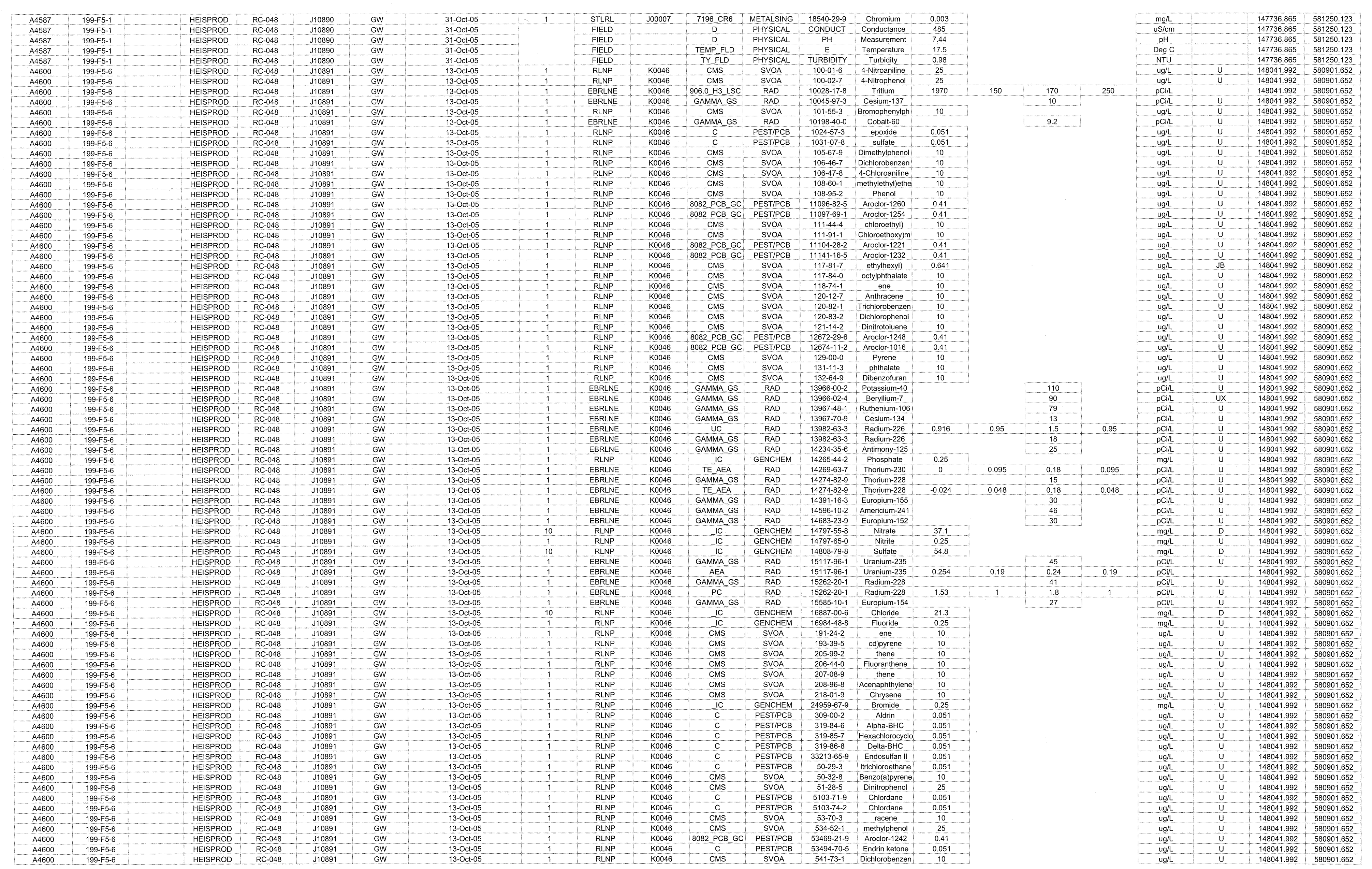




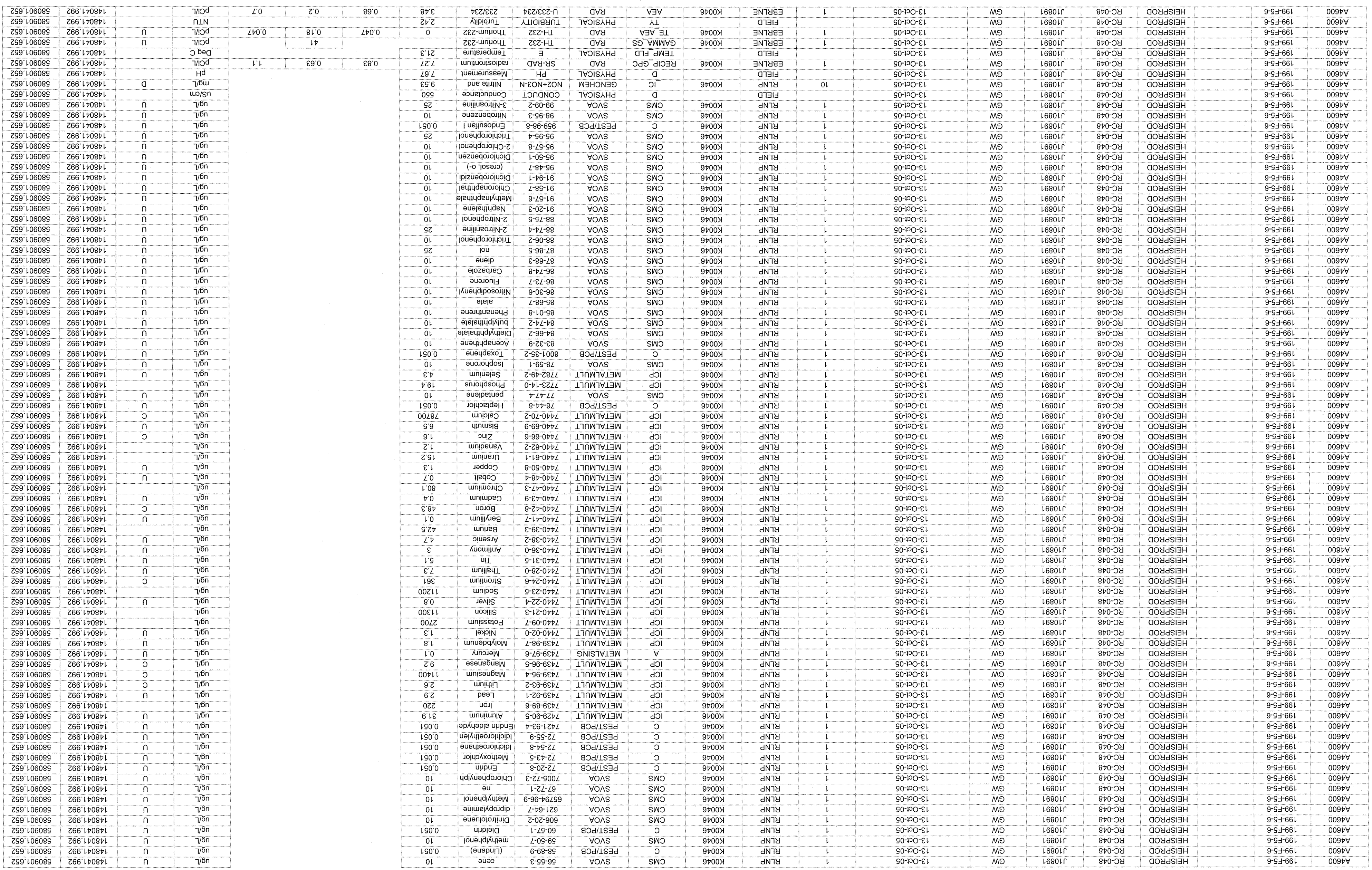




\begin{tabular}{|c|c|c|c|c|c|c|c|c|c|c|c|c|c|c|c|c|c|c|c|c|c|}
\hline A4600 & $199-55-6$ & HEISPROD & RC-048 & J10891 & GW & 13-0ct-05 & 1 & EBRLNE & K0046 & GAMMA_GS & RAD & U-238 & Uranium-238 & & & 1100 & & pcill & $u$ & 148041.992 & 580901.652 \\
\hline $\begin{array}{l}\text { A46600 } \\
\text { A4600 }\end{array}$ & $\begin{array}{l}199-5-5-6 \\
199-95-6\end{array}$ & HEISPROD & RC-048 & J108991 & $G W$ & 13-0ct-15 & 1 & $\begin{array}{l}\text { EBRLNE } \\
\text { STRPI }\end{array}$ & K0046 & $\begin{array}{l}\text { AEA } \\
\text { A196 }\end{array}$ & RAD & U-238 & Uranium-238 & 3.54 & 0.69 & 0.2 & 0.71 & pcil & & 148041.992 & 5809001.652 \\
\hline $\begin{array}{l}A 46000 \\
A 4600\end{array}$ & 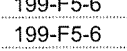 & $\begin{array}{l}\text { HESPRROD } \\
\text { HESPROD }\end{array}$ & 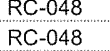 & $\begin{array}{l}\mathrm{J} 108922 \\
\mathrm{~J} 10892\end{array}$ & $\frac{G W}{G W}$ & $\begin{array}{l}\text { 13-OCt-0.5 } \\
\text { 13-coct-55 }\end{array}$ & & $\begin{array}{l}\text { STLRL } \\
\text { FIELD }\end{array}$ & J00002 & 7196 CR6 & METALSING & $\begin{array}{l}18540-29-9 \\
\text { CONDUCT }\end{array}$ & $\begin{array}{l}\text { Chromium } \\
\text { Conductance }\end{array}$ & $\frac{0.0825}{550}$ & & & & $\begin{array}{l}\text { mgll } \\
\text { ussm }\end{array}$ & & $\begin{array}{l}148041.992 \\
148041.92\end{array}$ & $\begin{array}{l}580901.652 \\
5880001652\end{array}$ \\
\hline A4600 & $199-55-6$ & HEISPROD & RC-048 & J10892 & GW & 13-OCc1-05 & & FIELD & & $\begin{array}{l}\text { D } \\
\text { D }\end{array}$ & $\begin{array}{l}\text { PHYSSICAL } \\
\text { PHYICAL }\end{array}$ & $\begin{array}{l}\text { PH } \\
\text { PH }\end{array}$ & $\begin{array}{c}\text { Connouctance } \\
\text { Measuremen }\end{array}$ & $\frac{50}{767}$ & & & & $\frac{\mathrm{wscm}}{\mathrm{vor}}$ & & 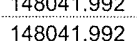 & 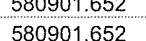 \\
\hline A4600 & $199-F 5-6$ & HEISPROD & RC-048 & J10892 & GW & 13-oct-05 & & FIELD & & TEMP FLD & $\begin{array}{l}\text { PHYSSICAL } \\
\text { PHYICAL }\end{array}$ & E & $\begin{array}{l}\text { Temperature } \\
\text { Temperate }\end{array}$ & $\frac{1.6}{21.3}$ & & & & $\begin{array}{c}\text { pH } \\
\text { Deg C }\end{array}$ & & 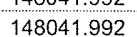 & $\begin{array}{l}5890001.1652 \\
580901.652\end{array}$ \\
\hline$A 4600$ & $199-F 5-6$ & HEISPROD & RC-048 & J10892 & GW & 13-Oct-05 & & FIELD & & TY & PHYSICAL & TURBIDITY & Turbidity & 2.42 & & & & NTU & & 148041.992 & 580901.652 \\
\hline A4650 & $199-k-21$ & HEISPROD & RC-048 & 110000 & GW & 24-Oct-15 & 1 & STLRL & J00003 & 7196 CR6 & METALSING & $18540-29-9$ & Chromium & 0.013 & & & & moll & & 147932.06 & 569769.902 \\
\hline A4650 & $\begin{array}{l}199-\mathrm{K}-21 \\
199-\mathrm{K}-21\end{array}$ & HEISPROD & RC-048 & J100008 & GW & 24-Oct-15 & & $\begin{array}{l}\text { FIELD } \\
\text { FIEIDD }\end{array}$ & & D & PHYSICAL & CONDUCT & $\begin{array}{l}\text { Conductance } \\
\text { opyen }\end{array}$ & $\begin{array}{l}361 \\
1079\end{array}$ & & & & uslcm & & $\begin{array}{l}147932.06 \\
1772023006\end{array}$ & 569769.902 \\
\hline $\begin{array}{l}\text { A44560 } \\
\text { A4650 }\end{array}$ & $\frac{199-k-21}{199-k-21}$ & HEISPROD & $\frac{R C-048}{R C-048}$ & $\begin{array}{l}\text { J10D008 } \\
10008\end{array}$ & $\frac{G W}{G W}$ & $\begin{array}{l}\text { 24-COC-1-55 } \\
\text { 24-Cct-15 }\end{array}$ & & $\begin{array}{l}\text { FIELD } \\
\text { FIELLD }\end{array}$ & & $\begin{array}{l}\text { FLD } \\
E_{F F L D}\end{array}$ & GENCHEM & $\begin{array}{l}\text { DO } \\
E H\end{array}$ & $\begin{array}{l}\text { oxygen } \\
\text { Reduction }\end{array}$ & $\begin{array}{l}10.79 \\
107\end{array}$ & & & & 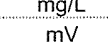 & & $\begin{array}{l}1449922.06 \\
19773206\end{array}$ & 569769.902 \\
\hline A4650 & $\begin{array}{l}1999-k-21 \\
199-k-21\end{array}$ & $\begin{aligned} \text { HESPROD D } \\
\text { HEISPROD }\end{aligned}$ & 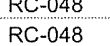 & $\begin{array}{l}100008 \\
10008\end{array}$ & $\frac{G W}{G W}$ & $\begin{array}{l}\text { 24-Coct-155 } \\
\text { 24-Cct-05 }\end{array}$ & & $\begin{array}{l}\text { FIELD } \\
\text { FIELLD }\end{array}$ & & D & PENCHEM & $\begin{array}{l}\mathrm{EH} \\
\mathrm{PH}\end{array}$ & $\begin{array}{l}\text { Keacuction } \\
\text { Measurement }\end{array}$ & 8.16 & & & & $\mathrm{mV}$ & & $\begin{array}{l}144992.00 \\
147932.06\end{array}$ & 5697699902 \\
\hline A4650 & $199-k-k-21$ & & RC-048 & J10008 & GW & 24-0ccl-05 & & FIELD & & TEMPFFLD & PHYSICAL & $E$ & Temperature & 18.1 & & & & Deg C & & 147932.06 & 5697699.902 \\
\hline A4650 & $199-k-21$ & HEISPROD & RC-048 & J10008 & GW & 24-Oct-05 & & FIELD & & D & PHYSICAL & TURBIDITY & Turbidity & 3.78 & & & & NTU & & 147932.06 & 569769.902 \\
\hline A4650 & $199-k-21$ & HEISPROD & RC-048 & J10009 & GW & 24-Oct-05 & 1 & STLRL & J00003 & 7196 CR6 & METALIING & 18540-29-9 & Chromium & 0.014 & & & & mol & & 1477932.06 & 569769.902 \\
\hline $\begin{array}{l}A 4660 \\
A 4650\end{array}$ & $\frac{199-k-21}{199-k-21}$ & HEISPROD & $\begin{array}{l}\text { RC-048 } \\
\text { RC-048 }\end{array}$ & $\begin{array}{l}\text { J10D009 } \\
\text { J10009 }\end{array}$ & $\frac{G W}{G W}$ & 24-COt-155 & & $\begin{array}{l}\text { FIIEDD } \\
\text { FIELLD }\end{array}$ & & $\begin{array}{l}\text { D } \\
\text { FLD }\end{array}$ & $\begin{array}{l}\text { PHYSIICAL } \\
\text { GENCHEM }\end{array}$ & CONDUCT & $\begin{array}{c}\text { Conductance } \\
\text { oxyyen }\end{array}$ & $\begin{array}{c}361 \\
10.79\end{array}$ & & & & $\begin{array}{l}\text { uslcm } \\
\text { moll }\end{array}$ & & $\begin{array}{l}1479922.06 \\
177932006\end{array}$ & 5697699.902 \\
\hline $\begin{array}{l}A 4650 \\
A 4650\end{array}$ & $\begin{array}{l}\frac{199-k-21}{199-K-21} \\
1\end{array}$ & $\begin{aligned} \text { HESPROOD } \\
\text { HEISPROD }\end{aligned}$ & 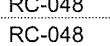 & $\begin{array}{l}J 10000909 \\
J 10009\end{array}$ & GW & 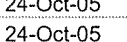 & & $\begin{array}{l}\text { FIELU } \\
\text { FIELD }\end{array}$ & & $\begin{array}{l}F L D \\
E F L D \\
E F\end{array}$ & GENCHEM & EH & $\begin{array}{l}\text { oxygen } \\
\text { Reduction }\end{array}$ & $\begin{array}{l}0.19 \\
0.19\end{array}$ & & & & mV & & $\begin{array}{l}144952.06 \\
147932.06\end{array}$ & 569769.902 \\
\hline A4650 & $199-k-21$ & HEISPROD & RC-048 & J10009 & $\mathrm{GW}$ & 24-0ct-05 & & FIELD & & & PHYSICAL & PH & Measurement & 8.16 & & & & $\mathrm{pH}$ & & 147932.06 & 569769.902 \\
\hline A4650 & $199-\mathrm{K}-21$ & HEISPROD & RC-048 & 5100009 & GW & 24-0ct-105 & & FIELD & & TEMP,FLD & PHYSICAL & E & Temperature & 18.1 & & & & $\operatorname{Deg} C$ & & 147932.06 & 569769.902 \\
\hline A4650 & $199-\mathrm{K}-21$ & HEISPROD & RC-048 & J100009 & GW & 24-0ct-05 & & FIELD & (50) & D & PHYSICAL & TURBIDITY & Turbidity & 3.78 & & & & NTU & & 147932.06 & 569769.902 \\
\hline $\begin{array}{l}\text { A4650 } \\
\text { A4560 }\end{array}$ & $199-K-21$ & HEISPROD & RC-048 & J10010 & GW & 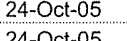 & & 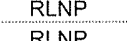 & 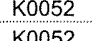 & $\begin{array}{l}\text { CMS } \\
\text { CMS }\end{array}$ & $\begin{array}{l}\text { SVOA } \\
\text { SVOA }\end{array}$ & $\begin{array}{l}100-01-6 \\
10027\end{array}$ & 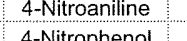 & $\begin{array}{l}25 \\
25\end{array}$ & & & & ugh & u & $\begin{array}{l}147932.06 \\
1140306\end{array}$ & 569769.902 \\
\hline $\begin{array}{l}A 4500 \\
A^{44650}\end{array}$ & 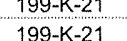 & $\begin{array}{l}\text { HeISPROD } \\
\text { HESPROD }\end{array}$ & 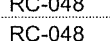 & S10D10 & GW & 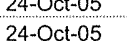 & & EBRINE & $\frac{10052}{k 0052}$ & $906.0 \mathrm{HB}$ L LSC & SAD & $\frac{1000-25-7}{1002-18}$ & 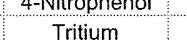 & ${ }_{274}^{274}$ & 110 & 170 & 110 & 然 & & $\begin{array}{l}147532.06 \\
1.49306\end{array}$ & $\begin{array}{l}5697699.902 \\
55976902\end{array}$ \\
\hline A4650 & $199-k-21$ & HEISPROD & RC-048 & J10D10 & $G W$ & 24-0ct-05 & & EBRLNE & Koos2 & GAMMA_GS & RAD & $10045-97-3$ & Cesium-137 & & & 21 & & pcil & u & 147932.06 & $\begin{array}{l}56996999.902 \\
569769.902\end{array}$ \\
\hline A4650 & $199-k-21$ & HEISPROD & RC-048 & $110 D 10$ & $G W$ & 24-0ct-05 & & RLNP & K0052 & cMS & SVOA & $101-55-3$ & Bromophenyph & 10 & & & & ugh & u & 147932.06 & 569769.902 \\
\hline A4650 & 199-k-21 & HEISPROD & RC-048 & J10010 & GW & 24-0ct-05 & & EBRLNE & Ko052 & & & $10198-40-0$ & & & & 25 & & pcill & $u$ & & 569769.902 \\
\hline A4650 & $199-K-21$ & HEISPROD & RC-048 & J10D10 & GW & 24-Oct-05 & & RLNP & Ko052 & $c$ & PESTPPCB & 1024-57-3 & epoxide & 0.05 & & & & ugl & u & 147932.06 & 569769.902 \\
\hline $\begin{array}{l}A 4500 \\
A 460\end{array}$ & $\frac{199-k-k 1}{199-k-21}$ & HEISPROD & RC-048 & J101010 & $G W$ & 24-0ct-05 & 1 & $\begin{array}{ll}\text { RRNP } \\
\text { ReNP }\end{array}$ & K0052 & $\begin{array}{cc}c \\
c k\end{array}$ & PESTIPCB & $\begin{array}{l}1031-71-8 \\
03-87\end{array}$ & sulfate & 0.05 & & & & ugh & $u$ & 147932.06 & 569769.902 \\
\hline A44650 & $\frac{|199-k-2|}{199-k-21}$ & $\begin{aligned} \text { HEISPROD } \\
\text { HEISPROD }\end{aligned}$ & $\begin{array}{l}\text { RC-0.08 } \\
\text { RC-08 }\end{array}$ & $\begin{array}{l}510010 \\
101070\end{array}$ & GW & $\begin{array}{l}24-0.0-105 \\
\text { 24-Cot-05 }\end{array}$ & & RLINP & $\begin{array}{l}K_{k 0052} \\
K_{0052}\end{array}$ & 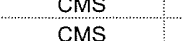 & $\begin{array}{l}\text { SOVA } \\
\text { SVIA }\end{array}$ & $\begin{array}{l}106-67-9 \\
1006-7\end{array}$ & DDmethyphenono: & 10 & & & & ugh & 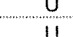 & $\begin{array}{l}174392.06 \\
1179306\end{array}$ & 569769.902 \\
\hline A4650 & $199-k-21$ & HEISPROD & RC-040 & J10D10 & GW & 24-Oct-05 & & RLNP & 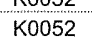 & CMS & $\begin{array}{l}\text { SVMA } \\
\text { sVoA }\end{array}$ & 106-47-8 & 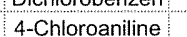 & 10 & & & & (1) & u & 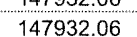 & 567696990202 \\
\hline A4650 & $199-K-21$ & HEISPROD & RC-048 & 510010 & $\mathrm{GW}$ & 24-Oct-05 & 1 & RLNP & K0052 & CMS & SVOA & $108-60-1$ & methylethyl) ethe & 10 & & & & ug/h & u & 147932.06 & 569769.902 \\
\hline $\begin{array}{l}A 4650 \\
A 450\end{array}$ & 199-k-21 & HEISPROD & RC-048 & J10D10 & GW & 24-Oct-05 & & RLNP & K0052 & CMS & SVOA & 108-95-2 & Phenol & 10 & & & & ugh & $u$ & 147932.06 & 569769.902 \\
\hline $\begin{array}{l}\text { A44560 } \\
\text { A4650 }\end{array}$ & $\frac{199-K-21}{109-k-21}$ & HEISPROD & RC-048 & J10D10 & GW & 24-Oct-05 & 1. & RLNP & K0052 & 8082_PCB_GC & $\begin{array}{l}\text { PESTIPCB } \\
\text { DESTTPOPB }\end{array}$ & $11096-82-5$ & Aroclor-1260 & 0.41 & & & & mgl & u & 147932.06 & 569769.902 \\
\hline A4650 & $199-k-21$ & $\begin{array}{l}\text { HESPROOD } \\
\text { HESPROD }\end{array}$ & 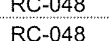 & $\begin{array}{l}5 \\
5100010\end{array}$ & $G \mathrm{~W}$ & 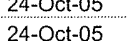 & & 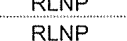 & $\begin{array}{l}K_{k 0052} \\
K_{0052}\end{array}$ & 年 & SWAB & 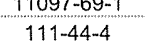 & 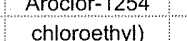 & $\frac{0.47}{10}$ & & & & mghn & 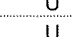 & $\begin{array}{l}144352.06 \\
1.49306\end{array}$ & 569769.902 \\
\hline A4650 & $199 \cdot-k-21$ & HEISPROD & RC-048 & $\frac{100 D 10}{15100}$ & $G \mathrm{G}$ & 24-Oct-05 & & RLNP & Ko052 & CMS & SVOA & $111-91-1$ & 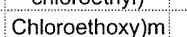 & 10 & & & & ug/h & u & 147932.06 & 5699699902 \\
\hline A4650 & $199-k-21$ & HEISPROD & RC-048 & J10010 & GW & 24-Oct-05 & & RLNP & K0052 & 8082_PCB_GC & PESTPCB & $11104-28-2$ & Aroclor-1221 & 0.41 & & & & $\mathrm{mglh}$ & u & 147932.06 & 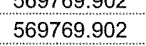 \\
\hline $\begin{array}{l}\text { A46650 } \\
\text { A4450 }\end{array}$ & $\frac{199-K-21}{199-K-21}$ & HEISPROD & RC-048 & J10D10 & GW & 24-Oct-05 & 1 & RLNP & K0052 & 8082_PCB_GC & PESTIPCB & $11141-16-5$ & Aroclor-1232 & 0.41 & & & & $\mathrm{mgll}$ & $u$ & 147932.06 & 569769.902 \\
\hline A46500 & $\frac{199-k-21}{199-k-21}$ & & $\begin{aligned} R C-048 \\
R C-083\end{aligned}$ & J10D10 & GW & 24-Cct-15 & & RLNP & K0052 & $\begin{array}{l}\text { CMS } \\
\text { CMS }\end{array}$ & SVOA & $1117-81-7$ & ethylhexyl) & 0.659 & & & & ugh & $\begin{array}{ll}B \mathrm{~B} \\
\mathrm{~B}\end{array}$ & 1479322.06 & 569769.902 \\
\hline$A 4650$ & $199-k-21$ & HEISPROD & $\frac{R C-48}{R C-048}$ & J10010 & GW & 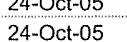 & 1 & S & K0052 & cys & SVA & 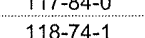 & 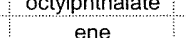 & 10 & & & & 年 & u & 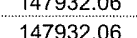 & 569769.902 \\
\hline A4650 & $1999-k-21$ & HEISPROD & RC-048 & J10D10 & GW & 24-0ct-05 & 1 & RLNP & Ko052 & cMs & SVOA & $120-12-7$ & Anthracene & 10 & & & & ugh/ & u & 1479322.06 & $\begin{array}{l}5699699902 \\
569769.902\end{array}$ \\
\hline A4650 & $199-k-21$ & HEISPROD & RC-048 & J10D10 & GW & 24-0ct-05 & 1 & RLNP & K0052 & CMS & SVOA & $120-82-1$ & Trichlorobenzen. & 10 & & & & ug/L & $u$ & 147932.06 & 569769.902 \\
\hline $\begin{array}{l}\text { A4660 } \\
\text { A4450 }\end{array}$ & $199-k-21$ & HEISPROD & RC-048 & J10010 & $\mathrm{GW}$ & 24-0ct-05 & 1 & RLNP & K0052 & CMS & SVOA & $120-83-2$ & Dichlorophenol & 10 & & & & ugh/ & $u$ & 147932.06 & 569769.902 \\
\hline $\begin{array}{l}A 4650 \\
A 4650\end{array}$ & $199-k-21$ & HEISPROD & RC-048 & J10D010 & $\mathrm{GW}$ & $\begin{array}{l}\text { 24-4coct-105 } \\
205\end{array}$ & & $\begin{array}{l}\text { RINP } \\
\text { RINP }\end{array}$ & K0052 & CNS & $\begin{array}{c}\text { SVAA } \\
\text { PESTPPCB }\end{array}$ & $\begin{array}{l}121-144-2 \\
.126720\end{array}$ & Dinifitotolluene & 10 & & & & ugh & u & $\begin{array}{l}1479322.06 \\
170206\end{array}$ & 569769.902 \\
\hline A44650 & 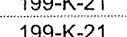 & HESTRODOD & 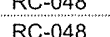 & (100010 & $G w$ & 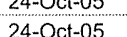 & 1 & RLN & Konse & 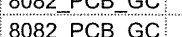 & P & 年 & 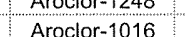 & 0.44 & & & & mal & u & 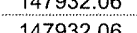 & 569769.902 \\
\hline A4t50 & 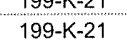 & HEISPROD & RC-0.08 & J10D10 & GW & 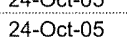 & 1 & RINP & Konse & CMS & SVOA & 129-00-0 & Pyrene & $\frac{1.40}{10}$ & & & & oul. & u & 14793206 & $\begin{array}{l}5699699902 \\
56976902\end{array}$ \\
\hline A4650 & $199-k-21$ & HEISPROD & RC-048 & 510010 & GW & 24-Oct-05 & 1 & RLNP & Ko052 & CMS & SVOA & 131-11-3 & phthalate & 10 & & & & ught & $\mathrm{u}$ & 147932.06 & 569769.902 \\
\hline A4650 & $199-k-21$ & HEISPROD & RC-048 & J10D10 & GW & 24-Oct-05 & 1 & RLNP & K0052 & CMS & SVOA & $132-64-9$ & Dibenzofuran & 10 & & & & ugh & $u$ & 147932.06 & 569769.902 \\
\hline A4650 & $\begin{array}{l}199-\mathrm{K}-21 \\
19-\mathrm{G}-\mathrm{k} 21\end{array}$ & $\begin{array}{l}\text { HEISPROD } \\
\text { HEISPROD }\end{array}$ & $\begin{array}{l}R C-048 \\
R C-0.08\end{array}$ & 110010 & GW & 24-Oct-05 & 1 & EBRLNE & Ko052 & GAMMA GS & RAD & $13966-00-2$ & $\begin{array}{l}\text { Potassium-40 } \\
\text { Rentumium }\end{array}$ & & & 370 & & pCill & U & $\begin{array}{l}1479323.06 \\
11702020\end{array}$ & 569769.902 \\
\hline $\begin{array}{l}A 4650 \\
A 4560\end{array}$ & $\frac{199-9-21}{199-k-21}$ & $\begin{array}{l}\text { HEISPROD } \\
\text { HEISPROD }\end{array}$ & $\begin{array}{l}\text { RC-0-048 } \\
\text { RC-048 }\end{array}$ & $\begin{array}{l}5100010 \\
j 10010\end{array}$ & GW & 24-OC-OLE & 1 & $\begin{array}{l}\text { EBRLLE } \\
\text { EBRLNEE }\end{array}$ & $\begin{array}{l}\text { K0052 } \\
\text { K0052 }\end{array}$ & $\begin{array}{l}\text { GAMMA GS GS } \\
\text { GAMMA GS }\end{array}$ & $\frac{R A D}{\text { RAD }}$ & $\begin{array}{l}13966-02-4-4 \\
13967-48-1\end{array}$ & $\begin{array}{l}\text { Berellum-I } \\
\text { Ruthenium-106 }\end{array}$ & & & 810 & & pcin & $v_{x}$ & $\begin{array}{l}149932.06 \\
149306\end{array}$ & 569769.902 \\
\hline$\frac{14060}{A 450}$ & 势 & HEISPROD & RC-048 & J10D10 & $G \mathrm{~W}$ & 24-Oct-05 & 1 & $\begin{array}{l}\text { EBRLLE } \\
\text { EBRLNEE }\end{array}$ & Koov2 & GAMMMAGG G & RAD & $139067-70-0$ & Cosium-134 & & & 24 & & pcil & u & 147932.06 & 56996999.902 \\
\hline A4650 & $199-k-21$ & HEISPROD & RC-048 & J10010 & $\mathrm{GW}$ & 24-Oct-05 & 1 & EBRLNE & Koos2 & UC & RAD & $13982-63-3$ & Radium-226 & 0.212 & 1.2 & 2.1 & 1.2 & pcil & $u$ & 147932.06 & 5699769.902 \\
\hline A4650 & $199-k-21$ & HEISPROD & RC-048 & 510010 & GW & 24-Oct-05 & 1 & EBRLNE & Ko052 & GAMMA_GS & RAD & $13982-63-3$ & Radium-226 & & & 44 & & pcill & $u$ & 147932.06 & 569769.902 \\
\hline A4650 & 199-K-21 & $\begin{array}{l}\text { HEISPRPOD } \\
\text { HEIPPR }\end{array}$ & $\begin{array}{l}\mathrm{RC}-048 \\
R C-048\end{array}$ & $\begin{array}{l}\text { J10D10 } \\
\text { J10D10 }\end{array}$ & GW & 24-OCt-05 & 1 & $\begin{array}{l}\text { EBRLNE } \\
\text { RLNP }\end{array}$ & K0052 & $\begin{array}{l}\text { GAMMA_GS } \\
\mathrm{IC}\end{array}$ & $\begin{array}{l}\text { RAD } \\
\text { GENCHEM }\end{array}$ & $\begin{array}{l}14234-35-6 \\
14255-44-2\end{array}$ & Antimony-125 & 025 & & 48 & & pCill & u & 147932.06 & $\begin{array}{r}569769.902 \\
550970007\end{array}$ \\
\hline$\frac{\mathrm{A} 46650}{\mathrm{~A} 4550}$ & $\frac{199-k-11}{199-k-21}$ & $\begin{array}{l}\text { HEIIPRPDD } \\
\text { HEISPROD }\end{array}$ & $\frac{R C-048}{R C-48}$ & $\begin{array}{l}\text { J10D10 } \\
\text { J10D10 }\end{array}$ & $\begin{array}{l}\text { GW } \\
\text { GW }\end{array}$ & $\begin{array}{l}\text { 24-OCt-1505 } \\
\text { 24-Cct-05 }\end{array}$ & $\frac{1}{1}$ & $\begin{array}{l}\text { RLNP } \\
\text { EBRLNE }\end{array}$ & $\begin{array}{l}\text { KK0052 } \\
\text { K00552 }\end{array}$ & $\begin{array}{c}\text { IC } \\
\text { TE AEA }\end{array}$ & GENCHEM & $\begin{array}{l}142655-44-2 \\
14269-63-7\end{array}$ & $\begin{array}{l}\text { Phosshhate } \\
\text { Thorium-230 }\end{array}$ & $\begin{array}{l}0.255 \\
-0.022\end{array}$ & 0.087 & 0.17 & 0.087 & $\begin{array}{l}\text { mgl } \\
\text { pcilh }\end{array}$ & u & $\begin{array}{l}1449923.06 \\
147932.06\end{array}$ & 569769.902 \\
\hline A4650 & $199-k-21$ & HEISPROD & RC- $\frac{-048}{R-048}$ & J10D10 & $G W$ & 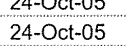 & 1 & $\begin{array}{l}\text { ESRLNE } \\
\text { EBRLNE }\end{array}$ & Koos2 & GAMMA_GS & RAD & $14274-82-9$ & $\begin{array}{l}\text { Thoroum-230 } \\
\text { Thorium-228 }\end{array}$ & & & 30 & 0.00 & pcill & u & $\begin{array}{l}144952.06 \\
147932.06\end{array}$ & 56996999.902 \\
\hline A4650 & $199 \cdot-k-21$ & HEISPROD & RC-048 & J10010 & GW & 24-Oct-05 & 1 & EBRLNE & Koo52 & TEAEA & RAD & $14274-82-9$ & Thorium-228 & 0.043 & 0.044 & 0.17 & 0.044 & pcil & $u$ & 147932.06 & 569769.902 \\
\hline A4650 & $199-k-21$ & HEISPROD & RC-048 & 510010 & oW & 24-Oct-05 & 1 & EBRLNE & K0052 & GAMMA GS & RAD & $14391-16-3$ & Europium-155 & & & 56 & & pCill & u & 1479322.06 & 569769.902 \\
\hline A4650 & $\begin{array}{l}199-K-21 \\
99-k-12\end{array}$ & $\begin{array}{l}\text { HEIIPROD } \\
\text { HEISPROD }\end{array}$ & $\begin{array}{l}\text { RC-048 } \\
\text { RC-0.08 }\end{array}$ & $\begin{array}{l}\text { J110D10 } \\
\text { J10D10 }\end{array}$ & GW & 24-Oct-05 & & EBRLNE & Ko052 & GAMMA GS & $\begin{array}{l}\text { RAD } \\
\text { RAD }\end{array}$ & $\begin{array}{l}\frac{14596-10-2}{14633-23-9} \\
\end{array}$ & Americium-241 & & & $\begin{array}{l}75 \\
51\end{array}$ & & pcill & u & $\begin{array}{l}147932.06 \\
147092006\end{array}$ & 569769.902 \\
\hline $\begin{array}{l}\text { A46650 } \\
\text { A4650 }\end{array}$ & $\begin{array}{l}199-K-21 \\
199-K-21\end{array}$ & $\begin{array}{l}\text { HEIIPROD } \\
\text { HEISPROD }\end{array}$ & $\begin{array}{l}R C-048 \\
R C-048\end{array}$ & $\begin{array}{l}\text { J1100010 } \\
\text { J10D10 }\end{array}$ & $\begin{array}{l}\text { GW } \\
\text { GW }\end{array}$ & 24-OCt-105 2400 & $\frac{1}{1}$ & $\begin{array}{l}\text { EBRLNE } \\
\text { EBRLNE }\end{array}$ & $\begin{array}{l}\text { K0052 } \\
\text { Ko052 }\end{array}$ & $\begin{array}{l}\text { GAMMA GS } \\
\text { C }\end{array}$ & $\begin{array}{l}\text { RAD } \\
\text { RAD }\end{array}$ & $\begin{array}{l}148635-23-9-9 \\
1462-75-5\end{array}$ & $\begin{array}{l}\text { Europlumin-1252 } \\
\text { Carbon-14 }\end{array}$ & -6.87 & 23 & $\begin{array}{l}51 \\
39\end{array}$ & 23 & $\begin{array}{l}\text { pClin } \\
\text { pcil }\end{array}$ & u & $\begin{array}{l}144392.06 \\
14930206\end{array}$ & $\begin{array}{l}55996769.902 \\
6596002\end{array}$ \\
\hline A4650 & $199-k-21$ & HEISPROD & RC-048 & J100010 & $G W$ & 24-COC-10.0 & 10 & RLNP & Koos2 & IC & GENCHEM & $147967-55-8$ & 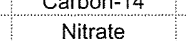 & 27.7 & & & & mels & $\mathrm{D}$ & $\begin{array}{l}14593200 \\
14923.06\end{array}$ & 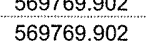 \\
\hline A4650 & $199-k-21$ & HEISPROD & RC-048 & J10D10 & GW & 24-Oct-05 & 1 & RLNP & Ko052 & IC & GENCHEM & $14797-65-0$ & Nirite & 0.25 & & & & mglh & $u$ & 147932.06 & 569769.902 \\
\hline A4650 & $199-K-21$ & HEISPROD & RC-043 & J10010 & GW & 24-Oct.-05 & 10 & $\begin{array}{l}\text { RLNP } \\
\text { Ret }\end{array}$ & K0052 & IC & GENCHEM & $\begin{array}{l}14808-79-8 \\
\end{array}$ & Sulfate & 65.4 & & & & mglt & $c$ & 147932.06 & 569769.902 \\
\hline $\begin{array}{c}A 4650 \\
A 450\end{array}$ & $\begin{array}{l}199-K-21 \\
199-k-22\end{array}$ & $\begin{array}{l}\text { HEISPROD } \\
\text { HEISPROD }\end{array}$ & 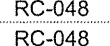 & J10D10 & GW & 24-Oct-05 & 1 & EBRLNE & Ko052 & GAMMA $6 S$ & $\begin{array}{l}\text { RAD } \\
P A D\end{array}$ & $\begin{array}{l}151717-96-1 \\
1517\end{array}$ & $\begin{array}{l}\text { Urantium-235 } \\
\end{array}$ & 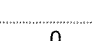 & 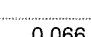 & 825 & (2066 & pcin & $u$ & $\begin{array}{l}1479922.06 \\
17702306\end{array}$ & 569769.902 \\
\hline $\begin{array}{l}\text { A44660 } \\
\text { A4650 }\end{array}$ & $\frac{199-K-21}{199-k-21}$ & HEISPROD & $\begin{array}{l}\text { RC-048 } \\
\text { RC-048 }\end{array}$ & $\begin{array}{l}\text { J10D10 } \\
\text { J10D10 }\end{array}$ & $\begin{array}{l}G W \\
G W\end{array}$ & $\begin{array}{l}\text { 24-OCt-0.5 } \\
\text { 24-Ccot-05 }\end{array}$ & 1 & $\begin{array}{l}\text { EBRRLE } \\
\text { EBRLNEE }\end{array}$ & $\begin{array}{l}\text { K0052 } \\
\text { Ko052 }\end{array}$ & $\begin{array}{l}\text { AEA } \\
\text { GAMMA GS }\end{array}$ & $\frac{R A D}{R A D}$ & $15262-20-1$ & $\begin{array}{l}\text { Uranium-235 } \\
\text { Radium-228 }\end{array}$ & 0 & 0.000 & 0.20 & & pcim & u & 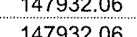 & 569769.902 \\
\hline A4650 & $\frac{|99-k-k|}{199-k-21}$ & HEISPROD & RC-048 & J10D10 & GW & 24-Ccte-0.5 & 1 & EBRRLNE & Koos2 & $P C$ & $\begin{array}{l}\mathrm{RAD} \\
\mathrm{RAD}\end{array}$ & $152062-20-1$ & Radium-228 & 4.43 & & 1.7 & & pcin & $x$ & $\begin{array}{l}149323200 \\
14932.06\end{array}$ & 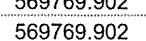 \\
\hline A4650 & $199-k-21$ & HEISPROD & RC-048 & J10D10 & GW & 24-Oct-05 & 1 & EBRLNE & Koo52 & GAMMA_GS & RAD & $15585-10-1$ & Europium-154 & & & 68 & & pCiL & ú & 1479322.06 & 569769.902 \\
\hline A4650 & $199-K-21$ & HEISPROD & RC-048 & J10D10 & GW & 24-Oct-05 & 1 & RLNP & K0052 & IC & GENCHEM & $16887-00-6$ & Chloride & 8.82 & & & & & 1. & 147932.06 & 569769.902 \\
\hline $\begin{array}{l}A 4560 \\
A 450\end{array}$ & $\frac{199-K-21}{199-K-21}$ & $\begin{array}{l}\text { HEISPRPD } \\
\text { HEISPR }\end{array}$ & $\begin{array}{l}\text { RC-0.048 } \\
\text { RC }\end{array}$ & J100010 & GW & $\begin{array}{l}24-0.0-105 \\
200105\end{array}$ & 1 & $\begin{array}{l}\text { RLNPP } \\
\text { RiNP }\end{array}$ & Ko052 & $\begin{array}{l}\text { If } \\
\text { cos }\end{array}$ & $\begin{array}{l}\text { GENCHEM } \\
\text { SWA }\end{array}$ & $\begin{array}{l}16984-48-8 \\
109-2\end{array}$ & Fluoride & 0.25 & & & & mol & $u$ & $\begin{array}{l}147932.06 \\
1472066\end{array}$ & 569769.902 \\
\hline 兒44560 & 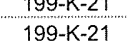 & HESPROD & 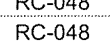 & J10010 & $\frac{G W}{G W}$ & 24-0.0-10505 & & RRN & 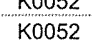 & cis & SSOA & 1919-3-24 & chene & $\frac{10}{10}$ & & & & 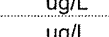 & 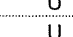 & 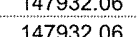 & 569769.902 \\
\hline A4650 & $\frac{199-k-21}{199-k-21}$ & HEISPROD & $\begin{array}{l}\text { KC- }-048 \\
\text { RC-048 }\end{array}$ & J100010 & Gw & 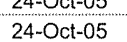 & 1 & RLNP & Koos2 & CMS & SVOA & $205-99-2$ & $\begin{array}{c}\text { conpprene } \\
\text { thene }\end{array}$ & 10 & & & & ug & u & 149932.06 & 56996999.902 \\
\hline A4650 & $199-k-21$ & HEISPROD & RC-048 & 100010 & GW & 24-Oct-05 & 1 & RLNP & Koo52 & CMS & SVOA & $206-44-0$ & Fluoranthene & 10 & & & & uglt & u & 147932.06 & 5699699.902 \\
\hline & 199-K-21 & HEISPROD & RC-048 & J10D10 & GW & 24-.oct-05 & & RLNP & K0052 & CMS & SVOA & $207-08-9$ & thene & 10 & & & & & $u$ & 147932.06 & 569769.902 \\
\hline A4650 & $199-k-21$ & HEISPROD & RC-048 & J10D10 & GW & 24-Oct-05 & 1 & RLNP & K0052 & CMS & SVOA & $208-96-8$ & Acenaphitylyene & 10 & & & & ugh & u & 147932.06 & 569769.900 \\
\hline
\end{tabular}




\begin{tabular}{|c|c|c|c|c|c|c|c|c|c|c|c|c|c|c|}
\hline $\begin{array}{l}A 4650 \\
A 6650\end{array}$ & $\begin{array}{l}199-\mathrm{K}-21 \\
199-\mathrm{K}-21\end{array}$ & HEISPROD & RC-048 & $\begin{array}{l}\frac{J 10010}{J 100010} \\
\end{array}$ & $\frac{G W}{G W}$ & $\begin{array}{l}24-\text { oct-05 } \\
24 \text { coct-0.5 }\end{array}$ & & RLNP & K0052 & CMS & SVOA & $218-01-9$ & Chrysene & 10 \\
\hline A4650 & $\frac{199-k-21}{199-k-21}$ & $\begin{array}{l}\text { HEISPROD } \\
\text { HEISPROD }\end{array}$ & $\begin{array}{l}\text { RC-048 } \\
\text { RC-048 }\end{array}$ & J100010 & GW & 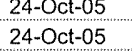 & & $\begin{array}{l}\text { RLNP } \\
\text { RLNP }\end{array}$ & $\begin{array}{l}\mathrm{K} 0052 \\
\text { K0052 }\end{array}$ & $\frac{1 C}{C}$ & $\begin{array}{l}\text { GENCHEM } \\
\text { PEST/PCB }\end{array}$ & $\frac{24959-67-9}{309-00-2}$ & $\begin{array}{l}\text { Bromide } \\
\text { Aldin }\end{array}$ & $\begin{array}{l}0.25 \\
0.05\end{array}$ \\
\hline A4650 & $199 \cdot k-21$ & HEISPROD & RC-048 & J10010 & GW & 24-Oct-05 & & RLNP & K0052 & & PESTIPCB & $319-84-6$ & & 0.05 \\
\hline A4650 & $199-k-21$ & HEISPROD & RC-048 & 510010 & GW & 24-.oct-05 & & RLNP & K0052 & $c$ & PEST/PCB & 319-85-7 & Hexachlorocyctlo & 0.05 \\
\hline $\begin{array}{l}A 4650 \\
A 4650\end{array}$ & $\frac{199-k-21}{199-k-21}$ & HEISPROD & RC-048 & 510010 & $G W$ & 24-Oct-05 & & RLNP & K0052 & c & PESTIPCB & $319 \cdot 86-8$ & Detta-BHC & 0.05 \\
\hline A4650 & $\frac{199-k-21}{199-k-21}$ & $\begin{array}{l}\text { HEISPROD } \\
\text { HEISPROD }\end{array}$ & 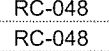 & $\begin{array}{l}J 100010 \\
J 10 D 10\end{array}$ & GW & $\begin{array}{l}\text { 24-OCt-055 } \\
\text { 24-Oct-05 }\end{array}$ & & $\begin{array}{l}\text { RLNP } \\
\text { RLNP }\end{array}$ & $\begin{array}{l}\text { K0052 } \\
\text { Ko052 }\end{array}$ & $\frac{c}{c}$ & $\begin{array}{l}\text { PEET/PCB } \\
\text { PEST/PCB }\end{array}$ & $\begin{array}{l}33213-65-9 \\
50-29-3\end{array}$ & $\begin{array}{l}\text { Endosulfan II } \\
\text { Itrichloroethane }\end{array}$ & $\begin{array}{l}0.05 \\
0.05\end{array}$ \\
\hline A4650 & $199-\mathrm{k}-21$ & HEISPROD & RC-048 & J10010 & Gw & 24-oct-05 & & RLNP & Ko052 & CMS & SVOA & $50-32-8$ & Benzo(a)pyrene & 10 \\
\hline A4650 & 199-K-21 & HEISPROD & 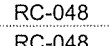 & $\begin{array}{l}510010 \\
\end{array}$ & GW & $\begin{array}{l}24-0 \mathrm{Oct}-05 \\
20.05\end{array}$ & & RLNP & K0052 & CMS & $\begin{array}{l}\text { SVOA } \\
\text { PESTIPCB }\end{array}$ & $\begin{array}{l}51-28-5 \\
51037-7 \cdot 9-9\end{array}$ & $\begin{array}{l}\text { Dinitrophenol } \\
\text { Chloricines }\end{array}$ & 25 \\
\hline $\begin{array}{l}A 4650 \\
A 4650\end{array}$ & $\begin{array}{l}\frac{199--k-11}{199-k-21} \\
\end{array}$ & $\begin{array}{l}\text { HEISPROD } \\
\text { HEISPROD }\end{array}$ & 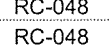 & $\begin{array}{l}J 100010 \\
J 10 D 10\end{array}$ & $\begin{array}{l}G W \\
G W\end{array}$ & 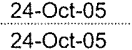 & & $\frac{\text { RLNP }}{\text { RLNP }}$ & $\begin{array}{l}\text { K0052 } \\
\text { Ko052 }\end{array}$ & $\begin{array}{l}c \\
c \\
c\end{array}$ & $\begin{array}{l}\text { PEST/PCB } \\
\text { PEST/PCB }\end{array}$ & $\begin{array}{l}5503-71-9 \\
5103-7-2\end{array}$ & $\begin{array}{l}\text { Chlordane } \\
\text { Cllordane }\end{array}$ & 0.05 \\
\hline A4650 & $199-k-21$ & HEISPROD & RC-048 & J100010 & Gw & 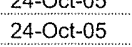 & & RLNP & Ko052 & CMS & SVOA & $53-70-3$ & $\begin{array}{l}\text { Cacene } \\
\text { racente }\end{array}$ & $\frac{0.05}{10}$ \\
\hline A4650 & $1999-k-21$ & HEISPROD & RC-048 & J10010 & Gw & 24-.oct-05 & & RLNP & K0052 & CMS & SVOA & $534-52-1$ & methylphenol & 25 \\
\hline A6450 & $199-\mathrm{K}-21$ & HEISPROD & RC-048 & J10D10 & Gw & 24-OCt-05 & & RLNP & K0052 & 8082_PCB_GC & PEST/PCB & 53469-21-9 & Arocolor-1242 & 0.41 \\
\hline $\begin{array}{l}A 4650 \\
A 4650\end{array}$ & $\frac{199-9-11}{199-k-21}$ & HEISPROD & 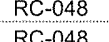 & $\begin{array}{l}110010 \\
100010\end{array}$ & GW & $\begin{array}{l}24-\text { Oct-05 } \\
24.0505\end{array}$ & & RLNP & K0052 & $\begin{array}{c}c \\
c M s\end{array}$ & $\begin{array}{l}\text { PESTPCB } \\
\text { SVOA }\end{array}$ & $\begin{array}{l}53494-70-5 \\
54173-1\end{array}$ & Endrin ketone & 0.05 \\
\hline A4650 & $199-k-21$ & HEISPROD & $\begin{array}{ll}R L-048 \\
R C-048\end{array}$ & J10010 & GW & 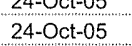 & & RLNP & K0052 & CMS & $\begin{array}{l}\text { SUVA } \\
\text { SVOA }\end{array}$ & 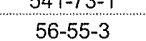 & Hetrivorove & $\frac{10}{10}$ \\
\hline A4650 & $199-k-21$ & HEISPROD & RC-048 & J10D10 & $\mathrm{GW}$ & 24-oct-05 & & RLNP & Ko052 & & PESTPCB & 58-8s-9 & & $\begin{array}{l}10 \\
0.05\end{array}$ \\
\hline $\begin{array}{c}A 4650 \\
A 4650\end{array}$ & $199-K-21$ & HEISPROD & RC-048 & J10D10 & GW & 24-0ct-05 & & RLNP & K0052 & CMS & SVOA & 59-50-7 & methylphenol & 10 \\
\hline $\begin{array}{l}A 4650 \\
A 4650\end{array}$ & $\begin{array}{l}199-k-21 \\
199-k-21\end{array}$ & HEISPROD & RC-048 & 510010 & GW & 24-Oct-05 & & RLNP & K0052 & $c$ & PESTPCB & $60-57-1$ & Dieldrin & 0.05 \\
\hline A4650 & 199-k-21 & $\begin{array}{l}\text { HEISPROD } \\
\text { HESPROD }\end{array}$ & $\begin{array}{ll}\text { RC- }-048 \\
\text { RC-048 }\end{array}$ & $\begin{array}{l}110010 \\
10 D 10\end{array}$ & GW & 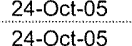 & & RLNP & K0052 & CMS & SVOA & $\begin{array}{l}606-20-2 \\
60.164-7\end{array}$ & Dinitrotoluene & $\frac{10}{10}$ \\
\hline A4650 & $199-k-21$ & HEISPROD & $\begin{array}{l}\text { RC-048 } \\
\text { RC-048 }\end{array}$ & J10010 & $G W$ & 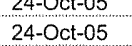 & & RLNP & K0052 & CMS & $\begin{array}{l}\text { SVOA } \\
\text { SVOA }\end{array}$ & $\begin{array}{l}0<1-1-44-1 \\
65794-96-9\end{array}$ & $\begin{array}{l}\text { alporoyylamine } \\
\text { Methylohenol }\end{array}$ & 10 \\
\hline A4650 & $199-K-21$ & HEISPROD & RC-048 & J10010 & GW & 24-.oct-05 & & RLNP & Koos2 & CMS & SVOA & & & 10 \\
\hline $\begin{array}{l}A 4650 \\
A 450\end{array}$ & 199-k-21 & HEISPROD & RC-048 & 110010 & GW & 24-.oct-05 & & RLNP & K0052 & CMS & SVOA & $7005-72-3$ & Chlorophenylph & 10 \\
\hline A4650 & 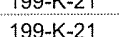 & HESPRDOD & RC-048. & 5100010 & GW & 24-Oct-05 & & RLNP & K0052 & c & PESTIPCB & $72-20-8$ & Endrin & 0.05 \\
\hline A4650 & $199-k-21$ & HEISPROD & 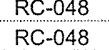 & $\begin{array}{l}5100010 \\
510010\end{array}$ & $\begin{array}{l}\text { GW } \\
\text { GW }\end{array}$ & $\begin{array}{l}24-0.0-1003 \\
24-0 c t-05\end{array}$ & & $\begin{array}{l}\text { RLNPP } \\
\text { RLNP }\end{array}$ & K0052 & ${ }_{c}^{c}$ & PESTIPCB & $\begin{array}{l}72-43-5 \\
72-54-8\end{array}$ & $\begin{array}{l}\text { Methoxyychlor } \\
\text { |lichloroethane }\end{array}$ & $\begin{array}{l}0.05 \\
0.05\end{array}$ \\
\hline A4650 & $199-K-21$ & HEISPROD & RC-048 & $J 10010$ & GW & 24-Oct-05 & & RLNP & K0052 & c & PESTIPCB & $72-55-9$ & Idichloroethylen & 0.05 \\
\hline $\begin{array}{l}A 4650 \\
A 4650\end{array}$ & $\frac{199-K-21}{199-k-21}$ & $\begin{array}{l}\text { HEIIPROD } \\
\text { HEISPROD }\end{array}$ & $\frac{\mathrm{RC}-048}{\mathrm{RC}-048}$ & $\begin{array}{l}\frac{510010}{J 10010} \\
\end{array}$ & GW & $\begin{array}{l}\text { 24-OCt-0.05 } \\
\text { 24-coct-55 }\end{array}$ & & $\frac{R L N P}{R L N P}$ & K0052 & $\begin{array}{c}c \\
c P\end{array}$ & $\begin{array}{l}\text { PESTIPCB } \\
\text { METAMUIT }\end{array}$ & $\begin{array}{l}7421-93-4 \\
7729-900-5\end{array}$ & Endrin aldehyde & 0.05 \\
\hline $\begin{array}{l}\text { A44505 } \\
A 4650\end{array}$ & $\begin{array}{l}199-k-21 \\
199-k-21\end{array}$ & $\begin{array}{l}\text { HEISPROD } \\
\text { HEISPROD }\end{array}$ & $\begin{array}{l}\text { RC-048 } \\
\text { RC-048 }\end{array}$ & $\begin{array}{l}5100010 \\
J 10010\end{array}$ & GW & $\begin{array}{l}24-0.0-05 \\
24-0 c t-05 \\
\end{array}$ & & $\begin{array}{l}\text { RLN } \\
\text { RLNP }\end{array}$ & K0.052 & TF & 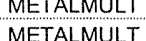 & 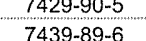 & $\begin{array}{l}\text { Aluminum } \\
\text { Irron }\end{array}$ & $\begin{array}{l}49.1 \\
287\end{array}$ \\
\hline A4650 & 199-K-21 & HEISPROD & RC-048 & J10010 & GW & 24-0ct-05 & & RLNP & K0052 & ICP & METALMULT & The & Lead & 3.1 \\
\hline A4650 & $199-k-21$ & HEISPROD & RC-048 & J10010 & GW & 24-0ct-05 & & RLNP & K0052 & ICP & METALMULT & $7439-93-2$ & Lithium & 2.9 \\
\hline A4650 & $199-\mathrm{K}-21$ & HEISPROD & RC-048 & 510010 & GW & 24-Oct-05 & & RLNP & K0052 & ICP & METALMULT & 74399-95-4 & Magnesium & 12000 \\
\hline $\begin{array}{l}A 4650 \\
\text { A6550 }\end{array}$ & $\begin{array}{l}199-K-21 \\
199-k-21\end{array}$ & $\begin{array}{l}\text { HEISPROD } \\
\text { HEISPROD }\end{array}$ & $\begin{array}{ll}R C-048 \\
\text { RC-048 }\end{array}$ & $\begin{array}{ll}510010 \\
1\end{array}$ & GW & $\begin{array}{l}\text { 24-Oct-05 } \\
\text { 24-cost-5 }\end{array}$ & & RLNP & K0052 & ICP & METALMULT & $\begin{array}{l}74399.96-5 \\
7320906\end{array}$ & Manganese & 120 \\
\hline A4650 & $\begin{array}{l}\frac{199-k-21}{199-k-21} \\
1\end{array}$ & $\begin{array}{l}\text { HESPROD } \\
\text { HEISPROD }\end{array}$ & $\begin{array}{l}\text { RC- }-048 \\
\text { RC-048 }\end{array}$ & $\begin{array}{l}5100010 \\
J 10010\end{array}$ & GW & $\begin{array}{l}24-0.0-5 \\
24-0 c t-05\end{array}$ & & $\begin{array}{l}\text { RLN P } \\
\text { RLNP }\end{array}$ & $\begin{array}{l}k 0052 \\
k 0052\end{array}$ & A & $\begin{array}{l}\text { MEIALIISG } \\
\text { METALMULT }\end{array}$ & $\begin{array}{l}74499-9-6-6 \\
7439-98-7\end{array}$ & $\begin{array}{l}\text { Melcrury } \\
\text { Molydenum }\end{array}$ & $\frac{0.1}{1.3}$ \\
\hline A4650 & $199-k-21$ & HEISPROD & $\begin{array}{l}\text { RC- }-048 \\
\text { RC-048 }\end{array}$ & $\begin{array}{ll}5100010 \\
J 10\end{array}$ & GW & 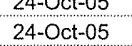 & & RLNP & Ko052 & $\mathrm{ICP}$ & METALMULT & $7440-02-0$ & Wolyodenum & $\begin{array}{l}1.3 \\
3.8 \\
\end{array}$ \\
\hline A4650 & $199-\mathrm{k}-21$ & HESPROD & RC-048 & 5100010 & GW & 24-.oct-05 & & RLNP & K0052 & ICP & METALMULT & $7440-09-7$ & Potassium & 1910 \\
\hline A4650 & 199-K-21 & HEISPROD & RC-048 & 510010 & GW & 24-Oct-05 & & RLNP & K0052 & ICP & METALMULT & $7440-21-3$ & Silicon & 6170 \\
\hline $\begin{array}{l}A 4650 \\
A 4650\end{array}$ & $\begin{array}{l}199-9-21 \\
199-k-21\end{array}$ & $\begin{array}{l}\text { HEISPROD } \\
\text { HEIPRODD }\end{array}$ & $\begin{array}{l}\text { RC-048 } \\
\text { RC-048 }\end{array}$ & $\begin{array}{l}J 10010 \\
J 100010\end{array}$ & GW & $\begin{array}{l}24 \text {-OCt-0.05 } \\
24-0 \text { cot-05 }\end{array}$ & & $\frac{R L N P}{R I N P}$ & K0052 & ICP & METALMULT & $7440-22-4$ & $\begin{array}{l}\text { Silver } \\
\text { Sdium }\end{array}$ & 1.4 \\
\hline A4650 & 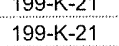 & $\begin{array}{l}\text { HESPRPOD } \\
\text { HEISPROD }\end{array}$ & $\begin{array}{l}\text { RC-048 } \\
\text { RC-048 }\end{array}$ & $\begin{array}{ll}100010 \\
100\end{array}$ & GW & $\begin{array}{l}24-0 \text { - } 20.05 \\
24-0 c t-05\end{array}$ & & RLNP & Koo52 & ICP & $\begin{array}{l}\text { MEIALMULT } \\
\text { METALMULT }\end{array}$ & $\begin{array}{l}7440-33-5 \\
7440-24-6\end{array}$ & $\begin{array}{l}\text { SSodumm } \\
\text { Strontium }\end{array}$ & $\begin{array}{l}4090 \\
284\end{array}$ \\
\hline A4650 & $199 \cdot-\mathrm{k}-21$ & HEISPROD & RC-048 & J100010 & GW & 24-Oct-05 & & RLNP & Koos2 & ICP & METALMUTT & $7440-28-0$ & Thallium & $\begin{array}{l}254 \\
6.4\end{array}$ \\
\hline A4650 & $199-k-21$ & HEISPROD & RC-048 & 510010 & GW & 24-Oct-05 & & RLNP & K0052. & ICP & METALMULT & $7440-31-5$ & Tin & 5.2 \\
\hline A4650 & $\begin{array}{l}199-K-21 \\
\end{array}$ & & $\begin{array}{ll}\text { RC-048 } \\
\text { RC-048 }\end{array}$ & $\begin{array}{ll}110010 \\
10010\end{array}$ & GW & 24-oct-05 & & RLNP & K0052 & ICP & METALMULT & 7440-36-0 & Antimony & 4 \\
\hline $\begin{array}{l}A 4650 \\
A 4650\end{array}$ & $\begin{array}{l}\frac{199-k-21}{199-k-21} \\
\end{array}$ & $\begin{array}{l}\text { HEIIPROD } \\
\text { HEISPROD }\end{array}$ & 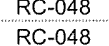 & $\begin{array}{l}\frac{510010}{510010} \\
\end{array}$ & GW & $\begin{array}{l}24-0.0-05 \\
24-0.05\end{array}$ & & $\begin{array}{l}\text { RLNP } \\
\text { BNNP }\end{array}$ & $\begin{array}{l}K_{0.052} \\
\text { Ko52 }\end{array}$ & $\frac{I C P}{1 C P}$ & $\begin{array}{l}\text { METALMULT } \\
\text { METAMIT }\end{array}$ & 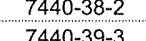 & $\begin{array}{l}\text { Arsenic } \\
\text { Barim }\end{array}$ & $\begin{array}{l}3.4 \\
16.1\end{array}$ \\
\hline A4650 & 199-k-21 & HEISPROD & RC-048 & J150010 & GW & $24-0.0505$ & & RLNP & $\begin{array}{l}\text { Kovose } \\
\text { Ko }\end{array}$ & WP & 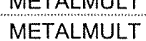 & 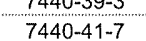 & $\begin{array}{l}\text { Berlumilum } \\
\text { Bente }\end{array}$ & $\begin{array}{l}16.1 \\
0.1 \\
0.1\end{array}$ \\
\hline A4650 & $199-\mathrm{k}-21$ & HEISPROD & RC-048 & $J 10010$ & GW & $24-\mathrm{Oct}-05$ & & RLNP & Ko052 & ICP & METALMULT & $7440-42-8$ & Boron & $\frac{0.1}{15.6}$ \\
\hline A4650 & $199-k-21$ & HEISPROD & RC-048 & 510010 & GW & 24-0ct-05 & & RLNP & K0052 & ICP & METALMULT & $7440-43-9$ & Cadmium & 0.7 \\
\hline A4650 & $199-K-21$ & HEISPROD & RC-048 & $\begin{array}{l}310010 \\
10010\end{array}$ & GW & 24-.oct-05 & & RLNP & K0052 & ICP & METALMULT & $7440-47-3$ & Chromium & \\
\hline $\begin{array}{l}A 4650 \\
A 4650\end{array}$ & $\frac{199-k-21}{199-K-21}$ & $\begin{array}{l}\text { HEISPROD } \\
\text { HEISPROD }\end{array}$ & $\begin{array}{l}\text { RC-048 } \\
\text { RC-048 }\end{array}$ & $\begin{array}{l}\frac{J 10010}{J 10010} \\
\end{array}$ & GW & $\begin{array}{l}24.010-05 \\
24-0.05-5.5\end{array}$ & & $\begin{array}{l}\text { RLNP } \\
\text { RINP }\end{array}$ & K05052 & ICP & METALLULT & $7440-48-4$ & $\begin{array}{l}\text { Cobalt } \\
\text { Cogper }\end{array}$ & $\begin{array}{l}1.2 \\
12\end{array}$ \\
\hline A4650 & $199-\mathrm{k}-21$ & HEISPROD & RC-048 & J150010 & $G w$ & $24-0 \mathrm{ct}-05$ & & $\begin{array}{l}\text { RLNP } \\
\text { RLN }\end{array}$ & K0052 & WCP & METALUUTT & $7440-61-1$ & 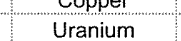 & $\frac{1.2}{20.6}$ \\
\hline A4650 & $199-\mathrm{k}-21$ & HEISPROD & RC-048 & J10010 & GW & 24-.oct-05 & & RLNP & K0052 & ICP & METALMULT & $7440-62-2$ & Vanadium & 0.94 \\
\hline A4650 & $199-k-21$ & HEISPROD & RC-048 & 510010 & ow & 24-.0ot-05 & & RLNP & K0052 & ICP & METALMULT & $7440-66-6$ & Zinc & 5.6 \\
\hline A4650 & 199-K-21 & HEIPPROD & 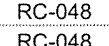 & $\begin{array}{r}110010 \\
10010\end{array}$ & GW & $\begin{array}{l}24-\text { Oct-.05 } \\
24-0.050 .5\end{array}$ & & RLNP & K0052 & ICP & METALMULT & $\begin{array}{l}7440-69-9 \\
7440-70-2\end{array}$ & $\begin{array}{l}\text { Bismuth } \\
\text { Calcium }\end{array}$ & 6.1 \\
\hline A6450 & 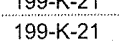 & $\begin{array}{l}\text { HEISPROD } \\
\text { HEISPROD }\end{array}$ & $\begin{array}{l}\text { RC-048 } \\
\text { RC-048 }\end{array}$ & $\begin{array}{l}J 10010 \\
J 10010\end{array}$ & GW & $\begin{array}{l}24 \text {-OCtC.05 } \\
\text { 24-oct-05 }\end{array}$ & & $\begin{array}{l}\text { RLNP } \\
\text { RLNP }\end{array}$ & K0052 & & $\begin{array}{l}\text { METALMULT } \\
\text { PEST/PCB }\end{array}$ & $\begin{array}{l}7440-70-2 \\
76-44-8\end{array}$ & $\begin{array}{l}\text { Calcium } \\
\text { Heptachior }\end{array}$ & 56900 \\
\hline A4650 & $199-k-21$ & HEISPROD & RC-048 & J10010 & GW & $24-\mathrm{Oct}-05$ & & RLNP & 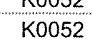 & CMS & SVOA & $\begin{array}{l}10-44-0 \\
77-47-4\end{array}$ & pentadiene & $=10$ \\
\hline A4650 & $199-k-21$ & HEISPROD & RC-048 & J10010 & $G W$ & 24-oct-05 & & RLNP & K0052 & ICP & METALMULT & $7723-4-0$ & Phosphorus & $\frac{16.4}{16.4}$ \\
\hline A4650 & $199-\mathrm{K}-21$ & HEISPROD & RC-048 & 110010 & ow & 24-Oct-05 & & RLNP & K0052 & ICP & METALMULT & $7782-49-2$ & Selenium & 3.6 \\
\hline $\begin{array}{l}A 4650 \\
\text { A4650 }\end{array}$ & 199-K-21 & HEISPROD & 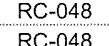 & $\begin{array}{l}110010 \\
10010\end{array}$ & GW & $\begin{array}{l}24-\text { Oct-0.05 } \\
\text { 24-0.05 }\end{array}$ & & RLNP & K0052 & CMS & $\begin{array}{l}\text { SVOA } \\
\text { SCOIPC }\end{array}$ & $\begin{array}{l}78-59-1 \\
78-52\end{array}$ & $\begin{array}{l}\text { Isophororne } \\
\text { Iste }\end{array}$ & 10 \\
\hline A4650 & 199-k-21 & $\begin{array}{l}\text { HEISPROD } \\
\text { HEISPROD }\end{array}$ & $\begin{array}{l}\text { RC-048 } \\
\text { RC-048 }\end{array}$ & $\begin{array}{l}J 100010 \\
10010\end{array}$ & GW & $\begin{array}{l}24 \text {-OCtC-05 } \\
\text { 24-Coct-05 }\end{array}$ & & $\begin{array}{l}\text { RLNP } \\
\text { RINP }\end{array}$ & 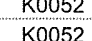 & CMS & $\begin{array}{l}\text { PESTPCB } \\
\text { SVOA }\end{array}$ & $\begin{array}{l}8001-35-2 \\
83-32-9\end{array}$ & $\begin{array}{l}\text { Toxaphenene } \\
\text { Acapathe }\end{array}$ & 0.05 \\
\hline A4650 & $199-k-21$ & HEISPROD & RC-048 & J10010 & GW & 24-0ct-05 & & RLNP & K0052 & CMS & $\begin{array}{l}\text { SVOA } \\
\text { SOAA }\end{array}$ & 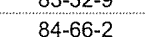 & Diethrypthtalat & 10 \\
\hline A4650 & $199-k-21$ & HEISPROD & RC-048 & J10010 & GW & 24-Oct-05 & & RLNP & Ko052 & CMS & SVOA & $84-74-2$ & butyphthalate & 0.593 \\
\hline A4650 & $199-\mathrm{k}-21$ & HEISPROD & RC-048 & 110010 & GW & 24-Oct-05 & & RLNP & K0052 & CMS & SVOA & $85-01-8$ & Penanthrene & 10 \\
\hline $\begin{array}{l}A 4650 \\
A 4650\end{array}$ & 199-K-21 & $\begin{array}{l}\text { HEISPROD } \\
\text { HEISROD }\end{array}$ & $\begin{array}{ll}\text { RC- }-048 \\
\text { BC-048 }\end{array}$ & $\begin{array}{l}110010 \\
\text { J110010 }\end{array}$ & $G W$ & $\begin{array}{l}24-\text { Oct- } 05 \\
\text { 24-OC-0.5 }\end{array}$ & & RLNP & K0052 & CMS & SVOA & $85-68-7$ & alate & 10 \\
\hline $\begin{array}{lll}A 4650 \\
A 6650\end{array}$ & $\frac{199-k-21}{199-K-21}$ & $\begin{array}{l}\text { HEISPROD } \\
\text { HEISPROD }\end{array}$ & 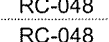 & $\begin{array}{l}110010 \\
\text { J110010 }\end{array}$ & GW & $\begin{array}{l}24-\text { Oct-05 } \\
\text { 24-OCt-05 }\end{array}$ & & $\frac{R L N P}{R L N P}$ & K0052 & $\begin{array}{l}\text { CMS } \\
\text { CMS }\end{array}$ & $\begin{array}{l}\text { SVOA } \\
\text { SVOOA }\end{array}$ & $\begin{array}{l}86-3-3-6 \\
86-73.7\end{array}$ & $\begin{array}{l}\text { Nitrosodiphenyl } \\
\text { Florene }\end{array}$ & 10 \\
\hline A4650 & 199-k-21 & HEISPROD & RC-048 & J150010 & GW & 24-oct-05 & & $\begin{array}{l}\text { RLNP } \\
\text { RLN }\end{array}$ & K0052 & CMS & SVOA & 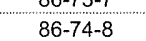 & Carbazole & $\frac{10}{10}$ \\
\hline A4650 & $199-\mathrm{k}-21$ & HEISPROD & RC-048 & $J 10010$ & $\mathrm{gw}$ & 24-oct-05 & & RLNP & K0052 & CMS & SVOA & $87.68 \cdot 3$ & diene & 10 \\
\hline A4650 & $199-k-21$ & HEISPROD & RC-048 & 110010 & GW & 24-Oct-05 & & RLNP & K0052 & CMS & SVOA & $87-86-5$ & nol & 25 \\
\hline A4650 & 199-K-21 & HEISPROD & RC-048 & $\begin{array}{l}110010 \\
110010\end{array}$ & GW & 24-.oct-05 & & RLNP & K0052 & CMS & SVOA & 88-06-2 & Trichlorophenol] & 10 \\
\hline A4t50 & | & HESPROD & 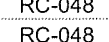 & $\begin{array}{l}510010 \\
J 10010\end{array}$ & GW & 24-0.0-10.05 & & RLNP & K05052 & ccs & SUAA & 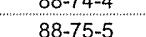 & 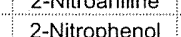 & 25 \\
\hline A4650 & $199-\mathrm{k}-21$ & HEISPROD & RC-048 & J10D10 & GW & 24-oct-05 & & RLNP & Ko052 & CMS & SVOA & 91-20-3 & Naphthalene & 10 \\
\hline A4650 & $199-k-21$ & HEISPROD & RC-048 & J10010 & GW & 24-oct-05 & & RLNP & K0052 & CMS & SVOA & $91-57-6$ & Methyrnaphthale & 0 \\
\hline A4650 & & & $\mathrm{RC}-048$ & 110010 & & 24-.0ct-05 & & RLNP & K0052 & CMS & SVOA & & Chloronaphthal & \\
\hline
\end{tabular}




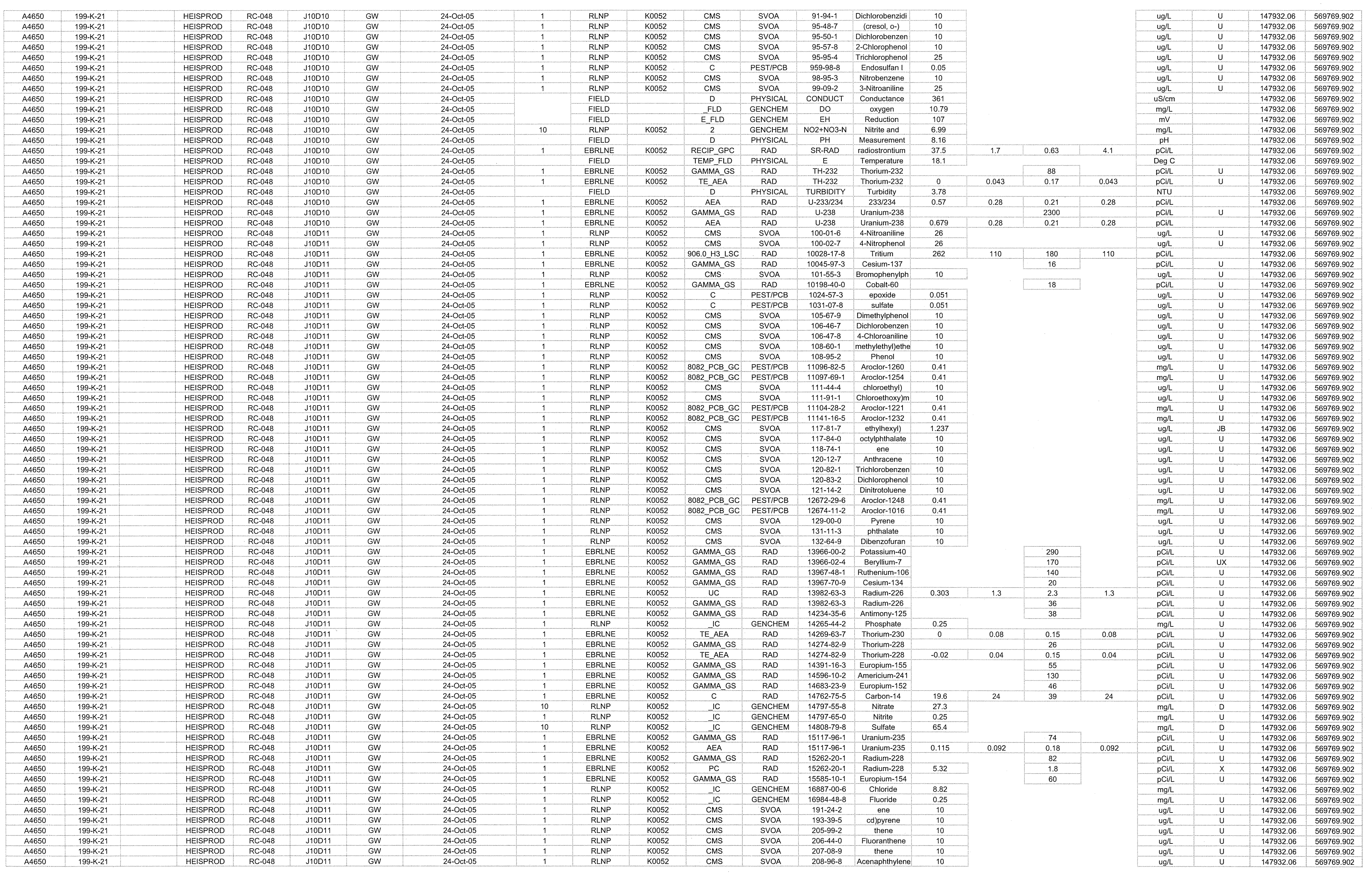




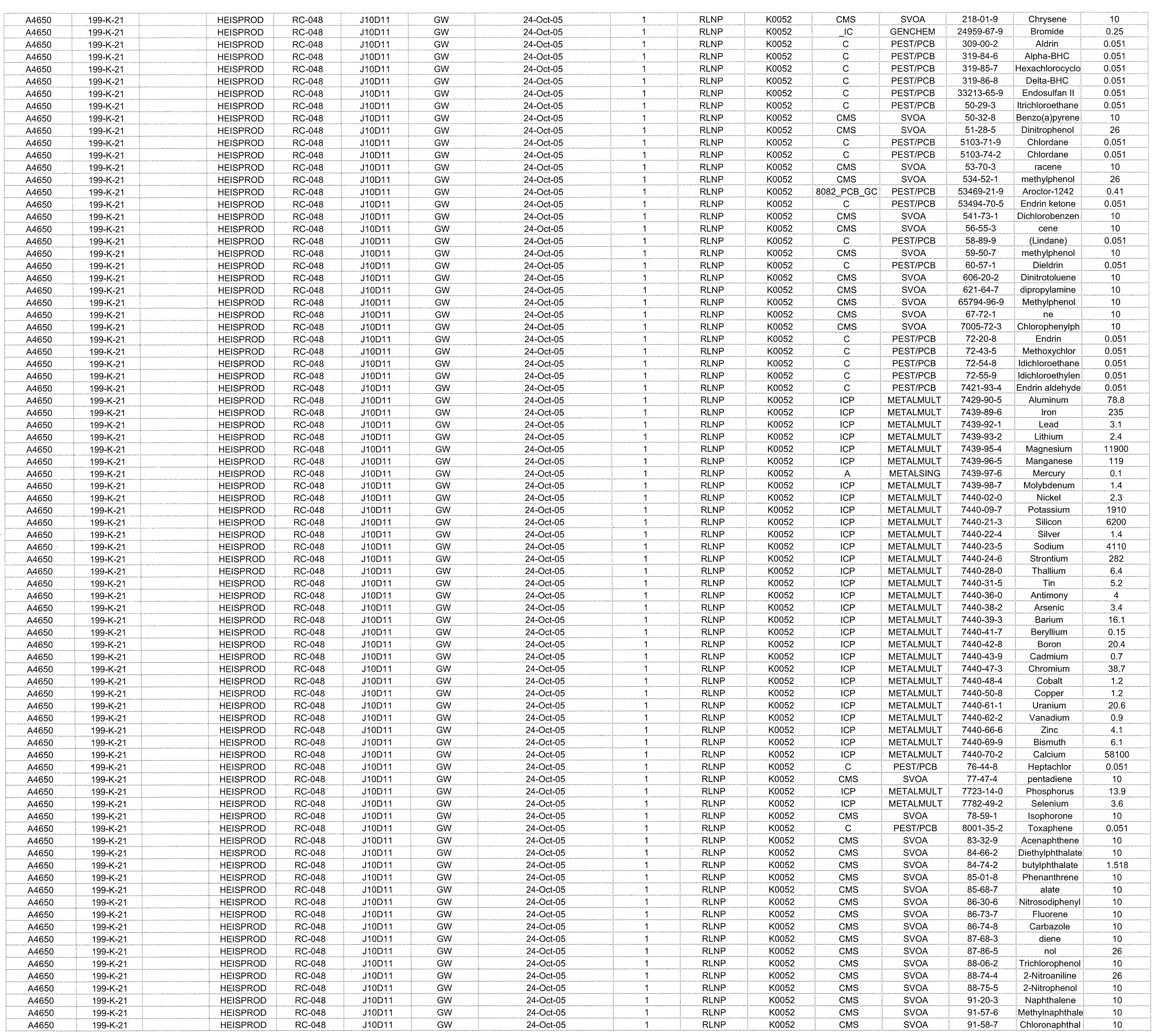




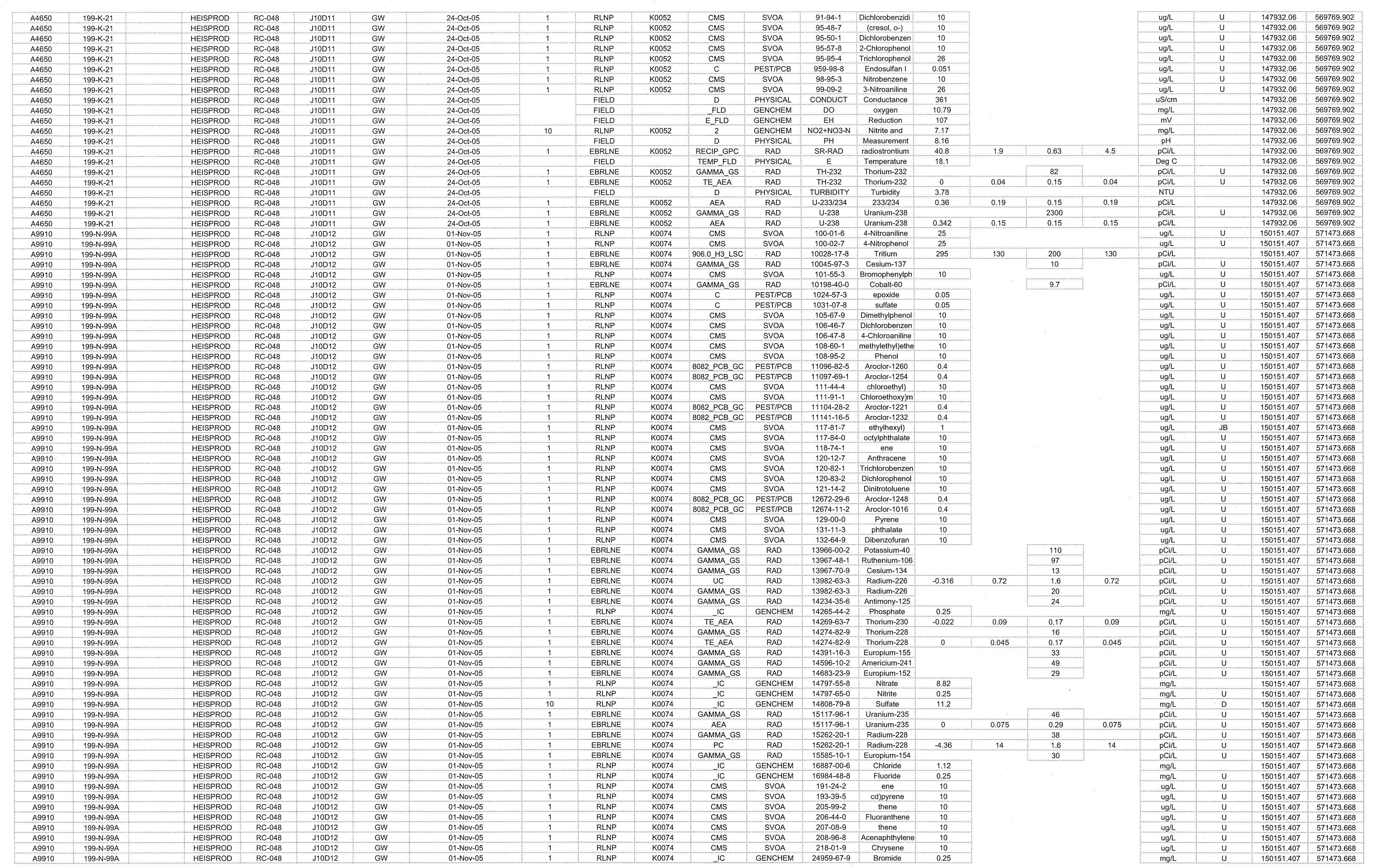



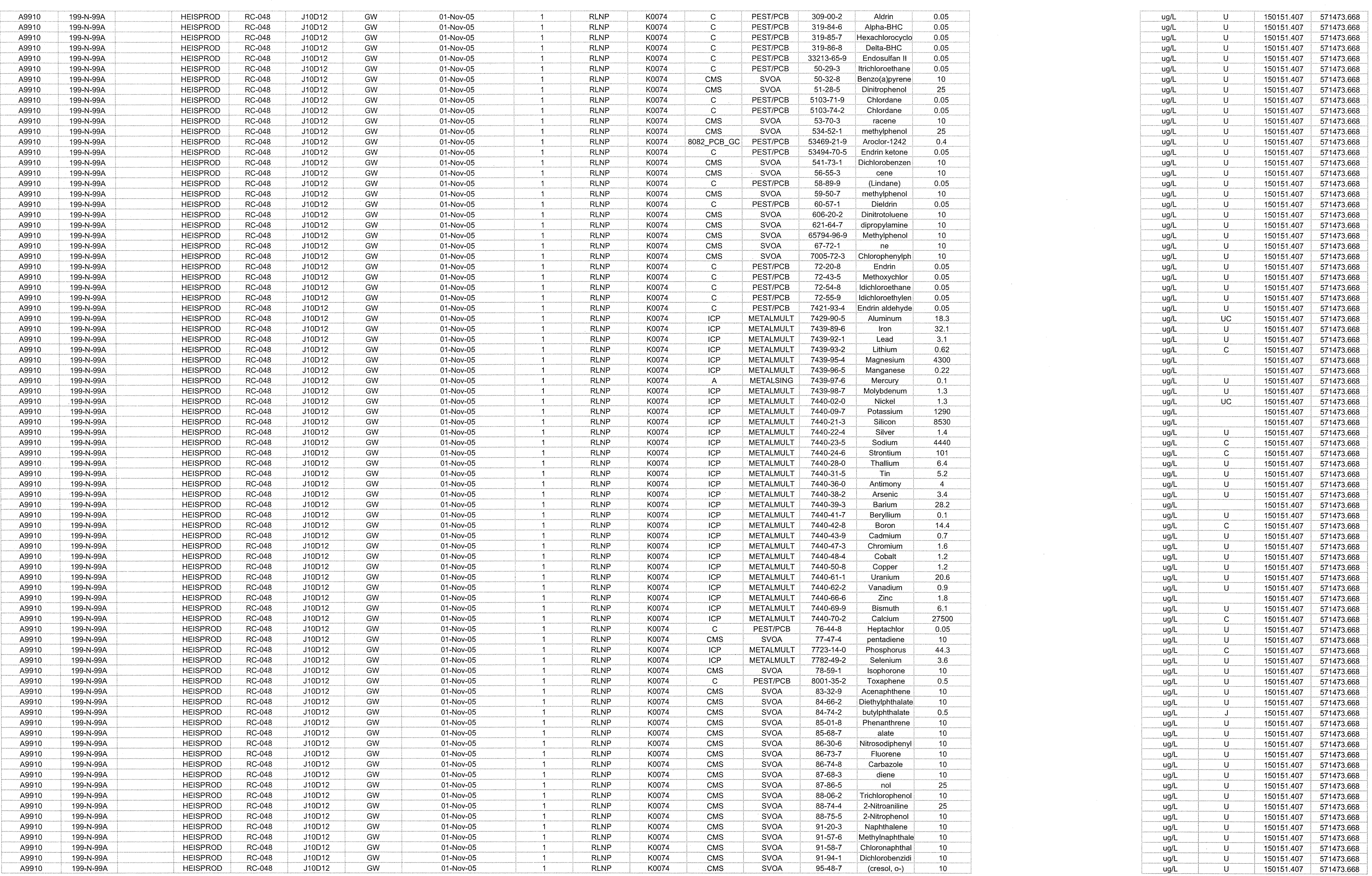


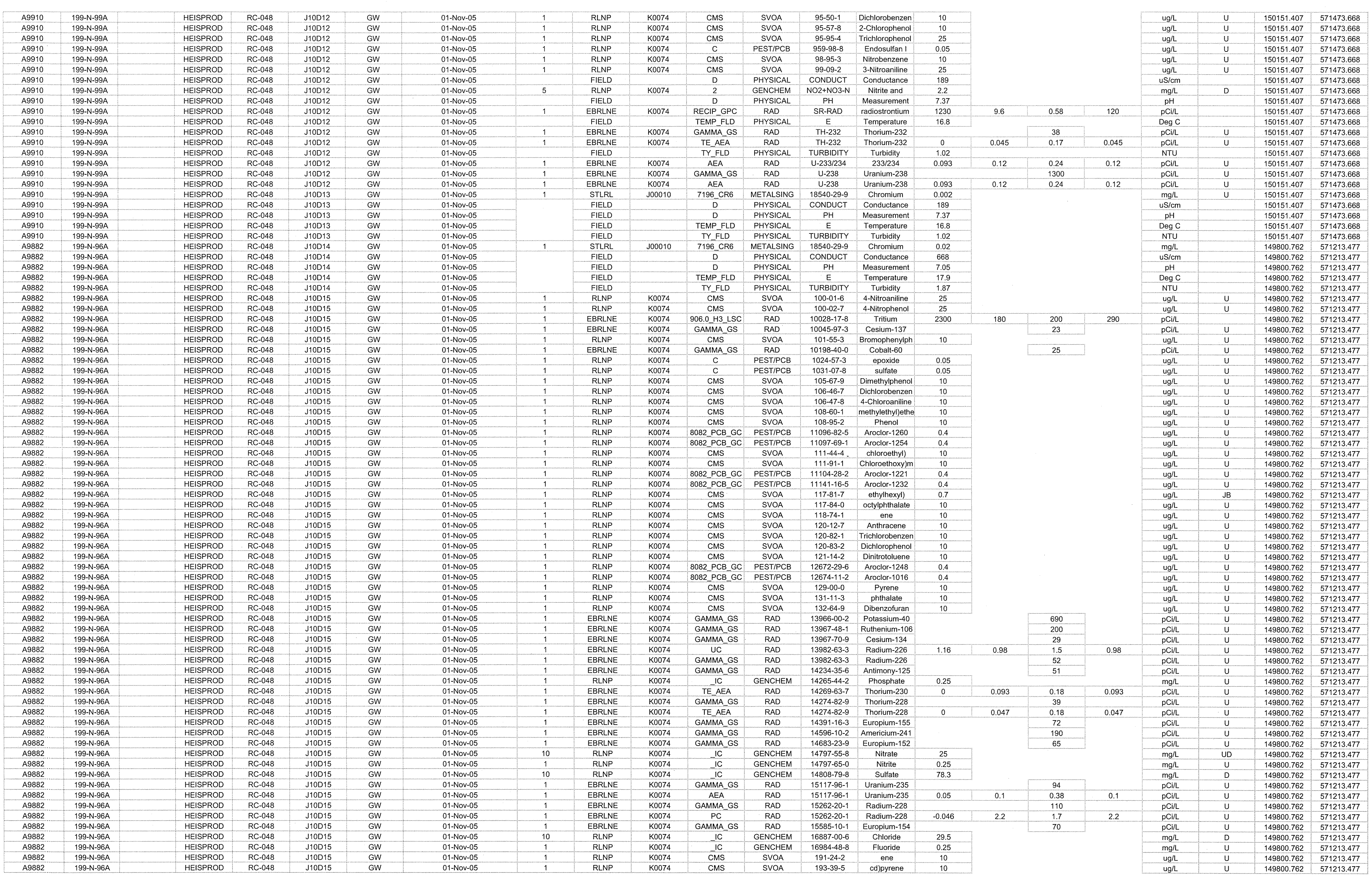



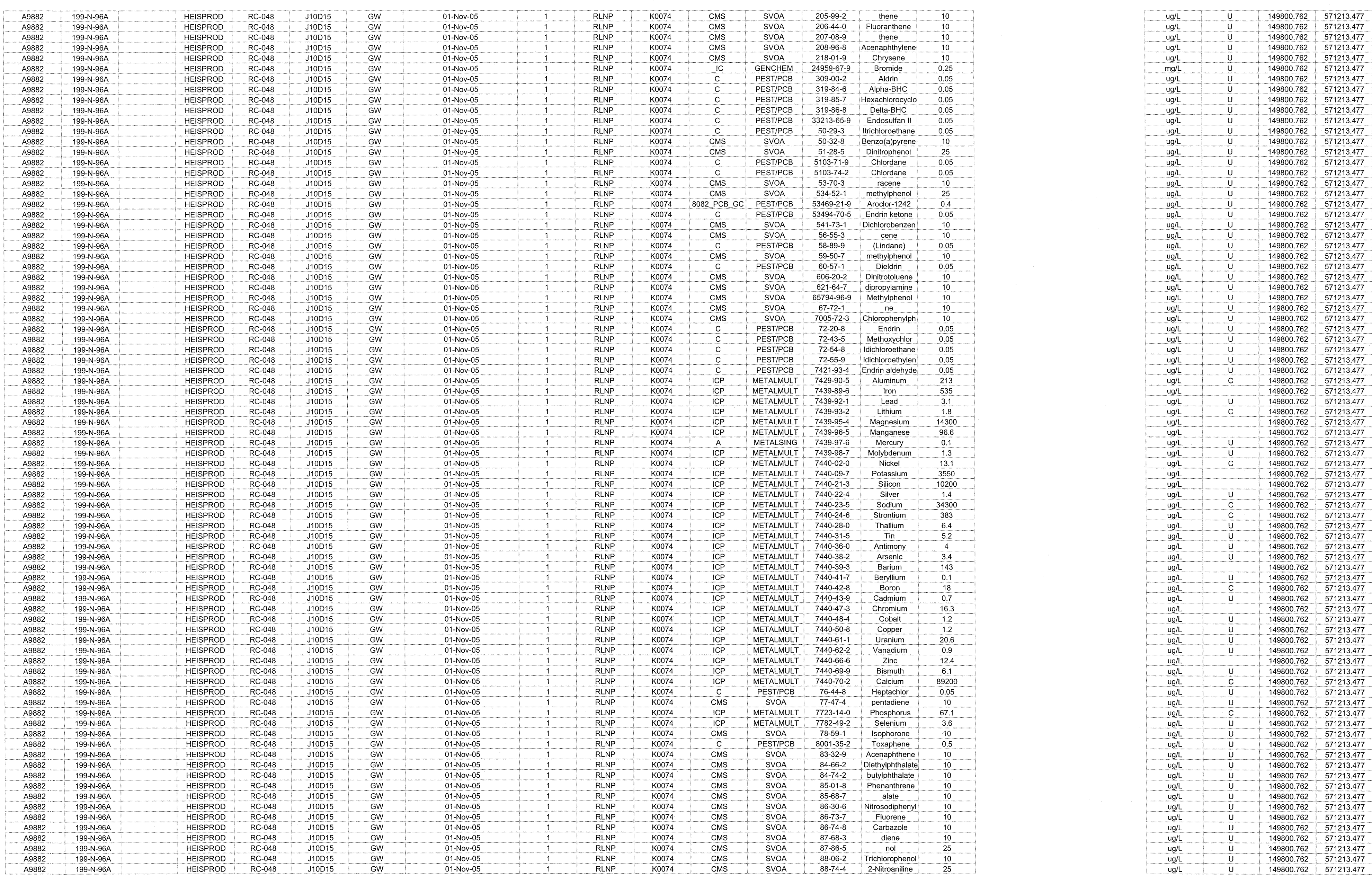


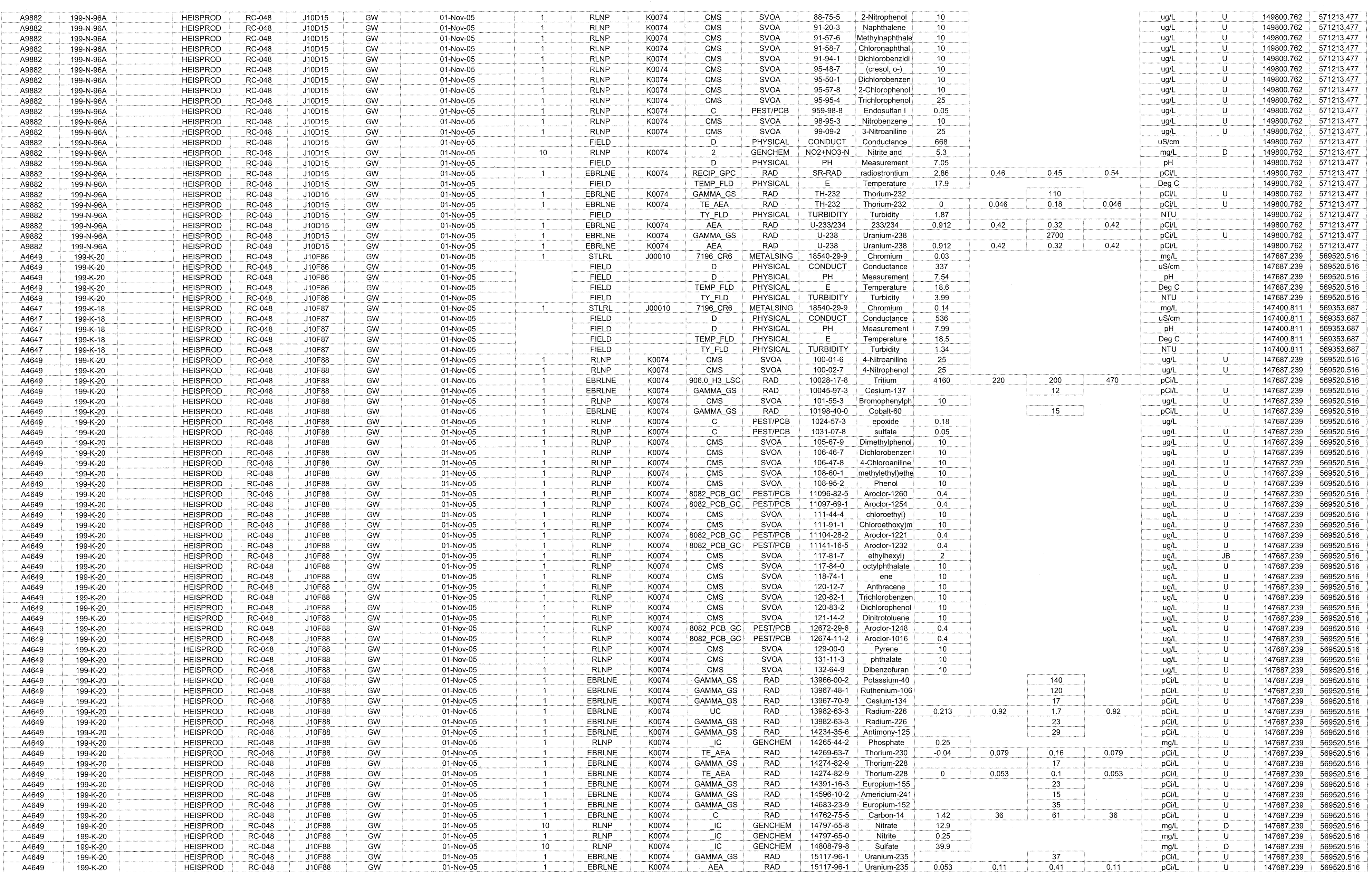



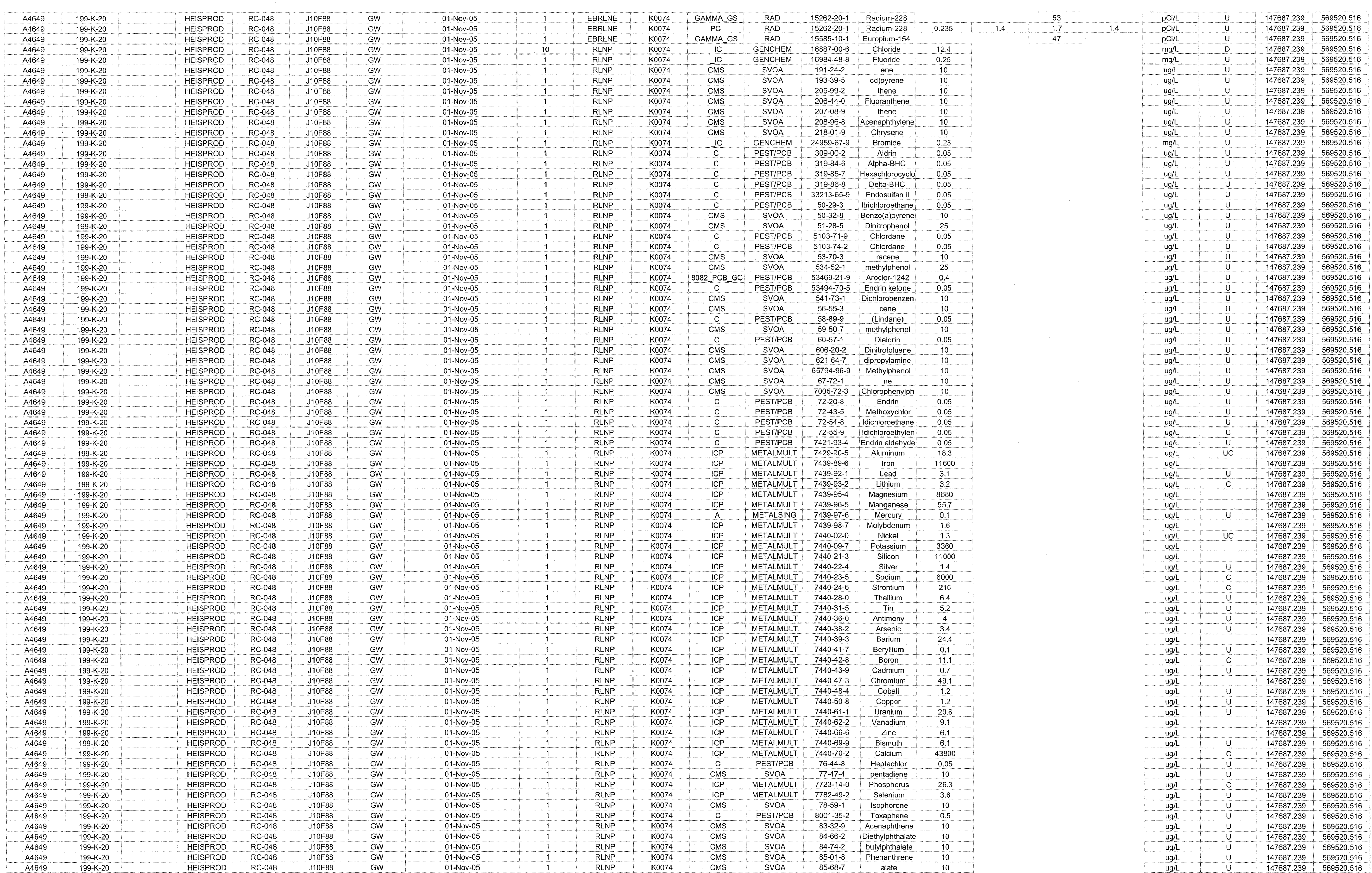


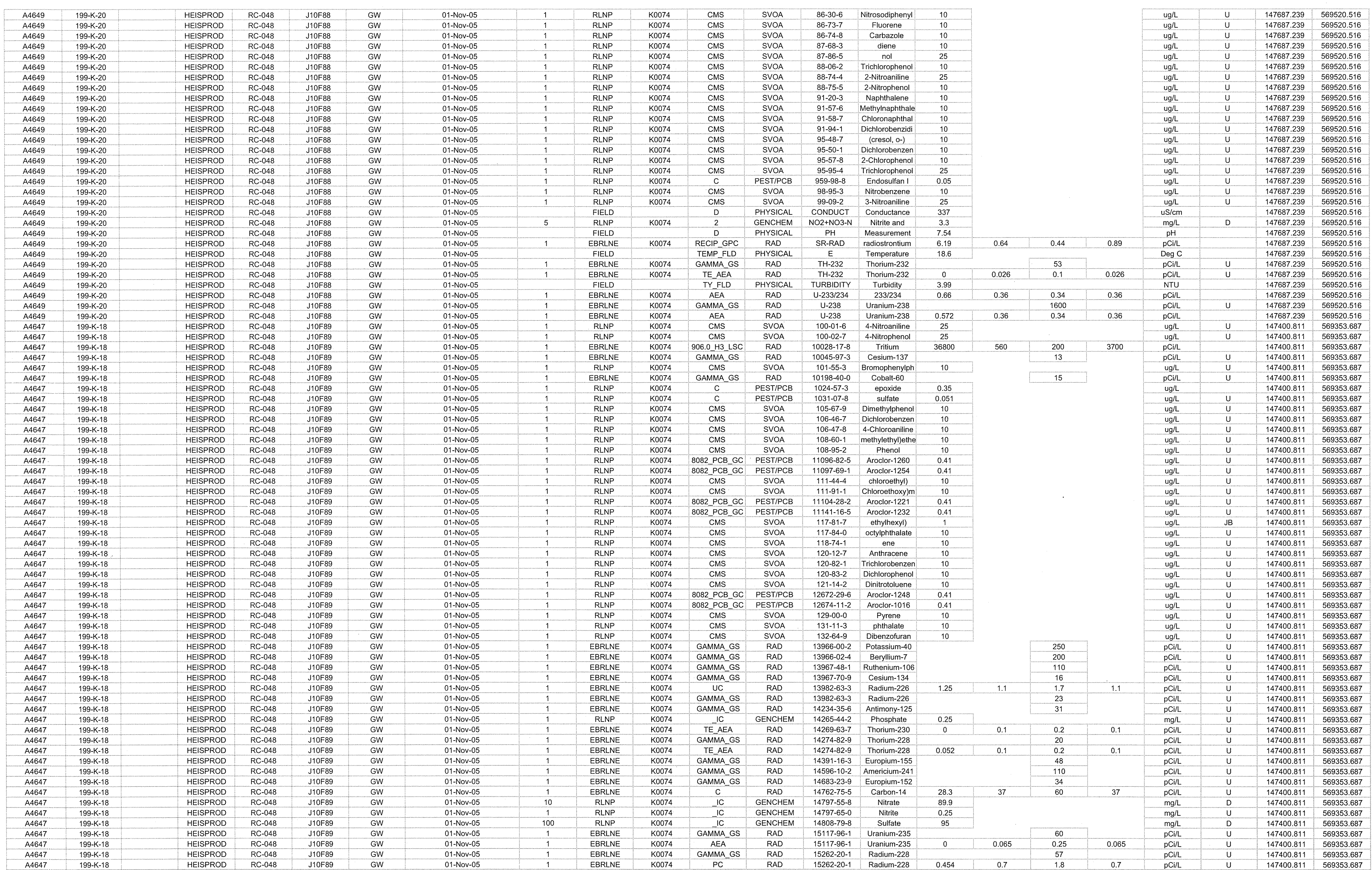



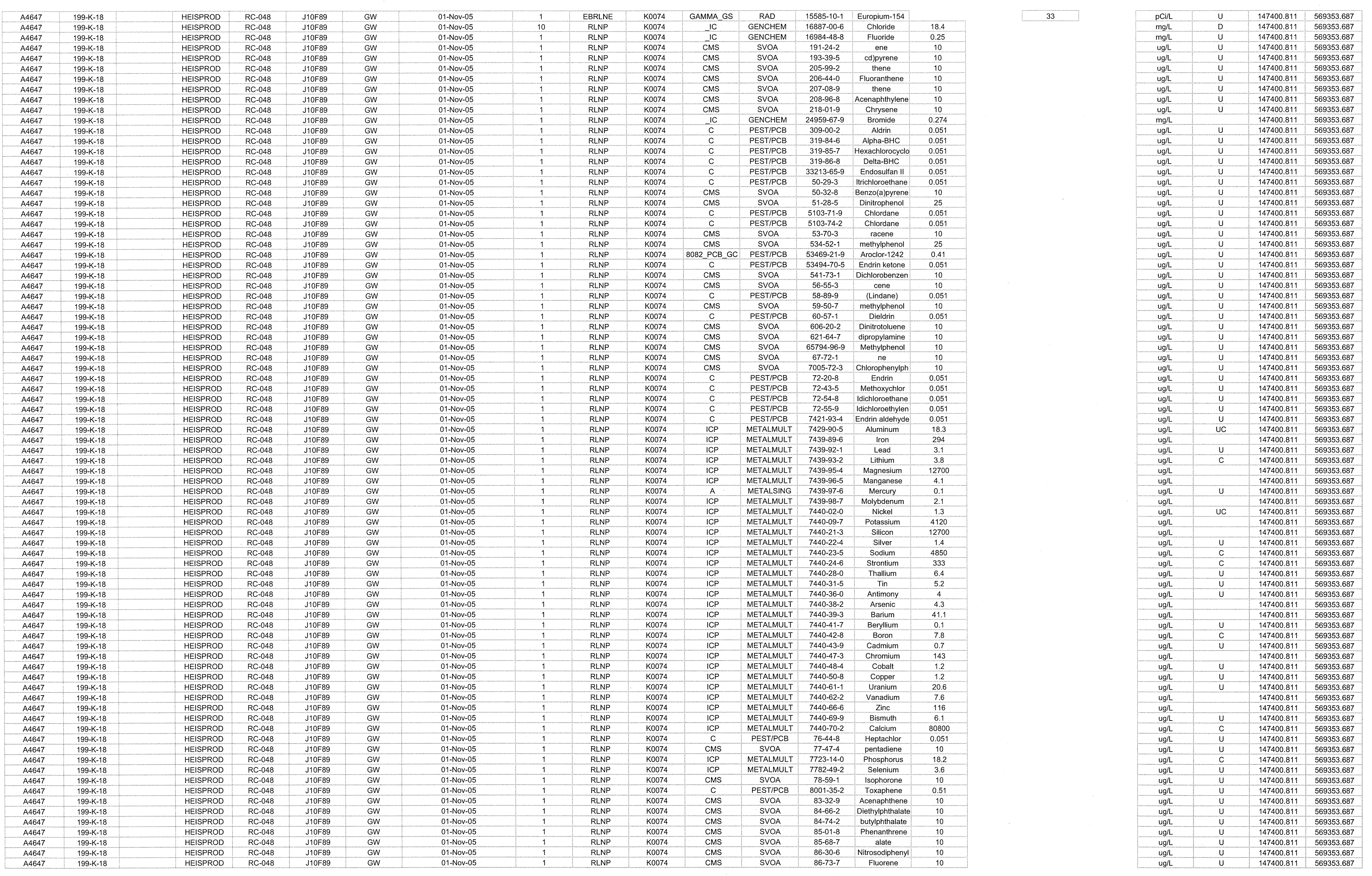


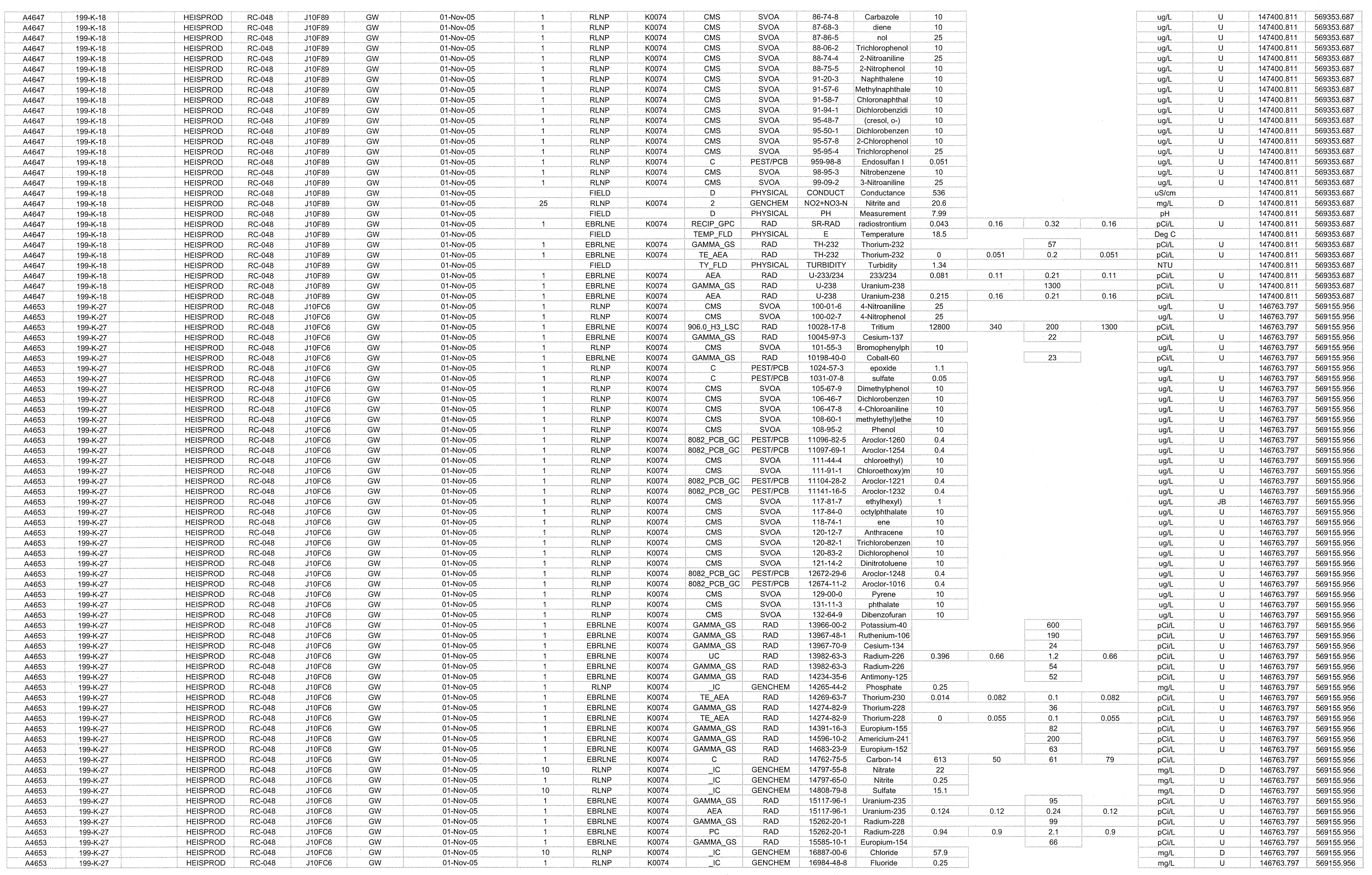




\begin{tabular}{|c|c|c|c|c|c|c|c|c|c|c|c|c|c|c|}
\hline $\begin{array}{l}\mathrm{A} 46653 \\
\mathrm{~A} 4653\end{array}$ & $\begin{array}{l}199-1-27 \\
199-K-27\end{array}$ & $\begin{array}{l}\text { HEIIPROD } \\
\text { HEISPROD }\end{array}$ & 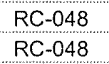 & $\begin{array}{l}\text { J10FCC } \\
\text { J10FC6 }\end{array}$ & $\frac{G W}{G W}$ & 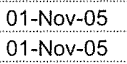 & & $\begin{array}{l}\frac{R L N P}{R L N P} \\
\text { RINP }\end{array}$ & K0074 & $\frac{\text { CMS }}{\text { CMS }}$ & $\begin{array}{l}\text { SVOA } \\
\text { SVOA }\end{array}$ & $\begin{array}{l}\frac{191-24-2}{193-39-5} \\
190\end{array}$ & $\begin{array}{l}\text { ene } \\
\text { colpryene }\end{array}$ & $\begin{array}{l}10 \\
10\end{array}$ \\
\hline A4653 & $199-K-27$ & HEISPROD & RC- 048 & J10FC6 & Gw & 年- Nov-05 & & RLNP & Koort & CMS & SVOA & $2005-99-2$ & thene & $\frac{10}{10}$ \\
\hline $\begin{array}{ll}A 4653 \\
\text { A4653 }\end{array}$ & $199-K-27$ & $\begin{array}{l}\text { HEISPROD } \\
\text { HEISPROD }\end{array}$ & $\begin{array}{l}\text { RC-048 } \\
\text { RC-048 }\end{array}$ & $\begin{array}{lll}\text { J10FC6 } \\
\text { J10FC }\end{array}$ & Gw & 01-Nov-05 & & RLNP & K0074 & CMS & SVOA & $206-44-0$ & Fluoranthene & 10 \\
\hline $\begin{array}{l}A 4653 \\
\text { A4653 }\end{array}$ & $\begin{array}{l}199-K-27 \\
199-K-27\end{array}$ & HEISPROD & RC-048 & $\begin{array}{lll}110 F C 6 \\
10565\end{array}$ & GW & 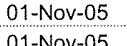 & & RLNP & K0074 & CMS & SVOA & $207-08-9$ & thene & 10 \\
\hline A4653 & $\begin{array}{l}199-k-27 \\
199-k-27\end{array}$ & $\begin{array}{l}\text { HEISPROD } \\
\text { HEISPROD }\end{array}$ & $\begin{array}{l}\text { RC-048 } \\
\text { RC-048 }\end{array}$ & $\begin{array}{l}\text { J10FCE } \\
\text { J10FC6 }\end{array}$ & $\frac{G W}{G W}$ & 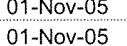 & & $\begin{array}{l}\text { RLNP } \\
\text { RINP }\end{array}$ & K0074 & $\begin{array}{l}\text { CMS } \\
\text { CMS }\end{array}$ & $\begin{array}{l}\text { SVOA } \\
\text { SVOA }\end{array}$ & $\begin{array}{l}208-96-8 \\
218-18-9\end{array}$ & $\begin{array}{l}\text { Accenaphitylene } \\
\text { chrosene }\end{array}$ & $\begin{array}{l}10 \\
10\end{array}$ \\
\hline A4653 & 1999-k-27 & HEISPROD & $\begin{array}{l}\text { RC-048 } \\
\text { RC-048 }\end{array}$ & 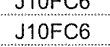 & GW & 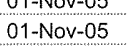 & & RLNP & Koort & 16 & GENCHEM & $24959-67-9$ & $\begin{array}{l}\text { Cinsune } \\
\text { Bromide }\end{array}$ & 0.25 \\
\hline A4653 & $199-k-27$ & HEISPROD & RC-048 & J10FC6 & GW & 01-Nov-05 & & RLNP & Ko074 & $c$ & PESTIPCB & $309-00-2$ & Aldrin & \\
\hline A4653 & $199-K-27$ & HEISPROD & RC-048 & J10FC6 & GW & 01-Nov-05 & & RLNP & K0074 & $\mathrm{c}$ & PESTIPCB & $319-84-6$ & Alpha-BHC & 0.05 \\
\hline $\begin{array}{l}\text { A46653 } \\
\text { A4653 }\end{array}$ & $\begin{array}{l}199-k-27 \\
199-k-27\end{array}$ & $\begin{array}{l}\text { HEISPROD } \\
\text { HEISPROD }\end{array}$ & $\begin{array}{l}\text { RC-048 } \\
\text { RC-048 }\end{array}$ & $\begin{array}{ll}\text { J110FC6 } \\
\text { J10FC } 6\end{array}$ & GW & $\begin{array}{l}\text { 01-Nov-05 } \\
\text { 01-1-0V-5 }\end{array}$ & & $\frac{R L N P}{R L N P}$ & K0074 & $\begin{array}{l}c \\
c \\
c\end{array}$ & $\begin{array}{l}\text { PEST/PCB } \\
\text { PEST/PCB }\end{array}$ & $\frac{319-85-7}{319-86-8}$ & $\begin{array}{l}\text { Hexachlorocycyco } \\
\text { Delta:BCC }\end{array}$ & 0.05 \\
\hline & & $\begin{array}{l}\text { HEISPROD } \\
\text { HEISPROD }\end{array}$ & $\begin{array}{l}\text { RC-048 } \\
\text { RC-048 }\end{array}$ & $\begin{array}{l}\text { JuFCL } \\
\text { J10FC }\end{array}$ & GW & 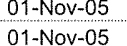 & & $\begin{array}{l}\text { RLNP } \\
\text { RLNP }\end{array}$ & K0074 & $\begin{array}{l}\mathrm{c} \\
\mathrm{c}\end{array}$ & $\begin{array}{l}\text { PESTPCB } \\
\text { PESTPCB }\end{array}$ & $\begin{array}{c}319-86-8 \\
3323-65-9\end{array}$ & $\begin{array}{l}\text { Delta-BHC } \\
\text { Endouffand }\end{array}$ & 0.05 \\
\hline A4653 & 1999-k-27 & HEISPROD & $\begin{array}{l}\text { RL-048 } \\
\text { RC- }-048\end{array}$ & J10FE & Gw & 年-Nove-05 & & RLNP & K0074 & $\mathrm{c}$ & PESTIPCB & $\frac{50-29-3}{50-3}$ & 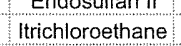 & 0.05 \\
\hline A4653 & $199-k-27$ & HEISPROD & RC-048 & J10FC6 & GW & 01-Nov-05 & & RLNP & K0074 & CMS & SVOA & $50-32-8$ & Benzo(a)pyrene: & 10 \\
\hline A4653 & $199-K-27$ & HEISPROD & RC-048 & J10FC6 & GW & 01-Nov-05 & & RLNP & K0074 & CMS & $\begin{array}{l}\text { SVOA } \\
\text { SVETPCB }\end{array}$ & $\begin{array}{l}51-28.5 \\
5102710\end{array}$ & Dinitrophenol & 25 \\
\hline $\begin{array}{l}A 4653 \\
A 4653\end{array}$ & $\begin{array}{l}199-k-27 \\
199-k-27\end{array}$ & $\begin{array}{l}\text { HEISPROD } \\
\text { HESPROD }\end{array}$ & $\begin{array}{l}\text { RC-048 } \\
\text { RC-048 }\end{array}$ & $\begin{array}{l}\text { J110FC6 } \\
\text { J10FC }\end{array}$ & GW & $\begin{array}{l}\text { 01-Nov-05 } \\
0 \text { 0-1-Nov-05 }\end{array}$ & & $\frac{R L N P}{R L P}$ & K0074 & $\frac{\mathrm{c}}{\mathrm{c}}$ & $\begin{array}{l}\text { PESTIPCB } \\
\text { PESTPCB }\end{array}$ & $\begin{array}{l}5103-71-9 \\
55103-7-2-2\end{array}$ & $\begin{array}{l}\text { Chlordane } \\
\text { Chlordane }\end{array}$ & 0.05 \\
\hline A4653 & $\begin{array}{l}1999-K-27 \\
199-K-27\end{array}$ & HEISPROD & $\begin{array}{l}\text { RC-048 } \\
\text { RC-048 }\end{array}$ & $\begin{array}{l}\text { JuFC } \\
\text { J10FC6 }\end{array}$ & GW & 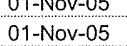 & & $\begin{array}{l}\text { RLN } \\
\text { RLNP }\end{array}$ & Koort & CMS & SVOA & $\begin{array}{l}5103-1-1-2-2 \\
53-70-3\end{array}$ & $\begin{array}{l}\text { Chlordane } \\
\text { racene }\end{array}$ & $\frac{0.05}{10}$ \\
\hline A4653 & 199-k-27 & HEISPROD & RC-048 & J10FCG & GW & 01-Nov-05 & & RLNP & K0074 & CMS & SVOA & $534-52-1$ & $\begin{array}{l}\text { methyiphenol } \\
\text { ment }\end{array}$ & 25 \\
\hline A4653 & $199-k-27$ & HEISPROD & RC-048 & J10FCG & GW & 01-Nove-05 & & RLNP & K0074 & 8082 PCB_GC & PEST/PCB & $53469-21-9$ & Aroclor-1242 & 0.4 \\
\hline $\begin{array}{ll}A 4653 \\
A 4653\end{array}$ & $199-K-27$ & HEISPROD & RC-048 & 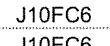 & GW & 01-Nov-05 & & RLNP & K0074 & $c$ & PESTIPCB & 534944-70-5 & Endrin ketone & 0.05 \\
\hline$\frac{14663}{A 4653}$ & $\frac{199-2-27}{199-K-27}$ & $\begin{array}{l}\text { HEIIPRODD } \\
\text { HEISPROD }\end{array}$ & $\begin{array}{l}\text { RC- }-048 \\
\text { RC-048 }\end{array}$ & $\begin{array}{l}\text { J10FC } \\
\text { J10FC }\end{array}$ & $\begin{array}{l}G W \\
G W\end{array}$ & $\begin{array}{l}\text { O1-Nov-05 } \\
\text { 01-Nov-05 }\end{array}$ & & $\begin{array}{l}\text { RLNP } \\
\text { RLN }\end{array}$ & K0074 & $\begin{array}{l}\text { CMS } \\
\text { CMS }\end{array}$ & $\begin{array}{l}\text { SVOA } \\
\text { SVOA }\end{array}$ & $\frac{541-73-1}{56-55-3}$ & Dichlorobenzen & 10 \\
\hline A4653 & $\begin{array}{l}199-K-27 \\
199-K-27\end{array}$ & HEISPROD & $\begin{array}{ll}R C-048 \\
R C-048\end{array}$ & $\begin{array}{l}\mathrm{JuFC6} \\
\mathrm{J10FC6}\end{array}$ & GW & 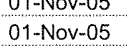 & & $\begin{array}{l}\text { RLN } \\
\text { RLNP }\end{array}$ & Koort & $\mathrm{c}$ & PEST/PCB & $\begin{array}{l}50-35-5 \\
58-89-9\end{array}$ & \begin{tabular}{|l|l} 
(Linnene \\
\end{tabular} & $\begin{array}{l}10 \\
0.05\end{array}$ \\
\hline A4653 & $\begin{array}{l}199-k-k-27 \\
199-K-27\end{array}$ & HEISPROD & $\begin{array}{l}\text { RL-048 } \\
\text { RC-048 }\end{array}$ & J10FC6 & Gw & 年-Nov-05 & & RLNP & K0074 & CMS & SVOA & 59-50-7 & $\begin{array}{l}\text { (uncare) } \\
\text { methylphenol }\end{array}$ & 10 \\
\hline A4653 & $1999-k-27$ & HEISPROD & RC-048 & J10FC6 & GW & 01-Nov-05 & & RLNP & Ko074 & $c$ & PESTIPCB & $60-57-1$ & Dieldrin & 0.05 \\
\hline A4653 & $199-\mathrm{K}-27$ & HEISPROD & RC-048 & 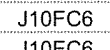 & GW & 01-Nov-05 & & RLNP & K0074 & CMS & SVOA & $606-20-2$ & Dinitrotoluene & 10 \\
\hline $\begin{array}{ll}A 4653 \\
A 4635\end{array}$ & 199-K-27 & HEISPROD & 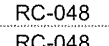 & $\begin{array}{ll}\text { J110FC6 } \\
\end{array}$ & GW & 01-Nov-05 & & RLNP & K0074 & CMS & SVOA & $621-64-7$ & dipropylamine & 10 \\
\hline $\begin{array}{l}\text { A46653 } \\
\text { A4653 }\end{array}$ & $\frac{199--1-27}{199-k 27}$ & $\begin{array}{l}\text { HEISPROD } \\
\text { HEISPROD }\end{array}$ & $\begin{array}{l}\text { RC-048 } \\
\text { RC-048 }\end{array}$ & $\begin{array}{l}\text { J10FCG } \\
J 10 F C 6\end{array}$ & GW & 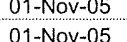 & & RLNP & K0074 & $\begin{array}{l}\text { CMS } \\
\text { CMS }\end{array}$ & $\begin{array}{l}\text { SSOA } \\
\text { SVOA }\end{array}$ & $\begin{array}{l}65794-96-9 \\
67-72-1\end{array}$ & $\begin{array}{l}\text { Methylphenol } \\
\text { ne }\end{array}$ & $\frac{10}{10}$ \\
\hline $\begin{array}{l}A 46653 \\
A 4653\end{array}$ & 199-k-k-27 & HEISPROD & RC-048 & J10FC 6 & ow & 11-Nov-05 & & RLNP & $\begin{array}{l}\text { Koort4 } \\
\text { Koor }\end{array}$ & CMS & SVOA & $7005-72-3$ & Chlorophenyiph & 10 \\
\hline A4653 & $1999-K-27$ & HEISPROD & RC-048 & J10FC6 & Gw & 01-Nov-05 & & RLNP & K0074 & $c$ & PEST/PCB & $72-20-8$ & Endrin & 0.05 \\
\hline A4653 & $199-K-27$ & HEISPROD & RC-048 & J10FC6 & GW & 01-Nov-05 & & RLNP & K0074 & $c$ & PEST/PCB & $72-43-5$ & Methoxychlor & 0.05 \\
\hline $\begin{array}{l}A 4653 \\
453\end{array}$ & $199-k-27$ & $\begin{array}{l}\text { HEISPROD } \\
\text { HITPODF }\end{array}$ & 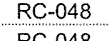 & J10FC6 & $G W$ & 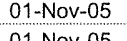 & & RLNP & $\begin{array}{l}K 0074 \\
K\end{array}$ & $c$ & $\begin{array}{l}\text { PEETIPCB } \\
\text { PFTPC }\end{array}$ & $\begin{array}{l}72.54-8 \\
725-8\end{array}$ & Ilichloroethane & 0.05 \\
\hline $\begin{array}{l}A_{44653} \\
4463\end{array}$ & $\begin{array}{l}199-27 \\
190.27-27\end{array}$ & $\begin{array}{l}\text { HESPROD } \\
\text { HESPROD }\end{array}$ & 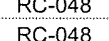 & 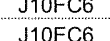 & $G w$ & 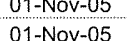 & & RINP & 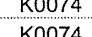 & $c$ & FEIPCB & $\frac{12-55-9}{742034}$ & 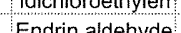 & 0.055 \\
\hline A4t553 & 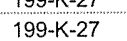 & HEISPROD & $\frac{1 C-048}{R C-048}$ & J10FCG & & - & & RLN & Ko074 & ICP & $\begin{array}{l}\text { PESTIPCB } \\
\text { METAMUT }\end{array}$ & $\begin{array}{l}7241-39-4 \\
7720 .-5\end{array}$ & Endrin aldehyde & 0.055 \\
\hline A4653 & $199-k-27$ & HEISPROD & RC-048 & J10FCG & GW & 01-Nov-05 & & RLNP & Koo74 & ICP & $\begin{array}{l}\text { MEALTLUULT } \\
\text { METALTT }\end{array}$ & 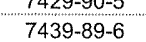 & Autminum & $\frac{18.3}{67}$ \\
\hline A4653 & $1999-\mathrm{K}-27$ & HEISPROD & RC-048 & J10FC6 & GW & 01-Nov-05 & & RLNP & K0074 & ICP & METALMULT & 74339-92-1 & Lead & $\frac{3.1}{3.1}$ \\
\hline A4653 & $199-\mathrm{K}-27$ & HEISPROD & RC-048 & J10FC6 & GW & 01-Nov-05 & & RLNP & K0074 & ICP & $\begin{array}{l}\text { METALMULT } \\
\end{array}$ & 7439-93-2 & Lithium & 5.5 \\
\hline A4653 & 199-k-27 & HEISPROD & RC-048 & J10FC6 & GW & 01-Nov-05 & & RLNP & K0074 & $1 C P$ & METALMULT & $7439-95-4$ & Magnesium & 13900 \\
\hline $\begin{array}{l}A_{44653} \\
A 4653\end{array}$ & $\begin{array}{l}199-k-27 \\
190.27\end{array}$ & $\begin{array}{l}\text { HESPROD } \\
\text { HESPROD }\end{array}$ & 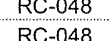 & 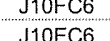 & $\frac{6 w}{G w}$ & 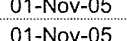 & & RINP & K0074 & ICP & METLAMULT & $\begin{array}{l}734996-6-5 \\
732906\end{array}$ & Manganese & 0.92 \\
\hline A46653 & 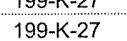 & HESPROD & $\begin{array}{l}\text { RC-048 } \\
\text { RC-048 }\end{array}$ & STHFC & Gw & 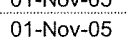 & & RLNP & Konor4 & ${ }_{1 C P}$ & METALMULT & 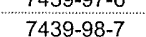 & 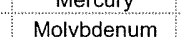 & $\frac{0.1}{13}$ \\
\hline A4653 & $199-k-27$ & HEISPROD & RC-048 & J10FCG & GW & 01-Noy-05 & & RINP & K0074 & ICP & METALMULT & $7400-02-0$ & Nickel & 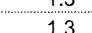 \\
\hline A4653 & $199-k-27$ & HEISPROD & RC-048 & J10FC6 & GW & 01-Nov-05 & & $\frac{R L N P}{R ! N P}$ & Ko074 & ICP & METALMULT & $\begin{array}{l}74400-20-0 \\
7440-09-7\end{array}$ & Potassium & 5080 \\
\hline A4653 & $199-k-27$ & HEISPROD & RC-048 & J10FC6 & ow & 01-Nov-05 & & RLNP & Ko074 & $\mathrm{ICP}$ & $\begin{array}{l}\text { METALMULT } \\
\end{array}$ & 7440-21-3 & Silicon & 12300 \\
\hline & & & & & & & & & & $1 C P$ & METALMULT & $7440-22-4$ & Silver & 1.4 \\
\hline A4653 & $199 \cdot-k-27$ & HEISPROD & RC-048 & J10FC6 & Gw & 01-Nov-05 & & RLNP & K0074 & ICP & METALMULT & $7440-23-5$ & Sodium & 8420 \\
\hline A4653 & $199-K-27$ & HESPRPOD & RC-048 & J10FC6 & GW & 01-Nov-05 & & RLNP & K0074 & ICP & $\begin{array}{l}\text { METALMULT } \\
\text { METMLT }\end{array}$ & $7440-24-6$ & Strontium & 306 \\
\hline A45053 & 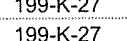 & $\begin{array}{l}\text { MESRROD } \\
\text { HESPROD }\end{array}$ & 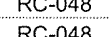 & 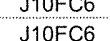 & Giv & 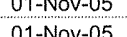 & & 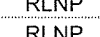 & Kovort & 种 & $\begin{array}{l}\text { MEIALMULT } \\
\text { METAMUIT }\end{array}$ & 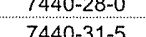 & Thalum & $\begin{array}{c}6.4 \\
5.4\end{array}$ \\
\hline A4t50 & 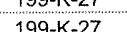 & HEISTROD & BC.048 & 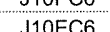 & Giw & (2) & & BIN & K0074 & 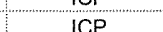 & METAMULIT & $7400-36-0$ & (n) & 5.2 \\
\hline A4653 & 1999-k-27 & HEISPROD & RC-048 & J10Fce & GW & 01-Nov-05 & & RINP & K0074 & ICP & METALMULT & $7440-38-2$ & A Assenic & $\begin{array}{l}4 \\
35\end{array}$ \\
\hline A4653 & $1999-k-27$ & HEISPROD & RC-048 & J10FC6 & Gw & 01-Nov-05 & & RLNP & K0074 & ICP & METALMULT & 7440-39-3 & $\begin{array}{l}\text { A Astellic } \\
\text { Barium }\end{array}$ & 32.6 \\
\hline A4653 & $199 \cdot-k-27$ & HEISPROD & RC-048 & J10FC6 & GW & 01-Nov-05 & & RLNP & K0074 & ICP & METALMULT & 7440-41-7 & Beryllium & 0.1 \\
\hline $\begin{array}{l}A 4653 \\
A 653\end{array}$ & $199-K-27$ & HEISPROD & RC-048 & J110FC6 & GW & 01-Nov-05 & & RLNP & K0074 & ICP & METALMULT & 7440-42-8 & Boron & 54.1 \\
\hline A4653 & $\begin{array}{l}199-1-27 \\
100-2-27\end{array}$ & $\begin{array}{l}\text { HEISPROD } \\
\text { HIFCDD }\end{array}$ & $\begin{array}{l}\text { RC- }-048 \\
\text { RC }\end{array}$ & J10FC6 & GW & 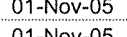 & & RLNP & K0074 & ICP & METALMULT & 7440-43-9 & Cadmium & 0.7 \\
\hline $\begin{array}{l}\text { A4653 } \\
\text { A4553 }\end{array}$ & $\begin{array}{l}\frac{199-9-27}{199-k-27} \\
10\end{array}$ & HESPROD & 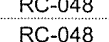 & $\begin{array}{l}\text { JiterC6 } \\
\text { J10FC }\end{array}$ & GW & 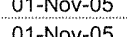 & & RLNP & K0074 & ICP & $\begin{array}{l}\text { METAMLULT } \\
\text { METLLT }\end{array}$ & 7440-47-3.3 & Chromium & 2.4 \\
\hline A4toss & 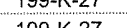 & HESPROD & RC-048 & sures & w & & & RLN & Kovory & 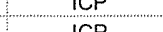 & MEIALMULI & $1740-48-4$ & & 1.2 \\
\hline A4b53 & $199-k-27$ & HEISPROD & RC-048 & S sures & ow & S & & RLNP & K0074 & Wh & METALMULT & $7440-50-8$ & Copper & 1.2 \\
\hline A4653 & $199-k-27$ & HESPROD & RC-048 & J10FC6 & GW & 01-Nov-05 & & RLNP & K0074 & ICP & METALMULT & $7440-661-1$ & Uranium & 20.6 \\
\hline 等4563 & $190-k-2 / 2$ & HESPROOU & KC-048 & Norec & Gw & & & RLN & Kovory & (WT & MEIALMULI & $7440-62-2$ & Vanaluam & 5.5 \\
\hline A4bs3 & $199-\mathrm{k}-27$ & HEISPROD & RC-048 & siveles & ow & 01-Nov-05 & & RLNP & Rourt & & METALWULLT & $7440-66-6$ & Zinc & 2.4 \\
\hline A4653 & $199 \cdot-27$ & HESPPROD & RC-048 & J10FCE & GW & 01-Nov-05 & & RLNP & K0074 & ICP & METALMULT & $7440-69-9$ & Bismuth & 6.1 \\
\hline 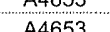 & 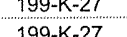 & HEISPROD & BC.048 & (1)ECG & Gw & & & STN & Kovit & TF & & 76-414-8 & 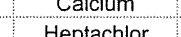 & 65000 \\
\hline A4655 & 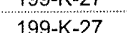 & HEISPPOD & Bcos & 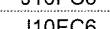 & wiw & 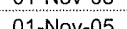 & & BiN & K0174 & CMS & sones & 77.40 & enplachior & 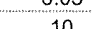 \\
\hline A4653 & 10007 & HEFTSPOD & Bcusto & toreco & Giw & O1: Nove & & Ner & novit & sos & STro & $77-47-4$ & pentaciene & 10 \\
\hline 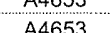 & $199-1-27$ & HEISTROD & RC-048 & (1)ECG & . & & & RINP & 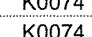 & we & 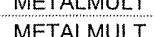 & $\begin{array}{l}77233-14-0 \\
7230-0\end{array}$ & Phosphorus & $\frac{16.6}{6.6}$ \\
\hline A4653 & $109 \times-27$ & HEISPROD & RC-048 & 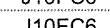 & $G W$ & & & PIN & K0071 & cMs & & $782-40-2$ & Selentium & 3.6 \\
\hline A6453 & $199-\mathrm{K}-27$ & HEISPROD & RC-048 & J10FC6 & GW & 01-Nov-05 & & RiNP & K0074 & $\frac{c_{0}}{c}$ & PESTPCB & $\frac{80-59-1}{8001-35-2}$ & 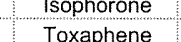 & 10 \\
\hline A4653 & $199-K-27$ & HEISPROD & RC- -48 & J10FC6 & GW & 01-Nov-05 & & RLNP & K0074 & CMS & SOAA & $83-32-9$ & Accanonthe & 0.5 \\
\hline A4653 & $199-K-27$ & HEISPROD & RC-048 & J10FC6 & GW & & & RLNP & Ko074 & CMS & & & 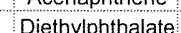 & 10 \\
\hline A4653 & $199-k-27$ & HEISPROD & RC-048 & J10FC6 & GW & 01-Nov-05 & & RLNP & Ko074 & cMS & SVOA & $84-74-2$ & butylyhthalate & 0.7 \\
\hline A4653 & $199-K-27$ & HEISPROD & RC-048 & J10FC6 & GW & 01-Nov-05 & & RLNP & K0074 & CMS & SVOA & & Penenanthrene & 10 \\
\hline A4653. & $199-k-27$ & HEISPROD & RC-048 & J10FC6 & GW & 01-Nov-05 & & RLNP & K0074 & CMS & SVOA & $85-68-7$ & alate & 10 \\
\hline A4653 & $199-K-27$ & HEISPROD & RC-048 & J10FC6 & GW & 01-Nov-05 & & RLNP & K0074 & CMS & SVOA & $86-30-6$ & Nitrosodiphenyl & 10 \\
\hline A4653 & $199-k-27$ & HEISPROD & RC-048 & J10FC6 & GW & 01-Nov-05 & & RLNP & Koo & CMS & SVOA & & Fluorene & 10 \\
\hline A4653 & $199-K-27$ & $\begin{array}{l}\text { HESTSROD } \\
\text { HITSODOD }\end{array}$ & $\begin{array}{ll}R C-048 \\
R C\end{array}$ & J10FC6 & GW & 01-Nov-05 & & RLNP & K0074 & CMS & SVOA & $\begin{array}{l}86-74-8 \\
80-8\end{array}$ & Carbazole & 10 \\
\hline $\begin{array}{l}\frac{14663}{A 4653} \\
A\end{array}$ & $\begin{array}{l}199-k-k 7 \\
199-k 27\end{array}$ & $\begin{array}{l}\text { HEISPROD } \\
\text { HEISPROD }\end{array}$ & $\begin{array}{l}\text { RC-048 } \\
\text { RC-048 }\end{array}$ & $\begin{array}{l}\text { 110FC6 } \\
\text { J10FC6 }\end{array}$ & $\frac{G W}{G W}$ & $\begin{array}{l}01 \text {-Novos } 05 \\
01-\text { Nov-05 }\end{array}$ & & $\begin{array}{l}\text { RLLPP } \\
\text { RLNP }\end{array}$ & K0074 & $\begin{array}{l}\text { CMS } \\
\text { CMS }\end{array}$ & $\begin{array}{l}\text { SVOA } \\
\text { SVOA }\end{array}$ & $\begin{array}{l}87-768-3 \\
87-86-5\end{array}$ & $\begin{array}{l}\text { diene } \\
\text { nol }\end{array}$ & $\begin{array}{l}10 \\
25\end{array}$ \\
\hline
\end{tabular}

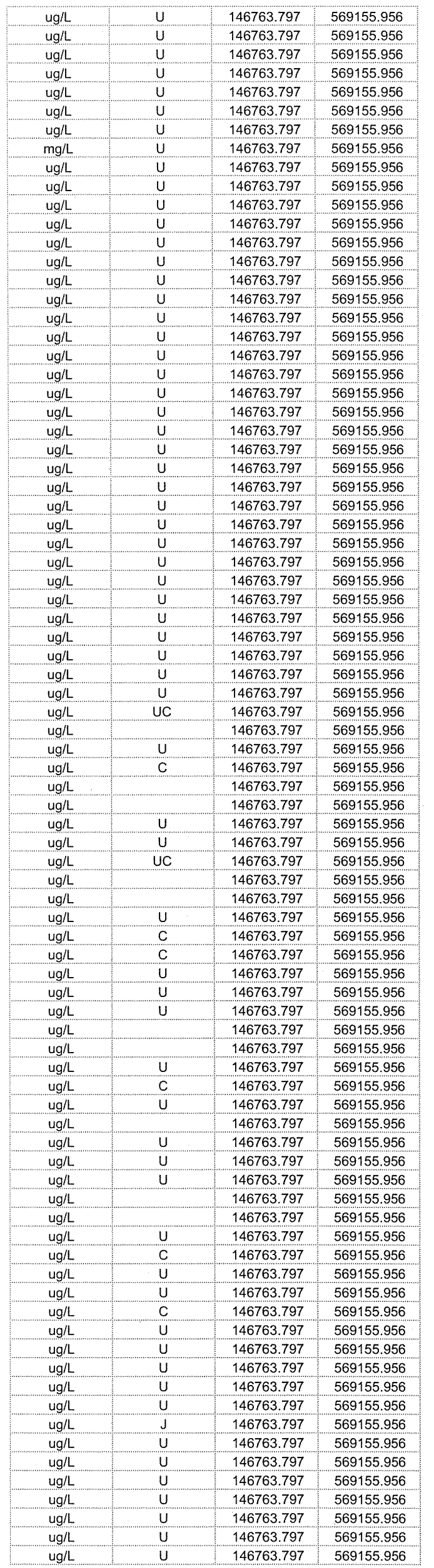




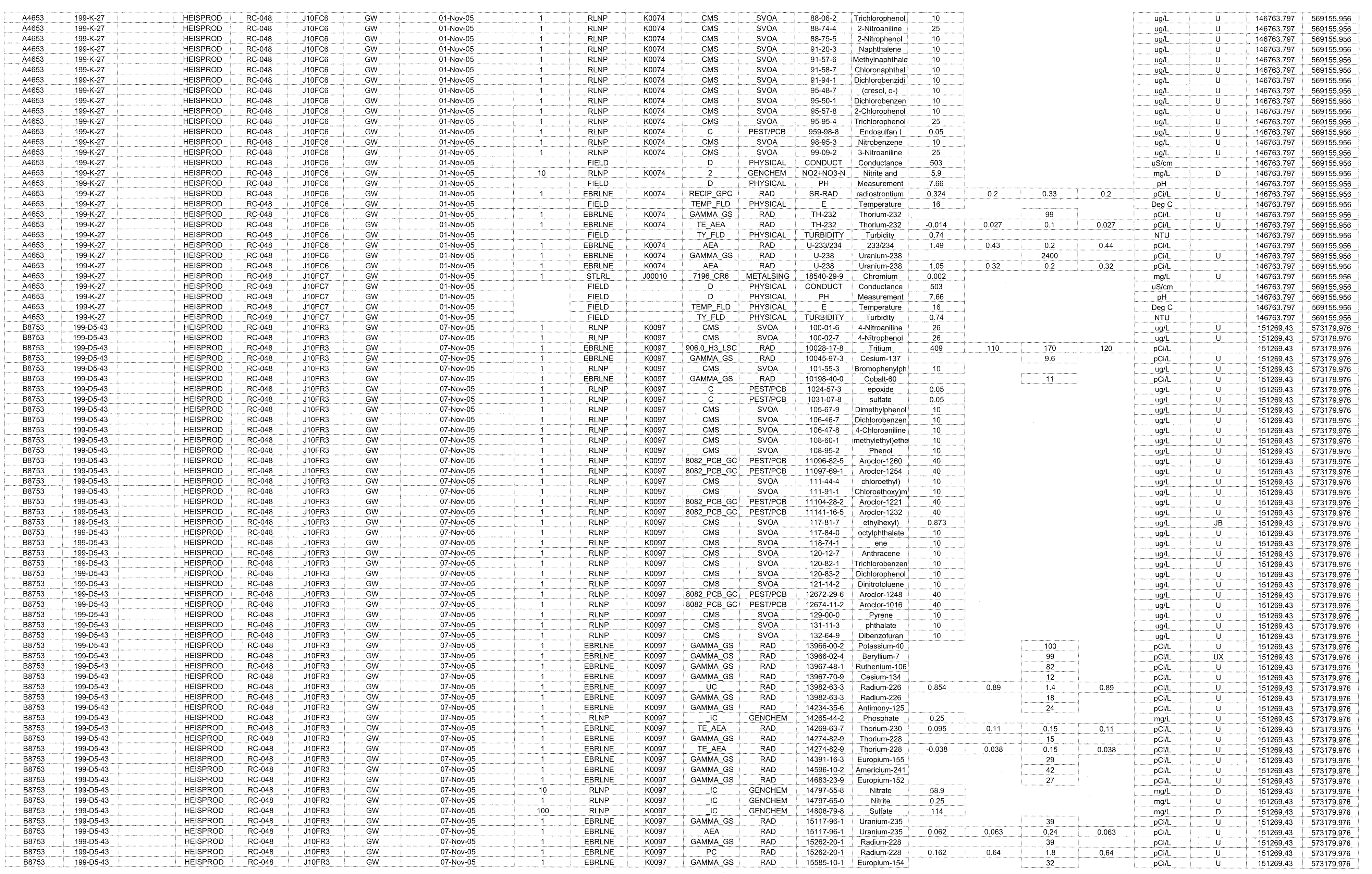



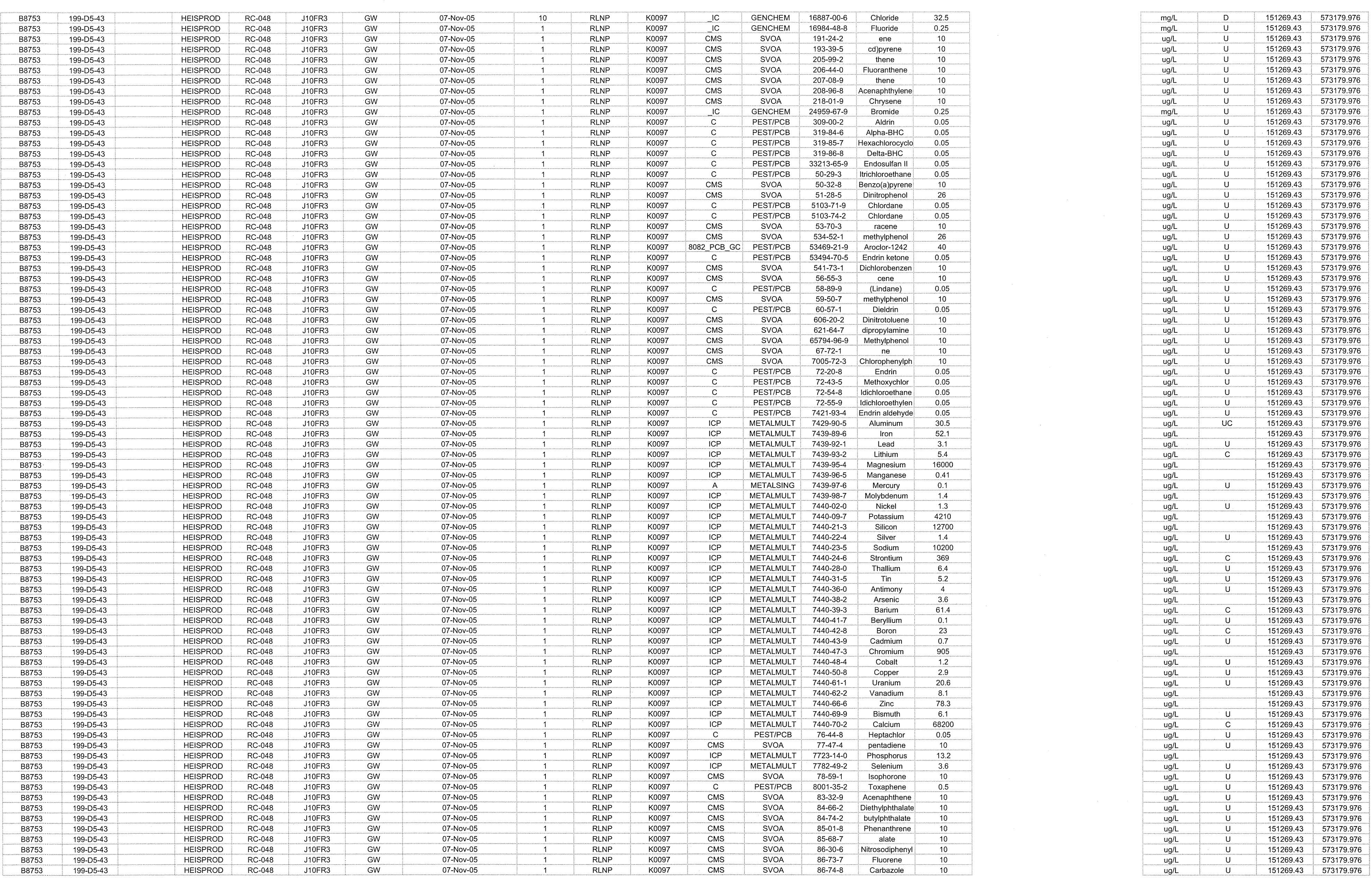


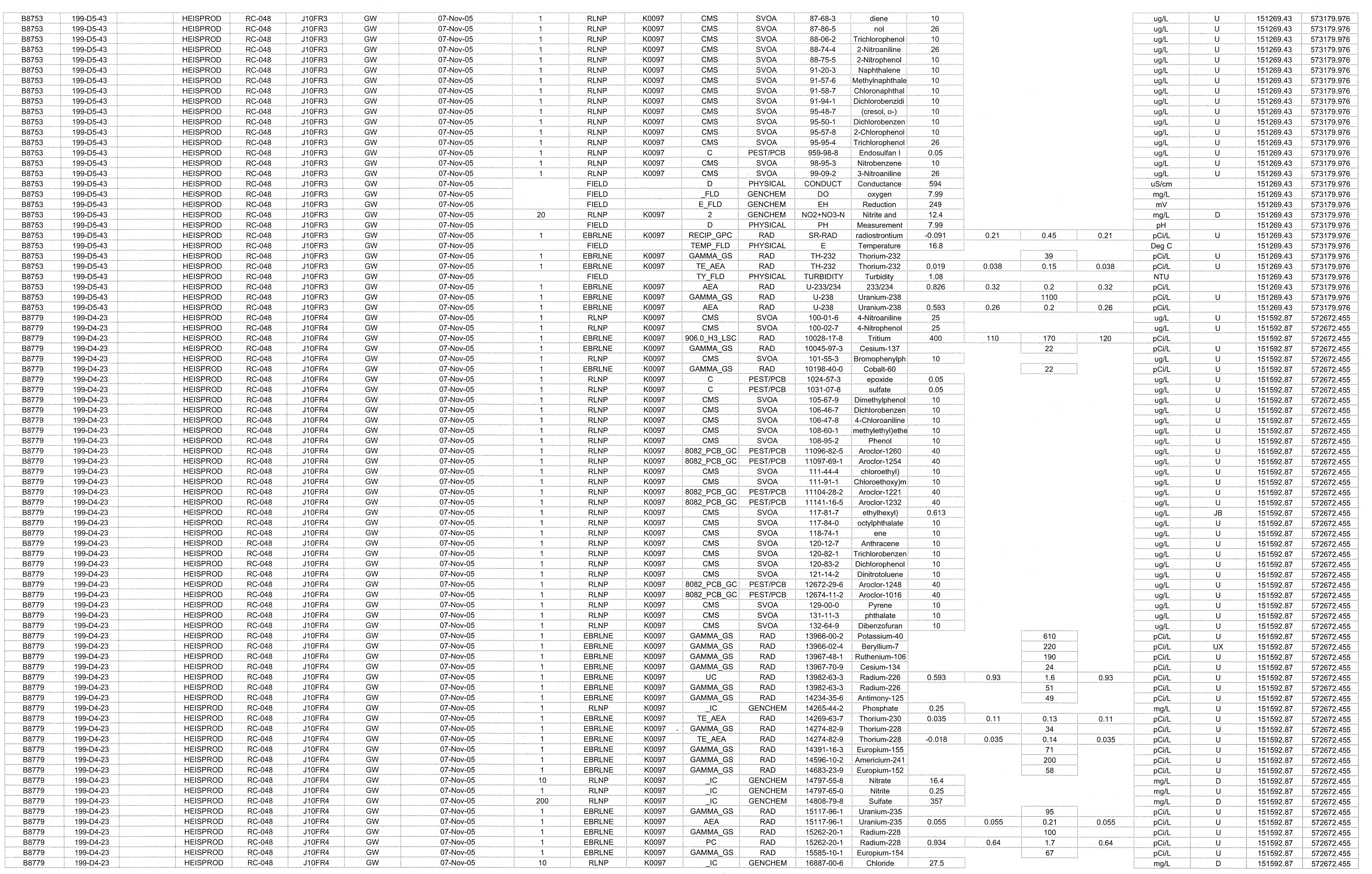



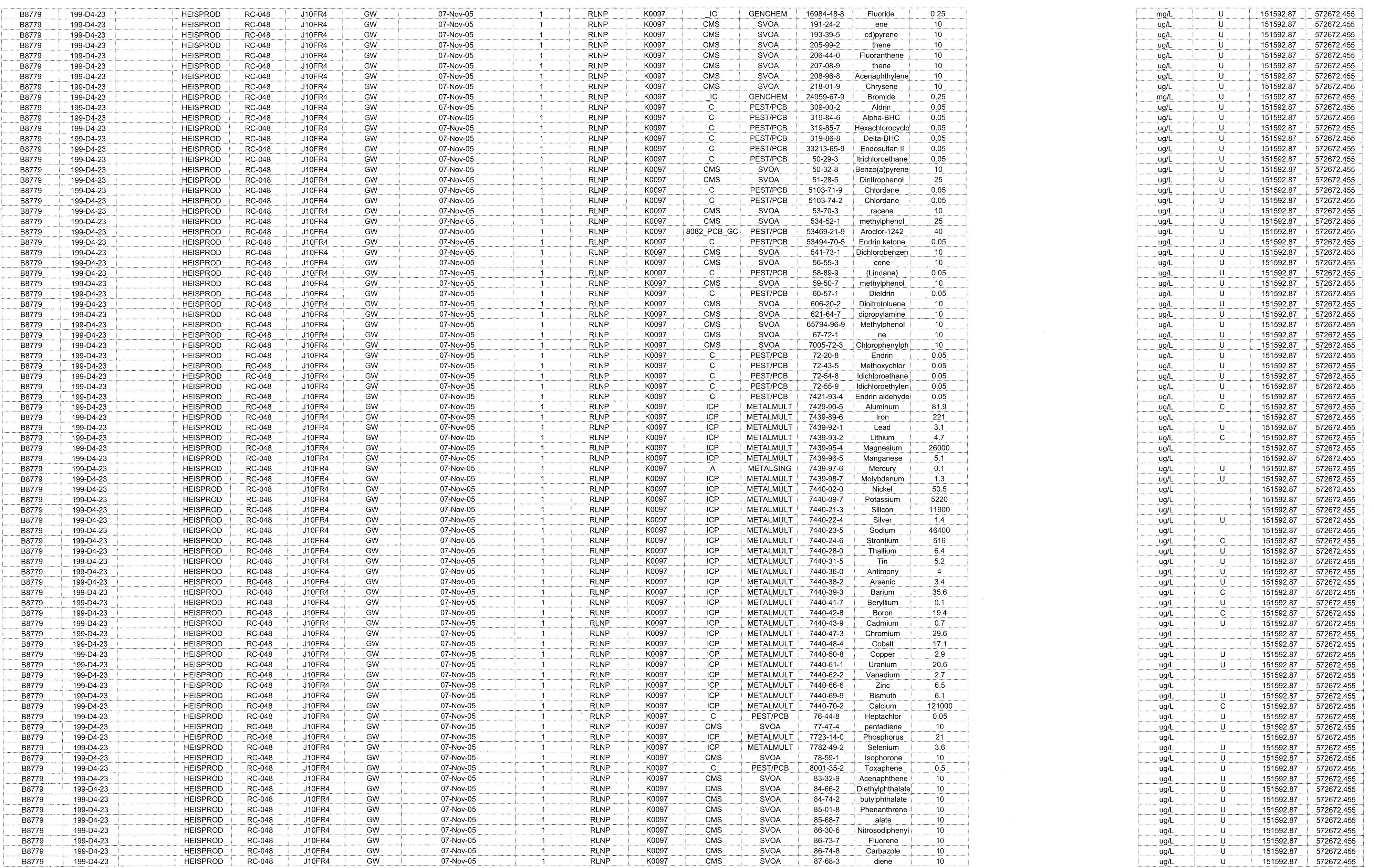


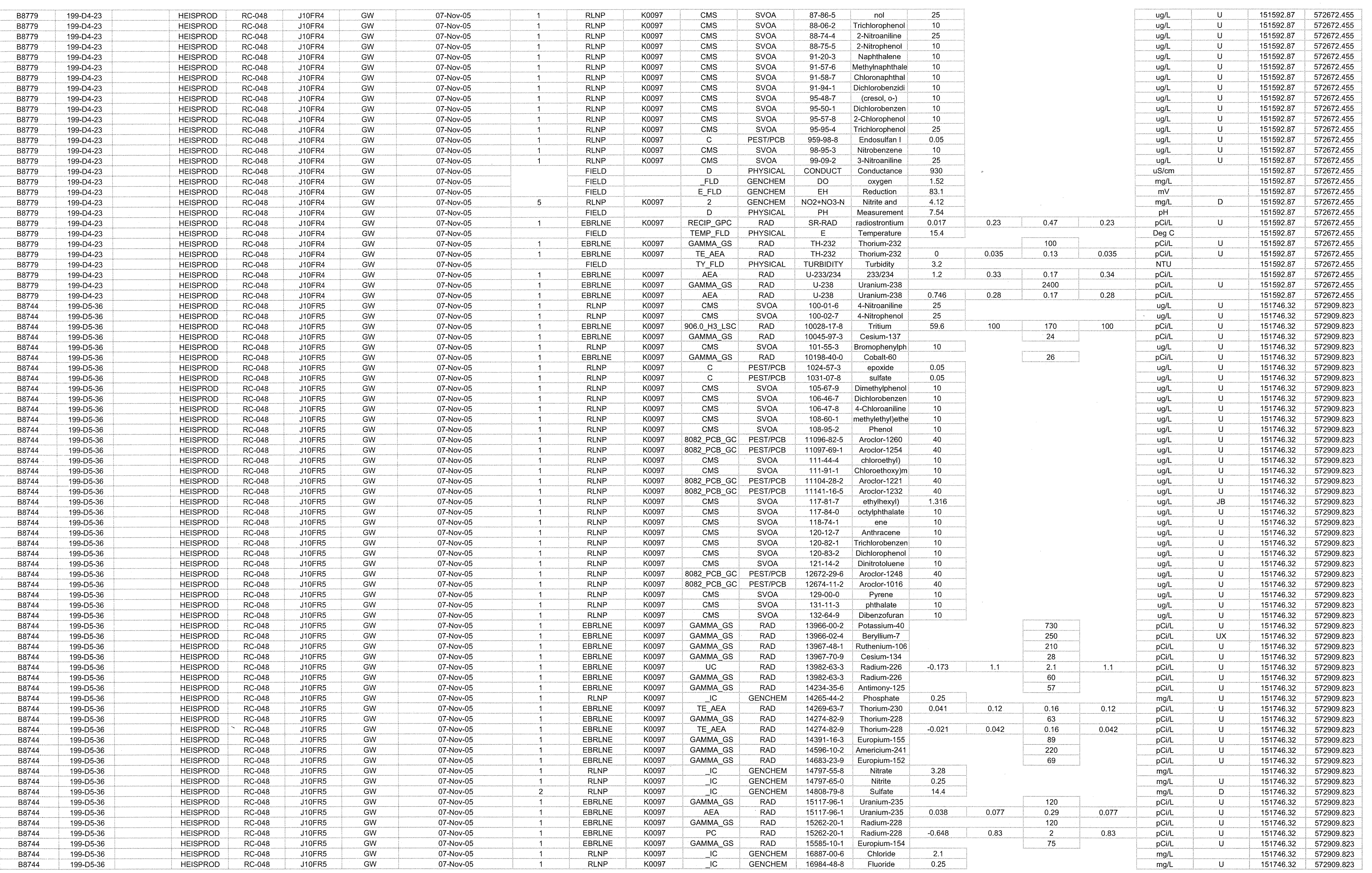



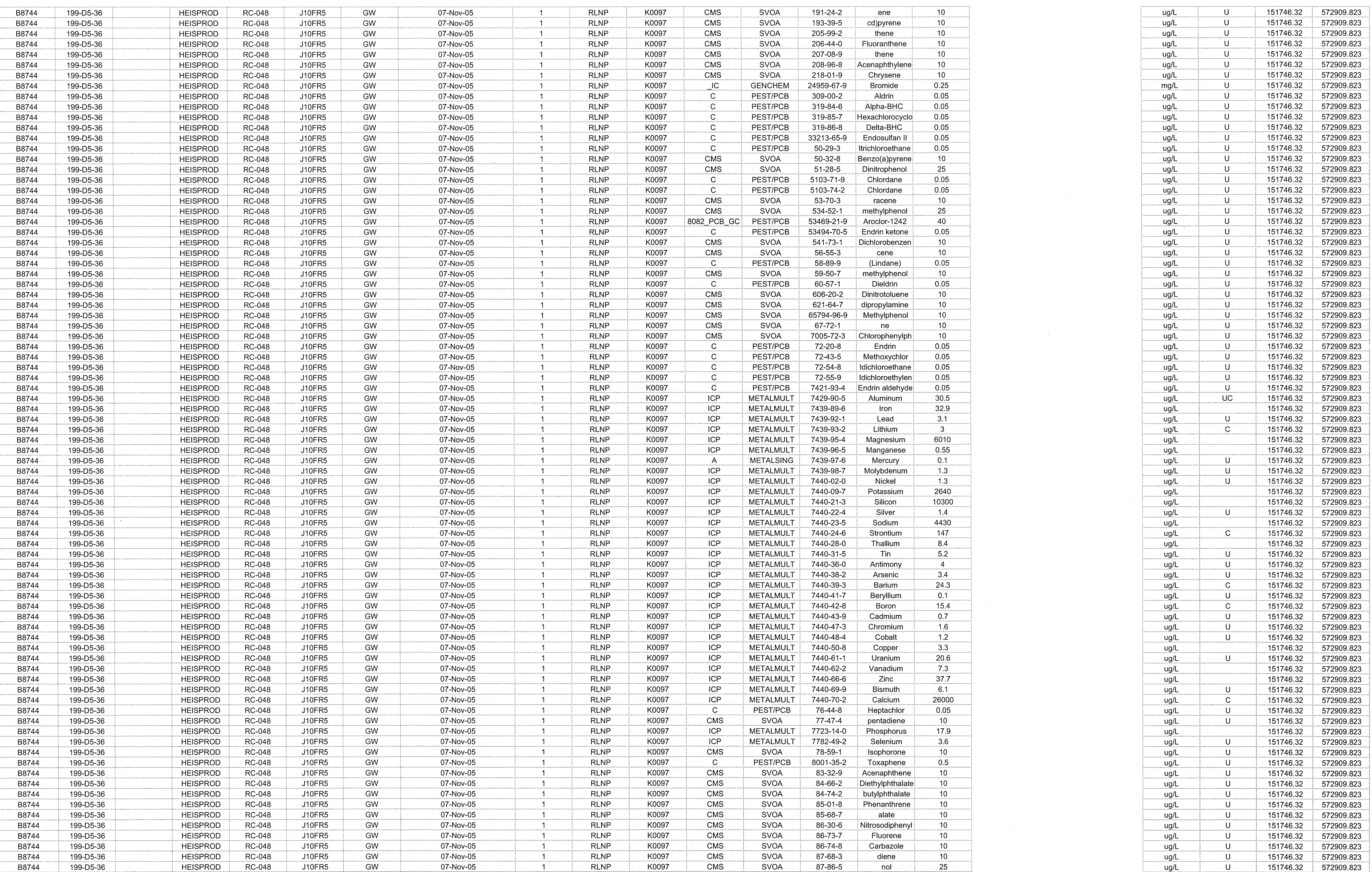


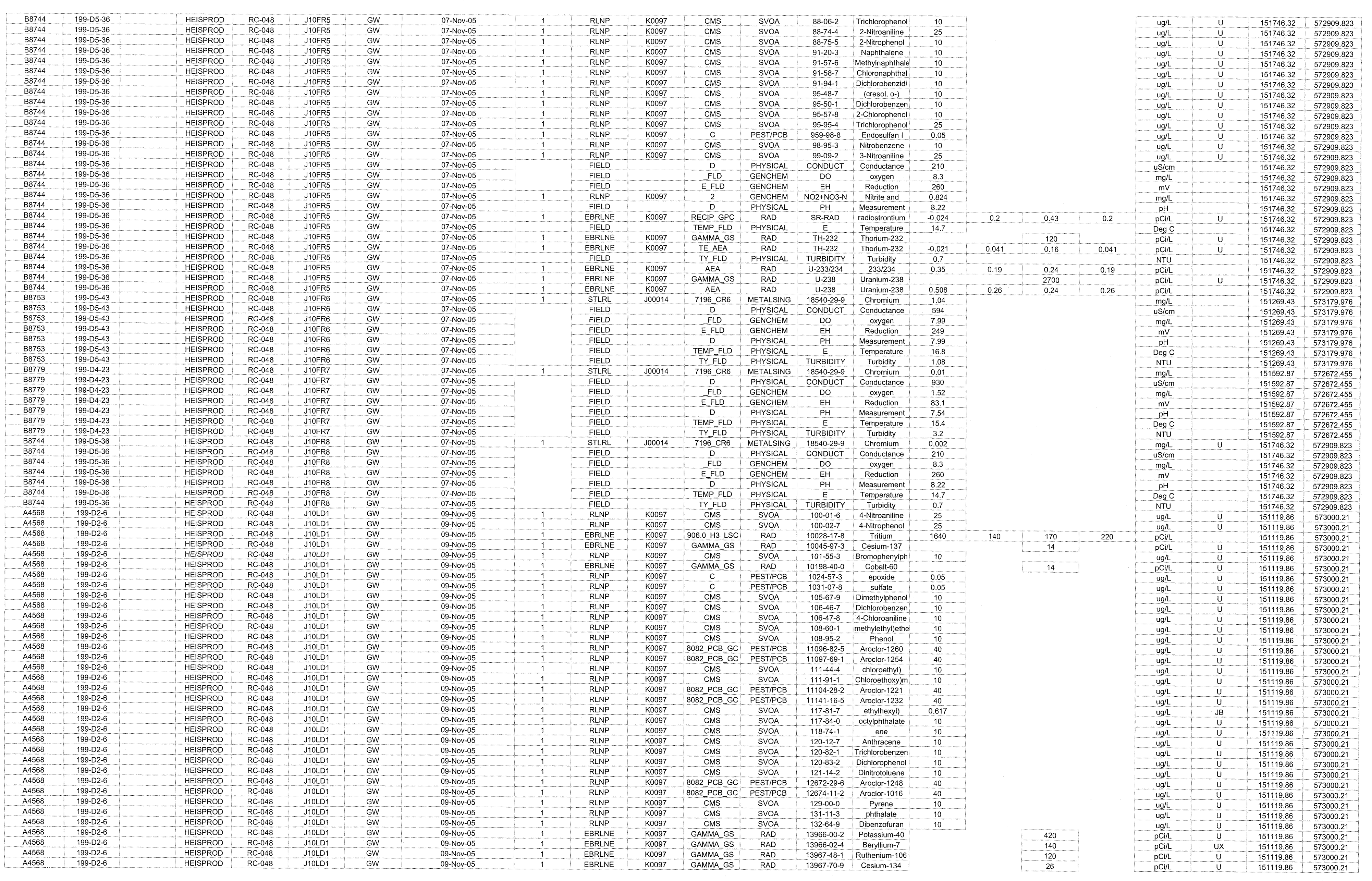




\begin{tabular}{|c|c|c|c|c|c|c|c|c|c|c|c|c|c|c|c|c|c|c|c|c|c|}
\hline A4568 & 199-D2-6 & HEISPROD & RC-048 & J10LD1 & GW & 09-Nov-05 & & EBRLNE & K0097 & uc & RAD & $13982-63-3$ & Radium-226 & -0.076 & 1 & 1.9 & 1 & pcill & $u$ & 151119.86 & 573000.21 \\
\hline A4568 & $\begin{array}{l}199-D 2-6 \\
1002-6\end{array}$ & HEISPROD & RC-048 & J10LD1 & $G W$ & og-Nov-05 & & $\begin{array}{l}\text { EBRLNE } \\
\text { ERPRIE }\end{array}$ & K0097 & GAMMAGSS & RAD & $13982-63-3$ & Radium-226 & & & 34 & & pcill & $u$ & 151119.86 & 5730000.21 \\
\hline $\begin{array}{l}\text { A45568 } \\
\text { A4568 }\end{array}$ & $\begin{array}{l}199-D 2-6 \\
199-D 2-6\end{array}$ & $\begin{array}{r}\text { HEISPROD } \\
\text { HEISPROD }\end{array}$ & $\begin{array}{l}\text { RC-048 } \\
\text { RC-048 }\end{array}$ & J10LD1 & GW & og-Nov-05 & & EBRLNE & Ko097 & GAMMA_GS & RAD & $14234-35-6$ & Antimony-125 & & & & & pcil & $u$ & 151119.86 & 573000.21 \\
\hline $\begin{array}{l}A 45686 \\
A 4568\end{array}$ & $\begin{array}{l}199-02.6 \\
190.026\end{array}$ & HEISPROD & $\begin{array}{l}\text { RC-048 } \\
\text { RC-048 }\end{array}$ & St1001 & $\frac{G N}{G N}$ & O9-Nov-05 & & $\begin{array}{l}\text { RLNP } \\
\text { EBRINE }\end{array}$ & K0097 & $\begin{array}{l}\text { IC } \\
\text { IE }\end{array}$ & GENCHEM & $14265-44-2$ & $\begin{array}{l}\text { Phosphate } \\
\text { Thoriu-230 }\end{array}$ & 0.25 & 0069 & 013 & 8069 & mgl & 4 & $\begin{array}{l}1517119.968 \\
1511968\end{array}$ & 573000.21 \\
\hline A & $\begin{array}{l}1999-D 2-6 \\
199-D 2-6\end{array}$ & \begin{tabular}{rl|l|l}
$H$ \\
HEISPROODOD
\end{tabular} & $\begin{array}{l}\text { RC-048 } \\
\text { RC-488 }\end{array}$ & $\begin{array}{l}\text { J10LDD1 } \\
\text { J10D1 }\end{array}$ & $\frac{G W}{G W}$ & 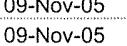 & 1 & $\begin{array}{l}\text { EBRLNE } \\
\text { EBRLNEE }\end{array}$ & $\begin{array}{l}\text { Kove97 } \\
\text { Koog7 }\end{array}$ & $\begin{array}{l}\text { IEAEAA } \\
\text { GAMMA GS }\end{array}$ & $\begin{array}{l}\text { RAD } \\
\text { RAD }\end{array}$ & $\begin{array}{l}14269-63-1 \\
1427482-9\end{array}$ & $\begin{array}{l}\text { Thorlum-230 } \\
\text { Thorium-228 }\end{array}$ & & 0.069 & 0.133 & 0.069 & $\begin{array}{l}\text { PClih } \\
\text { pcil }\end{array}$ & u & $\begin{array}{l}151119.986 \\
151119.86\end{array}$ & $\begin{array}{l}5730000.21 \\
573000.21\end{array}$ \\
\hline A45560 & 199-D2-0 & HEISPROD & RC-048 & J10LD1 & GW & O9-Nov-05 & 1 & EBRLNE & Koog7 & TE_AEA & RAD & $14274-82-9$ & Thorium-228 & 0.017 & 0.035 & 0.13 & 0.035 & pCill & $u$ & 151119.86 & 5730000.21 \\
\hline A4568 & $199-D 2-6$ & HEISPROD & RC-048 & J10LD1 & GW & 09-Nov-05 & 1 & EBRLNE & Ko097 & GAMMA_GS & RAD & $14391-16-3$ & Europium-155 & & & 49 & & pcil & $u$ & 151119.86 & 5730000.21 \\
\hline A4568 & 199-D2-6 & HEISPROD & $R C-048$ & HOLD1 & GW & o9-Nov-05 & 1 & EBRLNE & K0097 & GAMMA_GS & RAD & $14596-10-2$ & Americium-241 & & & 130 & & pcilih & $u$ & 151119.86 & 573000.21 \\
\hline A4568 & $199-D 2-6$ & HEISPROD & 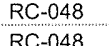 & J10L1 & $G W$ & o-Nov-05 & $\frac{1}{10}$ & EBRLNE & Ko097 & GAMMA_GS & RAD & $\begin{array}{l}14663-23-9 \\
478-569\end{array}$ & Europium-152 & & & & & pcin & u & $\begin{array}{l}1511119.96 \\
1511968\end{array}$ & 573000.21 \\
\hline $\begin{array}{l}\text { A45658 } \\
\text { A4568 }\end{array}$ & 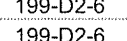 & 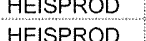 & 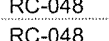 & 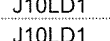 & GW & 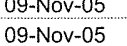 & 10 & RINP & Kolog7 & $\frac{\pi}{16}$ & 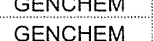 & 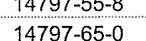 & $\begin{array}{l}\text { Nulfale } \\
\text { Nitrite }\end{array}$ & 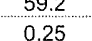 & & & & 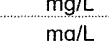 & u & 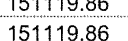 & $\begin{array}{l}5730000.21 \\
573000021\end{array}$ \\
\hline & $199-D 2-6$ & HEISPROD & $\frac{\pi-40}{R C-048}$ & J10LD1 & GW & O9-Nov-05 & 20 & RLNP & Koog7 & $\frac{16}{16}$ & GENCHEM & $14808-79-8$ & Sulfate & 122 & & & & mg/h & D & 151119.86 & $\begin{array}{l}575000.21 \\
573000.21\end{array}$ \\
\hline A4568 & $199-D 2-6$ & HEISPROD & RC-048 & J10LD1 & GW & 09-Nov-05 & 1 & EBRLNE & K0097 & GAMMA_GS & RAD & $15117-96-1$ & Uranium-235 & & & 66 & & pcill & $u$ & 151119.86 & 573000.21 \\
\hline & & & & & GW & & & EBRLNE & & & RAD & & & 0.042 & 0.084 & & 0.084 & pCil & u & & 573000.21 \\
\hline A4568 & $199-D 2-6$ & HEISPROD & RC-048 & J10LD1 & GW & 09-Nov-05 & & EBRLNE & Koo97 & GAMMA_GS & RAD & $15262-20-1$ & Radium-228 & & & & & pCilh & u & 151119.86 & 5730000.21 \\
\hline A4568 & 199-D2-6 & HEISPROD & RC-048 & J10LD1 & GW & 09-Nov-05 & & EBRLNE & K0097 & $P C$ & RAD & $15262-20-1$ & Radium-228 & 0.913 & 0.68 & 1.8 & 0.68 & pCilL & $u$ & 151119.86 & 573000.21 \\
\hline A4568 & 199-D2-6 & HESPROD & RC-048 & J10LD1 & GW & 09-Nov-05 & 1 & EBRLNE & K0097 & GAMMA_GS & RAD & $15585-10-1$ & Europium-154 & 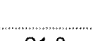 & & 44 & & pCil & u & 151119.86 & 573000.21 \\
\hline A4566 & 199-D2-6 & HEISPROD & $\begin{aligned} R C-048 \\
R C-48\end{aligned}$ & J10LD1 & $G W$ & o- o-Nov-05 & 10 & $\begin{array}{l}\text { RLLPP } \\
\text { PNDP }\end{array}$ & Ko097 & $\frac{16}{-16}$ & 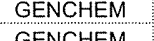 & $\begin{array}{l}16887-0.06-6 \\
608048\end{array}$ & $\begin{array}{l}\text { Chloride } \\
\text { Conide }\end{array}$ & $\begin{array}{l}21.8 \\
205\end{array}$ & & & & mol & D & $\begin{array}{l}151111.96 \\
1511.96\end{array}$ & 573000.21 \\
\hline $\begin{array}{l}\text { A45656 } \\
\text { A4568 }\end{array}$ & $\frac{199-D 2-6}{199-D 2-6}$ & $\begin{array}{l}\text { HESPRODD } \\
\text { HEISPROD }\end{array}$ & 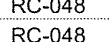 & $\begin{array}{l}\text { J10D1 } \\
\text { J10LD1 }\end{array}$ & GW & 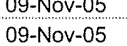 & $\frac{1}{1}$ & $\begin{array}{l}\text { RLNP } \\
\text { RLNP }\end{array}$ & Ko097 & $\begin{array}{l}\text { IC } \\
\text { CMS }\end{array}$ & $\begin{array}{l}\text { GENCHEM } \\
\text { SVOA }\end{array}$ & $16984-48-8$ & $\begin{array}{l}\text { Huonoe } \\
\text { - nene }\end{array}$ & $\frac{0.25}{10}$ & & & & yolh & $u$ & $\begin{array}{l}151119.86 \\
151119.86\end{array}$ & $\begin{array}{l}5730000.21 \\
5730000.21\end{array}$ \\
\hline $\begin{array}{l}\text { A45000 } \\
\text { A4568 }\end{array}$ & 199-D2-6 & HEISPROD & RC-048 & J10LD1 & Gw & O9-Nov-05 & 1 & RLNP & Koog7 & CMS & SVOA & $193-39-5$ & colppyene & 10 & & & & ugh & U & 151119.86 & 5 \\
\hline A4568 & $199-02-6$ & HEISPROD & RC-048 & J10LD1 & $G W$ & 09-Nov-05 & 1 & RLNP & K0097 & cMS & SVOA & $205-99-2$ & thene & 10 & & & & ugl & $u$ & 151119.86 & 5730000.21 \\
\hline A4568 & $199-D 2-6$ & & RC-048 & J10LD1 & GW & 09-Nov-05 & 1 & RLNP & K0097 & CMS & SVOA & $206-44-0$ & Fluoranthene & 10 & & & & ug/L & $u$ & 151119.86 & 573000.21 \\
\hline A4568 & 199-D2-6 & HEISPROD & RC-048 & J10LD1 & GW & o9-Nov-05 & 1 & RLNP & K0097 & CMS & SVOA & $207-08-9$ & thene & 10 & & & & ugll & u & 151119.86 & 5730000.21 \\
\hline $\begin{array}{l}{ }^{445686} \\
\text { A4568 }\end{array}$ & $\frac{199-D 2-6}{199-D 2-6}$ & HEISPROD & RC-048 & 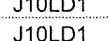 & GW & o-Nov-05 & 1 & $\begin{aligned} \text { RLNP } \\
\text { RNAP }\end{aligned}$ & K0097 & CMS & SVOA & $208-96-8$ & ccenaphthylene: & 10 & & & & ught & $u$ & 1511119.86 & 573000.21 \\
\hline A4568 & $\frac{199-26}{190-62-6}$ & $\begin{array}{l}\text { HESPRODD } \\
\text { HEISPROD }\end{array}$ & 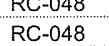 & J10LD1 & & 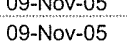 & 1 & $\begin{array}{l}\text { RLNP } \\
\text { RLNP }\end{array}$ & Ko097 & CMS & $\begin{array}{l}S{ }_{S} \text { SVA } \\
G E C H A M\end{array}$ & 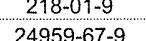 & $\begin{array}{l}\text { Chrysenen } \\
\text { Crense }\end{array}$ & $\begin{array}{l}10 \\
0.25\end{array}$ & & & & wgh & 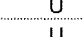 & $\begin{array}{l}151119.966 \\
5111.08\end{array}$ & 573000.21 \\
\hline A4568 & 199-D2-6 & HEISPROD & RC-048 & J10LD1 & Gw & 09-Nov-05 & 1 & RLNP & Kongr & $\frac{10}{C}$ & PESTPEB & 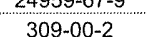 & 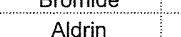 & 0.0 .25 & & & & ogl & u & 151119.86 & $\begin{array}{l}5730000.21 \\
573000021\end{array}$ \\
\hline A4568 & 199-D2-6 & HEISPROD & RC-048 & J10LD1 & GW & 09-Nov-05 & 1 & RLNP & Ko097 & & PEST/PCB & $3199-84-6$ & Alpha-BHC & 0.05 & & & & ug/L/L & u & 1515119.86 & $\begin{array}{l}5 / 3000.21 \\
573000.21\end{array}$ \\
\hline A44568 & $199-02-6$ & HEISPROD & RC-048 & J10LD1 & GW & 09-Nov-05 & 1 & RLNP & K0097 & & PESTIPCB & 319-85-7 & Hexachlorocycolo & 0.05 & & & & ugh & $u$ & 151119.86 & 573000.21 \\
\hline$\frac{A 45668}{\text { A4568 }}$ & 199-D2-6 & HEISPROD & RC-048 & J10LD1 & sw & 09-Nov-05 & 1 & RLNP & K0097 & $c$ & PESTIPCB & $319-86-8$ & Detha-BHC & 0.05 & & & & ugh & $u$ & 151119.8.86 & 573000.21 \\
\hline A45568 & 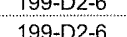 & HEISPROD & $\begin{array}{l}\text { RC-048 } \\
\text { RC-048 }\end{array}$ & J10LD1 & $G \mathrm{GW}$ & 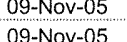 & $\frac{1}{1}$ & $\begin{array}{l}\text { RLNP } \\
\text { RLNP }\end{array}$ & $\begin{array}{l}\text { Konog7 } \\
\text { Kong }\end{array}$ & $\begin{array}{l}c \\
c\end{array}$ & $\begin{array}{l}\text { PESTPCB } \\
\text { PEST/PCB }\end{array}$ & $\begin{array}{l}33213-65-9 \\
50-25-3\end{array}$ & 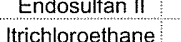 & 0.05 & & & & ugh & un & $\begin{array}{l}151119.966 \\
1511968\end{array}$ & 573000.21 \\
\hline A45568 & 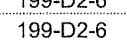 & HESPROD & RC-068 & J10L1 & $G \mathrm{G}$ & 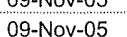 & 1 & RLNP & Kongr & CMS & sVoa & 50 & Bentrovataprenen & $\frac{0.05}{10}$ & & & & . & $u$ & $\begin{array}{l}157119.968 \\
1511986\end{array}$ & 573000.21 \\
\hline A45686 & 199-D2-6 & HEIIPRROD & RC-068 & J10L1 & GW & O9-Nove-5s & 1 & RLNP & Koog7 & CMS & syou & (51-28-6 & 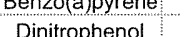 & 25 & & & & woll & 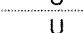 & 15111906 & 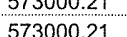 \\
\hline A4568 & $199-D 2-6$ & HEISPROD & RC-048 & JoLD1 & sw & o9-Nov-05 & 1 & RLNP & Ko097 & $c$ & PESTIPCB & $5103-71-9$ & Chlordane & 0.05 & & & & . & u & 151119.86 & 573000.21 \\
\hline A4568 & 199-D2-6 & HEISPROD & RC-048 & J10LD1 & & og-Nov-05 & 1 & RLNP & K0097 & c & PESTIPCB & $\begin{array}{l}\frac{5103-74-2}{5} \\
5=7-703\end{array}$ & Chlordane & 0.05 & & & & ugh & $u$ & 151119.86 & 573000.21 \\
\hline $\begin{array}{l}\text { A45668 } \\
\text { A4568 }\end{array}$ & $\frac{199-92-6}{199-D 2-6}$ & $\begin{array}{l}\text { HEISPROD } \\
\text { HESPROD }\end{array}$ & $\begin{array}{l}\frac{R C-048}{R C-088} \\
\text { RC }\end{array}$ & $\begin{array}{l}\text { J10LD1 } \\
\text { J10D1 }\end{array}$ & GW & 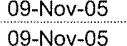 & & $\begin{array}{l}\text { RLNP } \\
\text { RLNP }\end{array}$ & K0097 & $\begin{array}{l}\text { CMS } \\
\text { CMS }\end{array}$ & $\begin{array}{l}\text { SVOA } \\
\text { SVA }\end{array}$ & $\begin{array}{l}53-70-3 \\
54-52-1\end{array}$ & 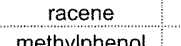 & & & & & ugh & $u$ & $\begin{array}{l}151119.86 \\
151119.986\end{array}$ & $\begin{array}{l}573000.21 \\
553200021\end{array}$ \\
\hline 年 & 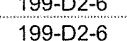 & $\begin{array}{l}\text { HEIIPRODD } \\
\text { HEISPROD }\end{array}$ & $\begin{array}{l}\text { RC-048 } \\
\text { RC-048 }\end{array}$ & $\begin{array}{l}\text { J10LD1 } \\
\text { J10LD1 }\end{array}$ & GW & $\begin{array}{l}\text { o-Nov-05 } \\
\text { 0-N-Nov-05 }\end{array}$ & $\frac{1}{1}$ & $\begin{array}{l}\text { RLNPP } \\
\text { RLNP }\end{array}$ & $\begin{array}{l}\text { K0097 } \\
\text { Ko0997 }\end{array}$ & $\begin{array}{c}\text { 8082 PCB } \\
80 C\end{array}$ & $\begin{array}{l}\text { SVIA } \\
\text { PEST/PCB }\end{array}$ & $\begin{array}{l}534-52-1 \\
5369-21-9\end{array}$ & $\begin{array}{l}\text { methylyhenol } \\
\text { Arroclor-1242 }\end{array}$ & $\frac{25}{40}$ & & & & $\frac{\mathrm{ughl}}{\mathrm{g} \text { g/h }}$ & u & $\begin{array}{l}1511199.86 \\
151119.86\end{array}$ & $\begin{array}{l}5730000.21 \\
573000.21\end{array}$ \\
\hline A45658 & $\frac{199-D L-6}{199-D 2-6}$ & HEISPROD & RC-048 & J10LD1 & GW & 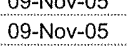 & 1 & RLNP & Koog7 & 然 & PEST/PCB & $\frac{53469-2-70-5}{3}$ & $\begin{array}{l}\text { Aloctorl-242 } \\
\text { Endrin ketone }\end{array}$ & 0.05 & & & & $\frac{g g h}{\text { ugh }}$ & $u$ & 151119.86 & 5730000.21 \\
\hline A4568 & $199-D 2-6$ & HEISPROD & RC-048 & J10LD1 & GW & 09-Nov-05 & 1 & RLNP & K0097 & CMS & SVOA & $541-73-1$ & Dichlorobenzen & 10 & & & & uglh & $U$ & 151119.86 & 5730000.21 \\
\hline A4568. & 199-D2-6 & HEISPROD & RC-048 & HOLD1 & GW & 09-Nov-05 & 1 & RLNP & K0097 & CMS & SVOA & $56-55-3$ & cene & 10 & & & & ug/h & $u$ & 151119.86 & \\
\hline A4568 & 199-D2-6 & HEISPROD & RC-048 & J10LD1 & GW & 09-Nov-05 & 1 & RLNP & K0097 & $c$ & PESTIPCB & $58-89-9$ & (Lindane) & 0.05 & & & & uglt & u & 151119.86 & 573000.21 \\
\hline A4568 & $\frac{199-92-6}{19-D 2-6}$ & HEISPROD & 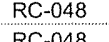 & J10DT & GW & 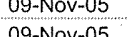 & $\frac{1}{1}$ & RLPP & K0097 & CMS & 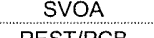 & $\begin{array}{l}59-50-7 \\
6057.7\end{array}$ & methylphenol & 10 & & & & ugh/ & $u$ & 151119.86 & 573000.21 \\
\hline A4t560 & 199-D2- & HESPRDD & 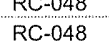 & J10LD1 & GW & 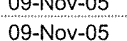 & 1 & RLP & 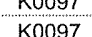 & $\begin{array}{c}c \\
c c\end{array}$ & PESTPCB & $60-57-1$ & Dieldrin & 0.05 & & & & ugh & $u$ & $\begin{array}{l}151119.966 \\
511.86\end{array}$ & 5730000.21 \\
\hline A4568 & $199-D 2-6$ & HEISPROD & 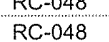 & J10LD1 & GW & 09-Nov-05 & & RLNP & Konog7 & cis & SWUA & - & 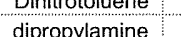 & 10 & & & & agh & 0 & 1511.960 & 573000.21 \\
\hline A45568 & 199-D2-6 & HESPROD & RC-068 & J10LD1 & $\mathrm{GW}$ & 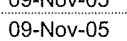 & 1 & RLNPP & Kong & cMs & SVOA & $657964-96-9$ & Methylathener & 10 & & & & ogh & u & 15111906 & $\begin{array}{l}573000.21 \\
573000211\end{array}$ \\
\hline A4568 & $199-\mathrm{D} 2-6$ & HEISPROD & RC-048 & J10LD1 & GW & 09-Nov-05 & 1 & RLNP & Ko097 & CMS & SVOA & $67-72-1$ & ne & 10 & & & & ugh & u & 151119.86 & 573000.21 \\
\hline A4568 & $199-D 2-6$ & HEISPROD & RC-048 & J10LD1 & GW & 09-Nov-05 & 1 & RLNP & K0097 & CMS & SVOA & $7005-72-3$ & Chlorophenylph & 10 & & & & ug/L & $u$ & 151119.86 & 573000.21 \\
\hline A4568 & $199-D 2-6$ & HEISPROD & RC-048 & J10LD1 & GW & o9-Nov-05 & 1 & RLNP & K0097 & c & PEST/PCB & $\begin{array}{l}72-20-8 \\
770.35\end{array}$ & Endrin & 0.05 & & & & ugh & u & 151119.86 & 573000.21 \\
\hline $\begin{array}{l}\text { A4568 } \\
\text { A4568 }\end{array}$ & $\frac{199-20-6}{190-02-6}$ & $\begin{array}{l}\text { HEIIPROD } \\
\text { HEISPROD }\end{array}$ & $\begin{array}{l}\text { RC-048 } \\
\text { RC-048 }\end{array}$ & J10DD1 & GW & 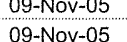 & $\frac{1}{1}$ & $\frac{R \text { RLNP }}{\text { RINP }}$ & $\begin{array}{l}\text { K0097 } \\
\text { Ko097 }\end{array}$ & $\begin{array}{l}c \\
c \\
c\end{array}$ & $\begin{array}{l}\text { PESTIPCB } \\
\text { PESTPCB }\end{array}$ & $\begin{array}{l}72-234-5 \\
72-54-8\end{array}$ & $\begin{array}{l}\text { Methoxychlor } \\
\text { Idichlorethane }\end{array}$ & $\begin{array}{l}0.05 \\
0.05\end{array}$ & & & & gigh & u & $\begin{array}{l}151119.966 \\
1511.086\end{array}$ & $\begin{array}{l}573000.211 \\
573022\end{array}$ \\
\hline $\begin{array}{l}\text { A4500 } \\
A 4568\end{array}$ & $199-D 2-6$ & HEISPROD & RC- -048 & J10LD1 & GW & o- & 1 & RLNP & Koog7 & $c$ & PEST/PCB & $72-55-9$ & 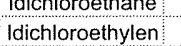 & 0.05 & & & & goll & u & 151119.86 & $\begin{array}{l}5 / 3000.21 \\
5730002.21\end{array}$ \\
\hline A4568 & $199-22-6$ & HEISPROD & RC-048 & J10LDI & GW & o9-Nov-05 & 1 & RLNP & K0097 & $c$ & PESTIPCB & $7421-93-4$ & Endrin aldehyde & 0.05 & & & & ugg/h & u & 151119.86 & 573000.21 \\
\hline A4568 & $199-02-6$ & HEISPROD & RC-048 & J10.LD1 & GW & 09-Nov-05 & 1 & RLNP & K0097 & ICP & METALMULT & $7429-90-5$ & Aluminum & 32.1 & & & & ugh & $c$ & 151119.86 & 5730000.21 \\
\hline A4568 & & HEISPROD & RC-048 & J10LD1 & GW & O9-Nov-05 & 1 & $\begin{array}{l}\text { RLNP } \\
\text { PanP }\end{array}$ & K0097 & ICP & METALMULT & $\begin{array}{l}7439-89-6 \\
77209091\end{array}$ & Iron & 266 & & & & ugh & 4 & $\begin{array}{l}151119.86 \\
151110.086\end{array}$ & 573000.21 \\
\hline $\begin{array}{l}\text { AA5566 } \\
\text { A4568 }\end{array}$ & $\frac{199-20-6}{19-02-6}$ & $\begin{array}{l}\text { HEIIPROD } \\
\text { HEISPROD }\end{array}$ & 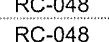 & $\begin{array}{l}\text { J10LDD1 } \\
\text { J10D1 }\end{array}$ & $\frac{G W}{G W}$ & 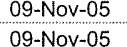 & $\frac{1}{1}$ & $\begin{array}{l}\text { RLNP } \\
\text { RLNP }\end{array}$ & $\begin{array}{l}\text { K0097 } \\
\text { Ko0997 }\end{array}$ & ICP & 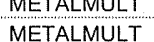 & $\begin{array}{l}74399-9-1 \\
7739-93-2\end{array}$ & $\begin{array}{l}\text { Lead } \\
\text { Lithium }\end{array}$ & $\begin{array}{l}3.1 \\
5.6\end{array}$ & & & & gigh & 0 & $\begin{array}{ll}151119.966 \\
1511086\end{array}$ & $\frac{573000.21}{573000}$ \\
\hline $\begin{array}{l}\text { AA568 } \\
\text { A4568 }\end{array}$ & $\frac{199-22-6}{199-D 2-6}$ & $\begin{array}{l}\text { HEISPODOD } \\
\text { HEISPRO }\end{array}$ & $\begin{array}{l}\text { RC- }-048 \\
\text { RC-048 }\end{array}$ & J10LD1 & GW & 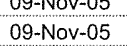 & $\frac{1}{1}$ & RLNP & Koog7 & ICP & $\begin{array}{l}\text { MEALALMULT } \\
\text { METALMUT }\end{array}$ & 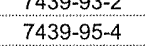 & $\begin{array}{l}\text { Linfium } \\
\text { Manesium }\end{array}$ & $\begin{array}{l}3.600 \\
15600\end{array}$ & & & & 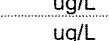 & & 151119.968 & $\begin{array}{l}5=5730.0 .21 \\
5\end{array}$ \\
\hline A4568 & $199-02-6$ & HEISPROD & RC-048 & J10LD1 & GW & 09-Nov-05 & 1 & RLNP & Ko097 & ICP & METALMULT & $74399-96-5$ & Manganese & 5.7 & & & & uggh & & 151119.86 & 5730000.21 \\
\hline A4568 & $199-D 2-6$ & HEISPROD & RC- 048 & J10LD1 & GW & 09-Nov-05 & 1 & RLNP & K0097 & A & METALSING & 74339-97-6 & Mercury & 0.1 & & & & ugh & $u$ & 151119.86 & 573000.21 \\
\hline A44568 & $199-22-6$ & HEISPROD & RC-048 & J10LD1 & GW & & & RLNP & K0097 & ICP & METALMULT & $\begin{array}{l}7439-98-7 \\
740020\end{array}$ & Molybdenum & 1.4 & & & & ugh & & $\begin{array}{l}1511119.96 \\
1511.86\end{array}$ & 573000.21 \\
\hline $\begin{array}{l}\frac{A 4568}{4} \\
A 568\end{array}$ & 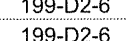 & $\begin{array}{l}\text { HILSPRDD } \\
\text { HIISPROD }\end{array}$ & 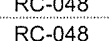 & $\begin{array}{l}\text { J10LDD } \\
\text { J10LD1 }\end{array}$ & $G W$ & $\begin{array}{l}\text { o9-NNov-05 } \\
\text { 0-9-0y-0.05 }\end{array}$ & & 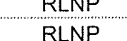 & $\begin{array}{l}\text { K0097 } \\
\text { Ko0997 }\end{array}$ & ICP & 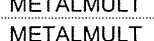 & 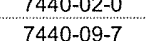 & $\begin{array}{l}\text { PNiche } \\
\text { Prtasium }\end{array}$ & $\begin{array}{r}8.1 \\
4920\end{array}$ & & & & gigh & & 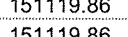 & 5 \\
\hline $\begin{array}{l}\text { AA5568 } \\
\text { A }\end{array}$ & $\frac{19-92-6}{199-22-6}$ & $\begin{array}{l}\text { HESPROD } \\
\text { HEISPROD }\end{array}$ & $\frac{R C-048}{R C-048}$ & J10LD1 & GW & 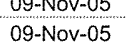 & $\frac{7}{1}$ & $\begin{array}{l}\text { RLNP } \\
\text { RLNP }\end{array}$ & Koog7 & ICP & $\begin{array}{l}\text { MEIALMULLT } \\
\text { METALMULT }\end{array}$ & $\begin{array}{l}7440-90-1 \\
7744-21-3\end{array}$ & $\begin{array}{l}\text { Polosassium } \\
\text { Silicon }\end{array}$ & $\begin{array}{l}49200 \\
13400\end{array}$ & & & & $\begin{array}{l}\text { ugh } \\
\text { ug/h }\end{array}$ & & 1511199.86 & $\begin{array}{l}5 / 3000.21 \\
5730002.21\end{array}$ \\
\hline A4568 & 199--D2-6 & HEISPROD & RC-048 & J10LD1 & ow & 09-Nov-05 & & RLNP & Ko097 & ICP & METALMULT & $7440-22-4$ & Silver & 1.4 & & & & uggh & $u$ & 151119.86 & 5730000.21 \\
\hline A4568 & $199-D 2-6$ & HEISPROD & RC- 048 & J10LD1 & GW & 09-Nov-05 & 4 & RLNP & K0097 & ICP & METALMULT & $7440-23-5$ & Sodium & 12100 & & & & uglh & & 151119.86 & 573000.21 \\
\hline A4568 & 199-D2-6 & HEISPROD & RC-048 & J10LD1 & GW & o9-Nov-05 & & RLNP & K0097 & ICP & METALMULT & $7440-24-6$ & Strontium & 372 & & & & ugh & c & $\begin{array}{l}151119.86 \\
15110.086\end{array}$ & 573000.21 \\
\hline $\begin{array}{l}A 45668 \\
A 4568\end{array}$ & $\frac{199-2-6}{19-22-6}$ & HEISPROD & $\frac{R C-048}{R C-048}$ & $\begin{array}{l}\text { J10LDD1 } \\
\text { J10LDD }\end{array}$ & $\frac{G W}{G W}$ & 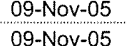 & & $\begin{array}{l}\text { RLNPP } \\
\text { RLNP }\end{array}$ & $\begin{array}{l}\text { K0097 } \\
\text { Ko097 }\end{array}$ & $\frac{\mid}{\text { ICP }}$ & $\begin{array}{l}\text { MEIALMLLT } \\
\text { METALMULT }\end{array}$ & $\begin{array}{l}7440-28-0 \\
7442-31-5\end{array}$ & Thallium & $\begin{array}{l}6.4 \\
5.2 \\
\end{array}$ & & & & $\frac{\text { ugh }}{\text { noll }}$ & u & $\begin{array}{l}151119.86 \\
5111986\end{array}$ & 573000.21 \\
\hline A & 199-20-6. & HEISPROD & RC-048 & J10LD1 & $G W$ & 09-Novo-05 & 1 & $\begin{array}{l}\text { RLNP } \\
\text { RLNP }\end{array}$ & Koo97 & ICP & METALMULT & $\begin{array}{l}7440-15-5 \\
7740-36-0\end{array}$ & Antimony & $\frac{0.2}{4}$ & & & & $\frac{\text { ugh }}{\text { uolt }}$ & $u$ & 1515119.86 & $\begin{array}{l}5 / 3000.21 \\
5730002.21\end{array}$ \\
\hline A4568 & $199-02-6$ & HEISPROD & RC-048 & J10LD1 & GW & 09-Nov-05 & 1 & RLNP & K0097 & ICP & METALMULT & $7440-38-2$ & Arsenic & 4.6 & & & & uglh & & 151119.86 & 573000.21 \\
\hline A4568 & $199-02-6$ & HEISPROD & RC-048 & J10LD1 & GW & 09-Nov-05 & 7 & RLNP & K0097 & ICP & METALMULT & $7440-39-3$ & Barium & 54.1 & & & & uggh & c & 151119.86 & 573000.21 \\
\hline A4568 & 199-D2-6 & & RC-048 & J10LD1 & & 09-Nov-05 & & RLNP & K0097 & ICP & METALMULT & $7440-41-7$ & Beryllium & 0.1 & & & & & & & \\
\hline A4568 & 199-D2-6 & HEISPROD & RC-048 & J10LD1 & GW & 09-Nov-05 & 1 & RLNP & K0097 & ICP & METALMULT & $7440-42-8$ & Boron & 28.3 & & & & ug/h & $c$ & 151119.86 & S57300.0.21 \\
\hline A4568 & 199-D2-6 & HEISPROD & RC-048 & J10LD1 & GW & 09-Nov-05 & & RLNP & K0097 & ICP & METALMULT & $7440-43-9$ & Cadmium & 0.7 & & & & ught & $u$ & 151119.86 & 573000.21 \\
\hline & & & & & & & & & & ICP & METALMULT & $7440-47-3$ & & & & & & woll & & & \\
\hline A4568 & $199-02-6$ & HEIIPROD & RC-048 & J10LD1 & GW & 09-Nov-05 & 1 & RLNP & Ko097 & ICP & $\begin{array}{l}\text { METALMULT } \\
\end{array}$ & $7440-48-4$ & Cobalt & 1.2 & & & & ugh & u & & 5730000.21 \\
\hline A4568 & & HEISPROD & RC-048 & J10LD1 & GW & 09-Nov-05 & 1 & RLNP & Ko097 & $I C P$ & METALMULT & $7440-50-8$ & Copper & 2.9 & & & & uglh & u & 51119.86 & 573000.21 \\
\hline A45668 & 199-D2-6 & HEISPROD & RC-048 & S10LD1 & GW & 09-Nov-05 & & RLNP & K0097 & ICP & METALMULT & $7440-61-1$ & Uranium & 20.6 & & & & yolh & $u$ & 151119.86 & (573000.21 \\
\hline
\end{tabular}




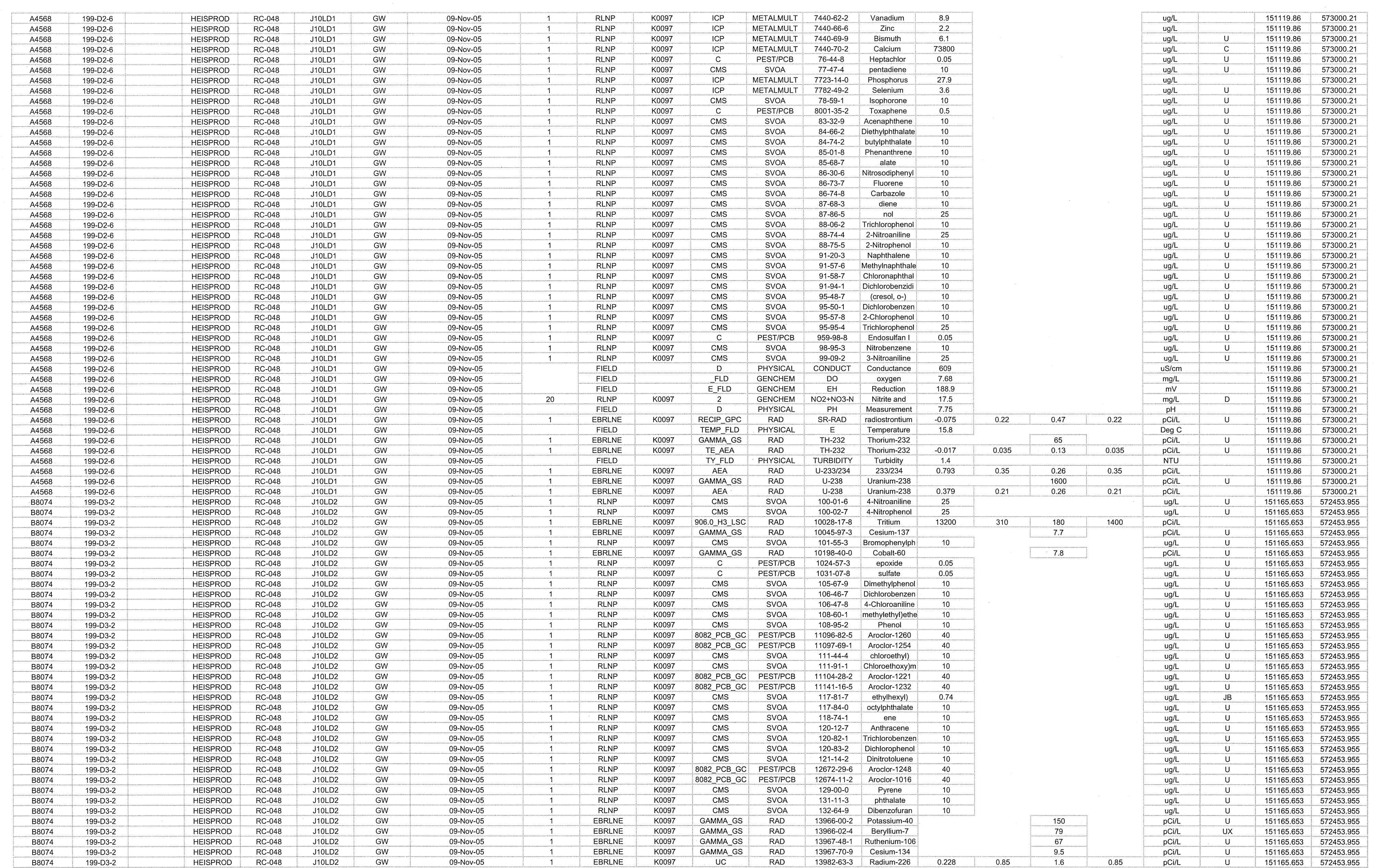




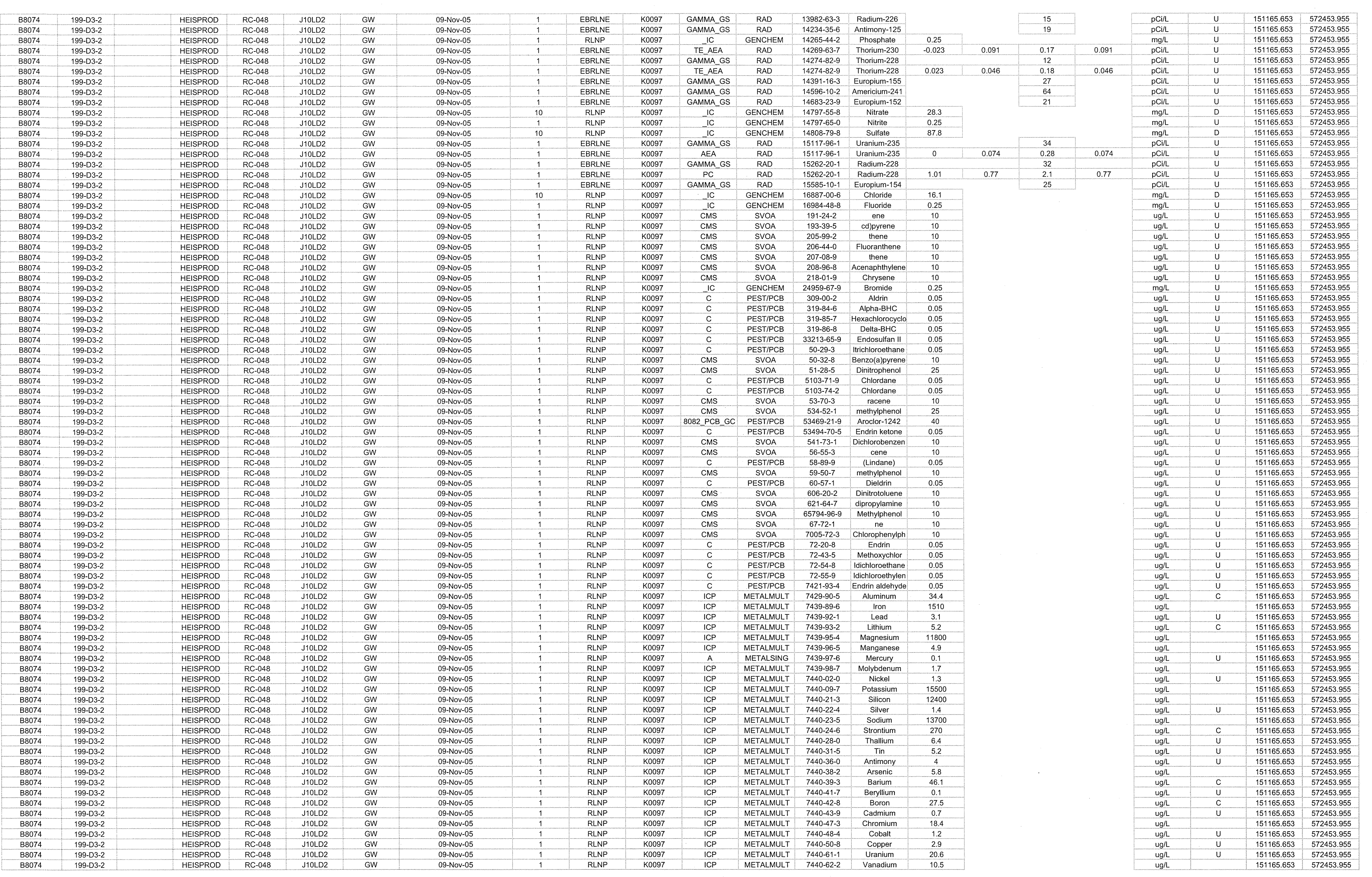



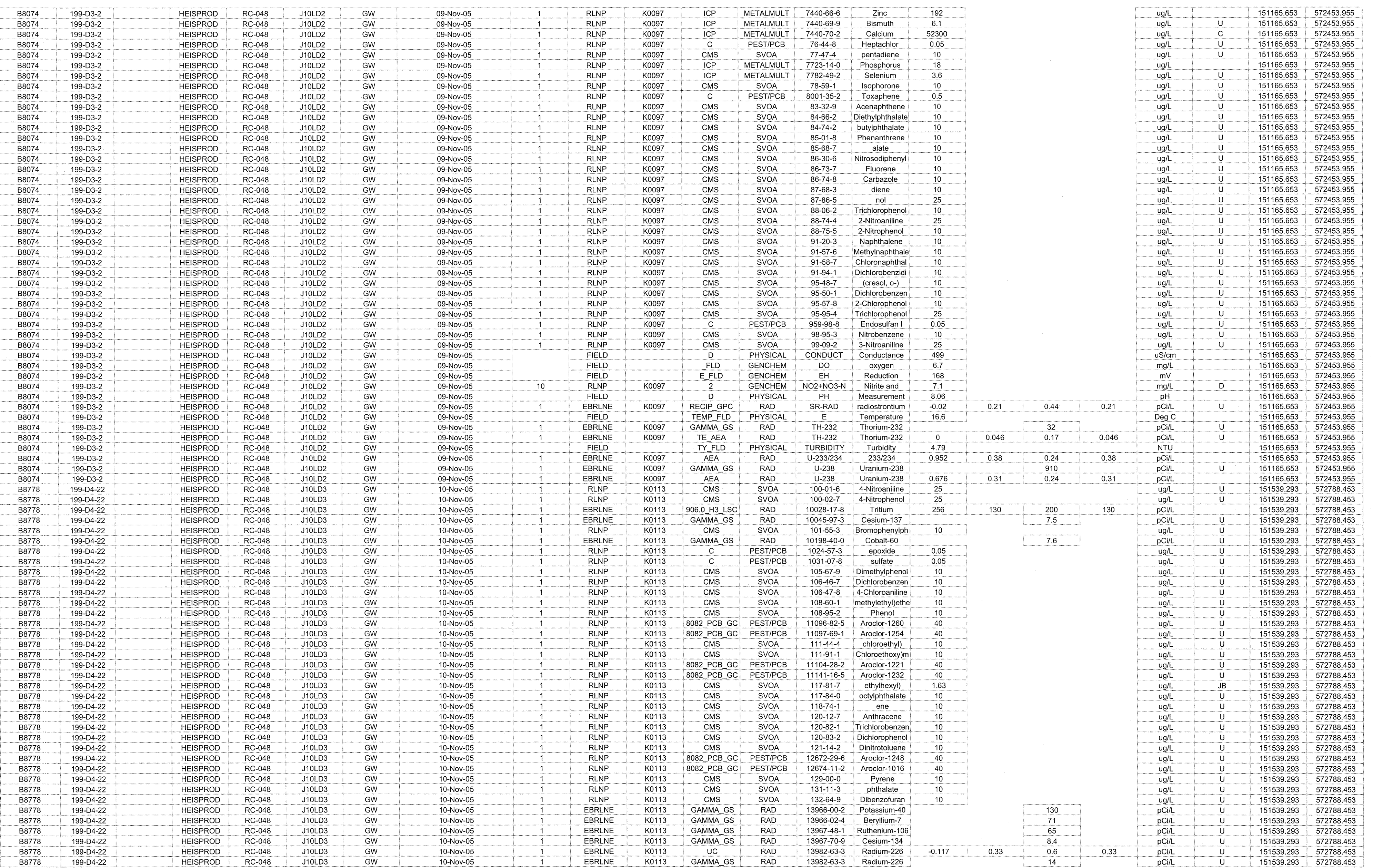

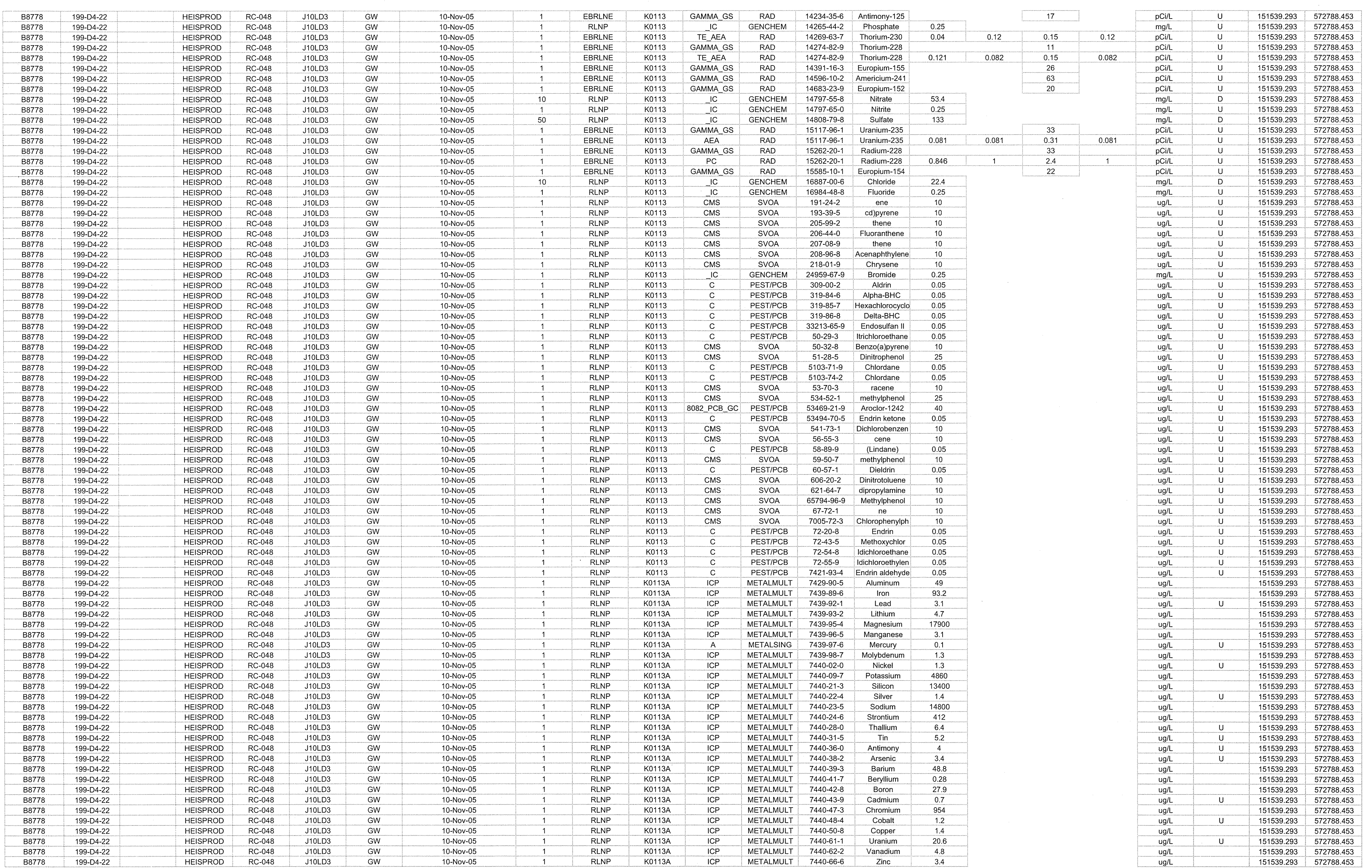

32 


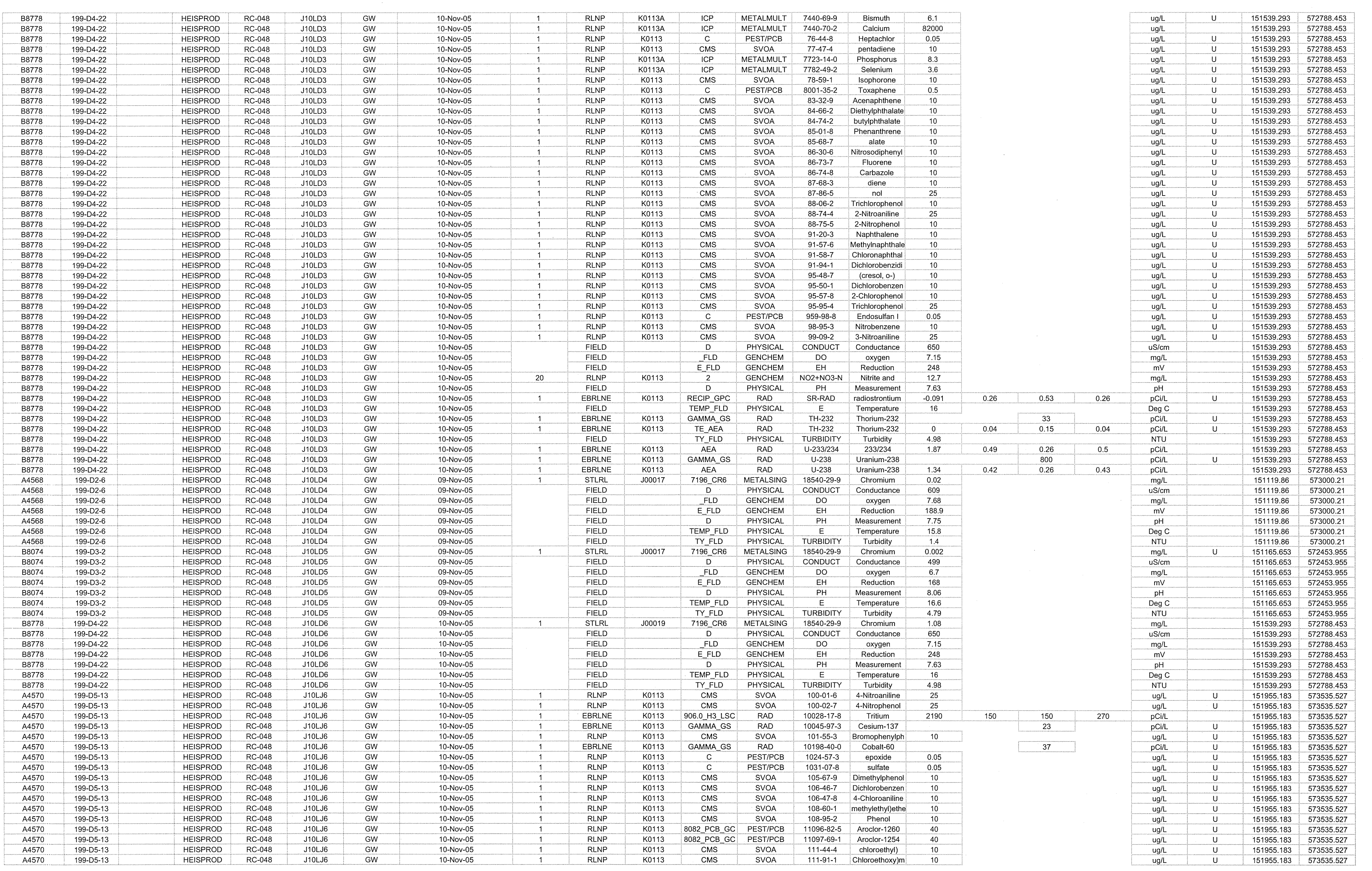




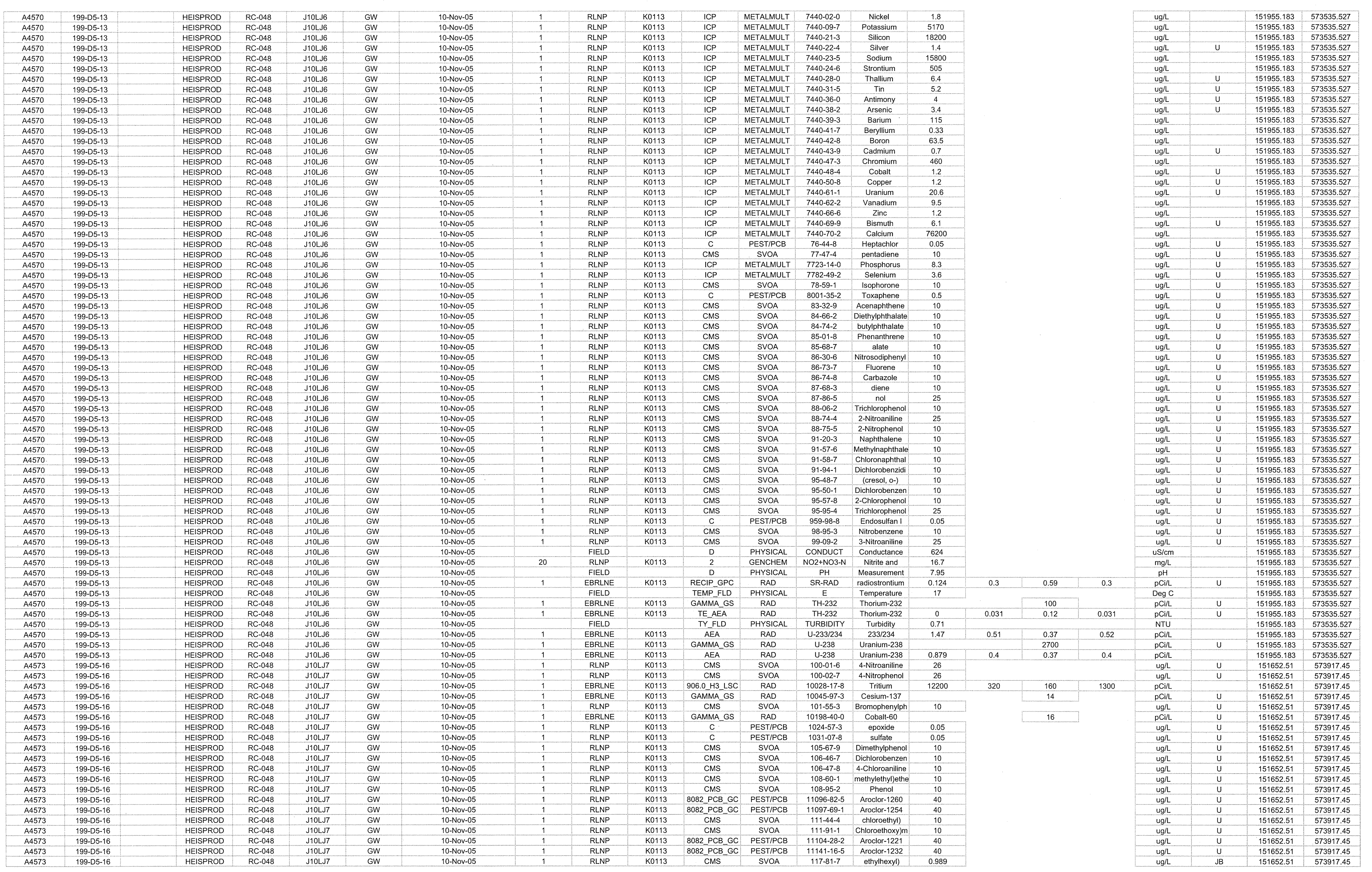




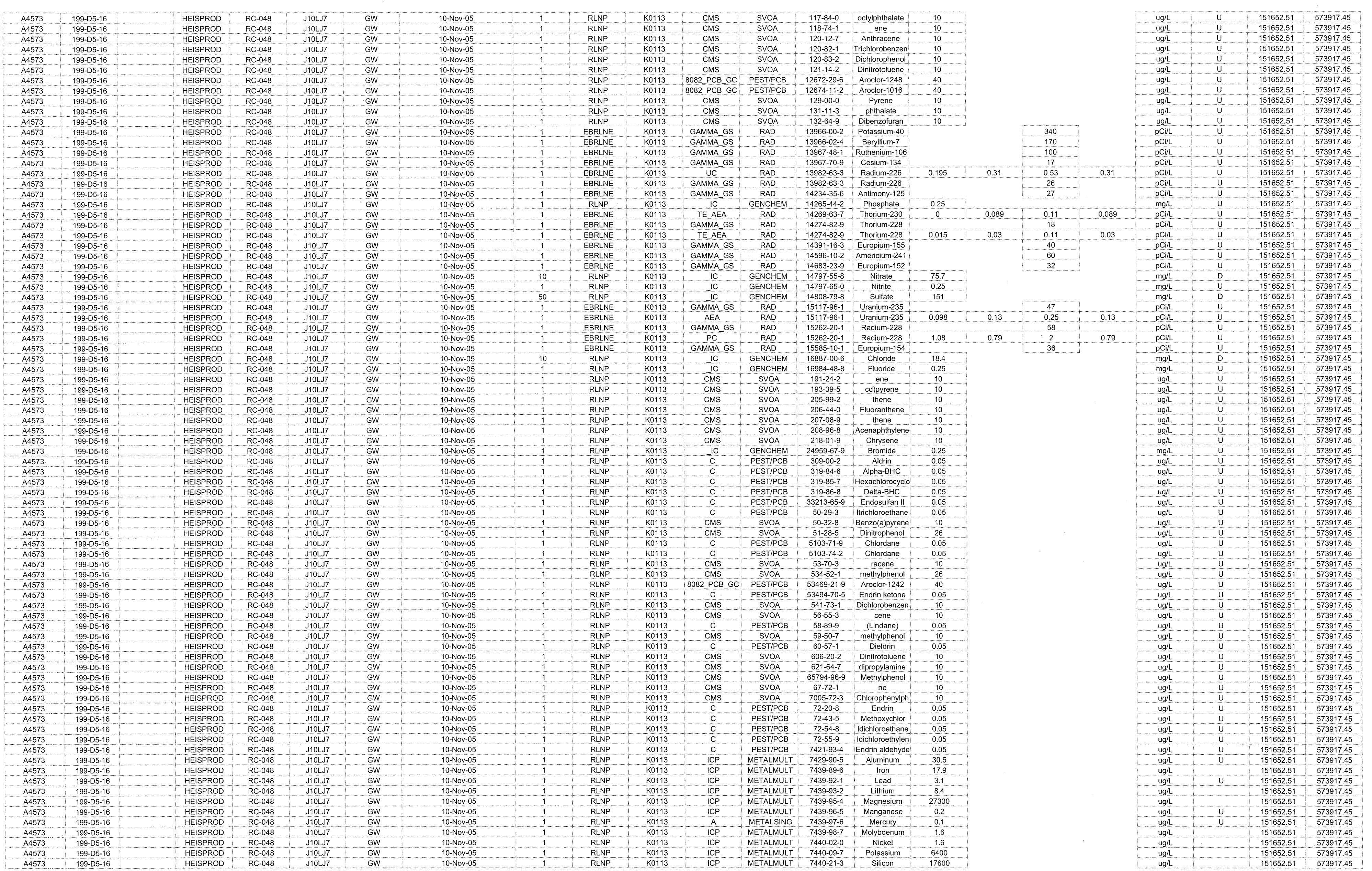




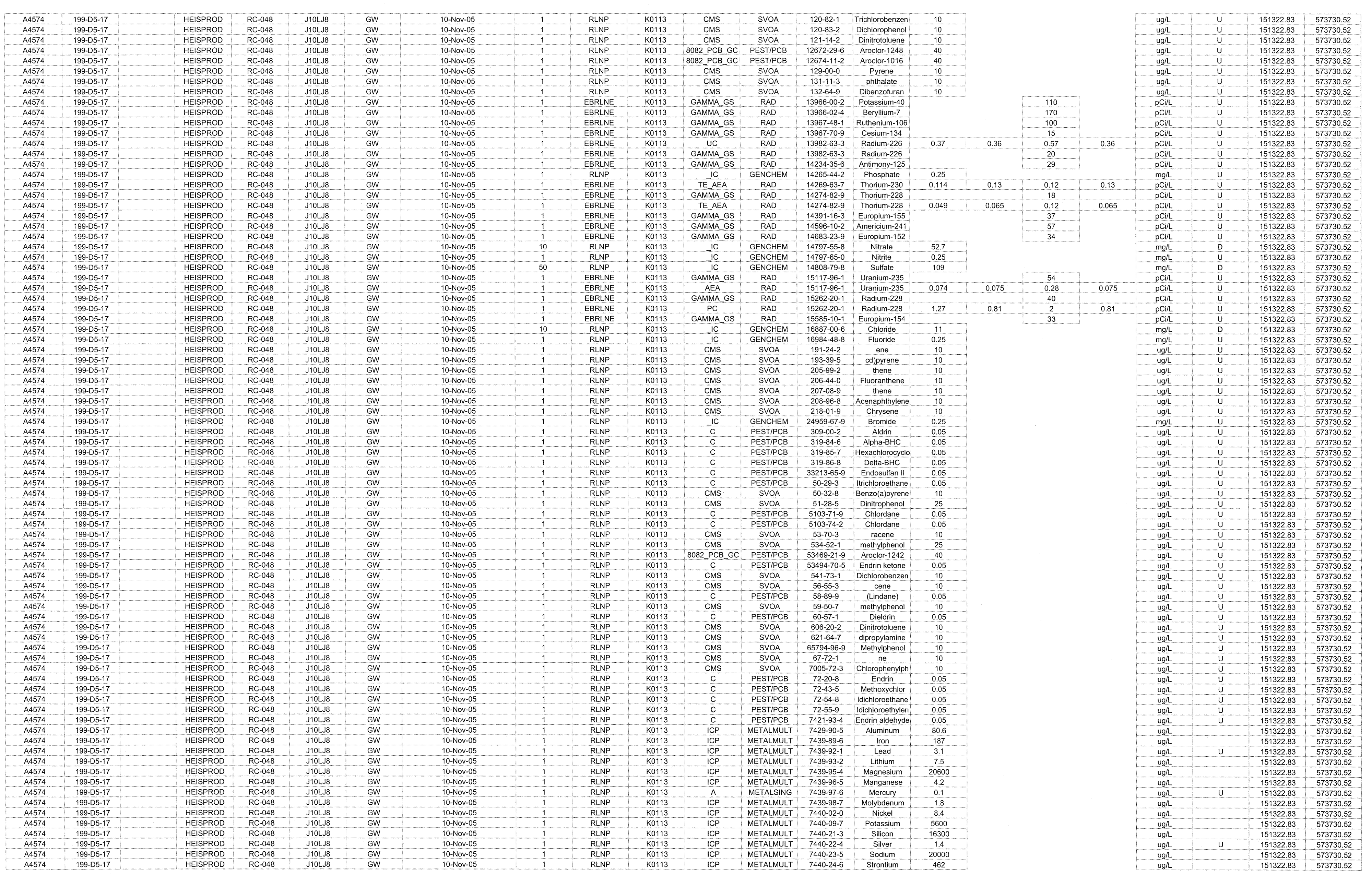




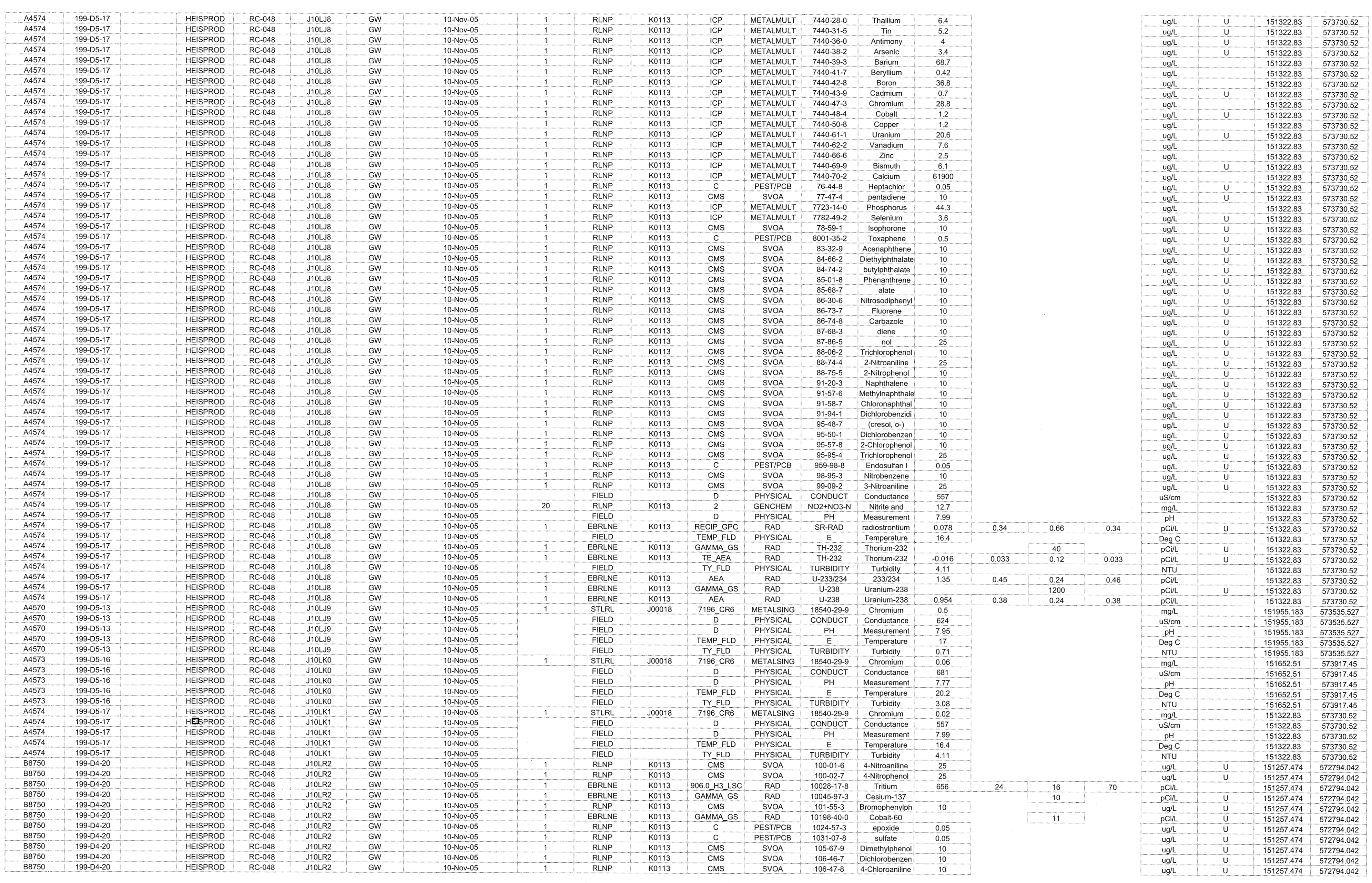



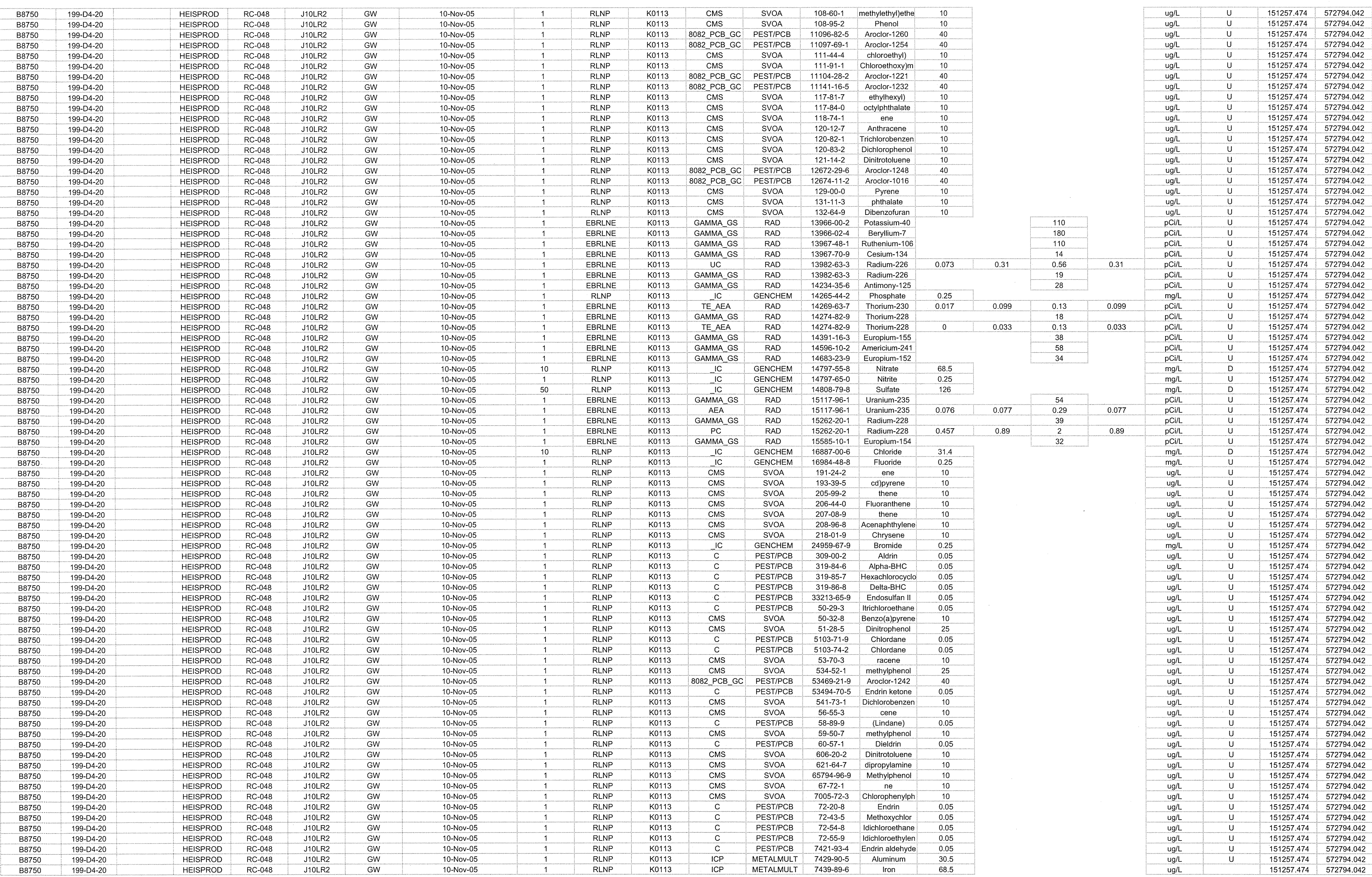


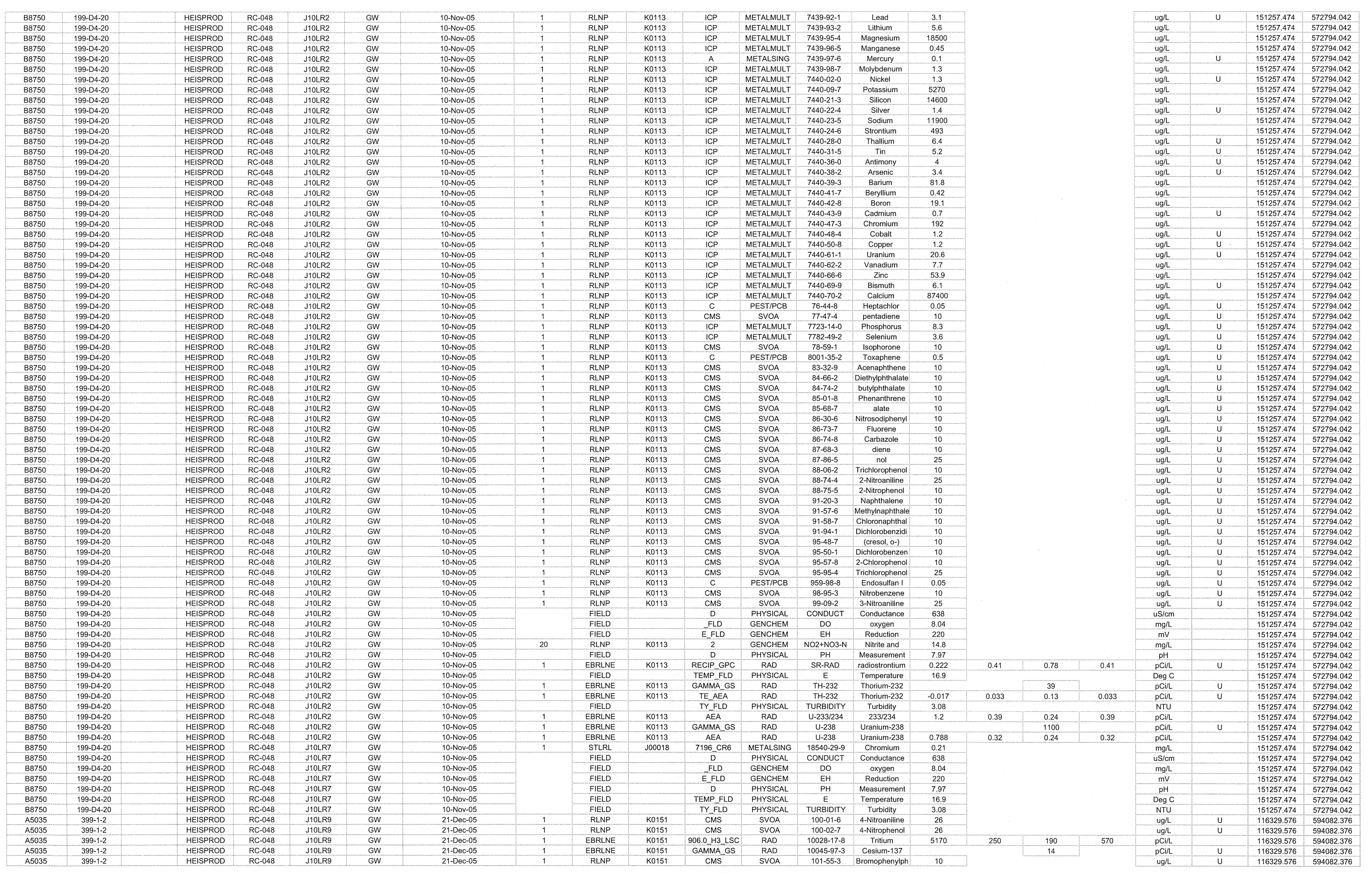




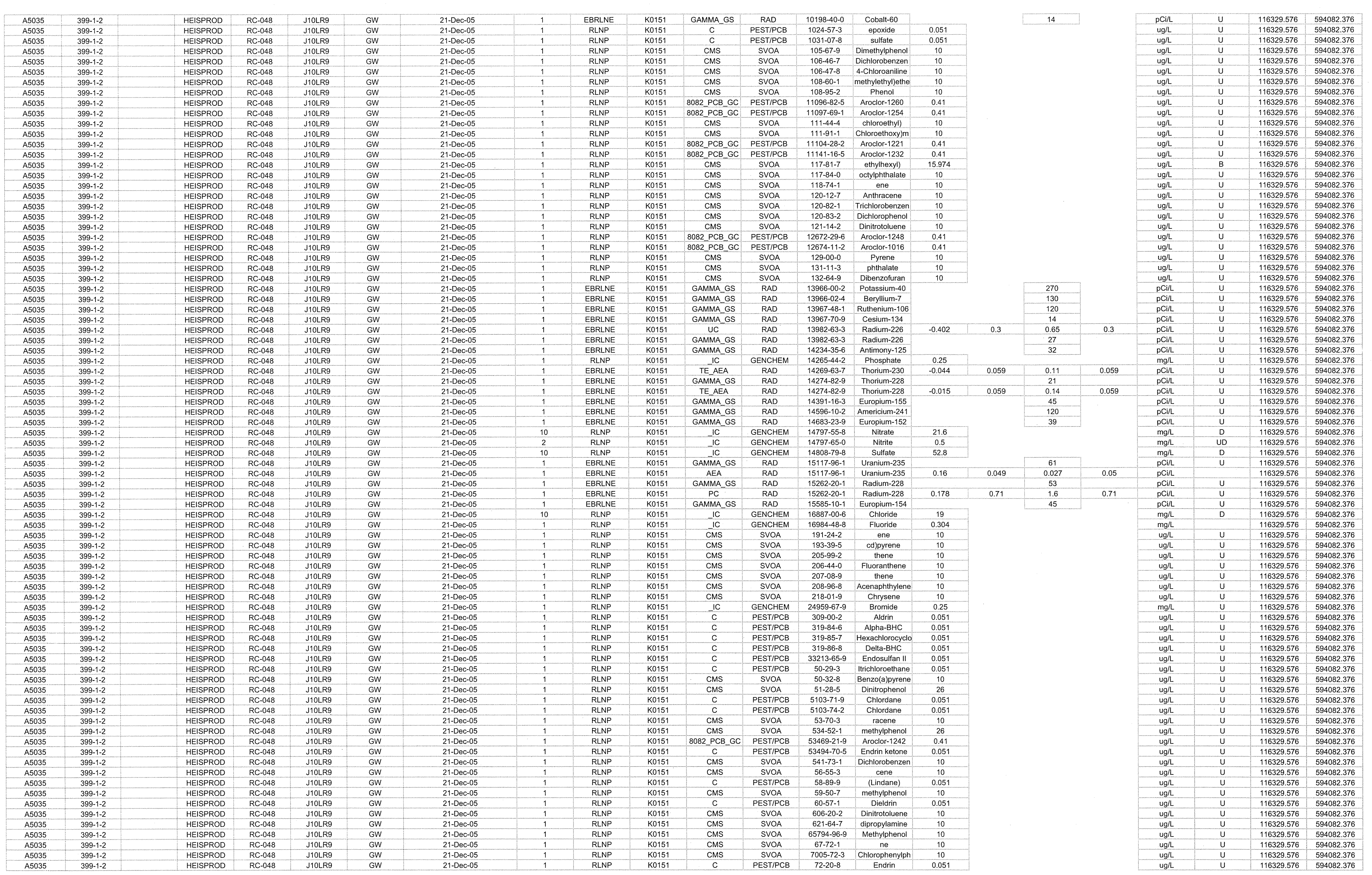




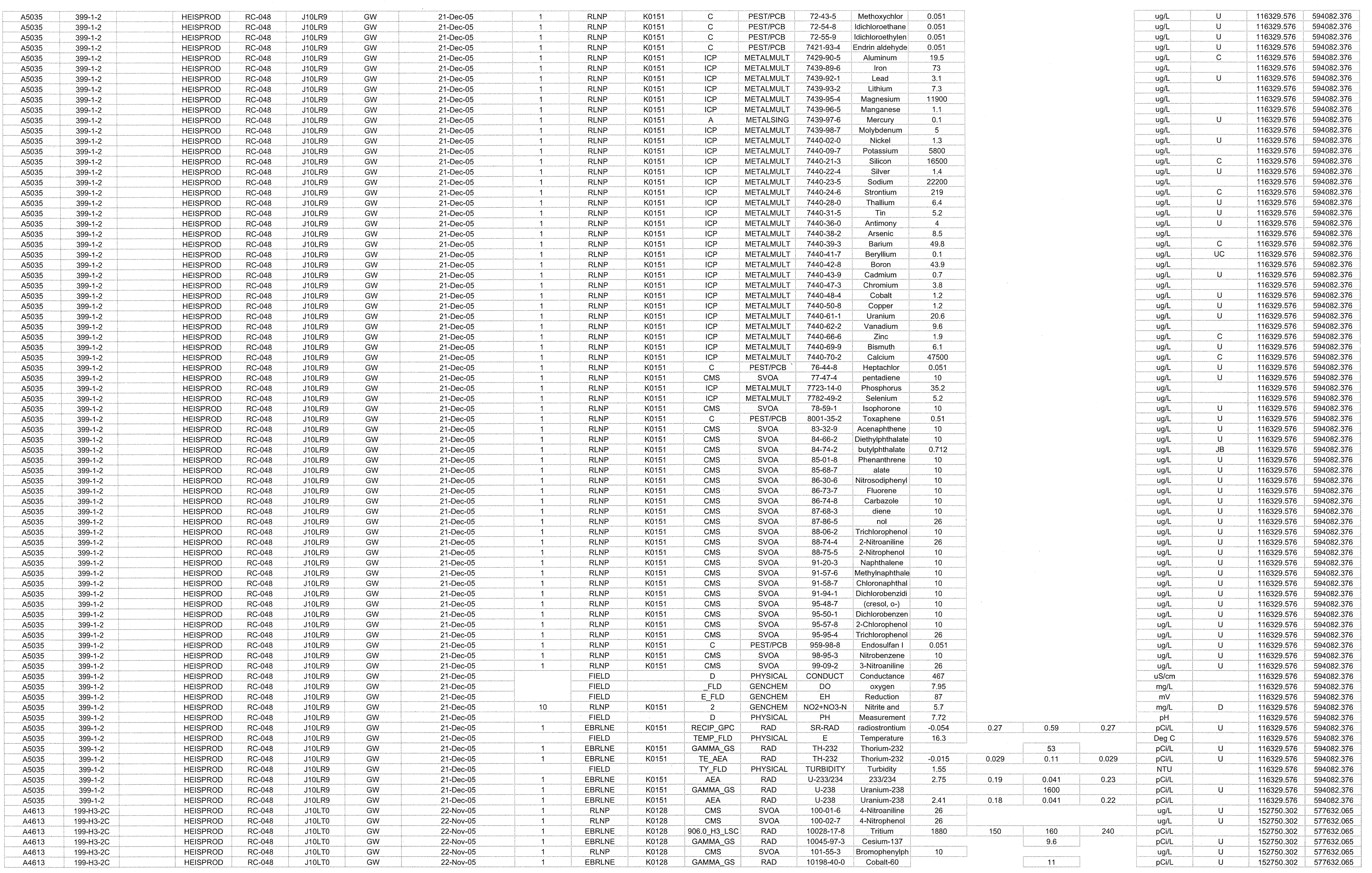




\begin{tabular}{|c|c|c|c|c|c|c|c|c|c|c|c|c|c|c|c|c|c|c|c|c|c|c|}
\hline A4613 & $199-13-2 C$ & HEISPROD & RC-048 & J10LTO & GW & 22-Nov-05 & 1 & RLNP & k0128 & $c$ & PEST/PCB & $1024-57-3$ & epoxide & 0.05 & & & & & ugl & $u$ & 152750.302 & 577632.065 \\
\hline $\begin{array}{ll}A 4613 \\
A 4613\end{array}$ & $199-13-2 C$ & HEISPROD & $\begin{array}{ll}R C-048 \\
R C-084\end{array}$ & 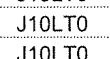 & $G W$ & 22-Nov-05 & 1 & RLNP & Ko128 & $\mathrm{c}$ & PEST/PCB & $1031-07-8$ & $\begin{array}{l}\text { sulfate } \\
\text { simothes }\end{array}$ & 0.05 & & & & & ugh & $u$ & 152750.302 & 577632.065 \\
\hline $\begin{array}{l}A 6413 \\
A 4613\end{array}$ & $\begin{array}{l}199-H 3-2 C \\
199-3 / 2 C\end{array}$ & HEISPROD & $\begin{array}{l}\mathrm{RC}-048 \\
R C-088\end{array}$ & $\begin{array}{l}\text { J10LtT } \\
\text { J10TO }\end{array}$ & $\frac{G W}{G W}$ & 22-Nov-05 & 1 & $\frac{R L N P}{\text { RLNP }}$ & $\begin{array}{l}\text { K0128 } \\
\text { K0128 }\end{array}$ & $\begin{array}{l}\text { CMS } \\
\text { CMS }\end{array}$ & $\begin{array}{l}\text { SVOA } \\
\text { SVOA }\end{array}$ & $\begin{array}{l}105-67-9 \\
10-46.7\end{array}$ & Dimethylphenol & 10 & & & & & ugh & $\mathrm{J}$ & $\begin{array}{l}152750.302 \\
152750302\end{array}$ & 577632.065 \\
\hline A4613 & $199-\mathrm{H} 3-2 \mathrm{C}$ & HEISPROD & RC-048 & J10LTO & GW & 22-Novor-05 & 1 & RLNP & K0128 & $\begin{array}{l}\text { CMS } \\
\text { CMS }\end{array}$ & $\begin{array}{l}\text { SOVA } \\
\text { SVOA }\end{array}$ & $\begin{array}{l}10 .-66-7 \\
00-67-8\end{array}$ & $\begin{array}{l}\text { Dichlorobenenzen } \\
\text { 4-Chloroaniline }\end{array}$ & $\frac{10}{10}$ & & & & & $\begin{array}{l}\text { ugh } \\
\text { uolu } \\
\text { vol }\end{array}$ & & $\begin{array}{l}\frac{152750.302}{1525302} \\
503\end{array}$ & $\begin{array}{l}577632.065 \\
57672065\end{array}$ \\
\hline A4613 & $199-\mathrm{H} 3-2 \mathrm{C}$ & HEISPROD & RC-048 & J10LTO & GW & 22-Nov-05 & 1 & RLNP & Ko128 & CMS & SVOA & 108-60-1 & 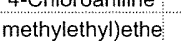 & $\frac{10}{10}$ & & & & & wgh & u & $\frac{10257.002}{15275.302}$ & 5 \\
\hline A4613 & 199-H3-2C & HEISPROD & RC-048 & J10LTO & GW & 22-Nov-05 & 1 & RLNP & Ko128 & CMS & SVOA & $108-95-2$ & Phenol & 10 & & & & & ugh. & u & 152750.302 & 577632.065 \\
\hline $\begin{array}{l}\text { A4613 } \\
\text { A6613 }\end{array}$ & $199-\mathrm{H} 3-2 \mathrm{C}$ & HEISPROD & RC-048 & J10LTO & GW & 22-Nov-05 & $\frac{1}{1}$ & RLNP & Ko128 & 8082_PCB_GC & PEST/PCB & 11096-82-5 & Aroclor-1260 & 0.4 & & & & & ugh & $u$ & 152750.302 & 577632.065 \\
\hline A6413 & 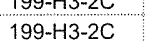 & $\begin{array}{l}\text { HEISPROD } \\
\text { HESPROD }\end{array}$ & $\begin{array}{l}\text { RC- }-48 \\
\text { R- }-48\end{array}$ & $\begin{array}{l}\text { J10LTO } \\
\text { J10LTO }\end{array}$ & $\begin{array}{l}\text { GW } \\
\text { GW }\end{array}$ & $\begin{array}{l}\text { 22-Nov-No5 } \\
22 \text {-Nov-05 }\end{array}$ & & $\frac{R \text { RLP }}{\text { RLNP }}$ & K10128 & 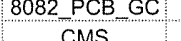 & PESTIPCB & $\begin{array}{l}11097-69-1 \\
111-444-4\end{array}$ & 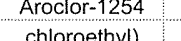 & 0.4 & & & & & ug/h & 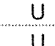 & $\begin{array}{l}152750.302 \\
155750 \\
\end{array}$ & 577632.065 \\
\hline$A 4613$ & 199--13-2C & $\begin{array}{l}\text { HESPROD } \\
\text { HEISPROD }\end{array}$ & $\begin{array}{l}\frac{R C-0.08}{R C-048} \\
R\end{array}$ & J10uto & $\mathrm{GW}$ & 22-Nov-Nos & 1 & RLNP & $\frac{K 0128}{K 0128}$ & $\begin{array}{l}\text { CMS } \\
\text { CMS }\end{array}$ & $\begin{array}{l}\text { SVVA } \\
\text { SVOA }\end{array}$ & (11-194-4 & 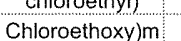 & 10 & & & & & ugh & ja & $\begin{array}{l}\frac{152750.0302}{152750.302} \\
-\end{array}$ & $\begin{array}{l}577632.065 \\
5767632005\end{array}$ \\
\hline A4613 & $199-13-2 C$ & HEISPROD & RC-048 & J10LTO & GW & 22-Nov-05 & 1 & RLNP & K0128 & 8082 PCB GC & $\begin{array}{l}\text { SUAA } \\
\text { PEST/PCB }\end{array}$ & $111104-28-2$ & Aroclor-1221 & 0.4 & & & & & ugh & 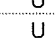 & 15272750.00202 & 577632.0655 \\
\hline A4613 & $199-H 3-2 C$ & HEISPROD & RC-048 & J10LTO & GW & 22-Nov-05 & 1 & RLNP & K0128 & 8082_PCB_GC & PEST/PCB & $11141-16-5$ & Aroclor-1232 & 0.4 & & & & & ugh & u & 152750.302 & 577632.20065 \\
\hline A6613 4432 & 199-1H3-2C & HEISPROD & RC-048 & J10LTO & Gw & 22-Nov-05 & 1 & RLNP & K0128 & CMS & SVOA & $117-81-7$ & ethylhexyl) & 6 & & & & & ugh & JB & 152750.302 & 577632.065 \\
\hline $\begin{array}{l}\text { A46613 } \\
\text { A4613 }\end{array}$ & 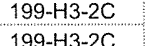 & $\begin{array}{l}\text { HEISPROD } \\
\text { HESPROD }\end{array}$ & $\begin{array}{ll}\text { RC-048 } \\
\text { BC.048 }\end{array}$ & J10LTO & GW & 22-200-05 & $\frac{1}{1}$ & RLNP & K0128 & CMS & SVOA & $\begin{array}{ll}111-84-0 \\
11184.0\end{array}$ & octylphthalate: & 10 & & & & & ugh & $\frac{u}{u}$ & 1572750.30302 & 577632.065 \\
\hline A4613 & 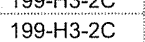 & $\begin{array}{l}\text { HESPROD } \\
\text { HEISPROD }\end{array}$ & $\frac{R C-0-048}{R C-48}$ & S10LTO & GW & 22-NOON-No5 & 1 & $\begin{array}{l}\text { KLLN } \\
\text { RLNP }\end{array}$ & K0128 & $\begin{array}{l}\text { CMS } \\
\text { CMS }\end{array}$ & $\begin{array}{l}\text { SVOA } \\
\text { SVOA }\end{array}$ & 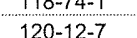 & Antine & $\begin{array}{l}10 \\
10\end{array}$ & & & & & (a) & & $\begin{array}{l}152755.302 \\
15027502\end{array}$ & \\
\hline A46613 & $199-13-2 C$ & HEISPROD & $\frac{\mathrm{R} C-048}{\mathrm{RC}}$ & 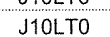 & $\mathrm{GW}$ & 22-NOOV-05 & & RLNP & Ko128 & CMS & $\begin{array}{l}\text { SWUA } \\
\text { SVOA }\end{array}$ & $120-82-1$ & Trichlorobenzzen & 10 & & & & & ugh & & 15275750.003 & $\frac{57632.065}{577632.065}$ \\
\hline A4613 & 199--33-2C & HEISPROD & RC-048 & J10LTO & $\mathrm{GW}$ & 22-Nov-05 & 1 & RLNP & K0128 & CMS & SVOA & $120-83-2$ & Dichlorophenol: & 10 & & & & & uglh & & 152750.302 & $\frac{177632.065}{5}$ \\
\hline A4613 & $199-133-2 C$ & HEISPROD & RC-048 & J10LTO & GW & 22-Nov-05 & & RLNP & K0128 & CMS & SVOA & $121-14-2$ & Dinitrotoluene & 10 & & & & & ugh & & 152750.302 & 577632.065 \\
\hline $\begin{array}{l}A 4613 \\
A 4631\end{array}$ & 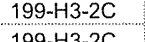 & HEISPROD & $\begin{aligned} R C-048 \\
B C-08\end{aligned}$ & J10LTO & $G W$ & 22-Nov-05 & & RLNP & K0128 & 8082 PCB_GC & $\begin{array}{l}\text { PESTPCB } \\
\text { PESTPCPCB }\end{array}$ & $\begin{array}{l}12672-29-6 \cdot 6 \\
1272-6\end{array}$ & Aroctor-1248 & 0.4 & & & & & ugh & & $\begin{array}{l}152750.302 \\
1527550302\end{array}$ & $\begin{array}{l}5777632.065 \\
577202055\end{array}$ \\
\hline $\begin{array}{l}A 6613 \\
A 4613\end{array}$ & $\begin{array}{l}1999-H-H-C C \\
199-3-2 C\end{array}$ & $\begin{aligned} H E S P P O D \\
\text { HEISPROD }\end{aligned}$ & $\frac{R C-0.048}{R C-048}$ & J10LLTO & GW & 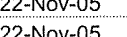 & & FLNP & 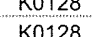 & 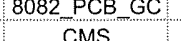 & SYAB & (1) & 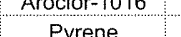 & $\frac{0.4}{10}$ & & & & & 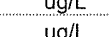 & & 1572756.302 & $\begin{array}{l}5777632.065 \\
75776320655\end{array}$ \\
\hline A4613 & 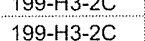 & HESPROD & 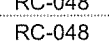 & J10ito & $G \mathrm{G}$ & 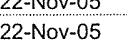 & 1 & RLNPP & K0128 & ciss & $\begin{array}{l}\text { SNOA } \\
\text { SVOA }\end{array}$ & - & $\begin{array}{l}\text { phtininate } \\
\text { phthate }\end{array}$ & 10 & & & & & & & 1572750.002 & 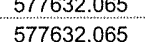 \\
\hline A4613 & $199-\mathrm{H} 3-2 \mathrm{C}$ & HEISPROD & RC-048 & J10LTO & GW & 22-Nov-05 & 1 & RLNP & K0128 & CMS & SVOA & $132-64-9$ & Dibenzofuran & 10 & & & & & ugh/ & & 152750.302 & 5 \\
\hline A4613 & $199-133-2 \mathrm{C}$ & HEISPROD & RC-048 & J10LTO & GW & 22-Nov-05 & 1 & EBRLNE & K0128 & GAMMA_GS & RAD & $13966-00-2$ & Potassium-40 & & & & 220 & & pcill & & 152750.302 & 577632.065 \\
\hline A4613 & 199-H3-2C & HEISPROD & RC-048 & J10LTO & GW & 22-Nov-05 & 1 & EBRLNE & K0128 & GAMMA_GS & RAD & $13966-02-4$ & $\begin{array}{l}\text { Beryllium-7 } \\
\text { - }\end{array}$ & & & & 100 & & pcill & & 152750.302 & 577632.065 \\
\hline A4613 & $\begin{array}{l}199-H 3-2 C \\
1\end{array}$ & $\begin{array}{l}\text { HEISPRODD } \\
\text { HISPOD }\end{array}$ & RC-048 & J10LTT & $G W$ & 22-200v-05 & 1 & $\begin{array}{l}\text { EBRLNE } \\
\text { EPLLET }\end{array}$ & K0128 & $\begin{array}{l}\text { AAMMA GS } \\
\text { GAM GS }\end{array}$ & $\begin{array}{l}R^{A R D} \\
{ }^{A}\end{array}$ & $\begin{array}{l}13967-48-1 \\
1307-7\end{array}$ & Ruthenium-1060 & & & & 10 & & pciin & 5 & $\begin{array}{l}152755.0302 \\
1527302\end{array}$ & \\
\hline $\begin{array}{l}A 6413 \\
A 4613\end{array}$ & $\frac{199-H 3-2 C}{199-B-2 C}$ & 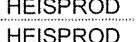 & $\frac{R C-048}{R C-048}$ & $\begin{array}{l}\text { J10LLTTO } \\
\text { J10LTO }\end{array}$ & $\frac{G W}{G W}$ & 22-2-Nov-05 & $\frac{1}{1}$ & EBLREN & $\begin{array}{l}\mathrm{K} 0128 \\
\mathrm{~K} 0128\end{array}$ & GAMMA GS & RAD & 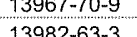 & 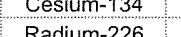 & 0008 & 0.42 & & $\frac{11}{17}$ & 0.42 & pein & 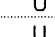 & $\begin{array}{l}152250.302 \\
157302\end{array}$ & $\begin{array}{l}577632.065 \\
5.77203055\end{array}$ \\
\hline A4613 & $199-\mathrm{H}-2 \mathrm{-2C}$ & HEISPROD & RC-048 & J10LLTO & GW & 22-Nov-05 & 1 & EBLINE & Kol128 & GAMMA GS & RAD & 13982-63-3 & 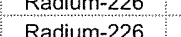 & 0.108 & 0.42 & & 18 & 0.42 & 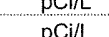 & & $\begin{array}{l}152755.302 \\
515750302\end{array}$ & $\begin{array}{l}5777332.065 \\
572055\end{array}$ \\
\hline $\mathrm{A4613}$ & $199-H 3-2 C$ & HEISPROD & RC-048 & J10LTO & GW & 22-Nov-05 & 1 & EBRLNE & Ko128 & GAMMA_GS & RAD & 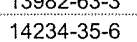 & Antimony-20 125 & & & & 19 & & 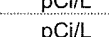 & & 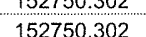 & $\begin{array}{l}577632.065 \\
557732065\end{array}$ \\
\hline A4613 & $199-\mathrm{H}-2 \mathrm{C}$ & HEIIPPROD & RC-048 & J10LTO & GW & 22-Nov-05 & 1 & RLNP & K0128 & IC & GENCHEM & 14265-44-2 & $\begin{array}{l}\text { Mhousphate } \\
\text { Phoshate }\end{array}$ & 0.25 & & & & & $\mathrm{mght}$ & & $\frac{152750.302}{1527502}$ & $\begin{array}{l}571 / 3632.2065 \\
577632.065\end{array}$ \\
\hline A4613 & 199-H3-2C & HEISPROD & RC-048 & J10LTO & GW & 22-Nov-05 & 1 & EBRLNE & Ko128 & TE_AEA & RAD & 142669-3-7. & Thorium-230 & 0.074 & 0.11 & & b.14 & 0.11. & pcill & & 152750.302 & 577632.065 \\
\hline 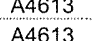 & $199-H 3-2 C$ & HEISPROD & RC- -48 & $\begin{array}{l}\text { S110LTO } \\
\text { J10LTO }\end{array}$ & GW & 22-Nov-05 & 1 & EBRLNE & K0128. & GAMMA_GS & RAD & 142274-82-9 & Thorium-228 & (1) & & & 12 & & pCill & & 152750.302 & 577632.065 \\
\hline A4613 & (199-H3-2C & $\begin{array}{l}\text { HEESPRDOD } \\
\text { HESPROD }\end{array}$ & $\begin{array}{l}\text { RC-048 } \\
R C-048\end{array}$ & J10LTO & $G \mathrm{G}$ & 22-NoN-Nov-05 & & 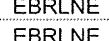 & K0128 & $\begin{array}{l}\text { TEAEA } \\
\text { GEMBA GS }\end{array}$ & RAD & 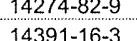 & $\begin{array}{l}\text { hinorum-2.28 } \\
\text { Euroum }\end{array}$ & -0.019 & 0.037 & & 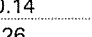 & 0.037 & pcin & & 152755.30202 & $\begin{array}{l}577632.065 \\
5577320055\end{array}$ \\
\hline A4613 & $199-H 3-2 C$ & HEISPROD & $\begin{array}{l}\text { RC- } \\
\text { RC-048 }\end{array}$ & J10LTO & GW & 22-Nov-05 & 1 & F & K0128 & GAMMA GS & RAD & 14596-1-1-2 & Americum-241 & & & & 42 & & pcil & & $\begin{array}{l}152750.306 \\
1502\end{array}$ & $\begin{array}{l}577632.005 \\
577632.065\end{array}$ \\
\hline A4613 & $199-\mathrm{H} 3-2 \mathrm{C}$ & HEISPROD & RC-048 & J10LTO & ow & 22-Nov-05 & 1 & EBRLNE & Ko128 & GAMMAGS & RAD & 14683-23-9 & Europium-152 & & & & 22 & & pcil & & 152750.302 & 577632.065 \\
\hline $\begin{array}{l}A 4613 \\
A 4613\end{array}$ & 199-H3-2C & HEISPROD & RC-048 & J10LTO & ow & 22-Nov-05 & 10 & RLNP & K0128 & IC & GENCHEM & 14497-55-8. & Nitrate & 25.8 & & & & & mgll & 5 & 152750.302 & 577632.065 \\
\hline $\begin{array}{l}A 4613 \\
A 4613\end{array}$ & $\begin{array}{l}1999-\mathrm{H} 3-2 \mathrm{C} \\
199-\mathrm{H}-2 \mathrm{C}\end{array}$ & HEISPROD & RC-048 & J10LTO & $\frac{G w}{G w}$ & 22-NON-05 & 10 & RLNP & K0128 & $\frac{1 C}{10}$ & GENCHEM & $\begin{array}{l}14797-65-0 \\
102087-7.98\end{array}$ & $\begin{array}{l}\text { Nititite } \\
\text { Selvitor }\end{array}$ & 0.25 & & & & & mgll & & $\begin{array}{l}152750.302 \\
15275520202\end{array}$ & 577632.065 \\
\hline A4613 & $199-\mathrm{H} 3-2 \mathrm{CC}$ & $\begin{array}{l}\text { HEISPROD } \\
\text { HEISPROD }\end{array}$ & $\begin{array}{l}\text { RC-048 } \\
\text { RC-048 }\end{array}$ & $\begin{array}{l}\text { J10LLTO } \\
\text { J10LTO }\end{array}$ & GW & 22-2-Nov-05 & 1 & $\begin{array}{l}\text { RRINP } \\
\text { EBRINE }\end{array}$ & $\begin{array}{l}K 0128 \\
K 0128\end{array}$ & GAMMA GS & GENCHEM & $\begin{array}{l}14880-7-7-8 \\
1517-96-1\end{array}$ & $\begin{array}{l}\text { Sulfate } \\
\text { Uranium-235 }\end{array}$ & 44.3 & & & 32 & & mgl & & 152756.30202 & $\begin{array}{l}5777632.065 \\
5577302065\end{array}$ \\
\hline A4613. & $199-13-2 C$ & HESPROD & $\frac{1 C-068}{R C-088}$ & J10LTO & GW & 22-Nov-05 & & LBLE & K0128 & AEA & $\frac{R A D}{R A D}$ & $15117-96-1$ & $\begin{array}{l}\text { Uranium-235 } \\
\text { Uranium }\end{array}$ & 0.04 & 0.081 & & .0.31 & 0.081 & pcili & j & $\begin{array}{l}152750.0302 \\
150202\end{array}$ & $\begin{array}{l}5777632.2065 \\
57632.065\end{array}$ \\
\hline A4613 & $199-43-2 \mathrm{C}$ & HEISPROD & RC-048 & J10LTO & GW & 22-Nov-05 & & EBRLNE & Ko128 & GAMMA_GS & RAD & $15262-20-1$ & Radium-228 & & & & 39 & & pCill & u & 152750.302 & 577632.065 \\
\hline A4613 & $199-\mathrm{H} 3-2 \mathrm{C}$ & HEISPROD & RC-048 & J1OLTO & GW & 22-Nov-05 & & EBRLNE & K0128 & $P C$ & RAD & $15262-20-1$ & Radium-228 & -0.548 & 5.3 & & 2 & 5.3 & pcill & & 152750.302 & 577632.065 \\
\hline A4613 & $199 \cdot-13 \cdot 2-2$ & HEISPROD & RC-048 & J10LTO & GW & 22-Nov-05 & 1 & EBRLNE & K0128 & GAMMA_GS & $\begin{array}{l}\text { RAD } \\
\text {. }\end{array}$ & $\begin{array}{l}15585-10-1 \\
16828700-1\end{array}$ & Europium-154 & 209 & & & 25 & & pciil & 1 & $\begin{array}{l}152750.302 \\
1520.0202\end{array}$ & 577632.065 \\
\hline $\begin{array}{l}A 4613 \\
A 4613\end{array}$ & $\begin{array}{l}1999-H 3-C C \\
199-H 3-2 C\end{array}$ & $\begin{array}{l}\text { HEISPROD } \\
\text { HEISPROD }\end{array}$ & $\begin{array}{l}\text { RC-048 } \\
\text { RC-048 }\end{array}$ & J10LLTOO & $G W$ & 22-Nov-Nov-05 & $\frac{10}{1}$ & KLNP & K0128 & $\pi$ & $\begin{array}{l}\text { GENCHEM } \\
\text { GNCHEM }\end{array}$ & 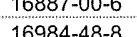 & 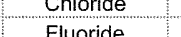 & 20.9 & & & & & mghn & & 152757.0.302 & $\begin{array}{l}5776322.065 \\
6777202055\end{array}$ \\
\hline A613 & $199-\mathrm{H}-2 \mathrm{-2C}$ & HESPROD & RC-048 & J10LTO & Gw & (2-2-Nov-05 & 1 & RLNP & K0128 & crss & SYoA & $191-24-2$ & ene & $\frac{6.90}{10}$ & & & & & vol & & 152750.0302 & $\begin{array}{l}5777302.065 \\
577632065\end{array}$ \\
\hline A6413 & $199+13-2 C$ & HEISPROD & RC-048 & J10LTO & $\mathrm{GW}$ & 22-Nov-05 & 1 & RLNP & Ko128 & CMS & SVOA & $193-39-5$ & cdlpyrene & 10 & & & & & ogli & j & 152750.302 & 577632.065 \\
\hline A4613 & $199-13-2 C$ & HEISPROD & RC-048 & J10LTO & GW & 22-Nov-05 & 1 & RLNP & K0128 & CMS & SVOA & $205-99-2$ & thene & 10 & & & & & ugl & j & 152750.302 & 577632.065 \\
\hline A4613 & 199-H3-2C & HEISPROD & RC-048 & J10LTO & GW & 22-Nov-05 & 1 & RLNP & K0128 & CMS & SVOA & $206-44-0$ & Fluoranthene & 10 & & & & & ugh & 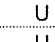 & 152750.302 & 5776332.065 \\
\hline A4613 & $199-13-2 C$ & HEISRROD & RC-048 & J110LTTO & GW & & 1 & $\begin{array}{l}\text { RLNNP } \\
\text { RLNP }\end{array}$ & K0128 & CMS & SVOA & $207-0889$ & thene & 10 & & & & & ugh & & 152750.302 & 577632.065 \\
\hline 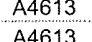 & $199-\mathrm{H} 3-2 \mathrm{CC}$ & $\begin{array}{l}\text { HEISPROD } \\
\text { HEISPR }\end{array}$ & $\begin{array}{l}\text { RC- }-048 \\
\text { RC-048 }\end{array}$ & $\begin{array}{l}\text { S110LTTO } \\
\text { J10LITO }\end{array}$ & $\frac{G W}{G W}$ & 22-2-No-0-5 & 1 & RLNP & $\frac{K 0128}{K 0128}$ & $\begin{array}{l}\text { CMS } \\
\text { CNS }\end{array}$ & $\begin{array}{l}\text { SSOA } \\
\text { SWOA }\end{array}$ & $\begin{array}{l}200-96-8 \\
238-8\end{array}$ & Acenaphithylene & 10 & & & & & ugh & 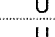 & $\begin{array}{l}152750.302 \\
157275020202\end{array}$ & $\begin{array}{l}577632.065 \\
-77702007\end{array}$ \\
\hline A4613 & $199-H 3-2 C$ & HEISPROD & RC-048 & J10LLTO & Gw & 22-Nov-05 & 1 & RLNP & K0128 & ic & $\begin{array}{l}\text { SNDA } \\
\text { GECHEM }\end{array}$ & $218-510.9-9$ & $\begin{array}{l}\text { Chrysenen } \\
\text { Ensend }\end{array}$ & 0.25 & & & & & ugh & & $\begin{array}{l}152750.302 \\
1572553202\end{array}$ & 5776332.065 \\
\hline A4613 & $199-\mathrm{H} 3-2 \mathrm{C}$ & HEISPROD & RC-048 & J10LTO & GW & 22-Nov-05 & & RLNP & K0128 & $c$ & $\begin{array}{l}\text { PENCHM } \\
\text { PEST/PCB }\end{array}$ & 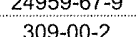 & $\begin{array}{l}\text { Bromber } \\
\text { Aldrien }\end{array}$ & $\begin{array}{l}0.25 \\
0.05\end{array}$ & & & & & mgin & & 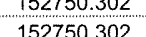 & $\begin{array}{l}577632.065 \\
55773202065\end{array}$ \\
\hline A4613 & & HEISPROD & RC-048 & J10LTO & GW & 22-Nov-05 & & RLNP & K0128 & $c$ & PEST/PCB & $319-84-6$ & Alpha-BHC & 0.05 & & & & & ught & & $\frac{152750.302}{1502}$ & $\begin{array}{l}57773632.2065 \\
57632.065\end{array}$ \\
\hline A4613. & $199-43-2 C$ & HEISPROD & RC-048 & J10LTO & GW & 22-Nov-05 & & RLNP & K0128 & c & PESTPCB & 319-85-7 & Hexachlorocyclo & 0.05 & & & & & ught & & 152750.302 & 577632.2005 \\
\hline A4613. & $199-H 3-2 C$ & HEISPROD & RC-048 & J10LTO & GW & 22-Nov-05 & & RLNP & K0128 & $c$ & PEST/PCB & $319-86-8$ & Delta-BHC & 0.05 & & & & & uggh & $\mathrm{J}$ & 152750.302 & 577632.065 \\
\hline A4613 & $199-H 3-2 C$ & HEISPROD & RC-048 & J10LTO & GW & 22-Nov-05 & 1 & RLNP & K0128 & S & PESTIPCB & $\begin{array}{l}33213-65-9 \\
60-20.9\end{array}$ & Endosulfan II & 0.05 & & & & & ugh & & $\begin{array}{l}152750.302 \\
152750.0202\end{array}$ & 5776332.065 \\
\hline A & 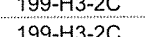 & HESTSPOD & RC-048 & 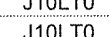 & Gw & 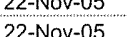 & & RINP & K0128 & C & SYOA & 年 & tricichloroethanee & 0.05 & & & & & gigh & & 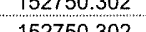 & $\begin{array}{l}5577332.2065 \\
5772055\end{array}$ \\
\hline 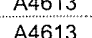 & 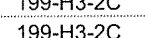 & HESPROD & RC-048 & 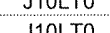 & $G w$ & 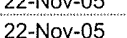 & & RLNPP & K0128 & sis & SWA & 5 & 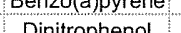 & 10 & & & & & . & 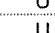 & 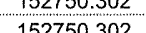 & $\begin{array}{l}5577332.2655 \\
5.7732065\end{array}$ \\
\hline A4613 & $199-H 3-2 C$ & HEISPROD & RC-048 & J10LLTO & GW & 22-Nov-05 & 1 & RLNP & K0128 & c & PEST/PCB & $5103-71-9$ & Chlordane & 0.05 & & & & & uglh & 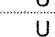 & $\begin{array}{l}1525250.3020 \\
152502\end{array}$ & $\begin{array}{l}5777632.2065 \\
577632065\end{array}$ \\
\hline A4613 & 199-H3-2C & HEISPROD & RC-048 & J10LTO & GW & 22-Nov-05 & 1 & RLNP & K0128 & $c$ & PESTIPCB & $5103-74-2$ & Chlordane & 0.05 & & & & & ugh & u & $\frac{152750.302}{1502}$ & 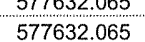 \\
\hline A4613 & $199-H 3-2 C$ & & & & GW & 22-Nov-05 & & RLNP & K0128 & CMS & SVOA & $53-70-3$ & racene & 10 & & & & & ugh & u & 152750.302 & $\begin{array}{l}571703.2005 \\
577632.065\end{array}$ \\
\hline A4613 & $199-H 3-2 C$ & HEISPROD & RC-048 & J10LTO & GW & 22-Nov-05 & & RLNP & K0128 & CMS & SVOA & $534-52-1$ & methylphenol & 26 & & & & & ught & $\mathrm{J}$ & 152750.302 & 577632.065 \\
\hline A4613. & $199-H 3-2 C$ & HEISPROD & RC-048 & J10LTO & GW & 22-Nov-05 & 1 & RLNP & K0128 & 8082_PCB_GC & PEST/PCB & $53469-21-9$ & Aroclor-1242 & 0.4 & & & & & ugil & u & 152750.302 & 577632.065 \\
\hline A4613. & $199-H 3-2 C$ & HEISPROD & RC-048 & J10LTO & GW & 22-Nov-05 & 1 & RLNP & K0128 & c & PEST/PCB & $\begin{array}{l}53494-70-5 \\
5-147-1\end{array}$ & Endorin ketone & 0.05 & & & & & uglt & u & 152750.302 & 5777632.065 \\
\hline 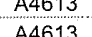 & 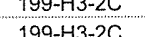 & HESTSPOD & 甚C.048 & 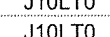 & Gw & 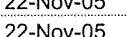 & 1 & RINP & K01728 & CMS & SVWA & 告 & Dicnorovenzzent & 10 & & & & & ogh & 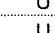 & 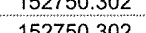 & $\begin{array}{l}5577332.2065 \\
5572065\end{array}$ \\
\hline 然 & 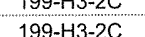 & HESTSROD & RC-048 & J1900T0 & Gw & 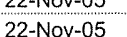 & 1 & RLNP & K0128 & chos & PESTACB & 58-58-9.99 & (lindane) & 0.05 & & & & & , & u & 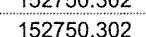 & $\begin{array}{l}5776320.065 \\
577632065\end{array}$ \\
\hline A6413 & $199-H 3-2 C$ & HEISPROD & RC-048 & J10LTO & Gw & 22-Nov-05 & 1 & RLNP & K0128 & CMS & SVOA & 59-50-7 & methylplonol & 10 & & & & & ught & u & $\frac{152750.302}{1502}$ & (577632.2005 \\
\hline A4613 & $199-13-2 C$ & HEISPROD & RC-048 & J10LTO & Gw & 22-Nov-05 & 1 & RLNP & K0128 & $c$ & PESTPCB & $60-57-1$ & Dieldrin & 0.05 & & & & & ugh & u & 152750.302 & 577632.065 \\
\hline A4613 & $199-\mathrm{H} 3-2 \mathrm{C}$ & HEISPROD & RC-048 & S10LTO & Gw & 22-Nov-05 & & RLNP & K0128 & CMS & SVOA & $606-20-2$ & Dinitrotoluene & 10 & & & & & ught & $u$ & 152750.302 & 577632.065 \\
\hline A6613 & $199-\mathrm{H} 3-2 \mathrm{C}$ & HEISPROD & $\begin{array}{l}\text { RC- }-448 \\
\text { BC-048 }\end{array}$ & $\begin{array}{l}310 L T 0 \\
\text { S1101T0 }\end{array}$ & $G W$ & 202-200-05 & & RLNP & K0128 & CMS & $\begin{array}{l}\text { SVOA } \\
\text { SWOA }\end{array}$ & $\begin{array}{l}6211-64-7 \\
65754-96-9\end{array}$ & dipropyyamine & 10 & & & & & ugl & 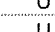 & $\begin{array}{l}152575.0302 \\
1525302\end{array}$ & 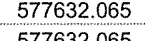 \\
\hline 其4413 & $\begin{array}{l}1999-\mathrm{H} 3-2 \mathrm{C} \\
199-\mathrm{H}-2 \mathrm{~T}\end{array}$ & 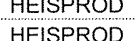 & $\begin{array}{l}\frac{1 C-048}{R C-048} \\
\text { Re }\end{array}$ & 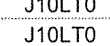 & GW & 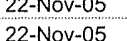 & 1 & RLNP & Ko128 & cms & SUOA & (6) & Netmperion & $\begin{array}{l}10 \\
10\end{array}$ & & & & & woh & u & 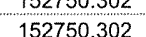 & 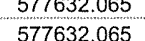 \\
\hline A4613 & $199-\mathrm{H} 3-2 \mathrm{C}$ & HEISPROD & RC-048 & J10LTO & Gw & 22-Nov-05 & 1 & RLNP & Ko128 & CMS & SVOA & $7005-72-3$ & Chlorophenylph & 10 & & & & & ugl & u & 152750.302 & 5 \\
\hline A4613 & $199-13-2 C$ & HEISPROD & RC-048 & J10LTO & GW & 22- Nov-05 & 1 & RLNP & K0128 & c & PESTIPCB & $72-20-8$ & Endrin & 0.05 & & & & & ugl & u & 152750.302 & 577632.065 \\
\hline A4613 & 199-43-2C & HEISPROD & RC-048 & & GW & 22-Nov-05 & & RLNP & K0128 & & PEST/PCB & $72-43-5$ & Methoxychlor & 0.05 & & & & & ug/L & u & 152750.302 & 577632.065 \\
\hline
\end{tabular}




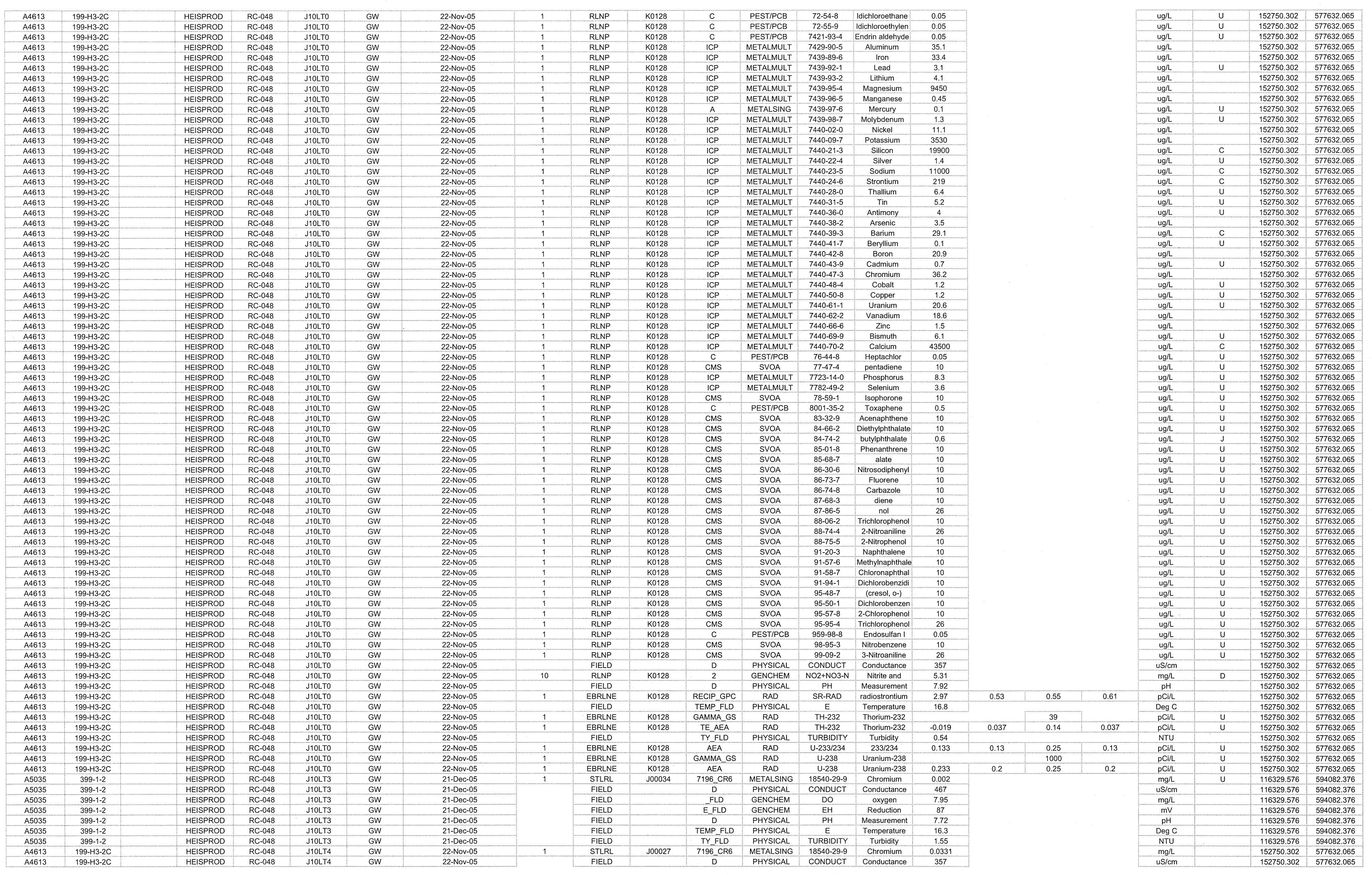



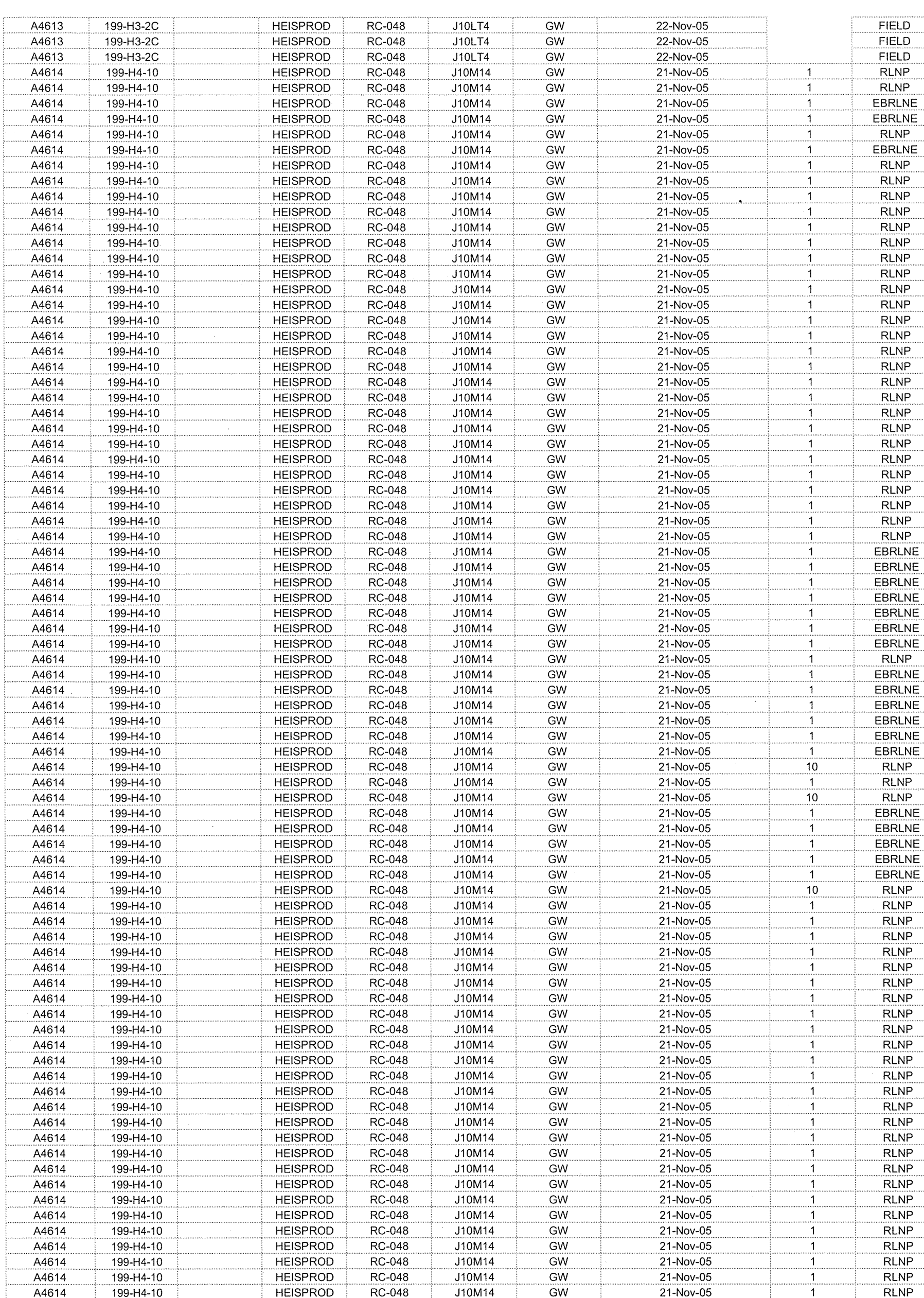

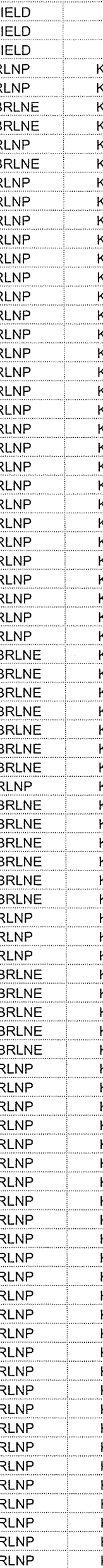
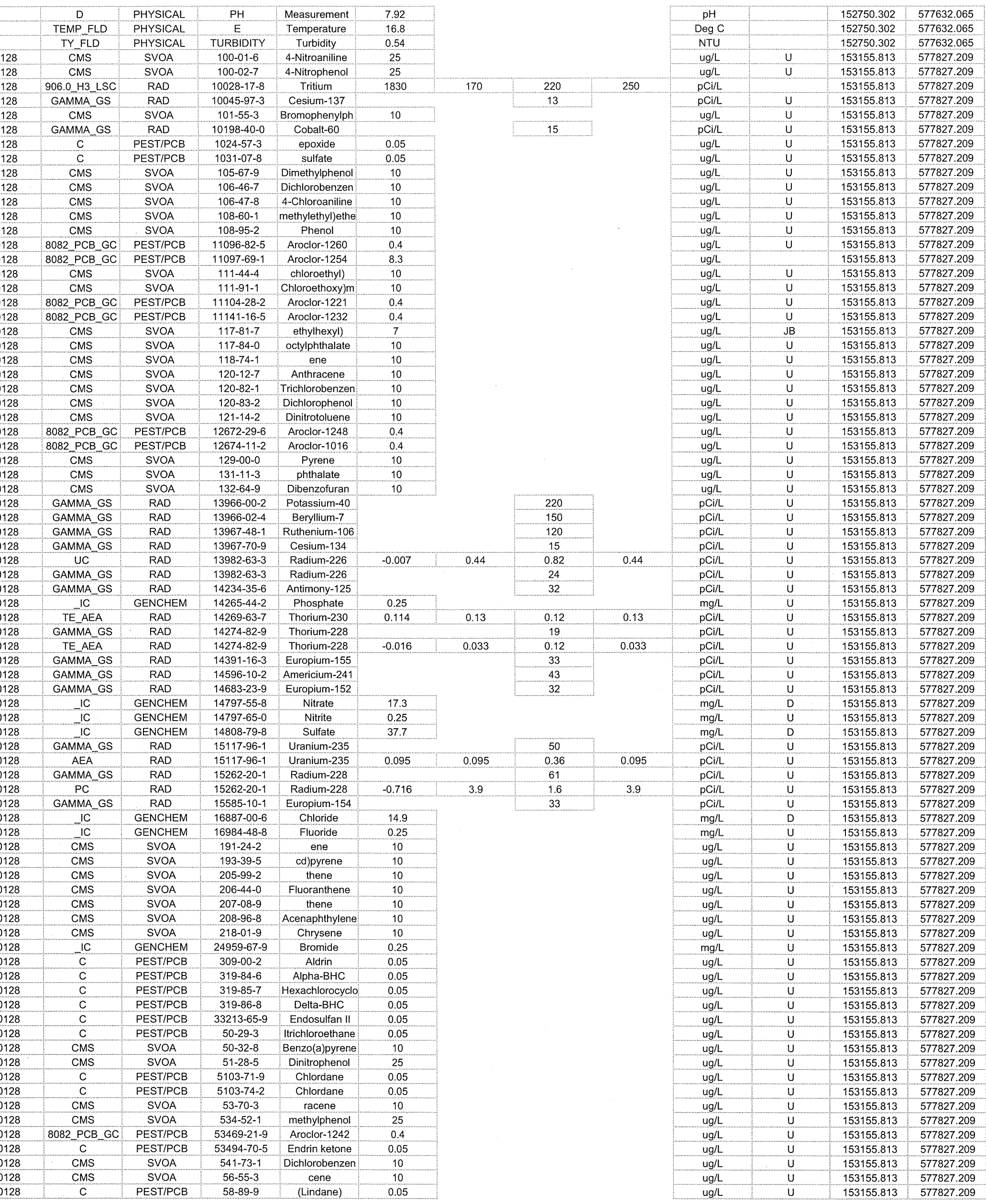


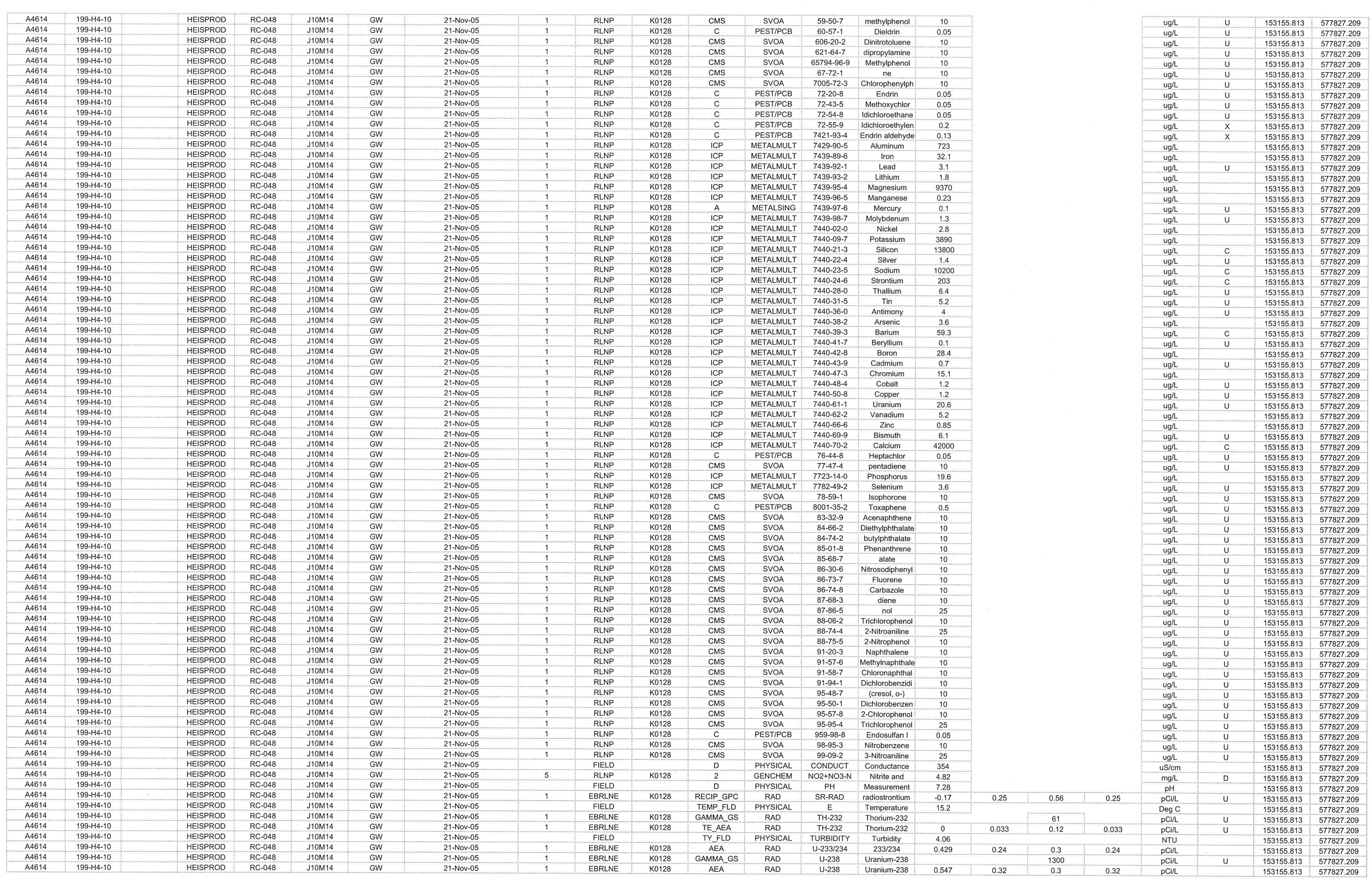




\begin{tabular}{|c|c|c|c|c|c|c|c|c|c|c|c|c|c|c|c|c|c|c|c|c|c|}
\hline A4619 & $199 \cdot-44-13$ & HEISPROD & RC-048 & J10M16 & GW & 21-Nov-05 & & RLNP & Ko128 & CMs & SVOA & $100-01-6$ & 4-Nitroaniline & 25 & & & & ug/ & $u$ & 152595.273 & 578219.304 \\
\hline A4619 & $199-144-13$ & HEISPROD & $\begin{array}{ll}\text { RC-048 } \\
\text { RC-048 }\end{array}$ & $\begin{array}{l}\text { J10M16 } \\
\text { J10M16 }\end{array}$ & $\frac{G W}{G W}$ & $\frac{21 \text { Nov-0.05 }}{\text { 21-Nov-55 }}$ & & $\begin{array}{l}\text { RLNP } \\
\text { ERRLNE }\end{array}$ & $\begin{array}{l}\text { K0128 } \\
\text { K0128 }\end{array}$ & $\begin{array}{c}\text { CMS } \\
9060 \mathrm{H}^{3} \mathrm{LSC}\end{array}$ & $\begin{array}{l}\text { SVAA } \\
\text { RAD }\end{array}$ & $\begin{array}{l}100-02-7 \\
10028-17-8 \\
\end{array}$ & 4-Nitrophenol & $\frac{25}{2140}$ & 180 & 220 & 280 & $\begin{array}{l}\text { ug/h } \\
\text { pCili }\end{array}$ & & $\begin{array}{l}\frac{152595.273}{152555273} \\
150\end{array}$ & $\begin{array}{l}578219.304 \\
5782190404\end{array}$ \\
\hline A4619 & $1999-44-13$ & HEISPROD & RC-048 & J10m16 & sw & 21-Nov-05 & & ERLLNE & K0128 & GAMMA GS & RAD & $10045-97-3$ & Cesium-137 & & & 9.7 & & pcil & u & 152595.273 & 578219.304 \\
\hline A4619 & $1999-14-13$ & HEISPROD & RC-048 & Jiom16 & $\mathrm{GW}$ & 21-Nov-05 & & RLNP & K0128 & CMS & SVOA & $101-55-3$ & Bromophenylph & 10 & & & & ugh & & 152595.273 & 578219.304 \\
\hline A4619 & $199-44-13$ & HEISPROD & RC-048 & J10M16 & $\mathrm{GW}$ & 21-Nov-05 & & $\begin{array}{l}\text { EBRLNE } \\
\text { RNP }\end{array}$ & Ko128 & GAMMA GS & $\begin{array}{l}\text { RAD } \\
\text { PESTPCB }\end{array}$ & $\begin{array}{l}10198-40-0 \\
1024-57-3\end{array}$ & 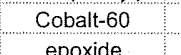 & & & 11 & & pcin & u & $\begin{array}{l}152595.273 \\
152555273 \\
\end{array}$ & $\begin{array}{l}578219.304 \\
578291904\end{array}$ \\
\hline A4619 & 199--H4-13 & HEISPROD & RC-048 & J10M16 & GW & 21-Nov-05 & & RLNP & K0128 & c & PESTIPCB & $1024.57-3$ & $\begin{array}{l}\text { epoxide } \\
\text { estrote }\end{array}$ & 0.05 & & & & ugh & $u$ & $\begin{array}{r}152595.273 \\
152552073\end{array}$ & 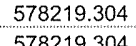 \\
\hline $\begin{array}{l}A 4619 \\
A 4619\end{array}$ & $199-44-13$ & $\begin{array}{l}\text { HESPRROD } \\
\text { HESPROD }\end{array}$ & $\begin{array}{l}\text { RC-048 } \\
\text { RC-048 }\end{array}$ & J10M16 & GW & $\begin{array}{l}21-N \text { Nov-05 } \\
\text { 21-No-05 }\end{array}$ & & $\frac{R \text { RINP }}{\text { RLNP }}$ & $\begin{array}{l}K 0128 \\
K 0128\end{array}$ & C & PESIPCB & $\begin{array}{l}1001-01-8 \\
105-67-9\end{array}$ & $\begin{array}{l}\text { suluate } \\
\text { Dimethvithenol }\end{array}$ & 0.05 & & & & $\begin{array}{l}\text { gugh } \\
\text { uglh }\end{array}$ & 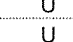 & $\begin{array}{l}1525555.273 \\
152595273\end{array}$ & $\begin{array}{l}57722919.304 \\
55829304\end{array}$ \\
\hline A4619 & 1999-14-13 & HEISPROD & RC- -048 & Jiom16 & GW & 21-Nov-05 & & RLNP & Ko128 & cMs & SVOA & $106-46-7$ & Dichlorobenzzen & 10 & & & & ugh & $u$ & 152595.273 & 578219.304 \\
\hline A4619 & $199--44-13$ & HEISPROD & RC-048 & J10M16 & $\mathrm{GW}$ & 21-Nov-05 & & RLNP & Ko128 & cMs & SVOA & $106-47-8$ & 4-Chloroaniline & 10 & & & & ugh & $u$ & 152595.273 & 578219.304 \\
\hline A4619 & 199-H4-13 & HEISPROD & $\begin{array}{ll}\text { RC-048 } \\
\text { BC. }\end{array}$ & J10M16 & GW & 21-Nov-05 & & RLNP & K0128 & CMS & SVOA & $\begin{array}{l}108-60-1 \\
0.085-2\end{array}$ & $\begin{array}{l}\text { methylethyly the } \\
\text { phenel }\end{array}$ & 10 & & & & ugh & & 152595.273 & 578219.304 \\
\hline A4619 & $199 \cdot-44-13$ & HEISPROD & RC-048 & J10M16 & GW & 21-Nov-05 & & RLNP & K0128 & CMS & $\begin{array}{l}\text { SVOA } \\
\text { DESTIDCSE }\end{array}$ & $\begin{array}{l}108-95-2 \\
10090.25\end{array}$ & $\begin{array}{l}\text { Phenol } \\
\text { Pans }\end{array}$ & 10 & & & & ugh & $u$ & $\begin{array}{r}152595.273 \\
152552073\end{array}$ & 578219.304 \\
\hline $\begin{array}{l}A 4619 \\
A 419\end{array}$ & $199-14-13$ & $\begin{array}{l}\text { HESPRROD } \\
\text { HESPPROD }\end{array}$ & $\begin{array}{l}\text { RC-048 } \\
\text { RC-048 }\end{array}$ & J10M16 & GW & 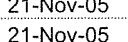 & & $\begin{array}{l}\text { RLNP } \\
\text { RINP }\end{array}$ & $\begin{array}{l}K 0128 \\
K 0128\end{array}$ & $\begin{array}{l}8002 \text { PCE GC } \\
8082 \text { PCB GC }\end{array}$ & $\begin{array}{l}\text { PESTIPCB } \\
\text { PEST/PCB }\end{array}$ & $\begin{array}{l}10.1069-82-5 \\
11097-69-1\end{array}$ & $\begin{array}{l}\text { Aracolor-1260 } \\
\text { Arroctor-1254 }\end{array}$ & $\begin{array}{l}0.4 \\
0.4\end{array}$ & & & & $\begin{array}{c}\text { oggh } \\
\text { oglh }\end{array}$ & u & $\begin{array}{l}\frac{152555.2 / 3}{152595.273} \\
\end{array}$ & 5 \\
\hline A4619 & 1999-14-13 & HEISPROD & RC-048 & J10m16 & GW & 21-Nov-05 & & RLNP & K0128 & CMS & SVOA & $111-44-4$ & chlorothethyli) & $\begin{array}{l}0.4 \\
10\end{array}$ & & & & $\begin{array}{l}\text { gll } \\
\text { ugl }\end{array}$ & $u$ & 152595.273 & 578219.304 \\
\hline A4619 & $199 \cdot-44-13$ & HEISPROD & RC-048 & J10M16 & GW & 21-Nov-05 & & RLNP & K0128 & cMS & SVOA & $111-91-1$ & chloroethoxylmm & 10 & & & & ugl & $u$ & 152595.273 & 578219.304 \\
\hline A4619 & $199-44-13$ & HEISPROD & RC-048 & J10M16 & GW & 21-Nov-05 & & RLNP & K0128 & 8082 PCB $6 C$ & PEST/PCB & 11104-28-2 & Aroclor-1221 & 0.4 & & & & ug/h & & 152595.273 & 578219.304 \\
\hline A4619 & $\begin{array}{l}199-144-13 \\
199-13\end{array}$ & HEISPROD & $\begin{array}{l}\text { RC- } 0488 \\
\text { RC }\end{array}$ & J10M16 & GW & 21-1-Nov-05 & & $\begin{array}{l}\text { RINPP } \\
\text { LNDP }\end{array}$ & K0128 & 8082 PCB GC & PESTPCB & 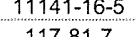 & Aroctor-123232 & 0.4 & & & & ugl & 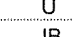 & $\begin{array}{l}1525555.2273 \\
15250573\end{array}$ & 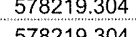 \\
\hline $\begin{array}{l}\text { A46619 } \\
\text { A4619 }\end{array}$ & $\frac{199-94-13}{190-14-13}$ & $\begin{array}{r}H E S P R P O D \\
H E S P R O D\end{array}$ & $\frac{R C-048}{R C-048}$ & J10M16 & GW & 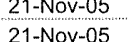 & & $\begin{array}{l}\text { RLNP } \\
\text { RINP }\end{array}$ & $\begin{array}{l}\text { KO128 } \\
\text { K0128 }\end{array}$ & $\begin{array}{l}\text { CMS } \\
\text { CMS }\end{array}$ & $\begin{array}{l}\text { SVOA } \\
\text { SVOA }\end{array}$ & $\begin{array}{l}111-8-1-1 \\
117-84-0\end{array}$ & 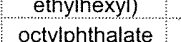 & $\begin{array}{l}4 \\
10\end{array}$ & & & & $\begin{array}{l}\text { ugh } \\
\text { ugh }\end{array}$ & $U$ & $\begin{array}{l}1525255.273 \\
152952.273\end{array}$ & 5778219.304 \\
\hline A4619 & $199-44-13$ & HEISPROD & RC- -048 & J10m16 & $G W$ & 211-Nov-05 & & RLNP & K0128 & CMS & SVOA & $118-74-1$ & ene & $\frac{10}{10}$ & & & & ugh/ & u & 152595.273 & 5 \\
\hline A4619 & $199--44-13$ & HEISPROD & RC-048 & J10M16 & $\mathrm{GW}$ & 21-Nov-05 & & RLNP & K0128 & CMS & SVOA & $120-12-7$ & Anthracene & 10 & & & & ugh & $u$ & 152595.273 & 578219.304 \\
\hline A4619 & $199-44-13$ & HEISPROD & RC-048 & J10M16 & GW & 21-Nov-05 & & RLNP & K0128 & CMS & & $120-82-1$ & Trichlorobenzen: & 10 & & & & ugh & u & 152595.273 & $\begin{array}{l}578219.0 .94 \\
5\end{array}$ \\
\hline A4619 & $199-144-13$ & HEISPROD & RC-048 & J10M16 & GW & 21-Nov-05 & & RLNP & K0128 & CMS & SVOA & $120-83-2$ & Dichlorophenol & 10 & & & & ug/L & u & 152595.273 & 578219.304 \\
\hline A4619 & $199--44-13$ & HEISPROD & RC-048 & J10M16 & GW & 21-Nov-05 & & RLNP & K0128 & cMs & SVOA & $121-14-2$ & Dinitrotoluene & 10 & & & & ugh & $u$ & 152595.273 & 578219.304 \\
\hline A4619 & $199-44-13$ & HEISPROD & RC-048 & J10M16 & GW & 21-Nov-05 & & RLNP & K0128 & 8082 PCB_GC & PESTIPCB & $\begin{array}{l}12672-29-6 \\
10270711-6\end{array}$ & Aroclor-1248 & 0.4 & & & & ugl & $u$ & 1525955.273 & $\begin{array}{l}578219.304 \\
6782010204\end{array}$ \\
\hline A4619 & $\begin{array}{l}199 \cdot-14-13 \\
109-14-13\end{array}$ & HEISPROD & $\begin{array}{l}\text { RC-048 } \\
\text { RC-048 }\end{array}$ & J10M16 & GW & 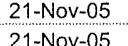 & & $\begin{array}{l}\text { RLNP } \\
\text { RLNP }\end{array}$ & K0128 & 8082_PCB_GC & PESTIPCB & $\begin{array}{l}12674-11-2 \\
120-00-0\end{array}$ & $\begin{array}{l}\text { Arocolor-1016 } \\
\text { Prrenes }\end{array}$ & $\begin{array}{l}0.4 \\
10\end{array}$ & & & & $\begin{array}{l}\mathrm{ugh/ \textrm {h }} \\
\mathrm{ug} / \mathrm{h}\end{array}$ & $u$ & 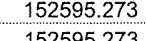 & $\begin{array}{l}578219.304 \\
57820190304\end{array}$ \\
\hline $\begin{array}{l}\text { A46619 } \\
\text { A4619 }\end{array}$ & 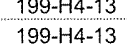 & $\begin{array}{l}\text { HESPRROD } \\
\text { HEISPROD }\end{array}$ & $\begin{array}{l}\text { RC-048 } \\
\text { RC-048 }\end{array}$ & J10M16 & $\begin{array}{l}G W \\
G W\end{array}$ & $\frac{21 \text { 21-Nov-05 }}{21 \text {-Nov-05 }}$ & & $\begin{array}{l}\text { RLNP } \\
\text { RLNP }\end{array}$ & $\begin{array}{l}\text { KO128 } \\
\text { K0128 }\end{array}$ & $\begin{array}{l}\text { CMS } \\
\text { CMS }\end{array}$ & $\begin{array}{l}\text { SVAA } \\
\text { SVOA }\end{array}$ & $\begin{array}{l}2 \begin{array}{l}29-0.0-0 \\
13-11-3\end{array} \\
0\end{array}$ & $\begin{array}{l}\text { Pyrene } \\
\text { phthalate }\end{array}$ & $\begin{array}{l}10 \\
10\end{array}$ & & & & $\begin{array}{l}\text { ug/h } \\
\text { ugh/ }\end{array}$ & u & 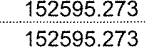 & $\begin{array}{l}57828219.304 \\
55821904\end{array}$ \\
\hline A4619 & $199-14-13$ & HEISPROD & RC-048 & J10m16 & GW & 21-Nov-05 & & RLNP & Ko128 & CMS & SVOA & $132-64-9$ & 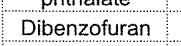 & 10 & & & & ugh & u & 1525595.273 & 578219.304 \\
\hline A4619 & $199--44-13$ & HEISPROD & RC-048 & Jiom16 & GW & 21-Nov-05 & & EBRLNE & K0128 & GAMMA GS & RAD & $13966-00-2$ & Potassium-40 & & & 220 & & pcill & $u$ & 152595.273 & 578219.304 \\
\hline A4619 & $199-144-13$ & HEISPROD & RC-048 & J10M16 & GW & 21-Nov-05 & & EBRLNE & K0128 & GAMMA GS & RAD & $13966-02-4$ & Beryllium-7 & & & 110 & & pCli & $u$ & $\begin{array}{l}1525955.273 \\
1525.272\end{array}$ & 578219.304 \\
\hline A4619 & $199-14-13$ & HESPROOD & RC- -048 & J10M16 & GW & 211-Nov-05 & & EBRLNE & K0128 & GAMMA_GS & RAD & $\begin{array}{l}13967-48-1 \\
1307-70\end{array}$ & Ruthennium-106 & & & & & pcil & u & 152595.273 & 578219.304 \\
\hline $\begin{array}{l}A 4619 \\
A 4619\end{array}$ & $\begin{array}{l}199-144-13 \\
190.44 .13\end{array}$ & HEISPROD & Rc-048 & STlompo & $G W$ & 21-Nov-05 & & EBRRNA & K0128 & GAMMA_GS & RAD & $13967-70-9$ & Cesium-134 & 0125 & & 12 & & pcill & $u$ & 152595.273 & 578219.304 \\
\hline A4619 & $199 \cdot-44 \cdot 13$ & HEISPROD & RC-048 & J10M16 & $\frac{G w}{G w}$ & 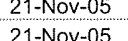 & & $\begin{array}{l}\text { EBRLNE } \\
\text { EBRR }\end{array}$ & 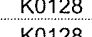 & UC & $\begin{array}{l}\text { RaA } \\
\text { PAD }\end{array}$ & $\begin{array}{l}19382-63-3 \\
1020253\end{array}$ & $\begin{array}{l}\text { Radiumm-226 } \\
\text { Radium }\end{array}$ & 0.125 & 0.3 & 0.055 & 0.3 & 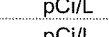 & 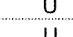 & $\begin{array}{l}1525555.273 \\
15255273\end{array}$ & $\begin{array}{l}578219.304 \\
57872010304\end{array}$ \\
\hline A4619 & $199-14-13$ & HEISPROD & RC-048 & J10M16 & $G w$ & 21-Noveros & & EBRE & K0128 & GAMMA GS GS & $\begin{array}{l}\text { RAD } \\
\text { RAD }\end{array}$ & 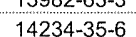 & Antimonof-125 & & & 24 & & 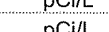 & 4 & 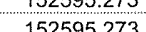 & 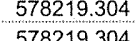 \\
\hline A4619 & $199-144-13$ & HESPROD & RC-048 & J10M16 & Gw & 21-Novoros & & $\begin{array}{l}\text { EDLLNE } \\
\text { RLNP }\end{array}$ & K0128 & II & GENCHEM & $\frac{14205-44-2}{1026-2}$ & Phosphate & 0.25 & & & & mgli & u & $\begin{array}{l}1525595.273 \\
15253\end{array}$ & $\begin{array}{l}57782919.304 \\
57821904\end{array}$ \\
\hline A4619 & $199-44-13$ & HEISPROD & RC-048 & J10m16 & GW & 21-Nov-05 & & EBRLLNE & Ko128 & TEAEEA & RAD & $14269-63-7$ & Thorium-230 & 0.016 & 0.09. & 0.12 & 0.094 & poil & u & 152595.273 & 578219.304 \\
\hline A4619 & $199 \cdot-44-13$ & HEISPROD & RC-048 & J10M16 & GW & 21-Nov-05 & & EBRLNE & K0128 & GAMMA GS & RAD & $\begin{array}{l}14274-82-9 \\
10270329\end{array}$ & Thorium-228 & & & 012 & & pcill & u & 1525955.273 & 578219.304 \\
\hline A4619 & $199 \cdot-44-13$ & HEISPROD & RC-048 & J10M16 & GW & 21-Nov-05 & & EBRLNE & K0128 & TEAAEA & RAD & $14274-82-9$ & Thorium-222 & 0 & 0.03 & 0.12 & 0.031 & pCill & $u$ & 152595.273 & 578219.304 \\
\hline A4619 & $\begin{array}{l}199-H 4-13 \\
199-13\end{array}$ & HEISPROD & $\begin{array}{l}\text { RC-048 } \\
\text { RC- }\end{array}$ & J10M16 & $G W$ & 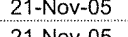 & & $\begin{array}{l}\text { EBLLNE } \\
\text { ERDLNE }\end{array}$ & K0128 & GAMMA GS & RAD & 14391-16-3 & Europium-155 & & & 32 & & pCilL & $u$ & 152595.273 & 578219.304 \\
\hline A46619 & $\frac{199-144-13}{199-144-13}$ & 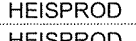 & $\begin{array}{l}\frac{R C-048}{R C-048} \\
\text { RC. }\end{array}$ & $\frac{510 M 16}{J 10 M 16}$ & $\frac{G W}{G W}$ & 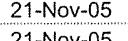 & & $\begin{array}{l}\text { EBRRNE } \\
\text { EPRDINE }\end{array}$ & K0128 & GAMMAGS & RAD & 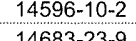 & Amercicum-241 & & & $\begin{array}{l}85 \\
28\end{array}$ & & pcin & 0 & 152595.273 & 578219.304 \\
\hline A4t69 & $199-44-13$ & HESPRDO & 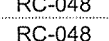 & J10M16 & $G w$ & 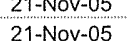 & 10 & $\begin{array}{l}\text { EBELNE } \\
\text { BIND }\end{array}$ & K0.128 & AAMMACSOS & READ & 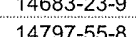 & Futropium-152 & 371 & & 28 & & pelin & 0 & 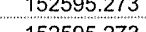 & 578219.304 \\
\hline A4619 & 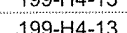 & HESPROOD & RC-048 & 然 & Giv & 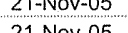 & & PLNP & K0128 & $\frac{10}{10}$ & ENCAR & 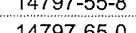 & Nivrale & 37.1 & & & & mgin & D & 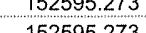 & 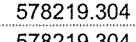 \\
\hline A4619 & $\frac{19-14-145}{199-H 4-13}$ & HEISPROD & RC-048 & J10M16 & GW & 21-Nov-05 & 10 & $\begin{array}{l}\text { RLNPP } \\
\text { RLNP }\end{array}$ & $\begin{array}{l}\text { KO128 } \\
\text { KO128 }\end{array}$ & $\frac{1}{16}$ & GENCHEM & $\begin{array}{l}14496-155-0 \\
14808-79-8\end{array}$ & $\begin{array}{l}\text { Nitrite } \\
\text { Sulfate }\end{array}$ & $\begin{array}{l}.0 .20 \\
54.8\end{array}$ & & & & 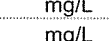 & D & 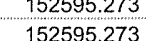 & 578219.304 \\
\hline A4619 & $199-44-13$ & HEISPROD & RC-048 & J10M16 & Gw & 21-Nov-05 & & EBRLNE & K0128 & GAMMA_GS & RAD & $15117-96-1$ & Uranium-235 & & & 46 & & pcilh & $u$ & 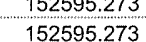 & 5 \\
\hline & $199-14-13$ & & RC-048 & & GW & 21-Nov-05 & & EBRLLE & K0128 & AEA & RAD & $15117-96-1$ & Uranium-235 & 0.067 & 0.06 & 0.26 & 0.068 & Coil & u & 152555273 & 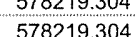 \\
\hline A46619 & $199-H 4-13$ & HEISPROD & RC-048 & J10M16 & GW & 21-Nov-05 & & EBRLNE & K0128 & GAMMA_GS & RAD & $15262-20-1$ & Radium-228 & & & 50 & 0 & pcil & $u$ & 152595.273 & 5 \\
\hline A4619 & $199 \cdot-44-13$ & HEISPROD & RC-048 & J10m16 & GW & 21-Nov-05 & & EBRLNE & & PC & RAD & $15262-20-1$ & Radium-228 & 0.173 & 0.55 & 1.5 & 0.55 & pcil & u & 1525955.273 & 578219.304 \\
\hline A4619 & $199 \cdot-44-13$ & HEISPROD & RC-048 & J10M16 & GW & 21-Nov-05 & & EBRLNE & Ko128 & GAMMA_GS & RAD & $155555-10-1$ & Europium-154 & 20 & & 33 & & pcil & $u$ & 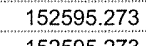 & 578219.304 \\
\hline A4619 & $\frac{199-44-13}{10-94-13}$ & HEISPROD & $\begin{array}{l}\text { RC-048 } \\
\text { RC-048 }\end{array}$ & J10016 & $\frac{G W}{G W}$ & $\begin{array}{l}21 \text {-Nov-05 } \\
21-\text { Nove-05 }\end{array}$ & & $\begin{array}{l}\text { RLNP } \\
\text { RNPP }\end{array}$ & K0128 & $\frac{1 C}{1 C}$ & $\begin{array}{l}\text { GENCHEM } \\
\text { GNCHEM }\end{array}$ & $\begin{array}{l}16887-00-6 \\
10684-48-8\end{array}$ & $\begin{array}{l}\text { Chloride } \\
\text { Col }\end{array}$ & 25.1 & & & & mglh & D & $\begin{array}{l}1525555.273 \\
152505\end{array}$ & 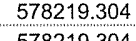 \\
\hline$\frac{A 4669}{A 4619}$ & $\frac{199-94-13}{199-14-13}$ & $\begin{array}{l}\text { HEISPROD } \\
\text { HEISPROD }\end{array}$ & $\begin{array}{l}\text { RC-048 } \\
\text { RC-048 }\end{array}$ & J10M16 & $\frac{G W}{G W}$ & $\begin{array}{l}21 \text { 21-Nov-05 } \\
\text { 21-Nov-05 }\end{array}$ & & $\begin{array}{l}\text { RLNP } \\
\text { RLNP }\end{array}$ & $\begin{array}{l}\text { KO1128 } \\
\text { Ko128 }\end{array}$ & $\begin{array}{l}\text { IC } \\
\text { CMS }\end{array}$ & $\begin{array}{l}\text { CENCHEM } \\
\text { SVOA }\end{array}$ & $\begin{array}{l}16684-48-8 \\
191-24-2\end{array}$ & $\begin{array}{l}\text { Fluoride } \\
\text { ene }\end{array}$ & $\frac{0.25}{10}$ & & & & $\begin{array}{l}\text { mggh } \\
\text { uglh }\end{array}$ & u & $\begin{array}{l}1525555.273 \\
152555.273 \\
\end{array}$ & 5778219.304 .304 \\
\hline A4619 & $199-14-13$ & HEISPROD & RC-048 & J10m16 & Gw & 21-Nov-05 & & RLNP & Ko128 & cMs & SVOA & 193-49-5 & codpyrene & 10 & & & & ugh & $u$ & 1525955.273 & 578219.304 \\
\hline A4619 & $199-44-13$ & HEISPROD & RC-048 & J10M16 & GW & 21-Nov-05 & & RLNP & & cMs & SVOA & $205-99-2$ & thene & 10 & & & & ugh & $u$ & 152595.273 & 578219.304 \\
\hline A4619 & $199-44-13$ & HEISPROD & RC-048 & J10M16 & GW & & & RLNP & K0128 & CMS & SVOA & $206-44-0$ & Fluoranthene & 10 & & & & ugh & $u$ & $\begin{array}{l}152555.273 \\
1565057272\end{array}$ & 578219.304 \\
\hline $\begin{array}{l}\text { A4619 } \\
\text { A44619 }\end{array}$ & 199-H4-13 & HEISPROD & $\begin{array}{l}\text { RC- }-048 \\
\text { BC-048 }\end{array}$ & J10M16 & GW & $\begin{array}{l}21 \text {-Novo- } 05 \\
21 \text {-Nove-05 }\end{array}$ & & $\frac{R L N P}{R L N P}$ & K0128 & $\begin{array}{c}\text { cMs } \\
\text { cMs }\end{array}$ & $\begin{array}{l}\text { SVA } \\
\text { SVOA }\end{array}$ & $\begin{array}{r}207-08-9 \\
208-96-8\end{array}$ & $\begin{array}{l}\text { Achene } \\
\text { Acenanthentiven }\end{array}$ & 10 & & & & ugh & $u$ & $\begin{array}{l}1525595.273 \\
1525552733\end{array}$ & 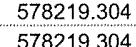 \\
\hline $\begin{array}{l}\text { A46619 } \\
\text { A4619 }\end{array}$ & $\frac{199-94-13}{199-44-13}$ & $\begin{array}{l}\text { HEISPROD } \\
\text { HEISPROD }\end{array}$ & $\begin{array}{l}\text { RC-048 } \\
\text { RC-048 }\end{array}$ & J10M16 & GW & $\begin{array}{l}\text { 21-Nov- } \\
\text { 21-Nov- }\end{array}$ & & $\begin{array}{l}\text { RLNP } \\
\text { RLNP }\end{array}$ & $\begin{array}{l}\text { KO128 } \\
\text { Ko128 }\end{array}$ & $\begin{array}{l}\text { MSS } \\
\text { CMS }\end{array}$ & $\begin{array}{l}\text { SVOA } \\
\text { SVOA }\end{array}$ & $\begin{array}{l}208-9-9-8 \\
218-01-9\end{array}$ & Acecanaphithyene & $\begin{array}{l}10 \\
10\end{array}$ & & & & $\begin{array}{l}\mathrm{gugh} \\
\mathrm{gghl}\end{array}$ & $u$ & 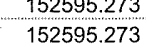 & 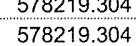 \\
\hline A4619 & $199-44-13$ & HEISPROD & RC- -048 & J10m16 & GW & 21-Nov-05 & & RLNP & Ko128 & 16 & GENCHEM & $24959-67-9$ & Bromide & 0.25 & & & & mglh & $u$ & 1525955.273 & 578219.304 \\
\hline A4619 & $199-44-13$ & HEISPROD & RC-048 & J10m16 & GW & & & RLNP & & & PESTIPCB & $309-00-2$ & Aldrin & 0.05 & & & & ugh & $u$ & 152595.273 & 578219.304 \\
\hline A4619 & $1999-44-13$ & HEISPROD & RC-048 & J10M16 & GW & & & RLNP & ko & & PESTIPCB & $\begin{array}{l}319-84-6 \\
210967\end{array}$ & Alpha-BHC & 0.05 & & & & ugh & $u$ & $\begin{array}{l}152595.273 \\
1.525057272\end{array}$ & 578219.304 \\
\hline A4619 & $199-44-13$ & HEISPROD & $\begin{array}{l}\text { RC- }-048 \\
\text { BC-048 }\end{array}$ & J10016 & $G W$ & $\frac{21 \text {-Nov }}{21 \text { Nowo }}$ & & RLNP & K012 & & $\begin{array}{l}\text { PESTPCB } \\
\text { PESTPCB }\end{array}$ & & 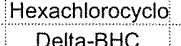 & 0.05 & & & & ugh & $u$ & $\begin{array}{l}1525595.273 \\
1555505\end{array}$ & 578219.304 \\
\hline $\begin{array}{l}\text { A46619 } \\
\text { A4619 }\end{array}$ & $\begin{array}{l}199-944-13 \\
199-144-13\end{array}$ & $\begin{array}{l}\text { HEISPROD } \\
\text { HEISPROD }\end{array}$ & $\begin{array}{l}\frac{R C-048}{R C-048} \\
\text { Re }\end{array}$ & J100M16 & $\begin{array}{l}G W \\
G W\end{array}$ & 21-Nov- 21 -Nov- & & $\begin{array}{l}\text { RLN } \\
\text { RLNP }\end{array}$ & $\begin{array}{l}\text { K0128 } \\
\text { K0128 }\end{array}$ & $\frac{c}{c}$ & $\begin{array}{l}\text { PESTIPCB } \\
\text { PESTIPCB }\end{array}$ & $\begin{array}{l}339-6-6-8 \\
33213-65-9\end{array}$ & $\begin{array}{l}\text { Dellab-BHC } \\
\text { Endoutifin }\end{array}$ & $\begin{array}{l}0.05 \\
0.05\end{array}$ & & & & $\frac{\text { ught }}{\text { oulh }}$ & u & $\begin{array}{l}1552559.273 \\
1525555273 \\
\end{array}$ & $\begin{array}{l}5772891.304 \\
57821904\end{array}$ \\
\hline A4619 & $199-44-13$ & $\begin{array}{l}\text { HEISPROD } \\
\text { HET }\end{array}$ & RC-048 & J10m16 & GW & 21-Nov-05 & & RLNP & K0128 & c & PESTPCB & 50-29-3-3 & 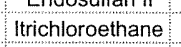 & 0.05 & & & & ught & $\mathrm{u}$ & 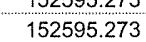 & 578219.304 \\
\hline A4619 & $199 \cdot-14-13$ & HEISPROD & RC-048 & J10m16 & GW & 21-Nover & & RLNP & K0128 & CMS & SVOA & $50-32-8$ & Bezzo(a)pyrene & 10 & & & & ugh & u & 152595.273 & 578219.304 \\
\hline A4619 & $199 \cdot-44-13$ & HEISPROD & RC-048 & J10M16 & ow & 21-Nov & & RLNP & K0128 & CMS & 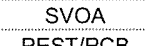 & & Dinitrophenol & 25 & & & & ugh & $u$ & $\begin{array}{l}152595.273 \\
1.5050-2727\end{array}$ & 57829.304 \\
\hline A4619 & $199-44-13$ & HEISPROD & $\begin{array}{l}\text { RC- }-048 \\
\text { BC-048 }\end{array}$ & J10M16 & GW & 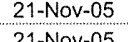 & & RLNP & $\frac{K 0}{k 0}$ & $\mathrm{C}$ & $\begin{array}{l}\text { PESTPCB } \\
\text { DETSTIDCB }\end{array}$ & $\begin{array}{l}5103-71-9 \\
5152072712\end{array}$ & $\begin{array}{l}\text { Chlordane } \\
\text { Sordpon }\end{array}$ & 0.05 & & & & ugh & u & $\begin{array}{l}1525955.273 \\
15505552723\end{array}$ & 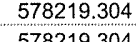 \\
\hline $\begin{array}{l}\text { A46619 } \\
\text { A4619 }\end{array}$ & $\frac{199-94-13}{199-14-13}$ & $\begin{array}{l}\text { HEISPROD } \\
\text { HEISPROD }\end{array}$ & $\begin{array}{l}\frac{1 C-048}{R C-048} \\
\text { RC }\end{array}$ & $\begin{array}{l}\text { J10M16 } \\
\text { J10M16 }\end{array}$ & $\frac{G W}{G W}$ & $\begin{array}{l}21-\mathrm{N} \text {-Nov-05 } \\
\text { 21-Nov-05 }\end{array}$ & & $\begin{array}{l}\text { RINP } \\
\text { RLNP }\end{array}$ & $\begin{array}{l}\text { K0128 } \\
\text { K0128 }\end{array}$ & CMS & SVOA & $\begin{array}{c}-51303-74-2 \\
53-70-3 \\
\end{array}$ & $\begin{array}{l}\text { Chlordane } \\
\text { racene }\end{array}$ & $\begin{array}{l}0.05 \\
10\end{array}$ & & & & $\frac{\text { ugh }}{\text { noll }}$ & u & $\begin{array}{l}\frac{1525595.273}{152595273} \\
\end{array}$ & $\begin{array}{l}5782529.304 \\
57821904\end{array}$ \\
\hline A4619 & $199-\mathrm{H} 4-13$ & HEISPROD & RC-048 & J10M16 & GW & $21-\mathrm{Nov}$ & & RLNP & Ko128 & cMs & SVOA & 534-52-1 & methylphenol & 25 & & & & ugh & U & 1052595.273 & 578219.304 \\
\hline A4619 & $199-44-13$ & HEISPROD & RC-048 & J10M16 & GW & 21-Nov-05 & & RLNP & K0128 & 8082_PCB_GC & PESTIPCB & $53469-21-9$ & Aroclor-1242 & 0.4 & & & & ugh & U & 152595.273 & 578219.304 \\
\hline A4619 & $199 \cdot-44-13$ & HEISPROD & RC-048 & J10M16 & GW & 21-Nov-05 & & RLNP & K0128 & $c$ & PESTPCB & 534494-70-5 & Endrin ketone & 0.05 & & & & ugl & $u$ & 152595.273 & 578219.304 \\
\hline A4619 & $199 \cdot-44-13$ & $\begin{array}{l}\text { HEISPROD } \\
\text { HIFPODOD }\end{array}$ & $\begin{array}{l}\text { RC-048 } \\
\text { PC. }\end{array}$ & J110016 & GW & $\begin{array}{l}21-\mathrm{N}-\mathrm{N}-0.05 \\
2025\end{array}$ & & RLNP & $\begin{array}{l}\text { K0128 } \\
\text { Ko128 }\end{array}$ & CMS & $\begin{array}{l}\text { SVOA } \\
\text { SVA }\end{array}$ & $\begin{array}{l}551-7-7-1 \\
465-3\end{array}$ & Dichlorobonzzen & 10 & & & & 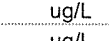 & $u$ & $\begin{array}{l}152555.2 .273 \\
15250.72\end{array}$ & 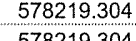 \\
\hline A4tis & 195-94-18 & HESPRPOD & RC-040 & 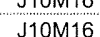 & $G w$ & 21-Nove-0.05 & & RLN & K0128 & c & PESTIPCB & 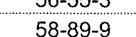 & 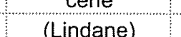 & 0.05 & & & & van & 4 & 155505.2573 & 5. \\
\hline A4619 & $199-44-13$ & HEISPROD & RC-048 & J10m16 & GW & 21-Nov-05 & & RLNP & Ko128 & CMS & SVOA & $59-50-7$ & methylphenol & 10 & & & & ugh & u & 152595.273 & 578219.304 \\
\hline A4619 & $199-44-13$ & HEISPROD & RC-048 & s10m16 & GW & 21-Nov-05 & & RLNP & K0128 & $c$ & PESTIPCB & $60-57-1$ & Dieldrin & 0.05 & & & & ugh & $u$ & 1525955.273 & 578219.304 \\
\hline A4619 & $199-44-13$ & HEISPROD & RC-048 & J10M16 & GW & 21-Nov-05 & & RLNP & K0128 & CMS & & & Dinitrotoluene & & & & & uggh & u & 152595.273 & 578219.304 \\
\hline
\end{tabular}




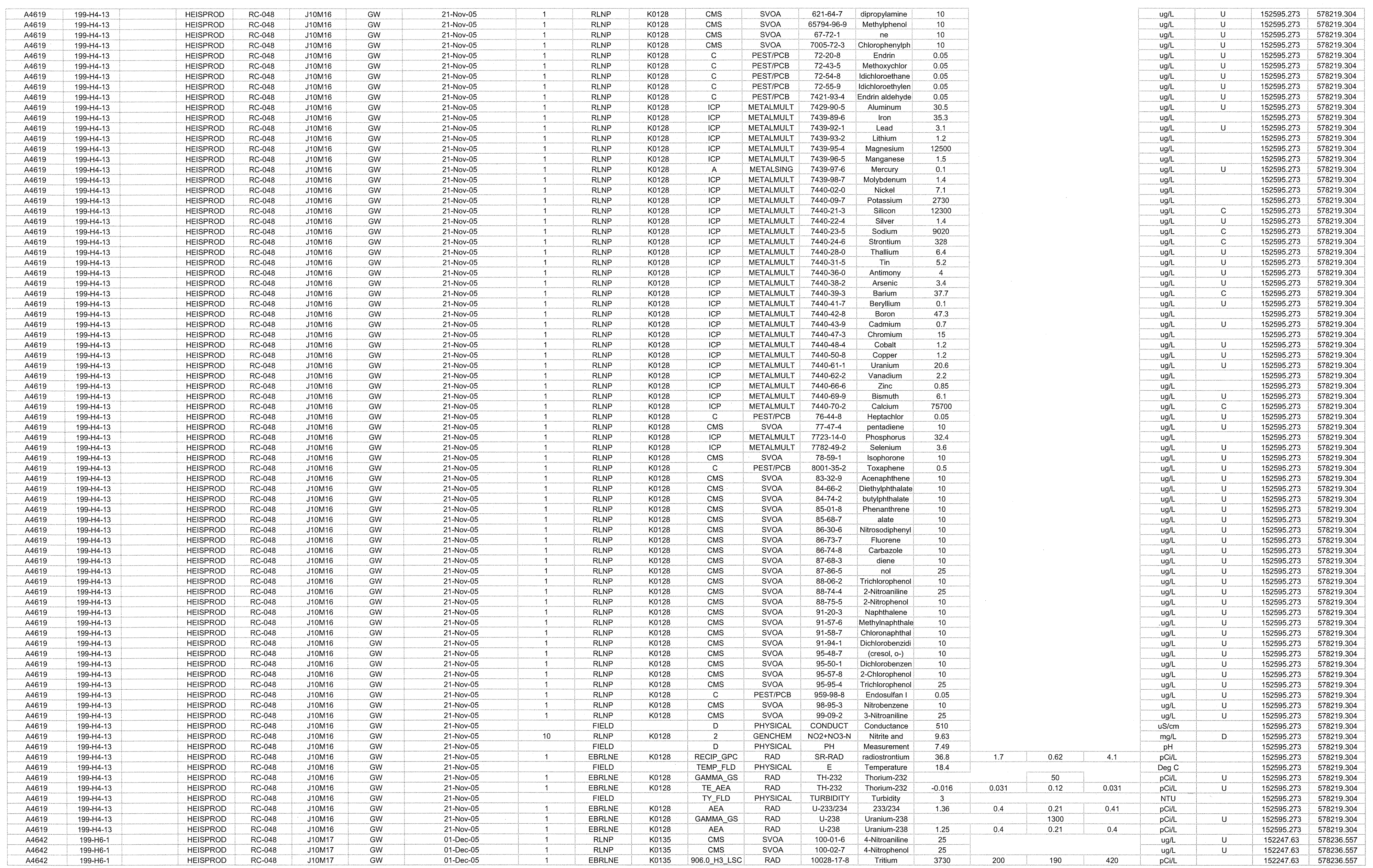




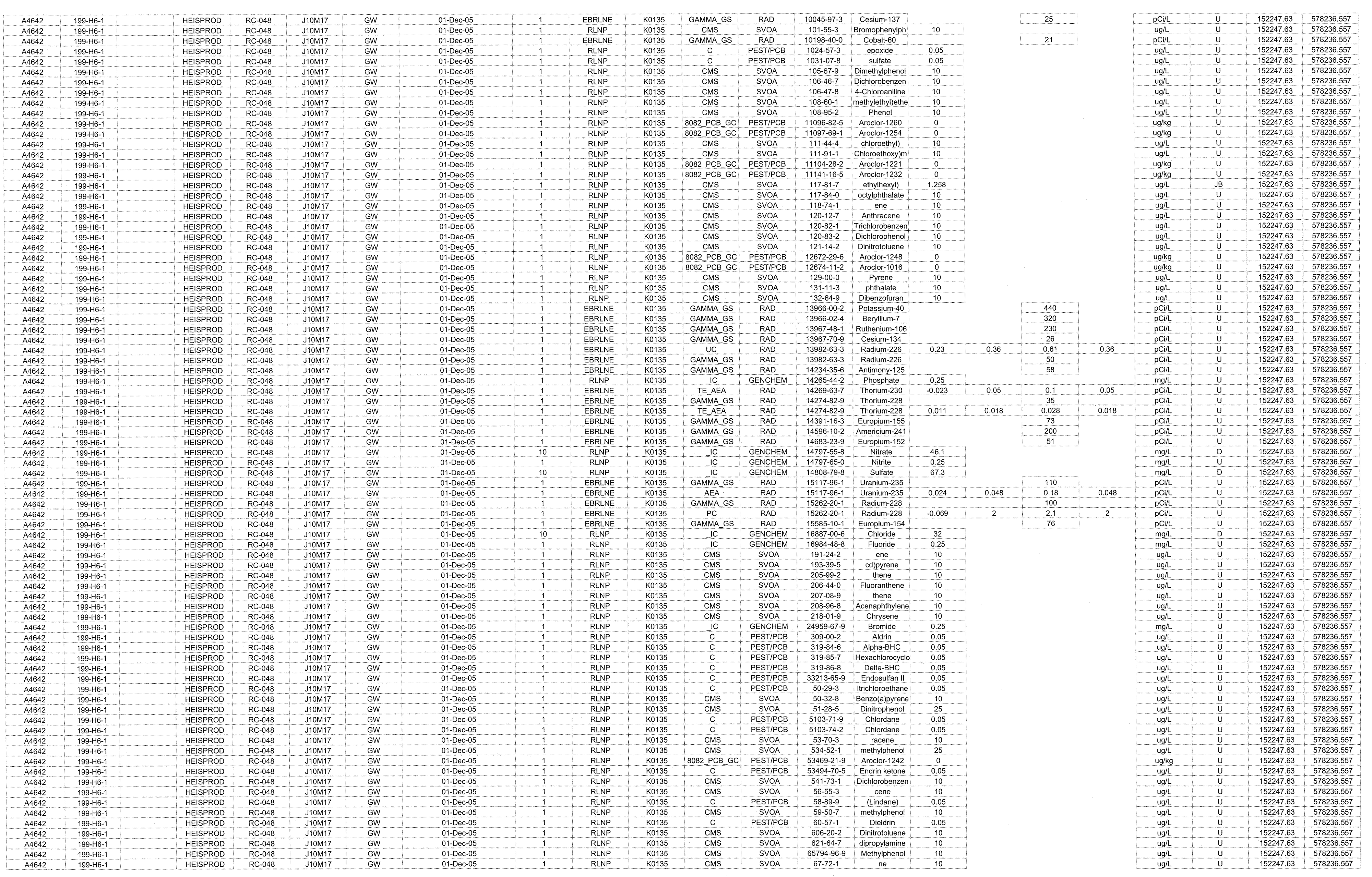




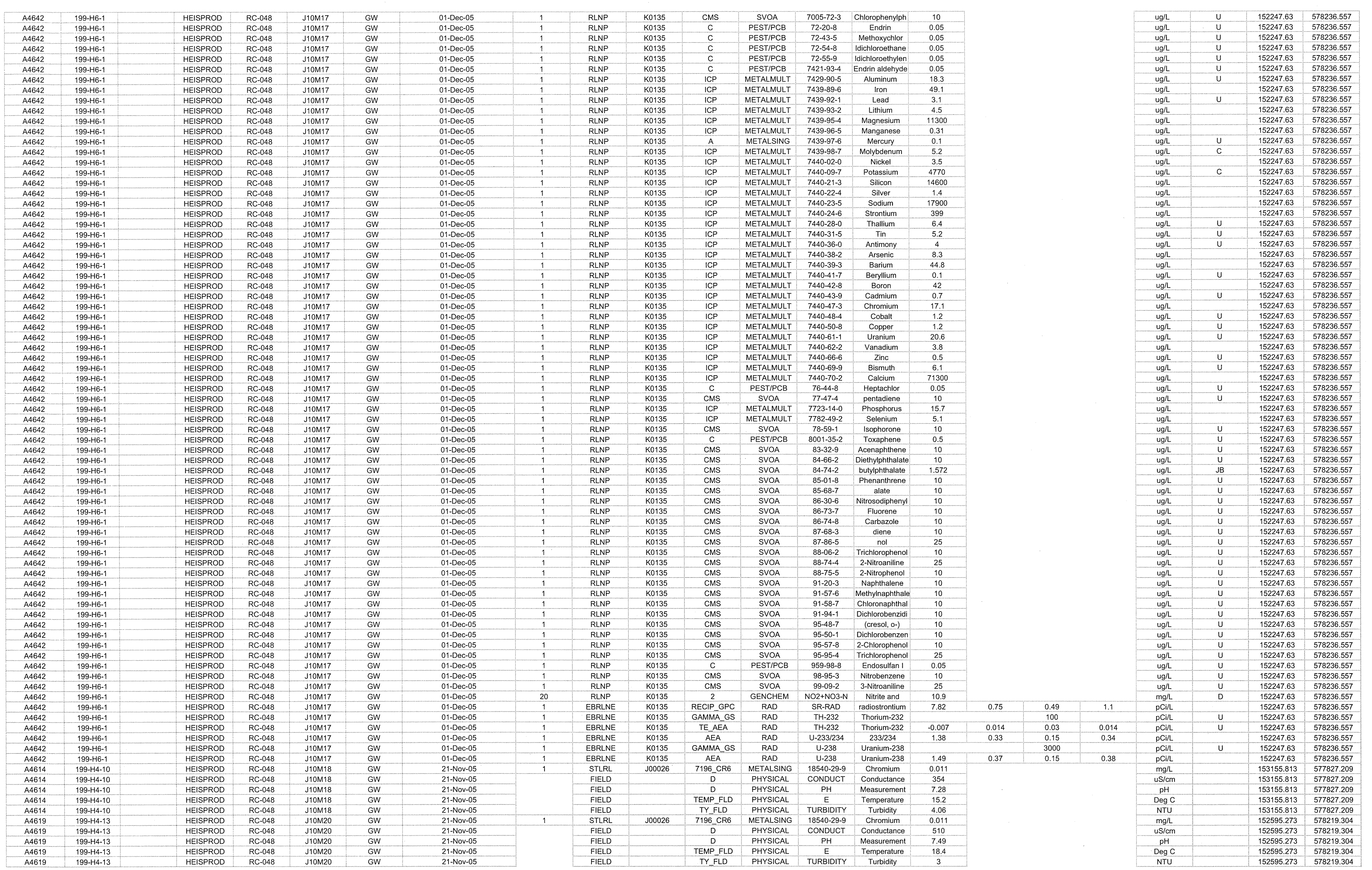




\begin{tabular}{|c|c|c|c|c|}
\hline A4642 & 199--H6-1 & HEISPROD & RC-048 & \\
\hline & 199-N-N4 & $\begin{array}{l}\text { HEISRROD } \\
\text { HITPODOD }\end{array}$ & $\frac{R C-048}{R C-048}$ & J10M48 \\
\hline $\begin{array}{l}\text { A4t08 } \\
\text { A4700 }\end{array}$ & 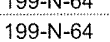 & $\begin{array}{l}\text { HEISRROD } \\
\text { HEISPROD }\end{array}$ & Re-40 & $\begin{array}{l}J 10 \mathrm{M} 48 \\
\mathrm{J10M48}\end{array}$ \\
\hline A4708 & $199-N-64$ & HEISPROD & & \\
\hline A4708 & $\begin{array}{l}1999-N-64 \\
100-64\end{array}$ & HEISPROD & RC-048 & J10M48 \\
\hline & $\begin{array}{l}1999-N-64 \\
199-N-64\end{array}$ & HESPROD & RC-048 & \\
\hline & $199-\mathrm{N}-64$ & HEIIPROD & 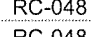 & J10M48 \\
\hline A4708 & $199-N-64$ & HEISPROD & $\mathrm{RC}-048$ & $\frac{\text { J10M48 }}{\text { J10M48 }}$ \\
\hline A4708 & $199-N-64$ & & RC-048 & J10M48 \\
\hline $\begin{array}{l}\text { A4408 } \\
\text { A4708 }\end{array}$ & $1999-\mathrm{N}-64$ & $\begin{array}{l}\text { HEISPROD } \\
\text { HEISPROD }\end{array}$ & $\frac{R C-48}{R C-048}$ & $\frac{510 M 48}{J 10 M 48}$ \\
\hline A4703 & $199-N-64$ & & RC-048 & $\begin{array}{l}\text { J10M448 } \\
\text { J10M8 }\end{array}$ \\
\hline $\begin{array}{l}A 4708 \\
A 4708\end{array}$ & $\begin{array}{l}1999-\mathrm{N}-64 \\
199-\mathrm{N} 64\end{array}$ & $\begin{array}{l}\text { HEIIPROD } \\
\text { HEISPROD }\end{array}$ & $\frac{R_{C-1}-48}{\mathrm{RC}-48}$ & J100M48 \\
\hline A4708 & 199-N-64 64 & HEISPROD & RC- -448 & \\
\hline A4708 & 199-N- -64 & HEISPROD & & \\
\hline & 1999-N-64 & HEISPROD & RC-048 & J10M48 \\
\hline & & $\begin{array}{l}\text { HEISPROD } \\
\text { HEISPROD }\end{array}$ & $\begin{array}{l}\text { RCC-0.08 } \\
\text { oc }\end{array}$ & J10M48 \\
\hline A4708 & 199-N-64 & HEISPROD & $\frac{\mathrm{R}-40}{\mathrm{R}-048}$ & $\begin{array}{l}\text { J10M48 } \\
\text { J10M48 }\end{array}$ \\
\hline A4708 & 199-N-N4 & HEISPROD & RC-048 & J10M48 \\
\hline$\frac{A 408}{A 4708}$ & $\begin{array}{l}1999-N-64 \\
199-N-64\end{array}$ & $\begin{array}{l}\text { HEIIPROOD } \\
\text { HEISPROD }\end{array}$ & $\begin{array}{l}\text { RC-0-084 } \\
\text { RC-048 }\end{array}$ & $\begin{array}{l}\text { J10M48 } \\
\text { J10M48 }\end{array}$ \\
\hline A4708 & $199-N-64$ & HEISPROD & RC-048 & \\
\hline A4t08 & 1999-N-64 & HEISPROD & & J10M48 \\
\hline & & HEISPROD & $R C-048$ & J10M48 \\
\hline A4708 & $\begin{array}{l}1999-N-1-04 \\
199-N-64\end{array}$ & $\begin{aligned} \text { HEIIPROD D } \\
\text { HEISPROD }\end{aligned}$ & $\begin{array}{l}\frac{R C-048}{R C-08} \\
R C-08\end{array}$ & J10M48 \\
\hline A4708 & $199-N-64$ & HEISPROD & RC-048 & $\begin{array}{l}\text { J110M48 } \\
\text { J10M48 }\end{array}$ \\
\hline A4708 & $199-N-24$ & HEIPPROD & RC-048 & J10M48 \\
\hline & & & & J10M48 \\
\hline A4708 & 1999-N-64 & HESSRODD & 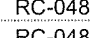 & J10M48 \\
\hline A4708 & $1099-1-04$ & $\begin{array}{l}\text { HEISPROD } \\
\text { HEISPROD }\end{array}$ & RC-048 & J10M48 \\
\hline A4708 & $199-N-64$ & HEISPROD & & $\begin{array}{l}\text { J10M48 } \\
\text { J10M48 }\end{array}$ \\
\hline & $199-N-64$ & HEISPROD & RC-048 & J10M48 \\
\hline 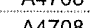 & & HEISPROD & RC-048 & J10M48 \\
\hline A4708 & 1000 & 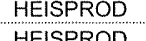 & RC-048 & J10M48 \\
\hline A4708 & $199-N-64$ & $\begin{array}{l}\text { HLISRODD } \\
\text { HESPROOD }\end{array}$ & . & J10M48 \\
\hline & & HEISPROD & RC-048 & J10M48 \\
\hline & $199-N-64$ & HEISPROD & RC-048 & J10M48 \\
\hline 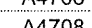 & $199-N-64$ & HEISPROD & RC-048 & J10M48 \\
\hline A4t00 & 199-N-64 & HEISPROD & RC-048 & J10M48 \\
\hline A4708 & 199-N-N64 & $\begin{array}{l}\text { MLISPODD } \\
\text { HESPROD }\end{array}$ & RC-0.-168 & $\begin{array}{l}510 \mathrm{~N} 48 \\
\text { J10M48 }\end{array}$ \\
\hline A4708 & $199-N \cdot 64$ & HEIPROD & & \\
\hline & 199-N-64 & HEISPROD & RC-048 & J10M48 \\
\hline A4708 & 199-N-64 & HEISPROD & RC-048 & J10M48 \\
\hline A4708 & 199-N-64 & HEISPROD & RC-048 & J10M48 \\
\hline A4708 & 1959-N-64 & HESTSPOD & RC-048 & \\
\hline A4708 & 199-N-64 & HEISPROD & RC-048 & J10M48 \\
\hline & 199-N-64 & HEISPROD & RC-048 & J10M48 \\
\hline A4708 & $199-N-64$ & HESPROD & RC-048 & J10M48 \\
\hline A4708 & 199-N-64. & HESPROD & RC-048 & J10M48 \\
\hline A4408 & 199-N-N64 & HESPPROD & & \\
\hline A4708 & $199-N-64$ & HEISPROD & RC-048 & J10M48 \\
\hline A4708 & 199-N-64 & HEISPROD & RC-048 & J10M48 \\
\hline A4708 & 199-N-64 & HEISPROD & RC-048 & J10M48 \\
\hline A4708 & 199-N-64 & HEISPROD & RC-048 & J10M48 \\
\hline A44008 & 195N-N-64 & HESPSROD & BC.048 & \\
\hline & $199-N-64$ & HEISPROD & RC-048 & J100448 \\
\hline A4708 & 199-N-64 & HEISPROD & RC-048 & J10M48 \\
\hline A4708 & 199-N-64 & HEISPROD & RC-048 & J10M48 \\
\hline A4708 & 199-N-N-64 & HEISPROD & RC-048 & J100M48 \\
\hline A44008 & 1995-N-N-64 & HESPROD & BC.-048 & 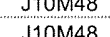 \\
\hline & & & & \\
\hline A4708 & 199-N-64 & HEISPROD & RC-048 & J10M48 \\
\hline A4708 & 199-N-64 & HEISPROD & RC-048 & J10M48 \\
\hline A4708 & 199-N-64 & HESPROD & RC- -048 & J10M48 \\
\hline A4t108 & 1959N-104-64 & 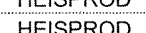 & BC- & \\
\hline & $199-N-64$ & HEISPROD & & \\
\hline & 199-N-64 & ISPROD & RC-048 & J10M48 \\
\hline A4708 & 199-N-64. & HEISPROD & RC-048 & J10M48 \\
\hline A4708 & & HESPROD & RC-048 & $\begin{array}{l}110 \mathrm{M} 48 \\
10048\end{array}$ \\
\hline
\end{tabular}
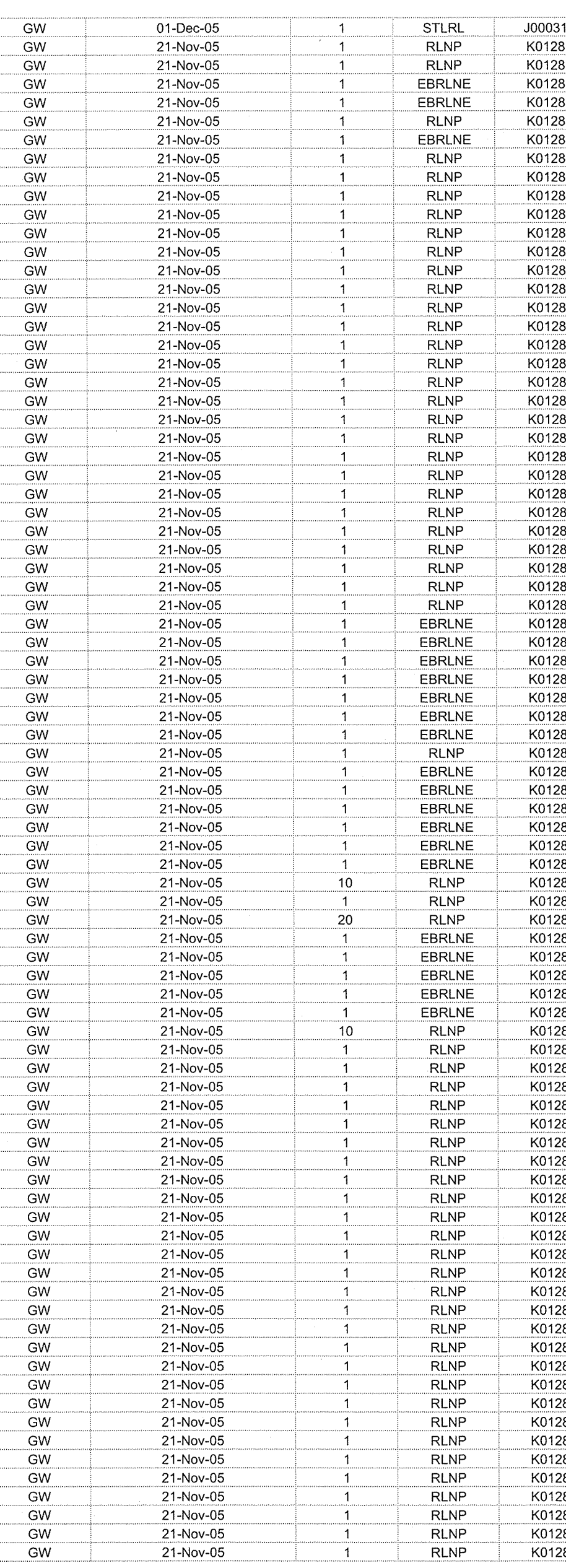

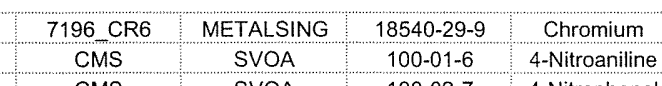

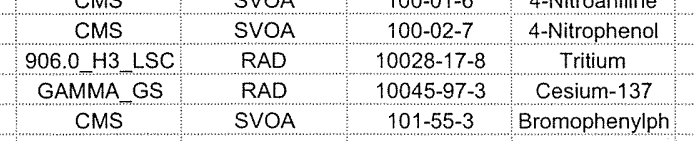

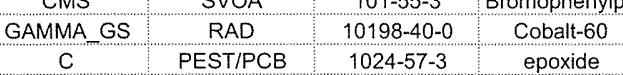

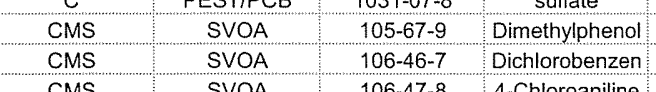

082 PCB GC PESTIPCB

\begin{tabular}{c|c}
$1096-82-5$ & Aroctor-1220 \\
\hline & Aroclor-1254
\end{tabular}

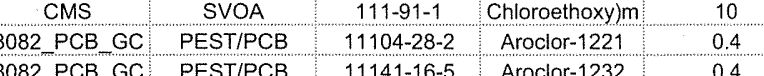

\begin{tabular}{l|l|l|l|l}
\hline CMS & SVOA & $117-81-7$ & ethylhexyl) \\
\hline CMS & SVOA & $117-84-0$ & octyphthalate \\
\hline
\end{tabular}

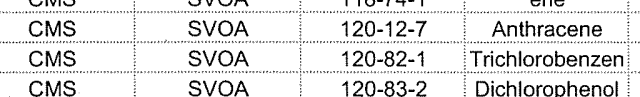

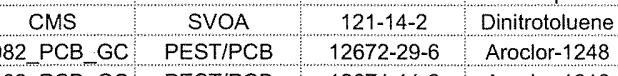

O82 PCB GC PESTIPCB

\begin{tabular}{c|c|c|c|c} 
CMS & SVOA & $129-000.0$ & Pryene & 10 \\
\hline CuS & SVOA & $131-3$ & phthalate & 10 \\
\hline
\end{tabular}

\begin{tabular}{l|l|l|l|}
\hline GAMMAGS & RAD & $13266-00-2$ & Potassium-40 \\
\hline GAMMAGS & RAD & $13966-02-4$ & Beryllium-7 \\
\hline
\end{tabular}

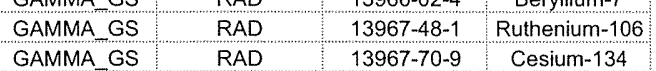

\begin{tabular}{c|c|c|c} 
UL & RAD & $13982-63-3$ & Radium-226 \\
\hline GAMMA GS & RAD & $13982-63-3$ & Radium-226 \\
\hline GAMMA_GS & RAD & $14234-35-6$ & Antimony-125 \\
\hline
\end{tabular}

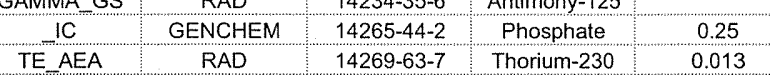

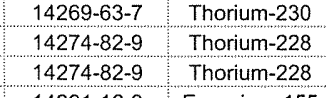

(0)

9 Europium-152

\begin{tabular}{|c|c|c|c|c|}
\hline IC & GENCHEM & $14797-55-8$ & Nitrate & 69 \\
\hline IC & GENCEMM & $14797-6-0$ & Nitite & 0.25 \\
\hline IC & GENCHEM & $14808-79-8$ & Suffate & 104 \\
\hline
\end{tabular}

\begin{tabular}{c|c|cc} 
GAMMA_GS & RAD & $15011-796-1$ & Uranium-235 \\
AEA & RAD & $1517-96-1$ & Uranium-235 \\
GAMMA_GS & RAD & $15262-20-1$ & Radium-222 \\
\hline
\end{tabular}

\begin{tabular}{|l|l|l|l|}
\hline 0.103 & 0.14 & 70 & 0.26 \\
\hline
\end{tabular}

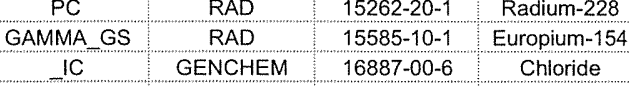

\begin{tabular}{|l|l|l|l|l|}
\hline IC & GENCCHEM & $16887-00-6$ & Chloride & 21.5 \\
\hline
\end{tabular}

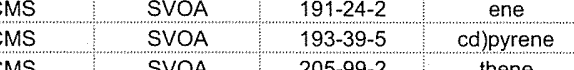

\begin{tabular}{l|l|l|l|l|}
\hline CMS & SVAA & $205-99-2$ & thene & 10 \\
\hline CMS & SVG & FVA & Fluoranthen & 10 \\
\hline
\end{tabular}

\begin{tabular}{c|c|c|c} 
CMS SVA & $208-96-8$ & Acenaphithylene \\
$218-01-9$ & Chysyene
\end{tabular}

\begin{tabular}{c|ccc} 
& $24959-67-9$ & Chyssene & 10 \\
\hline
\end{tabular}

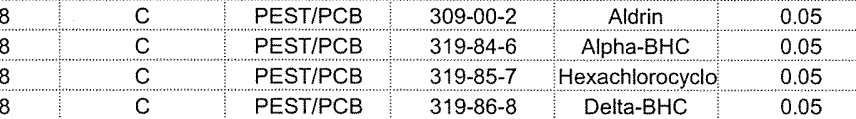

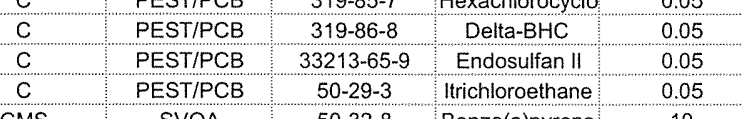

\begin{tabular}{c|c|c|c|} 
CMS & SVOA & $50-32-8$ & Benzo(a) pyrene \\
\hline CMS & SVOA & $51-28-5$ & Dinitrophenol \\
\hline
\end{tabular}

\begin{tabular}{|l|l|l|l|l} 
C & PESTTPCB & $5103-71-9$ & Chlordane & 0.05 \\
\hline C & PESTPCB & $5103-74-2$ & Chlordane & 0.05 \\
\hline
\end{tabular}

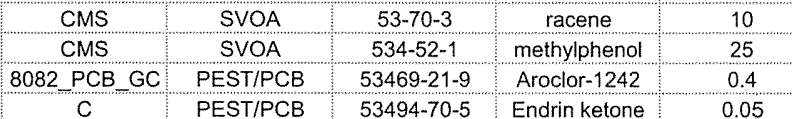

CMS SVOA 541-73-1 Dichtorbotone

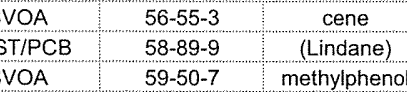

\begin{tabular}{cc} 
& 10 \\
\hline & 0.05 \\
\hline & 10 \\
\hline & 0.5
\end{tabular} 


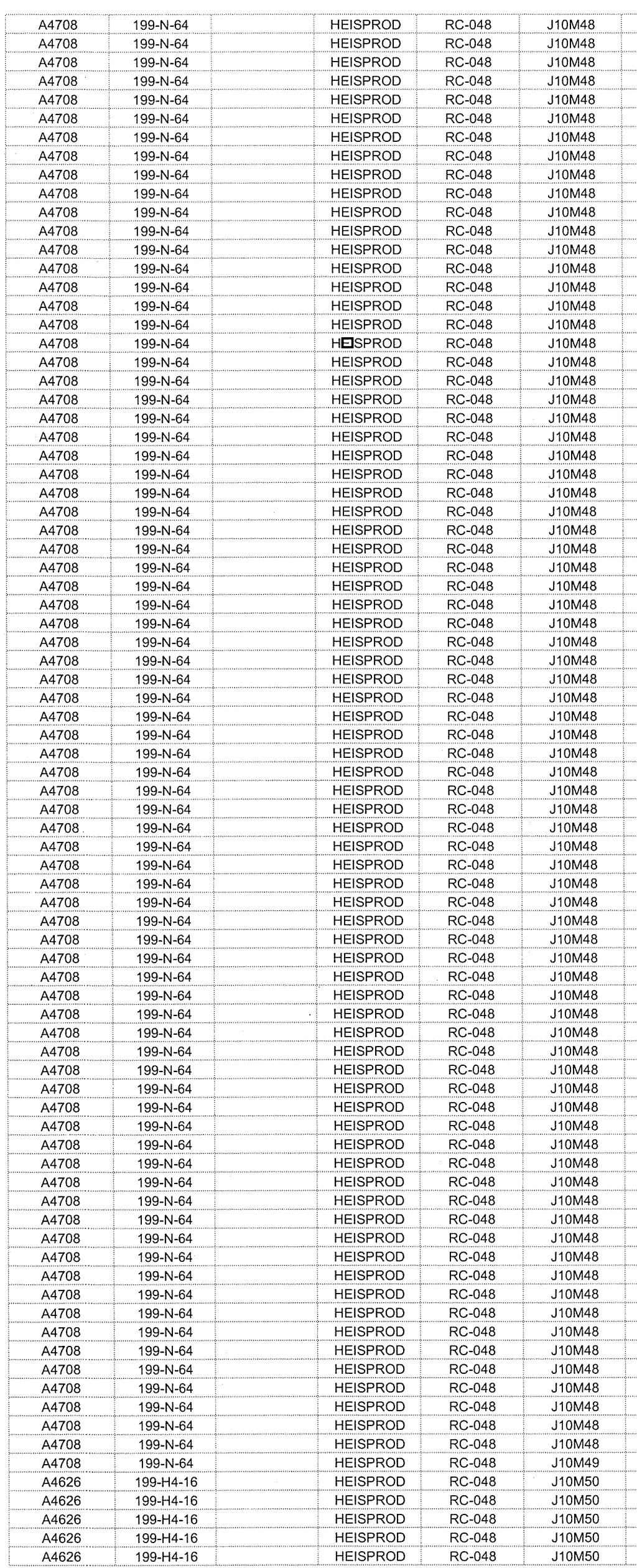
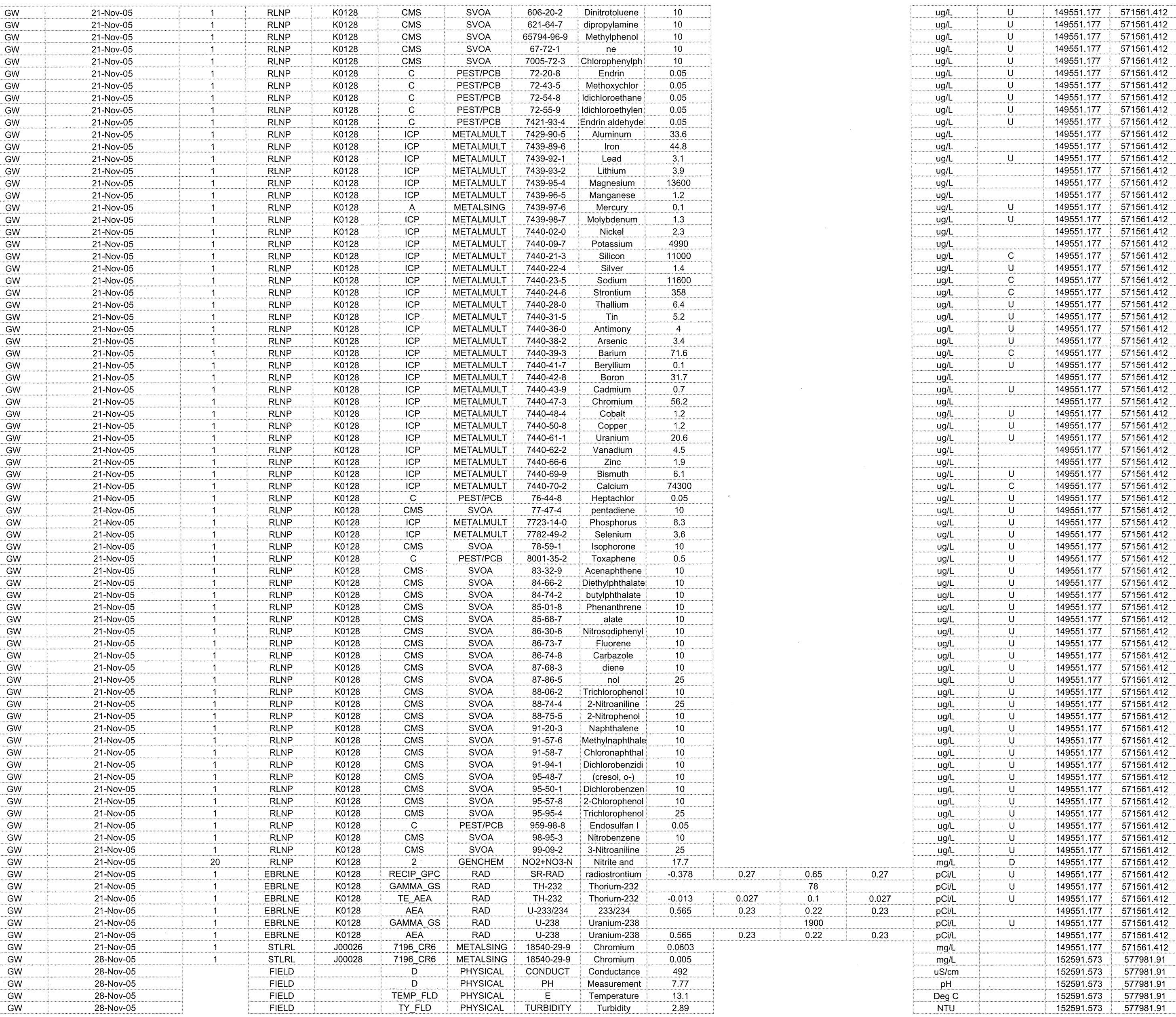


\begin{tabular}{|c|c|c|c|c|c|c|c|c|c|c|c|c|c|c|c|c|c|c|c|c|c|}
\hline A4630 & $199-H 4-4$ & HEISPROD & RC-048 & J10M51 & cW & 28-Nov-05 & 1 & STLRL & J00028 & 7196 CR6 & METALSING & $18540-29-9$ & Chromium & 0.018 & & & & mglt & & 152853.956 & 5780600.859 \\
\hline $\begin{array}{l}{ }^{A 4630} \\
A 4630\end{array}$ & $\begin{array}{l}1999-44-4 \\
09094-4\end{array}$ & HEISPROD & $\begin{array}{l}\text { RC- }-048 \\
\text { RC-048 }\end{array}$ & J10M51 & GW & 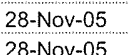 & & $\begin{array}{l}\text { FIELD } \\
\text { FIED }\end{array}$ & & $\begin{array}{l}D \\
D\end{array}$ & $\begin{array}{l}\text { PHYSICAL } \\
\text { PHSIIACL }\end{array}$ & $\begin{array}{l}\text { CONDUCT } \\
\text { PH }\end{array}$ & $\begin{array}{l}\text { Conductance } \\
\text { Measuremen }\end{array}$ & ${ }^{488}$ & & & & $\mathrm{us} / \mathrm{cm}$ & & 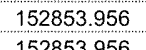 & 578060.8599 \\
\hline $\begin{array}{l}A 4630 \\
A 4630\end{array}$ & $\frac{199-14-4}{199-H 4-4}$ & $\begin{array}{l}\text { HEIIPRROD } \\
\text { HEISPROD }\end{array}$ & 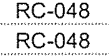 & $\begin{array}{l}\text { JoM51 } \\
\text { J10M51 }\end{array}$ & $\frac{G W}{G W}$ & $\begin{array}{l}\text { 28-NON-No-5 } \\
\text { 28-05 }\end{array}$ & & $\begin{array}{l}\text { FFEDD } \\
\text { FIELD }\end{array}$ & & $\begin{array}{c}D \\
\text { TEMP FLD }\end{array}$ & $\begin{array}{l}\text { PHHSIIAL } \\
\text { PHYSICAL }\end{array}$ & $\begin{array}{l}\mathrm{PH} \\
\mathrm{E}\end{array}$ & $\begin{array}{l}\text { Measurement } \\
\text { Temperature }\end{array}$ & $\begin{array}{l}7.04 \\
16.3 \\
\end{array}$ & & & & $\mathrm{pH}$ & & $\frac{1528533956}{152853.56}$ & 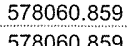 \\
\hline$A 4630$ & $199 \cdot-44-4$ & HEISPROD & RC-048 & J10M51 & $\mathrm{GW}$ & 28-Nov-05 & & FIELD & & TY FLD & PHYISAL & TURBDITY & $\begin{array}{l}\text { Temperafure } \\
\text { Turbidite }\end{array}$ & $\begin{array}{l}0.34 \\
0.04\end{array}$ & & & & $\begin{array}{l}\text { Degu } \\
\text { NTU }\end{array}$ & & 152535.39056 & $\begin{array}{l}5780060.859 \\
578060.859\end{array}$ \\
\hline A4626 & 199-H4-16 & HEISPROD & RC-048 & J10M54 & GW & 28-Nov-05 & & RLNP & K0135 & cms & SVOA & $100-01-6$ & 4-Nitiraaniline & 25 & & & & uglh & u & 152591.573 & $\begin{array}{l}578000.589 \\
577981.91\end{array}$ \\
\hline A4626 & $199 \cdot-44-16$ & HEISPROD & RC-048 & J10M54. & GW & 28-Nov-05 & & RLNP & K0135 & cMS & SVOA & $100-02-7$ & 4-Nitrophenol & 25 & & & & ugll & $u$ & 152591.573 & 577981.91 \\
\hline A4626 & $199--14-16$ & HEISPROD & RC-048 & J10M54 & GW & 28-Nov-05 & 1 & EBRLNE & Ko135 & 906.0 H3 LSC & RAD & $10028-17-8$ & Trifium & 2630 & 180 & 190 & 320 & pcil . & & 152591.573 & 577981.91 \\
\hline A4626 & $199-14-16$ & HEISPROD & RC-048 & J10M54 & GW & 28-Nov-05 & 1 & EBRLINE & Ko 0135 & GAMMAGS & RAD & 100045-97-3 & Cesium-137 & 10 & & 21 & & poin & $u$ & 152591.573 & 577981.91 \\
\hline A4626 & 1999-144-16 & HEISPROD & RC-048 & J10M54 & $\frac{G W}{G W}$ & 年-800-05 & 1 & 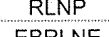 & Ko135 & CMS & SVOA & 101-155-3 & Bromophenylph & 10 & & & & ggh & u & 152591.573 & 577981.91 \\
\hline $\begin{array}{l}A_{44626} \\
A 4626\end{array}$ & 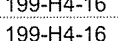 & $\begin{array}{l}\text { HEIIPROD } \\
\text { HEISPROD }\end{array}$ & $\frac{R C-048}{R C-048}$ & $\begin{array}{l}\text { J10M54 } \\
\text { J10M54 }\end{array}$ & $\frac{G W}{G W}$ & 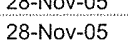 & 1 & $\begin{array}{c}\text { EBRLNE } \\
\text { RINP }\end{array}$ & K0135 & GAMMA_GS & RAD & 10198-40-0.0 & Cobalt:-60 & & & ${ }_{18}$ & & pcin & $u$ & 152591.573 & 577981.91 \\
\hline A4626 & $199-14-16$ & HEISPROD & RC-048 & J10M54 & GW & 28-Nov-05 & 1 & $\begin{array}{l}\text { RLNPP } \\
\text { RLNP }\end{array}$ & $\begin{array}{l}K 01135 \\
K 0135\end{array}$ & $c$ & $\begin{array}{l}\text { PESTPCB } \\
\text { DESTPTPBP }\end{array}$ & 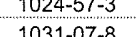 & $\begin{array}{l}\text { eepoxde } \\
\text { sultate }\end{array}$ & 0.05 & & & & gighe & U & 152599.5/3 & 577981.91 \\
\hline A4626 & 199-H4-16 & HEISPROD & RC-048 & J10M54 & ow & 28-Nov-05 & 1 & RLNP & K0135 & CMS & SVA & 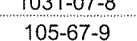 & $\begin{array}{l}\text { Dimaturer } \\
\text { Dimbenol: }\end{array}$ & $\frac{0.05}{10}$ & & & & $\frac{\text { ogh }}{\text { uch }}$ & u & 15250.1573 & $\begin{array}{l}577981.91 \\
57798191\end{array}$ \\
\hline A4626 & 199-H4-16 & HEISPROD & RC-048 & J10M54 & ow & 28-Nov-05 & 1 & RLNP & K0135 & CMS & SVOA & $106-46-7$ & Dichlorobenzen: & 10 & & & & gull & u & $\begin{array}{l}1525991.573 \\
1573\end{array}$ & 5779881.91 \\
\hline A4626 & 199-H4-16 & HEISPROD & RC-048 & J10M54 & ow & 28-Nov-05 & & RLNP & Ko135 & CMS & SVOA & $106-47-8$ & 4-Chloroaniline : & 10 & & & & ugll & $u$ & 152591.573 & 577981.91 \\
\hline $\begin{array}{l}A 4626 \\
A 4426\end{array}$ & 199-14-16 & $\begin{array}{l}\text { HEISRROD } \\
\text { HITPOD }\end{array}$ & 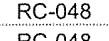 & J10M54 & Gw & 28-Nov-05 & 1 & RLNP & K0135 & CMS & SVOA & $108-60-1$ & methylethyl) ethe & 10 & & & & ugh & $u$ & 152591.573 & 577981.91 \\
\hline $\begin{array}{l}A 4626 \\
A 4626\end{array}$ & 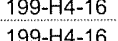 & $\begin{array}{l}\text { HESPRODD } \\
\text { HESPROD }\end{array}$ & 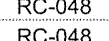 & 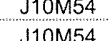 & $\frac{G w}{G w}$ & 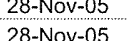 & & $\begin{array}{l}\text { RLP } \\
\text { RINP }\end{array}$ & 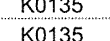 & CMS & $\begin{array}{l}S V A \\
\text { DEFTPCB }\end{array}$ & 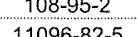 & Astenol & 10 & & & & gigh & 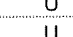 & 152599.5/3 & 577981.91 \\
\hline A4626 & 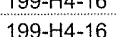 & HESPROD & RC-048 & J10M54 & $\frac{G W}{G W}$ & (2) & $\frac{1}{1}$ & RINP & K0135 & 8082 PB GC & PEST/PCB & $\begin{array}{l}71069-82-5 \\
11097-69-1\end{array}$ & 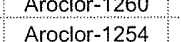 & 0 & & & & 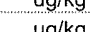 & u & 152591573 & $\begin{array}{l}5779191.91 \\
5779191\end{array}$ \\
\hline A462026 & $199-44-16$ & HEISPROD & RC-048 & J10M54 & GW & 28-Nov-05 & 1 & RLNP & Ko135 & CMS & SWOA & $111-44-4$ & 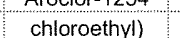 & 10 & & & & ugl & u & $\frac{152591.573}{15253}$ & 577981.91 \\
\hline A4626 & $199 \cdot-14-16$ & HEISPROD & RC-048 & Jrom54 & Gw & 28-Nov-05 & 1 & RLNP & Ko135 & CMS & SVOA & $111-91-1$ & chlorothoxyly m & 10 & & & & uglh & $u$ & 152591.573 & 577981.91 \\
\hline A4626 & $199-H 4-16$ & HEISPROD & RC-048 & J10M54 & GW & 28-Nov-05 & 1 & RLNP & Ko135 & 8082 PCB_GC & PEST/PCB & $11104-28-2$ & Aroclor-1221 & 0 & & & & ug $/ \mathrm{kg}$ & $u$ & 152591.573 & 577981.91 \\
\hline $\begin{array}{c}A 4626 \\
A 62626\end{array}$ & $199-44-16$ & $\begin{array}{l}\text { HEISPROD } \\
\text { HEISPROD }\end{array}$ & $\begin{array}{l}\text { RC-048 } \\
\text { RC-048 }\end{array}$ & J10M54 & $G W$ & $\begin{array}{l}28 \text {-Nov-05 } \\
\text { 2-8ON-05 }\end{array}$ & $\frac{1}{1}$ & RLNP & $\begin{array}{l}\text { Ko135 } \\
\text { Ko135 }\end{array}$ & 8082 8PC__CC & PESTIPCB & $\begin{array}{l}11141-16-5 \\
1178017\end{array}$ & Aroclor-1232 & 0 & & & & ug/kg & $u$ & $\begin{array}{l}152591.573 \\
152501\end{array}$ & 577981.91 \\
\hline $\begin{array}{l}A 4626 \\
A 4626\end{array}$ & $\begin{array}{l}199-14-16 \\
199-144-16\end{array}$ & $\begin{array}{l}\text { HEIIPROD } \\
\text { HEISPROD }\end{array}$ & $\frac{R_{C C-048}}{\text { RC-048 }}$ & $\begin{array}{l}\text { J10M54 } \\
\text { JitoM54 }\end{array}$ & $\frac{G W}{G W}$ & $\begin{array}{l}\text { 28-NN-Nov-05 } \\
\text { 2-No-05 }\end{array}$ & $\frac{1}{1}$ & $\frac{R L N P}{R L N P}$ & $\begin{array}{l}\text { K0135 } \\
\text { Ko135 }\end{array}$ & $\begin{array}{l}\text { CMS } \\
\text { CMS }\end{array}$ & $\begin{array}{l}\text { SVOA } \\
\text { SVOA }\end{array}$ & $\begin{array}{l}\frac{117-178-7}{117-84-0} \\
\end{array}$ & $\begin{array}{l}\text { ethylyexyl) } \\
\text { octylyhthalate }\end{array}$ & 0.838 & & & & $\frac{\text { uggh }}{\text { uglh }}$ & $\begin{array}{ll}\text { JB } \\
u\end{array}$ & $\begin{array}{l}1525991.1 / 13 \\
15259.573\end{array}$ & $\begin{array}{l}577981.91 \\
5779019101\end{array}$ \\
\hline A462026 & $\begin{array}{l}1999-14-16 \\
199-H 4-16\end{array}$ & $\begin{array}{l}\text { MESRPOD } \\
\text { HESPOD }\end{array}$ & $\begin{array}{l}\text { RC-048 } \\
\text { RC-048 }\end{array}$ & J10M54 & $G w$ & 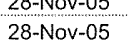 & 1 & RNP & Ko135 & cms & $\begin{array}{l}\text { SVIA } \\
\text { svoA }\end{array}$ & 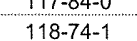 & - & 10 & & & & valh & u & 152595.1573 & 年 \\
\hline A4626 & $199-144-16$ & HEISPROD & RC-048 & J10M54 & GW & 28-Nov-05 & & RLNP & K0135 & CMS & SVOA & $120-12-7$ & Anthracene & 10 & & & & ugh & $u$ & 1525991.573 & 577981.91 \\
\hline A4626 & $199 \cdot-44-16$ & HEISPROD & RC-048 & J10M54 & GW & 28-Nov-05 & & RLNP & Ko135 & cMs & SVOA & $120-82-1$ & Trichlorobenzen: & 10 & & & & uglh & $u$ & 152591.573 & 577981.91 \\
\hline A4626 & 199-14-16 & HEISPROD & RC-048 & J10M54 & GW & 28-Nov-05 & & RLNP & Ko135 & CMS & SVOA & $120-83-2$ & Dichlorophenon: & 10 & & & & ug/L & $u$ & 152591.573 & 577981.91 \\
\hline A4626 & $199 \cdot-44-16$ & HEISPROD & RC-048 & J10M54 & GW & 28-Nov-05 & & RLNP & Ko135 & CMS & SVOA & $121-14-2$ & Dinitrotoluene & 10 & & & & $\mathrm{ug} / \mathrm{L}$ & u & 152591.573 & 577981.91 \\
\hline $\begin{array}{l}A 4626 \\
A 4426\end{array}$ & 1999-44-16 & HEISPROD & RC- 048 & J10M54 & GW & 28-Nov-05 & & RLNP & Ko135 & 8082_PCB_GC & PESTPCB & 12672-29-6 & Arocolor-1248 & 0 & & & & ug/kg. & $u$ & 152591.573 & 577981.91 \\
\hline $\begin{array}{l}A 4626 \\
A 4626\end{array}$ & $\begin{array}{l}199-144-16 \\
1004-16\end{array}$ & 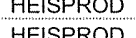 & RC-048 & $\begin{array}{l}\text { J10M54 } \\
\text { J10M54 }\end{array}$ & GW & $\begin{array}{l}\text { 28-N-NVV-05 } \\
\text { 28-Nov-05 }\end{array}$ & & $\frac{R L N P}{\text { RLNP }}$ & $\begin{array}{l}\text { KU135 } \\
\text { Ko135 }\end{array}$ & 8082 & SEOTEA & 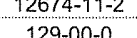 & Arocolor-1016 & 0 & & & & 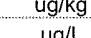 & 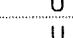 & $\begin{array}{l}152599.1573 \\
1525.153\end{array}$ & $\begin{array}{l}577981.91 \\
5 \\
577981.91\end{array}$ \\
\hline A4626 & 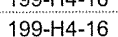 & $\begin{array}{l}\text { HEIPRODD } \\
\text { HESR }\end{array}$ & $\frac{R^{R C-048}}{\mathrm{RC}-048}$ & J10M54 & $G w$ & 28-Novo-05 & & RLNP & K0135 & cMs & SVOA & (131-11-3 & phthalate & 10 & & & & (1) & u & 152559.1573 & $\begin{array}{l}577981.91 \\
577981.91\end{array}$ \\
\hline A4626 & $199-H 4-16$ & HEISPROD & RC-048 & J10M54 & $G W$ & 28-Nov-05 & & RLNP & Ko135 & CMS & SVOA & $132-64-9$ & Dibenzofouran & 10 & & & & wghth & u & 152591.573 & $\begin{array}{l}577981.91 \\
57791.91\end{array}$ \\
\hline A4626 & $199-44-16$ & HEISPROD & RC-048 & J10M54 & GW & 28-Nov-05 & & EBRLNE & Ko135 & GAMMA_GS & RAD & $13966-00-2$ & Potassium-40 & & & 190 & & pcill & u & 152591.573 & 577981.91 \\
\hline A4626 & $199-144-16$ & HEISPROD & $\begin{array}{l}\text { RC-048 } \\
\text { RC-048 }\end{array}$ & J10M54 & GW & 28-Nov-05 & 1 & EBRLNE & Ko135 & GAMMA GS & $\frac{R A D}{R A D}$ & $\begin{array}{l}13966-02-4 \\
13076-48-1\end{array}$ & Berllium-7 & & & 270 & & pcilh & $u$ & 152591.573 & 5779881.91 \\
\hline $\begin{array}{l}{ }^{A 462626} \\
A 4626\end{array}$ & $\begin{array}{l}199-14-16 \\
199-14-16\end{array}$ & $\begin{array}{l}\text { HEISPROD } \\
\text { HEIPROD }\end{array}$ & 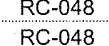 & $\begin{array}{l}\text { J10M54 } \\
\text { J10M54 }\end{array}$ & $\begin{array}{l}G W \\
G W\end{array}$ & 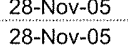 & & EBRLNE & K0135 & GAMMA GS & $\frac{R A D}{R A D}$ & $\begin{array}{l}13967-48-1 \\
13067-70-9\end{array}$ & Ruthenium-106 & & & $\frac{190}{25}$ & & pCiL & $u$ & $\begin{array}{l}152595.1573 \\
1525.153\end{array}$ & $\begin{array}{l}577981.91 \\
577981.91\end{array}$ \\
\hline A4626 & 195-14-160 & $\begin{array}{l}\text { MESPROD } \\
\text { HESPROD }\end{array}$ & RC-048 & J10M54 & Gw & & & EBRLNE & K0135 & UC & RAD & $\begin{array}{l}15306-2-63-3 \\
1392-3\end{array}$ & 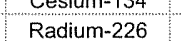 & 0.116 & 0.3 & 0.67 & 0.37 & pin & u & $\begin{array}{l}152559.1573 \\
15253\end{array}$ & 5779889.91 \\
\hline A4626 & $199-44-16$ & HEISPROD & RC-048 & J10M54 & GW & 28-Nov-05 & 1 & EBRLNE & Ko135 & GAMMA_GS & RAD & 13982-63-3 & Radium-2026 & & & 35 & & pcil & u & 152591.573 & 577981.91 \\
\hline A4626 & $199-44-16$ & HEISPROD & RC-048 & J10M54 & GW & 28-Nov-05 & 1 & EBRLNE & Ko135 & GAMMAGS & RAD & $14234-35-6$ & Antimony-125 & & & 50 & & pcill & $u$ & 152591.573 & 577981.91 \\
\hline A4626 & $199-14-16$ & HEISPROD & $\begin{array}{l}\text { RC-048 } \\
\text { RC-048 }\end{array}$ & $\begin{array}{l}510 M 54 \\
100954\end{array}$ & $G W$ & 28-Nov-05 & 1 & $\begin{array}{l}\text { RLNP } \\
\text { ERPE }\end{array}$ & K0135 & IC & GENCHEM & $14265-44-2$ & Phosphate & 0.25 & & & & molh & $u$ & 152591.573 & 577981.91 \\
\hline A4626 & $199-144-16$ & $\begin{array}{l}\text { HEISPROD } \\
\text { HIFPODOD }\end{array}$ & $\begin{array}{l}\text { RC-048 } \\
\text { Rc }\end{array}$ & J100054 & $G W$ & 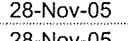 & 1 & 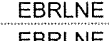 & 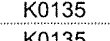 & TEAEA & $\begin{array}{l}\text { RAD } \\
\text { PAD }\end{array}$ & $\begin{array}{l}14266-63-7 \\
1427-8-70\end{array}$ & Thorium-230 & -0.029 & 0.04 & 0.097 & 0.043 & pcil & $u$ & 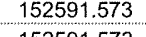 & \\
\hline $\begin{array}{l}A 4626 \\
A 4626\end{array}$ & $\frac{199-94-16}{199-14-16}$ & $\begin{array}{l}\text { HEISPROD } \\
\text { HEISPROD }\end{array}$ & $\begin{array}{l}\frac{1}{2 C-048} \\
\text { RC-048 }\end{array}$ & J10M544 & $\frac{G W}{G W}$ & 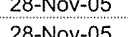 & 1 & EBRENE & K0135 & GAMMA GS & RAD & 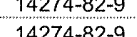 & Thorume-2828 & 0002 & 0.01 & 30 & fop & (c) & 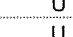 & $\begin{array}{l}152595.1 .573 \\
15572\end{array}$ & 577981.91 \\
\hline A46260 & $199-44-16$ & HEISPROD & RC-048 & J10M54 & GW & 28-Nov-05 & 1 & EBRLNE & Ko135 & GAMMA GS & RAD & $14391-16-3$ & EForimium-2825 & 0.002 & 0.0 & & 0.019 & sont & u & $\begin{array}{l}1525251.1753 \\
1525951573\end{array}$ & $\begin{array}{l}577981.91 \\
577090101\end{array}$ \\
\hline A4626 & $199-44-16$ & HEISPROD & RC-048 & Jrom54 & GW & 28-Nov-05 & 1 & EBRLLE & K0135 & GAMMA_GS & RAD & 145596-10-3 & 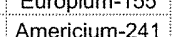 & & & . & & cin & u & 1525951.575 & $\begin{array}{l}5779787.91 \\
5.7798 .91\end{array}$ \\
\hline & $199-H 4-16$ & HEISPROD & RC-048 & J10M54 & GW & 28-Nov-05 & & EBRLNE & Ko135 & GAMMA GS & RAD & $14683-23-9$ & Europium 1 & & & 61 & & DCiL & u & 152591.1573 & $\begin{array}{l}5 / 7988.91 \\
577981.91\end{array}$ \\
\hline A4626 & $199 \cdot-44-16$ & HEISPROD & RC-048 & Jrom54 & GW & 28-Nov-05 & 10 & RLNP & Ko135 & ic & GENCHEM & $14797-55-8$ & Nitrate & 35 & & & & mglh & $\mathrm{D}$ & 152591.573 & 577981.91 \\
\hline A4626 & $199 \cdot-44-16$ & HEISPROD & RC-048 & J10M54 & GW & 28-Nov-05 & 1 & RLINP & Ko135 & IC & GENCHEM & $14797-65-0$ & Nitite & 0.25 & & & & mglh & $u$ & 152591.573 & 577981.91 \\
\hline $\begin{array}{l}A 4626 \\
A 4626\end{array}$ & $199-14-16$ & HESSROD & RC-048 & J100054 & GW & 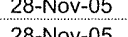 & 10 & RNPP & 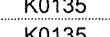 & $\begin{array}{l}\text { IC } \\
\text { s. }\end{array}$ & GENCHEM & $\begin{array}{l}14808-79-8 \\
1517170651\end{array}$ & $\begin{array}{l}\text { Sulfate } \\
\text { s. }\end{array}$ & 58.4 & & 88 & & $\mathrm{mglh}$ & D & $\begin{array}{l}1.52595 .1573 \\
15257.573\end{array}$ & 577981.91 \\
\hline A4426 & $199-94-16$ & HESPROD & RC-048 & J150m54 & Gw & 28-Nover-05 & & EBRNE & K0135 & AEA & RAD & $15117-96-1$ & $\begin{array}{l}\text { Uraniumim-235 } \\
\text { Uranium-235 }\end{array}$ & 0.025 & 0.0 & 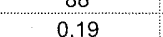 & 0.05 & (2) & 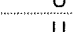 & 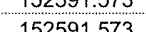 & $\begin{array}{l}577981.91 \\
5779891\end{array}$ \\
\hline A4tero & 1950-74-1060 & HFSTRPOD & RC-048 & J10M5454 & Gw & 28 - & 1 & EBRLNE & K0135 & GAMMA GS & RAD & $15262-20-1$ & $\begin{array}{l}\text { Rranumim-253 } \\
\text { Radium-228 }\end{array}$ & 0.020 & 0.0 & $\frac{75}{75}$ & 0.05 & (n) & 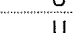 & 1.5259 .1573 & $\begin{array}{l}5 / 79787.91 \\
57798.91\end{array}$ \\
\hline A4626 & $199-44-16$ & HEISPROD & RC-048 & J10M54 & sw & 28-Nov-05 & 1 & EBRLNE & Ko135 & PC & RAD & 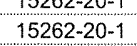 & $\begin{array}{l}\text { Radulumm-2808 } \\
\text { Radiu-220 }\end{array}$ & -0.053 & 1.3 & 2.1 & 1.3 & pcin & $u$ & 1525991.573 & $\begin{array}{l}577968.91 \\
57791.91\end{array}$ \\
\hline A4626 & $199--44-16$ & HEISPROD & RC-048 & J10M54 & GW & 28-Nov-05 & & EBRLNE & Ko135 & GAMMAGS & RAD & & Europium-154 & & & 54 & & pcill & u & 152591.573 & $\begin{array}{l}5779789.91 \\
55798.91\end{array}$ \\
\hline A4626 & $199-144-16$ & HEISPROD & RC-048 & J10M54 & Gw & 28-Nov-05 & 10 & RLNP & Ko135 & 10 & GENCHEM & $16887-00-6$ & Chloride & 23.7 & & & & mg/h & $\mathrm{D}$ & 152591.573 & 577981.91 \\
\hline A4626 & $199-44-16$ & HEISPROD & RC-048 & J10M54 & Gw & 28-Nov-05 & & RLNP & k0135 & 10 & GENCHEM & $16984-48-8$ & Fluoride & 0.25 & & & & $\mathrm{mg} / \mathrm{h}$ & u & 152591.573 & 577981.91 \\
\hline A4626 & $1999-44-16$ & HEISPROD & RC-048 & J10M54 & GW & 28-Nov-05 & & RLNP & Ko135 & CMS & SVOA & $191-24-2$ & ene & 10 & & & & uglh & $u$ & 152591.573 & 577981.91 \\
\hline A4626 & $199-44-16$ & HEISPROD & RC-048 & J10M54 & GW & 28-Nov-05 & & RLNP & K0135 & CMS & SSOA & $1933-39-5$ & cd)pyrene & 10 & & & & ugh & $u$ & 1525951.573 & 577981.91 \\
\hline $\begin{array}{l}A 4626 \\
A 4626\end{array}$ & $\frac{199-94-16}{199-44-16}$ & $\begin{array}{l}\text { HEISPROD } \\
\text { HEISROD }\end{array}$ & $\frac{R C-048}{\text { RC- } 048}$ & $\begin{array}{l}J 10 M 54 \\
\text { J10M54 }\end{array}$ & GW & 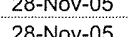 & & RLN & 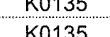 & cins & SWOA & $2005-9-2-2$ & $\begin{array}{l}\text { thene } \\
\text { Fluoranthene }\end{array}$ & 10 & & & & gon & 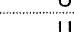 & $\begin{array}{l}1.52595 .1573 \\
1.52515\end{array}$ & $\begin{array}{l}5779791.91 \\
5779891\end{array}$ \\
\hline A4426 & $\begin{array}{l}\frac{19-944-16}{199-H 4-16} \\
\end{array}$ & HEISPROD & RC-048 & J10M54 & GW & 28-Novo-05 & & RLNP & Ko135 & cMs & SVOA & $207-08-9$ & $\begin{array}{l}\text { Fuorantinene } \\
\text { thene }\end{array}$ & 10 & & & & volh & u & 1525991.1573 & 5779881.91 \\
\hline$A 4626$ & $199-44-16$ & HEISPROD & RC-048 & J10M54 & GW & 28-Nov-05 & & RLNP & K0135 & CMS & SVOA & $208-96-8$ & Acenaphthylene & 10 & & & & uglh & u & 152591.573 & 577981.91 \\
\hline A4626 & $199 \cdot-44-16$ & HEISPROD & RC- -48 & J10M54 & GW & 28-Nov-05 & & RLNP & Ko135 & cMs & SVOA & $218-01-9$ & Chrrsene & 10 & & & & uglh & $u$ & 1525991.573 & 577981.91 \\
\hline A4626 & $199-44-16$ & HEISPROD & $\begin{array}{l}\text { RC-048 } \\
\text { SC-018 }\end{array}$ & J10M54 & GW & 28-Nov-05 & & RLNP & 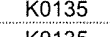 & $=$ & $\begin{array}{l}\text { GENCHEM } \\
\text { DESTPOPCS }\end{array}$ & $\begin{array}{l}24959-67.9 \\
3000-9-9\end{array}$ & Bromide & 0.25 & & & & moll & u & 1525951.573 & $\begin{array}{r}577981.91 \\
57708.101\end{array}$ \\
\hline $\begin{array}{l}A 4626 \\
A 4626\end{array}$ & $\frac{199-14-16}{199-H 4-16}$ & $\begin{array}{l}\text { HEISPROD } \\
\text { HEISROD }\end{array}$ & $\frac{R \text { RC- }-048}{\text { RC-048 }}$ & $\begin{array}{l}\text { J11005454 } \\
\text { J10M54 }\end{array}$ & $\frac{G W}{G W}$ & 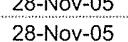 & & RLN & Ko135 & $\begin{array}{c}c \\
c\end{array}$ & $\begin{array}{l}\text { PESTIPCE } \\
\text { PESTPCB }\end{array}$ & $\begin{array}{l}3099-0 .-2 \\
319-848-6\end{array}$ & $\begin{array}{l}\text { Aldrin } \\
\text { Alpha-BHC }\end{array}$ & 0.055 & & & & gogh & 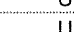 & $\begin{array}{l}1.52595 .1573 \\
15251573\end{array}$ & $\begin{array}{l}5779191.91 \\
57789191\end{array}$ \\
\hline A4626 & $\frac{199--14-16}{199-H 4-16}$ & HEISPROD & RC-048 & J10M544 & GW & 2 & & RLNP & Ko135 & $c$ & PESTPCB & 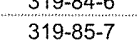 & 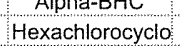 & 0.05 & & & & val & $u$ & 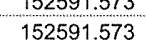 & $\begin{aligned} 577988.91 \\
57798.91\end{aligned}$ \\
\hline A4626 & $199-44-16$ & HEISPROD & RC-048 & JHOM54 & ow & 28-Nov-05 & & RLNP & Ko135 & $c$ & PESTPCB & $319-86-8$ & Delta-BHC & 0.05 & & & & uglh & $u$ & 152591.573 & 577981.91 \\
\hline A4626 & $1999-44-16$ & HEISPROD & RC-048 & J10M54 & ow & 28-Nov-05 & & RLNP & Ko135 & $c$ & PESTIPCB & $33213-65-9$ & Endosulfian II & 0.05 & & & & uglh & $u$ & 152591.573 & 577981.91 \\
\hline A4626 & $199-H 4-16$ & HEISPROD & 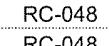 & J10M54 & GW & 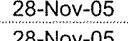 & & $\begin{array}{l}\text { RRNPP } \\
\text { RND }\end{array}$ & 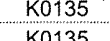 & CMS & $\begin{array}{l}\text { PESTPCB } \\
\text { SWOA }\end{array}$ & $\begin{array}{l}50-29-3 \\
55-323.8\end{array}$ & Iltrichloroethane & 0.05 & & & & uglh & $u$ & $\begin{array}{l}152591.573 \\
1.5651 .1772\end{array}$ & 577981.91 \\
\hline $\begin{array}{l}A 426 \\
A 4266\end{array}$ & $\frac{199-94-16}{199-14-16}$ & $\begin{array}{l}\text { HEESPROD } \\
\text { HEISPROD }\end{array}$ & $\begin{array}{l}\text { RC-048 } \\
\text { RC-048 }\end{array}$ & $\begin{array}{l}\text { J110054 } \\
\text { J10M54 }\end{array}$ & $\frac{G W}{G W}$ & 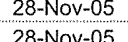 & & RNP & $\begin{array}{l}\text { K0135 } \\
\text { K0135 }\end{array}$ & $\begin{array}{l}\text { CMS } \\
\text { CMS }\end{array}$ & $\begin{array}{l}\text { SVOA } \\
\text { SVOA }\end{array}$ & $\frac{50-128-8}{55-28-5}$ & Benzonaplaprene & 10 & & & & ugh & 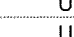 & 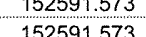 & $\begin{array}{l}577981.91 \\
577901.91\end{array}$ \\
\hline A44626 & $199-H 4-16$ & HESPROD & RC-048 & J10M54 & GW & $28-\mathrm{Nov}-05$ & & RLNP & Ko135 & Sus & PEST/PCB & $5103-71-9$ & Chlordane & $\begin{array}{l}25 \\
0.05\end{array}$ & & & & volh & u & 152559.1573 & 5779881.91 \\
\hline A4626 & $199-44-16$ & HEISPROD & RC-048 & J10M54. & Gw & 28-No & & RLNP & ko1 & C & PESTPCB & $5103-74-2$ & Chlordane & 0.05 & & & & uglh & $u$ & 152591.573 & 577981.91 \\
\hline A4626 & $1999-44-16$ & HEISPROD & RC-048 & J10M54 & GW & 28-Nov-05 & & RLNP & Ko135 & CMS & SVOA & $53-70-3$ & racene & 10 & & & & ugh & $u$ & 152591.573 & 577981.91 \\
\hline A4626 & $199-.44-16$ & HEISPROD & RC-048 & $\begin{array}{l}310054 \\
100454\end{array}$ & $G W$ & 28-Nov-055 & & RLNP & Ko135 & CMS & 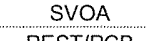 & $534-52-1$ & methylphenol & 25 & & & & ught & 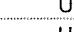 & 1525251.573. & 577981.91 \\
\hline $\begin{array}{l}A 4626 \\
{ }^{A 4426}\end{array}$ & $\frac{199-94-16}{19-44-16}$ & HESTROD & $\frac{R C-048}{R C-048}$ & J100M544 & GW & $\frac{28-\mathrm{N} \text {-Nov-05 }}{28 \text {-Nov-05 }}$ & & $\begin{array}{l}\text { RLN } \\
\text { RLNP }\end{array}$ & Ko135 & 8082 PCE GC & $\begin{array}{l}\text { PESTIFCB } \\
\text { PESTPCB }\end{array}$ & $\begin{array}{l}53464-21-9 \\
53494-70-5\end{array}$ & $\begin{array}{l}\text { Arrocolor-1242 } \\
\text { Endrin Ketone }\end{array}$ & 0.05 & & & & & 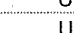 & 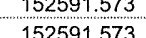 & 577981.91 \\
\hline A4626 & $199 \cdot-44-16$ & HEISPROD & RC-048 & J100M54 & GW & 28-Novo-05 & 1 & RLNP & K0135 & CMS & SVOA & $\frac{5444-70-5}{541-73-1}$ & 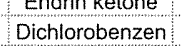 & $\frac{.05}{10}$ & & & & ugh & u & $\begin{array}{l}152595.1 .753 \\
152591.573\end{array}$ & 5 \\
\hline
\end{tabular}




\begin{tabular}{|c|c|c|c|c|}
\hline & $199-H 4-16$ & HEISPROD & & \\
\hline & & & & \\
\hline A4626 & $199-H 4-16$ & HEISPROD & RC-048 & J10M54 \\
\hline & $199-44-16$ & HEISRODD & 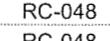 & J100054 \\
\hline & $\begin{array}{l}199-44-16 \\
199-44-16\end{array}$ & & 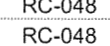 & $\begin{array}{l}310054 \\
\text { J110054 }\end{array}$ \\
\hline A4t626 & $\begin{array}{l}1999-444-16 \\
199-H 4-16\end{array}$ & HEISPROD & $\begin{array}{l}\text { RC-048 } \\
\text { RC-048 }\end{array}$ & J10M5454 \\
\hline A4626 & $199--44-16$ & & RC-048 & \\
\hline$A 4626$ & $199-44-16$ & HEISPROD & RC-048 & Jiom54 \\
\hline $\begin{array}{l}\text { A4626 } \\
\text { A4626 }\end{array}$ & $199-14-16$ & $\begin{array}{l}\text { HEISPROD } \\
\text { HESPRPOD }\end{array}$ & $\begin{array}{l}R C-048 \\
R C-048\end{array}$ & J10M54 \\
\hline & & $\begin{array}{l}\text { HEISPROD } \\
\text { HEISPROD }\end{array}$ & $\begin{array}{l}\text { RC-048 } \\
\text { RC-048 }\end{array}$ & $\begin{array}{l}\text { J10054 } \\
\text { J10M54 }\end{array}$ \\
\hline A4626 & $199-44-16$ & HESPROD & RC-048 & J1500454 \\
\hline A4626 & $199-14-16$ & HEISPROD & RC-048 & \\
\hline A4626 & $199-44-16$ & HEISPROD & RC-048 & J10M54. \\
\hline & $199-14-16$ & $\begin{array}{l}\text { HEISPROD } \\
\text { HESPRPOD }\end{array}$ & $\begin{array}{l}R C-048 \\
R C-048\end{array}$ & $510 M 54$ \\
\hline A4626 & $\begin{array}{l}199-44-16 \\
199-H 4-16\end{array}$ & $\begin{array}{l}\text { HESSRPOD } \\
\text { HEISPROD }\end{array}$ & 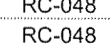 & $\begin{array}{l}\frac{110054}{J 10 M 54} \\
\text { Jom }\end{array}$ \\
\hline & $199-444-1$ & HESPRPOD & & J5040454 \\
\hline A4626 & $199-\mathrm{H} 4-16$ & HEISPROD & RC-048 & \\
\hline A4626 & $199-H 4-16$ & HEISPROD & RC-048 & J10M54 \\
\hline & $\begin{array}{l}199-144-16 \\
199-44-16\end{array}$ & $\begin{array}{l}\text { HESISPOD } \\
\text { HEISPROD }\end{array}$ & $\frac{R C-048}{R C-048}$ & J10M54 \\
\hline $\mathrm{A} 4626$ & & & & $\begin{array}{l}\text { J10M54 } \\
\text { J10M54 }\end{array}$ \\
\hline A4626 & $199-444-1$ & HESPROD & RC-048 & J1500454 \\
\hline A4626 & $199--44-16$ & HEISPROD & RC. -048 & J100M54 \\
\hline A4626 & $199-44-16$ & HEISPROD & RC-048 & J10M54 \\
\hline & $\frac{199-H 4-16}{199-H 4-16}$ & $\begin{array}{l}\text { HEISPROD } \\
\text { HESSPROD }\end{array}$ & & J10M54 \\
\hline A4626 & $199-444-1$ & $\begin{array}{l}\text { HESISFOD } \\
\text { HEISPROD }\end{array}$ & $\begin{array}{l}\text { RC-048 } \\
\text { RC-048 }\end{array}$ & $\frac{510054}{310 M 54}$ \\
\hline A4626 & $199-44-1$ & HEISPROD & RC-048 & J10M54 \\
\hline A4626 & $199-H 4-16$ & HEISPROD & RC-048 & J10M54 \\
\hline A442626 & & HEISPROD & & J10M54 \\
\hline $\begin{array}{l}. \\
A 462626 \\
\text { A4t26 }\end{array}$ & $\begin{array}{l}199-144-16 \\
109-44-16\end{array}$ & $\begin{array}{l}\text { HEISPROD } \\
\text { HEISPROD }\end{array}$ & $\begin{array}{l}\text { RC-048 } \\
\text { RC-048 }\end{array}$ & J10M54 \\
\hline A4626 & $\begin{array}{l}1959-14-16 \\
199-16\end{array}$ & $\begin{array}{l}\text { MESTRUD } \\
\text { HEISRODO }\end{array}$ & $\begin{array}{l}\text { RC-1-048 } \\
\text { RC-048 }\end{array}$ & $\begin{array}{l}\text { J10M0M54 } \\
\text { J10M54 }\end{array}$ \\
\hline A4626 & $199-44-16$ & HEISPROD & RC-048 & 5100454 \\
\hline A4626 & $199--44-16$ & HEISPROD & RC- 048 & J10M54 \\
\hline $\begin{array}{l}A^{A 4626} \\
4426\end{array}$ & $\begin{array}{l}199-144-16 \\
199-44-16\end{array}$ & $\begin{array}{l}\text { HESPROD } \\
\text { HESPRPOD }\end{array}$ & $\begin{array}{l}\text { RC-048 } \\
\text { RC-048 }\end{array}$ & \\
\hline A4626 & $199-44-1$ & HEISPROD & RC-048 & J10M54 \\
\hline A4626 & $199-44-1$ & HEISPRO & RC-018 & 51000544 \\
\hline A4626 & $199-44-16$ & HEISPROD & RC-048 & J10M54 \\
\hline A4626 & $199-44-16$ & HEISPROD & RC-048 & J10M54 \\
\hline & & & & \\
\hline A4t26 & $1907-1$ & 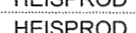 & Bu- & Jom54 \\
\hline A46626 & $199 \cdot-444-1$ & HEISPROD & RC-048 & 5 \\
\hline A4626 & $199-\mathrm{H} 4-16$ & HEISPROD & RC-048 & J10M54 \\
\hline A4626 & & HEISPROD & & J10M54 \\
\hline A4t6626 & $199-144-16$ & HELSPRD & & J1100054 \\
\hline$\frac{14626}{A 426}$ & $199-14$ & HESTPRO & RC-040 & 5 \\
\hline A4626 & $199-44-16$ & HEISPROD & RC-048 & J10M54 \\
\hline A4626 & 199-H4-16 & HEISPROD & RC-048 & J10M54 \\
\hline 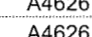 & & & & \\
\hline A46626 & 195-74-44 & 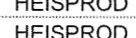 & RC- & J10M54 \\
\hline & 199--44-16 & & RC-048 & 3 \\
\hline A4626 & $199-44-16$ & HEISPRPD & RC-048 & J1500054 \\
\hline$A 4626$ & $199--44-16$ & HEISPROD & RC- 048 & J10M54 \\
\hline $\begin{array}{l}A 4626 \\
A 426\end{array}$ & $199-44-16$ & HESPROD & RC-048 & J110054 \\
\hline & & & & J10M54 \\
\hline A4626 & $199+44-1$ & & 8900 & 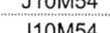 \\
\hline A46626 & 1906-14t-1 & HESPRP & CN-040 & J10M54 \\
\hline A4626 & $199-44-16$ & HEISPROD & RC-048 & J10M54 \\
\hline & $199-\mathrm{H} 4-16$ & & & \\
\hline A4626 & $199-44-16$ & HEISPROD & RC-048 & J10M54 \\
\hline A4626 & $199-44-16$ & HEISPROD & RC-048 & J10M54 \\
\hline 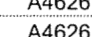 & 199-74-16 & HF & RLC-048 & J150M54 \\
\hline & & & & \\
\hline A4626 & $109-44$ & & & 5100054 \\
\hline & & & & JTOMBS \\
\hline & & HESPROD & & 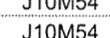 \\
\hline$A 4626$ & $109-14$ & & & 5 \\
\hline & & & & 5100054 \\
\hline A4626 & $199-44-16$ & HEISPROD & RC-048 & J10M54 \\
\hline A4626 & $199-44-16$ & & & J10M54 \\
\hline A4626 & $199-H 4-16$ & HEISPROD & RC-048 & J150M54 \\
\hline A4626 & & HEISPROD & & J10M54 \\
\hline & & & & \\
\hline
\end{tabular}

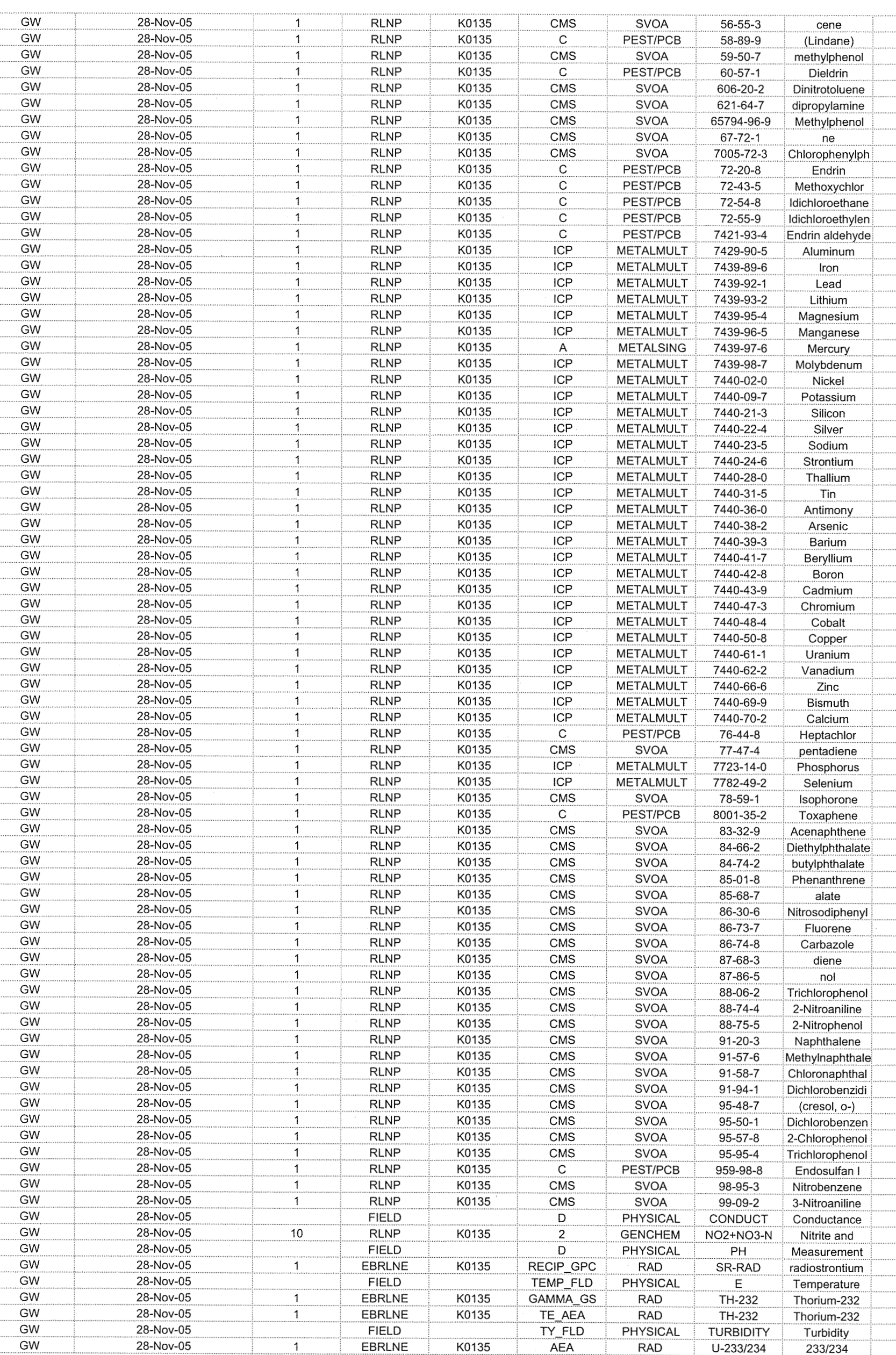

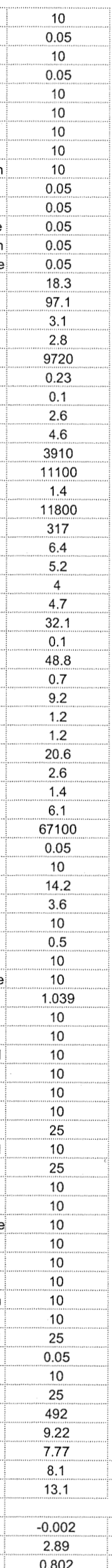

\begin{tabular}{|c|c|c|c|}
\hline & u & 152591.573 & 577981.91 \\
\hline & u & $\begin{array}{l}152591.573 \\
15259153\end{array}$ & 577981.91 \\
\hline & u & $\begin{array}{l}152595.1573 \\
152575\end{array}$ & $\begin{array}{l}577981919 \\
57798919\end{array}$ \\
\hline & & & \\
\hline & $u$ & & \\
\hline ugl & u & & \\
\hline ugh/ & u & $\begin{array}{l}152591.573 \\
15259153\end{array}$ & \\
\hline ug/h & u & $\begin{array}{l}1525951.1753 \\
152591.573\end{array}$ & 577981.91 \\
\hline & & 1525991.573 & \\
\hline uglt & $u$ & 152591.573 & 577981.91 \\
\hline ug/h & $u$ & 152591.573 & 577981.91 \\
\hline ught & u & $\begin{array}{l}152591.573 \\
1552595.1573\end{array}$ & 577981.91 \\
\hline & & $\begin{array}{l}1525951.15 / 3 \\
152591.573\end{array}$ & $\begin{array}{l}577981.91 \\
577981.91\end{array}$ \\
\hline & u & 152591.573 & 5777981.91 \\
\hline & & 152591.573 & 577981.91 \\
\hline ugh & & 152591.573 & 577981.91 \\
\hline vol & 4 & $\begin{array}{l}152591.573 \\
1552501573\end{array}$ & 577981.91 \\
\hline ugh & $\mathrm{c}$ & & \\
\hline & & 152591.573 & 5779881.91 \\
\hline & $c$ & 152591.573 & 577981.91 \\
\hline & & 1525991.573 & 577981.91 \\
\hline & 0 & $\begin{array}{l}152599.1573 \\
155967\end{array}$ & $\begin{array}{l}577981.91 \\
577819\end{array}$ \\
\hline ught & & $\frac{152591.1573}{157}$ & $\begin{array}{l}577981.91 \\
55791.91\end{array}$ \\
\hline & $u$ & 152591.573 & 577981.91 \\
\hline & & 152591.573 & 577981.91 \\
\hline ugl L & $u$ & & \\
\hline von & & 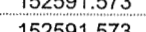 & $\begin{array}{l}577981.91 \\
5770191\end{array}$ \\
\hline & u & 152591.573 & 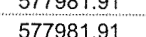 \\
\hline ugh & & 152591.573 & 577981.91 \\
\hline & $u$ & 152591.573 & 577981.91 \\
\hline ught & & 152591.573 & 577981.91 \\
\hline wol & u & $\begin{array}{l}1525951.173 \\
152591.573\end{array}$ & $\begin{array}{l}577981.91 \\
57798191\end{array}$ \\
\hline ug/h & u & 152591.573 & 577981.91 \\
\hline & & 152591.573 & 577981.91 \\
\hline ugh & $c$ & 152591.573 & 577981.91 \\
\hline ugh & & 152599.57.573. & 5 577981.91 \\
\hline ugh & u & 152599.1573 & $\begin{array}{l}5 / 7981.91 \\
577981.91\end{array}$ \\
\hline & & 152591.573 & 577981.91 \\
\hline uglh & & 152591.573 & 577981.91 \\
\hline ugh & $u$ & 152591.573 & 577981.91 \\
\hline & 0 & 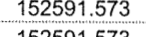 & 5777981.91 \\
\hline & & & \\
\hline ug/h & u & 152591.573 & 577981.91 \\
\hline & JB & 152599.573 & 577981.91 \\
\hline woll & $u$ & 152591.573 & 5777981.91 \\
\hline vall & 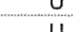 & $\begin{array}{l}152599.1573 \\
1559.572\end{array}$ & 5777981.911 \\
\hline & & & $\begin{array}{l}5 \\
5\end{array}$ \\
\hline uglh & u & 152591.573 & 57798191 \\
\hline & $u$ & 152591.573 & 577981.91 \\
\hline ugh & $u$ & 152591.573 & 577981.91 \\
\hline wol & 4 & $\begin{array}{l}152599.573 \\
5152573\end{array}$ & $\begin{array}{l}577981.91 \\
57701911\end{array}$ \\
\hline & & & $\begin{array}{l}5 \\
5\end{array}$ \\
\hline & $u$ & 152591.573 & 577981.91 \\
\hline ug/L & u & 152591.573 & 577981.91 \\
\hline woll & $u$ & 152591.573 & 5779881.91 \\
\hline wall & 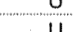 & 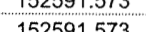 & $\begin{array}{l}5777891.91 \\
5670191\end{array}$ \\
\hline & & & 57798191 \\
\hline & u & 152591.573 & 577981.91 \\
\hline ug/h & $u$ & 152591.573 & 577981.91 \\
\hline & u & 152599.573 & 5777981.91 \\
\hline & u & 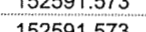 & $\begin{array}{l}577881.91 \\
6770191\end{array}$ \\
\hline $\mathrm{us} / \mathrm{cm}$ & & 152591.573 & 57798191 \\
\hline & D & 152591.573 & 577981.91 \\
\hline & & 152591.573 & 577981.91 \\
\hline pcin & & 152591.573 & 5777981.91 \\
\hline & & (5) & $\begin{array}{l}779781.91 \\
7709191\end{array}$ \\
\hline & $u$ & 25591.573 & 7798191 \\
\hline & & 52591.573 & \\
\hline & & & \\
\hline
\end{tabular}



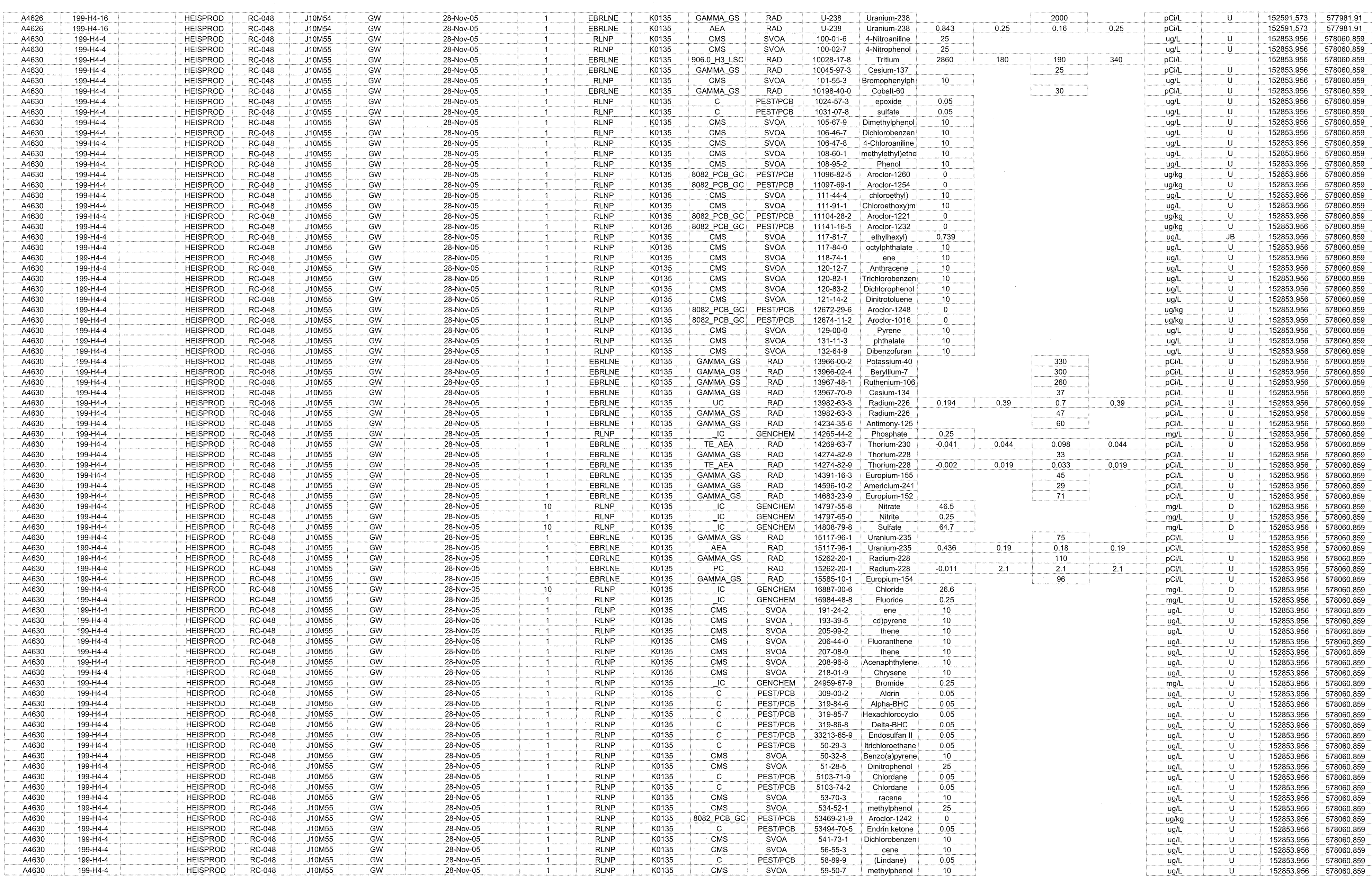


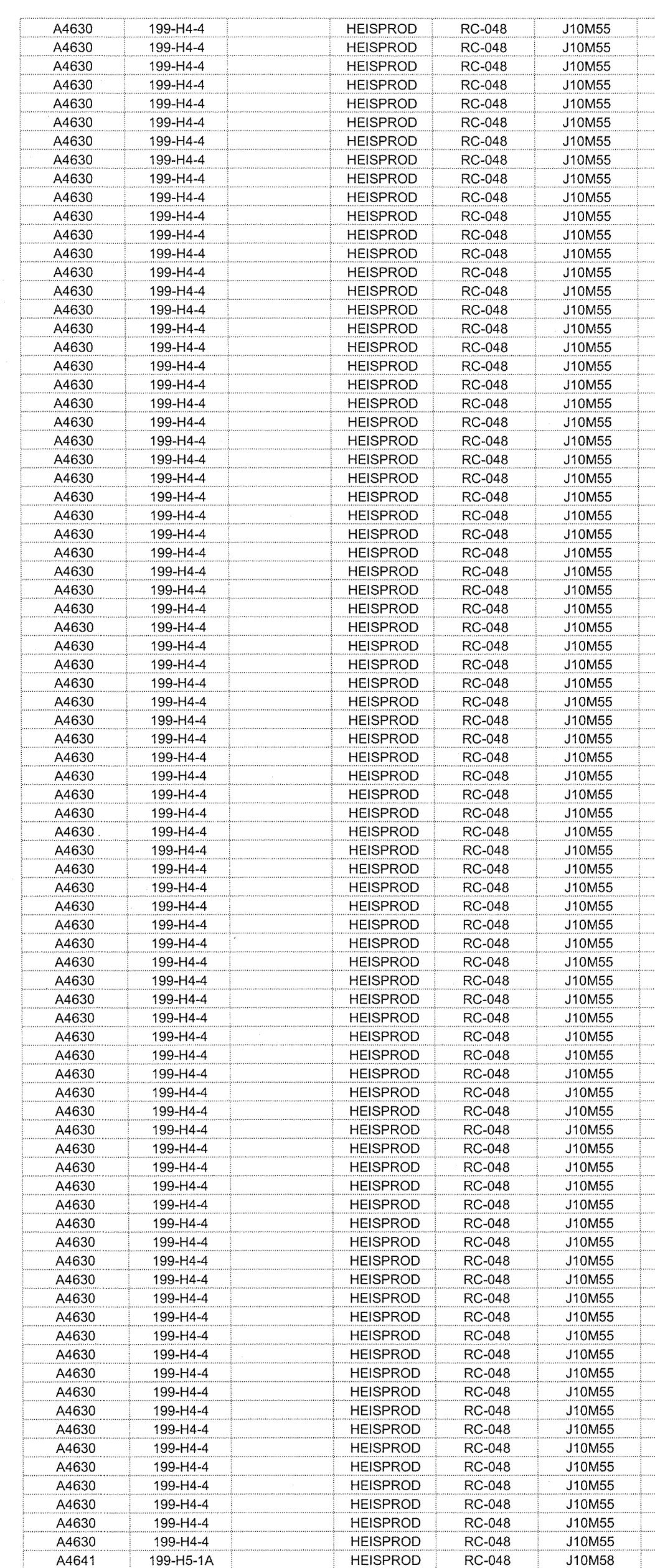

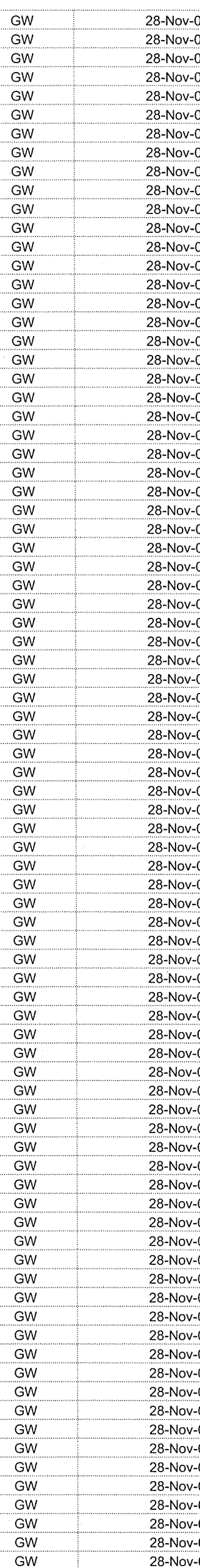
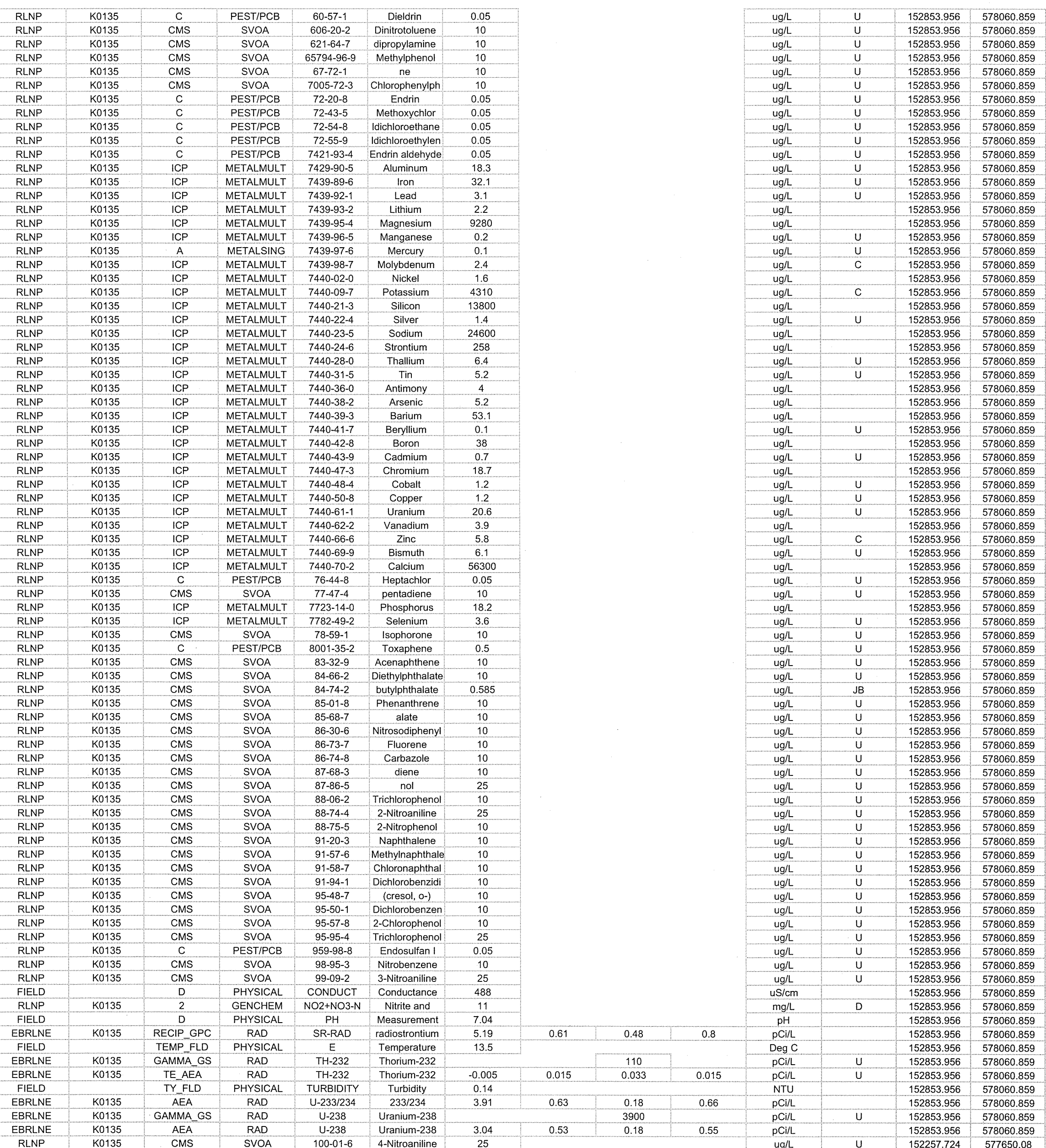

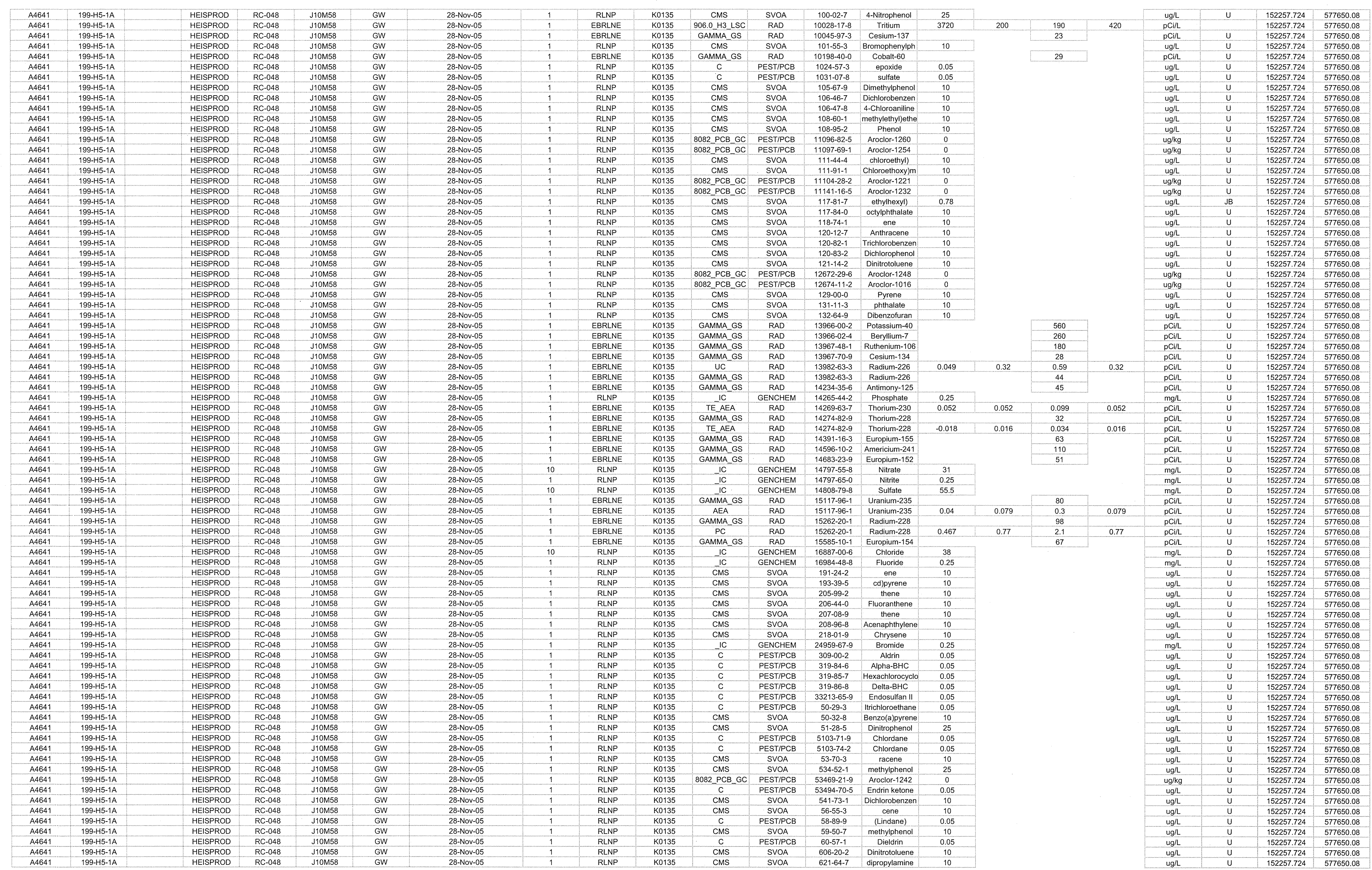


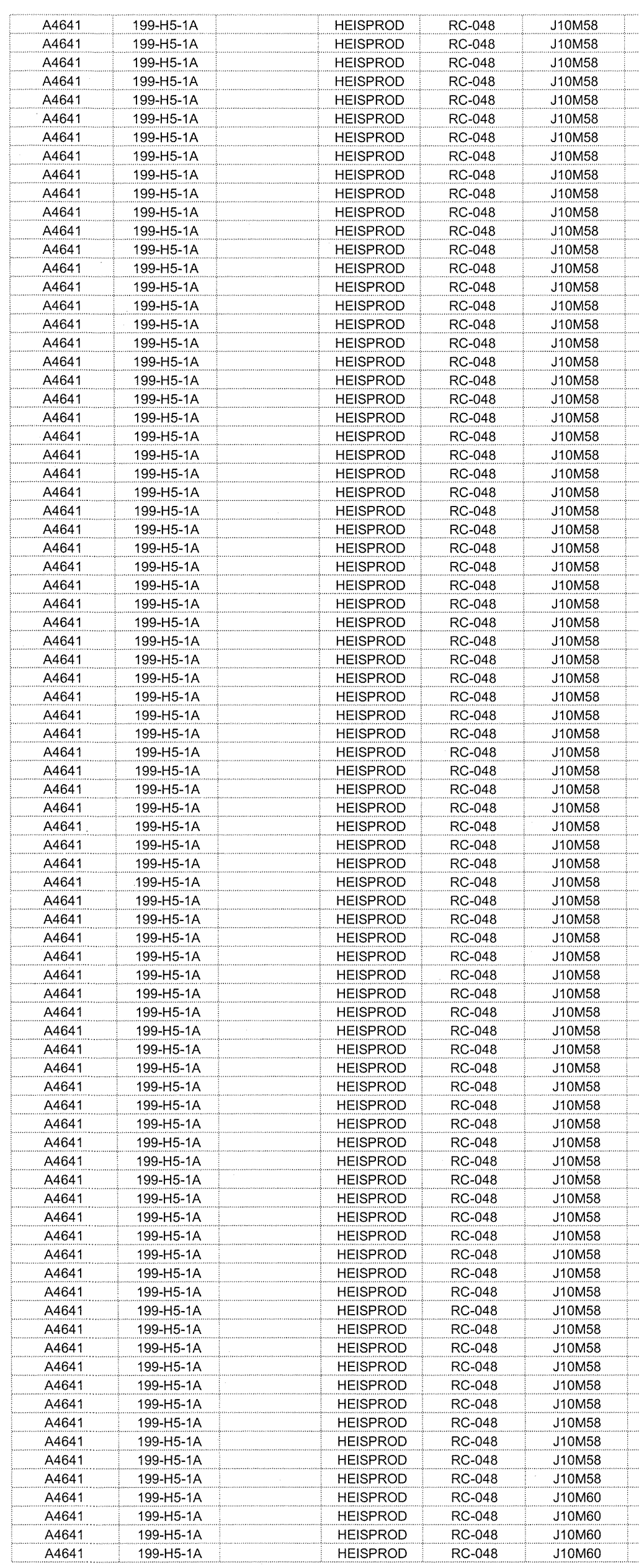

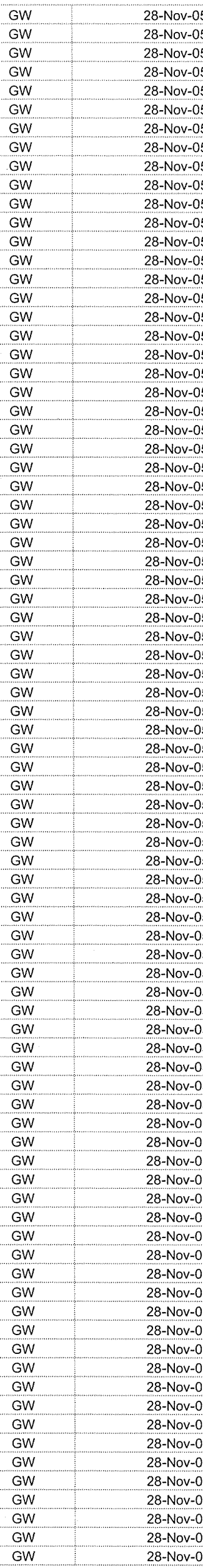
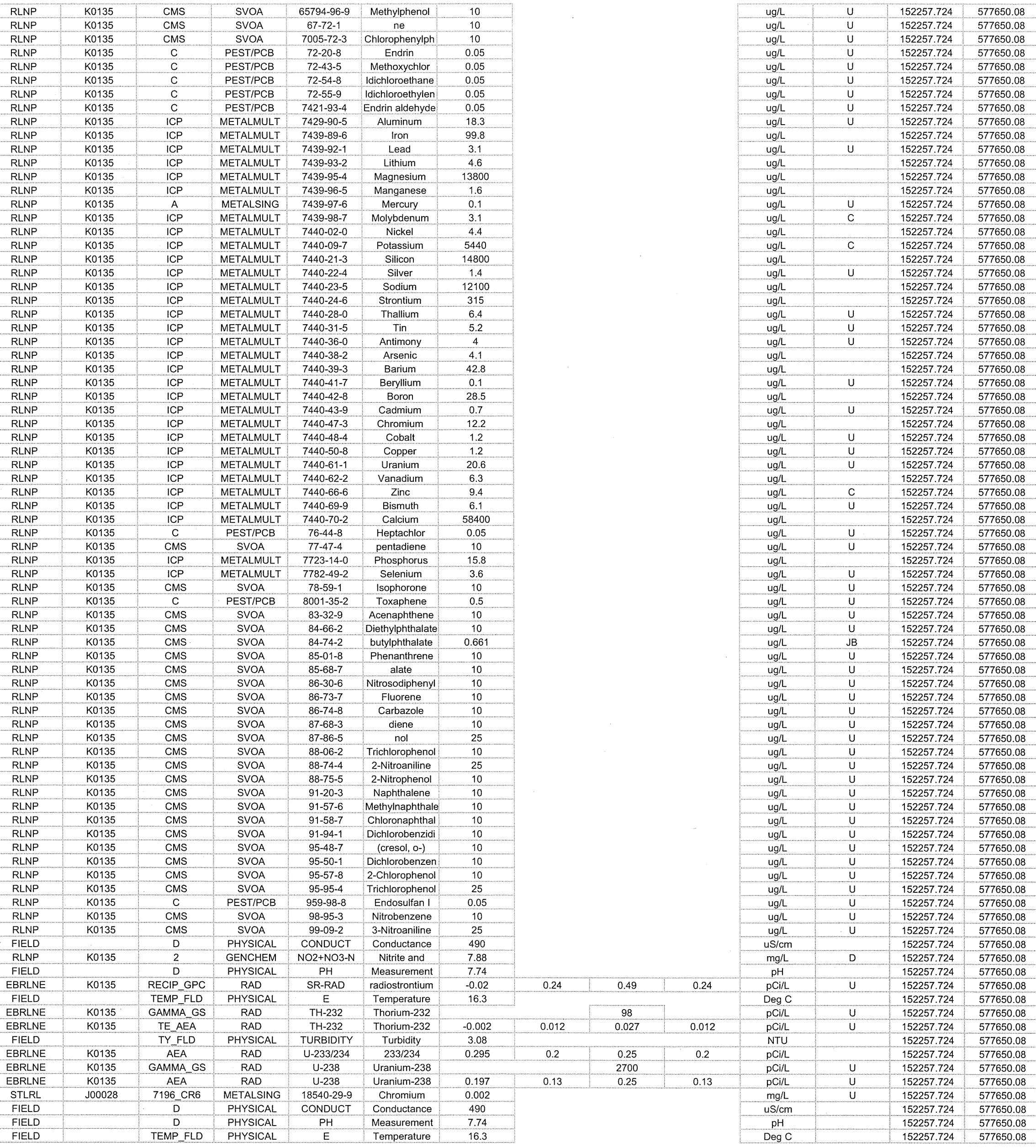

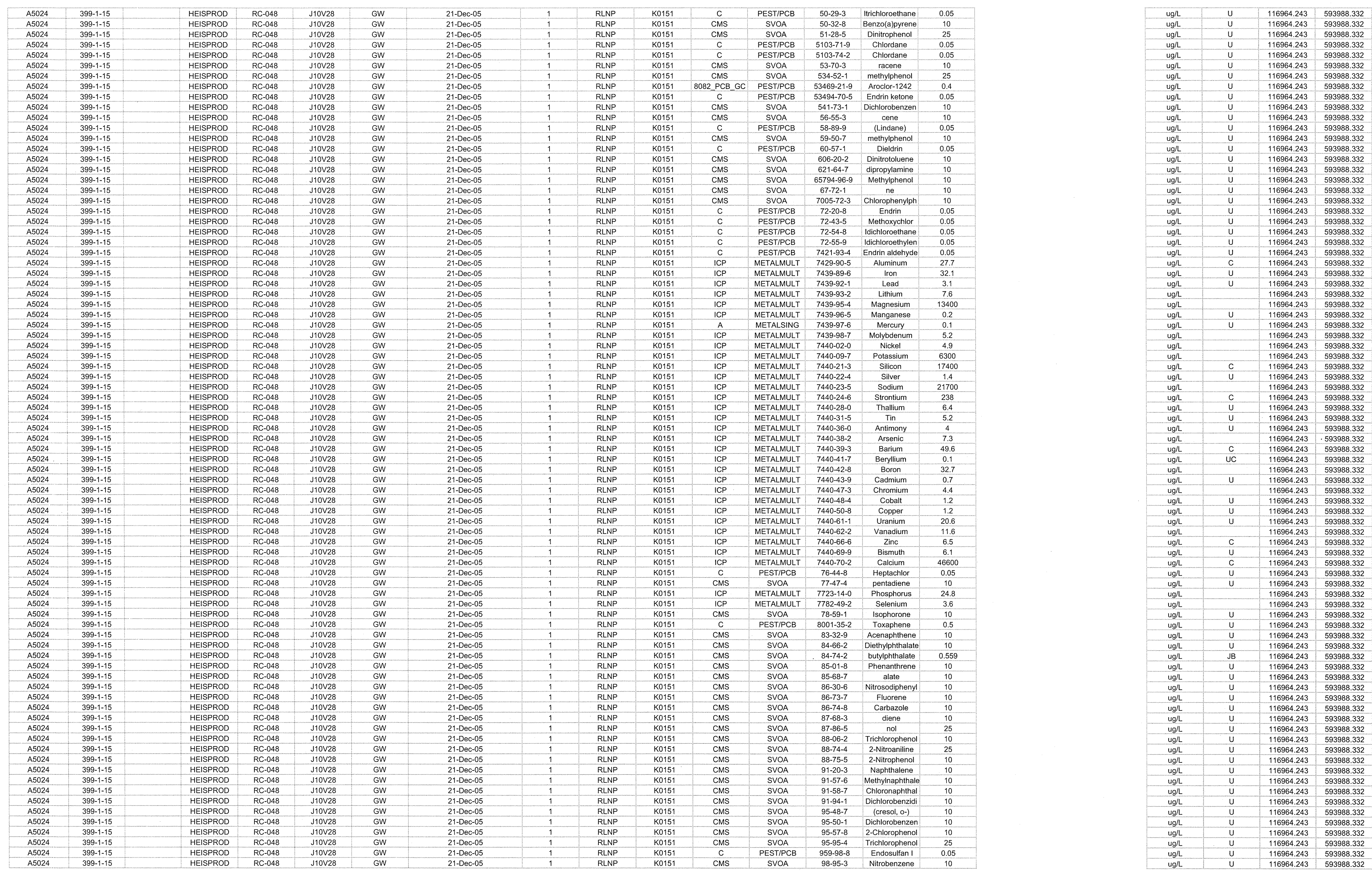

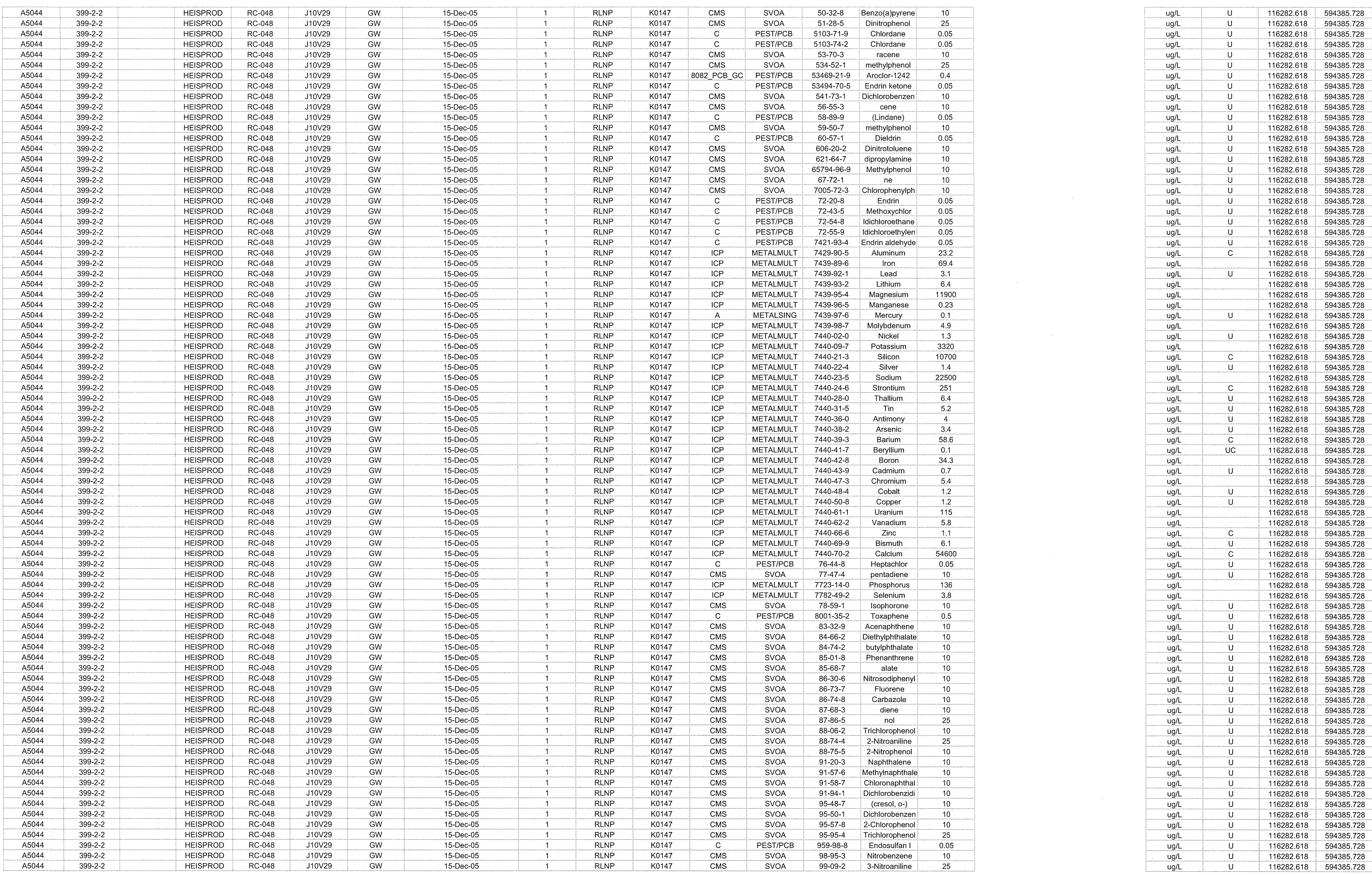


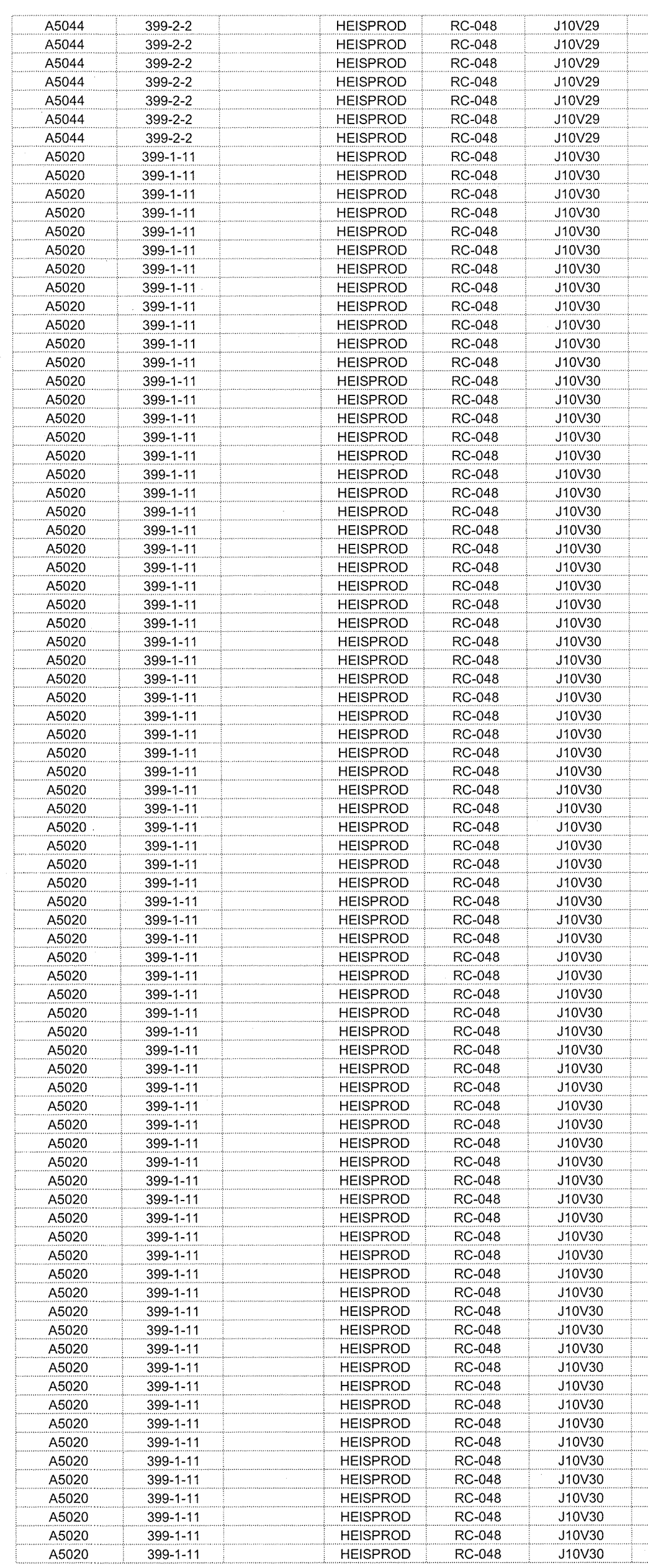

\begin{tabular}{|c|c|c|c|}
\hline$G W$ & 15-Dec-05 & $\frac{250}{1}$ & $\begin{array}{l}\text { RLNP } \\
\text { EBRLEE }\end{array}$ \\
\hline$G W$ & & & EBRLNE \\
\hline GW & 15-Dec-05 & & EBRLNE \\
\hline & 15-15-Dec-0.05 & & $\begin{array}{l}\text { EBRLNE } \\
\text { EBRINE }\end{array}$ \\
\hline GW & 15-Decc-05 & & $\begin{array}{l}\text { EBRRLNE } \\
\text { EBRLINE }\end{array}$ \\
\hline GW & 04-Dec-05 & & $\begin{array}{l}\text { EDLLNE } \\
\text { RLNP }\end{array}$ \\
\hline GW & 04-Dec-05 & & \\
\hline GW & 04-Dec-05 & & EBRLNE \\
\hline GW & O4-Dedec-005 & & $\begin{array}{l}\text { EBRLNEE } \\
\text { RINP }\end{array}$ \\
\hline GW & 04-Dec-05 & & $\begin{array}{l}\text { RENPP } \\
\text { EBRLNE }\end{array}$ \\
\hline$\frac{G W}{G W}$ & 04-4-Dec-05 & & $\begin{array}{l}\text { RLNP } \\
\text { PNDP }\end{array}$ \\
\hline GW & & & $\begin{array}{l}\text { RLNPP } \\
\text { RLNP }\end{array}$ \\
\hline GW & 04-Dec-05 & & $\begin{array}{l}\text { RLNP } \\
\text { RLNP }\end{array}$ \\
\hline GW & 04-Dec-05 & & RLNP \\
\hline GW & 04-Dec-05 & & RLNP \\
\hline GW & 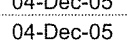 & & \\
\hline & 04-Dec-05 & & $\begin{array}{l}\text { RLNP } \\
\text { RLNP }\end{array}$ \\
\hline & & & RLNP \\
\hline GW & O4-Dec-05 & & RLNP \\
\hline GW & O4-D-Dec-05 & 1 & RLNP \\
\hline & 04-Dec-05 & & \\
\hline & 04-Dec-05 & & $\begin{array}{l}\text { RLNP } \\
\text { RLNP }\end{array}$ \\
\hline & & & RLNP \\
\hline GW & o & & RLNP \\
\hline GW & 0 & & RLNP \\
\hline & & & \\
\hline & 04-Dec-05 & & RLNP \\
\hline$G w$ & 04-Dec-05 & & RLNP \\
\hline GW & 04-4edc-05 & & \\
\hline GW & 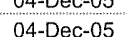 & & $\frac{R L N P}{R L N P}$ \\
\hline & 04-Dec-05 & & EBRLNE \\
\hline GW & 04-Dec-05 & & EBRLNE \\
\hline GW & O4-Dec-0 -0 & 1 & $\begin{array}{l}\text { EBRRNEE } \\
\text { EBRLLNE }\end{array}$ \\
\hline GW & 04-Dec-00 & & \\
\hline GW & 04-Dec-05 & & EBRLNE \\
\hline GW & 04-Dec-05 & 1 & EBRLNE \\
\hline GW & 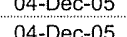 & 1 & $\begin{array}{l}\text { RLNP } \\
\text { ERNP }\end{array}$ \\
\hline GW & 04-Dec-05 & & \\
\hline & & & EBLLNE \\
\hline sw & 04-Dec-05 & 1 & EBRLNE \\
\hline$s w$ & 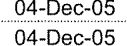 & 1 & EBRLNE \\
\hline GW & 04-Dec-05 & 10 & RLNP \\
\hline$\frac{G W}{6 w}$ & O4-Dec-05 & 1 & RLNP \\
\hline GW & 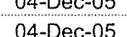 & 1 & \\
\hline & 04-Dec-00 & & 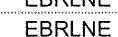 \\
\hline$E w$ & 04-Dec-05 & & EBLINE \\
\hline GW & 04-Dec--0 & 1 & EBRLNE \\
\hline GW & 04-Dec-05 & 1 & EBRLNE \\
\hline$G w$ & 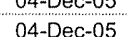 & 1 & \\
\hline & 04-Dec-0 & & RLNP \\
\hline$G W$ & 04-Dec-05 & & RLNP \\
\hline GW & 04-Dec-00 & & RLNP \\
\hline GW & 04-Dec-05 & & RLNP \\
\hline$G w$ & 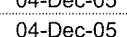 & & \\
\hline & & & \\
\hline GW & 04--есc-05 & & RLNP \\
\hline GW & 04-Dec- 05 & & RLNP \\
\hline GW & 04-Dec-05 & 1 & RLNP \\
\hline & & & \\
\hline & & 1 & \\
\hline sW & 04-Dec-05 & & RLNP \\
\hline GW & 04-Dec-05 & & RLNP \\
\hline GW & 04-Dec- 05 & & RLNP \\
\hline$G W$ & 0 & & \\
\hline & & & \\
\hline sw & 04-Dec-05 & & \\
\hline GW & 04-Dec-05 & 1 & RLNP \\
\hline
\end{tabular}
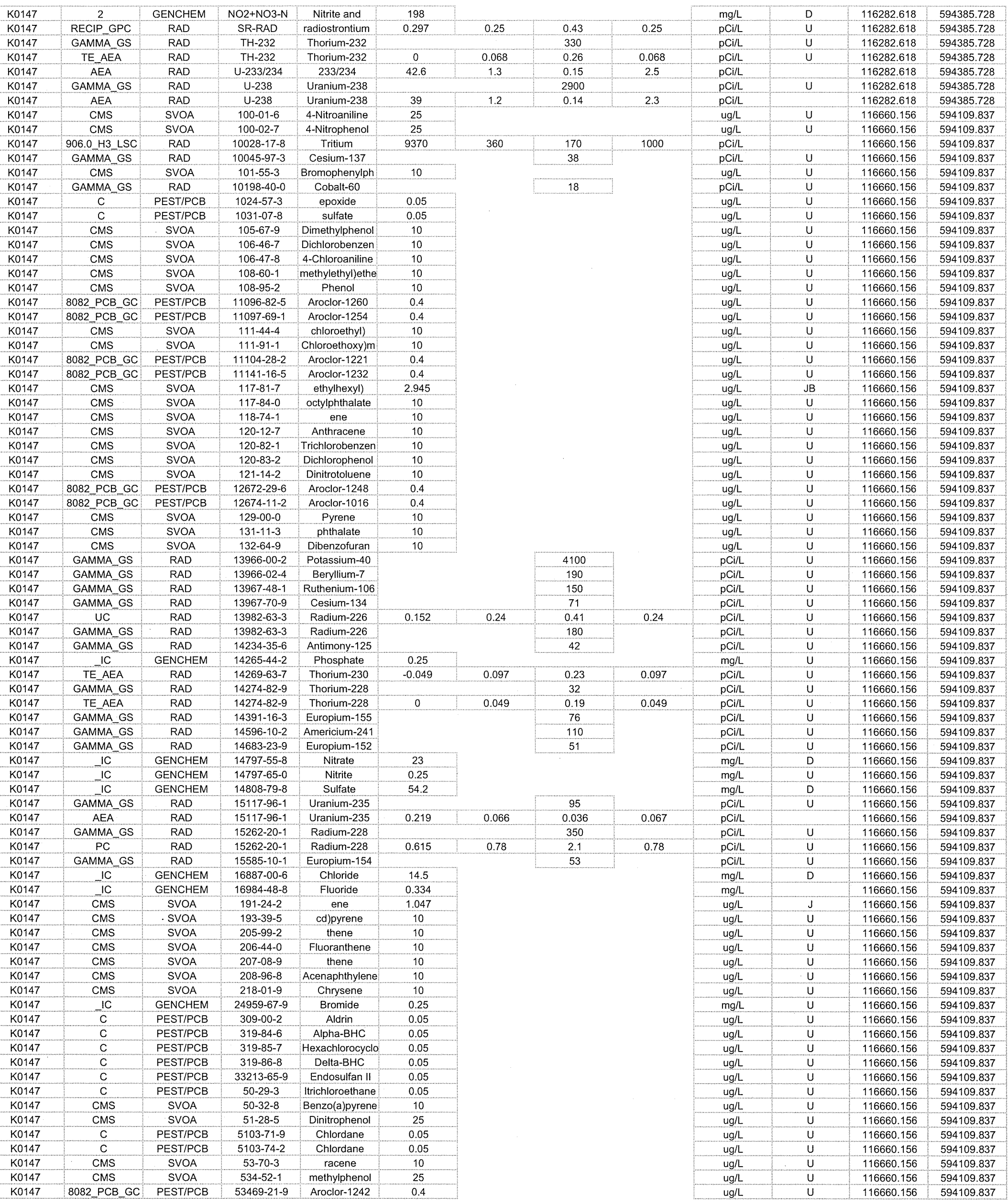


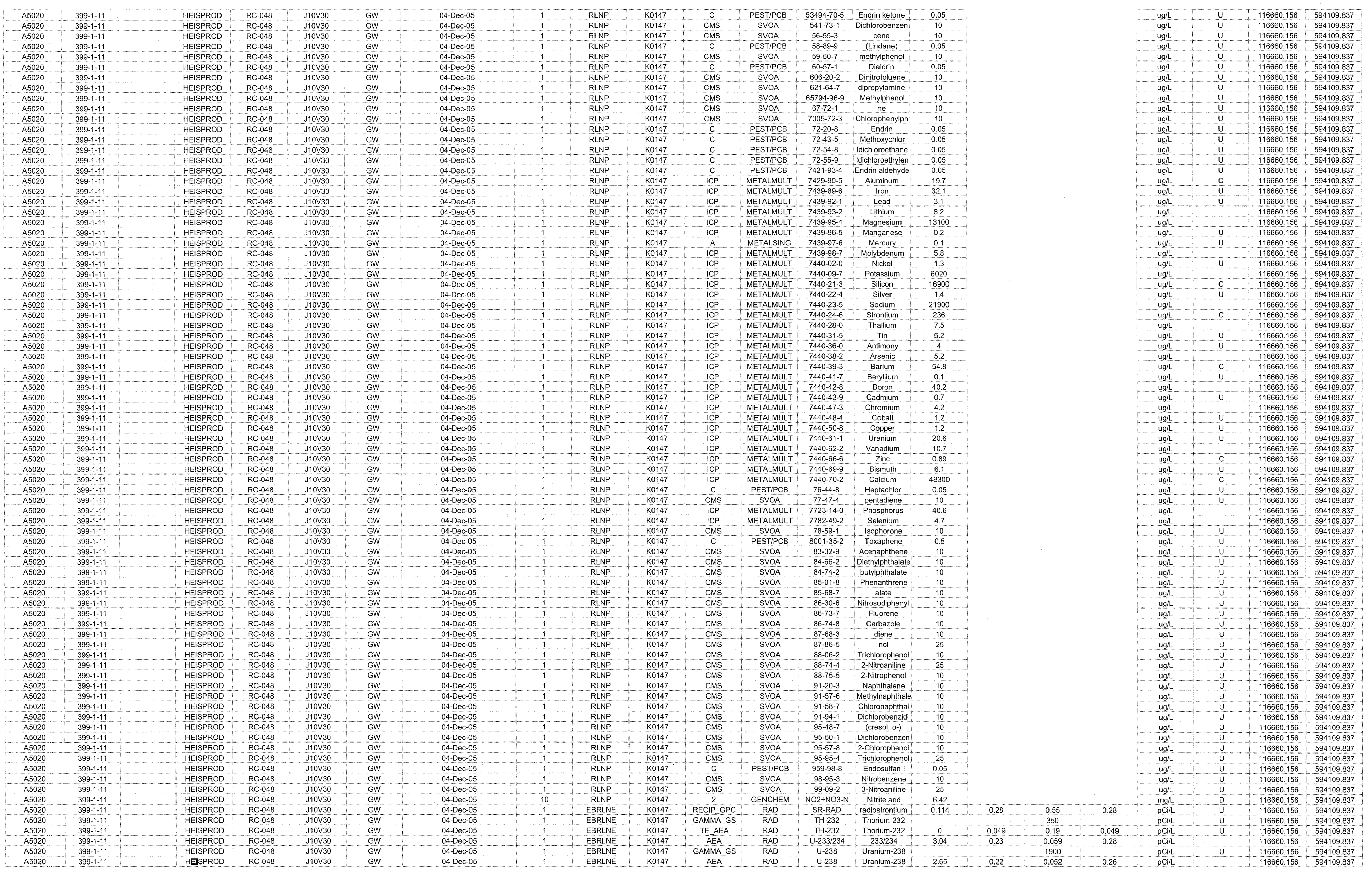




\begin{tabular}{|c|c|c|c|c|c|c|c|c|c|c|c|c|c|c|c|c|c|c|c|c|c|}
\hline${ }_{A 4632}^{A 4632}$ & $\frac{199-H A-46}{199-H 4-46}$ & $\begin{array}{l}\text { HEISPROD } \\
\text { HEIPRRD }\end{array}$ & $\begin{array}{l}\text { RC-048 } \\
\text { RC-048 }\end{array}$ & $\frac{5100231}{510 v 31}$ & $\begin{array}{c}G W \\
G W\end{array}$ & 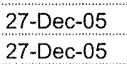 & & $\begin{array}{l}\text { RLNP } \\
\text { RNPP }\end{array}$ & $\begin{array}{l}\text { Ko158 } \\
\text { KK1588 }\end{array}$ & $\begin{array}{l}\text { CMS } \\
\text { CMS }\end{array}$ & $\begin{array}{l}\text { SVOA } \\
\text { SOOA }\end{array}$ & $\begin{array}{l}100-11-6 \\
100-02-7 \\
\end{array}$ & $\begin{array}{l}\text { 4-Nitroaniline } \\
4-\text { Nitrohent }\end{array}$ & $\begin{array}{l}25 \\
25\end{array}$ & & & & ugl & u & $\begin{array}{l}152439.868 \\
152393868\end{array}$ & $\begin{array}{l}577883.861 \\
55783896\end{array}$ \\
\hline A4632 & $199-44-46$ & HEISPROD & RC-048 & Jiov31 & GW & 27-Decer-05 & & $\begin{array}{l}\text { RLNP } \\
\text { EBRLNE }\end{array}$ & Ko158 & $\begin{array}{c}\text { CNS } \\
906 . \mathrm{H} \text { L LSC }\end{array}$ & $\begin{array}{l}\text { SVOA } \\
\text { RAD }\end{array}$ & $\begin{array}{l}1000-02-7 \\
10028-17-8\end{array}$ & $\begin{array}{l}\text { 4-N-NIrofoneno } \\
\text { Tritium }\end{array}$ & 3340 & 150 & & 370 & pcin & & 1524399.8688 & $\begin{array}{l}577883.861 \\
57783.861\end{array}$ \\
\hline A4632 & $199-44-46$ & HEISPROD & RC-048 & J10v31. & ow & 27-Dec-05 & & EBRLNE & K0158 & GAMMAGS & RAD & $10045-97-3$ & Cesium-137 & & & & & pcin & u & 152439.868 & 577883.861 \\
\hline A4632 & $199-44-46$ & HEISPROD & RC-048 & s10031 & GW & 27-Dec-05 & & RLNP & K0158 & CMS & SVOA & $101-55-3$ & Bromophenylph & 10 & & & & uglh & $u$ & 152439.868 & 577883.861 \\
\hline A4632 & $199-414-46$ & HEISPROD & RC-048 & J100v31 & GW & 27-Dec-05 & & EBRLNE & K0158 & GAMMA GS & $\begin{array}{l}\text { RAD } \\
\text { PDSTPPB }\end{array}$ & $101988-40-0$ & Coballt-60 & & & 28 & & poill & $\begin{array}{llll}u & \end{array}$ & 152439.868 & 577883.861 \\
\hline $\begin{array}{l}A 4632 \\
A 4632\end{array}$ & $\begin{array}{l}199-444-46 \\
199-4-46\end{array}$ & $\begin{array}{r}\text { HEISPROD } \\
\text { HESPROD }\end{array}$ & $\begin{array}{l}\text { RC-048 } \\
\text { RC-048 }\end{array}$ & J10V31 & $\begin{array}{c}G W \\
G W\end{array}$ & 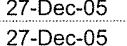 & & $\begin{array}{l}\text { RLNP } \\
\text { RLNP }\end{array}$ & $\begin{array}{l}\text { K0158 } \\
\text { K0158 }\end{array}$ & $\begin{array}{l}\mathrm{c} \\
\mathrm{c}\end{array}$ & $\begin{array}{l}\text { PESTPRCB } \\
\text { PEST/PCB }\end{array}$ & $\begin{array}{l}1024-57-3-3 \\
1031-78-8\end{array}$ & $\begin{array}{l}\text { epoxide } \\
\text { sulfate }\end{array}$ & 0.05 & & & & ugh & U & $\begin{array}{r}152439.868 \\
15234988\end{array}$ & $\begin{array}{l}577883.861 \\
577838\end{array}$ \\
\hline A463232 & $\begin{array}{l}199-144-46 \\
199-44-46\end{array}$ & & $\begin{array}{l}\text { RC-048 } \\
\text { RC-048 }\end{array}$ & J10v31 & GW & $\begin{array}{l}\text { 27-Ded-cos } \\
\text { 27-Dec-05 }\end{array}$ & & RLNP & K0158 & $\begin{array}{l}\text { C } \\
\text { CMS }\end{array}$ & $\begin{array}{l}\text { PESTPCB } \\
\text { SVOA }\end{array}$ & $\begin{array}{l}10311.07-8 \\
105-67-9\end{array}$ & $\begin{array}{l}\text { suffite } \\
\text { Dimethylphenol }\end{array}$ & $\frac{0.05}{10}$ & & & & gol & $u$ & 1524399.8688 & $\begin{array}{r}5777883.861 \\
577883.861\end{array}$ \\
\hline A4632. & $199-144-46$ & HEISPROD & RC-048 & J10V31 & GW & 27-Dec-05 & & RLNP & Ko158 & CNS & SVOA & $106-46-7$ & Dichlorobenzen & 10 & & & & ugl & $u$ & 152439.868 & 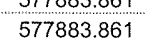 \\
\hline A4632 & $199-44-46$ & HEISPROD & RC- -048 & J10V31 & GW & 27-Dec-05 & & RLNP & Ko158 & CMS & SVOA & $106-47-8$ & 4-Chloroaniline & 10 & & & & ugh & $u$ & 152439.868 & 577883.861 \\
\hline A4632 & $199-H 4-46$ & HEISPROD & RC-048 & J10v31 & GW & 27-Dec-05 & & RLNP & K0158 & $\begin{array}{l}\text { CMS } \\
\text { CMS }\end{array}$ & SVOA & 108-60-1 & $\begin{array}{l}\text { methylethy) ethe } \\
\text { penone }\end{array}$ & 10 & & & & ugh & U & 152439.868 & 577883.861 \\
\hline $\begin{array}{l}A 4632 \\
A 4632\end{array}$ & $\begin{array}{l}199-144-46 \\
199-H 4-46\end{array}$ & $\begin{array}{r}\text { HEISPROD } \\
\text { HESPROD }\end{array}$ & 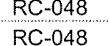 & $\begin{array}{l}\text { J10VV31 } \\
\text { J10Y31 }\end{array}$ & $\begin{array}{l}G W \\
G W\end{array}$ & $\begin{array}{l}\text { 27-Dec-0.05 } \\
\text { 22-Dep-05 }\end{array}$ & & $\begin{array}{l}\text { RLNPP } \\
\text { RLNP }\end{array}$ & $\begin{array}{l}\text { K0158 } \\
\text { K0158 }\end{array}$ & $\begin{array}{c}\text { CMS } \\
8082 \text { PCB GC }\end{array}$ & $\begin{array}{l}\text { SVAA } \\
\text { PEST/PCB }\end{array}$ & $\frac{180-95-2}{11806-82-5}$ & $\begin{array}{l}\text { Prenol } \\
\text { Aroclor-1260 }\end{array}$ & $\begin{array}{l}10 \\
0.4\end{array}$ & & & & $\begin{array}{l}\text { uggh } \\
\text { oglh }\end{array}$ & & $\begin{array}{l}1524349968 \\
15250808\end{array}$ & $\begin{array}{r}577883.861 \\
5778838\end{array}$ \\
\hline A4632 & $199-44-46$ & HEISPROD & $\begin{array}{l}\text { RL-048 } \\
\text { RC-048 }\end{array}$ & J10v31 & $G W$ & 27-Dec-05 & & RLNP & K0158 & 8082 PCB_GC & $\begin{array}{l}\text { PESTIPCB } \\
\text { PESTEB }\end{array}$ & 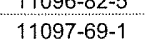 & 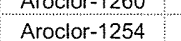 & & & & & ug/h & & 152543906808 & $\begin{array}{r}57778838.861 \\
577883.861\end{array}$ \\
\hline A4632 & $199-44-46$ & HEISPROD & RC-048 & J10V31 & GW & 27-Dec-05 & & RLNP & Ko158 & CMS & SVOA & $111-44-4$ & 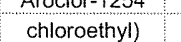 & 10 & & & & uglh & u & 152439.868 & 577883.861 \\
\hline A4632 & $199--44-46$ & HEISPROD & RC-048 & J10V31 & GW & 27-Dec-05 & & RLNP & K0158 & CMS & SVOA & $111-91-1$ & Chloroethoxy)m & 10 & & & & ugh & $u$ & 152439.868 & 577883.861 \\
\hline A4632 & 199-H4-46 & HEISPROD & RC-048 & J10v311 & GW & $\begin{array}{l}27-\text { Dec-05 } \\
27\end{array}$ & & RLNP & K0158 & 80082_PCB_GC & PEST/PCB & $11104-28-2$ & Arroclor-1221 & 0.4 & & & & ugh & $u$ & 152439.868 & 577883.861 \\
\hline $\begin{array}{l}{ }^{A 4432} 2 \\
A 4632\end{array}$ & $\begin{array}{l}199-144-46 \\
199-44-46\end{array}$ & HEISPROD & $\frac{R C-048}{\text { RC-048 }}$ & 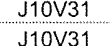 & $\frac{G W}{G W}$ & 27-Dec-0.05 & & $\begin{array}{l}\text { RLNP } \\
\text { RLNP }\end{array}$ & K0158 & 8082_PCB_GC & $\begin{array}{c}\text { PEST/PCB } \\
\text { SVOA }\end{array}$ & $\begin{array}{l}111141-16-5 \\
117-81-5\end{array}$ & $\begin{array}{l}\text { Arocolor-12323 } \\
\text { ethrhhexy) }\end{array}$ & $\begin{array}{l}0.4 \\
0.618\end{array}$ & & & & $\begin{array}{l}\text { ug/h } \\
\text { ull }\end{array}$ & $\begin{array}{l}U \\
\text { JB }\end{array}$ & $\begin{array}{r}152439.868 \\
15234988\end{array}$ & $\begin{array}{r}577883.861 \\
577838\end{array}$ \\
\hline & $\begin{array}{l}1999-144-46 \\
199-H 4-46\end{array}$ & HEISPROD & $\begin{array}{l}\text { RC-0.048 } \\
\text { RC-048 }\end{array}$ & J10V31 & GW & 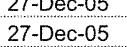 & & RLNP & K0158 & $\begin{array}{l}\text { CMS } \\
\text { CMS }\end{array}$ & $\begin{array}{l}\text { SVOA } \\
\text { SVOA }\end{array}$ & $117-84-0$ & $\begin{array}{l}\text { eryryinexy) } \\
\text { ocylyhtrate }\end{array}$ & $\frac{0.618}{10}$ & & & & goll & u & $\begin{array}{l}1524349.868 \\
152439.868\end{array}$ & $\begin{array}{r}5777883.861 \\
577883.861\end{array}$ \\
\hline A4632 & $199-44-46$ & HEISPROD & RC-048 & J10v31 & GW & 27-Dec-05 & & RLNP & K0158 & CMS & SVOA & $118-74-1$ & $\begin{array}{l}\text { ene } \\
\text { ene }\end{array}$ & 10 & & & & $\begin{array}{c}\text { sgli } \\
\text { ugl }\end{array}$ & u & 152439.868 & 577883.861 \\
\hline A4632 & $199-44-46$ & HEISPROD & RC-048 & J10V31 & GW & 27-Dec-05 & & RLNP & K0158 & CMS & SVOA & $120-12-7$ & Anthracene & 10 & & & & uglh & u & 152439.868 & 577883.861 \\
\hline A4632 & 199-14-46 & HEISPROD & RC-048 & J10V31 & GW & 27-Dec-05 & & RLNP & K0158 & CMS & SVOA & $\begin{array}{l}120-82-1 \\
202032\end{array}$ & Trichlorobenzzzen & 10 & & & & ugl & $u$ & 152439.868 & 577883.861 \\
\hline$\frac{A 4632}{A 4632}$ & $\begin{array}{l}199-144-46 \\
199-H 4-46\end{array}$ & $\begin{array}{l}\text { HEIISPROD } \\
\text { HEISPROD }\end{array}$ & $\frac{R C-048}{R C-048}$ & $\frac{510 V 31}{J 10 Y 31}$ & $\frac{G W}{G W}$ & 27-Dec-0.05 & 1 & $\begin{array}{l}\text { RLNPP } \\
\text { RLNP }\end{array}$ & $\begin{array}{l}\text { K0158 } \\
\text { K0158 }\end{array}$ & $\begin{array}{l}\text { CMS } \\
\text { CMS }\end{array}$ & $\begin{array}{l}\text { SVOA } \\
\text { SVOA }\end{array}$ & $\begin{array}{l}\frac{120-838-2}{121-14-2} \\
1\end{array}$ & Dicihlorophenonl & $\begin{array}{l}10 \\
10\end{array}$ & & & & $\frac{\text { uglh }}{\text { uglh }}$ & 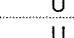 & $\begin{array}{l}152439.868 \\
15239\end{array}$ & $\begin{array}{r}577883.861 \\
55788381\end{array}$ \\
\hline & $\begin{array}{l}199-1-14-46 \\
199-44-46\end{array}$ & & $\begin{array}{l}\text { RC-0.048 } \\
\text { RC-048 }\end{array}$ & & GW & $\begin{array}{l}\text { 27-1-De-cos } \\
\text { 27-Dec-05 }\end{array}$ & r & $\begin{array}{l}\text { RLNP } \\
\text { RLNP }\end{array}$ & K0158 & 8082_PCB_GC & PEST/PCB & $12672-29-6$ & 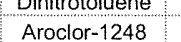 & & & & & oght & u & 152439.868 & $\begin{array}{l}5777883.861 \\
577883.861\end{array}$ \\
\hline A4632 & $199-44-46$ & HEISPROD & RC-048 & J10V31 & GW & 27-Dec-05 & & RLNP & K0158 & 8082 PCB,GC & PEST/PCB & $\frac{1267-41-2}{12674-1-2}$ & A Arochlor-1016 & $\begin{array}{l}0.4 \\
0.4\end{array}$ & & & & ogl & u & 1525439.068 & 5 \\
\hline A4632 & $199-14-46$ & HEISPROD & RC-048 & J10v31 & GW & 27-Dec-05 & & RLNP & Ko158 & CMS & SVOA & $129-00-0$ & Pyrene & 10 & & & & ughl & u & 152439.868 & 577883.861 \\
\hline A4632 & $199-44-46$ & HEISPROD & RC-048 & J10V31 & GW & $\begin{array}{l}\text { 27-Dec-05 } \\
\text { 27-05 }\end{array}$ & 1 & RLNP & K0158 & $\begin{array}{l}\text { CMS } \\
\text { CMS }\end{array}$ & SVOA & $\begin{array}{l}131-11-3 \\
132-649\end{array}$ & $\begin{array}{l}\text { phihalate } \\
\text { Piphonofiran }\end{array}$ & 10 & & & & ught & $u$ & 152439.868 & 577883.861 \\
\hline $\begin{array}{l}\text { A44632 } \\
A 4632\end{array}$ & $\begin{array}{l}199-444-46 \\
199-44-46\end{array}$ & $\begin{array}{l}\text { HEIIPROD } \\
\text { HEISPROD }\end{array}$ & $\begin{array}{l}\text { RC-048 } \\
\text { RC-048 }\end{array}$ & 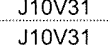 & GW & $\begin{array}{l}\text { 27-Dec-0.05 } \\
\text { 27-Dec-05 }\end{array}$ & 1 & $\begin{array}{l}\text { RLNP } \\
\text { EBRLNE }\end{array}$ & $\begin{array}{l}\text { K0158 } \\
\text { K0158 }\end{array}$ & $\begin{array}{l}\text { CMS } \\
\text { GAMMA GS }\end{array}$ & $\begin{array}{l}\text { SVOA } \\
\text { RAD }\end{array}$ & $\begin{array}{l}132-64-9 \cdot 9 \\
1396-00-2\end{array}$ & 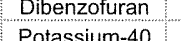 & 10 & & 690 & & ogh & u & $\begin{array}{l}1524349.968 \\
1523868\end{array}$ & $\begin{array}{l}577883.861 \\
557838361\end{array}$ \\
\hline A4632 & $\begin{array}{l}199--144-46 \\
199-46-46\end{array}$ & & $\begin{array}{ll}R C-048 \\
R C-048\end{array}$ & J10v31 & GW & 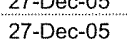 & & EBKRLNE & Kol58 & GAMMMA GS & RAD & $\frac{15900-12-2}{1396-02-4}$ & Borstlium-7 & & & 260 & & pin & u & 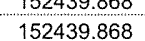 & $\begin{array}{l}5777883.861 \\
57783.861\end{array}$ \\
\hline A4632 & $199-44-46$ & HEISPROD & RC-048 & J10V31 & gw & 27-Dec-05 & 1 & EBRLNE & K0158 & GAMMA GS & RAD & $13967-48-1$ & Ruthenium-106 & & & 210 & & pcilh & u & $\begin{array}{l}152439.000 \\
152439.868\end{array}$ & 577883.861 \\
\hline A4632 & $199-H 4-46$ & HEISPROD & RC-048 & J100v31 & GW & 27-Dec-05 & 1 & EBRLNE & K0158 & GAMMA_GS & RAD & $13967-70-9$ & Cesium-134 & & & 29 & & pcill & $u$ & 152439.868 & 577883.861 \\
\hline $\begin{array}{l}A 4362 \\
A^{A 4332}\end{array}$ & $199-14-46$ & HEISPROD & RC-048 & 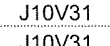 & GW & $\begin{array}{l}\text { 27-Dec-05 } \\
\text { 27-05 }\end{array}$ & 1 & EBRLNE & K0158 & UC & $\frac{R A D}{R A D}$ & $\begin{array}{l}13982-63-3 \\
\end{array}$ & Radium-226 & 0.31 & 0.33 & 0.54 & 0.33 & pcil & u & $\begin{array}{r}152439.868 \\
\end{array}$ & 577883.861 \\
\hline 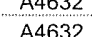 & $\begin{array}{l}199-H 44-46 \\
199-H 4-46\end{array}$ & $\begin{array}{l}\text { HEIIPROD } \\
\text { HEISPROD }\end{array}$ & $\begin{array}{l}\text { RC-048 } \\
\text { RC-048 }\end{array}$ & $\begin{array}{l}\text { J10V131 } \\
\text { J10V31 }\end{array}$ & & $\begin{array}{l}\text { 27-Dec-05 } \\
\text { 27-Dec-5 }\end{array}$ & $\frac{1}{1}$ & $\begin{array}{l}\text { EBRLNE } \\
\text { EBRLEE }\end{array}$ & K0158 & $\begin{array}{l}\text { GAMMA GS } \\
\text { GAMMA GS }\end{array}$ & $\begin{array}{l}\text { RAD } \\
\text { RAD }\end{array}$ & $\begin{array}{r}13982-63-33 \\
1434-35-6\end{array}$ & $\begin{array}{l}\text { Radium-226 } \\
\text { Antimonv-125 }\end{array}$ & & & $\begin{array}{r}57 \\
58 \\
58\end{array}$ & & pein & u & $\begin{array}{l}152439.868 \\
1523986\end{array}$ & $\begin{array}{l}577883.6361 \\
577806\end{array}$ \\
\hline $\begin{array}{l}\text { A46332 } \\
\text { A4632 }\end{array}$ & $\begin{array}{l}199-144-46 \\
199-H 4-46\end{array}$ & $\begin{array}{l}\text { HEISPROD } \\
\text { HEISPROD }\end{array}$ & $\begin{array}{l}\text { RC-048 } \\
\text { RC-048 }\end{array}$ & J10V31 & GW & $\begin{array}{l}\text { 22-1-0ec-0.05 } \\
\text { 27-Dec-05 }\end{array}$ & $\frac{7}{1}$ & $\begin{array}{l}\text { EBRLNE } \\
\text { RLNP }\end{array}$ & $\begin{array}{l}\text { K0158 } \\
\text { Ko158 }\end{array}$ & $\begin{array}{l}\text { AAMMAGS } \\
\text { IC }\end{array}$ & $\begin{array}{l}\text { RAD } \\
\text { GENCHEM }\end{array}$ & 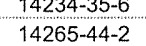 & $\begin{array}{l}\text { Antimony-125 } \\
\text { Phosphate }\end{array}$ & 0.25 & & 58 & & $\begin{array}{l}{ }_{\text {poll }} \\
\text { mglL }\end{array}$ & u & $\begin{array}{l}1522349.868 \\
152439.868\end{array}$ & $\begin{array}{l}5777838.861 \\
577883.861\end{array}$ \\
\hline A4632 & $199-144-46$ & HEISPROD & RC-048 & $5100 \sqrt{31}$ & GW & 27-Dec-05 & & EBRLNE & Ko158 & TE_AEA & RAD & $14269-63-7$ & Thorium-230 & $\begin{array}{c}-.0 .053 \\
-0.053 \\
\end{array}$ & 0.053 & 0.2 & 0.053 & pcil & u & 152439.868 & 577883.861 \\
\hline A46332 & $199-44-46$ & HEISPROD & RC-048 & J10V31 & Gw & 27-Dec-05 & 1 & EBRLNE & K0158 & GAMMA GS & RAD & $14277-82-9$ & Thorium-228 & & & 40 & & pCill & u & 152439.868 & 577883.861 \\
\hline A4632 & $199-14-46$ & $\begin{array}{l}\text { HEISPROD } \\
\text { HEIPROD }\end{array}$ & $\begin{array}{ll}\text { RC-048 } \\
\text { RC-048 }\end{array}$ & 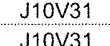 & GW & $\begin{array}{l}\text { 27-Dec-05 } \\
\text { 27-peca05 }\end{array}$ & 1 & EBRLNE & K0158 & $\begin{array}{l}\text { TEAEA } \\
\text { GAMMA GS }\end{array}$ & RAD & $\begin{array}{r}14274-82-9 \\
\end{array}$ & Thorium-228 & 0.106 & 0.11 & 0.2 & 0.11 & pcill & $u$ & $\begin{array}{r}152439.868 \\
\end{array}$ & 577883.861 \\
\hline $\begin{array}{l}{ }^{A 46362} \\
A 4632\end{array}$ & $\frac{199-944-46}{199-44-46}$ & $\begin{array}{l}\text { HEISPROD } \\
\text { HEISPROD }\end{array}$ & $\begin{array}{l}\text { RC- }-048 \\
\text { RC-048 }\end{array}$ & $\begin{array}{l}\text { J10Vist } \\
\text { J10V31 }\end{array}$ & GW & $\begin{array}{l}\text { 27-Dece-05 } \\
\text { 27-Dec-05 }\end{array}$ & $\frac{1}{1}$ & $\begin{array}{l}\text { EBRLNE } \\
\text { EBRLNEE }\end{array}$ & $\begin{array}{l}\text { KO158 } \\
\text { K0158 }\end{array}$ & $\begin{array}{l}\text { GAMMAGGS } \\
\text { GAMMA GS }\end{array}$ & $\frac{R A D}{R A D}$ & $\begin{array}{l}\frac{143991-16-3}{14391-65-2} \\
\end{array}$ & $\begin{array}{c}\text { Europium-155 } \\
\text { metastable }\end{array}$ & & & $\begin{array}{l}77 \\
19\end{array}$ & & pcil & u & $\frac{152439.8688}{15243988}$ & $\begin{array}{l}577883.861 \\
577883861\end{array}$ \\
\hline A46032 & $199-44 \cdot 46$ & HEISPROD & RC-048 & J10v31 & Gw & 27-Dec-05 & 1 & EBRLNE & Ko158 & $\begin{array}{l}\text { AAMMAGSS } \\
\text { GAMMAGS }\end{array}$ & RAD & $\begin{array}{r}143599-65-2-2 \\
1456-10-2\end{array}$ & $\begin{array}{l}\text { melastable } \\
\text { Americium-241 }\end{array}$ & & & $\begin{array}{r}19 \\
200\end{array}$ & & $\begin{array}{l}\text { plith } \\
\text { pcil }\end{array}$ & u & $\begin{array}{r}1524399.868 \\
152439.868\end{array}$ & 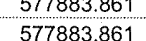 \\
\hline A4632 & $199-44-46$ & HEISPROD & RC-048 & J10V31 & GW & 27-Dec-05 & 1 & EBRLNE & Ko158 & GAMMA_GS & RAD & $14683-23-9$ & Europium-152 & & & 61 & & pcill & u & 152439.868 & 577883.861 \\
\hline A4632 & $199 \cdot-44-46$ & HEISPROD & RC-048 & J100V31 & GW & 27-Dec-05 & 10 & RLNP & K0158 & IC & GENCHEM & $14797-55-8$ & Nitrate & 46.7 & & & & mgll & D & 152439.868 & 577883.861 \\
\hline $\begin{array}{l}A_{44632} \\
A 4352\end{array}$ & $\begin{array}{l}199-144-46 \\
199-44-46\end{array}$ & $\begin{array}{l}\text { HEISPROD } \\
\text { HEISPROD }\end{array}$ & $\begin{array}{l}\frac{R C-048}{R C-088} \\
\text { RC }\end{array}$ & $\begin{array}{l}\text { J10V31 } \\
\text { J10V31 }\end{array}$ & GW & 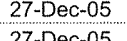 & $\frac{2}{10}$ & $\begin{array}{l}\text { RenNP } \\
\text { PINP }\end{array}$ & K0158 & $\frac{\text { II }}{1 C}$ & $\begin{array}{l}\text { GENNHEM } \\
\text { SFNCHEF }\end{array}$ & $\begin{array}{l}17497-65-0 \\
1980-208\end{array}$ & $\begin{array}{l}\text { Nititi } \\
\text { Sittition }\end{array}$ & 813 & & & & moll & UD & 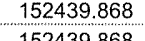 & 577883.861 \\
\hline A4t352 & $\begin{array}{l}199-744-46 \\
199-94-46\end{array}$ & $\begin{array}{l}\text { HEIISROD } \\
\text { HEISPROD }\end{array}$ & 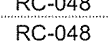 & $\begin{array}{l}\text { J110V31 } \\
\text { J10v31 }\end{array}$ & $\frac{G W}{G W}$ & $\begin{array}{l}\text { 27-DDC-05 } \\
\text { 27-Dec-05 }\end{array}$ & $\frac{10}{1}$ & EERLNE & $\begin{array}{l}\text { K0158 } \\
\text { K0158 }\end{array}$ & $\begin{array}{c}\text { GAMA } \\
\text { GAMMA GS }\end{array}$ & $\begin{array}{l}\text { GENCHEM } \\
\text { RAD }\end{array}$ & 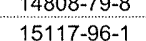 & $\begin{array}{l}\text { Sulfate } \\
\text { Uranium-235 }\end{array}$ & 84.3 & & & & mgle & D & $\begin{array}{r}1524399688 \\
1523080\end{array}$ & $\begin{array}{l}577883.861 \\
5778838\end{array}$ \\
\hline A4632 & $199-14-46$ & HEISPROD & RC-048 & J10v31 & sw & $27-\operatorname{Dec}-05$ & 1 & EBRLNE & Ko158 & $A E A$ & RAD & $15117-96-1$ & Uranium-235 & 0.096 & $\begin{array}{l}0.038 \\
\end{array}$ & 0.024 & 0.038 & pCill & & 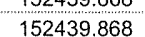 & 577883.861 \\
\hline A4632 & $199-44-46$ & HEISPROD & RC-048 & J10v31 & ow & 27-Dec-05 & 1 & EBRLNE & K0158 & GAMMA_GS & RAD & $15262-20-1$ & Radium-228 & & & 120 & & pcill & u & 152439.868 & 577883.861 \\
\hline A4632 & $199-H 4-46$ & HEISPROD & RC-048 & J100v31 & GW & 27-Dec-005 & 1 & EBRLNE & K0158 & $P C$ & RAD & $15262-20-1$ & Radium-228 & -0.407 & 1.4 & 2 & 1.4 & pCill & u & 152439.868 & 577883.861 \\
\hline A4632 & $\begin{array}{l}199-94-46 \\
199-44-46\end{array}$ & $\begin{array}{l}\text { HEISPROD } \\
\text { HEISPROD }\end{array}$ & $\begin{array}{l}\text { RC-048 } \\
\text { RC-048 }\end{array}$ & $\begin{array}{l}\text { J110V31 } \\
\text { J10Vy31 }\end{array}$ & $\frac{G W}{G W}$ & 27-Dec-.05 & $\frac{1}{10}$ & $\begin{array}{l}\text { EBRLNE } \\
\text { RLNP }\end{array}$ & K0158 & GAMMA_GS & $\begin{array}{l}\text { RAD } \\
\text { GENCHEM }\end{array}$ & $\begin{array}{l}\frac{155858-10-1}{1687-00-6} \\
\end{array}$ & Europium-154 & 208 & & 73 & & poin & u & $\begin{array}{l}152439.868 \\
15230.8\end{array}$ & 577883.861 \\
\hline $\begin{array}{l}\text { A46562 } \\
\text { A4632 }\end{array}$ & $\begin{array}{l}199-944-46 \\
199-44-46\end{array}$ & HEISPROD & $\begin{array}{l}\text { RC-0.048 } \\
\text { RC-048 }\end{array}$ & J10vev31 & sw & $\begin{array}{l}\text { 27-JUec-05 } \\
\text { 27-Dec-05 }\end{array}$ & 1 & $\begin{array}{l}\text { RLNP } \\
\text { RLNP }\end{array}$ & KO158 & $\frac{16}{10}$ & $\begin{array}{l}\text { GENCHCM } \\
\text { GENCHEM }\end{array}$ & $168984-400-68$ & $\begin{array}{l}\text { Chloride } \\
\text { Fluoride }\end{array}$ & $\begin{array}{l}29.8 \\
0.25\end{array}$ & & & & moli & 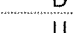 & $\begin{array}{l}15243998868 \\
152439868\end{array}$ & 577883.861 \\
\hline A4632 & $199 \cdot-144-46$ & HEISPROD & RC-048 & J100v31 & Gw & 27-Dec-05 & 1 & RLNP & K0158 & CMS & SVOA & 191-24-2 & ene & $\frac{.60}{10}$ & & & & uglt & u & 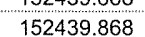 & 577883.861 \\
\hline A4632 & $1999-44-46$ & HEISPROD & RC-048 & siov31 & GW & 27-Dec-05 & 1 & RLNP & K0158 & CMS & SVOA & $193-39-5$ & colpyrene & 10 & & & & uglh & u & 152439.868 & 577883.861 \\
\hline A4632 & $199-44-46$ & HEISPROD & RC-048 & 5100031 & ow & 27-Dec-05 & 1 & RLNP & K0158 & CMS & SVOA & $205-99-2$ & thene & 10 & & & & ugh & $\begin{array}{lll}u & \end{array}$ & 152439.868 & 577883.861 \\
\hline A4632 & $\begin{array}{l}\frac{99-944-46}{199-44-46} \\
1\end{array}$ & $\begin{array}{l}\text { HEISPROD } \\
\text { HEISPROD }\end{array}$ & $\begin{array}{l}\frac{R C-048}{R C-048} \\
\text { RC }\end{array}$ & 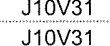 & $\frac{G w}{G w}$ & $\begin{array}{l}\text { 27-Dec-.05 } \\
\text { 27-0.05 }\end{array}$ & $\frac{1}{1}$ & $\begin{array}{l}\text { RLNP } \\
\text { Ren }\end{array}$ & K0158 & CMS & SVOA & $\begin{array}{l}206-44-0 \\
2070-0.9-9\end{array}$ & $\begin{array}{l}\text { Fluoranthene } \\
\end{array}$ & $\begin{array}{l}10 \\
10\end{array}$ & & & & gigh & 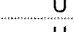 & 152439.868 & 577883.861 \\
\hline A4632 & $199-44-46$ & HEISPROD & $\begin{array}{l}\text { RL-048 } \\
\text { RC-048 }\end{array}$ & J10val & Gw & 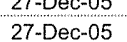 & 1 & RLNP & KO158 & CMS & SVOA & $\begin{array}{l}207-80-9-9 \\
208-96-8\end{array}$ & $\begin{array}{l}\text { Acenenen } \\
\text { Acentylene }\end{array}$ & 10 & & & & voll & u & $\begin{array}{l}1524399.868 \\
152439.868\end{array}$ & 5778838661 \\
\hline A4632 & $199-44-46$ & HEISPROD & RC-048 & J10v31 & GW & 27-Dece-05 & 1 & RLNP & K0158 & cMS & SVOA & $218-01-9$ & Chrsene & 10 & & & & voll & u & 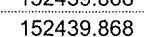 & 5 \\
\hline A4632 & $199-H 4-46$ & HEISPROD & RC- -48 & J10v31 & ow & 27-Dec-05 & 1 & RLNP & K0158 & IC & GENCHEM & $24959-67-9$ & Bromide & 0.25 & & & & $\mathrm{mg} / \mathrm{L}$ & u & 152439.868 & 577883.861 \\
\hline A4632 & $199-14-46$ & HEISPROD & RC-048 & $1100 v 31$ & GW & 27-Dec-05 & 1 & RLNP & K0158 & $c$ & PESTPPCB & $309-00-2$ & Aldrin & 0.05 & & & & ugh & u & 152439.868 & 577883.861 \\
\hline $\begin{array}{l}\text { A44362 } \\
\text { A4632 }\end{array}$ & $\frac{199-14-46}{199-44-46}$ & $\begin{array}{l}\text { HEISPROD } \\
\text { HEISPROD }\end{array}$ & $\frac{R C-048}{R C-048}$ & $\frac{5100031}{510 v 31}$ & $\frac{G W}{G W}$ & 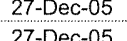 & 1 & $\begin{array}{l}\text { RRNP } \\
\text { RNP }\end{array}$ & K0158 & & PESTIPCB & $\begin{array}{l}319-84-6 \\
330.5-7.6\end{array}$ & 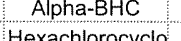 & 0.05 & & & & ught & $\underline{u}$ & 152439.8688 & 577883.861 \\
\hline $\begin{array}{l}A_{44532} \\
A 4632\end{array}$ & $\begin{array}{l}199-9-44-46 \\
199-44-46\end{array}$ & HEISPROD & RC-048 & J10val & $\frac{G W}{G W}$ & $\begin{array}{l}\text { 27-DLCe-15 } \\
\text { 27-Dec-05 }\end{array}$ & 1 & RLNP & K0158 & c & PEST/PCB & $319-86-8$ & rexachiorocectio & 0.05 & & & & . & 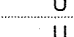 & $\begin{array}{l}1524349.8688 \\
1525080\end{array}$ & 577883.861 \\
\hline A4632 & $199-44-46$ & HEISPROD & RC-048 & J10v31 & GW & 27-Dec-05 & 1 & RLNP & Ko158 & c & PEST/PCB & 33213-65-9 & Endosulfan II & 0.05 & & & & ught & u & $\frac{1524249.068}{152988}$ & 577883.861 \\
\hline A4632 & $199-44-46$ & HEISPROD & RC-048 & J10v31 & GW & 27-Dec-05 & 1 & RLNP & Ko158 & $c$ & PESTIPCB & $50-29-3$ & Itrichloroethane & 0.05 & & & & ugh & u & 152439.868 & 577883.861 \\
\hline A4632 & $1999-44-46$ & HEISPROD & RC-048 & 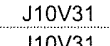 & $G W$ & 27-Dec-05 & 1 & RLNP & K0158 & CMS & SVOA & $50-32-8$ & Benzo(a)pyrene & 10 & & & & ugh & $u$ & 152439.868 & 577883.861 \\
\hline $\begin{array}{l}A 4632 \\
A 4632\end{array}$ & $\begin{array}{l}199-94-46 \\
199-44-46\end{array}$ & $\begin{array}{l}\text { HEISPROD } \\
\text { HEISPROD }\end{array}$ & $\begin{array}{l}\text { RC-048 } \\
\text { RC.048 }\end{array}$ & $\frac{5100031}{510 v 31}$ & GW & 27-Dec-05 & 1 & RLNP & K0158 & CMS & $\begin{array}{l}\text { SVOA } \\
\text { PESTIPCB }\end{array}$ & $\begin{array}{r}51-28-5 \\
5 \\
5010327-1-9\end{array}$ & $\begin{array}{l}\text { Dinitrophenol } \\
\text { chlortanene }\end{array}$ & 25 & & & & $n^{2}$ & U & 152439.868 & 577883.861 \\
\hline A4632 & $\begin{array}{l}199-4-446 \\
199-94-46\end{array}$ & $\begin{array}{l}\text { MESPROD } \\
\text { HESPROD }\end{array}$ & RC-048 & J190v31 & Gw & 27-0e-10.05 & 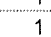 & RNAP & K0158 & 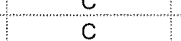 & PESTPCB & 5 & $\begin{array}{l}\text { Chiodone } \\
\text { Chlorane }\end{array}$ & $\begin{array}{l}0.035 \\
0.05\end{array}$ & & & & & & 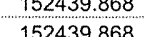 & 577883.861 \\
\hline A4632 & $199-44-46$ & HEISPROD & RC-048 & J10v31 & GW & 27-Dec-05 & 1 & RLNP & Ko158 & CMS & SVOA & $53-70-3$ & racene & $\frac{10}{10}$ & & & & ugl & $u$ & $\frac{152429.068}{152968}$ & 577883.861 \\
\hline A4632 & $199-44-46$ & HEISPROD & RC-048 & J10uv31 & GW & 27-Dec-05 & 1 & RLNP & K0158 & CMS & SVOA & $534-52-1$ & methylphenol & 25 & & & & uglh & u & 152439.868 & 577883.861 \\
\hline A4632 & $199-144-46$ & HEISPROD & RC-048 & J10v31 & GW & 27-Dec-05 & 1 & RLNP & K0158 & 8082 PCB_GC & PEST/PCB & $53469-21-9$ & Aroclor-1242 & 0.4 & & & & ug/ & $\begin{array}{lll}u & \end{array}$ & 152439.868 & 577883.861 \\
\hline 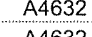 & $199-14-46$ & $\begin{array}{l}\text { HEISPROD } \\
\end{array}$ & $\begin{array}{l}\text { RC-048 } \\
\text { RC. } 048\end{array}$ & -0.00031 & $G W$ & $\begin{array}{l}27-\text {-Dec-05 } \\
27-0.05\end{array}$ & 1 & RLNP & K0158 & C & $\begin{array}{l}\text { PESTPCB } \\
\text { SWOA }\end{array}$ & $53494-70-5$ & Enddin ketone & 0.05 & & & & $n$ & & 152439.868 & 5777883.861 \\
\hline A44532 & $\begin{array}{l}199-144-46 \\
199-14-46\end{array}$ & HEISPROD & RC.-048 & (1) & Giv & 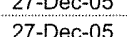 & & RLNP & K0158 & ciss & $\begin{array}{l}\text { SNOA } \\
\text { SVOA }\end{array}$ & -56-55-3-3 & Dichlorobenenzen & 10 & & & & , & & 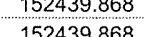 & 5 \\
\hline A46632 & $199 \cdot-44-46$ & HEISPROD & RC-048 & J10v31 & GW & 27-Decc-05 & 1 & RLNP & K0158 & $w_{c}$ & PEST/PCB & 58-89-9 & (Lindane) & 0.05 & & & & & & 1524399.0680 & 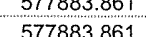 \\
\hline A4632 & $199-44-46$ & HEISPROD & RC-048 & J10v31 & GW & 27-Dec-05 & 1 & RLNP & K0158 & CMS & SVOA & 59-50-7 & methyphenol & 10 & & & & uglh & u & 152439.868 & 577883.861 \\
\hline A4632 & 199-H4-46 & HEISPROD & RC-048 & J10V31 & GW & 27-Dec-05 & & RLNP & K0158 & & PEST/PCB & $60-57-1$ & Dieldrin & 0.05 & & & & ualh & u & 152439.868 & 577883888 \\
\hline
\end{tabular}



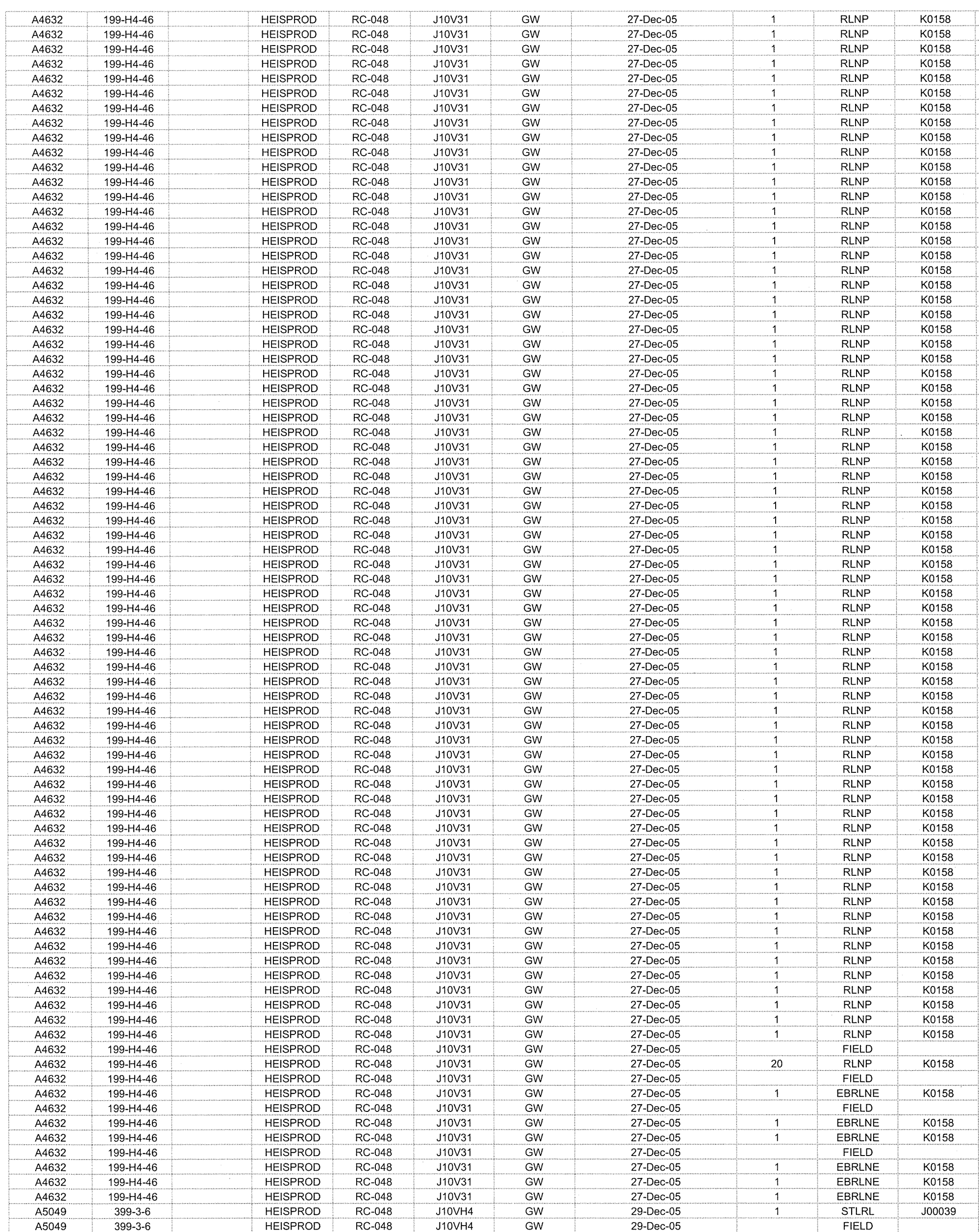

\begin{tabular}{l|l|l|l} 
& SVOA & $606-20-2$ & Dinitrotoluene \\
\hline SVIS & SVOA & $621-64-7$ & dipropylamine \\
\hline
\end{tabular}

\begin{tabular}{|c|c|c|c|}
\hline igh & & 152439.868 & 577883.861 \\
\hline ug/L & $u$ & 152439.868 & 577883.861 \\
\hline & $u$ & $\begin{array}{l}15242499.688 \\
152439.868\end{array}$ & \\
\hline & $u$ & 1524339.8688 & \\
\hline & $u$ & 152439.868 & 5777883.801 \\
\hline & $u$ & 152439.868 & \\
\hline il & $u$ & 152439.868 & \\
\hline & u & $\begin{array}{l}15243499.868 \\
52439.863\end{array}$ & 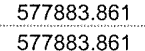 \\
\hline . & $c$ & & \\
\hline & & 152439.868 & 5777883.861 \\
\hline & u & 152439.8688 & 577883.861 \\
\hline & & $\begin{array}{r}152439.968 \\
152439.868\end{array}$ & $\begin{array}{c}5778838.861 \\
557783.611\end{array}$ \\
\hline & & & \\
\hline & $u$ & $\begin{array}{l}1524399.868 \\
15152328968\end{array}$ & $\begin{array}{l}5778838.861 \\
5778838961\end{array}$ \\
\hline ugh & u & 152439.8608 & $\begin{array}{l}5777838380101 \\
5\end{array}$ \\
\hline & & & 577883.861 \\
\hline & $c$ & 152439.868 & 577883.861 \\
\hline & 0 & 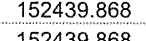 & 577883.861 \\
\hline & $c$ & $\begin{array}{l}15242439006 \\
152988\end{array}$ & 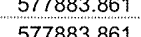 \\
\hline & u & 152439.868 & 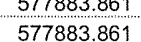 \\
\hline & $u$ & 152439.868 & 577883.861 \\
\hline & & $\begin{array}{l}15243439.868 \\
15268\end{array}$ & 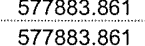 \\
\hline & & 152439.868 & 577883.861 \\
\hline $19 / 1 / 2$ & UC & 152439.868 & 577883.861 \\
\hline & & 152433.868 & 577883.861 \\
\hline & 0 & $\begin{array}{l}1524399.868 \\
15152328968\end{array}$ & 577883.8611 \\
\hline & u & & \\
\hline & u & 152439.868 & 5778833.861 \\
\hline & $u$ & 152439.868 & 577883.861 \\
\hline & & & 577883.861 \\
\hline & 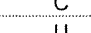 & 152435.8088 & 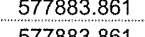 \\
\hline & c & 152439.868 & 577883.861 \\
\hline & u & & 577883.861 \\
\hline She & $u$ & 15243 & 577883.861 \\
\hline & & & 577883.861 \\
\hline & 4 & & \\
\hline & & 152439.868 & 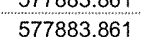 \\
\hline & u & & 577883.861 \\
\hline & u & 15243 & 57788 \\
\hline & $J_{B}$ & 15243 & 57788 \\
\hline 4 & u & & \\
\hline & & & 57788 \\
\hline & u & & \\
\hline uoll & u & 152439.868 & 577883.861 \\
\hline . & 0 & .8.868 & 577883.861 \\
\hline ugl & u & 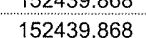 & 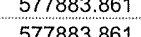 \\
\hline . & & & \\
\hline & & & 577883.861 \\
\hline & u & 152439.868 & 577883.861 \\
\hline an & 0 & 152439.868 & 577883.861 \\
\hline & u & 1525439.0808 & 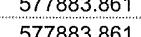 \\
\hline g? & & & \\
\hline & & & \\
\hline & u & 15243: & 5778 \\
\hline 9 & & 152439.868 & 577883.861 \\
\hline & u & 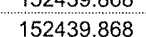 & \\
\hline ugh & $u$ & .868 & 577883.861 \\
\hline $\lg$ & $\mathrm{D}$ & & \\
\hline & & 152439.868 & 577883.861 \\
\hline & & $15243 C^{1}$ & 5778833.861 \\
\hline & & 152439.50608 & $\begin{array}{l}577080.061 \\
5\end{array}$ \\
\hline & $u$ & & 577883.861 \\
\hline & & & 3.861 \\
\hline & & 8688 & 5777883.861 \\
\hline pCii & 0 & 152439.0008 & (577883.8.861 \\
\hline & & $\frac{115944.403}{100400}$ & 593927.103 \\
\hline & & & \\
\hline
\end{tabular}




\begin{tabular}{|c|c|c|c|c|c|c|c|c|c|c|c|c|c|c|}
\hline $\begin{array}{l}A 5049 \\
5049\end{array}$ & $\begin{array}{l}399-3 \cdot 6 \\
30.3-6\end{array}$ & HEISPROD & $\begin{array}{l}\text { RC-048 } \\
\text { RC-048 }\end{array}$ & 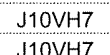 & GW & 29-Dec-05 & & RLNP & K0158 & CMS & SVOA & $51-28-5$ & Dinitrophenol & 25 \\
\hline $\begin{array}{l}\text { A55049 } \\
\text { A5049 }\end{array}$ & $\begin{array}{l}399-3-6 \\
399-3-6\end{array}$ & $\begin{array}{l}\text { HEIIPRODD } \\
\text { HEISPROD }\end{array}$ & 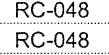 & $\begin{array}{l}\text { J10VVHT } \\
\text { J10VIT }\end{array}$ & $\frac{G W}{G W}$ & $\begin{array}{l}\text { 29-D-DC-05 } \\
\text { 20-Dedc-505 }\end{array}$ & $\frac{1}{1}$ & $\begin{array}{l}\text { RLNP } \\
\text { RNPP }\end{array}$ & $\begin{array}{l}\text { Ko158 } \\
\text { KK15158 }\end{array}$ & $\begin{array}{l}c \\
c\end{array}$ & $\begin{array}{l}\text { PESTIPCB } \\
\text { PETTPCB }\end{array}$ & $\begin{array}{l}\frac{5103-71-9}{5103-74-2} \\
\end{array}$ & $\begin{array}{l}\text { Chlordane } \\
\text { Chlorddane }\end{array}$ & $\begin{array}{l}0.05 \\
0.05\end{array}$ \\
\hline A5049 & $399 \cdot-3-6$ & HEISPROD & RC- -048 & J10VVH? & GW & $\begin{array}{l}29-0.0 c-05 \\
29-D e c-05\end{array}$ & 1 & $\begin{array}{l}\text { RLN } \\
\text { RLNP }\end{array}$ & K0158 & CMS & SVOA & $\frac{5113-7-12-2}{53-70-3}$ & $\begin{array}{l}\text { Chlordane } \\
\text { racene }\end{array}$ & $\frac{0.05}{10}$ \\
\hline A5049 & $399-3-6$ & HEISPROD & RC-048 & J10VH7 & ow & 29-Dec-05 & & RLNP & Ko158 & CMS & SVOA & $534-52-1$ & methylphenol & 25 \\
\hline $\begin{array}{l}\text { A50049 } \\
\text { A55049 }\end{array}$ & $\begin{array}{l}399-3-6 \\
303-3-6\end{array}$ & 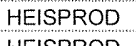 & RC-048 & J10VH7 & GW & 29-Dec-05 & & RLNP & K0158 & 8082 PCB GC & PESTPCB & $53469-21-9$ & Aroclor-1242 & 0.4 \\
\hline $\begin{array}{l}\text { A55049 } \\
\text { A55049 }\end{array}$ & $\begin{array}{l}399-3-6 \\
309-3-6\end{array}$ & HEISPRRD & RC-048 & J10VH? & GW & $\begin{array}{l}\text { 29-Dec- }-05 \\
\text { 20-DDC-05 }\end{array}$ & & RLNP & K0158 & C & PESTPCB & $534944-70-5$ & Endrin ketone & 0.05 \\
\hline A5049 & 399-3-6 & $\begin{array}{l}\text { HESPPROD } \\
\text { HEISPROD }\end{array}$ & 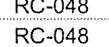 & $\begin{array}{l}\text { J10VH7 } \\
\text { J10VH7 }\end{array}$ & ow & $\begin{array}{l}\text { 29-DDc-05 } \\
\text { 29-Dec-05 }\end{array}$ & & & K0158 & $\begin{array}{l}\text { CMS } \\
\text { CMS }\end{array}$ & $\begin{array}{l}\text { SVOA } \\
\text { SVOA }\end{array}$ & $\begin{array}{l}54-7.3-1 \\
55-55.3\end{array}$ & $\begin{array}{l}\text { Dichlorobenzzen } \\
\text { cenen }\end{array}$ & $\begin{array}{l}10 \\
10\end{array}$ \\
\hline A5049 & $399-3-6$ & HEISPROD & RC.-048 & Jiovit? & ow & 29-Dec-05 & & $\begin{array}{l}\text { RLN } \\
\text { RLNP }\end{array}$ & K0158 & CMS & PEST/PCB & 56-58-3 & $\begin{array}{l}\text { cene } \\
\text { (Lindane) }\end{array}$ & $\begin{array}{l}10 \\
0.05\end{array}$ \\
\hline A5049 & 399-3-6 & HEISPROD & RC-048 & J10VHT & ow & 29-Dec-05 & & RLNP & Ko158 & CMS & SVOA & 59-50-7 & methyphenol & 10 \\
\hline A5049 & $\begin{array}{l}399-3-6 \\
3003-6-6\end{array}$ & HEISPROD & RC-048 & J10VH? & GW & 29-Dec-05 & & RLNP & K0158 & $c$ & PEST/PCB & $60-57-1$ & Dieldrin & 0.05 \\
\hline $\begin{array}{l}\text { A504099 } \\
\text { A55049 }\end{array}$ & $\begin{array}{l}399-3-6 \\
399-3-6\end{array}$ & HEISPROD & RC-048 & J10VH7 & Gw & 29-Dec-.05 & & RLNP & K0158 & CMS & $\begin{array}{l}\text { SVOA } \\
\text { GVOA }\end{array}$ & $606-20-22$ & $\begin{array}{l}\text { Dinitrotoluene } \\
\text { diprowle }\end{array}$ & 10 \\
\hline A5049 & 399-3-6 & $\begin{array}{l}\text { HEISPRODD } \\
\text { HEISPROD }\end{array}$ & $\frac{\text { RC-048 }}{\text { RC-048 }}$ & $\begin{array}{l}\text { J10VVH } \\
\text { J10V7? }\end{array}$ & ow & 29-Dec-05 & & $\begin{array}{l}\text { RLNP } \\
\text { RINP }\end{array}$ & $\begin{array}{l}\text { K0158 } \\
\text { K0158 }\end{array}$ & $\begin{array}{l}\text { MS } \\
\text { CMS }\end{array}$ & $\begin{array}{l}\text { SVOA } \\
\text { SVOA }\end{array}$ & $\begin{array}{l}6211-4-7-1 \\
65774-96-9\end{array}$ & $\begin{array}{c}\text { dipropplamine } \\
\text { Methylphon }\end{array}$ & $\begin{array}{l}10 \\
10\end{array}$ \\
\hline A5049 & $399-3-6$ & HEISPROD & RC-048 & J10VHT & ow & 29--Dec-05 & & $\begin{array}{l}\text { RLN } \\
\text { RLNP }\end{array}$ & Ko158 & CMS & $\begin{array}{l}\text { SUVA } \\
\text { SSOA }\end{array}$ & $667-72-1$ & $\begin{array}{l}\text { Meinyphenol } \\
\text { ne }\end{array}$ & $\begin{array}{l}10 \\
10\end{array}$ \\
\hline A5049 & $399-3-6$ & HEISPROD & RC-048 & J10VHT & GW & 29-Dec-05 & & RLNP & K0158 & CMS & SVOA & 7005-72-3 & Chlorophenylph & $\frac{10}{10}$ \\
\hline 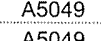 & $\begin{array}{l}399-3-6 \\
3090\end{array}$ & HEISPROD & RC-048 & J10VH7 & $\frac{G W}{6 w}$ & 29-Dec-05 & 1 & RLNP & K0158 & $c$ & PEST/PCB & $72-20-8$ & Endrin & 0.05 \\
\hline 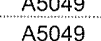 & 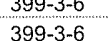 & HEISPROD & $\begin{array}{l}\text { RC-048 } \\
\text { RC }\end{array}$ & $\begin{array}{l}\text { J10VH7 } \\
\text { J10VH7 }\end{array}$ & GW & 29-Dec-.05 & & RLNP & K0158 & $c$ & PESTIPCB & $72-43-5$ & Methoxychlor & 0.05 \\
\hline A5049 & $399-3-6$ & $\begin{array}{l}\text { HEISPROD } \\
\text { HEISPROD }\end{array}$ & $\frac{R C-048}{\text { RC-048 }}$ & $\begin{array}{l}\text { J10VHT } \\
\text { J10VH7 }\end{array}$ & Gw & 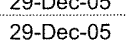 & & $\frac{R L N P}{R I N P}$ & $\begin{array}{l}\text { K0158 } \\
\text { K0158 }\end{array}$ & & $\begin{array}{l}\text { PESTIPCB } \\
\text { PEST/PCB }\end{array}$ & $\begin{array}{l}2.544-8 \\
7.559\end{array}$ & docichloroethane & 0.05 \\
\hline A5049 & $3999-3-6$ & HEISPROD & RC-048 & J10VHT & GW & 29-Dec-05 & 1 & RLNP & K0158 & c & PEST/PCB & $7421-93-4$ & Endrivi aldehter & $\begin{array}{l}0.05 \\
0.05\end{array}$ \\
\hline A55049 & $3999-3-6$ & HEISPROD & RC-048 & J10VHT & ow & 29-Dec-05 & 1 & RLNP & K0158 & ICP & METALMULT & $7429-90-5$ & Aluminum & 19.5 \\
\hline$\frac{15049}{A 5009}$ & 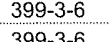 & HEISPROD & RC-048 & J10VH7 & GW & 29-Ded-05 & 1 & RLNP & K0158 & ICP & METALMULT & $7439-89-6$ & Iron & 424 \\
\hline A5049 & $\begin{array}{l}399-3-6 \\
399-3.6\end{array}$ & HEISPROD & RC-048 & 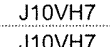 & GW & $\begin{array}{l}\text { 29-Dec-.05 } \\
\text { 20-0.0c-05 }\end{array}$ & 1 & RLNP & K0158 & ICP & METALMULT & $7439-92-1$ & Lead & 3.1 \\
\hline A5049 & $399-3-6$ & $\begin{array}{l}\text { HEISPROD } \\
\text { HEISPROD }\end{array}$ & 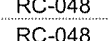 & $\begin{array}{l}\text { S10VHT } \\
\text { J10VH7 }\end{array}$ & GW & $\begin{array}{l}\text { 29-DDec.05 } \\
\text { 29-Dec-005 }\end{array}$ & & RIN & $\begin{array}{l}k_{00158} \\
k_{0158}\end{array}$ & icP & 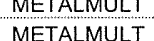 & 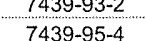 & 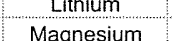 & $\frac{5.2}{10200}$ \\
\hline A5049 & $399 \cdot-3-6$ & HEISPROD & RC-048 & J10VVt7 & GW & 29-Dec-05 & 1 & RLNP & K0158 & ICP & METALMUTT & $74399.96-5$ & Manganesese & $\begin{array}{ll}10200 \\
10.2\end{array}$ \\
\hline A5049 & $3999-3-6$ & HEISPROD & RC-048 & J10VH? & GW & 29-Dec-05 & 1 & RLNP & K0158 & A & METALSING & $74399-97-6$ & Mercury & 0.1 \\
\hline 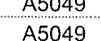 & $\begin{array}{l}399-3-6 \\
3093-6-6\end{array}$ & HEISPROD & RC-048 & J10VH? & GW & 29-Dec-05 & 1 & RLNP & K0158 & ICP & METALMULT & $7439-98-7$ & Molybdenum & 4.5 \\
\hline A5049 & $\begin{array}{l}395-5.6 \\
399-3-6\end{array}$ & HEISPROD & RC-048 & $\begin{array}{l}\text { S10VH7 } \\
\text { J10VH7 }\end{array}$ & GW & $\begin{array}{l}\text { 29-Dec-.05 } \\
\text { 29-Decc.05 }\end{array}$ & 1 & RLNP & K0158 & ICP & METALMULT & $7440-02-0$ & Nickel & 1.3 \\
\hline A5049 & $399 \cdot 3 \cdot 6$ & $\begin{array}{l}\text { HEISPROD } \\
\text { HEISPROD }\end{array}$ & 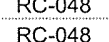 & $\begin{array}{l}\text { J10VAT } \\
\text { J10VH7 }\end{array}$ & GW & 29.0ecosos & & $\begin{array}{l}\text { RLNPP } \\
\text { RINP }\end{array}$ & $\begin{array}{l}\text { K0158 } \\
\text { K0158 }\end{array}$ & ICP & $\begin{array}{l}\text { MEIALMULT } \\
\text { METALMULT }\end{array}$ & $\begin{array}{l}7440-09-1 \\
7440-21-3\end{array}$ & $\begin{array}{l}\text { Potasssum } \\
\text { Silicon }\end{array}$ & $\frac{5220}{16200}$ \\
\hline A5049 & $399-3-6$ & HEISPROD & RC-048 & J10Vht7 & GW & 29-Dec-05 & 1 & RLNP & Ko158 & ICP & METALMULT & $7440-22-4$ & $\begin{array}{l}\text { micon } \\
\text { Silver }\end{array}$ & $\frac{16200}{1.4}$ \\
\hline A5049 & 399-3-6 & HEISPROD & RC-048 & J10VH7 & Gw & 29-Dec-05 & 1 & RLNP & K0158 & ICP & METALMULT & $7440-23-5$ & Sodium & 20900 \\
\hline 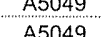 & $\begin{array}{l}399-5-6 \\
399-3-6\end{array}$ & HEISPROD & RC-048 & J10VH? & GW & 29-Dec-05. & 1 & RLNP & K0158 & ICP & METALMULT & $7440-24-6$ & Strontium & 207 \\
\hline A5049 & 399-3-6 & $\begin{array}{r}\text { HEIIPROD } \\
\text { HEISPROD }\end{array}$ & $\begin{array}{l}\text { RC-048 } \\
\text { RC- }\end{array}$ & $\begin{array}{l}\text { J10VH7 } \\
\text { J10VY77 }\end{array}$ & GW & $\begin{array}{l}\text { 29-Dece- } 05 \\
\text { 29-Dece-05 }\end{array}$ & & $\begin{array}{l}\text { RLNP } \\
\text { RuNP }\end{array}$ & K0158 & I IPP & $\begin{array}{l}\text { MEEALMUTT } \\
\end{array}$ & $\begin{array}{l}7440-2-2-0 \\
774-2.5\end{array}$ & Thallium & 6.4 \\
\hline A5049 & 399--3-6 & HEISPROD & $\begin{array}{l}\text { RC-048 } \\
\text { RC-048 }\end{array}$ & $\begin{array}{l}\text { JTOVAT } \\
\text { J10VHT }\end{array}$ & GW & 29-Dec-005 & & $\begin{array}{l}\text { RLN } \\
\text { RLNP }\end{array}$ & K0158 & $\mathrm{ICP}$ & $\begin{array}{l}\text { MEIALALULTT } \\
\text { METALM }\end{array}$ & $\begin{array}{l}74404-31-5 \\
7440-36-0\end{array}$ & Antimony & 5.2 \\
\hline A5049 & 399-3-6 & HEISPROD & RC-048 & J10VVH7 & GW & 29-Dec-.05 & 1 & $\frac{1 L N P}{R L N}$ & K0158 & ICP & METALMULT & $7440-38-2$ & Arsenic & $\begin{array}{l}4 \\
7.6\end{array}$ \\
\hline A5049 & $\begin{array}{l}399-3-6 \\
3036\end{array}$ & HEISPROD & RC-048 & J10VH7 & GW & 29-Dec-05 & 1 & RLNP & K0158 & ICP & METALMULT & $7440-39-3$ & Barium & 46.5 \\
\hline$\frac{15049}{A 5049}$ & $\begin{array}{l}399-9-6 \\
399-3-6\end{array}$ & HEISPROD & RC-048 & J10VH7 & GW & 29-Dec-05 & 1 & RLNP & K0158 & ICP & METALMULT & $7440-41-7$ & Beyllium & 0.1 \\
\hline A5049 & 399-3-6 & $\begin{aligned} \text { HEIIPROD } \\
\text { HEISPROD }\end{aligned}$ & 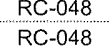 & $\begin{array}{l}\text { JTHVHA } \\
\text { J10VH }\end{array}$ & GW & $\begin{array}{l}\text { 29-Dece-105 } \\
\text { 29-Dec-05 }\end{array}$ & & $\begin{array}{l}\text { RINP } \\
\text { RINP }\end{array}$ & $\begin{array}{l}K_{k 0158} \\
K_{0058}\end{array}$ & IIPP & 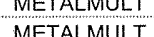 & $\begin{array}{l}7440-2-2-8 \\
740-3\end{array}$ & $\begin{array}{l}\text { Boron } \\
\text { Sorpim }\end{array}$ & 28.9 \\
\hline A5049 & $399-3-6$ & HEISPROD & RC-048 & J10VVH7 & GW & 29-Dec-05 & & RLNP & 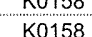 & WFP & MITETANULIT & 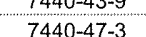 & Chromium & 0.7 \\
\hline & & HEISPROD & RC-048 & J10VH77 & GW & 29-Dec-05 & 1 & RLNP & K0158 & ICP & METALMULT & $7440-48-4$ & Cobalt & $\begin{array}{l}3 \\
1.2\end{array}$ \\
\hline A5049 & $399-3-6$ & HEISPROD & RC-048 & J10VH7 & GW & 29-Dec-05. & & RLNP & K0158 & ICP & METALMULT & $7440-50-8$ & Copper & 1.2 \\
\hline $\begin{array}{l}A 5049 \\
5049\end{array}$ & $399-3-6$ & HEISPROD & RC-048 & J10VH7 & GW & 29-Dec-05 & & RLNP & K0158 & ICP & METALMULT & $7440-61-1$ & Uranium & 20.6 \\
\hline 年 & $\begin{array}{l}399-3-6 \\
303-6-6\end{array}$ & HESPRODD & $\frac{R C-048}{B C-048}$ & J10VH? & GW & 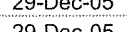 & & RLNP & K0158 & & $\begin{array}{l}\text { MEETALUULT } \\
\end{array}$ & $7440-62-2$ & Vanadium & $\begin{array}{l}8.4 \\
8.7\end{array}$ \\
\hline A50049 & 年 & FESTERO & RLC.048 & 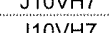 & sw & 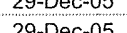 & & Ktw & nutso & W & TIVTALUVLI & $7440-00-0$ & Linc & $5 \pi, 4$ \\
\hline A & 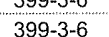 & HEISPROD & RC-048 & J10vent & $G w$ & 29-Dec-05 & 1 & RLNP & 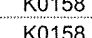 & ICP & $\begin{array}{l}\text { MEIALALULLLT } \\
\text { METAMULT }\end{array}$ & $74040.70-3$ & Gissun & $\begin{array}{l}6.1 \\
490000\end{array}$ \\
\hline A5049 & $3999-3 \cdot 6$ & HEISPROD & RC-048 & J10VH7 & GW & 29-Dec-05 & 1 & RLNP & K0158 & $c$ & PESTIPCB & $76-44-8$ & Heptachlor & $\frac{4500}{0.05}$ \\
\hline A5049 & 399-3-6. & HEISPROD & RC-048 & J10VH7 & GW & 29-Dec-05 & & RLNP & K0158 & CMS & SVOA & $77-47-4$ & pentadiene & 10 \\
\hline A5049 & $399-3-6$ & HEISPROD & RC-048 & JiOVH? & GW & 29-Dec-05 & & RLNP & K0158 & ICP & METALMULT & $7723-14-0$ & Phosphorus & 42.8 \\
\hline A5049 & 399-3-6.6 & HESPROD & RC-048 & J10VHT & GW & 29-Dec-05 & & RLNP & Ko158 & ICP & METALMULT & $7782-49-2$ & Selenium & 3.6 \\
\hline $\begin{array}{l}\text { AA5099 } \\
\text { A }\end{array}$ & $\begin{array}{l}399-3 \cdot-6 \\
\text { 3ag-6. }\end{array}$ & HESPROD & $\begin{array}{l}\text { RC-048 } \\
\text { BC. }\end{array}$ & J110VH? & $G W$ & $\begin{array}{l}29-\mathrm{Dec}-05 \\
\text { 200055 }\end{array}$ & & RLNP & K0158 & CMS & SVOA & $78-59-1$ & Isophorone & 10 \\
\hline A & 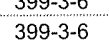 & HESPROD & $\begin{aligned} R C-048 \\
R C\end{aligned}$ & J10VAT & $G w$ & 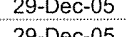 & & RLNP & Kon & C & PESTPCB & $8001-35-2$ & Toxaphene & 0.5 \\
\hline A5049 & 399-3-6 & HESPROD & RC-048 & J150VHT & $G w$ & 29-Dec-05 & & RLNP & K0158 & cms & $\begin{array}{l}S \text { SVOA } \\
\text { SNOA }\end{array}$ & 8 & 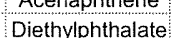 & 10 \\
\hline A5049 & 399-3-6 & HEISPROD & RC-048 & J10VHH & Gw & 29-Dec-05 & & RLNP & K0158 & cMs & svo & $84-74-2$ & 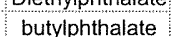 & 0.931 \\
\hline A5049 & 399-3-6 & HEISPROD & RC-048 & J10VH7 & ow & 29-Dec-05 & & RLINP & K0158 & CMS & SVOA & $85-01-8$ & Phenanthrene & 10 \\
\hline A5049 & $399-3-6$ & HEISPROD & RC-048 & J10VH7 & GW & 29-Dec-05 & & RLNP & K0158 & CMS & SVOA & $85-68-7$ & alate & 10 \\
\hline A5049 & 399-3-6.6 & HEISPROD & RC-048 & J10VH7 & GW & 29-Dec-05 & & RLNP & K0158 & CMS & & $86-30-6$ & Nitrosododipheny| & 10 \\
\hline A & $399-3-6$ & HEISPROD & RC-048 & $\begin{array}{l}\text { J10VH7 } \\
\text { H10VNH }\end{array}$ & GW & 29-Dec-05 & & $\frac{R L N P}{P R P P}$ & 然 & CMS & SVOA & 86-73-7. & Fluorene & 10 \\
\hline A5049 & 399-3-6 & $\begin{aligned} \text { HEISPROD } \\
\text { HEISPROD }\end{aligned}$ & RC-048 & $\begin{array}{l}\text { Jivert } \\
\text { J10VH }\end{array}$ & GW & $\begin{array}{l}\text { 29-Dede-05 } \\
\text { 29-Dec-05 }\end{array}$ & & $\begin{array}{l}\text { RLNP } \\
\text { RLNP }\end{array}$ & Ko158 & ciss & SVOA & $\begin{array}{l}86-74-8 \\
87-6-3-3\end{array}$ & Carbazole & 10 \\
\hline A5049 & 399-3-6 & HEISPROD & RC-048 & J10VH7 & GW & 29-Dec-05 & & RLNP & Ko158 & cMS & $\begin{array}{l}\text { SUAA } \\
\text { SVOA }\end{array}$ & $\begin{array}{l}87-60-5 \\
87-86-5\end{array}$ & not & 25 \\
\hline A5049 & $399-3-6$ & HEISPROD & RC-048 & J10VH7 & GW & 29-Dec-05 & & RLNP & K0158 & cMS & SWAA & $\begin{array}{l}88-06-2 \\
88-06\end{array}$ & Trichloroophenol & 10 \\
\hline$A 5049$ & $399 \cdot-3-6$ & HEISPROD & RC-048 & J10VH7 & GW & 29-De & & RLNP & K0158 & CMS & SVOA & $88-74-4$ & 2-Nitroaniline & 25 \\
\hline A5049 & $399 \cdot-3-6$ & HEISPROD & RC-048 & J10VH7 & GW & 29-Dec-05 & & RLNP & K0158 & CMS & SVOA & $88-75-5$ & 2-Nitrophenol & 10 \\
\hline A5049 & $399-3-6$ & HEISPROD & RC-048 & N10VH7 & ow & 29-De & & RLNP & K0158 & CMS & SVOA & & Naphthalene & 10 \\
\hline A5049 & 399-3-6.6 & HEISPROD & & & GW & & & & & CMS & & & & 10 \\
\hline A5049 & 399-3-6 & HEISRODD & RC- -048 & J10VH7 & GW & 29-Dec-05 & & RLNP & Ko158 & CMS & SVOA & 91-58-7 & Chloronaphthal & 10 \\
\hline A5549 & $\begin{array}{l}399-3-6 \\
303-6\end{array}$ & HEISPROD & RC- -048 & J10VHT & GW & 29-De & & RLNP & Ko158 & CMS & SVOA & 91-94-1 & Dichlororobenziditi & 10 \\
\hline A & 年 & (5) & (2)-048 & 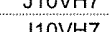 & swo & 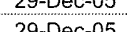 & & KLNT & & chs & SWOA & 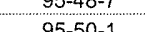 & coresol, of & 10 \\
\hline A5049 & 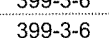 & HESPROD & RC-048 & Jiovit & Gw & 29-Dec-05 & & RLNP & K0158 & cMs & & & D. & 10 \\
\hline A5049 & 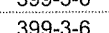 & HESTROD & BC-048 & 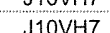 & $G W$ & 20-Decos.05 & & BINP & K0158 & cMs & SWOA & 950-95-4 & Trichlorophen & 05 \\
\hline & & HESPROD & RC-048 & J10ynt7 & GW & (20)ec05 & & PINP & Ko158 & 5 & SEAA & 050.098 & 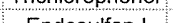 & 25 \\
\hline A5049 & $399 \cdot-6$ & HEISPROD & RC-048 & J10VH7 & GW & 29-Dec-05 & & & K0158 & CMS & SWOA & $98-95-3$ & Nitrobenzene & 10 \\
\hline A5049 & & HEISPROD & RC-048 & J10VH7 & GW & 29-Dec-05 & & RLNP & K0158 & & SVOA & $99-09-2$ & 3-Nitroaniline & 25 \\
\hline & & & & J10VH7 & & 29-Dec-05 & & & & & PHYSICAL & CONDUCT & Conductance & 475 \\
\hline
\end{tabular}

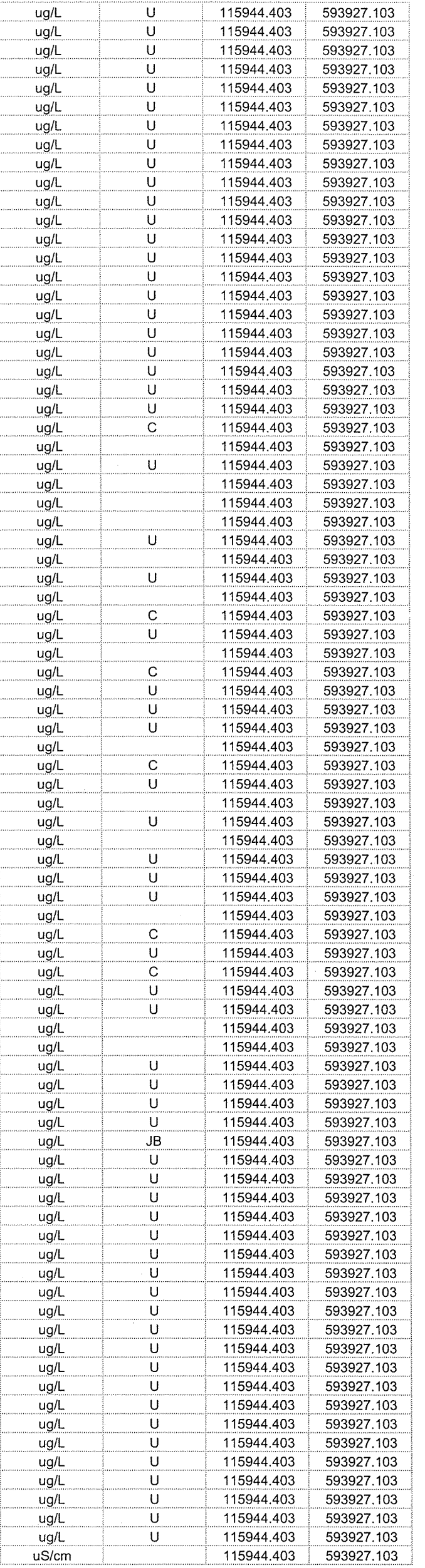




\begin{tabular}{|c|c|c|c|}
\hline $1+2 \geq 2+69$ & 6†E LESGLI & & wo/sn \\
\hline & & $n$ & 7/6n \\
\hline $1+\angle 2+669$ & 6†E LESSLL & $n$ & $7^{76 n}$ \\
\hline 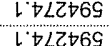 & 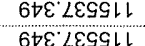 & $n$ & $7 / 7$ n \\
\hline 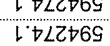 & & $n$ & ר. \\
\hline 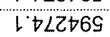 & 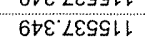 & $n$ & 7 76n \\
\hline $1+\angle 2+69$ & 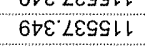 & $n$ & $7 / 6 n$ \\
\hline $1 \div \leqslant 2+699$ & $6 \rightarrow \varepsilon<\varepsilon q S L$ & $n$ & $7 / 6 n$ \\
\hline 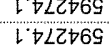 & 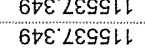 & $\begin{array}{l}n \\
n\end{array}$ & $\frac{776 n}{7 / 6 n}$ \\
\hline $1+22+699$ & $6+\varepsilon<\mathcal{L} \in 9 L$ & n & $76 n$ \\
\hline 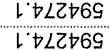 & 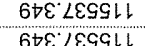 & $n$ & $7 / 6 n$ \\
\hline 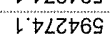 & 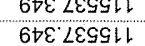 & $n$ & \\
\hline$\dot{1 \cdot D 2+69}$ & $6 চ \varepsilon<\varepsilon S$ SIL & $n$ & $7 / 6 n$ \\
\hline $1+\forall \angle Z 669$ & GDE LESGL & $n$ & $7 / 6 n$ \\
\hline $1+272769$ & G†E LESGLL & $n$ & $7^{6 / 6 n}$ \\
\hline 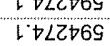 & $6 \nabla \varepsilon<\varepsilon S S L$ & $n$ & $\frac{7 / 70 n}{7 / 6 n}$ \\
\hline $1+\angle 2+695$ & $6+\varepsilon<\varepsilon 99 h$ & n & $7^{6} 6 n$ \\
\hline $1:+2 Z 669$ & $6+\varepsilon \angle E S G L$ & $g r$ & $7 / 7$ n \\
\hline & $6 \neq \varepsilon<\varepsilon g S 1$ & $n$ & \\
\hline 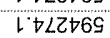 & 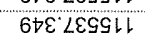 & $n$ & $7 / 6 n$ \\
\hline $1+\forall \angle 2669$ & 6దE LESSLL & n & $\eta / \hat{n}$ \\
\hline $1+227669$ & 6巾E LESSLL & $n$ & $7 / 6 n$ \\
\hline 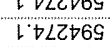 & 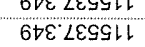 & ก & $7 / 7$. \\
\hline $1+2 \angle 26699$ & 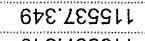 & $n$ & $7 / 6 n$ \\
\hline$\frac{1+2<699}{1+2<2669}$ & 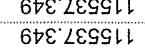 & $\frac{5}{n}$ & 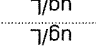 \\
\hline $1+2+2669$ & G+E LESGL & 0 & $7 / 6 n$ \\
\hline 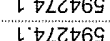 & 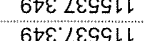 & 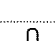 & 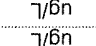 \\
\hline 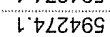 & $6+\varepsilon<\varepsilon q S \mid$ & $n$ & \\
\hline $1+\not 2769$ & $6 \nabla \varepsilon<\varepsilon q S \mathrm{~L}$ & n & $7 / 6 n$ \\
\hline $1+\angle 27669$ & $6+\varepsilon \mathcal{L}<\varepsilon S \subseteq L$ & & $7 / 6 n$ \\
\hline $1+22669$ & $67 \varepsilon$ LESSI & $n$ & $7^{7 / 6 n}$ \\
\hline 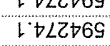 & 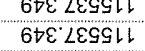 & on & $\frac{7}{7 / 6 n}$ \\
\hline $1+22+69$ & $6+\varepsilon \mathcal{L S S S L L}$ & & $7 / 6 n$ \\
\hline & 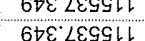 & $\Omega$ & \\
\hline $1 \cdot 12 \angle 699$ & 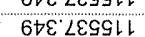 & 0 & 7/6n \\
\hline 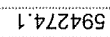 & 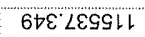 & $n$ & $7 / 6 \mathrm{n}$ \\
\hline $1 \forall \forall Z 1769$ & $6+\varepsilon<E S G L$ & 0 & $7 / 6 n$ \\
\hline 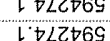 & 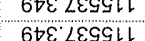 & $\Omega$ & $7 / 60 n$ \\
\hline $1+\angle 27669$ & $6+\varepsilon \angle E S S L$ & 0 & $7^{7 / 6 n}$ \\
\hline$\frac{1+2 \angle 669}{1+2 Z 669}$ & 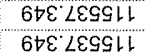 & $n$ & $7 / 60$ \\
\hline $1+2+2+69$ & $6 \oplus \varepsilon<\varepsilon S G L$ & & $7 / 6 n$ \\
\hline $1+2+2669$ & 6దE LESGL & $n$ & $77^{76 n}$ \\
\hline $1+\angle 2 \angle 669$ & 6†E L\&SG & 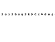 & $7 / 6 n$ \\
\hline $1+2727699$ & 6EE LESSLL & & $77^{6 / n}$ \\
\hline$\frac{120<09}{1-t<2769}$ & 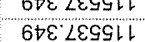 & $\pi$ & \\
\hline 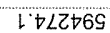 & $6 \downarrow \varepsilon \angle \mathcal{L S G L}$ & 0 & $76 n$ \\
\hline 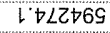 & $6 \varnothing \mathcal{L} \angle \mathcal{G S G l}$ & $n$ & $7^{6 \text { 6n }}$ \\
\hline $1+\angle Z Z 669$ & 6+E LESSLL & $n$ & $7 / 6 n$ \\
\hline 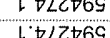 & OEE LEST & $n$ & (7) \\
\hline $1+2 \angle+69$ & 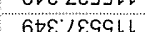 & 10 & \\
\hline $1 \cdot \square 2769$ & $6 \oplus \varepsilon<\varepsilon S G \mid b$ & n & $7,6 \mathrm{n}$ \\
\hline $1+\forall 2+669$ & G๑E LESGHL & $n$ & $7 / 6 n$ \\
\hline $1+212769$ & $6+\mathcal{L}$ LESSL & $n$ & $76 n$ \\
\hline 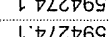 & GEE LESSH & $n$ & 760 \\
\hline $1+2+2+69$ & 6 & 10 & \\
\hline 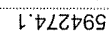 & 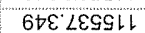 & $n$ & $76 \mathrm{n}$ \\
\hline 1†LZZ6G & 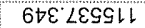 & $n$ & $76 n$ \\
\hline $1 \div \angle Z 769$ & $6 \oplus \varepsilon \mathcal{L E G S L L}$ & n & $7 / 6 n$ \\
\hline 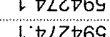 & OEE LEST & $n$ & $77^{160}$ \\
\hline $1+2<+6 s$ & 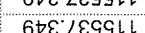 & r & \\
\hline 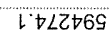 & $6 \oplus \varepsilon \mathcal{L} \varepsilon S \sqcup$ & $f^{2}$ & 7 76n \\
\hline 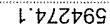 & $6+\varepsilon \dot{L \varepsilon S S L}$ & $n$ & $76 n$ \\
\hline $1+\forall \angle Z 669$ & $6+\varepsilon$ LESSLL & $n$ & $7 / 6 n$ \\
\hline 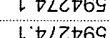 & 6tE LEGSH & $n$ & $\begin{array}{l}7 / 6 n \\
76 n\end{array}$ \\
\hline
\end{tabular}

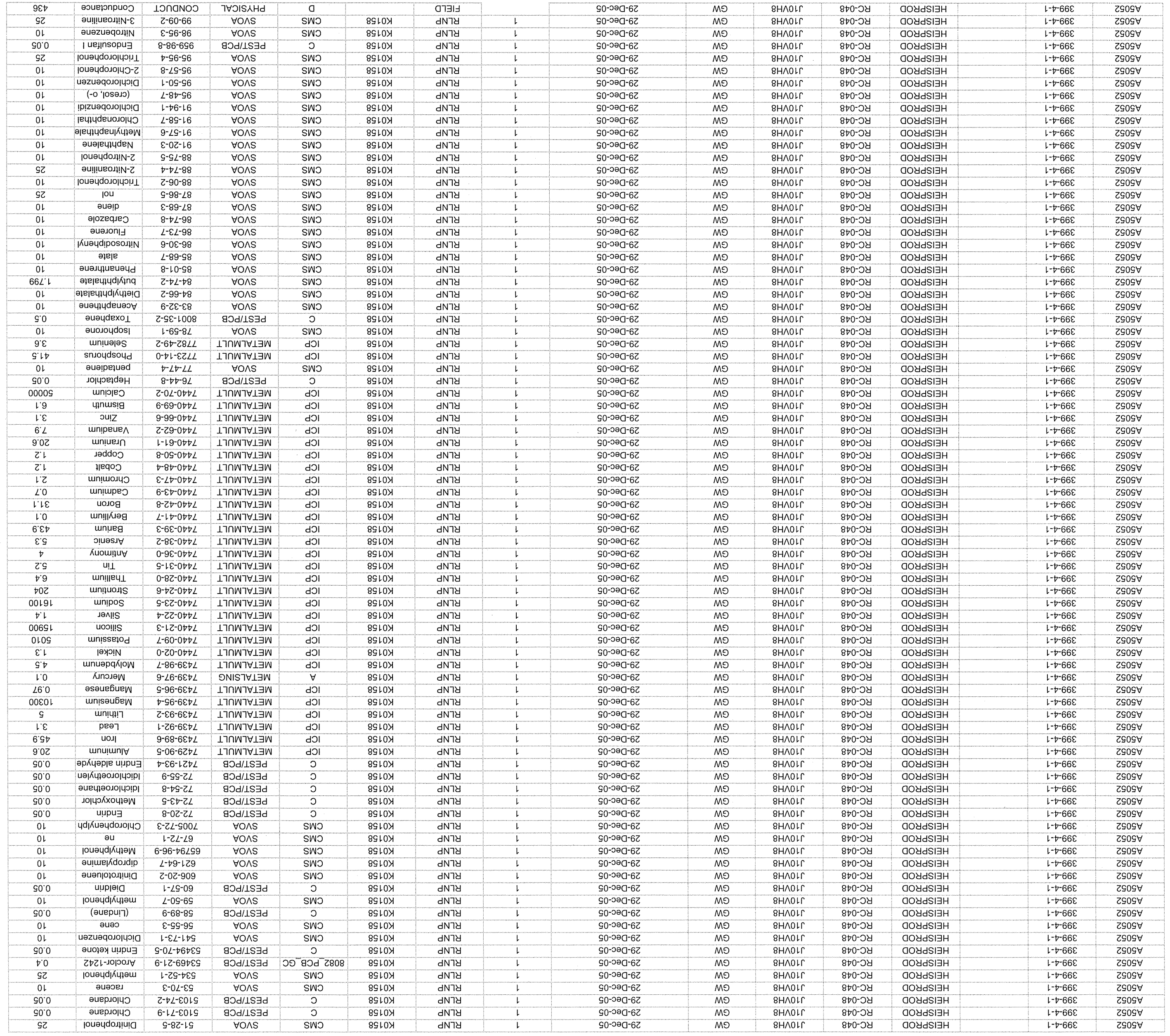




\begin{tabular}{|c|c|c|c|c|c|c|c|c|c|c|c|c|c|c|c|c|c|c|c|c|c|}
\hline $\begin{array}{l}{ }_{A}^{A 5052} \\
A 055252\end{array}$ & $399-4-1$ & $\begin{array}{r}\text { HEISPROD } \\
\text { HESTPOOD }\end{array}$ & RC-048 & J1OVH8 & GW & 29-Dec-05 & & $\begin{array}{l}\text { FIELD } \\
\end{array}$ & & $\begin{array}{l}F L D \\
E F D\end{array}$ & $\begin{array}{l}\text { GENCHEM } \\
\text { GENHEEM }\end{array}$ & DO & $\begin{array}{l}\text { oxygen } \\
\text { Redcuction }\end{array}$ & 6.48 & & & & $\mathrm{mg} / \mathrm{h}$ & & $\begin{array}{l}115537.349 \\
155737.34\end{array}$ & 594274.14 \\
\hline $\begin{array}{l}\text { A A505252 } \\
\text { A5052 }\end{array}$ & $\begin{array}{l}3999-4-1 \\
399-4-1\end{array}$ & $\begin{array}{l}\text { HESISROD } \\
\text { HEISPROD }\end{array}$ & $\begin{array}{l}\text { RC-048 } \\
\text { RC-048 }\end{array}$ & $\begin{array}{l}\text { J10VHB } \\
\text { J10VHB }\end{array}$ & GW & $\begin{array}{l}\text { 29-Dece-05 } \\
\text { 29-Dec-05 }\end{array}$ & 10 & $\begin{array}{l}\text { FELD } \\
\text { RLNP }\end{array}$ & K0158 & $\frac{1}{2}$ & $\begin{array}{l}\text { GENCHEM } \\
\text { GENCHEM }\end{array}$ & $\begin{array}{l}\mathrm{EH} \\
\mathrm{NO2}+\mathrm{NO}-\mathrm{N}\end{array}$ & $\begin{array}{l}\text { Reduction } \\
\text { Nitrite and }\end{array}$ & $\begin{array}{l}127 \\
5.9\end{array}$ & & & & $\frac{\mathrm{mV}}{\mathrm{mglh}}$ & D & $\begin{array}{l}1155377.399 \\
115537.349\end{array}$ & 594274.1 \\
\hline A5052 & 399-4-1 & HEISPROD & RC-048 & J10VH8 & GW & 29-Dec-05 & & FIELD & & & PHYSICAL & PH & Measurement & & & & & $\mathrm{pH}$ & & 115537.349 & \\
\hline A5052 & $\begin{array}{l}399-4-1 \\
309-4-1\end{array}$ & HE & & & GW & & 1 & $\begin{array}{l}\text { EBRLNE } \\
\text { FEDD }\end{array}$ & K0158 & 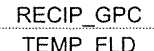 & $\begin{array}{l}\text { RAD } \\
\text { PHSICAI }\end{array}$ & $\begin{array}{l}\text { SR-RAD } \\
\text { E }\end{array}$ & $\begin{aligned} \text { radiostrontium } \\
\text { Temperatu }\end{aligned}$ & 0.168 & 0.25 & 0.45 & 0.25 & $\begin{array}{l}\text { PCil } \\
\text { Deac }\end{array}$ & u & $\begin{array}{r}115537.349 \\
15557\end{array}$ & 594274.1 \\
\hline $\begin{array}{l}\text { A5052 } \\
\text { A5052 }\end{array}$ & $\begin{array}{l}3999-4-1 \\
399-4-1\end{array}$ & $\begin{array}{l}\text { HEISPROD } \\
\text { HEISPROD }\end{array}$ & $\begin{array}{l}\text { RC- } \\
\text { RC-048 }\end{array}$ & $\begin{array}{l}\text { J10VHA } \\
\text { J10VHB }\end{array}$ & $\begin{array}{l}\text { GW } \\
\text { GW }\end{array}$ & $\begin{array}{l}\text { 29-Dece-05 } \\
\text { 29-Dec-05 }\end{array}$ & & $\begin{array}{l}\text { HEDD } \\
\text { EBRLNEE }\end{array}$ & K0158 & GAMMA GS & $\begin{array}{l}\text { PHSICAL } \\
\text { RAD }\end{array}$ & $\begin{array}{c}E \\
\text { TH-232 }\end{array}$ & $\begin{array}{l}\text { Temperature } \\
\text { Thorium-232 }\end{array}$ & & & $48 \quad$ & & $\begin{array}{l}\text { Lege } \\
\text { ocint }\end{array}$ & & 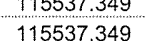 & $\begin{array}{l}59422474.1 \\
594274.1\end{array}$ \\
\hline & 399-4-1-1 & HEISPROD & & J10VhH & ow & 29-Dec-05 & 1 & EBRLNE & Ko158 & TE_AEA & RAD & TH-232 & Thorium-232 & 0 & 0.052 & 0.2 & 0.052 & pcill & u & 115537.349 & 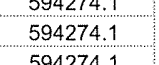 \\
\hline A5052 & $\begin{array}{l}399-4-1 \\
200.1-1\end{array}$ & HEISPROD & 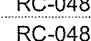 & 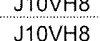 & $G W$ & 29-Dec-05 & 1 & 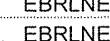 & K0158 & $\begin{array}{l}\text { AEA } \\
\text { GAMMA GS }\end{array}$ & $\begin{array}{l}\text { RAD } \\
\text { RAD }\end{array}$ & U-233/234 & 233/234 & 5.97 & 0.78 & $\begin{array}{l}0.15 \\
1000\end{array}$ & 0.84 & pcin & $u$ & $\begin{array}{l}115537.349 \\
11533749\end{array}$ & 5992727.1 \\
\hline $\begin{array}{l}\text { A }{ }^{A 5052} \\
\text { A5052 }\end{array}$ & $\begin{array}{l}399-4-1 \\
399-4-1\end{array}$ & $\begin{array}{l}\text { HESISROD } \\
\text { HEISRROD }\end{array}$ & RC-048 & J10VVH8 & sw & 29-Dec-05 & 1 & EBRLNE & Ko158 & AEA & $\begin{array}{l}\text { RAD } \\
\text { RAD }\end{array}$ & $\begin{array}{l}0.258 \\
\text { U.238 }\end{array}$ & 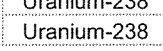 & 5.66 & 0.77 & (1400 & 0.82 & Cili & & 115537.349 & 594274.1 \\
\hline
\end{tabular}




\section{APPENDIX G}

\section{DATA SUMMARY OF BIOASSAY RESULTS}


WCH-85

Rev. 0 


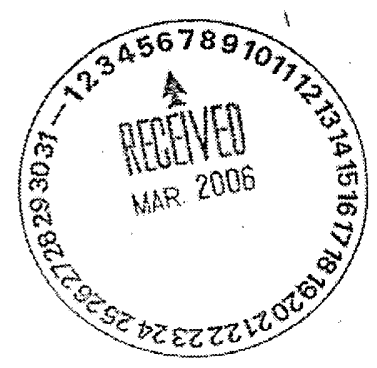

BIOASSAY REPORT ACUTE SCREENING BIOASSAYS

Conducted February 2 through 3

and February 13 though 14,2006

\author{
Prepared for \\ BLR CONSULTING, INC.
}

WASHESTON CLOSURE HANFORD

\author{
Preparea by \\ CH2M HILL \\ $2300 \mathrm{NW}$ Walnut Boulevard \\ Corvallis, Oregon 97330
}

March 3, 2006

Lab I.D. Nos. BN1542.01 thru 09

SDG Number BN1542

$-1-$ 
WCH-85

Rev. 0

\section{CONTENTS}

Section

Page

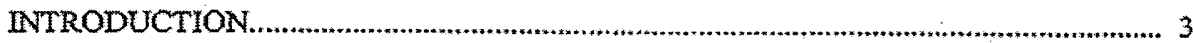

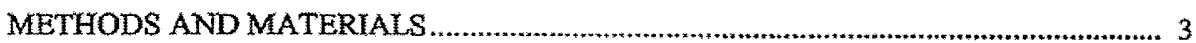

TEST METHODS $\ldots$

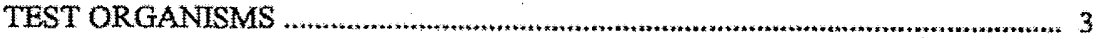

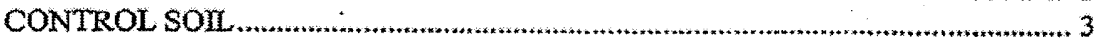

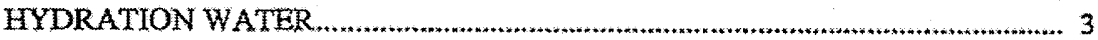

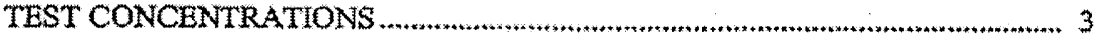

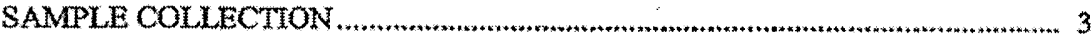

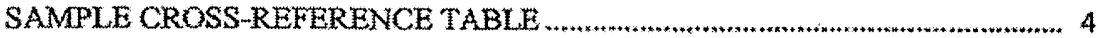

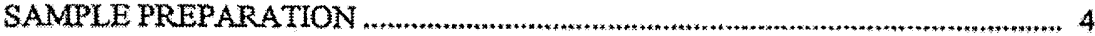

TEST INITLATION

TEST TERMINATION

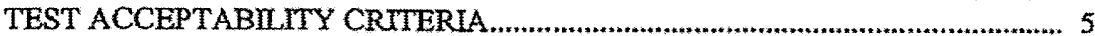

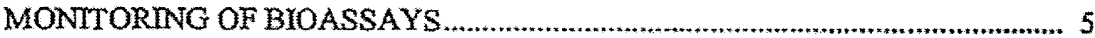

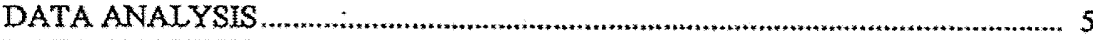

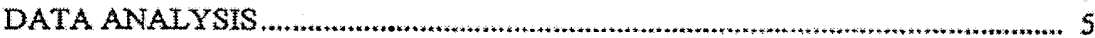

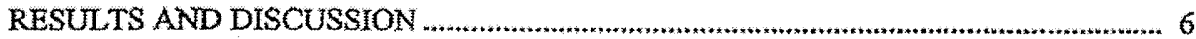

ACUTE RESUUL

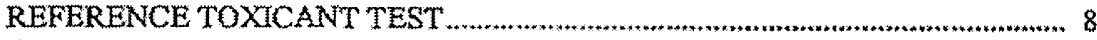

CERTIFICATION STATEMENT

APPENDIX A. RAW DATA SHEETS

APPENDIX B. REFERENCE TOXICANT RAW DATA SHEETS

APPENDIX C, CHAIN OF CUSTODY 


\section{INTRODUCTION}

CH2M HIII conducted acute screening bioassay tests using the nematode (Caenorhabditis elegans) on scil samples provided by the BLR Consulting for the Wastington Closure Fuanford project. The tests were conducted in two rounds from February 2 through 3,2006 , and February 13 through 14,2006 .

\section{METHODS AND MATERIALS}

\section{TEST METAODS}

The chronic test methods were performed according to: Standard Gutde for Conducting Laboratory Soil Toxictiy Tests with the Nematote Caenorhabditis elegans, ASTM E 2172-01 (2001).

\section{TEST ORGANISMS}

The nematodes used were obtained from CH2M MILL's in-house cultures and were age synchronized as 4 day old organisms at test initiation. All organisms tested were fed and maintained during culturing, acclimation, and testing as prescribed by the ASTM protocol. The test organisms appeared vigotous and in good condition prior to testing.

\section{CONTROL SOLL}

The control soil used in the tests was 70 grade sittea sand.

\section{HYDRATION WATER}

The water used to hydrate the control and test solls was Milli-Q equivalent de-ionized water.

\section{TEST CONCENTRATIONS}

The concentrations tested in the nematode test were 100 percent test sample with control soil alone for the control. For the nematode test, 30 organisms per concentration were used with three test chambers per concentration and 10 organistns per chamber.

\section{SAMPLE COLLECTION}

The soil samples were collected from Octobar 31, 2005, through December 6, 2005. The samples were stored in the dark at $4^{\circ} \mathrm{C}$ until test solutions were prepared and tested. Chain of Custody for sample collection is provided in Appentix $\mathrm{C}$. 
WCH-85

Rev. 0

\section{SAMRLE CROSS-REFERENCE TARLE}

Table 1 provides a cross-reference of the Client $\mathrm{D}$ numbers, sampling dates, sampling locations, Nernatode test sample identification (SDG) numbers, and Analytical Lab SDG numbers.

\begin{tabular}{|c|c|c|c|c|}
\hline \multicolumn{5}{|c|}{$\begin{array}{c}\text { Table } 1 \\
\text { Sample Cross-Reference }\end{array}$} \\
\hline Client ID & $\begin{array}{c}\text { Sample } \\
\text { Date }\end{array}$ & $\begin{array}{c}\text { Sample } \\
\text { Location } \\
\end{array}$ & $\begin{array}{l}\text { Nematode } \\
\text { test SDG }\end{array}$ & $\begin{array}{l}\text { Analytical } \\
\text { Lab SDG }\end{array}$ \\
\hline J10DW4 & $10 / 31 / 2005$ & $600-131$ & BN1542-01 & $\mathrm{E} 2748$ \\
\hline J10DV4 & $11 / 08 / 2005$ & PIT 23 & BN1542-02 & E2801 \\
\hline J10DT8 & $11 / 14 / 2005$ & Epland Backfill Elevated-100-F-2 & BN1542 03 & $\mathrm{E} 2831$ \\
\hline J10DV2 & $11 / 15 / 2005$ & $\begin{array}{l}\text { Upland Native Reference-Central } \\
\text { Platea: }\end{array}$ & BNI $542-04$ & E2846 \\
\hline J10DV1 & $11 / 15 / 2005$ & Upland Backfill Low-116-DR-182 & BNI542-05 & E2847 \\
\hline J10DV3 & $11 / 16 / 2005$ & Upland Native Elevated-JA Jones & BNI542-06 & E2857 \\
\hline J10DV0 & $11 / 21 / 2005$ & $\begin{array}{l}\text { Riparian Elevated-Site } \# 3 \\
\text { Upriver 100-D }\end{array}$ & BN1542-07 & E2877 \\
\hline 310235 & $11 / 28 / 2005$ & $\begin{array}{l}\text { Riparin Low-Site \#10 } \\
\text { Downtiver 100-D }\end{array}$ & BN1542-08 & $\mathrm{E} 2897$ \\
\hline J100T9 & $12 / 06 / 2005$ & $\begin{array}{l}\text { Riparian Reference-Site } 13 \\
\text { Vernita Bridge }\end{array}$ & BN1542-09 & $\mathrm{E} 2953$ \\
\hline
\end{tabular}

\section{SAMPLE PREPARATION}

Test soils and control soll were dried and homogenized prior to use. For each replicate, 2.33 $\mathrm{g}$ dry weight of soil was added to each test chamber. The soils were then hydrated to 40 percent of the dry weight by addition of hytration water $(0.93 \mathrm{ml})$ and test chambers were then covered. In addition, $23.3 \mathrm{~g}$ of soll was added to a surrogate chamber and hydrated to provide for $\mathrm{pH}$ measurements. All test chambers were allowed to equilibrate at test conditions for seven days prior to test inituation. 
WCH-85

Rev. 0

\section{TEST INITIATION}

Tests were initiated by the addition of 10 test organisnas to each test chamber. Organisms were added to test chambers in random oxder.

\section{TEST TERMINATION}

Tests were terminated after 24 hours. The contents of the test chambers were added to a centrifuge tube, $10 \mathrm{ml}$ of Lidox-AM silica solution added, and each tube was hand shaken to suspend the nematodes into the Ludox solution. The tubes were then centrifuged to concentrate the soil and the supernatant transferred to a $15 \mathrm{~cm}$ petri dish and allowed to sit for 15 minutes. The petri dish was then placed under a dissecting microscope and the nematodes were retrieved and inspected. The recovered test organisms were recorded as alive (responded with independent novement to tactile stimulation) or dead. Missing or unrecovered test organisms are scored as dead during data analysis.

\section{TEST ACCEPTABILTTY CRITERIA}

The test must meet the following two test acceptabitity criteria to be considered valid:

- A minimum of 80 percent of test organisms must be recovered, both in the control and each test concentration tested.

- The controls must achieve a minimum 90 percent survival.

\section{MONITORING OF BIOASSAYS}

The soil pH was measured from surrogate test chambers at test initiation. Temperature was monitored in the test incubator at test initiation and termination.

\section{DATA ANALYSIS}

The endpoints measured during the nematode test included survival over the 24 hour exposure period. The statistical analyses performed were those outlined in Standard Guide for Conducting Laboratory Soil Toxicity Tests with the Nematode Caenorhabditis elegans, ASTM E 2172-01, using CETS version 1.1.2. Equal Variance t Two-Sample Test was used to compare the survival data between the control and each test soil. When the assumptions of normality or homogeneity of yariance necessary for Equal variance 1 Two-Sample Test could not be met, Unequal Variance t Two-Sample Test was used to analyze the data. 
WCH-85

Rev. 0

\section{RESULTS AND DISCUSSION}

\section{ACUTE RESULTS}

Table 2 summarizes the survival data for the nematode acute test initiated on February 2 , 2006.

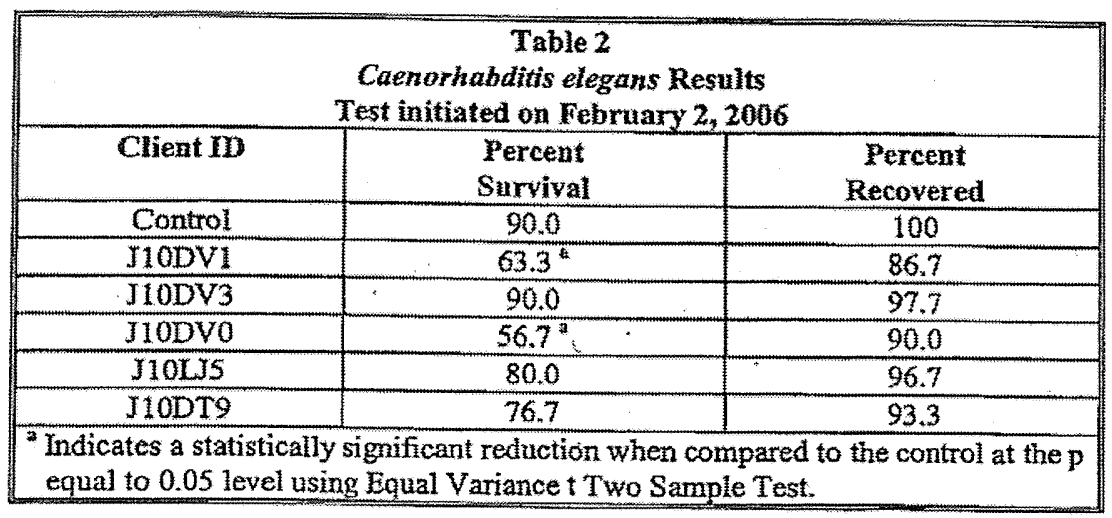

The nematode results indicated no statistically significant reduction in strvival in the J10DV3, J10L J5, and J10DT9 samples and a statistically significant reduction in survival in the J1ODV 1 and JIODVO samples when compared to the control.

Test acceptability criteria was met with control survival of 90.0 percent and recovery of test organisms was greater than 80 percent in all test concentrations. 
Table 3 summarizes the survival data for the rematode acute test initiated on February 13, 2006.

\begin{tabular}{|c|c|c|}
\hline \multicolumn{3}{|c|}{$\begin{array}{c}\text { Caenorhabaitis elegans Results } \\
\text { Test initiated on february 13,2006 }\end{array}$} \\
\hline Client ID & $\begin{array}{c}\text { Percent } \\
\text { Survival }\end{array}$ & $\begin{array}{c}\text { Percent } \\
\text { Recovered }\end{array}$ \\
\hline Control & 90.0 & 96.7 \\
\hline J10DW4 & 90.0 & 93.3 \\
\hline J10DV4 & 83.3 & 86.7 \\
\hline J10DT8 & $73.3^{3}$ & 83.3 \\
\hline J10DV2 & 83.3 & 93.3 \\
\hline $\begin{array}{l}\text { Indicates a statistically significant reduction whon compared to the control at the p } \\
\text { equal to O.0S level using Equal Variance tTwo Sample Test. }\end{array}$ \\
\hline
\end{tabular}

The nematode results indicated no statistically significant reduction in survival in the J10DW4, J10DV4, and $310 \mathrm{DV} 2$ samples and a statistically significant reiuction in survival in the J10DT'8 sample when compared to the control.

Test acceptability criteria was met with control suryival of 90,0 percent and vecovery of test organisms was greater than 80 percent in all test concentrations.

Test temperatures temained at $20 \mathrm{x} 1^{\circ} \mathrm{C}$. The tests proceeded without interruption or incidents that could have affected vest results. 


\section{REIEERENCE TOXICANT TEST}

The results of the reference toxicant test conducted in January with oupric chloride indicate that the test organisms were within their respective sensitivity range based on EPA guidelines (EPA 1994). The LC $\mathrm{Sol}_{50}$ value and control chart limits are listed in the table below.

\begin{tabular}{|c|c|c|}
\hline \multicolumn{3}{|c|}{$\begin{array}{c}\text { Table } 4 \\
\text { Chronie Reference Toxicant Tests (ug/L) } \\
\end{array}$} \\
\hline Species (test) & $\mathrm{LC}_{\mathrm{xg}}$ & Control Chart Limits \\
\hline Caenorhabditis elegans (survival) & 98.2 & 52.3 to 120.1 \\
\hline
\end{tabular}

\section{CERTINCATION STATEMENT}

I certify that this data package is in compliance with the Statement of Work, both technically and for completeness, for other than the conditions detailed above. Release of the data contained in this hard copy data package has been authorized by the Laboratory Manager or a designee, as verified by the following signature:

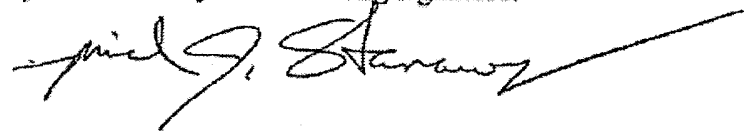




\title{
DISTRIBUTION
}

U.S. Department of Energy

Richland Operations Office

J. P. Sands

A3-04

D. C. Ward

A2-17

Washington Closure Hanford

C. S. Cearlock H0-23

K. R. Fecht H9-01

E. T. Feist H0-23

K. A. Gano H9-01

L. C. Hulstrom H0-23

T. E. Marceau HO-23

S. K. Muns HO-23

J. M. Queen H0-23

D. D. Teel H9-01

J. E. Thomson HO-23

S. G. Weiss (10) HO-23

Fluor Hanford, Inc.

R. G. Bauer E6-35

V. G. Johnson E6-35

Pacific Northwest National Laboratory

R. L. Dirkes K6-75

J. L. Downs K6-85

T. M. Poston K6-75

\author{
Mike Priddy \\ Washington Department of Health \\ Office of Radiation Protection \\ 309 Bradley Blvd \\ Suite 201 \\ MSIN B1-42 \\ Richland, WA 99352
}


Patrick Moran

U. S. Geological Service 1201 Pacific Avenue Suite 600

Tacoma, WA 98402

Greg Hughes

U. S. Fish and Wildlife Service

3250 Port of Benton Blvd

Richland, WA 99352

Lindsey Hayes

U. S. Fish and Wildlife Service

3250 Port of Benton Blvd

Richland, WA 99352

Brett Tiller

EAS

PO Box 265

Richland, WA 99352

Randy Ryti

Neptune \& Company, Inc.

$150515^{\text {th }}$ Street

Suite B

Los Alamos, NM 87544

Jim Markwiese

Neptune \& Company, Inc. $150515^{\text {th }}$ Street

Suite B

Los Alamos, NM 87544

Jennifer Linville

Neptune \& Company, Inc.

$150515^{\text {th }}$ Street

Suite B

Los Alamos, NM 87544

Natural Resource Trustees

Mary C. Baker

Department of Commerce

National Oceanic and Atmospheric Administration

7600 Sand Point Way NE

Seattle, WA 98115 
Craig O'Connor

Department of Commerce

National Oceanic and Atmospheric Administration

7600 Sand Point Way NE

Seattle, WA 98115

Larry Goldstein

Washington State Department of Ecology

300 Desmond Drive SE

Lacey, WA 98503

Stuart Harris

Confederated Tribes of the Umatilla Indian Reservation

750 Swift Blvd

Suite 12

Richland, WA 99352

Susan Hughs

Oregon Department of Energy

625 Marion Street NE

Salem, OR 97301-3737

Paul Shaffer

Oregon Department of Energy

625 Marion Street NE

Salem, OR 97301-3737

Tom Stoops

Oregon Department of Energy

625 Marion Street NE

Salem, OR 97301-3737

Jake Jakabosky

U. S. Bureau of Land Management

1103 North Fancher

Spokane, WA 99212

Dan Landeen

Nez Perce

PO Box 365

Lapwai, ID 83540

Jay McConnaughey

Yakama Nation

PO Box 6066

Kennewick, WA 99336-0066 
Douglas Robison

Washington Department of Fish and Wildlife 8702 N. Division Street

Spokane, WA 99203

Lauri Vigue

Washington Department of Fish and Wildlife 600 Capitol Way North

Olympia, WA 98501-1091

Don Steffeck

U. S. Fish and Wildlife Service

Portland Eastside Federal Complex

911 NE $11^{\text {th }}$ Avenue

Portland, OR 97232-4181

Records and Document Control

$\mathrm{HO}-30$

DOE-RL Public Reading Room

$\mathrm{H} 2-53$

Hanford Technical Library

P8-55 